\title{
Die Zukunft der Vergangenheit in der Gegenwart
}

\author{
Archive als Leuchtfeuer \\ im Informationszeitalter
}

Herausgegeben von

Elisabeth Schöggl-Ernst, Thomas Stockinger und Jakob Wührer

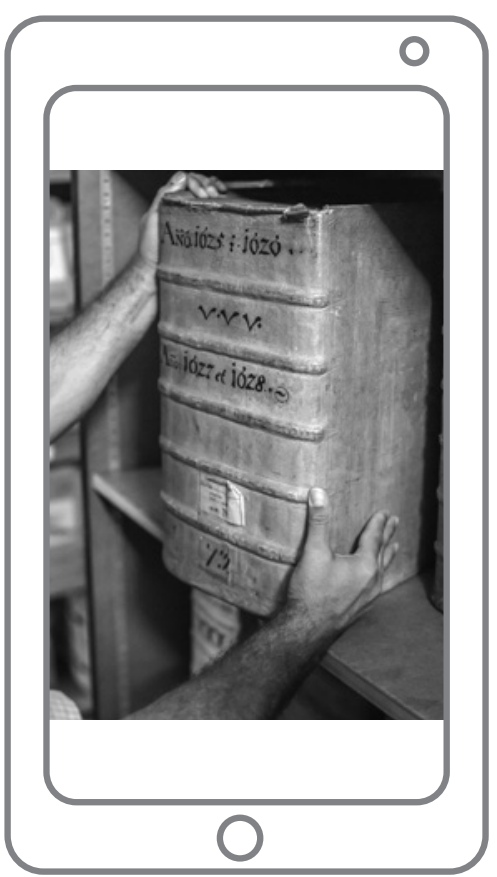


Die Zukunft der Vergangenheit in der Gegenwart 
Veröffentlichungen des Instituts
für Österreichische Geschichtsforschung

Band 71

IOG

2019

Böhlau Verlag Wien 


\title{
Die Zukunft der Vergangenheit in der Gegenwart
}

Archive als Leuchtfeuer im Informationszeitalter

\author{
Herausgegeben von
}

Elisabeth Schöggl-Ernst, Thomas Stockinger, Jakob Wührer 


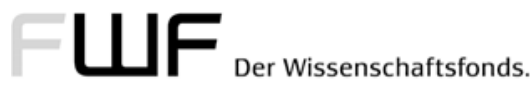 \\ Veröffentlicht mit Unterstützung des Austrian Science Fund (FWF): PUB 591-G28}

Open Access: Wo nicht anders festgehalten, ist diese Publikation lizenziert unter der Creative-Commons-Lizenz Namensnennung 4.0; siehe http://creativecommons.org/licenses/ by/4.0/

Diese Publikation wurde einem anonymen, internationalen Peer-Review-Verfahren unterzogen.

Bibliografische Information der Deutschen Nationalbibliothek:

Die Deutsche Nationalbibliothek verzeichnet diese Publikation in der Deutschen Nationalbibliografie; detaillierte bibliografische Daten sind im Internet über http://dnb.de abrufbar.

(C) 2019 by Böhlau Verlag GmbH \& Co. KG, Kölblgasse 8-10, A-1030 Wien

Umschlagabbildung: Foto: Oberösterreichisches Landesarchiv Umschlaggestaltung: Michael Haderer, Wien

Satz: Bettina Waringer, Wien

Vandenhoeck \& Ruprecht Verlage | www.vandenhoeck-ruprecht-verlage.com 


\section{Inhalt}

Elisabeth SchöggL-ERnst, Thomas StOckinger, Jakob WüHrer

Die Zukunft der Vergangenheit in der Gegenwart

Archive als Leuchtfeuer im Informationszeitalter

Einleitung . . . . . . . . . . . . . . . . . . 9

Luciana Duranti

The Right to Be Remembered and the Duty to Memorialize

The Role of Archives in an Increasingly Networked Society. . . . . . . . . . . . 31

Kategorisierung - Neue Quellenkunde im Archiv

\section{Robert Kretzschmar}

Archivalische Quellenkunde im frühen 21. Jahrhundert

Ein „Kleines Fach" mit potentiell großer Wirkung . . . . . . . . . . . . . . . . 41

Holger BERWINKEL

Probleme einer Aktenkunde der Zeitgeschichte . . . . . . . . . . . . . . . 57

Elizabeth KaTA

Archivalienkunde von unten. Möglichkeiten einer Archivalienkunde für

Unterlagen aus Neuen Sozialen Bewegungen am

Beispiel feministischen Archivguts . . . . . . . . . . . . . . . 71

Heather MacNeIL

Trusting Records in the Digital Age:

Changing Archival Perspectives . . . . . . . . . . . . . . . . . . . . . . 89 


\section{Überlieferungsbildung und Bewertung}

Helga Penz

Nil memorabile, sed tamen asservandum

Merkwürdigkeit und Überlieferungsbildung: Das Beispiel der Archive katholischer Ordensgemeinschaften . . . . . . . . . . . . . . . . . . . . . 101

Matthias BuchHOLZ

Alles doch ganz einfach? Archivische Bewertung zwischen

Wissenschaft und Bauchgefühl . . . . . . . . . . . . . . . . . . . . 113

Michel J. Pfeiffer

Digitale Bilder - Was darf, was kann bleiben? . . . . . . . . . . . . . . . . . 123

\section{Markus SCHMIDGALL}

Überlieferungsbildung und Erschließung im digitalen Bereich.

Ein Arbeitsbericht aus dem Vorarlberger Landesarchiv .

\section{Informationsaufbereitung und Vermittlung}

Andreas Kellerhals

Leuchtturm oder GPS - welche Orientierung in der Infosphäre?. . . . . . . . 155

\section{Brigitte KRENN}

Methoden der künstlichen Intelligenz und ihre Anwendung

in der Erschließung von Textinhalten . . . . . . . . . . . . . . . . . . 16

\section{Christoph Sonnlechner}

Parallelangebot oder Wegweiser in die Zukunft?

Das weltweit umfangreichste Stadtgeschichte-Wiki und seine Interaktion

mit dem Wiener Archivinformationssystem WAIS . . . . . . . . . . . . . . .

Georg Vogeler

Zum Verhältnis von klassischen Formen der

Archiverschließung und den Zugängen der Digital

Humanities zum Information Retrieval . . . . . . . . . . . . . . . . . . . 199

\section{Miroslav Novak}

Archival Information System:

A Bridge Between Past, Present and Future. . . . . . . . . . . . . . . . 21 
Das Archiv in seiner Umwelt

\section{Dietmar Schenk}

Das „neue“ Archivdenken und die geisteswissenschaftlichen

Grundlagen der Archivwissenschaft . . . . . . . . . . . . . . . . . . . 225

Joachim Kemper

Externe Kommunikation und digitale Vermittlung -

heraus aus der archivischen „Staubecke“?. . . . . . . . . . . . . . . . . . 247

\section{Mario Glauert}

Was ist ein erfolgreiches Archiv? . . . . . . . . . . . . . . . . . . . . . . 257

Autorinnen und Autoren. . . . . . . . . . . . . . . . . . . . . . . . . . . . 269

Stephanie Rosenkranz

Personen-, Sach- und Ortsregister . . . . . . . . . . . . . . . . . . . 273 



\title{
Die Zukunft der Vergangenheit in der Gegenwart
}

\author{
Archive als Leuchtfeuer im Informationszeitalter
}

\section{Einleitung}

\section{Elisabeth Schöggl-Ernst, Thomas Stockinger, Jakob Wührer}

Spricht man über Archive oder - schwieriger - über „das Archiv“, bemüht man gerne Metaphern. Schon Definitionen des Archivbegriffs zeigen sich „in hohem Maße anfällig für metaphorische Wendungen“, wie jüngst konstatiert wurde ${ }^{1}$. Tönt es vom Archiv, werden Ausdrücke wie „Erbe“, „Gedächtnis“, „Speicher“ und „Schatz“ nicht selten gleich mit in Schwingung versetzt. Der Begriff des Archivs reiht sich noch dazu in den „Metaphernvorrat der Kulturtheorie" ${ }^{\prime 2}$ ein, wobei die kulturwissenschaftlichen Bilder vom „Archiv“ oft kaum etwas gemein zu haben scheinen mit jenem, das ausgehend von der archivischen Praxis vor den Augen derer steht, die in Archiven arbeiten ${ }^{3}$. Doch auch in den Titeln dezidiert archivwissenschaftlicher Publikationen wird nicht mit Phantasie gegeizt, wenn es um die Reflexion der archivarischen Profession und ihres Tuns sowie um das Wesen ihres Bezugspunktes, des Archivs, geht. Sei es, dass von der „Kontrolle der Vergangenheit“ “4 oder von deren „Verarbeitung“ die Rede ist ${ }^{5}$, sei es, dass die Ausbildung archivischer Grundsätze als Gewinnung von „Neuland“ umschrieben wird ${ }^{6}$, das Archiv scheint zu wenig greifbar, zu wenig gedanklich klar fassbar zu sein, als dass man auf

\footnotetext{
${ }^{1}$ Marcel Lepper-Ulrich Raulff, Vorwort, in: Handbuch Archiv. Geschichte, Aufgaben, Perspektiven, hg. von Marcel Lepper-Ulrich RaulfF-Tanja KunZ-Simone Waidmann-Julia Katharina Waltke (Stuttgart 2016) VII-X, hier VIII.

2 Ebd. VIII; vgl. Martin Stingelin, Archivmetapher, in: Handbuch Archiv (wie Anm. 1) 21-27.

3 Gut sichtbar wird dies etwa bei der Lektüre der Literaturbesprechung von Annika Wellmann, Theorie der Archive - Archive der Macht. Aktuelle Tendenzen der Archivgeschichte. Neue Politische Literatur. Berichte über das internationale Schrifttum 57 (2012) 385-401. Weiters vgl. Alexandrina Buchanan, Strangely Unfamiliar: Ideas of the Archive from Outside the Discipline, in: The Future of Archives and Recordkeeping. A Reader, hg. von Jennie Hill (London 2011) 37-62.

${ }^{4}$ Controlling the Past. Documenting Society and Institutions. Essays in Honor of Helen Willa Samuels, hg. von Terence Gordon Cook (Chicago 2011). Der Titel erinnerte an: Helen Willa Samuels, Who Controls the Past. The American Archivist 49 (1986) 109-124, die sich ihrerseits auf George Orwell bezog. - Natürlich ist die Wahl eines Buchtitels in vielen Fällen vor allem aus Sicht des Produktmarketings zu sehen; die Auswahl der hier genannten Titel erfolgt zufällig und rein zum Zweck der Illustration.

5 Francis X. Blouin jr.-William G. Rosenberg, Processing the Past. Contesting Authority in History and the Archives (Oxford u. a. 2011).

${ }^{6}$ John Ridener, From Polders to Postmodernism. A Concise History of Archival Theory (Duluth 2009) 143 (zur Erklärung des Buchtitels).
} 
diese vielen Assoziationen verzichten könnte - allen voran auch eben jene des Archivs als "Gedächtnis " ${ }^{\text {, }}$, die auch von Archiven selbst für Public-Relations-Zwecke gerne verwendet wird ${ }^{8}$.

Das Institut für Österreichische Geschichtsforschung hat für seine Jahrestagung 2016 - zum ersten Mal im Laufe dieser Veranstaltungsreihe - ein Thema aus der Archivwissenschaft gewählt. Es lag daher nahe, sie in Kooperation mit österreichischen Archiven zu konzipieren und durchzuführen. Organisatorisch und finanziell beteiligten sich die Kulturabteilung der Stadt Wien (Magistratsabteilung 7) und das Wiener Stadt- und Landesarchiv, das Oberösterreichische Landesarchiv, das Steiermärkische Landesarchiv sowie der Verband Österreichischer Archivarinnen und Archivare. Besonderer Dank für ihre Leistungen in der administrativen und logistischen Vorbereitung und Begleitung der Veranstaltung gebührt Johanna Schiele und Stefanie Gruber vom Institut für Österreichische Geschichtsforschung sowie dem Personal des Wiener Stadt- und Landesarchivs, stellvertretend seien Monika Roither und Erwin Pold namentlich genannt.

Als wir gemeinsam mit Heinrich Berg ab dem ausklingenden Jahr 2015 die nachmaligen Vortragenden der Tagung und nunmehrigen Beitragenden dieses Bandes einzuladen begannen, wurden sie von uns nicht mit nur einer metaphorischen Assoziation zum „Archiv“, sondern mit einem metaphorischen Mehrklang konfrontiert: dem Arbeitstitel „Die Zukunft der Vergangenheit in der Gegenwart. Archive als Leuchtfeuer im Informationszeitalter“, bei dem es für die Veranstaltung wie für die vorliegende Publikation letztlich auch geblieben ist. Er war für uns Rahmen und roter Faden zugleich bei der Entwicklung des Tagungsprogramms. Der Haupttitel setzt die drei Zeitebenen Vergangenheit, Gegenwart und Zukunft in Beziehung und soll hinsichtlich des archivischen Tuns auf dessen in die Zukunft blickenden Gegenwartsbezug bei gleichzeitiger Beachtung von aus der Vergangenheit herreichenden Kontinuitätslinien aufmerksam machen”. Mehr noch als der Haupttitel drückt der Untertitel „Archive als Leuchtfeuer im Informationszeitalter" eine Hypothese und einen Anspruch aus: Archive seien

7 Vgl. Margaret Hedstrom, Archives and Collective Memory: More than a Metaphor, Less than an Analogy, in: Currents of Archival Thinking, hg. von Terence M. EAstwood-Heather MacNeil (Santa BarbaraDenver-Oxford 2010) 163-179; Randall C. Jimerson, Archives and Memory, in: Encyclopedia of Archival Science, hg. von Luciana Duranti-Patricia C. Franks (Lanham-Boulder-New York-London 2015) 99-102; Eric Ketelaar, Archives, Memories and Identities, in: Archives and Recordkeeping: Theory into Practice, hg. von Caroline Brown (Facet Books for Archivists and Records Managers, London 2014) 131-170; WeLLMann, Theorie der Archive (wie Anm. 3) 388-390.

8 Peter Csendes diskutiert diese Assoziation in Bezug auf „Gedächtnis“ als kulturwissenschaftlichen Leitbegriff: Peter Csendes, Metaphern für Archive - das Archiv als Metapher? Historisches Jahrbuch der Stadt Linz (2003/04) 49-56. Kritisch zur Gedächtnis-Assoziation Dietmar SchEnK, Kleine Theorie des Archivs (Stuttgart 2008) 22-29.

9 In Anknüpfung an Hermann LüввE, Die Zukunft der Vergangenheit. Kommunikationsnetzverdichtung und das Archivwesen, in: Die Archive am Beginn des 3. Jahrtausends - Archivarbeit zwischen Rationalisierungsdruck und Serviceerwartungen. Referate des 71. Deutschen Archivtages 2000 in Nürnberg, hg. von Jens Murken et al. (Der Archivar. Mitteilungsblatt für deutsches Archivwesen, Beiband 6, Siegburg 2002) 5-23, hier 14. Diese Beziehung zwischen den Zeitebenen reflektiert in kulturwissenschaftlicher Perspektive, aufbauend auf Überlegungen von Jacques Derrida: Georg GäNSER, Archive, Nachvollziehbarkeit und Vertrauen. Scrinium. Zeitschrift des Verbandes Österreichischer Archivarinnen und Archivare 71 (2017) 102-113, hier 107. Den Januskopf bezeichnet Leopold Auer als sinnvolles Symbol für Archive, da sie nach Vergangenheit und Zukunft ausgerichtet sind: Leopold AuER, Zur Rolle der Archive bei der Vernichtung und (Re-)Konstruktion von Vergangenheit, in: Speicher des Gedächtnisses. Bibliotheken, Museen, Archive 1: Absage an und Wiederherstellung von Vergangenheit. Kompensation von Geschichtsverlust, hg. von Moritz CsákY-Peter STACHel (Passagen Orte des Gedächtnisses, Wien 2000) 57-66, hier 64. 
Leuchtfeuer, also Orientierungsmarken, und böten Hilfe im Zeitalter des Informationsüberflusses ${ }^{10}$.

Die schon im Titel mitschwingenden Annahmen wurden in der Folge weiter ausformuliert, nicht zuletzt, um den Vortragenden Impulse zu geben. Wir stellten einige Diagnosen zur gegenwärtigen Situation der Archive in den Raum: Die „Sphäre des Archivischen" und insbesondere die Archivarinnen und Archivare erleben eine Zeit der Herausforderungen und Veränderungen. Archive werden in ihrer Funktion als Informationsspeicher von anderen Gedächtnisinstitutionen, Informationsanbietern beziehungsweise Informationsverwaltern - privaten wie öffentlichen - in Frage gestellt; die nahezu ubiquitäre Verwendung des Archivbegriffs mag dafür symptomatisch sein ${ }^{11}$. Die notwendige und schon ansatzweise vollzogene Hinwendung der Archive zur digitalen Welt verlangt nach neuen Strategien und erweiterten Fähigkeiten und Fertigkeiten des Archivpersonals, ohne dass traditionelle Fähigkeiten und Fertigkeiten obsolet würden, was bei abnehmenden Ressourcen Zielkonflikte eskalieren lässt, zu Priorisierungen und Spezialisierungen sowie vor allem zur Zusammenarbeit mit anderen Disziplinen und Institutionen zwingt.

Von dieser Ausgangslage leiteten wir zunächst weit gefasste Fragestellungen für die Tagung ab. Eine Frage war jene nach der Position der Archive in der Gegenwart, ihrer Bedeutung in den Gesellschaften des Informationszeitalters, wobei von der Existenz eines Spannungsfelds zwischen Sein, Sollen und Wollen ausgegangen wurde. Die Rollen der Archive hängen eng mit den in ihnen konzentrierten Fähigkeiten und Fertigkeiten zusammen; deren Schnittpunkt mit den Aufgaben und Zielen bilden die konkreten Tätigkeiten, denen Menschen - Archivarinnen und Archivare wie auch Benutzende - in Archiven oder in Beziehung zu ihnen nachgehen. Die einzelnen Sektionen der Tagung haben wir demnach entlang des Kanons archivischer Kerntätigkeiten wie Bewerten, Erschließen, Zugänglich-Machen gebildet. Diese galt es in einer Weise zu reflektieren, die sowohl die theoretische Dimension der kulturwissenschaftlichen wie auch der archivwissenschaftlichen Ansätze im Blick hat als auch in der Praxis, der Umsetzung im Archivalltag, verankert bleibt. Bevor wir nun auf diese Sektionen, aus denen die Hauptabschnitte dieses Bandes geworden sind, und auf die jeweiligen Beiträge näher eingehen, wollen wir zu den oben formulierten Grundannahmen noch etwas detaillierter Stellung nehmen.

Zunächst zu unserem eigenen Archivbegriff. Wie sich bereits angedeutet hat, ist für Thema und Zuschnitt der Tagung jenes „klassische“ Verständnis von „Archiv“ zentral, das in der archivwissenschaftlichen Standardliteratur, aber auch etwa in Handbüchern der historischen Hilfswissenschaften und Arbeitstechniken vielerorts nachzulesen ist: „die Gesamtheit der im Geschäftsgang oder im Privatverkehr organisch erwachsenen, zur dauernden Aufbewahrung bestimmten schriftlichen, bildlichen und anderen Überreste einer Behörde, Körperschaft, Familie oder einzelnen Person“12. Der Terminus meint ausdrücklich

${ }^{10}$ Nicht verschwiegen werden soll, dass auch diese Metapher schon ähnlich titelgebend genutzt wurde: Franz-Josef Zrwes, Archive als Leuchttürme. Die Erschließung mit Normdaten als Aufgabe und Chance, in: Archive ohne Grenzen. Erschließung und Zugang im europäischen und internationalen Kontext. 83. Deutscher Archivtag in Saarbrücken, hg. von Monika SтоRм et al. (Tagungsdokumentationen zum Deutschen Archivtag 18, Fulda 2014) 79-87.

11 Dietmar Schenk, „Aufheben, was nicht vergessen werden darf“. Archive vom alten Europa bis zur digitalen Welt (Stuttgart 2013) 49. Die Klage darüber von archivarischer Seite ist freilich nicht neu; sie findet sich beispielsweise schon bei Heinrich Otto MeIsner, Archivalienkunde vom 16. Jahrhundert bis 1918 (Leipzig 1969) $24 f$.

12 Christian Rohr, Historische Hilfswissenschaften. Eine Einführung (UTB 3755, Wien-Köln-Weimar 2015) 212. Ähnlich bei Angelika Menne-Haritz, Schlüsselbegriffe der Archivterminologie. Lehrmaterialien 
sowohl das verwahrte Material als auch die Institution, die für die Verwahrung zuständig ist $^{13}$ - wobei im deutschen archivwissenschaftlichen Sprachgebrauch meist die letztere Verwendung im Vordergrund steht, im Gegensatz etwa zur englischsprachigen Fachterminologie, in der die Bedeutung von „archives“ als überlieferte Bestände oft vorgeht ${ }^{14}$. Die Beitragenden sind überwiegend an Institutionen dieser Art tätig - mehrheitlich, aber keineswegs ausschließlich ${ }^{15}$, an staatlichen Archiven. Dieser Standpunkt gibt zwar einen Blickwinkel und Interessen vor, schließt aber eine Rezeption der Ansätze und Archivbegriffe anderer Disziplinen keineswegs aus, die für Archivarinnen und Archivare in der Gegenwart zumindest bereichernd, mitunter auch unverzichtbar ist, macht sie doch vieles, was an Archiven und ihrem Tun lange als selbstverständlich galt, erst „sichtbar“16.

Die oben postulierten „Herausforderungen“ aktueller Entwicklungen an die Archive könnten leicht als „Mühen“ verstanden werden - beide Ausdrücke gehen oft in einem gemeinsamen Gedankengang miteinander einher. Wir wollen uns besser der britischen Archivarin Jennie Hill anschließen, welche die Einleitung zu ihrem Reader über die Zukunft der Archive von vornherein enthusiastisch beginnt: „There has never been a more interesting time to be an archivist" ${ }^{17}$. Das Interesse der Postmoderne für das Archiv, der technologische Wandel, die Demokratisierung des Archivwesens und die interdisziplinäre Ausweitung der archivischen Sphäre in Richtung Informationswissenschaft und Records Management, so Hill, hätten in den letzten 50 Jahren diese Situation hervorgerufen ${ }^{18}$.

Die Digitalisierung der Gesellschaft und damit auch die Digitalisierung der archivischen Sphäre setzen die Archive inklusive der von ihnen - jetzt und in Zukunft - verwahrten Unterlagen gewissermaßen „unter Strom“. Mit der materiellen Diversifizierung und mit der Fragilität der Archivalien mussten sich Archive seit ihren Anfängen auseinandersetzen ${ }^{19}$, doch aktuell gilt es sich auf einen massiven Medienbruch einzustellen, der dazu zwingt, bewährte archivische Methoden, Konzepte und Strategien anzupassen beziehungsweise neue zu entwickeln ${ }^{20}$. Archivare und Archivarinnen brauchen neue Fähigkeiten, um in der digitalen Archivwelt ihre Arbeit verrichten zu können, doch eine

für das Fach Archivwissenschaft (Veröffentlichungen der Archivschule Marburg, Institut für Archivwissenschaft 20, Marburg 1992) 37. Siehe dazu auch den Beitrag von Dietmar Schenk.

13 Marcel Lepper-Ulrich Raulff, Idee des Archivs, in: Handbuch Archiv (wie Anm. 1) 1-9, hier 1.

${ }^{14}$ Luciana Duranti, Archives (material), in: Encyclopedia of Archival Science (wie Anm. 7) 95-99; Caroline Williams, Records and Archives: Concepts, Roles and Definitions, in: Archives and Recordkeeping (wie Anm. 7) 1-29.

15 Siehe insbesondere die Beiträge von Elizabeth Kata und Helga Penz.

16 Thomas Nesmith, Seeing Archives: Postmodernism and the Changing Intellectual Place of Archives. The American Archivist 65 (2002) 24-41.

17 Jennie Hill, Introduction: Seeing the Future of Archives and Recordkeeping, in: The Future of Archives (wie Anm. 3) XVII-XX, hier XVII.

18 Ähnlich beschreibt die Lage auch Terence M. Eastwood, A Contested Realm: The Nature of Archives and the Orientation of Archival Science, in: Currents of Archival Thinking (wie Anm. 7) 3-21, hier 10: „In the second half of the twentieth and into this century, deep and complex developments in scholarship, administration, information and communication technology, and society in general made a remarkable impact on archival institutions, the role they play in contemporary societies, and the expectations placed on them. These developments also promoted rethinking many traditional archival concepts, methods, and practices."

19 Andrea Pataki-Hundt, Bestandserhaltung, in: Handbuch Archiv (wie Anm. 1) 218-224, hier 219f. Zur Frühzeit vgl. Markus Friedrich, Die Geburt des Archivs. Eine Wissensgeschichte (München 2013) 160-163.

${ }^{20}$ Als gelungene Zusammenfassung zum Thema der digitalen Langzeitarchivierung vgl. Heinz Werner Kramsкi, Digitale Dokumente im Archiv, in: Handbuch Archiv (wie Anm. 1) 178-197. Weiters vgl. Michèle V. Cloonan, Preserving Records of Enduring Value, in: Currents of Archival Thinking (wie Anm. 7) 69-88, hier $83-85$. 
besondere Herausforderung dabei ist, dass deswegen alte Fertigkeiten nicht vergessen werden dürfen, werden doch auch jene Archivalien, deren archivische Bearbeitung dieser Fertigkeiten bedarf, nicht aufgegeben ${ }^{21}$. Es ist gerade eine Eigenart von Archiven: Neues kommt hinzu, ohne das Vorhandene zu verdrängen. Alleine aus diesem Grund wird die Aufgabenvielfalt größer, gleichzeitig auch die Notwendigkeit dringender, Strategien zur Aufgabenbewältigung zu entwickeln: Priorisierung, Spezialisierung und interdisziplinäre Zusammenarbeit erscheinen uns dabei als wichtige Schlagwörter ${ }^{22}$.

Gleichzeitig ist das Bereithalten von großen Informationsmengen archivalischen Charakters kein exklusives Merkmal von Archiven mehr - insofern es das je gewesen ist ${ }^{23}$ : sei es, weil alternative Anbieter aus Archiven stammende Informationen in aufbereiteter Form zur Verfügung stellen ${ }^{24}$, sei es, weil sie archivähnliche Informationsportale aufbauen und gleichzeitig auch noch den Archivbegriff für ihre Tätigkeit „kapern“25. Weder ist „Archiv" ein geschützter Begriff, noch haben Archive - welcher Kreis an Archiven dann immer - ein Monopol auf die Festlegung der Definition. Die Digitalisierung als allgemeiner Trend, der auch vor den Archiven natürlich nicht haltmacht, verschärft dieses Problem noch weiter: „Archiviert“ wird in E-Mail-Programmen ${ }^{26}$, Dateien werden im Rahmen eines „Archivierungsvorgangs“ von einem zum nächsten Dateiverzeichnis verschoben oder Online-Archive preisen ihre „Archivalien“" an ${ }^{27}$.

Wie können Archive - also: archivalische Institutionen im strengeren, „klassischen“ Sinne - auf diese Entwicklungen reagieren? Welche Rollen, welche Stellung sollen sie in den Gesellschaften der Gegenwart anstreben, und wie können sie diese realisieren? Einige erste Antworten dazu bietet der Text von Luciana Duranti, der aus einem als Höhepunkt im Tagungsprogramm angesetzten öffentlichen Abendvortrag hervorgeht. Gegenüber der - nicht ohne Grund - verbreiteten Wahrnehmung und Rede von Bedrängnis und Bedrohung der Archive schlägt Duranti einen bewusst optimistischen, anspruchsvollen

${ }^{21}$ Kai Naumann, Willkommene Einmischung. Über die Beziehungen von Archiven und Inhaltsproduzenten im digitalen Zeitalter, in: Kulturelles Kapital und ökonomisches Potential - Zukunftskonzepte für Archive. 82. Deutscher Archivtag in Köln, hg. von Heiner Sснмітт et al. (Tagungsdokumentationen zum Deutschen Archivtag 17, Fulda 2013) 35-46, hier 46; vgl. Nicole Convery, Information Management, Records Management, Knowledge Management: The Place of Archives in a Digital Age, in: The Future of Archives (wie Anm. 3) 191-212, hier 206; Richard J. Cox, Appraisal and the Future of Archives in the Digital Era, in: The Future of Archives (wie Anm. 3) 213-237, hier 231.

22 Martina WIECH, Strategisches Management für Archive, in: Archivmanagement in der Praxis, hg. von Mario Glauert-Hartwig Walberg (Veröffentlichungen der Landesfachstelle für Archive und öffentliche Bibliotheken im Brandenburgischen Landeshauptarchiv 9, Potsdam 2011) 13-35.

23 Natürlich konnten Archive auch früher keine Monopolstellung für sich reklamieren: vgl. AuER, Rolle der Archive (wie Anm. 9) 57; Csendes, Metaphern (wie Anm. 8) 49.

${ }^{24}$ Siehe als Auswahl Angebote wie Matricula, http://data.matricula-online.eu/de/ [1. 3. 2018]; Monasterium, http://monasterium.net/mom [1. 3. 2018]; Ancestry, https://www.ancestry.de/ [1. 3. 2018]; Familia Austria, https:/www.familia-austria.at/ [1. 3. 2018]. Zur Reaktion von Archiven auf solche Angebote vgl. Barbara Reed, Reconceptualising Access: Sustainability in the Digital Future, in: Digitalisierung im Archiv - Neue Wege der Bereitstellung des Archivguts. Beiträge zum 18. Archivwissenschaftlichen Kolloquium der Archivschule Marburg, hg. von Irmgard Christa Becker-Stephanie Oertel (Veröffentlichungen der Archivschule Marburg, Hochschule für Archivwissenschaft 60, Marburg 2015) 49-60, hier $59 f$.

25 Siehe dazu den Beitrag von Dietmar Schenk.

26 Patrick Sturm, Die E-Mail - ein Kommunikationsmedium des frühen 21. Jahrhunderts quellenkundlich betrachtet, in: Moderne Aktenkunde, hg. von Holger Berwinkel-Robert Kretzschmar-Karsten Uhde (Veröffentlichungen der Archivschule Marburg, Hochschule für Archivwissenschaft 64, Marburg 2016) 109_ 129.

27 Vgl. Schenk, Aufheben (wie Anm. 11) 46-52; Schenk, Theorie (wie Anm. 8) 11-13. 
Ton an. Sie streicht Stärken hervor, die es zu bewahren, an neue Gegebenheiten anzupassen, auszubauen und im öffentlichen Bewusstsein zu verankern gilt: voran die Reliabilität archivisch gesicherter Informationen und die Offenheit ihrer Bereitstellung. Aus der Kombination von beidem ergibt sich das Potential der Archive, nicht nur Hilfsorgane der öffentlichen Verwaltung oder Dienstleistungsstellen für die historischen Wissenschaften zu sein, sondern Orte der staatsbürgerlichen Partizipation und der Mitgestaltung gesellschaftlicher Diskurse über Vergangenheit, Gegenwart und Zukunft.

Reliabilität ${ }^{28}$ meint, dass das von Archiven bereitgehaltene Archivgut als Speicher von Informationen besonderer Güte zu erhalten und ihm Anerkennung zu verschaffen ist. In Zeiten der Verhandelbarkeit von Faktizität und sich verschiebender Standards des Vertrauens in der gesellschaftlichen Kommunikation ${ }^{29}$ sollten Archive die Verlässlichkeit der von ihnen angebotenen Informationen besonders hervorheben. Dabei geht es weder um einen absoluten Wahrheitsanspruch noch um das Pochen auf Autorität, die sich aus der Institutionalisierung durch staatliche oder andere Macht ergäbe. Vielmehr müssen Archive die Reliabilität ihres Archivguts durch die Verfahren herstellen, in denen sie es sammeln, bewahren, ordnen und benutzbar machen; auf diesem Wege ist dafür zu sorgen, dass die Herkunft der Information nachvollziehbar ist und sie auf dieser Basis einer inhaltlichen Prüfung unterzogen werden $\mathrm{kann}^{30}$. Dazu gehört neben der methodischen Reflektiertheit und der strengen Einhaltung ethischer Grundsätze ${ }^{31}$ auch an essentieller Stelle die transparente Dokumentation der Verfahren selbst.

Damit Archive in der alltäglichen Informationsflut wirkliche „Leuchtfeuer“ werden und archivische Standards für die Nachvollziehbarkeit von Informationsgüte ein gesellschaftlich relevanter Maßstab sein können, bedarf es aber auch noch großer Anstrengungen im Bereich der Zugänglichmachung und Vermittlung. Während die grundsätzliche Öffentlichkeit der staatlichen Archive in den letzten zweihundert Jahren allmählich erkämpft wurde ${ }^{32}$, ist diese Errungenschaft weiterhin als unvollkommen und brüchig anzusehen, denn es verbleiben viele implizite und praktische Schranken, die Menschen das Aufsuchen der Archive erschweren ${ }^{33}$. Hier bieten neue digitale Technologien enorme, noch vor kurzem ungeahnte Potentiale, doch sind die meisten Archive momentan im

\footnotetext{
28 Siehe dazu den Beitrag von Heather MacNeil.

29 Geoffrey Yeo, Trust and Context in Cyberspace. Archives and Records. The Journal of the Archives and Records Association 34 (2013) 214-234.

30 Siehe dazu den Beitrag von Dietmar Schenk; weiters vgl. Andreas Hedwig, Wozu brauchen wir authentische Archivalien? Archive im Dienste der Demokratiesicherung, in: Lebendige Erinnerungskultur für die $\mathrm{Zu}$ kunft. 77. Deutscher Archivtag 2007 in Mannheim, hg. von Heiner Schmiтt et al. (Tagungsdokumentationen zum Deutschen Archivtag 12, Fulda 2008) 269-279.

31 Vgl. Jeannette A. Bastian, Ethics for Archivists and Records Managers, in: Archives and Recordkeeping (wie Anm. 7) 101-129, hier 107.

32 Zur Schwierigkeit und Langwierigkeit dieser Entwicklung vgl. Anett LütTeken, Aufklärung und Historismus, in: Handbuch Archiv (wie Anm. 1) 45-56, hier 49f.; Jennifer S. Milligan, „What Is an Archive?“ in the History of Modern France, in: Archive Stories. Facts, Fictions and the Writing of History, hg. von Antoinette Burton (Durham-London 2005) 159-183; Philipp Müller, Archives and History: Towards a History of „the Use of State Archives“ in the $1^{\text {th }}$ Century. History of the Human Sciences 26/4 (2013) 27-49; Elizabeth Shepherd, Right to Information, in: Currents of Archival Thinking, hg. von Heather MacNeIL-Terence M. EASTwood (Santa Barbara-Denver ${ }^{2} 2017$ ) 247-269, hier 251-254.

33 Antoinette Burton, Introduction. Archive Fever, Archive Stories, in: Archive Stories (wie Anm. 32) 1-24, hier 9-13; Valerie Johnson, Solutions to the Silence, in: David Thomas-Simon Fowler-Valerie Johnson, The Silence of the Archive (Principles and Practice in Records Management and Archives, London 2017) 141-161, hier 144.
} 
Bereich netzbasierter Informationsbereitstellung kaum konkurrenzfähig. Um daran etwas zu ändern, bedarf es nicht nur der verstärkten zeitgemäßen Öffentlichkeitsarbeit ${ }^{34}$, sondern auch aufwendiger Verbesserungen an der Struktur der vorhandenen Informationen selbst und an den Mitteln des Zugangs zu ihnen. Probleme liegen etwa im meist zu wenig auf das Detail abzielenden Erschließungsgrad, aber auch darin, dass online zugängliche Informationssysteme nicht in die Suche von Suchmaschinen einbezogen werden. Erst durch gezielte Suche über das Webmodul eines Archivinformationssystems dringt man in die Bestände eines Archivs vor ${ }^{35}$. Wollen Archive sich Relevanz im gesellschaftlichen Diskurs sichern, dann genügt es also keinesfalls, Archivgut zu besitzen, es - sei es noch so gekonnt - zu bewahren und zu verwalten, aber passiv darauf zu warten, dass es benutzt wird. Vielmehr muss auch im Rahmen des Möglichen daran gearbeitet werden, es zu den Menschen zu bringen.

Die skizzierten Aufgaben werden in den meisten Archiven bereits in Angriff genommen; die Vorgehensweisen dabei sind oft sehr unterschiedlich und der Erfolg gleichfalls verschieden, denn die Herausforderungen sind anspruchsvoll und - wie nicht zu verkennen ist - ressourcenintensiv. Der Grund, weshalb sie dennoch angegangen werden sollten, warum die Ressourcen mobilisiert und bei Entscheidungsbefugten außerhalb der Archive unermüdlich um sie geworben werden sollte, liegt in den von Duranti hervorgehobenen Potentialen des gesellschaftlichen und insbesondere politischen Nutzens von Archiven ${ }^{36}$. Das Archiv ist von seinen Ursprüngen her, wie in den neueren Debatten immer wieder hervorgehoben wird, ein Instrument der Macht; schon das Wort leitet sich - über das

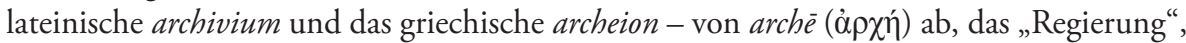
„Herrschaft“ oder „Amt“ bedeuten konnte. Der Zusammenhang ist ein doppelter: Archivgut ist der dokumentarische Niederschlag vergangener Machtausübung, und der Umgang mit Archivgut ist gegenwärtige Machtausübung. Wer Archive besaß, wie sie geführt wurden und wer Zugang hatte, waren, so lange es Archive gibt, Fragen der Verteilung von Gestaltungsmöglichkeiten und politischer Teilhabe.

Damit ist aber nicht nur ein herrschaftliches Potential angesprochen wie etwa bei den meisten frühneuzeitlichen Archiven, die als wachsam gehütete Speicher von Arkanwissen verbürgten, was Fürsten durften und was Untertanen schuldeten ${ }^{37}$, oder bei der Rolle von Archiven im Kolonialismus, für den sie nicht nur bürokratische Herrschaft, sondern auch die Konstruktion subalterner Andersartigkeit der Beherrschten unterstützten ${ }^{38}$. Gemeint

34 Siehe dazu den Beitrag von Joachim Kemper.

35 Als Schilderung des Idealzustands vgl. Angelika Menne-Haritz, Erschließung, in: Handbuch Archiv (wie Anm. 1) 207-217, hier 216f.

36 Die wissenschaftliche Erfassung dieses Nutzens - in konzeptueller wie empirischer Hinsicht - ist ein schwieriges Unterfangen, dem sich Archivwissenschaft und Archivwesen aber zwangsläufig stellen müssen. Für Ansätze dazu vgl. Wendy M. Duff-Andrew Fuinn-Karen Emily Suurtamm-David A. Wallace, Social Justice Impact of Archives: A Preliminary Investigation. Archival Science. International Journal on Recorded Information 13 (2013) 317-348.

37 Friedrich, Geburt (wie Anm. 19) 193-229; vgl. Anja Horstmann-Vanina Kopp, Archiv - Macht Wissen. Organisation und Konstruktion von Wissen und Wirklichkeiten in Archiven, in: Archiv - Macht Wissen. Organisation und Konstruktion von Wissen und Wirklichkeiten in Archiven, hg. von Anja HorstMANN-Vanina Kopp (Frankfurt am Main-New York 2010) 9-22, hier 14-16.

38 Ann Laura Stoler, Along the Archival Grain. Epistemic Anxieties and Colonial Common Sense (Princeton-Oxford 2009); weiters vgl. Patrick Joyce, Filing the Raj. Political Technologies of the Imperial British State, in: Material Powers. Cultural Studies, History and the Material Turn, hg. von Anthony BenneTt-Patrick JoyCe (Culture, Economy and the Social, London-New York 2010) 102-123; Adele Perry, The Colonial Archive on Trial. Possession, Dispossession, and History in Delgamuukw v. British Columbia, in: Archive Stories (wie 
ist auch das demokratisierende Potential von Archiven als Instrumente der Transparenz und der Verantwortlichkeit von Amtswaltung - was sich schon für das antike Athen zeigen lässt ${ }^{39}$ und auch in der Gegenwart zu den Hauptfunktionen und wesentlichen Existenzberechtigungen der Archive zählen muss. In heutigen Debatten über Archivrecht und Archivierung geht es um das Recht der Archive gegenüber den Ausübenden demokratisch mandatierter Macht als Recht der Öffentlichkeit auf Rechenschaft über die Ausübung des Mandats ${ }^{40}$. Ähnliches gilt aber auch für jene Einrichtungen, die explizit als „GegenGedächtnisse " 41 geschaffen werden, als Kontrapunkte gegen die Archive der staatlichen Stellen mit dem Ziel, das Erleben und die Kämpfe von Marginalisierten und Unterdrückten im gesellschaftlichen Gedächtnis zu sichern, wie etwa Archive von sozialen Bewegungen und Nichtregierungsorganisationen ${ }^{42}$. Auch sie können den Zusammenhang von Archiv und Macht zwar verschieben, erschüttern, manchmal sogar auf den Kopf stellen, aber sie bringen ihn nicht zum Verschwinden - und sollen das auch gar nicht. Die archè ist aus dem Archiv nicht wegzubringen.

Zuletzt sei noch - und in einer Veröffentlichung des Instituts für Geschichtsforschung ist dies als hervorgehobene, nicht als nachgeordnete Stellung zu verstehen - auf den $\mathrm{Zu}$ sammenhang von Archiven und historischen Wissenschaften hingewiesen. Auch er ist in der Gegenwart neu zu denken, sowohl im Hinblick auf technische Neuerungen als auch im Lichte der intensivierten kulturwissenschaftlichen Reflexion über Archive. Lange galt er als wenig reflexionsbedürftig; in mitunter recht unbedarfter Weise sahen Historikerinnen und Historiker Archive und Archivbestände als selbstverständlich vorauszusetzende Hilfsmittel respektive Gegenstände ihrer Forschung, oft mit zu wenig Respekt vor den Leistungen der Archive und mit zu wenig Nachdenken über deren Einfluss auf die Forschung und ihre Resultate. Und auch als der Archival Turn einsetzte und diese Fragen neu gestellt zu werden begannen, geschah dies zunächst - und geschieht allzu oft weiterhin - nahezu ohne Einbindung der Archivarinnen und Archivare selbst. Auf deren Seite wiederum wurden in den vergangenen Jahrzehnten Fachdebatten zunehmend in Abkopp-

Anm. 32) 325-350. Für einen Überblick zum Dialog zwischen postkolonialer Theorie und Archivwissenschaft vgl. Rachel Hardiman, Under the Influence: The Impact of Philosophy on Archives and Records Management, in: Archives and Recordkeeping (wie Anm. 7) 171-225, hier 209-212.

39 So, in berechtigter Kritik an der fragwürdigen Faktenbasis einiger berühmter Überlegungen Jacques Derridas: Stefan Rebenich, Altertum, in: Handbuch Archiv (wie Anm. 1) 29-40, hier 34; vgl. Adrian CunNInGHAM, Archives as a Place, in: Currents of Archival Thinking (wie Anm. 32) 53-79, hier 58.

${ }^{40}$ Livia Iacovino, Archives as Arsenals of Accountability, in: Currents of Archival Thinking (wie Anm. 7) 181-212; Udo SchäFer, Quod non est in actis, non est in mundo. Zur Funktion öffentlicher Archive im demokratischen Rechtsstaat, in: Alles was Recht ist. Archivische Fragen - juristische Antworten. 81. Deutscher Archivtag in Bremen, hg. von Heiner Sснмітт et al. (Tagungsdokumentationen zum Deutschen Archivtag 16, Fulda 2012) 57-78. Die Ermöglichung von Rechenschaft (accountability) kann generell als der vorrangige Wert der Bewahrung authentischer Dokumente gesehen werden: Bastian, Ethics (wie Anm. 31) 104.

41 Ulrich RaulfF, Gedächtnis und Gegen-Gedächtnis: das Archiv zwischen Rache und Gerechtigkeit, in: Handbuch Archiv (wie Anm. 1) 117-124, hier 123.

42 Siehe den Beitrag von Elizabeth Kata; weiters vgl. Jürgen BaCia-Cornelia Wenzel, Die Archive der Protest-, Freiheits- und Emanzipationsbewegungen. Ein Überblick. Archivar. Zeitschrift für Archivwesen 70 (2017) 130-141; Simon Fowler, Enforced Silences, in: Thomas-Fowler-Johnson, Silence (wie Anm. 33) 1-39, hier 18f.; Laura Mayhall, Creating the „Suffragette Spirit“: British Feminism and the Historical Imagination. Women's History Review 4 (1995) 319-344; Stefan Sudmann, „Archive von unten“, die Überlieferung der Neuen Sozialen Bewegungen und der schlanke Staat - eine Herausforderung für öffentliche Archive?, in: Archivarbeit - die Kunst des Machbaren. Ausgewählte Transferarbeiten des 39. und 40. wissenschaftlichen Kurses an der Archivschule Marburg, hg. von Volker Hirsch (Veröffentlichungen der Archivschule Marburg, Institut für Archivwissenschaft 47, Marburg 2008) 243-276. 
lung von der Frage nach Bedürfnissen, Interessen und möglichen Inputs der historischen Wissenschaften geführt. Aus beiderseitigen Unterlassungen hat sich eine schleichende Entfremdung entwickelt, ein ,archival divide“ ${ }^{43}$.

Diese gilt es heute im beiderseitigen Interesse zu überwinden oder zumindest zu überbrücken. Auf beiden Seiten sollte wieder das Bewusstsein einziehen, dass Archivarinnen und Archivare durch ihr gesamtes archivarisches Tun aktiv beitragen zur wissenschaftlichen Erforschung der Vergangenheit, ja dass sie darüber entscheiden, inwieweit diese überhaupt erforscht werden kann $^{44}$, dass sie dafür aber auch auf die Ergebnisse dieser Erforschung rekurrieren müssen - nicht nur zur faktographischen, sondern auch für wichtige Aspekte ihrer theoretischen und methodologischen Orientierung. Die wechselseitige Angewiesenheit wird durch technische Neuerungen keineswegs verringert, sondern noch akuter - überformen doch die neuen Medien nicht nur in zunehmendem Maße die angestammten Forschungstätigkeiten von Historikerinnen und Historikern in Archiven, sondern schaffen auch ganz neue Optionen in der Analyse und in der Darstellung von Ergebnissen ${ }^{45}$. Die vielfältigen Potentiale der Digital Humanities sind aber unweigerlich abhängig von Formaten, Strukturierung und Erschließung der zugrunde gelegten Daten. Dass ein sich wieder kräftigendes Bewusstsein für diese Angewiesenheiten in partnerschaftlichen Dialog mündet, zählt zu den zentralen Anliegen der Tagung und des nun vorliegenden Bandes. In diesem Sinne war es auch eine bewusste Entscheidung, das Programm mit einer Sektion zu eröffnen, deren Thema in besonders sichtbarer Weise im Überschneidungsbereich von Archivwissenschaft und historischen Wissenschaften liegt.

\section{Kategorisierung - Neue Quellenkunde im Archiv}

In Archiven werden die dort verwahrten Informationsträger nicht nur nach Provenienz geordnet und nach ihrem Inhalt erschlossen, sondern auch nach verschiedenen Kriterien kategorisiert oder typologisiert. Für das „konventionelle“ - im Gegensatz zum digitalen - Archivgut sind diese Kriterien traditionell in „innere“ und „äußere Merkmale“ gegliedert worden, wobei erstere die sprachliche und textuelle Gestaltung, letztere die physische Form und die optische Erscheinung betreffen ${ }^{46}$. Eine auf die Analyse dieser Merkmalstypen gestützte Typologisierung historischer Überlieferungen und Überlieferungsträger ist einer der Gegenstände der Quellenkunde, die unter die historischen

43 Blouin-Rosenberg, Processing the Past (wie Anm. 5) 63-93. Für einen ähnlichen Befund für den deutschen Sprachraum siehe den Beitrag von Dietmar Schenk.

44 Blouin-Rosenberg, Processing the Past (wie Anm. 5) 140-160.

45 Alexandra Chassanoff, Historians and the Use of Primary Source Materials in the Digital Age. The American Archivist 76 (2013) 458-480.

46 Neuere und neueste Standardwerke, die mit dieser Unterscheidung arbeiten, sind etwa Luciana DuRanti, Diplomatics: New Uses for an Old Science. Archivaria. The Journal of the Association of Canadian Archivists 28 (1989) 7-27; 29 (1989) 4-17; 30 (1990) 4-20; 31 (1990) 10-25; 32 (1991) 6-24; 33 (1991) 6-24, hier 32 6-16 („extrinsic elements“ und „intrinsic elements“); Michael Hochedinger, Aktenkunde. Urkunden- und Aktenlehre der Neuzeit (Historische Hilfswissenschaften, Wien-München 2009) 14, 118-170; Corinne Rogers, Diplomatics, in: Encyclopedia of Archival Science (wie Anm. 7) 176-180, hier 177; RoHr, Hilfswissenschaften (wie Anm. 12) 42. Zweifel an der Anwendbarkeit auf neuzeitliche und zeitgeschichtliche Quellen äußert hingegen Paola Carucci, Il documento contemporaneo. Diplomatica e criteri di edizione (Beni culturali 1, Roma 1987) 98f. 
Hilfs- oder Grundwissenschaften gezählt wird ${ }^{47}$; schon der Quellenbegriff ${ }^{48}$ im Namen der Disziplin verrät die Perspektivierung vornehmlich aus Sicht der Geschichtsforschung. Die tendenzielle Auseinanderentwicklung des geschichtswissenschaftlichen und des archivarischen Berufsfelds mag demnach auch einer der Gründe sein, weshalb für einige Jahrzehnte die nähere Befassung mit Quellenkunde in den Archiven wenig Priorität genoss, nachdem noch im frühen 20. Jahrhundert maßgebliche Beiträge von archivarischer Seite geleistet worden waren ${ }^{49}$. Dabei blieb die grundsätzliche Einteilung von Archivgut nach formalen und materiellen Gesichtspunkten - etwa in Urkunden, Handschriften, Akten, Photographien und so fort - schon aus konservatorischen Rücksichten unumgänglich.

Als weiterer Grund anzusprechen ist das zunehmende Übergewicht des neuesten und zeitgenössischen Archivguts unter den Gegenständen archivischer Arbeit. Die hilfswissenschaftliche Quellenkunde ist auch auf geschichtswissenschaftlicher Seite vor allem im Bereich der Mediävistik, zunehmend auch in der Frühneuzeit- und Neuzeitforschung etabliert; hingegen ist sie für die Belange der Zeitgeschichtsforschung noch wenig anerkannt und wenig entwickelt. Dass moderne und neueste Quellen hinsichtlich ihrer Auswertbarkeit keine Schwierigkeiten bereiten würden, ist freilich ein gefährlicher Trugschluss, der dadurch entsteht, dass sie im Gegensatz zu älteren Überlieferungen zumindest auf der vordergründigen Ebene einer ersten Texterfassung leicht lesbar sind. Mitunter fließt wohl auch, obwohl ausgebildete Historikerinnen und Historiker es besser wissen sollten, Anfälligkeit gegen den von Akten erhobenen Anspruch der Tatsächlichkeit ein ${ }^{50}$. Bei der Interpretation können allerdings durch mangelnde Kenntnis der Produktionsweise sowie der Merkmale, die einer Quellenkritik zugänglich sind, bedenkliche Irrtümer entstehen, was auch oft in der Praxis zu beobachten ist ${ }^{51}$. Deshalb ist in jüngsten Jahren im Rahmen der Debatten um den Stand und die Zukunft der historischen Hilfswissenschaften ${ }^{52}$ der Ruf nach einer Erweiterung und Vertiefung derselben im Allgemeinen und der Quellenkunde

47 RoHr, Hilfswissenschaften (wie Anm. 12) 15-36.

48 Verwendete Informationsträger als „Quellen“ zu bezeichnen, ist eine heute meist selbstverständliche, in ihrem Entstehen und ihrer Durchsetzung jedoch zu historisierende Metapher: vgl. Daniela SAXER, Die Schärfung des Quellenblicks. Forschungspraktiken in der Geschichtswissenschaft 1840-1914 (Ordnungssysteme. Studien zur Ideengeschichte der Neuzeit 37, München 2014) 15-19; Michael Zimmermann, Quelle als Metapher. Überlegungen zur Historisierung einer historiographischen Selbstverständlichkeit. Historische Anthropologie. Kultur - Gesellschaft-Alltag 5 (1997) 268-287. Aus archivarischer Sicht reflektiert den Quellenbegriff Schenk, Theorie (wie Anm. 8) 44-53.

49 Als Beispiel sei nur verwiesen auf Meisners Handbücher, zuletzt: Meisner, Archivalienkunde (wie Anm. 11). Zum breiteren forschungsgeschichtlichen Umfeld vgl. Eckart Henning, Wie die „Aktenkunde“ entstand. Zur Disziplingenese der Aktenkunde als Historischer Hilfswissenschaft, in: Archivistica docet. Beiträge zur Archivwissenschaft und ihres interdisziplinären Umfelds, hg. von Friedrich BECK-Wolfgang HeMPELEckart Henning (Potsdamer Studien. Schriftenreihe der gemeinnützigen Gesellschaft für Fortbildung, Forschung und Dokumentation Potsdam 9, Potsdam 1999) 439-461.

50 Vgl. Alexandra Kemmerer, Akten, in: Handbuch Archiv (wie Anm. 1) 131-143, hier 131-133; Alexandra Ortmann, Machtvolle Verhandlungen. Zur Kulturgeschichte der deutschen Strafjustiz 1879-1924 (Kritische Studien zur Geschichtswissenschaft 215, Göttingen 2014) 53-69; Cornelia Vismann, Akten. Medientechnik und Recht (Frankfurt am Main 2000) 89f.

51 Ein vergleichsweise harmloses Beispiel bietet eine ansonsten exzellente Untersuchung, in der Schreiben zwischen Behörden in offenbarer Unkenntnis aktenkundlicher Terminologie durchgehend unter der Bezeichnung „Brief“ zitiert werden: Vanessa Conze, Treue schwören. Der Konflikt um den Verfassungseid in der Weimarer Republik. HZ 297 (2013) 354-389. Für Beispiele weiterer Fallstricke siehe den Beitrag von Holger Berwinkel.

52 Eine knappe Übersicht bietet Andrea STIEldorf, Die historischen Grundwissenschaften an den deutschen Universitäten heute - eine Bestandsaufnahme. Archivar. Zeitschrift für Archivwesen 67 (2014) 257-264. 
im Besonderen auf die neuesten Quellen - einschließlich der digitalen - laut geworden ${ }^{53}$. „Digitale Hilfswissenschaften“ sind demnach einerseits digitale Anwendungen für die Analyse von Quellen jeglichen Alters, andererseits Hilfswissenschaften speziell für genuin digitale Überlieferungen. Die angestammten Kategorien und Begrifflichkeiten sind dabei grundlegend zu überdenken - bis hin zu der genannten Unterscheidung innerer von äußeren Merkmalen, die im Hinblick auf die für das digitale Zeitalter charakteristische Impermanenz der Bindung von Information und Träger ${ }^{54}$ neu zu formulieren ist.

Aber auch jenseits ihres zu erneuernden Zusammenwirkens mit geschichtswissenschaftlicher Forschung rufen die neuen oder neu erkannten gesellschaftlichen Aufgaben der Archive in der Gegenwart und für die Zukunft nach einer intensivierten Befassung mit Quellenkunde. Erst noch zu erarbeitende oder zu systematisierende Kenntnisse der Beschaffenheit neuer Quellengattungen sind als unverzichtbare Grundlagen ihrer Archivierung notwendig, sei es für die Bewertung, die Erschließung, die Ordnung oder die Erhaltung. Auch die verstärkt in den Vordergrund tretenden Publizitäts- und Vermittlungsaufgaben der Archive erfordern quellenkundliche Aufarbeitung und Beschreibung der Bestände, die allen Kategorien von Benutzenden ${ }^{55}$ Wegweiser durch das Verfügbare und einen „Werkzeugkasten für die [...] Analyse" 56 bieten müssen.

Zur aktuellen Situation der archivalischen Quellenkunde, vornehmlich im deutschsprachigen Raum, zu ihren Potentialen und Desideraten bietet der Beitrag von Robert Kretzschmar eine Überschau. Die daraus abgeleiteten Handlungsanforderungen richten sich an Archive sowie an Archivarinnen und Archivare; die Forderung, dem bereits seit Jahren von archivarischer Seite und inzwischen auch von der akademischen Geschichtswissenschaft öffentlich geäußerten Bedarf nun durch konkrete Projekte zu begegnen, richtet sich auch an die eigene Adresse: „da muss ich mir auch an die eigene Nase fassen“. Zur Einlösung schreiten Kretzschmar und das von ihm bis Anfang 2018 geleitete Landesarchiv Baden-Württemberg durch das Projekt einer „Südwestdeutschen Archivalienkunde“ im World Wide Web, dessen Parameter und Verlauf im zweiten Teil des Beitrags zur Darstellung kommen.

Holger Berwinkel stellt die Probleme vor, die es zu bewältigen gilt, um zu einer Ak-

53 Rainer Hering, Archive und Geschichtswissenschaft im digitalen Zeitalter. Probleme und Herausforderungen aus der Sicht eines deutschen Archivars und Historikers. MIÖG 120 (2012) 116-138, hier 127-130; Robert Kretzschmar, Hilflose Historikerinnen und Historiker in Archiven? Zur Bedeutung einer zukünftigen archivalischen Quellenkunde für die universitäre Forschung. Blätter für deutsche Landesgeschichte 147 (2011) 133-147; Kiran Klaus Patel, Zeitgeschichte im digitalen Zeitalter. Neue und alte Herausforderungen. Vierteljahrshefte für Zeitgeschichte 59 (2011) 331-351.

54 Grundlegend ist dazu das an den National Archives of Australia entwickelte „Performance Model“ der digitalen Überlieferung: Helen Heslop-Simon Davis-Andrew Wilson, An Approach to the Preservation of Digital Records (National Archives Green Paper, Canberra 2002). Analogien bestehen freilich zu manchen analogen Medien, etwa zur multiplen Umsetzung photographischer Negative in unterschiedliche Positive: Joan M. Schwartz, The Archival Garden: Photographic Plantings, Interpretive Choices, and Alternative Narratives, in: Controlling the Past (wie Anm. 4) 69-110.

55 Zur Diversität der Gruppen von Benutzenden, die in archivischen Planungen und Entscheidungen nicht immer hinreichend berücksichtigt wird, vgl. Wendy M. Duff-Elizabeth YAKeL, Archival Interaction, in: Currents of Archival Thinking (wie Anm. 32) 193-223, hier 196-199; Polona VILAR-Alenka ŠAuPERL, Archives, Quo Vadis et Cum Quibus? Archivists' Self-Perceptions and Perceptions of Users of Contemporary Archives. International Journal of Information Management 35 (2015) 551-560, hier 554f. Eine Übersicht der Forschung zu Benutzenden bietet Shadrack KatuU, User Studies and User Education Programmes in Archival Institutions. Aslib Journal of Information Management 67 (2015) 442-457.

56 Lorenz Friedrich Beck-Robert Kretzschmar, Zum Begriff „Aktenkunde“ - Verständnis und Abgrenzung als Disziplin, in: Moderne Aktenkunde (wie Anm. 26) 23-27, hier 27. 
tenkunde der Zeitgeschichte - hier vor allem des 20. Jahrhunderts - zu gelangen. Bürotechnische Neuerungen und Kanzleireformen ${ }^{57}$ hatten auf das Verwaltungsschriftgut tiefgreifende Auswirkungen, die im Allgemeinen zu einer Verringerung der Nachvollziehbarkeit von Verwaltungsvorgängen führten. Die dauerhafte Verschriftlichung auch der Vorgangssteuerung, die für den Höhepunkt des Aktenzeitalters im 19. Jahrhundert charakteristisch gewesen war, wurde erheblich reduziert. Dennoch, so Berwinkel, ist eine Aktenkunde des 20. Jahrhunderts sowohl möglich als auch notwendig; er bietet sowohl positive Beispiele des Erkenntnisgewinns aus ihrer Anwendung als auch abschreckende dafür, was bei unbedarfter Verwendung von Aktenmaterial herauskommen kann. Er plädiert nachdrücklich für eine „evolutionäre Fortentwicklung“ dieser Hilfswissenschaft ausgehend von den im frühen 20. Jahrhundert geschaffenen Grundlagen.

Einen ähnlichen Ansatz verfolgt in Anwendung auf gänzlich andere Unterlagen Elizabeth Kata, indem sie die Brauchbarkeit der Terminologie und Fragestellungen der „klassischen "Aktenkunde nach Meisner, Hochedlinger oder Kloosterhuis ${ }^{58}$ am Archivgut der österreichischen Frauen- und Lesbenbewegung seit den 1970er Jahren erprobt. Aktenförmig ist dieses Schriftgut klarerweise nicht ${ }^{59}$ und seine inneren und äußeren Merkmale unterscheiden sich teilweise markant davon, was in den Handbüchern jener Autoren beschrieben wird; aber die auf Meisner zurückgehende Trias von genetischer, analytischer und klassifizierender Untersuchung erweist sich doch als fruchtbar. Entstehungsprozesse und Entscheidungsstrukturen haben auch hier ihren Niederschlag in den Schriftstücken gefunden, der entschlüsselt werden und zur Einordnung und Deutung der Unterlagen als Archivalien und historische Quellen beitragen kann. Diese Hilfswissenschaft wird von Kata auf der Ebene der Archivalienkunde verortet - eines Teilbereichs der Quellenkunde, der im Sinne Meisners Akten, aber auch weitere in Archiven anzutreffende Quellen einschließ $\mathrm{t}^{60}$.

Mit einem aus archivischer wie aus geschichtswissenschaftlicher Sicht hochwichtigen Aspekt der Archivalien als Informationsquellen befasst sich Heather MacNeil, nämlich mit deren Reliabilität oder Vertrauenswürdigkeit. Die Geschichte der Vorstellungen und Debatten dazu sowie der damit verbundenen Praktiken skizziert sie von der Spätantike bis in die Gegenwart ${ }^{61}$, über diverse Medienrevolutionen und fundamentale Wandlungen in der gesellschaftlichen Rolle der Archive hinweg. Es geht dabei neben der historischen Quellenfunktion, die erst im 19. Jahrhundert in den Vordergrund rückte, auch weiterhin um die juristische Beweiskraft wie um die politisch und gesellschaftlich immer wichtiger gewordene

57 Holger Berwinkel, Zur Kanzleigeschichte des 20. Jahrhunderts - ein Versuch, in: Moderne Aktenkunde (wie Anm. 26) 29-50. Zur Bürotechnologie vgl. Delphine GARdeY, Écrire, calculer, classer. Comment une révolution de papier a transformé les sociétés contemporaines (1800-1940) (Textes à l'appui - Anthropologie des sciences et des techniques, Paris 2008); Harald Rösler, Bürokunde und ein Blick ins Archiv (Remscheid 2015).

58 Hochedlinger, Aktenkunde (wie Anm. 46); Jürgen Kloosterhuis, Amtliche Aktenkunde der Neuzeit. Ein hilfswissenschaftliches Kompendium. AfD 45 (1999) 465-563; Heinrich Otto Meisner, Aktenkunde. Ein Handbuch für Archivbenutzer mit besonderer Berücksichtigung Brandenburg-Preußens (Berlin 1935).

59 Zur Begrenzung des Gegenstands der Aktenkunde auf dasjenige, was im Rahmen eines geregelten Geschäftsgangs entstanden ist, vgl. Holger Berwinkel-Anette Meiburg, Die moderne Bundesverwaltung als Referenzmodell der Systematischen Aktenkunde - Erfahrungen aus der Praxis, in: Moderne Aktenkunde (wie Anm. 26) 81-92, hier 81 .

${ }^{60}$ Zur Terminologie vgl. Meisner, Archivalienkunde (wie Anm. 11) 21-24; für einen ähnlichen Gebrauch von „Archivalienkunde“, wie ihn Kata vorschlägt, vgl. BeCK-Kretzschmar, Aktenkunde (wie Anm. 56) 27; Berwinkel-Meiburg, Moderne Bundesverwaltung (wie Anm. 59) 81.

${ }^{61}$ Der Vortrag bietet somit eine Fortschreibung und Aktualisierung einiger Kernthesen aus ihrer früheren grundlegenden Arbeit: Heather MacNeIL, Trusting Records. Legal, Historical and Diplomatic Perspectives (The Archivist's Library 1, Dordrecht-Boston-London 2000). 
Transparenzfunktion. Alle sind gegenwärtig in Frage gestellt durch die Konsequenzen der Digitalisierung; weder Provenienz noch ununterbrochene Aufbewahrung reichen mehr aus als Basis einer vernünftigen Annahme der Vertrauenswürdigkeit. Vielmehr stehen Archive vor der ungleich schwierigeren Aufgabe, ungebrochene Kontinuität der Erhaltung zu gewährleisten und zu dokumentieren: Dazu müssen sämtliche Maßnahmen und Entscheidungen zur Bewertung, Übernahme, Speicherung und Migrierung archivalischer Daten nachvollziehbar aufgezeichnet und den Benutzenden zusammen mit diesen zur Verfügung gestellt werden. Diese Konklusion MacNeils leitet gleichzeitig zum Thema der folgenden Sektion über.

\section{Überlieferungsbildung und Bewertung}

Die zweite Sektion der Tagung war jenen archivischen Kerntätigkeiten gewidmet, die Vergangenheit, Gegenwart und Zukunft sinnfällig in Verbindung setzen ${ }^{62}$ : archivische Bewertung und Überlieferungsbildung. Vier der fünf Vorträge sind verschriftlicht in diesem Tagungsband vertreten, während jener von Christa Ehrmann-Hämmerle leider nicht erscheinen konnte.

Mit Hilfe der Bewertung treten Archivarinnen und Archivare der Informationsflut entgegen, indem verschiedene Methoden zur Auswahl und Verdichtung von Information eingesetzt werden. Mit der Entscheidung über Archivwürdigkeit oder Vernichtung von Informationen schaffen sie einen wesentlichen Teil der kulturellen Identität ${ }^{63}$. Deshalb wird die Bewertung als eine Königsdisziplin der Archivarbeit, jedenfalls als unverkennbar archivische Kerntätigkeit bezeichnet ${ }^{64}$. Sie fordert allen, die sie ausführen, hohes Verantwortungsbewusstsein, aber auch größtmögliche Umsicht und breites Wissen ab. Gleichzeitig ist die archivische Bewertung hinsichtlich Wirksamkeit und Erfolg schwer überprüfbar. Die Bewertungsarbeit kann nur an zuvor definierten Zielen gemessen werden, wie Matthias Buchholz in seinem Beitrag treffend festhält. Solche Ziele orientieren sich meist an der Überlieferungstradition eines Archivs.

Gesetzliche Grundlagen geben den groben Rahmen für die Überlieferungsbildung vor. Die allgemeinen Definitionen von Archiv- und Schriftgut, die beispielsweise im österreichischen Denkmalschutzgesetz festgehalten sind, werden durch ebenso breiten Raum gebende Formulierungen in den Bundes- und Landesarchivgesetzen ergänzt ${ }^{65}$. Die Archiv-

${ }^{62}$ Terence Gordon Соoк, Remembering the Future. Appraisal of Records and the Role of Archives in Constructing Social Memory, in: Archives, Documentation, and Institutions of Social Memory. Essays from the Sawyer Seminar, hg. von Francis X. Blouin jr.-William G. Rosenberg (Ann Arbor 2006) 169-181, hier 169: „Appraisal occurs primarily today on the records of yesterday to create a past for tomorrow. What kind of past should the future have?"

63 Terence Gordon Сooк, „We Are What We Keep; We Keep What We Are“: Archival Appraisal Past, Present and Future. Journal of the Society of Archivists 32 (2011) 173-189; vgl. Blouin-Rosenberg, Processing the Past (wie Anm. 5) 145-147.

${ }^{64}$ Exemplarisch Hartmut Weber, Bewertung im Kontext der archivischen Fachaufgaben, in: Bilanz und Perspektiven archivischer Bewertung. Beiträge eines archivwissenschaftlichen Kolloquiums, hg. von Andrea Wettmann (Veröffentlichungen der Archivschule Marburg, Institut für Archivwissenschaft 21, Marburg 1994) 63-81, hier 67: „Im Kreise der archivischen Kernaufgaben [...] kommt der Bewertung eine Schlüsselposition zu“. Ähnliche Topoi prägen auch seit bald einem halben Jahrhundert die Debatten in der englischsprachigen Archivistik: vgl. Cox, Appraisal (wie Anm. 21) 219-222; Ann J. Gilliland, Archival Appraisal: Practising on Shifting Sands, in: Archives and Recordkeeping (wie Anm. 7) 31-61, hier 31.

${ }_{65} \mathrm{Zu}$ den archivrechtlichen Rahmenbedingungen in Österreich vgl. Elisabeth ScHÖGGL-ERNST, Bewertung von Schriftgut. Tehnični in vsebinski problemi klasničnega in elektronskega arhiviranja 3 (2004) 96-102; 
gesetze legen dabei den Fokus auf Verwaltungsschriftgut. Die Entstehung von Unterlagen im digitalen Kontext schafft zudem für den Prozess der Überlieferungsbildung neue Herausforderungen ${ }^{66}$. Neueste gesetzliche Regelungen berücksichtigen bereits genuin digitales Schriftgut. Eine vielfach zu beobachtende nachlässige Schriftgutverwaltung erzeugt Probleme für die Bewertung ${ }^{67}$. Mangelhaft erstellte Metadaten der Behörden bei digitaler Protokollierung beziehungsweise im gesamten elektronischen Akt erschweren die Implementierung von Bewertungsplänen. Es bedarf daher einer gründlichen Schulung des Personals, um eine effektive Schriftgutverwaltung zu schaffen, welche die Voraussetzung für die Bewertung des analogen wie auch des elektronischen Aktes bildet. Somit muss Records Management als Führungs- sowie archivische Fachaufgabe einen zunehmend wichtigen Stellenwert einnehmen, wie Markus Schmidgall in seinem Beitrag betont.

Zur Bewältigung der Bewertung von Massenschriftgut aus der Verwaltung wurden verschiedene Theorien entwickelt, die sich entweder am Informationsgehalt oder an der aktenbildenden Stelle orientieren ${ }^{68}$. Die Anwendung dieser theoretischen Grundlagen in der Bewertungspraxis ist wiederum abhängig von der Überlieferungstradition eines Archivs und von den Unterlagen selbst. Manchmal klaffen Theorie und Praxis auch auseinander.

Betrachten wir die Zusammensetzung von Archivgut, dann sind Veränderungen der Bewertungsgrundsätze im Lauf der Geschichte unschwer zu erkennen. Dieser Wandel der Archivwürdigkeit ist ebenso als ein Zeichen des Zeitgeistes zu betrachten und wird damit Teil der Archivgeschichte, wie auch die Geschichtswissenschaft im Lauf der Zeit verschiedenen Trends folgte. Archivarinnen und Archivare sind in ihrer Bewertungstätigkeit bestrebt, die gängigen Forschungsschwerpunkte einzubeziehen. Sie allein dürfen aber nicht als Maßstab für die Archivwürdigkeit dienen ${ }^{69}$.

Abseits der öffentlichen Verwaltung unterliegen Privatarchive ganz anderen Grundsätzen der Überlieferungsbildung. Sofern für Privatarchive in Anwendung der Bestimmungen des Denkmalschutzgesetzes ein öffentliches Interesse festgestellt wurde, sind in der Regel Richtlinien für die Überlieferungsbildung erstellt worden. Dies trifft etwa auf die

Jakob Wührer, Die Auswahl des Essentiellen. Archivrechtliche Grundlagen für die archivische Bewertung in Österreichischen Archivgesetzen, in: Zeitgeschichtsforschung im Spannungsfeld von Archiv-, Datenschutzund Urheberrecht, hg. von Iris Eisenberger-Daniel EnNöckL-Ilse Reiter (Wien 2018) 83-122. Zur Rechtslage in Deutschland im Überblick Irmgard Christa BECKER, Bewertungshoheit - Bewertungskompetenz, in: Archivrecht für die Praxis. Ein Handbuch, hg. von Irmgard Christa Becker-Clemens Rehm (Berliner Bibliothek zum Urheberrecht 10, München 2017) 58-71.

${ }^{66}$ Hering, Archive und Geschichtswissenschaft (wie Anm. 53) 123f., 132-137; Robert Kretzschmar, Alles neu zu durchdenken? Archivische Bewertung im digitalen Zeitalter. Archivpflege in Westfalen-Lippe 80 (2014) 9-15; Ciaran B. Trace, On or Off the Record? Notions of Value in the Archive, in: Currents of Archival Thinking (wie Anm. 7) 47-68, hier 60-62.

${ }^{67}$ Hering, Archive und Geschichtswissenschaft (wie Anm. 53) 119-121; David Thomas, The Digital, in: Thomas-Fowler-Johnson, Silence (wie Anm. 33) 65-100, hier 78-82.

68 Zur Entwicklung der Bewertungstheorien im Überblick: Barbara CraIG, Appraisal, in: Encyclopedia of Archival Science (wie Anm. 7) 14-18; Fiorella Foscarini, Archival Appraisal in Four Paradigms, in: Currents of Archival Thinking (wie Anm. 32) 107-133; Robert Kretzschmar, Die „neue archivische Bewertungsdiskussion" und ihre Fußnoten. Zur Standortbestimmung einer fast zehnjährigen Kontroverse. Archivalische Zeitschrift 82 (1999) 7-40; Jürgen Treffeisen, Zum aktuellen Stand der archivischen Bewertungsdiskussion in Deutschland - Entwicklungen, Trends und Perspektiven. Scrinium. Zeitschrift des Verbandes Österreichischer Archivarinnen und Archivare 70 (2016) 58-92; Bodo UHL, Die Geschichte der Bewertungsdiskussion: Wann gab es neue Fragestellungen und warum?, in: Bilanz und Perspektiven (wie Anm. 64) 11-35.

69 Wilfried SснӧNTAG, Archivische Bewertung und Ansprüche der Forschung, in: Bilanz und Perspektiven (wie Anm. 64) 129-145. 
Archive der römisch-katholischen Kirche und ihrer Orden zu, die teilweise auf eine jahrhundertelange Archivtradition zurückblicken können ${ }^{70}$. Wie der Beitrag von Helga Penz zeigt, wurde der Fokus nicht so sehr auf die Verwaltungsarbeit eines Ordens, sondern vielmehr auf die Gründungsgeschichte, rechtliche Grundlagen und die Leitung der Institution gerichtet. Das Klosterleben selbst stand nicht im Mittelpunkt des Interesses und galt daher oft nicht als erinnerungswürdig. Dennoch haben sich Zeugnisse des Ordensalltags erhalten - manchmal zufällig, teils aus der Umsicht der Archivare und Archivarinnen oder mangels eines Vernichtungsauftrages. Jüngere Ordensgemeinschaften legten zum Teil größeren Wert auf die Nachhaltigkeit ihres Wirkens, weshalb dank großzügigerer Bewertungsgrundlagen der Erhaltungsgrad umfangreicher ist.

Vor eine besondere Herausforderung sind Archive bei der Bewertung der digitalen Bilderflut gestellt. Bilder wurden lange Zeit als Beiwerk zum Zweck der Illustration betrachtet. Neben dem Bild als Kunstobjekt nahm die Entwicklung der Photographie als eigenständiges Archivgut erst spät ihren Anfang. Die digitale Photographie stellt Archive vor das Problem der ungeheuren Datenmenge, deren Bewältigung Michel Pfeiffer in seinem Beitrag zu meistern versucht. Er führt uns vor Augen, dass die Bewertung der Bildmengen nicht mit herkömmlichen Werkzeugen machbar ist, und fordert Archivarinnen und Archivare zu Mut zur Kreativität auf, dieses Problem zu lösen und diese Herausforderung als Chance für Archive zu sehen. Auch bei Bilddaten bildet das Erstellen eines strategischen Sammlungskonzepts die Voraussetzung für einen Bewertungsansatz. Daran schließt die Frage nach methodischen und technischen Konzepten an, mit denen das Sammlungsziel erreicht und die Daten in der Folge gesichert, erschlossen und präsentiert werden können. Pfeiffer analysiert US-amerikanische und kanadische Bewertungsmodelle für Bilder, welche die Ermittlung des Entstehungszusammenhangs präferieren. In Anlehnung an die Bewertungstheorie von Theodore Schellenberg ${ }^{71}$ sollen Primär- und Sekundärwert der Bilder untersucht werden, wobei der Sekundärwert und hier vor allem der Evidenzwert als maßgeblich für die Archivwürdigkeit erachtet wird. Auch monetäre Werte werden in der Analyse berücksichtigt ${ }^{72}$. Dem Interesse der Benutzenden wird breiter Raum gegeben, was Pfeiffer kritisch hinterfragt. In Anlehnung an Bewertungsmodelle analoger Daten sollen auf Algorithmen basierende Systeme geschaffen werden, die das Archivpersonal in der Bewertung digitaler Daten unterstützen. Schließlich fordert er eine bessere Qualifikation desselben in Fragen der Bewertung.

Damit Archive dem Postulat der Bewertung als Königsdisziplin gerecht werden, wird die Dokumentation der Bewertungstätigkeit und der Bewertungsmethoden in größerem $\mathrm{Maße}$, als sie bisher umgesetzt wurde, gefordert. Denn nur dadurch ist Bewertung „messbar" und schafft Transparenz in der Überlieferungsbildung eines Archivs.

${ }^{70}$ Helga Penz, Die Prälatenarchive, in: Quellenkunde der Habsburgermonarchie (16.-18. Jahrhundert). Ein exemplarisches Handbuch, hg. von Josef Pauser-Martin Scheutz-Thomas Winkelbauer (MIÖG Ergbd. 44, Wien-München 2004) 686-695.

71 Theodore R. Schellenberg, The Appraisal of Modern Public Records (Bulletins of the National Archives 8, Washington 1956). Zur Bedeutung Schellenbergs in der Geschichte der Bewertungsdiskussionen vgl. Terence Gordon Сook, What is Past is Prologue: A History of Archival Ideas Since 1898, and the Future Paradigm Shift. Archivaria. The Journal of the Association of Canadian Archivists 43 (1997) 17-63, hier 27-29; GilliLAND, Archival Appraisal (wie Anm. 64) 40f.; Ridener, From Polders to Postmodernism (wie Anm. 6) 75-99.

$72 \mathrm{Zu}$ den Debatten über die Konzeptualisierung des „Werts“ von Dokumenten vgl. Trace, On or Off the Record (wie Anm. 66) $56 f$. 


\section{Informationsaufbereitung und Vermittlung}

Ein Kernbereich der Archivierung ist die Erschließung von Archivgut, bestehend im Ordnen und im Verzeichnen ${ }^{73}$. Ersteres bedeutet die Strukturierung und Kontextualisierung von Information, Letzteres das Anreichern mit Metadaten ${ }^{74}$. „Ohne Erschließung bleibt Archivgut unzugänglich und kann nicht genutzt werden. "75 Ordnung und Erschließung ermöglichen also erst die Nutzung von Archivgut; zugleich strukturieren sie diese vor, erleichtern manche Wege zum Material und behindern andere, und geben damit Deutungen und Narrative mehr oder weniger sichtbar vor ${ }^{76}$.

Diese Prozesse bilden sich aktuell schon in elektronischen Archivinformationssystemen nach internationalen Standard $s^{77} \mathrm{ab}$ - was auch eine internationale Vernetzung über Portalverbünde ${ }^{78}$ ermöglicht. Archivische Erschließung entstand, bewährte und erweiterte sich in Abgleich mit ihrem Gegenstand, dem zu erschließenden Archivgut. Wenn dieses Archivgut nun um neue, insbesondere digitale Archivguttypen weiter ausdifferenziert wird, die durch die elektronische Aktenführung auch in einem veränderten Umfeld der Schriftgutverwaltung entstehen, stellt sich die Frage, ob Bewährtes noch zukunftsträchtig ist.

Genuin digitales Archivgut eröffnet neue Nutzungsmöglichkeiten, da sein Inhalt für automationsunterstützte Auswertung direkt verwendbar ist. Solche Nutzungsmöglichkeiten müssen aber mittels Erschließung auch eins zu eins an die Benutzenden weitergegeben werden und dürfen nicht in eine Nutzungsumgebung gepresst werden, welche die Entfaltung des von vornherein vorhandenen Auswertungspotentials verhindern oder einschränken würde. Die so unmittelbarer zugängliche digitale Information, oder oft geradezu Informationsflut, stellt die Archive aber vor die Herausforderung der zielführenden

73 Menne-Haritz, Schlüsselbegriffe (wie Anm. 12) 46.

74 Siehe zuletzt als Überblick Menne-Haritz, Erschließung (wie Anm. 35); Sharon Thibodeau, Archival Description, in: Encyclopedia of Archival Science (wie Anm. 7) 39-42; Geoffrey Yeo, Debates about Description, in: Currents of Archival Thinking (wie Anm. 7) 89-114.

75 Menne-Haritz, Erschließung (wie Anm. 35) 207; vgl. Simon Fowler, Inappropriate Expectations, in: Thomas-Fowler-Johnson, Silence (wie Anm. 33) 41-63, hier 53-60.

76 Regina KeYLER, Der Zusammenhang zwischen Erschließung und Benutzung. Eine Untersuchung an Beständen des Hauptstaatsarchivs Stuttgart, in: Archivierung und Zugang. Transferarbeiten des 34. wissenschaftlichen Kurses der Archivschule Marburg, hg. von Nils BRÜBACH (Veröffentlichungen der Archivschule Marburg, Institut für Archivwissenschaft 36, Marburg 2002) 81-109; Heather MAcNeIL, What Finding Aids Do: Archival Description as Rhetorical Genre in Traditional and Web-Based Environments. Archival Science. International Journal on Recorded Information 12 (2012) 485-500; Jennifer MeEHAN, Arrangement and Description: Between Theory and Practice, in: Archives and Recordkeeping (wie Anm. 7) 63-99, hier 80; Elizabeth YAKEL, Archival Representation. Archival Science. International Journal on Recorded Information 3 (2003) 1-25.

77 Hans Hoffman, Archival Standards, in: Encyclopedia of Archival Science (wie Anm. 7) 86-90, hier 87f. Das International Council on Archives (ICA) betreibt maßgeblich die Ausarbeitung und Verbreitung von Verzeichnungsstandards wie ISAD $(\mathrm{G})$ oder zuletzt des neuen, noch in Entwicklung befindlichen Standards Records in Context. Zahlreiche dieser Standards stehen (mehrsprachig) auf der Website des ICA zur Verfügung: International Standards, in: ICA. International Council on Archives, https://www.ica.org/en/public-resources/ standards [1. 3. 2018]. Zum Stand der Umsetzung in Österreich vgl. Karin SperL, Die archivischen ErschlieBungsstandards ISDIAH, ISAD(G), ISAAR(CPF) in der Praxis - Möglichkeiten der Umsetzung. Scrinium. Zeitschrift des Verbandes Österreichischer Archivarinnen und Archivare 70 (2016) 43-57.

$78 \mathrm{Zu}$ Archivportalen bietet eine Momentaufnahme für Deutschland: Netz werken. Das Archivportal-D und andere Portale als Chance für Archive und Nutzung. Beiträge zum 19. Archivwissenschaftlichen Kolloquium der Archivschule Marburg, hg. von Irmgard Christa BeCKeR-Gerald MAIER-Karsten Uhde-Christina Wolf (Veröffentlichungen der Archivschule Marburg, Hochschule für Archivwissenschaft 61, Marburg 2015). 
Aufbereitung. Ob eine solche mit Archivinformationssystemen aktuellen Zuschnitts und der Strukturierung der archivischen Information nach dem Provenienzprinzip erreicht werden kann, muss dabei in Frage gestellt werden ${ }^{79}$, konditionieren doch viel eher Informationsportale wie Google, Wikipedia und Social-Media-Plattformen die Erwartungen hinsichtlich des Umgangs mit Informationen in Suche, Aufbereitung und Vernetzung ${ }^{80}$. Mit diesen Diensten und ihren Oberflächen sind Archivinformationssysteme nicht vergleichbar, und Benutzende reagieren darauf mit Unverständnis und Kritik. Müssen deshalb Archive ihre Disseminationsstrategien überdenken oder die Benutzenden besser mit typisch archivischen Erschließungsmethoden vertraut machen, wodurch es dann eher um ein Kommunikations- denn um ein Performanceproblem ginge ${ }^{81}$ ?

Andreas Kellerhals greift in seinem Beitrag die Metapher vom Archiv als „Leuchtturm“ auf, spinnt sie zum „GPS“ in der „Infosphäre“ fort und begleitet das Archiv auf seinem Weg in die Informationsgesellschaft. Etablierte Ziele archivischer Erschließung, namentlich Findbücher, die oft schon in der Vergangenheit im Archivalltag eher nicht erreichbarer Anspruch denn Erschließungswirklichkeit waren, hinterfragt er hinsichtlich ihrer Gegenwartstauglichkeit und lädt zu fünf „Grenzüberschreitungen“ ein, sodass Archive durch eine Neugestaltung des Suchens und Findens von Informationen ihre Stellung in der Informationsgesellschaft neu gestalten könnten.

Im Zuge der Digitalisierung übernehmen Archive zunehmend Informationen in maschinenlesbarer Form. Reichen die Metadaten nicht aus, um diese Informationen sinnvoll zu erfassen, oder sind Metadaten nur unzulänglich vorhanden, so bieten die Methoden der Computerlinguistik neue Möglichkeiten zur Extraktion wichtiger Aussagen. Sprachtechnologie als eine Subdisziplin der Künstlichen Intelligenz stellt dafür eine Reihe von Algorithmen, Methoden und Werkzeugen zur Verfügung. So können unter anderem Dokumente mit ähnlichen Inhalten identifiziert, Texte nach bestimmten Informationen durchsucht und deren Inhalte auch automatisch zusammengefasst werden. Brigitte Krenn stellt verschiedene sprachtechnologische Verfahren und Werkzeuge vor und weist auf deren Potential für die Anwendung im archivischen Bereich hin.

Anhand eines konkreten Beispiels zeigt Christoph Sonnlechner, wie Archive in Verbindung mit traditionell strukturierten Archivinformationssystemen ihr Informationsangebot erweitern können. Er stellt das Wien Geschichte Wiki vor, das unter Federführung des Wiener Stadt- und Landesarchivs seit 2014 erfolgreich, unter ständig wachsendem

79 Duff-Yakel, Archival Interaction (wie Anm. 55) 209f.; Fowler, Inappropriate Expectations (wie Anm. 75) 58-60; Wendy ScheIR, First Entry: Report on a Qualitative Exploratory Study of Novice User Experience with Online Finding Aids. Journal of Archival Organization 3/4 (2006) 49-85. Aus archivarischer Sicht ist hingegen die in Provenienz und Archivtektonik enthaltene Kontextinformation ein hoher Wert - und diese Sicht wird auch von erfahrenen Forschenden oftmals geteilt: Sylvia Necker, Wenn ich mir was wünschen dürfte. Wunsch(t)raum Archiv für NutzerInnen im digitalen Zeitalter, in: Digitalisierung im Archiv (wie Anm. 24) 117-134, hier 124-126. Zu dieser Problematik siehe den Beitrag von Georg Vogeler.

80 Thomas, The Digital (wie Anm. 67) 71-74.

81 Zum Erlernen archivrelevanter Fähigkeiten durch die Benutzenden und zu dessen Unterstützung von archivarischer Seite vgl. Katud, User Studies (wie Anm. 55) 447-451; Annegret Wenz-Haubfleisch, Fit für den Archivbesuch. Nutzerseminare des Staatsarchivs Marburg im Kontext seiner Öffentlichkeitsarbeit und Serviceorientierung, in: Transparenz für die Bürger? Perspektiven historischer Öffentlichkeits- und Bildungsarbeit in Archiven. Beiträge zum 17. Archivwissenschaftlichen Kolloquium der Archivschule Marburg, hg. von Jens Aspelmeier (Veröffentlichungen der Archivschule Marburg, Hochschule für Archivwissenschaft 57, Marburg 2014) 255-275; Elizabeth Yakel-Deborah A. Torres, AI: Archival Intelligence and User Expertise. The American Archivist 66 (2003) 51-78. 
Zuspruch der Nutzenden, betrieben wird. Im Wiki-Format erfolgt eine vernetzte und themenorientierte Aufbereitung der verfügbaren Informationen, welche die Interessierten letztendlich auch zu relevanten Verzeichnungsrepräsentationen im Archivinformationssystem geleitet - mitunter auch zu dort zur Verfügung gestellten Archivgut-Digitalisaten. Dem Archivinformationssystem bleibt mehr und mehr nur noch die korrekte archivische Nachweisführung überlassen.

Den Potentialen von in den Digital Humanities entwickelten Techniken des Information Retrieval für die Zwecke der archivischen Erschließung widmet sich Georg Vogeler. Sowohl die traditionellen als auch die aktuellsten archivischen Standards unterscheiden sich durch ihr hierarchisches Ordnungsprinzip, beruhend auf der Beständestruktur, erheblich von den meisten heute sonst üblichen Erschließungs- und Retrieval-Techniken, die überwiegend auf der Ebene der Einzelobjekte funktionieren. Vorschlägen, auch die archivische Erschließung zumindest schwerpunktmäßig auf diese Ebene zu verlagern ${ }^{82}$, hält Vogeler entgegen, dass mit den Informationen über Bestände und bestandsbildende Stellen wichtige Orientierungshilfen geboten werden, die dann vernachlässigt würden. Er beschreibt Ansätze, wie beides miteinander verbunden werden könnte, indem etwa die in der klassischen archivischen Beschreibung festgehaltenen Provenienzzusammenhänge in die Reihung der Treffer von Volltext- oder Schlagwortsuchen eingebracht würden. Dazu müssen freilich auch diese Metainformationen in eine maschinell auswertbare Form gebracht werden, etwa indem sie als strukturierte Daten - Relationen zwischen einzelnen Knoten - erfasst werden. Der Standard „Records in Context“ (RiC) bietet ein Beispiel eines dafür entwickelten Datenmodells ${ }^{83}$.

Auch der Beitrag von Miroslav Novak handelt von den Leistungen, Schwächen und Potentialen von Archivinformationssystemen. Novak verweist zunächst auf die Kontinuitäten zwischen traditionellen analog-papierbasierten Informationssystemen und den heute in Verwendung stehenden digitalen Anwendungen. Am Beispiel des slowenischen Archivwesens, das auf jenes anderer Länder gut übertragbar sein dürfte, zeichnet er die Phasen der Entwicklung in den letzten Jahrzehnten nach und macht dabei nicht zuletzt deutlich, dass die „Gleichzeitigkeit des Ungleichzeitigen“ auch für die Findbehelfe gilt. Neue Systeme sind in so rascher Folge verfügbar geworden, dass stets nur kleine Teile der vorhandenen Information in das gerade aktuelle System eingespeist werden konnten und somit faktisch Instrumente auf höchst unterschiedlichen technischen Entwicklungsstufen nebeneinander benutzt wurden und werden. Inkompatibilitäten zwischen den Lösungen verschiedener Staaten oder einzelner Archive, die Vielzahl verwendeter Sprachen und Standards sowie das Fehlen akkordierter Schlagwortlisten und Normdatensätze ${ }^{84}$ sind weitere Faktoren, die dem Aufbau internationaler Portalverbünde hinderlich sind; die oft unerfahrenen Benutzenden werden mit Suchresultaten konfrontiert, die in teils höchst zufälliger Weise selektiv sind, was aber auf den ersten Blick nicht immer zu erkennen ist. Novaks Konklusion ist ein entschiedenes Plädoyer für die Erarbeitung und Durchsetzung gemeinsamer Standards, damit die europäische archivische Community der Öffentlichkeit ebenso leistungsfähige wie benutzungsfreundliche Gelegenheiten zum Auffinden und

${ }^{82}$ Geoffrey Yeo, Contexts, Original Orders, and Item-Level Orientation. Responding Creatively to Users' Needs and Technological Change. Journal of Archival Organization 12 (2015) 170-185.

83 Bogdan Florin Popovici, Records in Contexts. Towards a New Level in Archival Description? Tehnični in vsebinski problemi klasičnega in elektronskega arhiviranja 15 (2016) 13-31, http://www.pokarh-mb.si/uploaded/datoteke/Radenci/radenci_2016/013-031_popovici_2016.pdf [1.3. 2018].

${ }^{84} \mathrm{Vgl}$. Ziwes, Archive als Leuchttürme (wie Anm. 10). 
Einsehen von Archivgut bieten kann. Mit der Perspektive der Benutzenden ist auch bereits das Thema der letzten Sektion angesprochen.

\section{Das Archiv in seiner Umwelt}

Archive übernehmen mit ihrem Engagement in der digitalen Welt eine Erweiterung ihres Aufgabenbereichs; Archivarinnen und Archivare müssen ihre Fähigkeiten verbreitern, sich eventuell im Arbeitsalltag auf gewisse Kernbereiche konzentrieren und spezialisieren. Blickt man auf „die Archive“, muss aber konstatiert werden, dass ohnehin wohl nicht von „dem Archiv“ gesprochen werden kann - bestenfalls als Idee, die aber gleichfalls nicht vor Veränderungen gefeit ist oder jemals war. Ein Archiv als reale Institution kann und konnte wohl nie in allen archivischen Betätigungsfeldern, die möglich und erwünscht sind, tätig sein: Archivierung von Informationen aller Erscheinungsformen und auf allen Trägermedien, historische Forschung, Vermittlung an ein außerwissenschaftliches Publikum und so fort. Werden Archive sich also in Abhängigkeit von ihrem Umfeld - von Erwartungen des Archivträgers, jenen des gesellschaftlichen Umfelds und speziell der Archivbenutzenden, oder auch in Fortschreibung einer traditionellen Rolle spezialisieren müssen: als „Bürgerarchiv“" ${ }^{\text {, }}$, Forschungsarchiv, Archive für bestimmte Archivguttypen oder in noch andere Richtungen? Dafür scheint zu sprechen, dass das „Universalarchiv“ schon vom Anspruch her nicht leistbar ist, respektive ein Archiv in der Praxis angesichts immer gegebener Ressourcenknappheit zwar alles wahrzunehmen versuchen kann, aber in Folge dann die Qualität unzumutbar nach unten gedrückt wird. Gilt es in dieser Hinsicht vor allem zu unterschieden zwischen „kleinen“ und „großen“ Archiven? Oder kann man eben ohnehin nicht von „dem Archiv“ sprechen, vielleicht nicht einmal Archive der gängigen Kategorien - Landesarchive, Kommunalarchive, kirchliche Archive und so fort - in ihrer Ausrichtung, ihrer Schwerpunktsetzung, ihren Problemen gleichsetzen, da jedes Archiv für sich im Spannungsfeld von Tradition und Reaktion auf Umweltbedingungen steht, also individuell sein Profil aus seinem Umfeld heraus entwickelt hat und ständig weiterentwickelt? Was ist dann eigentlich ein „Archiv“? Was sind die Kernaufgaben, die ein Merkmal aller Archive sind, oder gibt es selbst solch einen gemeinsamen Nenner nicht? Welche Prioritäten müssen gesetzt werden, um Erfolg zu haben? Was zeichnet ein erfolgreiches Archiv aus, gegenüber welchen Adressatinnen und Adressaten mit welchen Erwartungen ist man erfolgreich, und wie kommuniziert man Erfolg?

Die Vorträge der vierten Sektion der Tagung nahmen jeweils Bezug auf einzelne der gerade aufgeworfenen Fragen zum Wesen des Archivs, der Archivwissenschaft, des Archivbegriffs oder des archivischen Tuns. Drei von vier Vorträgen sind in diesem Tagungsband als Beiträge vertreten. Leider ist der Vortrag des Erstredners der Sektion, des britischen Historikers Patrick Joyce, nicht im Sammelband wiedergegeben. Joyce setzte mit seiner kulturhistorischen Sichtweise einen Kontrapunkt ${ }^{86}$. In seinen Überlegungen zum Wesen

85 Zum Konzept des „Bürgerarchivs“ vgl. Volker Hirsch-Jens Aspelmeier, Zur Einführung: Archivdidaktik als Aufgabe des Bürgerarchivs, in: Transparenz für die Bürger (wie Anm. 81) 13-30, hier 15-21; Andrea Wendenburg-Max Plassmann, Fachkonzept für das Historische Archiv der Stadt Köln bis zum Jahr 2050. Mitteilungen aus dem Stadtarchiv von Köln 100 (2014) 63-114, hier 75-80.

86 Der Vortrag vertiefte Ansätze, die bereits in früheren Publikationen vorgestellt wurden: Patrick JoyCE, The State of Freedom: A Social History of the British State since 1800 (Cambridge u. a. 2013); Ders., Filing the Raj (wie Anm. 38). 
des Staates und zu dessen Funktionsweise sieht er den Staat als Anordnung (,assemblage“) verschiedener Handlungsträger. Ein staatlicher Handlungsträger ist auch dessen Archiv, doch für Joyce ist der Staat im Grunde ein Archiv für sich, da man im Archiv nachvollziehen kann, wie bürokratische Autorität und somit Staatsgewalt mithilfe bürokratischer Schriftlichkeit, Schriftgutverwaltung sowie Archivierung etabliert wird ${ }^{87}$. Archive und das in ihnen verwahrte Archivgut spielen bei der Konstruktion von Wirklichkeiten eine zentrale Rolle, beziehungsweise bietet Archivierung auch die Möglichkeit des Ausblendens derselben.

Seit nunmehr über zwei Jahrzehnten ist der Archivbegriff Teil des Begriffsrepertoires der Kultur- und Geisteswissenschaften. Professionelle Archivarinnen und Archivare stehen so mancher nunmehr gebräuchlichen Begriffsverwendung oft skeptisch, aber - und in ähnlicher Weise eröffnet der Archivar Dietmar Schenk seinen Beitrag - auch ohnmächtig gegenüber. Verlieren sie - Schenk geht von der Situation in Deutschland aus, die auch für Österreich angenommen werden kann - aber gänzlich die Definitionshoheit über den Archivbegriff, schwächt dies die Konturierung der eigenen Tätigkeit, der eigenen Institution, was gerade in Zeiten des Postfaktischen fatal erscheint. Schenk plädiert für eine Ausrichtung der Archivwissenschaft, die durch die Kombination von praktischen, theoretischen und historischen Fragestellungen und Zugängen gekennzeichnet ist.

Um die Sichtbarmachung des Archivs aus ganz anderer Perspektive geht es in weiterer Folge Joachim Kemper. Greift er im Titel seines Beitrags noch die „Staubecke“ als für das Laienpublikum gängige Assoziation mit dem Archiv auf, so führt er im Weiteren vor Augen, wie mithilfe gezielter Öffentlichkeitsarbeit, gestützt auf digitales Angebot und sozial-mediale Kommunikation, Wege aus dieser Ecke heraus gefunden werden können, sodass Archive im Vergleich mit anderen Kulturgut verwahrenden Institutionen konkurrenzfähiger werden ${ }^{88}$.

Die Reihe der Vorträge und so auch der daraus resultierenden Beiträge wird von jenem von Mario Glauert abgeschlossen, der - so der Autor einleitend - als Titel mit einer einfach formulierten, aber schwierig zu beantwortenden Frage überschrieben ist: Was ist ein erfolgreiches Archiv ${ }^{89}$ ? Kennzahlen, Effizienz und Effektivität, Qualitätskriterien, Produkte, Prozesse - alles Schlagwörter, die Archivarinnen und Archivare in ihrem Berufsalltag mittlerweile begleiten. Doch anstatt mithilfe der hinter diesen Begriffen stehenden, oftmals wenig aussagekräftigen Kenngrößen das „erfolgreiche Archiv“ zu konstruieren,

87 Zum Zusammenhang von Staatsbildung und Archivierungspraktiken vgl. Karin GotтschaLK, Wissen über Land und Leute. Administrative Praktiken und Staatsbildungsprozesse im 18. Jahrhundert, in: Das Wissen des Staates. Geschichte, Theorie und Praxis, hg. von Peter Collin-Thomas Horstmann (Rechtspolitologie 17, Baden-Baden 2004) 149-174, hier 159-166.

${ }^{88}$ Zum Einsatz der sozialen Medien durch Archive vgl. Wendy M. Duff-Catherine A. Johnson-Joan M. Cherry, Reaching Out, Reaching In: A Preliminary Investigation into Archives' Use of Social Media in Canada. Archivaria. The Journal of the Association of Canadian Archivists 75 (2013) 77-96; Duff-Yakel, Archival Interaction (wie Anm. 55) 204f.; Kate Theimer, Interactivity, Flexibility and Transparency: Social Media and Archives 2.0, in: The Future of Archives (wie Anm. 3) 123-143.

${ }^{89}$ Die Frage nach dem „guten“ Archiv ist die klassische Ausgangsfrage des Archivmanagements: Mario Glauert-Hartwig Walberg, Einleitung, in: Archivmanagement in der Praxis (wie Anm. 22) 7-11, hier 9; Andreas Hedwig, Moderne Steuerungsinstrumente in den Archiven - Fluch oder Chance? Versuch einer Standortbestimmung, in: Ziele, Zahlen, Zeitersparnis. Wie viel Management brauchen Archive? Beiträge zum 20. Archivwissenschaftlichen Kolloquium der Archivschule Marburg, hg. von Irmgard Christa Becker-Dominik Haffer-Valeska KoAl (Veröffentlichungen der Archivschule Marburg, Hochschule für Archivwissenschaft 63, Marburg 2016) 13-58, hier 39-43. 
macht Glauert auf eine viel nützlichere Sichtweise auf „das Archiv“ aufmerksam: das Archiv, das Nutzen und Wirken zeigen kann - verstanden in einem breiten gesellschaftlichen Sinn, der zwar schwieriger empirisch nachzuweisen ist als betriebswirtschaftliche Kennzahlen, aber letztlich maßgeblich für Urteile über die Existenzberechtigung, die gesellschaftliche Stellung und die Ausstattung von Archiven sein muss.

\section{Abschluss}

Am dritten Tag der Jahrestagung 2016 und nach insgesamt an die zwanzig Vorträgen, einer Filmvorführung, einem Festvortrag mit Podiumsdiskussion sowie durchgehend aktiver und anregender Besprechung der Vortragsinhalte ließ Gerhart Marckhgott, ehemals Direktor des Oberösterreichischen Landesarchivs, das Gehörte nochmals Revue passieren $^{90}$. Er tat dies zunächst, indem er die angesprochenen Themen und die häufigsten genannten Begriffe in Beziehung zueinander setzte, damit vernetzte und vor den Augen der Zuhörenden die Inhalte der Tagung als Beziehungsgeflecht, als semantisches Netz, erstehen ließ. Zwei Begriffe, die selbst stark in Verbindung miteinander stehen und im Beziehungsgeflecht eng beieinander liegen, ließ Marckhgott dabei besonders hervortreten: Zugang und Transparenz. Zugang und Transparenz sind jene zwei Konzepte, die man mit der Mehrzahl der vorgetragenen Inhalte in Verbindung bringen konnte, wobei es keineswegs nur um die transparente Gestaltung des Zugangs zu mittels Archivgut archivierten Informationen geht. Transparenz, die Möglichkeit und Fähigkeit, archivarisches Handeln möglichst lückenlos darstellen zu können ${ }^{91}$, betrifft beispielsweise genauso die Bereiche der archivischen Bewertung und Erschließung, auf welche wieder eine nachvollziehbare Zugänglichmachung und damit Nutzung aufbauen kann.

Das Archivpersonal sieht Marckhgott gefordert, mit Interesse und Neugier Entwicklungen zu verfolgen und auf seinen Arbeitsbereich anzuwenden, um die in den Archiven vorgehaltenen Informationen mit immer weniger Barrieren und Aufwand Benutzenden zugänglich zu machen. Idealerweise würden Archive ihre archivierten Informationen proaktiv den Benutzenden überall und immer dann zur Verfügung stellen, wenn diese Archiv-Informationen Interessen befriedigen, Fragen beantworten können.

Es wäre vermessen zu behaupten, die Tagung beziehungsweise dieser daraus resultierende Tagungsband hielte nun alle Antworten zu den eingangs gestellten Fragen bereit. Insofern wäre auch die Funktion von Impulsfragen falsch verstanden worden. Impulse wollten wir setzen, um die Gedanken der Vortragenden in eine bestimmte Richtung zu lenken und alle Zuhörenden in das Generalthema zu involvieren. Letztendlich geht es um einen Weg, der dadurch etwas weiter nach vorne ausgeleuchtet werden sollte, und inso-

90 Siehe zusammenfassend auch zwei mittlerweile erschienene Tagungsberichte: Robert KrETzsCHMAR, Archivalische Quellenkunde, archivische Arbeitsfelder, archivarische Fachkompetenzen. Zur Jahrestagung des Instituts für Österreichische Geschichtsforschung 2016. Archivar. Zeitschrift für Archivwesen 70 (2017) 208-210; Georg GäNSER, Tagungsbericht: Die Zukunft der Vergangenheit in der Gegenwart. Archive als Leuchtfeuer im Informationszeitalter, in: H-Soz-Kult (5. 1. 2017), http://www.hsozkult.de/conferencereport/id/tagungsberichte-6905 [1. 3. 2018].

${ }_{91}$ Zur nachvollziehbaren und transparenten Archivierung als vertrauensbildende Notwendigkeit, mit weiterführender Literatur vor allem aus der englischsprachigen Archivlandschaft, vgl. GäNSER, Archive (wie Anm. 9). Einen besonders weitreichenden Anspruch auf die umfassende Dokumentation archivischen Handelns formuliert Thomas Nesmith, Documenting Appraisal as a Societal-Archival Process: Theory, Practice, and Ethics in the Wake of Helen Willa Samuels, in: Controlling the Past (wie Anm. 4) 31-50. 
fern setzte für viele Teilnehmende die archivwissenschaftliche Jahrestagung 2016 ebenfalls einen Impuls zum Weiterdenken des Gehörten. Wir hoffen, dass nun auch der Tagungsband bei allen Lesenden diesen Effekt haben wird. 


\title{
The Right to Be Remembered and the Duty to Memorialize
}

\author{
The Role of Archives in an Increasingly Networked Society
}

\author{
Luciana Duranti
}

Archives are regarded as the trusted custodians of recorded evidence and memory. Since Roman antiquity, the place where societal records are kept has been as important to their authenticity and reliability as the knowledge of the professionals responsible for them. The sense of the importance of the place of preservation for the trustworthiness of archival materials has been transmitted to us through the mediation of Roman law and has since permeated the Western world's understanding of the functions of archival institutions. Emperor Justinian's Corpus Juris Civilis, which is the summa of Roman law and jurisprudence, refers to archives at various points as the public place where deeds are deposited $^{1}$, which assured that they remained uncorrupted ${ }^{2}$, provided trustworthy evidence $^{3}$, and constituted a continuing memory of what they attested ${ }^{4}$. In ancient Rome, the archives was a place of preservation under the jurisdiction of a public authority. This place, public as well, endowed the records that passed its threshold with trustworthiness, thereby giving them the capacity of serving as evidence and continuing memory of facts and acts.

The archives of the Roman republic, the Tabularium, rose on the Capitoline Hill as the imposing terminal point to the Forum, higher than the Senate, closer to the Courts than any other building, surrounded by the markets and the temples, the point of reference for anyone walking through the city, and the beating heart of the res publica. It preserved the records as well as the legal, jurisprudential, literary, and theatrical writings of Rome; it contained written evidence and memory of the people for the people; it was a permanent, unforgettable reminder of whom allegiance and accountability are owed to, and of the achievements of the people of Rome.

\footnotetext{
1 Dig. 48.19.9.6, cit. Iustiniani Digesta, ed. Theodor Mommsen, in: Corpus Iuris Civilis, 1: Institutiones - Digesta, ed. Paul KrÜGer-Theodor Mommsen (Berlin ${ }^{7} 1895$ ) 814: eo loci [...], quo in publico instrumenta deponuntur, archivo forte vel grammatophylacio.

${ }^{2}$ Nov. 15.5.2, cit. Corpus Iuris Civilis, 3: Novellae, ed. Rudolf Schöll-Wilhelm Kroll (Berlin ${ }^{5} 1928$ ) 113: habitatio quaedam publica [...], in qua conveniens est defensores monumenta recondere, [...] quatenus incorrupta maneant haec et velociter inveniantur a requirentibus.

3 Nov. 15 Praef., ibid. 110: cum nullum habeant archivum, in quo gesta apud se reponant, [...], quae quidem inveniunt, nulla fide sunt digna.

${ }^{4}$ Cod. I.4.30.2, cit. Corpus Iuris Civilis, 2: Codex Iustinianus, ed. Paul Krüger (Berlin $\left.{ }^{8} 1906\right)$ 46: gesta in ipsis sacrosanctae ecclesiae archivis deponi sancimus, ut perpetua rei memoria sit.
} 
This inextricable connection between the place of preservation and the trustworthiness of the records was upheld until the 17th century. In 1664, a German jurist, Ahasver Fritsch, specified that records did not acquire trustworthiness simply by crossing the archival threshold, but from the fact that 1) the place to which they were brought belonged to a public sovereign authority, as opposed to its agents or delegates; 2) the officer forwarding them to such a place was a public officer; 3) the records were placed both physically (i. e., by location) and intellectually (i. e., by description) among authentic records; and 4) this association was not meant to be broken ${ }^{5}$. The archival right, that is, the right to maintain a place capable of conferring trustworthiness, and therefore authority, to the documentary by-products of activities, was in time acquired by all those bodies to which sovereignty was delegated by the highest secular and religious powers - among these, cities and churches. In medieval times, corporations of every kind, including universities, deposited the records of their activities in the camera actorum (chamber of the acts) of the municipality having jurisdiction over them or in the archives chests of ecclesiastical institutions, before themselves acquiring the right to keep archives ${ }^{6}$.

This remained the state of the affairs until the French Revolution, when, with the law of 7 Messidor, Year II (25 June 1794), the records of defunct institutions and organizations, concentrated in the National Archives of France, were declared the patrimony of the nation and made accessible to the public. By virtue of this declaration, the State recognized its duty to preserve them on behalf of its citizens in order to ensure universal access. Since 1808, the place of preservation of such records, as well as those subsequently acquired by the national institution, has been a monumental edifice, built to remind the people of their past and their right to the documentary evidence and memory of it: it is the locus of recognition and empowerment, giving records their identity and authority, because, at the threshold of the archives, a trusted third party (the archivist) authenticates them and takes responsibility for them, thereby transforming them into sources, and later contextualises them, thereby perpetuating their identity and preserving their integrity.

Thus, by the 1794 decree, a dichotomy was born between records and archives management; current and historical records became two distinct bodies of material and began to be controlled according to separate methods and regimes, with a hiatus often forming between them. Creators, once they no longer needed specific records for current business, would stop managing them and ensuring their reliability and authenticity, but often merely stored them for decades, until old age transformed them into sources for history to be entrusted to a centralised archival institution and open to the public. This dichotomy between administrative and historical archives can still be observed in countries like the United States and Germany, which continue to link the type of material to the place where it is kept, also by using different terminology to refer to records kept by the creator and records kept in an archives (e. g. „records“ versus „archives“ or „archival records"). Elsewhere the concept of archives came to be increasingly linked to the material itself, rather than to the place where it was kept or the uses to which it was put, as revealed by the definitions provided by the Dutch archivists, Sir Hilary Jenkinson, Eugenio Casanova, and many other archival writers of the 20 th century $^{7}$.

\footnotetext{
5 Ahasver FrITSCH, Tractatus de iure archivi et cancellariae (Jena 1664).

${ }^{6}$ Elio Lodolıni, Lineamenti di storia dell'archivistica italiana. Dalle origini alla metà del secolo XX (Beni culturali 13, Roma 1991) 43

7 Luciana Duranti, Archives as a Place. Archives and Manuscripts 24 (1996) 242-255; Ernst Posner,
} 
Regardless of this clear conceptual separation between archives as bodies of records and archives as institutions holding them, the 1991 article by David Bearman, „An Indefensible Bastion. Archives as a Repository in the Electronic Age ${ }^{\text {"8 }}$, significantly shook the established understanding of archives, claiming that the existence of archival institutions or organizations having the physical and legal custody of archives had become unnecessary, due to conditions that no longer existed, that is, the need to protect the physical integrity of the records, the economic advantage of concentrating permanent records in one place, and the benefit to users of having related materials accessible in the same dedicated place. Bearman's proposal was for archivists to leave electronic records with their creators, as they could be easily copied and made accessible on disc, and limit their responsibilities to keeping paper records and providing creators with directions for preservation and dissemination of electronic records, monitoring the way in which they carried out such activities.

Some archival commentators readily accepted this scenario casting archivists in the role of auditors, and considered archival acquisition a measure of last resort, to be undertaken only in situations in which records creators are defunct, on the grounds that electronic records should remain in their native environment to keep their integrity, the cost of electronic preservation for archival institutions was too high, and archivists lacked the variety of expertise required by the many different proprietary systems put in place by the creators9. This trend, which came to be called „postcustodialism“, found much support in Australia ${ }^{10}$, but was not generally accepted by the international archival community ${ }^{11}$, and appeared to have been definitely buried in 1998 by the renaming of the Australia Commonwealth Archives Office „National Archives of Australia“ and the attribution to it of the primary mandate of „preserving and making publicly available the archival resources of the Commonwealth" ${ }^{12}$, regardless of medium.

These developments were entirely predictable, because the postcustodialist idea was based on four false premises. The first was that the reason why public archives have custody of public records is custody in its own right. This premise is easily disproved by a study of the historical development of archives, which shows that the primary justifica-

\footnotetext{
Some Aspects of Archival Development Since the French Revolution, in: IDEM, Archives and the Public Interest. Selected Essays, ed. Kenneth W. Munden (Washington 1967) 23-35; Samuel Muller-Johan Adriaan Feith-Robert Fruin, Manual for the Arrangement and Description of Archives [1898], trans. Arthur H. Leavitt (New York 1940); Hilary Jenkinson, A Manual of Archive Administration [1922] (London ${ }^{3} 1968$ ); Eugenio Casanova, Archivistica (Siena 1928); Terence Gordon Cook, What is Past is Prologue: A History of Archival Ideas Since 1898, and the Future Paradigm Shift. Archivaria. The Journal of the Association of Canadian Archivists 43 (1997) 17-63.

8 David Bearman, An Indefensible Bastion. Archives as a Repository in the Electronic Age, in: Archival Management of Electronic Records, ed. David Bearman (Archives and Museum Informatics Technical Report 13, Pittsburgh 1991) 14-24.

9 Charles M. Dollar, Archival Theory and Information Technologies. The Impact of Information Technologies on Archival Principles and Methods (Informatics and Documentation Series 1, Macerata 1992) 53.

${ }_{10}$ Glenda Acland, Archivist - Keeper, Undertaker, or Auditor? Archives and Manuscripts 19 (1991) 9-15, at 13s.; Frank Upward-Susan M. McKemmish, Somewhere Beyond Custody. Archives and Manuscripts 22 (1994) 136-149, at 138-147.

11 Kenneth Thibodeau, To Be or Not to Be: Archives for Electronic Records, in: Archival Management of Electronic Records (cit. n. 8) 1-13; Terence M. Eastwood, Should Creating Agencies Keep Electronic Records Indefinitely? Archives and Manuscripts 24 (1995) 256-267.

12 Archives Act 1983 [with amendments up to 2014], Part I, 2A.a.ii, in: Australian Government. Federal Register of Legislation, https://www.legislation.gov.au/Details/C2014C00417 [1. 3. 2018].
} 
tion for physical and legal custody is historical accountability: the citizens have a right to access the authentic documentary evidence of how they were governed, and for this to happen, the records must be in the physical and legal custody of a neutral third party, not merely under its intellectual control. As Ken Thibodeau wrote: „archival retention seeks to preserve the connection between records and the organization which created them, recognizing that an essential part of the meaning of the records is conveyed by information about how the organization used those records, and how it organised them in order to support its uses. If this essential part of the meaning is lost or diminished, the records, as such, would be lost. "13 The second premise, that archivists would professionalise their otherwise unrecognised role by acting as auditors, is easy to dismiss on the basis of the abundant evidence of archival professionalism. The third premise was that it would be more economical and trustworthy to keep the material in the original systems rather than to recreate such environments in an archival institution. Technological obsolescence disproves such an assumption, as electronic records, in order to remain accessible, have to be moved to a new digital environment on a regular basis. Moreover, it would be more economical to conduct periodic mass migration of all digital materials in an archival institution, for preservation purposes, than to migrate the records within the different systems maintained by each different creator, maintaining all the original functionalities. In other words, substituting trust in the original environment and in its integral reproduction in subsequent systems with trust in the archivists operating in one unified technological environment, built according to professional archival standards, is a better choice, also because it would eliminate redundancies of both records and system documentation. The fourth and final premise was that it would be easier for users to access, manipulate and extract records if they were given access to the original system than after the records had been transferred to an archival system and processed by the archivist. This last premise can be rejected on the basis of the same argument used for the first premise: without the measures taken by the archives to ensure the protection of the identity and integrity of the records and to establish intellectual control over them by making explicit all their contexts (i. e. the juridical-administrative, provenancial, procedural, documentary and technological contexts), the records do not have much value to their users ${ }^{14}$. Thus, it can easily be seen why the abandonment of custody by the archives was finally seen as unacceptable.

One of the reasons why the dichotomy between paper and digital records preservation does not work is that digital information is physical as well, because it resides in very material servers, though at the same time it is ubiquitous, due to its redundancy, which is often involuntary, but is also used as a key preservation strategy. It is possible to preserve digital material in one central place, and in many cases it is desirable: trusted digital repositories (TDRs) are particularly appropriate when data privacy or confidentiality of information are at issue, for example. TDRs are, however, complex technologies that must be managed by professionals with specific and costly qualifications. Furthermore, there are few „trusted“ repositories that are „trustworthy“. In fact, regardless of certification, which very few are able to achieve, TDRs appear to be trusted only when they are maintained in a place of preservation like an archives ${ }^{15}$.

13 Thibodeau, To Be or Not to Be (cit. n. 11) 2.

14 Eastwood, Agencies (cit. n. 11).

15 Devan Ray Donaldson-Paul Conway, User Conceptions of Trustworthiness for Digital Archival Documents. Journal of the Association for Information Science and Technology 66 (2015) 2427-2444. 
Online places of preservation are also physical: they are places in between those who provide access to archival material (the providers) and those who access it (the users). They are data centres, whose general name is the Cloud. The Cloud is defined as a „broad range of infrastructures and services distributed across a network (typically the Internet) that are scalable on demand and that are designed to support management of high volumes of digital materials"16. Many organizations and individuals generate and/or keep their records in the Cloud because access is possible from any location to anyone who can use a browser, a trusted digital repository satisfying international standards is not affordable, the knowledge to deal with records produced by and in complex technologies and with their cycles of obsolescence is not easily available and very expensive, and security measures are stronger. However, the place where records reside is an issue. It is natural to think of the Cloud as cyberspace - in our mind, a virtual space. This idea comes from a refusal to accept the physical extension of digital information in computer terminals and other machines, but "real-virtual dualism is nothing more than a fiction“, states PJ Rey, specifying that virtual spaces are places of separation rather than of communication and learning, unless they can be centralised under trusted control ${ }^{17}$.

The many issues related to storing records in the Cloud have been reduced to a question of trust, both technological and social. Traditionally, people's and organizations' trust in records and archives is based on four types of knowledge about their creator and/or their preserver: reputation, which results from an evaluation of the trustee's past actions and conduct; performance, which is the relationship between the trustee's present actions and the conduct required to fulfill his or her current responsibilities as specified by the truster; competence, which consists of having the knowledge, skills, talents, and traits required to be able to perform a task to any given standard; and confidence, which is an assurance of expectation of action and conduct the truster has in the trustee ${ }^{18}$. In general, trust may be defined as the confidence of one party in another, based on an alignment of value systems with respect to specific actions or benefits, and involving a relationship of voluntary vulnerability, dependence and reliance, based on risk assessment ${ }^{19}$ : the four types of knowledge necessary for establishing trust in records and archives are reflected in this definition of the trust relationship.

The greatest concern in storing records in the Cloud is location independence, which is a defining feature of the Cloud and allows for the highest security and economy. One may wonder to whom the place where records reside matter, if to anyone. Certainly it matters to governments, because of data sovereignty, involving data protection; to citizens in democratic societies, as they are guaranteed the certainty of their rights; and to society at large, as it relies on the authenticity, contextualization, and authority of written evidence and memory. Several governments have issued geographical location restrictions

16 Art. Cloud [en], in: InterPARES Trust Terminology Database, http://arstweb.clayton.edu/interlex/en/ term.php?term=cloud [1.3. 2018].

17 PJ Rey, The Myth of Cyberspace. The New Inquiry 3 (April 2012), http://thenewinquiry.com/essays/ the-myth-of-cyberspace/ [1.3. 2018].

18 Jennifer Borland, Trusting Archivists. Archivi e Computer. Automazione e beni culturali 19 (2009) 94-106; Luciana Duranti-Corinne Rogers, Educating for Trust. Archival Science. International Journal on Recorded Information 11 (2011) 373-390; Piotr Sztompкa, Trust. A Sociological Theory (Cambridge-New York 1999).

19 Art. Trust [en], in: InterPARES Trust Terminology Database, http://arstweb.clayton.edu/interlex/en/ term.php?term=trust [1.3. 2018]. 
for data centres; providers have issued data residency taxonomies to address such laws and policies; and archival professionals have resumed the custodialism/postcustodialism debate.

Storing the records in a Cloud environment is in fact regarded by many archivists as a form of postcustodialism. In fact, the motivations on which the idea is based are similar to those of the original movement: complexity of technologies; need for specialised and rapidly upgradable professional knowledge; economies of space, technology, and human resources; and easy access from anywhere in the world. Except that the present scenario is very different from the old one: while it does require that archives abandon physical custody, such responsibility is not given to the same entity (i. e. Cloud providers rather than records creators) or for the same material (i. e. the archival holdings acquired from the creators after appraisal, and processed by archivists, rather than the records held in their systems by the creators); and not for the performance of the same functions (i.e. storage, security, and access through the Internet rather than appraisal, arrangement, description and dissemination). Clearly, while the original postcustodialism was a form of distributed physical and legal custody of archival material, whose care would be entirely entrusted to those who have the highest interest in modifying or destroying it when it does not serve the image they wish to project of themselves (the creators), the new postcustodialism requires that the centralised legal custody and intellectual control responsibilities be left with the archives, but it delegates physical custody and technological access provisions to the Cloud provider, which can be the archives itself (private Cloud), an archival community (community Cloud), a commercial provider (public Cloud), or a mix of the three (hybrid Cloud) ${ }^{20}$.

Thus, entrusting archival material to a Cloud environment is the result of a compromise between archival preservation requirements and economic, security, and remote access pressures: the material is physically secure with the providers and under the legislated jurisdiction, and is accessible from anywhere via a browser. However, how certain are we of the records' availability and reliability?

Legislation in North America and Europe guarantees the right to information held by public bodies, and sometimes also by private organizations, and this information must be provided within a specific period of time. When the data are stored in a cloud environment, ,availability of the stored data implies also the availability of the infrastructure, hardware and software, which facilitates the retrieval and readability of the data", because technical difficulties might slow the process, and the owner of the data, being liable for providing access to them, may be sanctioned ${ }^{21}$. Where "availability“ is „the amount of time that a system is expected to be in service“, which can be expressed either statistically or as a percentage, „reliability " is the characteristic of behaving consistently with expectations. Thus, when storing records in the Cloud, one must consider not only availability but also "consistency and accuracy of access" 22 . This means that copies of the records must be distributed across several data centers - thereby ensuring redundancy, but also

${ }^{20}$ Luciana Duranti, Archival Science in the Cloud Environment: Continuity or Transformation? Atlanti. Review for Modern Archival Theory and Practice 23 (2013) 45-52.

21 Jessica Bushey-Marie Demoulin-Robert McLelland, Cloud Service Contracts: An Issue of Trust. The Canadian Journal of Information and Library Science 39 (2015) 128-153, at 137s.

22 William Lenr, Reliability and the Internet Cloud, in: Regulating the Cloud: Policy for Computing Infrastructure, ed. Christopher S. Yoo-Jean-François Blanchette (Information Policy Series, Cambridge [Massachusetts]-London 2015) 87-113, at 95. 
that such copies must remain consistent while users access the same records at the same time. This is not currently possible as „providers do not have explicit agreements with each other that help ensure the reliability of the overall Internet“. The latter will „require collaboration among multiple regulatory authorities [...] as well as key stakeholders", including service providers, users, „security/public safety communities and international trade and standardization communities" 23 . In the meanwhile, an archives using a Cloud environment for its holdings should inquire whether a provider has architecture and business processes that give some assurance of reliability and credible response strategies if a problem occurs, and whether it is audited by some authority.

With all the above said, even if availability and reliability were ensured, one cannot help wondering whether the location of the servers containing the records is in fact the place that matters to society. The sense of separation remains: by storing archival holdings in the Cloud communication may be faster, less taxing, and more user-friendly, but the wall of impenetrability is becoming thicker. People do not feel that they matter. The cost of upholding separation is letting people become disassociated with their society and their past. To avoid it, archival institutions need to make clear the distinction between archival preservation and storage and access.

The place of preservation is the locus where a neutral third party carries out the functions of identification, authentication, legal and intellectual control of the archival material, providing it with authority, and transforming it into a source of evidence and memory. It is also the place where the rights of the people are protected, for example, by keeping the records containing personal data in a dark trusted repository, rather than online, and ensuring that descriptive instruments for retrieval do not infringe those same rights to oblivion, privacy, benefits from exploitation of data, and economic gain that archives are supposed to protect. Finally, it is the place where historical accountability is served by stewardship of freedom of information, transparency, and the duty to keep evidence of actions and of their motivations. For the institutions of our societies to maintain their moral accountability in the court of the people, in addition to their legal accountability in a court of law, and for the people to remain associated with their past, their collective memory and culture, we still need visible imposing archival repositories close to the center of the city, to the offices of the authority, the public market and the religious sites. Now more than ever, archival institutions must be the pulsating heart of civic life, a point of reference and a symbol, active participants in the everyday vicissitudes of the common people.

Archival institutions are places vital to nurturing civic skills, providing a physical hub where the citizenry meet and share knowledge. Archives are key to forming a national and cultural identity where it does not exist, and to nurturing it where it does. Archives as places are essential instruments for fostering pluralism, diversity, and public cohesion, as well as empowering the disenfranchised and enabling them to affirm their own identity as they perceive it.

We experience alienation when social structures, by imposing some form of separation, disrupt the natural relations through which beliefs and values are upheld and understood and human institutions supported. Records become evidence and memory when preserved, accessed, recognized, empowered, shared, promoted, and passed on as a trusted social capital to the next generation. Today it is possible to reliably store and give access

\footnotetext{
23 Ibid. 100s.
} 
to records online but, without a physical place where people can exercise their role of citizens, archival material is only information.

Records attesting to actions and events are also called „muniments“. The word derives from munimentum, the Latin term for a bastion, a protective monument, and its root is the same as that of „monument“. Monuments/archives preserving and controlling muniments/records of the past will inspire those living today and tomorrow. Memorializing the past makes it visible and pervasive and will inspire civic values in the new generations. This is why I believe that the role of archival institutions in the future will be that of arenas for public debate, promoters of democracy, nurturers of free opinions and civic skills, places to share and learn the muniments and foundations of our society. 
KATEGORISIERUNG - NEUE QUELLENKUNDE IM ARCHIV 



\title{
Archivalische Quellenkunde im frühen 21. Jahrhundert
}

\author{
Ein „Kleines Fach“ mit potentiell großer Wirkung
}

\author{
Robert Kretzschmar
}

Ende 2015 hat das Ministerium für Wissenschaft, Forschung und Kunst Baden-Württemberg eine „Landesinitiative Kleine Fächer“ gestartet und zunächst einen „Zukunftsrat Kleine Fächer" gebildet ${ }^{1}$. Er soll Konzeptionen und Maßnahmen für den Erhalt und die Weiterentwicklung der sogenannten Kleinen Fächer entwickeln, die als „strukturprekäre Disziplinen“ an den Hochschulen definiert wurden; das bedeutet, dass ihr Fortleben unter den aktuellen Bedingungen gefährdet ist. „Wir sind auf die Kleinen Fächer dringend angewiesen. Sie gehören zum Kern der Wissenschaft, sie sind elementar für die Grundlagenforschung und für die Vielfalt des Denkens. Mit der Landesinitiative werden wir ihre Leistungsfähigkeit sichern“, sagte Ministerin Theresia Bauer, wie die Pressemitteilung zu berichten wusste ${ }^{2}$. Sehr kurzfristig erfolgte dann Anfang 2016 eine Ausschreibung des Wissenschaftsministeriums, in deren Rahmen man Anträge auf Förderung aus einem „Strukturfonds Kleine Fächer“ stellen konnte. Dieser sollte „Anreize schaffen für die Erarbeitung und Erprobung von exemplarischen, zukunftsweisenden Instrumenten, Maßnahmen und Strukturmodellen“, so wiederum die Pressemitteilung 3 .

Da sahen wir uns im Landesarchiv Baden-Württemberg angesprochen und haben bei sehr kurzen Fristen - einen Antrag mit dem Titel „Aufbau eines Moduls zu einer auf den deutschen Südwesten bezogenen archivalischen Quellenkunde im landeskundlichen Informationssystem $L E O-B W$-Landeskunde entdecken online eingereicht. Wir hatten ein solches Projekt schon seit Langem angedacht und wollten uns die Chance der Förderung nicht entgehen lassen ${ }^{4}$. Sehr erfreut waren wir dann, als im Juni 2016 die Förderung des Antrags vom „Zukunftsrat Kleine Fächer“ empfohlen und im Ministerium beschlossen wurde.

${ }^{1}$ Die Maßnahme der Landesinitiative beruhte auf den Empfehlungen einer Expertenkommission, die das Ministerium im Vorfeld eingesetzt hatte; vgl. Expertenkommission zur Situation der Kleinen Fächer in BadenWürttemberg. Empfehlungen für ein Zukunftsprogramm „Kleine Fächer“ in Baden-Württemberg, hg. von Markus Hilgert-Michaela BötTner (Stuttgart ${ }^{2} 2015$ ).

2 Landesinitiative „Kleine Fächer“ vorgestellt. Ministerium für Wissenschaft, Forschung und Kunst BadenWürttemberg. Pressemitteilungen (26. 3. 2015), https://mwk.baden-wuerttemberg.de/de/service/presse/ pressemitteilung/pid/landesinitiative-kleine-faecher-vorgestellt/ [1. 3. 2018].

3 Ebd.

${ }^{4}$ Der Verfasser und Christian Keitel hatten ein solches Vorhaben schon seit Langem vorbereitet, es aber wegen anderer Projekte immer wieder zurückgestellt. Die Ausschreibung des Ministeriums bot nun die Möglichkeit, es über Drittmittel zu realisieren; siehe dazu auch unten Anm. 57. 
Nachdem die beantragte Projektstelle personell besetzt werden konnte, startete das Vorhaben dann im Oktober 2016 mit dem Titel „Südwestdeutsche Archivalienkunde“5. Seine Ziele hatten wir im Projektantrag in Anlehnung an die Ausschreibung ${ }^{6}$ wie folgt zusammengefasst: „Das Projekt [...] soll den Erhalt wie auch die Weiterentwicklung der Wissensbestände und wissenschaftlichen Kompetenzen auf dem Feld der archivalischen Quellenkunde und der Historischen Grundwissenschaften kontinuierlich sichern. Erreicht werden soll dies durch die Schaffung eines nachhaltig vom Landesarchiv gepflegten Moduls im landeskundlichen Informationssystem $L E O-B W$ - Landeskunde entdecken online. Dieses Modul soll auf den südwestdeutschen Raum bezogen sein, zugleich aber über Vernetzungen, die darüber hinaus gehen, einen allgemeinen Beitrag zur Sicherung und Weiterentwicklung der Quellenkunde wie auch der Historischen Grundwissenschaften insgesamt leisten" "7.

„Archivalische Quellenkunde im frühen 21. Jahrhundert“ ist der Titel meines Beitrags auf dieser Tagung. Da mit unserem Projekt Anforderungen an eine solche umgesetzt werden sollen, werde ich das begonnene Vorhaben im zweiten Teil meines Referats näher vorstellen, um dann abschließend ein kurzes Fazit zu ziehen und einen Ausblick zu geben. Zunächst jedoch möchte ich etwas zur aktuellen Situation der archivalischen Quellenkunde und - eng verbunden damit - der Historischen Grundwissenschaften sagen.

\section{Zur aktuellen Situation der archivalischen Quellenkunde und der Historischen Grundwissenschaften}

In Anlehnung an den aktuellen Diskurs ${ }^{8}$ werde ich dabei im Folgenden diesen Begriff der „Grundwissenschaften“ gebrauchen, ohne mich freilich abschließend festlegen zu wollen, ob man den traditionellen Fächerkanon als Hilfs- oder Grundwissenschaften bezeichnen soll. Denn das sollte man, so meine ich, wohl erst dann wieder vertieft diskutieren, wenn wir bei seiner Weiterentwicklung vorangeschritten und die damit verbundenen Disziplinen neu aufgestellt sind. Vielleicht haben sich ja die Begriffe und vor allem der Fächerkanon als solcher überlebt? Ich möchte das hier nur vorsichtig mit einem Fragezeichen versehen, aber ich glaube in der Tat, dass dringender Bedarf besteht, die hergebrachten Abgrenzungen zwischen den einzelnen Grundwissenschaften und ihr Verhältnis zur Quellenkunde ergebnisoffen zu durchdenken. Dass zumindest Ergänzungen und neue Zuschnitte wie auch Verankerungen in völlig veränderten Kontexten notwendig sind, liegt auf der Hand und tritt auch in letzter Zeit schon in der Diskussion hervor ${ }^{9}$.

5 Laufende Informationen zu dem Projekt finden sich auf der Website des Landesarchivs Baden-Württemberg. Vgl. zuletzt Workshop mit Autorinnen und Autoren der Südwestdeutschen Archivalienkunde in LEO-BW. Das Projekt schreitet voran. Landesarchiv Baden-Württemberg. Aktuelles - Presse/Berichte (3. 3. 2017), https://www.landesarchiv-bw.de/web/61728 [1.3. 2018].

6 Landesinitiative „Kleine Fächer“ vorgestellt (wie Anm. 2).

7 Projektantrag, gezeichnet von den Kooperationspartnern Landesarchiv Baden-Württemberg und Universität Tübingen am 25./27. 1. 2016.

${ }^{8}$ Verwiesen sei dazu nur auf Eva Schlotheuber-Frank Bösch, Quellenkritik im digitalen Zeitalter. Die Historischen Grundwissenschaften als zentrale Kompetenz der Geschichtswissenschaft und benachbarter Fächer. Blog des Historikerverbands (30. 10. 2015), http://blog.historikerverband.de/2015/10/30/quellenkritik-im-digitalen-zeitalter-die-historischen-grundwissenschaften-als-zentrale-kompetenz-der-geschichtswissenschaft-und-benachbarter-faecher/ [1.3. 2018].

9 Dies hat unter anderem die derzeitige Vorsitzende des Historikerverbands in der Diskussion zu ihrem 
Man spricht ja jetzt auch manchmal von „Materialwissenschaft“, so zum Beispiel im Obertitel einer „Einführung in die Historischen Hilfswissenschaften“" ${ }^{10}$, was ich als Anzeichen einer erfreulichen Rückbesinnung auf die „Materialität“ der Quellen in historischen Kreisen verstehe. Es gab gerade in jüngster Zeit auch mehrere Tagungen und Veranstaltungen, die entsprechend ausgerichtet waren und manchmal sogar den Begriff des „Materiellen " aufgegriffen haben ${ }^{11}$, teils in Verbindung mit den Digital Humanities ${ }^{12}$, deren Gegenstand, Ziele und Zuschnitt aber wohl noch einer besonderen Reflexion bedürfen ${ }^{13}$.

Insgesamt ist dieses wieder erwachte Interesse am „Materiellen“ positiv zu sehen, denn angesichts der für das Digitale Zeitalter geradezu konstitutiven Trennung der Information vom Träger ${ }^{14}$ kann die Reflexion - in welchem Rahmen auch immer - über „Materialität“ und „Materialitäten“ oder auch „Materialisierungen“ digitaler Daten, einschließlich solcher, die realiter vor der Übernahme in das Archiv nie erfolgt sind, nur weiterführen.

Gleichwohl ist nach wie vor zu konstatieren, dass die Historischen Grundwissenschaften in ihrer Existenz bedroht sind. Dass unser Projektantrag beim „Zukunftsrat Kleine Fächer" Zustimmung fand, hat sicher eine wesentliche Ursache darin, dass er im Blick auf die gerade entstandene Diskussion über die allgemeine Situation der von ihm betroffenen Disziplinen zu einem sehr geeigneten Zeitpunkt eingereicht wurde. Denn die aktuelle Relevanz des Projekts konnte mit der bundesweit „prekären Lage“ der Historischen Grundwissenschaften begründet werden, die - wie im Projektantrag ausgeführt wurde - „mit ihrer traditionellen Fokussierung auf das Mittelalter und die frühe Neuzeit bei der historischen Forschung vor dem Hintergrund vielfältigster neuer Fragestellungen und Methoden während der zurückliegenden Jahrzehnte an Resonanz verloren haben und deren

Vortrag „Digitalisierung - für wen? Wissenschaft und Archive im Dialog“ auf dem 86. Deutschen Archivtag 2016 in Koblenz sowie in der Sektion „Grundwissenschaften in der digitalen Welt“ auf dem 51. Historikertag 2016 in Hamburg (wie Anm. 21) vertreten.

${ }^{10}$ Hiram KüMPER, Materialwissenschaft Mediävistik. Eine Einführung in die Historischen Hilfswissenschaften (UTB 8605, Paderborn 2014).

${ }_{11}$ Verwiesen sei nur auf folgende im zeitlichen Umfeld des Vortrags angesiedelten Veranstaltungen: „Vade mecum! Nächste Schritte in den Historischen Grundwissenschaften“ (Universität zu Köln, April 2016); „Illuminierte Urkunden - von den Rändern zweier Disziplinen ins Herz der Digital Humanities“ (Haus-, Hof- und Staatsarchiv Wien, September 2016); „Pragmatische Visualisierung. Herrschaft, Recht und Alltag in Verwaltungskarten“ (Hessisches Staatsarchiv Marburg, September 2016); „Der Rotulus im Gebrauch. Einsatzmöglichkeiten, Gestaltungsvarianz und Aussagekraft einer Quellengattung “ (Bergische Universität Wuppertal, September 2016); „Herrschaft durch Schrift. Schriftlichkeit der Verwaltung im deutschen Südwesten (14.18. Jahrhundert)“ (Landesarchiv Baden-Württemberg, Abt. Generallandesarchiv Karlsruhe, Oktober 2016); „Die Materialität von Schriftlichkeit - Bibliothek und Forschung im Dialog: Teil 3“ (Staatsbibliothek zu Berlin, Vortragsreihe: Oktober 2016-Juli 2017); „Materialität als Herausforderung: Der spätmittelalterliche Codex im Fokus der Historischen Grundwissenschaften“ (Universität Heidelberg, Februar 2017).

${ }_{12}$ Hinzuweisen ist auf die Einrichtung eines neuen Ressorts „Digital Humanities“ innerhalb des Mittelalterblogs: vgl. Neues Ressort „Digital Humanities“. Mittelalter. Interdisziplinäre Forschung und Rezeptionsgeschichte (20. 6. 2016), http://mittelalter.hypotheses.org/8236 [1.3. 2018].

13 Verwiesen sei hier nur auf Guido KolLer, Geschichte digital. Historische Welten neu vermessen (Stuttgart 2016). - Interessant ist in diesem Zusammenhang die Abgrenzung der Digitalen Geschichtswissenschaften von einer digitalhistorischen Perspektive der zeithistorischen Forschung, die Frank Bösch auf dem 51. Historikertag 2016 in Hamburg vorgenommen hat; dazu der Sektionsbericht: Martin Sснмітт, HT 2016: Wege in die Computergesellschaft. Folgen digitaler Datenerfassung seit den 1950er Jahren. H-Soz-Kult (15. 10. 2016), http://www.hsozkult.de/conferencereport/id/tagungsberichte-6755 [1. 3. 2018].

14 Dazu jetzt Christian Keitel, Herausforderungen durch die digitale Welt, in: Moderne Aktenkunde, hg. von Holger Berwinkel-Robert Kretzschmar-Karsten Uhde (Veröffentlichungen der Archivschule Marburg, Hochschule für Archivwissenschaft 64, Marburg 2016) 145-148. 
Lehrstühle in der Folge an den Hochschulen abgebaut wurden“15. Verweisen konnten wir dazu auf den 2015 publizierten Aufruf „Quellenkritik im digitalen Zeitalter. Die Historischen Grundwissenschaften als zentrale Kompetenz der Geschichtswissenschaft und benachbarter Fächer" von Eva Schlotheuber und Frank Bösch aus dem Verband deutscher Historikerinnen und Historiker (VHD), veröffentlicht in dessen Journal und auf dessen Website ${ }^{16}$ sowie bei H-Soz-Kult, wo auch unmittelbar eine interessante Diskussion darüber einsetzte ${ }^{17}$.

Der historischen Forschung und ihrem Verband sind damit Defizite bewusst geworden, die uns in den Archiven bereits seit der Jahrtausendwende umtreiben und aus unseren Kreisen heraus jetzt schon seit gut 15 Jahren thematisiert werden ${ }^{18}$. Sie haben vor allem zwei Aspekte. Erstens: Angesichts des Abbaus der Historischen Grundwissenschaften gehen Kompetenzen verloren, die für die Auswertung von Quellen unabdingbar sind und gerade im Digitalen Zeitalter angesichts der Zugänglichkeit von Quellen im Netz wichtiger denn je geworden sind. Und zweitens: Die Historischen Grundwissenschaften müssen fortgeschrieben und neu aufgestellt werden, um endlich dem Faktum Rechnung zu tragen, dass die Zeit nicht im ausgehenden Mittelalter (und schon gar nicht im hohen!) stehen geblieben ist ${ }^{19}$.

Dieser zweite Aspekt ist im Papier von Eva Schlotheuber und Frank Bösch deutlich genannt ${ }^{20}$, stand aber in der Diskussion darüber in einer Sektion auf dem 51. Historikertag $2016^{21}$ in Hamburg weniger im Vordergrund als die generelle Lage der traditionellen Grundwissenschaften an den Universitäten, was auch aus dem Publikum ganz am Ende der Veranstaltung angemerkt wurde. Umso erfreulicher ist, dass in Kreisen der historischen Forschung dieses neue Interesse an den Grundwissenschaften entstanden ist ${ }^{22}$ und Möglichkeiten bietet, den Diskurs in die Richtung des zweiten Aspekts zu lenken. Und es liegt nahe, dass gerade wir Archivarinnen und Archivare ihn aus unserer speziellen Fachkompetenz und Praxis heraus einbringen müssen; so haben ihn auch in Hamburg auf dem Podium zwei Archivarinnen mit ihren Impulsreferaten abgedeckt ${ }^{23}$.

15 Projektantrag (wie Anm. 7).

16 Schlotheuber-Bösch, Quellenkritik (wie Anm. 8). Der Aufruf lag auch lose dem VHD-Journal 4 vom Oktober 2015 bei.

17 Eva Schlotheuber-Frank Bösch, Quellenkritik im digitalen Zeitalter: Die Historischen Grundwissenschaften als zentrale Kompetenz der Geschichtswissenschaft und benachbarter Fächer. H-Soz-Kult (16. 11. 2015), http://www.hsozkult.de/debate/id/diskussionen-2866 [1.3. 2018].

18 Eckart Henning, Die aktuelle Lage der Historischen Hilfswissenschaften in der Bundesrepublik Deutschland, in: Archive und Forschung. Referate des 73. Deutschen Archivtags 2002 in Trier, red. von Robert Kretzschmar et al. (Der Archivar. Mitteilungsblatt für deutsches Archivwesen Beiband 8, Siegburg 2003) 59-69. - Dazu auch der Bericht von Robert Kretzschmar, Sektion II: Archivwissenschaft und Historische Hilfswissenschaften. Der Archivar. Mitteilungsblatt für deutsches Archivwesen 56 (2003) 7-9.

19 Robert Kretzschmar, Hilflose Historikerinnen und Historiker in Archiven? Zur Bedeutung einer zukünftigen archivalischen Quellenkunde für die universitäre Forschung. Blätter für deutsche Landesgeschichte 147 (2011) 133-147; dazu auch der Bericht von Janina Fuge, HT 2010: Im Grenzbereich zwischen Quellenproduzenten, Archiven und historischer Forschung. H-Soz-Kult (23. 10. 2010), http://www.hsozkult.de/conferencereport/id/tagungsberichte-3315 [1.3. 2018].

20 Schlotheuber-Bösch, Quellenkritik (wie Anm. 8).

${ }^{21}$ Vgl. Claudia Hefter, HT 2016: Grundwissenschaften in der digitalen Welt. H-Soz-Kult (12. 11. 2016), http://www.hsozkult.de/conferencereport/id/tagungsberichte-6819 [1.3. 2018].

22 Aus der Sicht der Zeitgeschichte hatte schon zuvor Kiran Patel auf den Diskussionsbedarf hingewiesen: Kiran Klaus PATEL, Zeitgeschichte im digitalen Zeitalter. Neue und alte Herausforderungen. Vierteljahrshefte für Zeitgeschichte 59 (2011) 331-351.

23 Bettina Joergens und Nicola Wurthmann; vgl. Hefter, Grundwissenschaften (wie Anm. 21). 
Wir sollten jetzt aber auch verstärkt liefern. Denn es ist selbstkritisch anzumerken, dass wir in den Archiven die Notwendigkeit der Weiterentwicklung der Grundwissenschaften, die auf archivalische Quellen bezogen sind, zwar schon lange erkannt und zum Beispiel 2004 in ein Positionspapier der Deutschen Forschungsgemeinschaft eingebracht haben ${ }^{24}$, aber bisher neben dem Grundsätzlichen erst wenig Konkretes dazu beigetragen haben ${ }^{25}$; da muss ich mir auch an die eigene Nase fassen.

Etwas vorangebracht haben wir in letzter Zeit immerhin die Aktenkunde für das 20. und 21. Jahrhundert, der für die ganze Neuausrichtung der auf Archivgut bezogenen Historischen Grundwissenschaften eine zentrale Bedeutung zukommen dürfte ${ }^{26}$. Da ist vor allem Holger Berwinkel zu nennen. Sein Blog trägt ganz wesentlich dazu bei, der Aktenkunde Aufmerksamkeit zu verleihen ${ }^{27}$. Seine soliden Publikationen haben auch schon sehr konkret Lücken geschlossen ${ }^{28}$. Hinweisen darf ich auch auf den Band „Moderne Aktenkunde“, der im unmittelbaren Vorfeld dieser Tagung als Ergebnis des Arbeitskreises „Aktenkunde des 20. und 21. Jahrhunderts“ im Verband deutscher Archivarinnen und Archivare erschienen ist; darin finden sich auch mehrere Beiträge zu digitalen Überlieferungen ${ }^{29}$.

Mit Akten und prozessgenerierten digitalen Aufzeichnungen müssen wir Archivarinnen und Archivare uns im Berufsalltag auf verschiedenen Arbeitsfeldern intensiv befassen,

24 Thekla Kluttig-Robert Kretzschmar-Karl-Ernst Lupprian-Wilfried Reininghaus-Udo SchäferBarbara Schneider-Kempf-Günther Wartenberg, Die deutschen Archive in der Informationsgesellschaft Standortbestimmung und Perspektiven. Der Archivar. Mitteilungsblatt für deutsches Archivwesen 57 (2004) 28-36, hier 34.

${ }^{25}$ Außer dem in Anm. 19 genannten Beitrag vgl. Robert Kretzschmar, Archivwissenschaft als Historische Hilfswissenschaft. Schnittstelle zur Forschung, in: Archivisches Arbeiten im Umbruch. Vorträge des Kolloquiums der Staatlichen Archivverwaltung Baden-Württemberg am 26. und 27. November 2002 im Staatsarchiv Ludwigsburg aus Anlass der Verabschiedung von Herrn Professor Dr. Gerhard Taddey, hg. von Norbert Hofmann-Stephan Molitor (Stuttgart 2004) 11-34; Lorenz Friedrich Beck, Die Historischen Hilfswissenschaften im Informationszeitalter. Vom zeitlosen wie zeitgemäßen Nutzen des quellenkundlichen Instrumentariums für Archivar und Historiker, in: Archive und Gedächtnis. Festschrift für Botho BrachmanN, hg. von Friedrich Beck-Eckart Henning-Joachim-Felix Leonhard-Susanne Paulukat-Olaf B. Rader (Potsdamer Studien 18, Potsdam 2005) 239-251; Rainer Hering, Archive in der digitalen Welt. Informationstransfer zwischen Verwaltung und Wissenschaft, in: Forschung in der digitalen Welt. Sicherung, Erschließung und Aufbereitung von Wissensbeständen. Tagung des Staatsarchivs Hamburg und des Zentrums „Geisteswissenschaften in der digitalen Welt“ an der Universität Hamburg am 10. und 11. April 2006, hg. von Rainer HeringJürgen SARnowsky-Christoph Schäfer-Udo Schäfer (Veröffentlichungen aus dem Staatsarchiv der Freien und Hansestadt Hamburg 20, Hamburg 2006) 153-160; Robert Kretzschmar, Werkzeug, Forschungsfeld, Lehrfach? Zur Bedeutung der Historischen Hilfswissenschaften für die Archive, in: Quellenarbeit und Schriftgutverwaltung - Historische Hilfswissenschaften im Kontext archivischer Aufgaben. Beiträge zum 12. Archivwissenschaftlichen Kolloquium der Archivschule Marburg, hg. von Karsten Uhde (Veröffentlichungen der Archivschule Marburg, Institut für Archivwissenschaft 48, Marburg 2009) 151-176; Robert KRETzschmar, Auf dem Weg in das 21. Jahrhundert: Archivische Bewertung, Records Management, Aktenkunde und Archivwissenschaft. Archivar. Zeitschrift für Archivwesen 63 (2010) 144-150; Rainer Hering, Archive und Geschichtswissenschaft im digitalen Zeitalter. Probleme und Herausforderungen aus der Sicht eines deutschen Archivars und Historikers. MIÖG 120 (2012) 116-138, hier 129f.; Robert Kretzschmar, Akten- und Archivkunde im Tübinger Netzwerk Landesgeschichte. Ein Plädoyer für eine zeitgemäße Archivalienkunde, in: Netzwerk Landesgeschichte. Gedenkschrift für Sönke Lorenz, hg. von Dieter R. Bauer-Dieter Mertens-Wilfried SetzLER-Susanne Borgards (Tübinger Bausteine zur Landesgeschichte 21, Ostfildern 2013) 91-109.

26 Vgl. Kretzschmar, Hilflose Historikerinnen und Historiker (wie Anm. 19) 137.

27 Holger Berwinkel, Über das Blog. Aktenkunde. Aktenlesen als Historische Hilfswissenschaft, https://aktenkunde.hypotheses.org/uber [1.3. 2018].

28 Holger Berwinkel, Der diplomatische Schriftverkehr im 20. Jahrhundert. AfD 61 (2015) 343-390.

29 Moderne Aktenkunde (wie Anm. 14). 
ganz besonders aber bei der Überlieferungsbildung, für die wir den Entstehungskontext und Auswertungsmöglichkeiten quasi spiegelbildlich zur Quellenkritik analysieren müs$\operatorname{sen}^{30}$. Wir verfügen deshalb über spezialisierte Kompetenzen und Kenntnisse, die wir als Grundlage grundwissenschaftlicher und quellenkundlicher Betrachtungen gezielt nutzen können und sollten. Und mit dem Aufbau digitaler Archive als Schritt in die Praxis haben in den letzten Jahren vielerorts viele Kolleginnen und Kollegen begonnen, sich sehr konkret mit digitalen Aufzeichnungen zu befassen und darüber zu publizieren. Ich verweise dazu nur exemplarisch auf die jüngsten Beiträge aus dem Arbeitskreis „Archivierung von Unterlagen aus digitalen Systemen "31 sowie die Veröffentlichungen von Rolf Dässler und Karin Schwarz zu Datenbanken und Fachverfahren ${ }^{32}$, Christian Keitel ${ }^{33}$ und Kai Nau$\operatorname{mann}^{34}$ zu prozessgenerierten digitalen Unterlagen und digitaler Archivierung oder Johannes Renz zur archivischen Behandlung von Blogs ${ }^{35}$.

Die Kolleginnen und Kollegen, die aktuell mit dem Aufbau digitaler Archive befasst sind, stellen somit ein großes Potential für die Bearbeitung grundwissenschaftlicher und quellenkundlicher Aspekte dar. Sie sind auch untereinander gut vernetzt und tauschen sich in letzter Zeit immer stärker in archivfachlichen Gremien ${ }^{36}$ und auf speziellen Tagungen $^{37}$ aus. Das gilt in gleicher Weise aber auch für alle, die sich in den letzten Jahrzehnten normativ aus der Sicht des Records Management mit Anforderungen an elektronische Systeme befasst haben. Sie bringen ebenfalls beste Voraussetzungen mit, sich auch deskriptiv und analytisch mit entstandenen und entstehenden digitalen Überlieferungen auseinander zu setzen ${ }^{38}$.

In welchem Maße besonders hier in der archivalischen Quellenkunde Ergänzungsund Aktualisierungsbedarf besteht, zeigt schon ein Blick auf die vorliegenden Grundlagenwerke und ein begonnenes Vorhaben. Die grundlegende und erfreulich regelmäßig aktualisierte Publikation „Die archivalischen Quellen“von Friedrich Beck und Eckart Henning spannt den Bogen bis in die Gegenwart, spricht aber eher allgemeine Entwick-

${ }^{30} \mathrm{Vgl}$. Robert Kretzschmar, Die „neue archivische Bewertungsdiskussion“ und ihre Fußnoten. Zur Standortbestimmung einer fast zehnjährigen Kontroverse. Archivalische Zeitschrift 82 (1999) 7-40, hier $24 \mathrm{f}$.

31 Die Tagungsunterlagen werden online veröffentlicht: Arbeitskreis „Archivierung von Unterlagen aus digitalen Systemen“, in: Kanton St. Gallen - Staatsarchiv St. Gallen, http://www.staatsarchiv.sg.ch/home/auds. html [1. 3. 2018]. Die Ergebnisse des Arbeitskreises werden auch regelmäßig in Gestalt von Tagungsbänden publiziert.

32 Rolf Dässler-Karin Schwarz, Archivierung und dauerhafte Nutzung von Datenbankinhalten aus Fachverfahren - eine neue Herausforderung für die digitale Archivierung. Archivar. Zeitschrift für Archivwesen 63 (2010) 6-18.

33 Vgl. Dr. Christian Keitel: Literaturliste, in: Landesarchiv Baden-Württemberg. Ansprechpartner/innen, https://www.landesarchiv-bw.de/sixcms/media.php/79/Keitel_Publikationen_2.pdf [1.3. 2018].

${ }^{34}$ Vgl. Veröffentlichungen Dr. Kai Naumann, in: Landesarchiv Baden-Württemberg. Ansprechpartner/ innen, https:/www.landesarchiv-bw.de/sixcms/media.php/79/Naumann_pub.pdf [1.3. 2018].

35 Johannes Renz, Wir nennen es Archivierung. Sicherung von Blogs für die historische Überlieferung. Archivar. Zeitschrift für Archivwesen 68 (2015) 44-49.

36 Neben dem Arbeitskreis „Archivierung von Unterlagen aus digitalen Systemen“ ist hier vor allem der Ausschuss „Digitale Archive“ zu nennen, der bei der „Konferenz der Leiterinnen und Leiter der Archivverwaltungen des Bundes und der Länder (KLA)“ eingerichtet ist.

37 Kreative digitale Ablagen und die Archive. Ergebnisse eines Workshops des KLA-Ausschusses Digitale Archive am 22./23. November 2016 in der Generaldirektion der Staatlichen Archive Bayerns, hg. von Kai Naumann-Michael Puchta (Sonderveröffentlichungen der Staatlichen Archive Bayerns 13, München 2017).

38 Vgl. Udo Schäfer, Amtliche Aktenkunde der Neuzeit - Records Management des 21. Jahrhunderts. Zur Schnittmenge zweier Disziplinen, in: Quellenarbeit und Schriftgutverwaltung (wie Anm. 25) 89-128. 
lungen des Archiv- und Bibliothekswesens im Digitalen Zeitalter an ${ }^{39}$. Die „Quellenkunde der Habsburgermonarchie (16. bis 18. Jahrhundert)“ von $2004^{40}$ und die „Quellenkunde zur westfälischen Geschichte vor 1800“, die von der Historischen Kommission für Westfalen seit einiger Zeit im Netz aufgebaut wird ${ }^{41}$, sind konzeptionell - wie schon die Titel besagen - von vorneherein auf Zeiträume vor dem 19. Jahrhundert ausgerichtet. Digitale Überlieferungen sind dementsprechend darin nicht berücksichtigt. Die vom Landesarchiv Nordrhein-Westfalen 2010 gestartete Reihe der „Massenakten des 20. Jahrhunderts" deckt schon mit ihren ersten drei Bänden, die bisher erschienen sind, für Nordrhein-Westfalen in vorbildlicher Weise zentrale Überlieferungen aus normierten Verwaltungsverfahren $\mathrm{ab}^{42}$, hat aber erst in einem Fall digitale Unterlagen einbezogen ${ }^{43}$.

Was gilt es zu beschreiben und analysieren? Prozessgenerierte Unterlagen entstehen heute als Überreste ${ }^{44}$ in hybriden Kontexten, die es schon als solche zu betrachten und darzustellen gilt ${ }^{45}$. Ein wesentlicher Gesichtspunkt dabei dürfte die Verknüpfung analoger und digitaler Aufzeichnungen sein, die aufeinander bezogen sind. Nur so können wir quellenkundlich dem Digitalen Zeitalter gerecht werden, wie es sich bisher - also bis in das frühe 21. Jahrhundert - realiter entwickelt hat. Ich möchte diesen aktuellen Entwicklungstand als „Frühes Digitales Zeitalter“ abgrenzen von einer theoretisch denkbaren späteren Phase, in der die Entstehung von Unterlagen in der Verwaltung vielleicht einmal tatsächlich, wie von uns in den Archiven zu Recht schon lange angestrebt, ganz nach unseren Vorgaben geprägt sein wird. Darin liegt ja für uns eine geradezu unglaubliche Chance im Digitalen Zeitalter ${ }^{46}$ !

Bis sie erreicht ist, müssen wir - und dies erfordert den Perspektivenwechsel vom normativen Blick des Records Management zur deskriptiven und analytischen Sicht der Quellenkunde - prozessgenerierte Überlieferungen so beschreiben, wie sie realiter entstanden sind beziehungsweise laufend entstehen und in den Archiven (oder anderswo) zu nutzbaren Beständen formiert und bewahrt werden: als Produkte beziehungsweise

39 Botho Brachmann, Moderne Quellengattungen. Neue Medien, Massenmedien und Internet, in: Die archivalischen Quellen. Mit einer Einführung in die Historischen Hilfswissenschaften, hg. von Friedrich BECKEckart Henning (Wien-Köln-Weimar ${ }^{5}$ 2012) 182-208.

${ }^{40}$ Quellenkunde der Habsburgermonarchie (16.-18. Jahrhundert). Ein exemplarisches Handbuch, hg. von Josef Pauser-Martin Scheutz-Thomas Winkelbautr (MIÖG Ergbd. 44, Wien-München 2004). Der Band wird beschlossen durch einen kulturwissenschaftlichen Beitrag, der als einziger Artikel im Abschnitt „Medienarchäologischer Ausblick“ eine Verbindung zur unmittelbaren Gegenwart herstellt: Wolfgang ERNST, Das Archiv als Gedächtnisort?, in: ebd. 1113-1130.

${ }^{41}$ Quellenkunde zur westfälischen Geschichte vor 1800. Online-Ausgabe, Stand: März 2016, hg. von Stefan Pätzold-Wilfried Reininghaus (Materialien der Historischen Kommission für Westfalen 6), http:// www.lwl.org/hiko-download/HiKo-Materialien_006_\%282016-03\%29.pdf [1.3. 2018].

42 Unbekannte Quellen: „Massenakten“ des 20. Jahrhunderts. Untersuchungen seriellen Schriftguts aus normierten Verwaltungsunterlagen, hg. von Jens HeckL, 3 Bde. (Veröffentlichungen des Landesarchivs Nordrhein-Westfalen 32, 43, 55, Düsseldorf 2010-2012, Duisburg 2015).

43 Martin Schlemmer-Ragna Boden, Datenbanken zu Altlasten und zur Bodenbelastung, in: Unbekannte Quellen (wie Anm. 42) 3 25-35.

44 Robert Kretzschmar, Absichtlich erhaltene Überreste. Überlegungen zur quellenkundlichen Analyse von Archivgut. Archivar. Zeitschrift für Archivwesen 67 (2014) 265-269.

45 Robert Kretzschmar, „Akten“- Begriff und Realitäten im zweiten Jahrzehnt des 21. Jahrhunderts, in: Moderne Aktenkunde (wie Anm. 14) 13-21.

46 Siehe dazu die Beiträge von Heather MacNeil und Luciana Duranti in diesem Band. Zudem vgl. Robert Kretzschmar, Appraisal and Selection in the Early Digital Age: Traditional Values and Changing Routines, in: Values in Transition. Perspectives on the Past, Present and Future of the Archival Profession, hg. von Hildo vaN ENGEN (Den Haag 2017) 109-123. 
Überreste pragmatischer Schriftlichkeit (mit - nebenbei bemerkt - zunehmender „Bildlichkeit“/Visualisierung) eines vorläufig abzugrenzenden „Frühen Digitalen Zeitalters“ in seiner hybriden Ausprägung. Eine „archivalische Quellenkunde im frühen 21. Jahrhundert" muss dies leisten.

Sie muss freilich auch weiterhin alle wesentlichen Quellengattungen und -typen aus früheren Zeiten einbeziehen, nicht zuletzt um lange Linien zu verfolgen. Und da bestehen durchaus Lücken, die es noch aufzuarbeiten gilt - insgesamt für das Alte Reich, vor allem aber auch regional für historische Landschaften. Das Projekt der „Westfälischen Quellenkunde" belegt mit den bisher vorliegenden Beiträgen beispielhaft, wie fruchtbar ein auf überschaubare historische Räume beschränktes Vorhaben sein kann.

Gefordert sind da neben den Archivarinnen und Archivaren besonders auch die Historikerinnen und Historiker, die über professionelle Spezialkenntnisse in der Auswertung von Unterlagen verfügen, die vor dem Digitalen Zeitalter entstanden sind. Eine „archivalische Quellenkunde des frühen 21. Jahrhunderts" sollte daher gemeinsam von beiden Kreisen im Dialog erarbeitet und gepflegt werden. Das neu erwachte Interesse der Forschung an den Grundwissenschaften und dem „Materiellen“ bietet für die Umsetzung jetzt auch - anders als noch vor wenigen Jahren - gute Bedingungen. Wie wir das in Baden-Württemberg gerade anpacken, möchte ich nun anhand unseres Projekts erläutern.

\section{Das Projekt „Südwestdeutsche Archivalienkunde“}

Die Kooperation zwischen Archiv und historischer Forschung haben wir realisiert. Beantragt wurde und durchgeführt wird das Projekt gemeinsam vom Landesarchiv BadenWürttemberg und dem Institut für Geschichtliche Landeskunde und Historische Hilfswissenschaften an der Universität Tübingen. Die Federführung liegt beim Landesarchiv.

Der Begriff „Archivalienkunde“ benennt die Objekte, deren quellenkundliche Analyse und Beschreibung Ziel des Vorhabens ist. Archivalien sind nach unserem Verständnis alle Unterlagen, die gemeinhin als Archivgut bezeichnet werden: prozessgenerierte Unterlagen von der Papsturkunde bis zum elektronischen Fachverfahren, aber auch ergänzende Unterlagen und Sammlungsgut unter Berücksichtigung eventueller Schnittmengen mit anderen Institutionen, die dann zu reflektieren sind. Gegenstand der Archivalienkunde ist somit schlichtweg nutzbares Archivgut, das in Archiven erhalten und zugänglich gemacht wird.

Viele Archivarinnen und Archivare werden hier sofort an Heinrich Otto Meisners „Archivalienkunde vom 16. Jahrhundert bis 1918“ aus dem Jahr 1969 denken ${ }^{47}$. Diese begriffliche Kontinuität war aber nicht ausschlaggebend für die Bezeichnung des Vorhabens. Vielmehr ging es uns darum, damit das geplante Modul einer „Südwestdeutschen Archivalienkunde in LEO-BW“ als abgegrenzten Teilbereich einer allgemeinen Quellenkunde des Südwestens auszuweisen, die perspektivisch in LEO-BW realisiert werden kann.

LEO-BW als landeskundliches Informationssystem ${ }^{48}$ für den deutschen Südwesten erschien uns deshalb als Ort einer Archivalienkunde im Netz besonders geeignet, weil

47 Heinrich Otto Meisner, Archivalienkunde vom 16. Jahrhundert bis 1918 (Leipzig 1969).

48 LEO-BW. Landeskundliches Informationssystem Baden-Württemberg, http://www.leo-bw.de/ [1. 3. 2018]. 
dort bereits verschiedene Gedächtnisinstitutionen und Einrichtungen (derzeit sind es 33) ihre Daten zusammenführen: auf den Südwesten bezogen, epochenübergreifend und kollaborativ ${ }^{49}$. Damit sind gute Bedingungen für Verknüpfungen mit anderen quellenkundlichen Angeboten gegeben, vor allem der Museen und Bibliotheken. So haben auch die südwestdeutschen Numismatiker im Rahmen eines weiteren kollaborativen Vorhabens, das derzeit ebenfalls vom „Zukunftsrat Kleine Fächer“ gefördert wird, bereits Überlegungen angestellt, in LEO-BW Informationen zu Münzen aufzubereiten.

Perspektivisch wäre eine kontinuierliche Erweiterung des quellenkundlichen Angebots in LEO-BW nur zu begrüßen, denn die archivalische Quellenkunde kann nur gewinnen, wenn spartenübergreifend weite Horizonte für die Reflexion über Entstehungskontexte und Auswertungsmöglichkeiten sichtbar werden und konkrete Verbindungen zwischen Archiv-, Bibliotheks- und Museumsgut thematisiert und mittels Verlinkungen abgebildet werden. Man denke nur an chronikalische und historische Aufzeichnungen, die in Bibliotheken, aber auch in Archiven erhalten sind, wozu exemplarisch auf den Bestand „Sammlung von ungedruckten Schriften zur Landesgeschichte“ im Hauptstaatsarchiv Stuttgart verwiesen sei $^{50}$.

Die Realisierung in LEO-BW bietet weitere Möglichkeiten sinnvoller Verknüpfungen. Wir werden unser Online-Informationssystem, mit dem wir die Beständeübersichten und Findmittel des Landesarchivs zugänglich machen ${ }^{51}$, mit dem quellenkundlichen Modul verlinken. Ziel dabei ist, Erschließungsdaten zu Archivgut mit quellenkundlichen Basisinformationen zu verknüpfen, die auf einfache Weise abrufbar sind. Die Archivalienkunde erweitert dann automatisiert unser archivisches Online-Informationssystem und erfüllt so eine Forderung, die Wilfried Reininghaus schon 2002 auf dem 73. Deutschen Archivtag in Trier erhoben hat: „Die Vorlage von Findbüchern oder ihre Präsentation im Internet“, so Reininghaus bereits damals, „ist auf Dauer zu wenig. Zum Wissensmanagement, das von den Archiven selbst betrieben wird, gehört mehr" ${ }^{\text {"52 }}$. Orientiert an den Quellengattungen kann unsere Archivalienkunde im Netz Teil eines Angebots sein, das besonders auch allen Interessierten ohne Archiverfahrung Orientierung geben soll. Dazu gehören in letzter Zeit auch Rechercheführer, die auf spezielle Themenfelder und Fragestellungen, wie beispielsweise Familienforschung, Zwangsarbeit und Heimerziehung, ausgerichtet sind und Hinweise zu Recherchewegen und auf einschlägige Bestände geben $^{53}$. Entsprechend erweitert, wird unser Informationssystem auf drei Säulen beruhen: der Tektonik der Bestände, dem themenbezogenen Rechercheweg und der archivalischen Quellenkunde.

Wie werden wir die „Südwestdeutsche Archivalienkunde“ aufbauen? Das Grundmuster orientiert sich an dem Band „Serielle Quellen in Südwestdeutschen Archiven“, den

49 Zu LEO-BW zuletzt Daniel FäHLE-Andreas Neuburger, Landesgeschichte im digitalen Wandel: das landeskundliche Informationssystem LEO-BW. Blätter für deutsche Landesgeschichte 150 (2014) 559-568.

${ }^{50}$ J1. Allgemeine Sammlung von ungedruckten Schriften zur Landesgeschichte, in: Landesarchiv Baden-Württemberg - Hauptstaatsarchiv Stuttgart, Beständeübersicht, http://www.landesarchiv-bw.de/ plink/?f=1-6946 [1.3. 2018].

51 Recherche und Bestellung, in: Landesarchiv Baden-Württemberg, https://www.landesarchiv-bw.de/ web//web/46734 [1.3. 2018].

52 Wilfried Reininghaus, Quellenkunde und Quellenkritik aus der Sicht der Archive und der neueren Geschichte, in: Archive und Forschung (wie Anm. 18) 91-98, hier 98. Siehe auch unten Anm. 59.

53 Rechercheführer, in: Landesarchiv Baden-Württemberg, https://www.landesarchiv-bw.de/web/57399 [1.3. 2018]. 
2005 Christian Keitel und Regina Keyler herausgegeben haben ${ }^{54}$ und der bald in bemerkenswerter Weise über den Südwesten hinaus eine standardisierende Wirkung entfaltet hat. Denn ihm sind bewusst gefolgt die Publikationsreihe „Unbekannte Quellen: ,Massenakten des 20. Jahrhunderts"“, die das Landesarchiv Nordrhein-Westfalen seit einiger Zeit publiziert ${ }^{55}$, wie auch die „Westfälische Quellenkunde bis 1800 “56.

Die Absicht, den Band der „Seriellen Quellen“ bezogen auf weitere Gattungen und Typen archivalischer Unterlagen fortzusetzen, stand am Anfang des Unternehmens. Sie verband sich mit dem Ziel, auch jüngere und jüngste Überlieferungen des Digitalen Zeitalters einzubeziehen, und mündete ein in das Vorhaben einer umfassenden Quellenkunde für den deutschen Südwesten, die bis in die unmittelbare Gegenwart reicht und darauf angelegt ist, kontinuierlich ausgebaut zu werden ${ }^{57}$.

Das Raster für die Gliederung der Artikel haben wir nur geringfügig modifiziert. Vorgesehen sind Abschnitte zu den folgenden Punkten:

1. Definition der Quellengattung (Entstehungskontext, Primärwert)

2. Historische Entwicklung der Quelle

3. Aufbau und Inhalt

4. Überlieferungslage und gegebenenfalls vorarchivische und archivische Bearbeitungsschritte

5. Quellenkritik und Auswertungsmöglichkeiten (Sekundärwert)

6. Hinweise zur Benutzung

7. Forschungs- und Editionsgeschichte

8. Literatur

9. Fußnoten

10. Zitierhinweis

11. Beispiele (Abbildungen/Verlinkungen)

Die Artikel selbst sollen wissenschaftlichen Anforderungen entsprechen, sich aber dies unterscheidet sie von der "Quellenkunde der Habsburgermonarchie“ ${ }^{\text {"58 }}$ - an alle Interessierten richten ${ }^{59}$. Dementsprechend sollen die Beiträge konzise und gut lesbar geschrieben sein und sich nicht im Detail verlieren, sondern zur Vertiefung weiterführende Hinweise geben. Zentrale und unverzichtbare Punkte der Darstellung sind die Definition der Quellengattung, die Genese der Unterlagen im Entstehungskontext und ihr Aufbau, schließlich der Quellenwert und die Auswertungsmöglichkeiten, verbunden mit Hinweisen zur Benutzung.

${ }^{4}$ Serielle Quellen in südwestdeutschen Archiven, hg. von Christian KeItel-Regina Keyler (Stuttgart 2005). Online-Fassung: Serielle Quellen in südwestdeutschen Archiven, hg. von Christian KeITEL-Regina Keyler, in: Baden-Württembergisches Online-Archiv, http://www.boa-bw.de/jspview/downloads/frei/ bsz306616858/0/ index.html [1.3. 2018].

55 Unbekannte Quellen (wie Anm. 42).

56 Quellenkunde zur westfälischen Geschichte (wie Anm. 41).

57 Dies waren die Überlegungen, die der Verfasser und Christian Keitel schon seit langem angestellt hatten; siehe dazu auch oben Anm. 4.

58 Josef Pauser-Martin Scheutz-Thomas Winkelbauer, Vorbemerkung, in: Quellenkunde der Habsburgermonarchie (wie Anm. 40) 9-12, hier 11. Dort sind ausgewiesen als „Zielpublikum einerseits Wisssenschaftlerinnen und Wissenschaftler, denen das Handbuch ein rasches Nachschlagen ermöglichen soll, andererseits auch Studierende, denen mit Hilfe der übersichtlichen Beiträge eine erste Orientierung geboten wird“.

59 Christian Keitel und Regina Keyler hatten ihre Publikation entsprechend angelegt - mit dem Ziel, allen Interessierten neben den an der Provenienz orientierten Findmitteln quellenkundliche Hilfestellungen zu geben; vgl. Christian Keitel-Regina Keyler, Einleitung, in: Serielle Quellen (wie Anm. 54) 9-11, hier 10. 
Im Raster erscheinen bewusst die archivwissenschaftlichen Fachtermini „Primär- und Sekundärwert“, die Schellenberg für die archivische Bewertung entwickelt hat ${ }^{60}$. Ich habe in der universitären Lehre immer wieder die Erfahrung gemacht, dass sie bestens geeignet sind, den „Entstehungszweck“ und die „Auswertungsmöglichkeiten“ von Unterlagen in Relation zu setzen und aufeinander bezogen zu betrachten.

Dazu kommt ein programmatischer Aspekt: Ich bin der Überzeugung, dass es gerade die Aufgabe der Archivarinnen und Archivare ist, quellenkundlich relevante Schnittmengen zwischen den verschiedenen Disziplinen, die auf Registratur- und Archivgut bezogen sind, synergetisch für die Archivalienkunde heranzuziehen: beginnend bei den Historischen Grundwissenschaften über die Kanzlei ${ }^{61}$ - und Archivgeschichte bis hin zur Archivwissenschaft, einschließlich der archivalischen Strukturlehre und des Records Management, die für die Quellenkunde von besonderer Relevanz sind. Die „Archivalienkunde“ ist geradezu dazu prädestiniert, die Perspektiven und Ergebnisse dieser Disziplinen zusammenzuführen und zusammenzubinden, um das Spezifische der archivalischen Quellengattungen und -typen herauszuarbeiten und allen Interessierten an die Hand zu geben. Damit kann sie den „strukturprekären“ Historischen Grundwissenschaften nicht nur in vielfältigen Kontexten neue Impulse geben, sondern ihnen eine neue Relevanz verleihen und sie stärken. Sie kann sich damit aber auch zugleich - und das ist perspektivisch von noch größerer Bedeutung - als eine eigene Disziplin profilieren und etablieren, die für die Forschung und alle, die Archivgut nutzen, unverzichtbar ist ${ }^{62}$.

Zurück zu unserem Projekt: In der ersten Phase des Projekts waren wir damit befasst, eine Liste der vorzusehenden Artikel zusammenzustellen und Autorinnen und Autoren zu gewinnen. Begleitet wurde dies von der Diskussion innerhalb der Projektgruppe, wie wir kategoriale Gliederungen und Abgrenzungen vornehmen sollen. Welche Möglichkeiten gibt es? Die „Quellenkunde der Habsburgermonarchie“ ist im ersten Teil an „Institutionen" orientiert, beginnend beim Kaiserhof, und in einem zweiten an „Gattungen“, beginnend bei Selbstzeugnissen. Ein dritter Abschnitt ist „Bildern und Dingen“ gewidmet $^{63}$. Die Artikel in der Reihe der Bände des Landesarchivs Nordrhein-Westfalen zu den „Massenakten des 20. Jahrhunderts“ sind auf gleichförmige Überlieferungskomplexe einzelner Provenienzen - etwa serielle Steuerakten der Finanzverwaltung - und normierte Verwaltungsverfahren als deren Grundlage ausgerichtet ${ }^{64}$.

Schon angesichts der territorialen Verhältnisse im deutschen Südwesten und der Breite unseres Vorhabens werden wir bei der "Südwestdeutschen Archivalienkunde“ nicht

60 Theodore R. Schellenberg, The Appraisal of Modern Public Records (Bulletins of the National Archives 8 = National Archives Publications 57/5, Washington 1956); deutsche Übersetzung: DERs., Die Bewertung modernen Verwaltungsschriftguts, hg. von Angelika Menne-HaRitz (Veröffentlichungen der Archivschule Marburg, Institut für Archivwissenschaft 17, Marburg 1990).

${ }_{61}$ Wie wichtig gerade die Kanzleigeschichte ist, zeigt der Beitrag von Holger Berwinkel, Zur Kanzleigeschichte des 20. Jahrhunderts - ein Versuch, in: Moderne Aktenkunde (wie Anm. 14) 29-50.

${ }^{6}$ Vgl. Kretzschmar, Hilflose Historikerinnen und Historiker (wie Anm. 19) 146; Lorenz Friedrich Beck-Robert Kretzschmar, Zum Begriff „Aktenkunde“ - Verständnis und Abgrenzung als Disziplin, in: Moderne Aktenkunde (wie Anm. 14) 23-27, hier 27; Kretzschmar, Auf dem Weg (wie Anm. 25); Kretzschmar, Akten- und Archivkunde (wie Anm. 25). Auch Holger Berwinkel weist auf die Notwendigkeit einer „überwölbenden Archivalienkunde“ hin, „die alle in Archiven verwahrten Schriftquellen einbezieht“: Holger Berwinkel, „Quellenkritik im digitalen Zeitalter“: Zum Positionspapier des Historikerverbands. Aktenkunde. Aktenlesen als Historische Hilfswissenschaft (28. 11. 2015), https://aktenkunde.hypotheses.org/438 [1. 3. 2018].

63 Pauser-Scheutz-Winkelbauer, Vorbemerkung (wie Anm. 58) 11.

64 Sina Westphal, Serielle Steuerakten, in: Unbekannte Quellen (wie Anm. 42) 2 166-178. 
von Institutionen ausgehen, sondern von Archivaliengattungen, teils weiter untergliedert nach Quellentypen, die nach unterschiedlichen Kriterien sachgerecht zu definieren sind. Vergleichbar ist dies am ehesten mit der „Westfälischen Quellenkunde"65, dann aber auch immer wieder mit der „Quellenkunde der Massenakten“ und ihrer Orientierung am Verfahren $^{66}$. Denn wir werden verschiedene Ebenen der Untergliederung abzudecken haben. Es wird zum Beispiel einen Artikel geben zu „Akten“, dann aber auch eigene Artikel zu „Fallakten“, „elektronischen Akten“ wie auch zu „Personalakten“ und „Entnazifizierungsakten“. Kurzum: Wir haben unterschiedliche Abstraktionsebenen vorgesehen mit Querverbindungen, die zu verlinken sind.

Auf der obersten Ebene finden sich die klassischen Rubriken für die traditionellen Gattungen (Urkunden, Amtsbücher, Akten), aber auch - und hier wollen wir die allgemeine Diskussion befördern - Vorschläge für eine mögliche Kategorisierung jüngerer und jüngster Überlieferungen wie zum Beispiel „Strukturierte Informationssammlungen“, „Schwach strukturierte Unterlagen“ oder „Web-Unterlagen“67.

Bewusst nicht intendiert ist in irgendeiner Weise eine dogmatische Lehre der Kategorisierung; vielmehr wird eine problemorientierte, reflektierte Multiperspektivität der Betrachtung angestrebt. Wichtig sind uns daher auch Querschnittsartikel, die Überblicke geben, wie zum Beispiel „Archivalien des Mittelalters“ oder „Digitale Archivalien“. Auch wollen wir problemorientiert bestimmte Fragestellungen aufgreifen; solche Artikel haben wir als „Blicköffnende Exkurse“ eingeplant. Stichworte hierfür sind zum Beispiel „Überlieferungschance und -zufall“, „Arkancharakter der Archive und Zugänglichkeit“, „Archivgut als Spiegel ,pragmatischer Schriftlichkeit“" oder „Heterogenität als Wesensmerkmal von Archivgut" ${ }^{\text {"68. }}$.

Alle Artikel, einschließlich der Überblicke und Exkurse, sollen auf den Südwesten Deutschlands bezogen sein und konkrete Belege bieten. Zugleich aber ist auch intendiert, dass sie dazu beitragen, die Quellenkunde insgesamt voranzubringen und fortzuschreiben; dies gilt insbesondere für die Überlieferungen des 20. und 21. Jahrhunderts. Der Südwesten ist ja auch kein isolierter Raum, und bei mancher Quelle wird der Blick in die Schweiz, nach Österreich oder auch in das Elsass sinnvoll sein. Insofern versteht sich die „Südwestdeutsche Archivalienkunde“ wie schon die „Quellenkunde der Habsburgermonarchie“ als „Grundstein einer bislang noch kaum existierenden komparatistischen, qualitativ operierenden Quellenkunde, deren Ziel etwa ein Vergleich von Urbaren oder Selbstzeugnissen in verschiedenen Regionen sein könnte“69.

„Auf den Südwesten bezogen“: das bedarf ohnehin noch einer kurzen Erläuterung. Der historischen und territorialen Entwicklung werden wir Rechnung tragen. Die historischen Landkarten werden sich in den Beiträgen spiegeln. Bei der Entwicklung im Digitalen Zeitalter wollen wir uns auf unser Bundesland mit seinen Besonderheiten in der Verwaltung konzentrieren, aber natürlich auch bundesweiten Anwendungen und Kontexten Beachtung schenken, die im Südwesten wirksam waren beziehungsweise sind. Es gibt

65 Quellenkunde zur westfälischen Geschichte (wie Anm. 41).

66 So in der Beschreibung der Steuerakten bei Westphal, Steuerakten (wie Anm. 64).

${ }^{67}$ Vgl. Christian KeItel, Vorschläge zur gemeinsamen Klassifikation konventioneller und digitaler $\mathrm{Ar}$ chivalien, in: Moderne Aktenkunde (wie Anm. 14) 131-144, dessen Überlegungen hier einen Ausgangspunkt bildeten.

68 Hierbei handelt es sich um Arbeitstitel, deren Tragfähigkeit zu überprüfen bleibt.

69 Pauser-Scheutz-Winkelbauer, Vorbemerkung (wie Anm. 58) 11. 
ja Verfahren des Bundes und der Länder, die übergreifend eingesetzt werden; verwiesen sei dazu nur auf die Arbeitsverwaltung.

Zum Stand der Umsetzung kann zum Stichtag 1. April $2017^{70}$ gegenüber dem in Wien gehaltenen Vortrag berichtet werden, dass am 24. Februar 2017 am Institut für Geschichtliche Landeskunde und Historische Hilfswissenschaften der Universität Tübingen ein Workshop mit allen bisher beteiligten Autorinnen und Autoren stattfand, auf dem die so weit vorliegende und gegliederte Artikelliste diskutiert und offene Fragen geklärt wurden. Teilgenommen haben daran über 50 Personen. Aktuell umfasst die Liste der zugesagten Beiträge 184 Artikel, von denen einige wenige sogar bereits vorliegen. Beteiligt sind 91 Autorinnen und Autoren, die sich auf 70 Archivarinnen und Archivare sowie 21 Beitragende aus der universitären Forschung oder anderen Forschungseinrichtungen aufteilen. Eingebunden sind neben den staatlichen Archiven auch andere Archivsparten: Stadt- und Kreisarchive, kirchliche Archive, Universitätsarchive und das Wirtschaftsarchiv Baden-Württemberg in Stuttgart-Hohenheim.

\section{Fazit und Ausblick}

Ich komme zum Schluss und fasse zusammen. Angesichts eines neu erwachten Interesses der historischen Forschung an den Grundwissenschaften und der sich gerade vielerorts etablierenden Praxis digitaler Archivierung in den Archiven bestehen heute beste Bedingungen, die archivalische Quellenkunde bis in die Gegenwart fortzuschreiben und neu zu positionieren. Eine auf historische Räume ausgerichtete Archivalienkunde im Netz, die als verknüpftes Informationsangebot der Archive an alle Interessierten realisiert und nachhaltig gepflegt wird, kann und soll dies nachhaltig unterstützen.

Für die längst überfällige Fortschreibung der archivalischen Quellenkunde bis in die Gegenwart empfiehlt sich eine Zusammenarbeit zwischen Archiven und der historischen Forschung, in die besonders auch gezielt Personen einbezogen werden, die in den Archiven auf den Arbeitsfeldern der Überlieferungsbildung, des Records Management und der Digitalen Archivierung tätig sind.

Breit angelegte Beteiligungen der verschiedenen Archivsparten wie auch der universitären und außeruniversitären Forschung, wie sie im Projekt der „Südwestdeutschen Archivalienkunde“ realisiert werden, entsprechen dem zentralen Ziel des Gesamtkonzepts, möglichst viele Kreise für die Quellenkunde zu gewinnen, um sie dauerhaft neu zu positionieren. Das Portal soll nach seiner Freischaltung kontinuierlich ausgebaut werden und Wirkungen entfalten. Dazu finden sich im Projektantrag die Sätze: „Die angedachte Informationsplattform würde nachhaltig der kollaborativen Weiterentwicklung dienen und eine Struktur für die Ergebnissicherung darstellen, auf die überörtlich in der Lehre zurückgegriffen werden könnte. Sie würde [...] den Austausch zwischen Archiven und historischer Forschung wie auch innerhalb der Forschung erleichtern und die Lehre instrumental stützen“71. Um eben diese Wirkungen nachhaltig zu entfalten, wird sein Aufbau von der „Landesinitiative Kleine Fächer“ gefördert. Für den Dialog soll dabei auch eine

${ }^{70}$ Die Druckfassung entspricht weitgehend dem auf der Jahrestagung des Instituts für Österreichische Geschichtsforschung am 9. November 2016 gehaltenen Vortrag. Einzelne Passagen wurden unter Berücksichtigung des weiteren Fortgangs des Projekts „Südwestdeutsche Archivalienkunde“ aktualisiert. Ergänzungen zum Stand nach Manuskriptabschluss finden sich unten als „Nachtrag“.

71 Projektantrag (wie Anm. 7). 
Kommentarfunktion eingerichtet werden. Und wir werden für jede Korrektur, Anregung und Diskussion dankbar sein.

Beim Stichwort „Lehre“ möchte ich nochmals auf die Defizite in den Historischen Grundwissenschaften zurückkommen, die im Papier von Schlotheuber und Bösch angesprochen $\operatorname{sind}^{72}$. In Deutschland wird die universitäre Lehre in den Grundwissenschaften und in der archivalischen Quellenkunde heute weitgehend von Archivarinnen und Archivaren auf der Basis von Lehraufträgen und Honorarprofessuren abgedeckt, so auch am Institut für Geschichtliche Landeskunde und Historische Hilfswissenschaften in Tübingen, wo Sönke Lorenz über Jahre hinweg ein „Netzwerk Landesgeschichte“ geschaffen hat ${ }^{73}$.

Das funktioniert auch in Tübingen recht gut. Ich glaube aber, dass für eine nachhaltige Neubelebung der Grundwissenschaften und der Quellenkunde an den Universitäten weitaus mehr geschehen muss, als dass sie - wie bisher - zergliedert in einzelne Fächer als mehr oder weniger optionales Addendum angeboten werden. Vielmehr bedarf es neuer Ansätze im „Kern“ der Forschung und Pflichtbereich der Lehre. Hierbei könnte gerade die „Archivalienkunde“, wie ich sie zuvor als ein neu zu profilierendes und etablierendes eigenes Fach skizziert habe, eine besondere Bedeutung gewinnen, da sie das Potential bietet, die auf Archivgut bezogenen Disziplinen in ihren Schnittmengen unter einem Dach $\mathrm{zu}$ vereinen - epochenübergreifend und problemorientiert offen für immer neue Fragestellungen.

Unser Modul, das ortsunabhängig zur Verfügung stehen wird, soll auch in diesem weiten Kontext einer Neuausrichtung der Quellenkunde in Verbindung mit den Historischen Grundwissenschaften Wirkungen entfalten.

Es richtet sich freilich, wie bereits erwähnt, an alle Interessierten, gerade auch an solche ohne Vorkenntnisse. Es gibt ja weite Kreise jenseits der Universitäten, die im Netz auf Archive und ihre Online-Findmittel stoßen. Eine wichtige Funktion des Moduls als Informationsplattform soll daher auch darin bestehen, durch Verlinkungen grundwissenschaftliche Angebote im Netz zusammenzuführen, um sie allen Interessierten bekannt und zugänglich zu machen. Ich verweise nur auf „Grotefend online "74, die neue „Digitale Schriftkunde“75 der Staatlichen Archive Bayerns und die „Archivwissenschaftliche Terminologie" "76 auf der Website der Archivschule Marburg.

Ein letzter Punkt: Er berührt die archivarische Fachkompetenz. Ich sehe - und das ergibt sich aus allem zuvor Gesagten - die archivalische Quellenkunde nach wie vor als einen wichtigen Gegenstand in der Ausbildung von Archivarinnen und Archivaren an. Unser Modul wird auch hierfür einsetzbar sein. Ich könnte mir sogar vorstellen, dass seine Pflege zukünftig gerade in der modularisierten Ausbildung eine Rolle spielen wird.

Die archivalische Quellenkunde zählt unbestreitbar zu den Kernkompetenzen der Archive und darf auch im Digitalen Zeitalter nicht in den Hintergrund gedrängt werden. Eine Quellenkunde, die sich jüngsten und jüngeren Überlieferungen zuwendet, wird

72 Schlotheuber-Bösch, Quellenkritik (wie Anm. 8).

73 Vgl. Netzwerk Landesgeschichte (wie Anm. 25).

${ }^{74}$ Hermann Grotefend, Zeitrechnung des deutschen Mittelalters und der Neuzeit. HTML-Version von Horst Ruth, http://bilder.manuscripta-mediaevalia.de/gaeste/grotefend/grotefend.htm [1. 3. 2018].

75 Digitale Schriftkunde, in: Die staatlichen Archive Bayerns, http://www.gda.bayern.de/DigitaleSchriftkunde/ [1.3.2018].

76 Terminologie der Archivwissenschaften, in: Archivschule Marburg. Hochschule für Archivwissenschaft, http://www.archivschule.de/uploads/Forschung/ArchivwissenschaftlicheTerminologie/Terminologie.html [1.3. 2018]. 
auch keine Disziplin sein, die mit einem obsoleten archivarischen Berufsbild vergangener Zeiten in Verbindung zu bringen ist. Sie wird vielmehr Schnittmengen mit der Archivwissenschaft haben, in denen wechselseitig Befruchtungen eintreten werden. Die archivalische Quellenkunde bleibt eine zentrale Fachkompetenz der Archivarinnen und Archivare, die entsprechend weiter zu profilieren ist.

Um es in Anlehnung an den Titel dieser Tagung auf den Punkt zu bringen: Sie vermag vielleicht kein „Leuchtfeuer im Informationszeitalter“ zu entzünden. Aber sie ist ein „Kleines Fach“ mit potentiell großer Wirkung.

\section{Nachtrag}

Gegenüber dem Projektstand zum Manuskriptabschluss ist zum Stichtag 1. Juli 2018 mitzuteilen, dass die „Südwestdeutsche Archivalienkunde“ als ein neues Themenmodul in LEO-BW am 22. Februar 2018 in Stuttgart freigeschaltet wurde und nunmehr öffentlich abrufbar ist ${ }^{77}$. Zum damit erreichten Stand der Umsetzung kann auf die dortigen Ausführungen unter „Über das Projekt“ und „Aufbau des Themenmoduls und Artikelgliederung “ sowie auf einen parallel erschienenen Artikel verwiesen werden ${ }^{78}$. Über die Freischaltung im Rahmen eines Workshops und die dort geführte Diskussion ist ein zusammenfassender Bericht bereits publiziert ${ }^{79}$. Die Referate, die auf dem Workshop von Thomas Stockinger zur Archivalienkunde in Österreich und Niklaus Bütikofer zur Archivalienkunde in der Schweiz gehalten wurden, sowie ein Statement von Eva Schlotheuber als Vorsitzende des Verbands der Historiker und Historikerinnen Deutschlands (VHD) wurden in den „Blättern für deutsche Landesgeschichte“ veröffentlicht ${ }^{80}$.

77 Themenmodul - Südwestdeutsche Archivalienkunde, in: LEO-BW. Landeskundliches Informationssystem Baden-Württemberg, https:/www.leo-bw.de/web/guest/themenmodul/sudwestdeutsche-archivalienkunde [20. 6. 2018].

78 Anna Aurast-Christian Keitel-Robert Kretzschmar-Andreas Neuburger, „Südwestdeutsche Archivalienkunde" - ein neues Angebot in LEO-BW zur Stärkung der Historischen Grundwissenschaften. Archivar. Zeitschrift für Archivwesen 71 (2018) 47-51.

79 Anna Aurast, Tagungsbericht: Workshop zur Freischaltung der Südwestdeutschen Archivalienkunde als Beitrag zur Stärkung der Historischen Grundwissenschaften, 22.02.2018 Stuttgart. H-Soz-Kult (9. 6. 2018), https://www.hsozkult.de/conferencereport/id/tagungsberichte-7671 [1. 7. 2018].

80 Thomas Stockinger, Zum gegenwärtigen Stand der Archivalienkunde in Österreich. Blätter für deutsche Landesgeschichte 153 (2017) 447-459; Niklaus BütıкоғER, Archivwissenschaft und Archivalienkunde in der Schweiz. Ebd. 461-464; Eva Schlotheuber, Das Gedächtnis der Gesellschaft. Zur Freischaltung des Moduls. Ebd. 465-467. 



\title{
Probleme einer Aktenkunde der Zeitgeschichte
}

\author{
Holger Berwinkel
}

Die Aktenkunde ist in der Krise. De facto ist sie eine Geheimwissenschaft der Archivarinnen und Archivare. Es praktizieren sie in der einen oder anderen Weise zwar alle, die im Archiv arbeiten; ein Beispiel geben Quelleneditionen ${ }^{1}$. Aber diese Forscherinnen und Forscher tun es situativ und wissen oft nicht, dass sie sich mit einer speziellen Hilfswissenschaft behelfen könnten. Die Archivare haben ihrerseits kaum noch die Zeit für Aufgaben, die aktenkundliche Expertise erfordern würden. Und wenn doch, reicht zur Bewältigung kleiner Alltäglichkeiten, etwa der Zuweisung eines Farbstifts ${ }^{2}$, aus der Archivarsausbildung behaltenes Grundwissen. Auseinandersetzung mit der Forschungsliteratur ist kaum nötig - warum auch? Der Kern des Problems ist, dass die Aktenkunde für den größten Teil des Archivguts, die Akten des 20. Jahrhunderts, des ausgehenden Papierzeitalters, wenig anzubieten hat. Heinrich Otto Meisner hat noch selbst festgestellt, dass seine stilistische Typologie der Aktenstücke auf diese Überlieferung nicht mehr anwendbar ist ${ }^{3}$. Füllen konnte er die Lücke nicht mehr.

Kann das vertrocknende Pflänzchen der Aktenkunde noch aufgepäppelt werden oder sollte man es ausreißen und mit archivwissenschaftlichem Samen neu pflanzen? In der jüngst erschienenen Veröffentlichung ${ }^{4}$ aus dem „Arbeitskreis Aktenkunde des 20. und 21. Jahrhunderts" des Verbands deutscher Archivarinnen und Archivare e. V. ${ }^{5}$ wurde beiden Positionen Gehör geschenkt, ohne sie zu gewichten. Das Defizit wurde damit noch nicht beseitigt, aber von gefühltem Unbehagen hoffentlich auf die Stufe umrissener Probleme angehoben.

Das Folgende ist ein Plädoyer für die evolutionäre Fortentwicklung des Fachs. Am Anfang muss eine Aufgabenkritik stehen, die Zweck und methodische Aktualität der Aktenkunde aus der Perspektive der Anwenderinnen und Anwender beurteilt. Diese sind

${ }^{1}$ Ilse Dorothee Pautsch, Die „Akten zur Auswärtigen Politik der Bundesrepublik Deutschland“ - Ein Arbeitsbericht über die Erschließung der Bestände des Politischen Archivs des Auswärtigen Amts. Archivar. Zeitschrift für Archivwesen 61 (2008) 26-32.

2 Vgl. Holger Berwinkel, Akten sind bunt: Farbstifte und ihr Wert für die Archivarbeit. Aktenkunde. Aktenlesen als historische Hilfswissenschaft (17. 8. 2016), https://aktenkunde.hypotheses.org/552 [1. 3. 2018].

3 Heinrich Otto MeIsner, Allgemeine archivische Schriftgutkunde und Fragen der Archivwissenschaft. Archivalische Zeitschrift 54 (1958) 49-73, hier 65.

${ }^{4}$ Moderne Aktenkunde, hg. von Holger Berwinkel-Robert Kretzschmar-Karsten Uhde (Veröffentlichungen der Archivschule Marburg, Hochschule für Archivwissenschaft 64, Marburg 2016).

5 Zu dessen Arbeit: Holger Berwinkel-Robert Kretzschmar-Karsten Uhde, Aus der Werkstatt der Aktenkunde. Der Arbeitskreis „Aktenkunde des 20. und 21. Jahrhunderts“ des VdA. Archivar. Zeitschrift für Archivwesen 67 (2014) 293-295. 
längst nicht nur in Archiven zu suchen. Die Aktenkunde ist kein geschichtswissenschaftliches Glasperlenspiel, sondern bedient einen gesellschaftlichen Bedarf, der bis zur Überführung von Diktatoren als Kriegsverbrechern geht ${ }^{6}$.

Die zentrale Methode ist die von Heinrich Otto Meisner treffend bezeichnete Genetische Aktenkunde 7 . Mit ihrer Hilfe lässt sich „das Werden der Entscheidung in den Akten“ nachvollziehen ${ }^{8}$. Anhand von inneren Merkmalen, Bearbeitungsspuren und Entstehungsstufen wird ein Schriftstück einem Urheber zugewiesen und sein Weg durch den Geschäftsgang einer Institution nachvollzogen. Begründet wurde diese Methodik 1904 von Friedrich Küch als Nebenprodukt archivischer Bestandsbildung? ${ }^{9}$. Mit seinen Mitarbeitern im Staatsarchiv Marburg hatte er Schriftstück für Schriftstück die zersplitterte Registratur des Landgrafen Philipp provenienzgerecht geordnet und in solcher Tiefe erschlossen, dass seine Erschließungsleistung heute als die eines Akteneditors bezeichnet wird ${ }^{10}$. Man mag darin eine archivwissenschaftliche Dimension der Aktenkunde erkennen und diese von einer hilfswissenschaftlichen Dimension abgrenzen ${ }^{11}$. Ob eine derartig feine Differenzierung nützt, erscheint fraglich; vor allem ist die erste Dimension keineswegs auf archivische Zwecke beschränkt. Jedenfalls zielt die in diesem Sinne hilfswissenschaftliche Seite darauf, Entscheidungsabläufe zu rekonstruieren und Verantwortung zuzuweisen. 1909 entwickelte der an den „Acta Borussica“ tätige Editor Martin Haß eine Aktenkunde Brandenburg-Preußens, die in den Umrissen schon dem späteren Lehrgebäude Meisners entsprach, aus der ebenso einfachen wie zentralen Frage, welche Weisungen, die im Namen des Landesherrn ergangen waren, wirklich von diesem stammten ${ }^{12}$.

Diese beiden einleuchtenden Ansätze wurden anhand mediävistischer Vorbilder mit und für Akten der Frühen Neuzeit entwickelt ${ }^{13}$. Sind sie auch für zeitgeschichtliche Quellen brauchbar? 2010 veröffentlichte WikiLeaks 250.000 diplomatische Berichte

\footnotetext{
${ }^{6}$ Holger Berwinkel, Wiener Nachträge III: Assads Geschäftsgang. Aktenkunde. Aktenlesen als historische Hilfswissenschaft (20. 12. 2016), https://aktenkunde.hypotheses.org/630 [1. 3. 2018].

7 Heinrich Otto MeIsner, Aktenkunde. Ein Handbuch für Archivbenutzer mit besonderer Berücksichtigung Brandenburg-Preußens (Berlin 1935) 126.

${ }^{8}$ Lorenz Friedrich BECK, Leistung und Methoden der Aktenkunde bei der Interpretation formalisierter Merkmale von historischem Verwaltungsschriftgut, in: Der Zugang zu Verwaltungsinformationen - Transparenz als archivische Dienstleistung. Beiträge des 5. Archivwissenschaftlichen Kolloquiums der Archivschule Marburg, hg. von Nils BRÜвACH (Veröffentlichungen der Archivschule Marburg, Institut für Archivwissenschaft 33, Marburg 2000) 67-79, hier 70.

9 Friedrich $\mathrm{KüCH}$, Politisches Archiv des Landgrafen Philipp des Großmütigen von Hessen. Inventar der Bestände (Publikationen aus den K. Preußischen Staatsarchiven 78, Leipzig 1904) XXIX-XXXVI.

10 Robert Kretzschmar, Akten- und Archivkunde im Tübinger Netzwerk Landesgeschichte. Ein Plädoyer für eine zeitgemäße Archivalienkunde, in: Netzwerk Landesgeschichte. Gedenkschrift für Sönke LorENZ, hg. von Dieter R. Bauer-Dieter Mertens-Wilfried Setzler-Susanne Borgards (Tübinger Bausteine zur Landesgeschichte 21, Ostfildern 2013) 91-109, hier 93.

11 Jürgen Kloosterhuis, Amtliche Aktenkunde der Neuzeit. Ein hilfswissenschaftliches Kompendium. AfD 45 (1999) 465-563, hier 471.

12 Martin Hass, Über das Aktenwesen und den Kanzleistil im alten Preußen. Forschungen zur Brandenburgischen und Preußischen Geschichte 22 (1909) 521-575.

13 Zur forschungsgeschichtlichen Einordnung des oben Dargestellten: Eckart Henning, Wie die Aktenkunde entstand. Zur Disziplingenese einer Historischen Hilfswissenschaft und ihrer weiteren Entwicklung im 20. Jahrhundert, in: DERs., Auxilia Historica. Beiträge zu den historischen Hilfswissenschaften und ihren Wechselbeziehungen (Köln-Weimar-Wien 22004) 105-127; Holger Berwinkel, Forschungsgeschichte der Aktenkunde I: Wegbereiter im frühen 20. Jh. Aktenkunde. Aktenlesen als historische Hilfswissenschaft (17. 1. 2015), https://aktenkunde.hypotheses.org/306 [1.3. 2018].
} 
amerikanischer Botschaften an das Außenministerium in Washington ${ }^{14}$. Die meisten dieser Unterlagen waren wenige Jahre alt. Das Computersystem, aus dem sie stammten, enthielt aber auch den Text wesentlich älterer Stücke, die nachträglich erfasst worden waren; einige datieren in die Siebzigerjahre zurück. Mittlerweile wird aus diesem Fundus ganz selbstverständlich in zeitgeschichtlichen Studien zitiert. Die Fundstelle lautet dann: WikiLeaks ${ }^{15}$. Es dürfte fraglich sein, ob zur Überprüfung noch jemals Forscher in das amerikanische Nationalarchiv fahren werden. Die Veröffentlichung sei für Historiker ein Traum, meinte ein so eminenter Kommentator wie Timothy Garton Ash ${ }^{16}$. Diesen Traum zu deuten erfordert freilich besondere Fähigkeiten: Um die Datenmenge durchschaubar zu machen, haben die Enthüller und ihre Berater in den Redaktionen von Spiegel und Guardian Aktenkunde betrieben, obwohl ihnen das Wort unbekannt war; zwar an sekundären Überlieferungsformen, nämlich Datenbankeinträgen, aber mit validen Ergebnissen. Sie haben mit Akribie das Berichtsformular analysiert, relevante innere Merkmale, die sie als „kryptische Codes“ bezeichnen, isoliert und diese mit quellenkritischen Fragestellungen verbunden, etwa um Absender und Empfänger zu identifizieren ${ }^{17}$. So haben sie mit neuen Werkzeugen an neuartigem Material bewährte Methoden angewandt. In diesem Zusammenhang können auch ein Dateiname und ein Platz in der Verzeichnisstruktur einer Festplatte Ansatzpunkte aktenkundlicher Betrachtung sein ${ }^{18}$.

US-Depeschen en gros gibt es auch legal direkt beim Urheber, im so genannten Elektronischen Lesesaal des Department of State, in den Dokumente eingestellt werden, die punktuell auf Antrag nach dem Freedom of Information Act herausgegeben wurden. Hier liegen die Stücke nicht nur als Text vor, sondern als Scans vom Papier-Original, sind aber aus dem Aktenzusammenhang gerissen und nur über eine Volltextsuche zugänglich. Giulia Barrera hat den Umgang mit dieser Überlieferung didaktisch veranschaulicht: Sie hat anhand öffentlich zugänglicher Regelwerke die Kanzleipraxis des Ministeriums in den Siebzigerjahren rekonstruiert, eine intensive Innenanalytik mit Blick auf die Entstehungsstufen von Fernschreiben angestellt, virtuell die Provenienzen wiederhergestellt und schließlich einen exemplarischen Entscheidungsvorgang Henry Kissingers zu rekonstruieren versucht ${ }^{19}$. Hinsichtlich des Zwecks und der Grundlinien der Methodik besteht kein Unterschied zu den Arbeiten von Küch und Haß. Nur ist die Arbeit schwieriger, weil die untersuchte Überlieferung fragmentiert ist. Fragmentierte Akten sind aber der Grundtatbestand, mit dem sich die zeitgeschichtliche Quellenkunde auseinanderzusetzen hat. Dies gibt Anlass zu me-

${ }_{14}$ Diese Berichte sind in die so genannte „Public Library of US Diplomacy“ eingegangen: Public Library of US Diplomacy, https://wikileaks.org/plusd/ [1.3. 2018].

15 Siehe z. B. Henning Türk, Kooperation in der Krise? Die Ölkrise von 1973/74 und die multilaterale Zusammenarbeit der westlichen Industrieländer in der Energiepolitik. Journal of European Integration History 22 (2016) 47-65, hier 53 Anm. 22.

16 Zitiert nach Kiran Klaus Patel, Zeitgeschichte im digitalen Zeitalter. Neue und alte Herausforderungen. Vierteljahrshefte für Zeitgeschichte 59 (2011) 331-351, hier 331.

17 Der Spiegel 48/2010, v. a. Schaubild auf 106. Vgl. Simon Rogers, Wikileaks und der investigative Datenjournalismus. Wie wir beim Guardian mit den Wikileaks-Dateien umgehen, in: Wikileaks und die Folgen. Netz - Medien - Politik. Die Hintergründe, die Konsequenzen, hg. von Heinrich GeIselberger (Berlin 2011) $118-127$.

${ }^{18}$ Die jüngst an WikiLeaks verratenen Unterlagen des NSA-Untersuchungsausschusses des Deutschen Bundestages sind dafür ein Beispiel, siehe Holger Berwinkel, Wiener Nachträge I: Das Problem Wikileaks. Aktenkunde. Aktenlesen als Historische Hilfswissenschaft (9. 12. 2016), https://aktenkunde.hypotheses.org/620 [1. 3. 2018].

19 Giulia Barrera, La diplomatica dei diplomatici. Leggere i telegrammi on line del Dipartimento di Stato. Archivi e Computer. Automazione e beni culturali 21 (2011) 5-39. 
thodologischen Fragen, die seitens der Zeitgeschichtsforschung bereits formuliert wurden, ohne dabei das bestehende Angebot der Historischen Hilfswissenschaften aufzugreifen ${ }^{20}$. Das mag freilich auch am zeithistorischen Defizit der Hilfswissenschaften liegen.

Vor über 50 Jahren konnte Ahasver von Brandt noch schreiben: „Im ,Aktenzeitalter wird nicht nur dieses Endprodukt [die Urkunde], sondern auch alle Zwischenstadien des verwaltungsmäßigen oder geschäftlichen Handelns schriftlich dokumentiert" ${ }^{21}$. Solches Aktenwesen sei Kennzeichen einer entwickelten Gesellschaft. Die Gleichung Heutige Aktenlage $=$ Früheres Verwaltungshandeln aber war immer schon eine Illusion .

Noch 2015 war der Frankfurter Allgemeinen Zeitung eine neue Interpretation zur Schuld am Ersten Weltkrieg eine ganze Spalte wert, die ein ausgewiesener Kenner der Materie aus einer einzelnen Unterstreichung Wilhelms II. entwickelt. Sie steht im archivalischen Original unter einem anderen Wort als in der Edition. Die Akribie ist anerkennenswert und mag bei flüchtiger Betrachtung als aktenkundliche Höchstleistung erscheinen. Doch im welchem Verhältnis steht der isolierte Erkenntnisgewinn zur Masse der Akten zur Julikrise, die zum Großteil bereits elektromagnetischen Systemen entstammt und an wichtigen Stellen von allenfalls knapp verschriftlichten Telefonaten durchkreuzt wurde ${ }^{22}$ ?

Das 20. Jahrhundert sah eine Disruption der Akten als Arbeitsspeicher der Verwaltung. Das Maß der Verschriftlichung, das von Brandt vor Augen hatte, war eben kein Endzustand der zivilisatorischen Entwicklung, sondern ein zeitweiliges Maximum in einer besonderen Epoche der Verwaltungsgeschichte zwischen tintenklecksenden Kollegialbehörden und monokratischem Kontrollbedürfnis. Nur für diese Referenzepoche konnte Heinrich Otto Meisner seinem Lehrgebäude die Annahme einer Einheit von Inhalt, Form und Funktion zugrunde legen, nämlich „daß bestimmte sachliche Aufgaben nur in bestimmtem schriftlichem Gewande vollzogen wurden“23.

Diese Disruption wurde von zwei Kräften getrieben, zunächst von der Verwaltung selbst. Akten unterscheidet vom Rest des Archivguts, dass sie nicht einfach Dokumente bestimmten Inhalts sind, sondern abgelegte Werkzeuge der Selbststeuerung, die irreversible Spuren der eigenen Bearbeitung tragen ${ }^{24}$ - sozusagen die Narben des Geschäftsgangs. Vollständig verschriftlicht wurde das Verwaltungshandeln nie, immer sprachen die Beamten auch miteinander, und sei es nur zur Regelung ihrer Zuständigkeit ${ }^{25}$.

Im 20. Jahrhundert begann die Verwaltung nach exponentiellem Aufgabenzuwachs aber, die Verschriftlichung insgesamt als Hemmschuh zu betrachten. Veraktet wurden oft

20 Patel, Zeitgeschichte (wie Anm. 16) 346.

${ }^{21}$ Ahasver von Brandt, Werkzeug des Historikers. Eine Einführung in die historischen Hilfswissenschaften (Stuttgart $\left.{ }^{3} 1963\right) 126$.

22 Gerd Krumeich-Britta Gillessen, Muss - bald - aufräumen. Kaiser Wilhelms Notate. Frankfurter Allgemeine Zeitung (9. 12. 2015) N 3. Kritik an der Vorgehensweise übt Holger Berwinkel, Als der Kaiser musste: Eine Unterstreichung und die Schuld am Ersten Weltkrieg. Aktenkunde. Aktenlesen als Historische Hilfswissenschaft (9. 1. 2016), https://aktenkunde.hypotheses.org/460 [1.3. 2018]; dort Quellen- und Literaturangaben. - Zu Telefonaten und ihrer Ersatzüberlieferung siehe: Die deutschen Dokumente zum Kriegsausbruch 1914. Vollständige Sammlung der von Karl Kautsky zusammengestellten amtlichen Aktenstücke mit einigen Ergänzungen, hg. von Max Graf Montgelas-Walter Sснüскіng, 4 Bde. (Charlottenburg 1919) z. B. $2164 f$. Nr. 441; 36 Nr. 562; 43 Nr. 739.

23 Meisner, Schriftgutkunde (wie Anm. 3) 54.

24 Angelika Menne-Haritz, Schriftgut oder Dokumente: Was sind die Spuren automatisierter Verwaltungsarbeit? Archivalische Zeitschrift 79 (1996) 1-36, hier 3-6.

${ }^{25} \mathrm{Vgl}$. Holger Berwinkel, Wiener Nachträge II: Eine Zeichnungsordnung und die Grenzen formaler Erkenntnis. Aktenkunde. Aktenlesen als Historische Hilfswissenschaft (15. 12. 2016), https://aktenkunde.hypotheses.org/625 [1.3. 2018]. 
nicht mehr alle acta, sondern nur noch die Meilensteine der Entscheidungsfindung, und zwar in möglichst einfachen Formen, die nicht mehr an bestimmte Zwecke gekoppelt waren. Die Planung und Abarbeitung der Entscheidungsfindung wurde immer weniger mit Vermerken und Verfügungen verschriftlicht ${ }^{26}$. Oft beschränken sich diese, für die klassische Aktenkunde so zentralen, Spuren auf bürotechnische Eingangs- und „zdA“-Vermerke.

Als zweite Triebkraft lieferte die bürotechnische Innovation diesem neuen Verwaltungsstil die passenden Mittel. Früher hatten Kanzleibeamte Entwürfe erstellt, die im Geschäftsgang bis zur abschließenden Genehmigung revidiert wurden. Dann wurde als neues Schriftstück die Ausfertigung erstellt. In der neuen Zeit tippte eine Sekretärin ein Diktat vom Tonband direkt in die Maschine. Zu den Akten ging ein Durchschlag mit Vermerken wie „Doppel als Konzept“ oder „Durchschlagentwurf“: ein bloßes Dokument ohne den prospektiven Charakter eines wirklichen Entwurfs ${ }^{27}$.

Auf der Empfängerseite sollte die Ausfertigung eigentlich ein unikales Authenticum sein, das alle Vermerke und Verfügungen aufnimmt. Nicht nur, dass diese spärlicher wurden, es wütete auch die Kopiertechnik. Ein Aktenstück lief nicht mehr der Reihe nach in der Behörde um, sondern konnte gestreut werden wie ein Schrotschuss: Je mehr Kopien, desto größer die Wahrscheinlichkeit, auch die richtige Stelle zu treffen. Damit verteilen sich die relevanten Bearbeitungsspuren aber auf eine Vielzahl von Überlieferungsträgern. Die Leitbegriffe der genetischen Erkenntnis, „revidierter Entwurf“ (oder „Konzept“) und „behändigte Ausfertigung “28 - sozusagen Castor und Pollux der Aktenkunde -, haben vor diesem Hintergrund an Aussagekraft verloren. Auch das Begriffspaar „Überlieferungsform“ und „Entstehungsstufe“ gerät ins Wanken, je weniger man das Authenticum eines Stücks identifizieren kann. In den Akten finden sich wertlose Überlieferungsformen immer zahlreicher, gehaltvolle Entstehungsstufen aber zunehmend selten ${ }^{29}$.

Noch ein Begriffspaar passt nicht mehr: Die klassische Lehre unterscheidet zwischen vornehmlich an externe Empfänger gerichteten „Verkehrsschriftstücken“ und „Memorienschreibwerk“, das im Geschäftsgang unbeweglich sei und nur dem Urheber diene ${ }^{30}$. Für die frühen Achtzigerjahre, also die Zeit, deren Akten jetzt allgemein zugänglich geworden sind, ist davon auszugehen, dass über $50 \%$ der in deutschen Bundesministerien erstellten Schriftstücke interner Natur waren ${ }^{31}$. In der aktenkundlichen Literatur geht es weit überwiegend um externen Schriftverkehr. Die internen Zuschriften, Büroverfügungen und Leitungsvorlagen ${ }^{32}$ werden ebenso unzureichend abgedeckt wie das Reich der pro memoria niedergelegten Aktenvermerke und Protokolle.

${ }^{26}$ Holger Berwinkel, Zur Kanzleigeschichte des 20. Jahrhunderts - ein Versuch, in: Moderne Aktenkunde (wie Anm. 4) 29-50, hier 48f.

27 Ebd. 31-39; Karsten Uhde, Schriftgut des 20. und 21. Jahrhunderts genetisch betrachtet, in: Moderne Aktenkunde (wie Anm. 4) 51-72, hier 57-61.

${ }_{28}$ Besonders konzise Gerhard Schmid, Akten, in: Die archivalischen Quellen. Eine Einführung in ihre Benutzung, hg. von Friedrich Beck-Eckart Henning (Weimar 1994) 51-85, hier 62.

29 Berwinkel, Kanzleigeschichte (wie Anm. 26) 38f.; Uhde, Schriftgut (wie Anm. 27$) 57$.

${ }^{30}$ Kurt DüLfer, Urkunden, Akten und Schreiben in Mittelalter und Neuzeit. Studien zum Formproblem. Archivalische Zeitschrift 53 (1957) 11-53, hier 49f.

31 Alfred Kieser-Günther Klatt-Edith Rost-Schaude, Schreibdienstorganisation - human und wirtschaftlich. Eine Untersuchung zur ökonomischen und sozialen Effizienz von Schreibdiensten in obersten Bundesbehörden (Humanisierung des Arbeitslebens 46, Frankfurt am Main-New York 1983) 171.

32 Dazu Holger Berwinkel, Leitungsvorlagen in Ministerien als aktenkundliche Kategorie. Ihre Bedeutung für die Bewertung, Erschließung und Auswertung des Archivgutes. Archivalische Zeitschrift 95 (2017) $261-285$. 
Nach herkömmlichem Verständnis bildet eine Kette klar voneinander abgegrenzter Schriftstücke einen Vorgang, der es erlaubt, die Entscheidungsfindung in der betreffenden Sache nachzuvollziehen. Seit der Büroreform konnte ein ganzer Vorgang aber durch eine einzige sogenannte Büroverfügung ersetzt werden, in der alle Arbeiten zur Erledigung des Geschäftsvorfalls minutiös durchgeplant wurden. Entwürfe ausgehender Schreiben sind nicht mehr als selbständige Schriftstücke überliefert, sondern wurden nachträglich als Durchschläge in dafür vorgesehene Lücken inseriert: Zur Fertigung eines Ausgangs wurde der Wortlaut direkt diktiert und die Büroverfügung mit dem fertigen Rahmentext jeweils unter Durchschlagpapier mit in die Maschine eingespannt ${ }^{33}$. Von einer Selbststeuerung des Vorgangs durch sukzessive aufeinander aufbauende Verfügungen und Vermerke kann keine Rede mehr sein ${ }^{34}$. Was aussieht wie prospektiv planend, ist nur rückwirkende Dokumentation. Einen Entscheidungsprozess spiegeln solche Dokumente nur noch bedingt.

Dieser Befund umfasst den Schwund von aktenkundlichen Ansatzpunkten an Schriftstücken, die immerhin noch vorhanden sind. Zusätzlich ist aber an allen Stellen, an denen technische Verfahren ins Spiel kamen, ein Schwund von Überlieferungsträgern gegenüber der Epoche von Papier und Feder einzukalkulieren. Es wurde der Begriff „Zwischenmaterial“ vorgeschlagen, um dieses Phänomen zu kennzeichnen: Schriftstücke oder andere Speicherformen, die nie für die Akten bestimmt waren ${ }^{35}$. Ein Beispiel sind Stenogramme als Mittler zwischen dem Diktat und seiner Verschriftlichung durch die Maschine. Nach der Abschrift waren sie wertlos und wurden entsorgt. Es ist nicht mehr ersichtlich, welche nachträglichen Korrekturen darauf vielleicht angebracht waren. Gerade deshalb ist es auch nicht zulässig, Stenogramme mit der Begründung, dass sie nicht bei den Akten seien, für aktenkundlich nicht relevant zu erklären ${ }^{36}$. Eine für die Zeitgeschichte taugliche Aktenkunde muss auch die Entleerung der Akten von Stücken einbeziehen, die Entstehungsstufen hätten sein können, aber Zwischenmaterial waren.

Ein anderes Beispiel ist der Fernschreiber. Der Entwurf eines Erlasses eines Außenministeriums an eine seiner Botschaften wurde chiffriert, auf Lochstreifen kodiert, in elektromagnetischen Impulsen abgesetzt, von Relaisstationen mit Metadaten angereichert, dekodiert, maschinell ausgefertigt und in der empfangenden Botschaft interlinear dechiffriert. Dann wurde eine Reinschrift erstellt, und nur diese ist in den Akten der Botschaft zu finden. Ein solches Stück in konventioneller Art als Ausfertigung und das Gegenstück als Entwurf zu bezeichnen, würde zu viele Faktoren ausblenden ${ }^{37}$.

Dies war wohlgemerkt der technische Stand schon des Jahres 1914. Seit den Siebzigerjahren standen Computer an den Enden des Telex-Netzes und erledigten die Zwischenstadien elektronisch. Die Entkörperlichung und Verflüchtigung von Aktenstoff in der elektronischen Bürokommunikation war kein revolutionärer Bruch, sondern ein

33 Holger Berwinkel-Karsten Uhde, Anhang 2: Beispielsammlung, in: Moderne Aktenkunde (wie Anm. 4) 153-183, hier 159-161 Nr. 3. Onlinefassung: DIES., Beispielsammlung Moderne Aktenkunde, in: Archivschule Marburg. Hochschule für Archivwissenschaft - Publikation - Veröffentlichungsreihe, http://archivschule.de/DE/publikation/veroeffentlichungsreihe/voe64-beispielsammlung.html [1. 3. 2018].

34 Dieses Referenzmodell wird besonders prägnant entwickelt bei Angelika Menne-Haritz, Schriftlichkeit im Entscheidungsprozeß der Verwaltung. Die Geschäftsordnung der preußischen Regierung Kassel von 1867. Jahrbuch für europäische Verwaltungsgeschichte 9 (1997) 83-96.

35 Berwinkel, Kanzleigeschichte (wie Anm. 26) $34 \mathrm{f}$.

36 So Gerhard Schmid, Aktenkunde des Staates (Lehrbriefe für das Fachschulfernstudium der Archivare, Potsdam 1959) 189.

37 Berwinkel, Kanzleigeschichte (wie Anm. 26) 44. 
nahtloser Übergang aus der Welt des papierenen Zwischenmaterials und der elektromagnetischen Nachrichtentechnik. Jüngst hat Patrick Sturm kenntnisreich das aktenkundliche Problem der E-Mail analysiert. Weder stünde sie einer systematischen Typisierung offen, weil sie ein neutrales Trägermedium in verschiedenen Korrespondenzverhältnissen sei, noch seien die gewohnten genetischen Kriterien anwendbar ${ }^{38}$. Das ist richtig erkannt - nur ist es keine Besonderheit erst der vollelektronischen Kommunikation, sondern baute sich Stück für Stück, im Laufe von 150 Jahren, bereits in der Telegrafie auf.

Wie soll es vor dem Hintergrund dieses Bildes aktenkundlicher Verwüstung noch möglich sein, das „Werden der Entscheidungen in den Akten“ nachzuvollziehen?

Archivarinnen und Archivare sollten sich selbstkritisch bewusstmachen, dass, nur weil allein sie die Aktenkunde als Disziplin pflegen, sie diese dennoch nicht auf ihren Handgebrauch, die Objektsicht ${ }^{39}$, reduzieren dürfen. Sie wollen zuallererst Phänomene genau benennen. Daher rührt in der archivarischen Aktenkunde die auffällige Betonung der systematischen Klassifizierung, à la „Kabinettsorder in Form eines Handschreibens“ versus „Handschreiben in Form einer Kabinettsorder" 40 .

Im Gegensatz zur archivwissenschaftlichen Strukturlehre ist die Aktenkunde kein deskriptives System für papierne Speicherformen ${ }^{41}$, sondern eine historische Methode, um aus Akten auf acta zurückzuschließen. Darin unterscheiden sich Aktenkunde und Archivalienkunde, die Analyse von Prozessen und die Beschreibung von Strukturen ${ }^{42}$. Zwar ist es geboten, Elemente der Archivalienkunde aufzunehmen, um den Überlieferungskontext aufzubereiten, in dessen Rahmen aktenkundliche Schlüsse gezogen werden können ${ }^{43}$, denn es gilt der Satz: „Form und Methode der Aktenbenutzung sind abhängig von Form und Methode der Aktenordnung “44. Doch ist die Vergewisserung über die „Organisationsformen des Schriftgutes in Kanzlei und Registratur “ ${ }^{\star 45}$ eine vorbereitende Handlung, nicht die eigentliche Tätigkeit.

38 Patrick STURM, Die E-Mail - ein Kommunikationsmedium des frühen 21. Jahrhunderts quellenkundlich betrachtet, in: Moderne Aktenkunde (wie Anm. 4) 109-129, hier 114-119.

39 Zum Begriff vgl. Robert Kretzschmar, Akten - Begriff und Realitäten im zweiten Jahrzehnt des 21. Jahrhunderts, in: Moderne Aktenkunde (wie Anm. 4) 13-21, hier 16f. Zurecht werden hier die Prozessund die Objektsicht auf Verwaltungsvorgänge gleichberechtigt nebeneinandergestellt.

${ }^{40}$ Der Erkenntniswert der Identifizierung stilistischer Widersprüche in Aktenstücken sei unbestritten; als locus classicus vgl. Hans-Enno Konn, Kabinettsordres. Ein Kapitel Aktenkunde. Der Archivar. Mitteilungsblatt für deutsches Archivwesen 26 (1973) 225-332.

${ }^{41}$ Dies ist das grundlegende Missverständnis bei Ernst Pitz, Schrift- und Aktenwesen der städtischen Verwaltung im Spätmittelalter. Köln - Nürnberg - Lübeck. Beitrag zur vergleichenden Städteforschung und zur spätmittelalterlichen Aktenkunde (Mitteilungen aus dem Stadtarchiv von Köln 45, Köln 1959) 466. Die große Bedeutung dieser Studie für das Verständnis von Amtsbüchern als Sonderform amtlicher Überlieferung wird davon nicht berührt.

42 Botho Brachmann, Zum Verhältnis von Archivwissenschaft und Aktenkunde im Rahmen des Workflow-Managements, in: Archive und Forschung. Referate des 73. Deutschen Archivtags 2002 in Trier, hg. von Robert Kretzschmar et al. (Der Archivar. Mitteilungsblatt für deutsches Archivwesen Beiband 8, Siegburg 2003) 99-103, hier 100.

43 Eindrücklich demonstriert dies Robert Kretzschmar, Der Kriminalprozess gegen Jud Süß Oppenheimer aus archivwissenschaftlicher und aktenkundlicher Sicht, in: Text und Kontext. Historische Hilfswissenschaften in ihrer Vielfalt, hg. von Sönke Lorenz-Stephan Molitor (Tübinger Bausteine zur Landesgeschichte 18, Ostfildern 2011) 489-523.

44 Brandt, Werkzeug (wie Anm. 21) 134.

45 So der Titel des Teils II im Hauptwerk von Johannes Papritz, Archivwissenschaft, 4 Bde. (Marburg $\left.{ }^{2} 1983\right)$. 
Auch einem Fehlgebrauch der Aktenkunde ist vorzubeugen. Es geht nicht darum, die endlose Fülle der inneren und äußeren Merkmale, die an Akten zu beobachten sind, widerspruchsfrei in ein System zu pressen und dadurch das reibungslose Schnurren einer Kanzlei-Maschinerie zu dokumentieren. Aktenkunde ist eine Heuristik, die sich in Erkenntniszirkeln einem wahrscheinlichen Befund annähert. Formfehler, Überlieferungslücken und alles im Einzelfall Irreguläre sind dafür von höherem Wert als die Wiederkehr der immer gleichen regelkonformen Merkmale. Das gilt umso mehr, je weniger die Erstellung und Bearbeitung von Aktenstücken noch Regeln folgt. Weil die Aussagekraft der Details, etwa einzelner Formularbestandteile und Bearbeitungsvermerke, deshalb sinkt, mag solches Schriftgut einer Aktenkunde, die auf die Systematisierung von Details ausgelegt ist, als unzugänglich erscheinen. Damit ist aber keine Aporie formuliert, sondern eine Herausforderung an die Aktenkunde, sich anzupassen.

Eine Analogie mag hilfreich sein: Wer zu dicht vor einem pointilistischen Gemälde steht, sieht nur unverbundene Details. Erst für den, der den richtigen Abstand einnimmt, schält sich die Szenerie heraus, und das Werk wird erkennbar.

Ahasver von Brandt nannte das Bild eines Vorgangs, der nur aus Urkunden besteht, „grob gerastert" ${ }^{\prime 4}$, weil die Elemente unverbunden nebeneinanderstünden. Die Verbindungslinien seien ja das Besondere bei den Akten, die es als Begriff darum auch nur im Plural gebe. Wenn diese formal definierten genetischen Verbindungslinien aus Entstehungsstufen, Bearbeitungsspuren und so weiter nun zunehmend verblassen: Entsprechen die Aktenstücke dann inhaltlich nicht immer mehr autarken Urkunden ${ }^{47}$ ? Nur wer im Sinne obiger Analogie einen Schritt zurücktritt, von widersprüchlichen, unverständlichen oder ins Leere laufenden Details in den Schriftstücken abstrahiert und den Vorgang als Ganzen betrachtet, kann ein Bild erkennen: Der Weg der Entscheidungsfindung ist dann maßgeblich nicht mehr in nahtlosen inhaltlichen Bezugnahmen innerer Merkmale von Schriftstücken aufeinander zu suchen, sondern in der Art und Abfolge dieser Schriftstücke. Dazu gehören die physische und inhaltliche Komposition gleichermaßen. Diskrepanzen dieser beiden Ordnungen machen Überlieferungslücken sichtbar und vielleicht heilbar.

Angenommen, eine Behörde berichtet an ihre vorgesetzte Stelle und bittet um eine Weisung. Beim Empfänger findet dazu eine Besprechung statt, über die ein Protokoll geschrieben und genehmigt wird. Dem Behörden-Chef wird in einer Leitungsvorlage ein Vorschlag unterbreitet. Zum Schluss ergeht ein Erlass mit der erbetenen Weisung an die nachgeordnete Behörde. Dazwischen wird viel gesprochen, telefoniert und auf Haftnotizen gekritzelt, ohne dass dies dauerhaften Niederschlag in den Akten hinterlassen hätte. An bestimmten Stellen zwingt der Geschäftsgang aber zu einer umfassenderen, teilweise retrospektiven Verschriftlichung, zum Beispiel, wenn die Behördenleitung pflichtgemäß über alle entscheidungserheblichen Umstände informiert werden muss. Ein zusammenfassender Sachstandsvermerk wird zur Ersatzüberlieferung vieler nichtschriftlicher Handlungen. Fehlt ein solches Dokument, obwohl die nachfolgenden Stücke auf seine Anfertigung schließen lassen, dann liegt eine Irregularität vor, an der die inhaltliche Auswertung kaum vorbeigehen wird.

So vorzugehen, könnte eine genetische Aktenkunde des Vorgangs genannt werden. Nicht anders werden die Akten in der Verwaltung gelesen. Welcher Beamte hat die Zeit,

\footnotetext{
46 Brandt, Werkzeug (wie Anm. 21) 127.

47 Ebd. 125.
} 
sich durch einen Wust von Kopien zu arbeiten, um unter hundert Eingangsstempeln eine aussagekräftige Verfügung zu finden? Er oder sie sucht zunächst nach Leitdokumenten, in denen sich die wesentlichen Etappen der Entwicklung des Vorgangs spiegeln.

Dazu ist eine gründliche Kenntnis der Funktion bestimmter Schriftstücktypen für die Arbeit einer Institution nötig. Es mag sein, dass die Bedeutung der Systematischen Aktenkunde innerhalb des gesamten Lehrgebäudes oft überschätzt wurde ${ }^{48}$. Für die Weiterentwicklung des Fachs ist sie nicht zu unterschätzen, nur darf sie sich nicht in Quisquilien der Aktenstilistik verlieren.

Für den externen Schriftverkehr kann weiter die Schreibrichtung als Kriterium herangezogen werden: es wird berichtet, mitgeteilt oder angewiesen ${ }^{49}$. Man muss diese Ausprägungen nicht strikt im Sinne behördlicher Hierarchie verstehen. Sie stehen für kommunikative Grundhaltungen, in denen ein Partner auf ein bestimmtes Verhalten des Gegenübers hinwirkt ${ }^{50}$. In der öffentlichen Verwaltung korrespondiert dieses Modell mit formalen Unterstellungsverhältnissen, es funktioniert aber auch in anderen Zusammenhängen ${ }^{51}$.

Die neue zentrale Aufgabe ist die Typisierung der internen Schriftstücke, mit denen der Geschäftsgang gesteuert wurde. Das ist nur inhaltlich möglich. Hier lohnt es, den Blick vom Staat auf die Wirtschaft schweifen zu lassen. Schon in den 1950er Jahren hat Erich Neuß für dieses Gebiet eine Aktenkunde geschrieben, die vom Inhalt ausgeht, weil er formale Ansatzpunkte nicht in der nötigen Ausprägung fand, um Meisners Methodik übertragen zu können ${ }^{52}$. Die Aktenkunde zielt unverändert auf die Erkenntnis der Funktionalität bestimmter Schriftstücktypen für den Geschäftserfolg eines Unternehmens oder die Aufgabenerledigung einer Behörde, nur ist dies auch im staatlichen Bereich seltener auf einen Blick an Formalien ablesbar, sondern muss aus dem Inhalt bestimmt werden. Die Integration inhaltlicher Aspekte wird damit zum Schlüssel für die Weiterentwicklung der Aktenkunde ${ }^{53}$ - insbesondere wenn diese für Institutionen unterschiedlichen Charakters brauchbar sein soll.

48 Eine vorsichtige, aber berechtigte Kritik an der hyperpräzisen Terminologie, die sich in der Nachfolge Meisners etabliert hat, übt Karsten Uhde, Alter Stil - Neuer Stil - Neuester Stil. Überlegungen zu einer systematischen Aktenkunde des 20. und frühen 21. Jahrhunderts, in: Quellenarbeit und Schriftgutverwaltung Historische Hilfswissenschaften im Kontext archivischer Aufgaben. Beiträge zum 12. Archivwissenschaftlichen Kolloquium der Archivschule Marburg, hg. von Karsten UhDE (Veröffentlichungen der Archivschule Marburg, Institut für Archivwissenschaft 48, Marburg 2009) 71-88, hier 81-84.

49 Holger Berwinkel-Anette Meiburg, Die moderne Bundesverwaltung als Referenzmodell der Systematischen Aktenkunde, in: Moderne Aktenkunde (wie Anm. 4) 81-92, hier 85.

50 Gerhard LeIDel, Über die Prinzipien der Herkunft und des Zusammenhangs von Archivgut. Archivalische Zeitschrift 86 (2004) 91-130, hier 99.

${ }^{51}$ Vgl. Ulrich Schludi, Das Schriftgut der Wirtschaft, in: Moderne Aktenkunde (wie Anm. 4) 93-108, hier $105 \mathrm{f}$.

52 Erich Neuss, Aktenkunde der Wirtschaft, 1: Kapitalistische Wirtschaft (Schriftenreihe der Staatlichen Archivverwaltung 5, Berlin 1954). Neuß hat den Bogen freilich überspannt und ist in eine Quellenkunde des Wirtschaftsschriftguts abgedriftet, vgl. Holger Berwinkel, Forschungsgeschichte der Aktenkunde V: Stand und Desiderate. Aktenkunde. Aktenlesen als historische Hilfswissenschaft (16. 3. 2016), https://aktenkunde.hypotheses.org/496 [1.3. 2018].

53 Lorenz Friedrich BECK, Die Historischen Hilfswissenschaften im Informationszeitalter. Vom zeitlosen wie zeitgemäßen Nutzen des quellenkundlichen Instrumentariums für Archivar und Historiker, in: Archive und Gedächtnis. Festschrift für Botho Brachmann, hg. von Friedrich Beck-Eckart Henning-Joachim-Felix Leonhard-Susanne Paulukat-Olaf B. Rader (Potsdamer Studien 18, Potsdam 2005) 239-251, hier 240, 246f. Zuvor bereits Henning, Aktenkunde (wie Anm. 13) 120. 
Die Leitfrage des funktionalen Paradigmas ist: Was sollte mit dem Stück bewirkt werden? Was bedeutet es heute, in der Rückschau, wenn an einer bestimmten Stelle in den Akten ein bestimmtes Schriftstück auftaucht oder eben nicht? Unter diesem Blickwinkel rücken die genetische und systematische Betrachtung zusammen ${ }^{54}$.

Eine solche Methodik setzt eine Erwartungshaltung gegenüber der Zusammensetzung von Akten voraus. Die Typologie der Schriftstücke muss durch eine Typologie der Akten ergänzt werden. Gefordert ist eine ganzheitliche Betrachtung der Aktengattungen, die Inhalt, verwaltungspraktische Merkmale und strukturelle Erkenntnisse zur Kompositionsform einbezieht ${ }^{55}$. Sie erlaubt es, das Einzelstück nach seiner Bedeutung im Vorgang zu verorten und, wenn es Fragment ist, daraus auf unbekannte Teile zu schließen.

Die klassische Aktenkunde Meisnerscher Art wird gelegentlich als strukturblinde Lehre vom Einzelschriftstück kritisiert ${ }^{56}$. Damit wird zunächst Meisner Unrecht getan, der sehr wohl die Komposition der Schriftstücke zu Akten und schließlich Registraturen berücksichtigte: an ihrem natürlichen Platz im Rahmen der genetischen Betrachtung ${ }^{57}$. Sodann ist zu bemerken, dass die archivalische Strukturlehre in der Epoche der Zeitgeschichte mit der Auflösung der Strukturen zu kämpfen hat. Nach der Disruption der Verschriftlichung durch die Kanzleitechnik haben die bereits seit der Büroreform nachlassende Registraturdisziplin und in jüngster Zeit die Bildung von Hybridakten die Aktenüberlieferung auf eine noch höhere Stufe der Fragmentierung gehoben, die zu einer rekonstruierenden Betrachtung zwingt. Die verschwommene Gestalt der Überlieferung wird erst aus der ineinandergreifenden Analyse der erhaltenen Einzelstücke in Umrissen wieder erkennbar.

Noch weiter entzwei werden die Strukturen der Überlieferung durch Bewertung und Kassation gerissen. Und aus der Perspektive einer Historischen Hilfswissenschaft ist schließlich die Fragmentierung der Verbreitung zu berücksichtigen, insbesondere durch die Digitalisierung nur von ausgewähltem Akteninhalt. Es ist sicherlich nicht wünschenswert, dass Forschungsprojekte auf eine solche Materialgrundlage gestellt werden, aber es ist Realität ${ }^{58}$. Valide Ergebnisse bedürfen unter diesen Bedingungen einer besonders sorgfältigen Methodik, in der eine Aktenkunde einen zentralen Platz einnehmen muss, die Einzelstücke in Unkenntnis des vollständigen Überlieferungszusammenhangs sinnvoll erschließt - ob im Lesesaal oder am persönlichen Computer.

Auf die Leitfrage, wie unter den Bedingungen der Zeitgeschichte eine genetische Betrachtung von Akten noch möglich ist, bietet die Archivwissenschaft angesichts dieser Probleme keine Antwort, aber Anregungen. Sie leistet Dienste bei der funktionalen Typisierung von Aktenschriftstücken, insbesondere wenn die Akten aus normierten Verwaltungsverfahren stammen. Exemplarisch ist hinzuweisen auf eine zu wenig beachtete Veröffentlichungsreihe aus dem Landesarchiv Nordrhein-Westfalen, die in mittlerweile drei Bänden konzise Typologien sogenannter „Massenakten“ von homogener Struktur und

54 Beck, Hilfswissenschaften (wie Anm. 53) 445.

55 Für die ältere Zeit hat Michael Hochedlinger, Aktenkunde. Urkunden- und Aktenlehre der Neuzeit (Historische Hilfswissenschaften, Wien-München 2009), den Blick in diese Richtung bereits deutlich erweitert.

56 Mit dieser Kritik von Pitz, Schrift- und Aktenwesen (wie Anm. 41), setzt sich Henning, Aktenkunde (wie Anm. 13) 119f., treffend auseinander.

57 Meisner, Aktenkunde (wie Anm. 7) Abschnitt 5: „Genetische Aktenkunde (das Aktenschriftstück in Kanzlei, Registratur und Archiv)“.

58 Vgl. Patel, Zeitgeschichte (wie Anm. 16) 344. 
Inhalt bietet ${ }^{59}$ : Vom Grundbuchwesen über Namensänderungen, staatliche Fürsorge und Zivilprozesse bis zu Hochverrat und Staatsbesuchen wird ein breites Spektrum von Akten beschrieben, die jeweils der Regelung eines bestimmten Bereichs des Gesellschaftslebens gewidmet sind. Für diese Funktionen wurden die Akten angelegt, aus ihnen bestimmt sich die Zusammenstellung der Schriftstücke.

Mutatis mutandis ist der funktionale Ansatz aus der Mediävistik als Untersuchung pragmatischer Schriftlichkeit in ihrer Bedeutung für Verfasstheit und Arbeitsweise von Institutionen bekannt. Udo Schäfer hat ihn für die Aktenkunde fruchtbar gemacht und Perspektiven einer umfassenden Diplomatik der Neuzeit aufgezeigt, die die Kultur- und Mentalitätsgeschichte des Umgangs mit Schrift einbezieht und darin forschungsgeschichtlich der Urkundenlehre nachfolgt ${ }^{60}$. Auch das im selben Jahr veröffentlichte Handbuch Michael Hochedlingers geht bereits in diese Richtung ${ }^{61}$.

Die Elemente und Richtungen einer so verstandenen Aktenkunde lassen sich am Beispiel eines wichtigen, aber vernachlässigten Schriftguttyps aufzeigen: des Protokolls. Für die Organisation von Entscheidungsprozessen sind Protokolle von zentraler Bedeutung, mit rein formaler Methodik gleichwohl kaum aussagekräftig zu definieren. Schon Meisner hat sie daher inhaltlich erfasst und sachgerecht unterschieden zwischen Niederschriften zu Verhandlungen vor einem Gericht oder Beratungen in einem Gremium. Seine Terminologie ist freilich nicht trennscharf, geboten wird ein Materialsteinbruch ${ }^{62}$. Erst eine historische Annäherung an die Arbeitstechnik des Protokollierens klärt das Bild: Rainer Polley hat anhand von Kollegialbehörden des 18. Jahrhunderts den Typ des Beratungsprotokolls als Verschriftlichung von Mündlichkeit beschrieben ${ }^{63}$. Im Vergleich mit Udo Schäfers Befund zur Entwicklung des Verhandlungsprotokolls im kanonischen Prozessrecht ergibt sich ein klarer Unterschied zur Verschriftlichung von bereits Verschriftlichtem, die bei Gericht in Form der Abschrift von den Parteien vorgelegter Urkunden breiten Raum einnahm ${ }^{64}$. Damit ist eine aussagekräftige, für die praktische Anwendung brauchbare Binnendifferenzierung des Typs Protokoll entlang der Funktionalitäten gefunden. Für ein

59 Unbekannte Quellen: „Massenakten“ des 20. Jahrhunderts. Untersuchungen seriellen Schriftguts aus normierten Verwaltungsverfahren, hg. von Jens HeckL, 3 Bde. (Veröffentlichungen des Landesarchivs Nordrhein-Westfalen 32, 43, 55, Düsseldorf 2010-2012, Duisburg 2015). Vgl. zudem Serielle Quellen in südwestdeutschen Archiven, hg. von Christian Keitel-Regina Keyler (Stuttgart 2005).

${ }^{60}$ Udo Schäfer, Amtliche Aktenkunde der Neuzeit - Records Management des 21. Jahrhunderts. Zur Schnittmenge zweier Disziplinen, in: Quellenarbeit und Schriftgutverwaltung (wie Anm. 48) 89-128.

${ }^{61}$ Hochedlinger, Aktenkunde (wie Anm. 55). Vgl. Julian Holzapfl, [Rezension zu:] Michael Hochedlinger: Aktenkunde. Sehepunkte. Rezensionsjournal für die Geisteswissenschaften (15. 7. 2009), http://www. sehepunkte.de/2009/07/15648.html [1. 3. 2018].

62 Heinrich Otto Meisner, Archivalienkunde vom 16. Jahrhundert bis 1918 (Leipzig 1969) 194-197.

63 Rainer Polley, Die Archivierung der Mündlichkeit: Protokollierung in kollegialen Gremien, in: Digitale Archive - ein neues Paradigma? Beiträge des 4. Archivwissenschaftlichen Kolloquiums der Archivschule Marburg, hg. von Andreas Metzing (Veröffentlichungen der Archivschule Marburg, Institut für Archivwissenschaft 31, Marburg 2000) 253-273, hier 261f.

${ }^{64}$ Udo SCHÄFER, Quod non est in actis, non est in mundo. Zur Funktion öffentlicher Archive im demokratischen Rechtsstaat, in: Alles was Recht ist. Archivische Fragen - juristische Antworten. 81. Deutscher Archivtag in Bremen, hg. von Heiner Sснмітт et al. (Tagungsdokumentationen zum Deutschen Archivtag 16, Fulda 2012) 57-78, hier 58-66; DERs., Verschriftlichung von Verfahrenshandlungen vor kirchlichen Gerichten durch Protokollierung. Der Kanon X 2.19.11 und seine Interpretation durch die mittelalterliche Kanonistik, in: Archiv - Recht - Geschichte. Festschrift für Rainer Polley, hg. von Irmgard Christa Becker-Dominik Haffer-Volker Hirsch-Karsten Uhde (Veröffentlichungen der Archivschule Marburg, Hochschule für Archivwissenschaft 59, Marburg 2014) 275-310, hier 293-308. 
plastisches Bild bedarf es aber auch der Erfassung von Beispielmaterial in Fallstudien, die den Unterlagentyp im Zusammenhang tatsächlicher Aktenüberlieferungen darstellen. Martin Schlemmer hat dies für die Kabinettsprotokolle im Zusammenhang der Kabinettsakten der nordrhein-westfälischen Landesregierung getan und dabei instruktiv aufgezeigt, wie ausführliche Verlaufs- zu fleischlosen Beschlussprotokollen degenerierten ${ }^{65}$.

Eine solcherart vertiefte und verbreiterte Materialkenntnis führt zu verfeinerten Differenzierungen, zum Beispiel zwischen echten Protokollen aus einer Sitzung, die sich am Verlauf orientieren, ob zusammenfassend oder im Wortlaut, und Aktenvermerken über eine Sitzung. Denn während (genehmigte) Protokolle für alle Teilnehmer das bindende Ergebnis sind, enthalten Aktenvermerke die in der Regel nur zum eigenen Gebrauch bestimmte subjektive Bewertung einer Seite ${ }^{66}$. Diese Beobachtung Gerhard Schmids lässt sich erweitern: Eine Stelle mit genügend Einfluss im Behördenapparat kann ihren zusammenfassenden Vermerk entgegen der eigentlichen Funktion den anderen Sitzungsteilnehmern zustellen, um sich die Definitionshoheit über das Ergebnis zu sichern. Ein solches Aktenstück kann durchaus „Protokoll“ überschrieben sein; die Selbstbezeichnungen aus der zeitgenössischen Verwaltungspraxis trügen bekanntlich oft ${ }^{67}$.

Ein Beispiel ist jener Aktenvermerk des Reichssicherheitshauptamtes von 1942, der als Protokoll der Wannseekonferenz in die Geschichte eingehen musste ${ }^{68}$. Dieses Stück ist offensichtlich kein Wortprotokoll; inwieweit es wenigstens den chronologischen Verlauf der Besprechung wiedergibt, ist ungewiss. Das Stenogramm der Sekretärin ist als Zwischenmaterial ebenso verloren wie Eichmanns erster Entwurf, den Heydrich (nach Eichmanns der eigenen Exkulpierung dienender Aussage $\left.{ }^{69}\right)$ intensiv revidiert habe ${ }^{70}$. Der Mangel an Entstehungsstufen erweist sich als quellenkritisches Problem.

Umso wichtiger ist es, den Schriftstücktyp zu beachten: Für einen Aktenvermerk über eine Besprechung ist es normal, Beiträge zu raffen, außerhalb der Abfolge des gesprochenen Worts zusammenzuziehen und nach den Interessen der Verfasser zu gewichten. Der scheinbar diskursive Abschnitt am Ende des Dokuments ist dafür exemplarisch: Es ist nicht klar, zu welchem Zeitpunkt und in welcher Reihenfolge die Wortmeldungen von Heydrichs Folgerednern wirklich getätigt wurden ${ }^{71}$. Diesen Vermerk zu verfassen und an die übrigen Teilnehmer zu verschicken, diente dem Reichssicherheitshauptamt dazu, im Völkermord an den europäischen Juden bürokratisch die Federführung zu behaupten und eine Einzelfrage, die Behandlung sogenannter Mischlinge, in seinem Sinne zu entschei-

65 Martin Schlemmer, Kabinettsakten, in: Unbekannte Quellen (wie Anm. 59) 2 30-55, hier $30 f$.

66 Schmid, Aktenkunde (wie Anm. 36) 329f.

67 Vgl. Beck, Leistung (wie Anm. 8) 69.

68 Politisches Archiv des Auswärtigen Amts, RZ 214, R 100857, fol. 166-180. Auch digitalisiert verfügbar: Protokoll der „Besprechung über die Endlösung der Judenfrage vom 20. Januar 1942, in: Haus der WannseeKonferenz. Gedenk- und Bildungsstätte - Dokumente zur Wannsee-Konferenz, http://www.ghwk.de/fileadmin/user_upload/pdf-wannsee/dokumente/protokoll-januar1942_barrierefrei.pdf [1. 3. 2018].

69 Eichmann äußerte sich zur Wannsee-Konferenz in Kenntnis des Protokolls, versuchte es gar prozesstaktisch zu instrumentalisieren. Seine Aussagen sind daher keine Ersatzüberlieferung für die verlorene Mitschrift, sondern eine eigene mündliche Tradition; vgl. Bettina Stangneth, Eichmanns Erzählungen, in: Die WannseeKonferenz am 20. Januar 1942. Dokumente, Forschungsstand, Kontroversen, hg. von Norbert Kampe-Peter KLEIN (Köln-Weimar-Wien 2013) 139-150.

70 Peter Longerich, Wannseekonferenz. Der Weg zur „Endlösung“ (München 2016) 93; Christian MeNTEL, Das Protokoll der Wannsee-Konferenz. Überlieferung, Veröffentlichung und revisionistische Infragestellung, in: Die Wannsee-Konferenz (wie Anm. 69) 116-138, hier 119.

71 Mark Roseman, Die Wannsee-Konferenz. Wie die NS-Bürokratie den Holocaust organisierte (München 2002) 103. 
den. Die im Wesen des Besprechungsvermerks angelegte Verknappung gab Teilnehmern wie dem Staatssekretär des Reichsministeriums des Innern, Wilhelm Stuckart, nach 1945 Raum zur Relativierung ihrer Mittäterschaft, der durch genaueste Quellenarbeit ausgeräumt werden musste ${ }^{72}$.

Die Aktenkunde schafft das Fundament historisch-philologischer Hermeneutik, in dem sie die Authentizität von Quellen wie dem Wannsee-Protokoll zweifelsfrei feststellt ${ }^{73}$. Darüber hinaus formuliert sie Erwartungen an die Quellen, die die inhaltliche Analyse lenken, erleichtern und verifizieren können. Eine dafür ertüchtigte Aktenkunde der Zeitgeschichte kann nicht nur durch ihren Wert als Hilfswissenschaft, sondern auch mit gesellschaftlicher Relevanz überzeugen.

Der Riss, der im 20. Jahrhundert zwischen der Beschaffenheit der Akten und der Methodenlehre klafft, muss geschlossen werden. Wichtiger als Handbücher sind dafür Einzeluntersuchungen, die sich der Problematik aus verschiedenen Richtungen annähern. Da gibt es „Inseln stabiler Schriftlichkeit“: Behörden mit traditionellem Geschäftsgang oder besonders regulierte Systeme schriftlicher Kommunikation. Ihre Schriftlichkeit sollte exemplarisch untersucht werden, um Kontrastfolien zu gewinnen, die auf die Verhältnisse weniger gut organisierter Kanzleien aufgelegt werden können. Wenn solche Urheber Aktenstücke mit dichten und eindeutigen formalen Merkmalen produzierten, können und sollten diese selbstverständlich mit hergebrachten Methoden untersucht werden ${ }^{74}$. Dringend benötigt werden auch Studien zu besonderen Schriftstücktypen und ihrer Funktion für die Schriftlichkeit von Institutionen ${ }^{75}$. Die Basis von allem ist Kanzleigeschichte, was für das 20. Jahrhundert als Zeitalter der Mechanisierung eine Art bürotechnischer Archäologie bedeutet. Aktenkunde der Zeitgeschichte ist auch eine Geschichte der Kopierverfahren zwischen Ormig und Xerox. Hierzu ist jüngst eine wissenschaftlich sehr nützliche Materialsammlung erschienen ${ }^{76}$.

Die Aktenkunde muss sich stärker ihres Charakters als genuin historische Disziplin bewusstwerden. Sie neigt dazu, Äußerlichkeiten diachron nebeneinander zu stellen, statt Entwicklungen zu konzeptualisieren und in ihrem Gang zu verfolgen. Die Geschichte des Fernschreibers als Medium der Schriftlichkeit zeigt, dass der Verlust der „Einheit von Information und Träger "77, der für das Computer-Zeitalter beklagt wird, früher eingetreten ist, als man meinen möchte. Es hilft nicht, von Meisners „monarchischer Zeit“ in die Gegenwart springen zu wollen. Damit die wirklichen Kontinuitäten und Brüche erkennbar werden, muss zunächst die Lücke zwischen 1900 und 1990 geschlossen werden.

Aktenkunde ist sinnlos, wenn sie keinen Sitz im Leben beanspruchen kann. Der Journalismus und die Informationsfreiheitsgesetze spülen heute Aktenstücke nicht nur aus deutscher Verwaltungstradition in die Forschung, die noch gar nicht archiviert sind ${ }^{78}$. Der

72 Hans-Christian Jasch, Staatssekretär Wilhelm Stuckart und die Judenpolitik. Der Mythos von der sauberen Verwaltung (Studien zur Zeitgeschichte 84, München 2012) 325-330.

73 Siehe zuletzt die wichtigen Erkenntnisse zur Überlieferungsgeschichte des Wannsee-Protokolls und den Anstreichungen im Text bei Martin KRÖGER, Beim Aufgabenziel des AA steht ein Ausrufezeichen. Frankfurter Allgemeine Zeitung (25. 1. 2017) N3.

74 Zum Beispiel Berwinkel-Meiburg, Moderne Bundesverwaltung (wie Anm. 49).

75 Siehe etwa Holger Berwinkel, Der diplomatische Schriftverkehr im 20. Jahrhundert. AfD 61 (2015) 343-390.

76 Harald Rösler, Bürokunde und ein Blick ins Archiv (Duisburg 2015).

77 BeCK, Hilfswissenschaften (wie Anm. 53) 441.

78 Vgl. Stephan Lehnstaedt-Bastian Stemmer, Akteneinsicht. Das Informationsfreiheitsgesetz und die Historiker. Zeitschrift für Geschichtswissenschaft 60 (2012) 493-512; DIEs., Informationsfreiheit. Über die Ein- 
Hinweis ist müßig, dass solche fragmentierte Überlieferung problematisch ist. Sie wird ausgewertet, weil sie verfügbar ist. Auch wenn hier kein Archiv im Spiel ist, sind Archivarinnen und Archivare aufgrund ihrer Nähe zu den unterschiedlichsten Akten berufen, das methodische Instrumentarium bereitzustellen. Die Interpretation authentischer Unterlagen ist und bleibt die Kernkompetenz der Archivarinnen und Archivare ${ }^{79}$. 


\title{
Archivalienkunde von unten. Möglichkeiten einer Archivalienkunde für Unterlagen aus Neuen Sozialen Bewegungen am Beispiel feministischen Archivguts
}

\author{
Elizabeth Kata
}

Archivalienkunde als historische Hilfswissenschaft dient, wie Holger Berwinkel in dem Abstrakt zu seinem Vortrag „Probleme einer Aktenkunde der Zeitgeschichte“ treffend schrieb, „der Klärung zeitloser Fragen der historischen Quellenkritik: Wie wurden Entscheidungen getroffen, wer trug Verantwortung?“ Er nannte als Ziel „eine zeitgenössische Aktenkunde, die als Hilfswissenschaft im Sinne des Wortes nicht nur für Archivar [*innen $]^{1}$, sondern auch und vor allem für Archivbenutzer[*innen] zugänglich und brauchbar ist" ${ }^{\text {" }}$. Anhand von Unterlagen der Neuen Frauenbewegung in Österreich möchte ich zeigen, wie eine solche zeitgenössische Archivalienkunde möglicherweise aussieht und was sie kann.

Die Neuen Sozialen Bewegungen, die in der zweiten Hälfte des 20. Jahrhunderts ihren Anfang nahmen, haben die heutige Gesellschaft mitgeprägt und führten zu manchen bleibenden Veränderungen. Die Unterlagen dieser Bewegungen (etwa Frauenbewegungen, LGBTQ-Bewegungen, Öko-Bewegungen, Studierendenbewegungen und so weiter) werden vermehrt für wissenschaftliche Arbeiten oder auch museale Ausstellungen ausgewertet und verwendet. Als Archivarin im STICHWORT, Archiv der Frauen- und Lesbenbewegung, erlebte ich immer wieder, welche Schwierigkeiten Nutzerinnen* mit den schriftlichen Quellen hatten. Zwar ist das Schriftbild feministischer Unterlagen aus den letzten Jahrzehnten leserlich, aber manche Nutzerinnen* sind mit der Symbolik nicht mehr vertraut, und die unterschiedlichen Entstehungsstufen (und ihre entsprechende Bedeutung im Sinne der Vollständigkeit und Authentizität) sind für sie schwer auseinanderzuhalten. Archivalienkunde bietet die Werkzeuge, um die Bedeutung der Unterlagen in ihrem Kontext zu erschließen.

Die Unterlagen sind aus archivalischer oder hilfswissenschaftlicher Perspektive in mehrfacher Hinsicht unkonventionell, und sie gelangen in Bewegungsarchive auf unterschiedliche Art und Weise. Manchmal übergibt eine Gruppe ihre Unterlagen, wenn sie

${ }^{1}$ Ich verwende in diesem Text das Sternchen $\left(^{*}\right)$ zum Gendern, um Menschen, die nicht in das Zweigeschlechtersystem passen, wie Transgender- oder Intersex-Personen, sichtbar zu machen. Wenn das Sternchen am Ende des Wortes steht, wie bei „Nutzerinnen*“, dann steht das für Frauen und diejenigen, die sich als Frauen identifizier(t)en, beziehungsweise all jene, die sich nicht als Cisgender-Männer identifizieren. Wenn das Sternchen vor dem „innen“ steht, bezieht sich das Wort auf Menschen aller Genders.

2 Holger Berwinkel, Probleme einer Aktenkunde der Zeitgeschichte. BIÖG. Blog des Instituts für Österreichische Geschichtsforschung (18. 10. 2016), https://bioeg.hypotheses.org/1875 [1. 3. 2018]. 
sich auflöst ${ }^{3}$. Manchmal übergeben einzelne Aktivist*innen ihre Mitschriften oder gesammelte Unterlagen, womit die Erhaltungskette nicht immer ganz klar ist und das Schriftgut einer Gruppe sich teilweise mit persönlichen Notizen vermischt. Manche Unterlagen werden bewusst (von den Archivar*innen) gesammelt, zum Beispiel indem alle Flugblätter auf einer Demonstration eingesammelt werden. Manche Gruppen liefern regelmäßig Veranstaltungsankündigungen, Plakate, Positionspapiere, Jahresberichte und Ähnliches von sich aus ins Archiv. Es gibt keine Abgabepflicht an Bewegungsarchive, und sie unterliegen auch keinen Archivgesetzen. Aktivist*innen und Gruppen schenken Bewegungsarchiven ihre Unterlagen, weil sie ihre Geschichte für wichtig und erhaltungswert halten und weil sie die Überlieferung nicht dem Zufall (und auch nicht den staatlichen Archiven) überlassen wollen. Doch obwohl es fallweise systematische Überlieferungen von Gruppen in Bewegungsarchiven gibt, kann das nicht als Regel vorausgesetzt werden.

So gesehen muss man in diesem Kontext von Archivalienkunde sprechen. Eine Archivalienkunde von Bewegungsunterlagen klingt möglicherweise nach einem Widerspruch in sich, und ich wähle bewusst den Begriff Archivalien- und nicht Aktenkunde. Es handelt sich hier nicht oder nicht nur um Archivgut im engeren Sinne nach Meisner, aber sein Begriff der Archivalienkunde schließt Archivgut im weiteren Sinne (und somit meiner Meinung nach auch den Gegenstand meiner Untersuchungen) mit ein, auch wenn er nicht an jene Ausdehnung der Archivalienkunde dachte, die ich hier vorschlage ${ }^{4}$. Der Begriff „Archivalienkunde“ eröffnet manche Spielräume, die der Begriff „Aktenkunde“ anscheinend nicht zulassen will. Engere Auslegungen der Aktenkunde verstehen Bewegungsquellen überhaupt nicht als „Akten“5. Holger Berwinkel und Anette Meiburg machen den Geschäftsgang zur Voraussetzung einer aktenkundlichen Untersuchung: „Schriftstücke, die außerhalb eines Geschäftsgangs erstellt, weitergeleitet und bearbeitet werden, sind nicht aktenmäßig und können deshalb mit den Methoden der Aktenkunde nicht bestimmt werden. Sie können aber im Rahmen einer übergreifenden Archivalienkunde untersucht werden" ${ }^{6}$. Es stellt sich die Frage, ob Bewegungsquellen aus Geschäftsgängen entstehen und ob sie als Handeln verstanden werden können. Ich würde dies in manchen, aber nicht allen Fällen bejahen, und sie haben auf alle Fälle identifizierbare Formen, die oft aus bestimmten Entscheidungsprozessen stammen. Eine Archivalienkunde, die auf den Lektüren und Methoden der enger gefassten Aktenkunde aufbaut, kann zu einem verbesserten Verständnis von Bewegungsunterlagen führen und sowohl für Archivar*innen als auch für Historiker*innen und andere Nutzer*innen dienlich sein.

\footnotetext{
${ }^{3}$ Ein Beispiel wäre der Verein Frauengetriebe, der sich 2007 auflöste: Elizabeth KATA, Vor dem Arlberg kein ruhiges Hinterland - Frauengetriebe und Frauenbewegung in Vorarlberg. STICHWORT Newsletter 42 (2016) 13-15.

${ }^{4}$ Meisner differenziert zwischen dem engen Archivgutbegriff: „das archivierte (dem Archiv einverleibte) Registraturgut, nicht das archivierte Sammlungsgut“, und dem weiterem Archivgutbegriff, der „den gesamten Archivbestand, einschließlich des archivischen Sammlungsgutes“ einschließt: Heinrich Otto Meisner, Archivalienkunde vom 16. Jahrhundert bis 1918 (Leipzig 1969) 21.

5 Siehe zum Beispiel die Definition von „Akten“ nach Michael Hochedlinger als „der (organisierte) Niederschlag von schriftlicher Geschäftstätigkeit, erwachsen aus dem Geschäftsgang einer Behörde, Dienststelle, eines Unternehmens usw. “: Michael Hocheduinger, Aktenkunde. Urkunden- und Aktenlehre der Neuzeit (Historische Hilfswissenschaften, Wien-München 2009) 38.

${ }^{6}$ Holger Berwinkel-Anette Meiburg, Die moderne Bundesverwaltung als Referenzmodell der Sytematischen Aktenkunde - Erfahrungen aus der Praxis, in: Moderne Aktenkunde, hg. von Holger BerwinkeL-Robert Kretzschmar-Karsten Uhde (Veröffentlichungen der Archivschule Marburg, Hochschule für Archivwissenschaft 64, Marburg 2016) 81-92, hier 81.
} 
Ich möchte beschreiben, wie genetische, analytische und systematische Archivalienkunde aussehen können, wenn sie auf Bewegungsunterlagen angewendet werden. Wer sich zu sehr an die klassischen Begriffe klammert, wird es damit schwer haben. Authentizität spielt eine völlig andere Rolle. Doch ist es möglich, Züge einer Archivalienkunde für Bewegungsquellen zu skizzieren, die für die Anwendung archivalienkundlicher Methoden auf alle Sorten von nicht-behördlichen Unterlagen von Bedeutung sein kann.

\section{Genetische Archivalienkunde}

Genetische Archivalienkunde untersucht die Prozesse, aus denen Dokumente entstehen. Bei Behördenunterlagen würden wir uns für Geschäftsgänge interessieren, die möglicherweise durch Behördeninstruktionen oder Büroordnungen geregelt werden. Diese Formalitäten fallen für Bewegungsunterlagen meistens weg beziehungsweise werden nicht aufgezeichnet. Je nach dem (politischen) Selbstverständnis einer Gruppe oder eines Projekts können deren Entscheidungsstrukturen unterschiedlich ausgeprägt sein. Genau wie Behördengeschichte mit klassischer Aktenkunde verbunden ist, müssen diejenigen, die sich mit Sozialen Bewegungen beschäftigen, sich auch mit der Bewegungsgeschichte auseinandersetzen, um die Entstehung von Unterlagen nachvollziehen zu können ${ }^{7}$. Die Gruppen- und Entscheidungsstrukturen können uns einiges über die Unterlagen vermitteln, und umgekehrt können wir Informationen über formelle und vor allem informelle Strukturen und Entscheidungsprozesse durch die Unterlagen erfahren.

Feministische Gruppen bilden und bildeten sich in verschiedenen Formen. Gerade in der autonomen Frauenbewegung wurden staatliche und religiöse Institutionen sowie Parteien abgelehnt und nicht-hierarchische beziehungsweise anti-hierarchische Gruppenstrukturen angestrebt ${ }^{8}$. In den Beständen des STICHWORT Archives findet man Gruppen, die nach Konsens, und solche, die nach dem Mehrheitsprinzip entscheiden ${ }^{9}$. Man findet Gruppenstrukturen, wo Entscheidungen - darunter manchmal auch solche, die die gesamte Struktur betreffen - in Arbeitsgruppen getroffen werden. Man findet Frauenprojekte, die - „trotz“ Autonomie-Anspruch - um Fördermittel von institutioneller Seite ansuchen und sie annehmen, wodurch manche Entscheidungen durch Forderungen von außen (mit)bestimmt werden ${ }^{10}$. Manchmal verwandelt sich eine nicht-hierarchische Gruppe in einen Verein mit einem Vorstand, der Entscheidungen trifft, die Mitarbeiterinnen umsetzen (müssen $)^{11}$. Weiters gibt es die Organisationsform Bündnis. Ein Bündnis ermög-

7 Für die Frauenbewegung in Österreich siehe etwa: Brigitte GeIger-Hanna Hacker, Donauwalzer Damenwahl. Frauenbewegte Zusammenhänge in Österreich (Edition Spuren, Wien 1989).

${ }^{8}$ Ich orientiere mich an der Definition von „autonomer Frauenbewegung" nach Hildegund Dick, die „autonom“ in diesem Sinne als „,unter Männerausschluß und parteiunabhängig“ bestimmt: Hildegund DiCK, Die autonome Frauenbewegung in Wien. Entstehung, Entfaltung und Differenzierung von 1972 bis Anfang der 80er Jahre (ungedr. Diss., Wien 1991) 45.

9 Auf die genauen Unterschiede beziehungsweise auch die Unterschiede zwischen Anspruch und Realität bezüglich Entscheidungsformen werde ich hier nicht eingehen können. Es sei nur angemerkt, dass „kollektive“ Entscheidungen nicht mit Konsensentscheidungen gleichzusetzen sind.

${ }^{10}$ Die Frage um das Annehmen von staatlichen Förderungen wurde kontrovers diskutiert. Einen wichtigen Beitrag zur Autonomie-Debatte in Österreich liefert der Band nach der gleichnamigen Frauensommeruniversität: Autonomie in Bewegung. 6. Österreichische Frauensommeruniversität. Texte, Reflexionen, SubVersionen (Wien 1991).

${ }^{11}$ Ein konfliktreiches Beispiel eines solchen Vorgangs stammt aus Linz: vgl. Maria Prieler-Woldan, Selbstverwaltet oder fremdbestimmt? Das Linzer Frauenhaus, seine Entstehung und Konfliktgeschichte aus der Sicht einer Mitarbeiterin (Diplomarbeit, Universität Strasbourg 1988). 
licht Zusammenarbeit von Gruppen und Organisationen mit teilweise sehr unterschiedlichen Sichtweisen für bestimmte Themen, wie beispielsweise die Bündnisse zum 8. März, dem Internationalen Frauen(kampf)tag ${ }^{12}$. Vertreter*innen der teilnehmenden Gruppen treffen Entscheidungen zum Beispiel über die Form und Route einer Demonstration und erarbeiten einen Minimalkonsens über Inhalte - es gibt oft sowohl Informationsmaterial vom Bündnis als auch Flugblätter von den einzelnen teilnehmenden Gruppen, die ihre jeweilige eigene Position zu aktuellen Themen ausdrücken. Je nach Form werden die Entscheidungsstrukturen, die dann auch Dokumenten Authentizität verleihen, unterschiedlich aussehen. Die Authentizitätsfrage in Bewegungsunterlagen beschäftigt sich dann entsprechend mit der Frage, wer Entscheidungen treffen darf und ob ein Dokument entsprechend dem vorgesehenen oder üblichen Entscheidungsprozess entstanden ist $^{13}$. Hat ein Dokument, das außerhalb dieses Prozesses entsteht, trotzdem Legitimität für die Gruppe oder die Bewegung allgemein? Die Beantwortung solcher Fragen wird durch manche Aspekte nicht-hierarchischer Organisationsformen verkompliziert - wenn Rollen nicht fest zugeschrieben werden, darf beispielsweise jedes Mitglied einer Gruppe einen Text verfassen, aber die Veröffentlichung im Namen der Gruppe bedarf wiederum eines Legitimationsprozesses.

Nehmen wir als Beispiel ein hypothetisches Flugblatt. Eine Gruppe, die sich als Kollektiv versteht, entschließt sich, an einer von einem Bündnis organisierten Demonstration teilzunehmen und mit einem eigenen Flugblatt aufzutreten. Die ursprüngliche Entscheidung, das Flugblatt zu schreiben, fällt im Plenum und ist somit im Plenumsprotokoll aufgezeichnet. Eine Frau meldet sich freiwillig oder wird von der Gruppe gebeten, einen Entwurf zu schreiben. Die Frau bringt den Entwurf zum nächsten Plenum. Er wird im Plenum diskutiert, Änderungsvorschläge werden eingebracht und möglicherweise direkt auf dem Entwurf oder im Protokoll festgehalten. Der Entwurf wird ausgebessert und umgeändert. Der Text wird nochmals Korrektur gelesen, dann wird eine weitere Frau mit der graphischen Gestaltung und dem Druck betraut. Die druckfertige Version weist unter anderem ein Gruppenlogo und ein Impressum auf. Dieses Flugblatt gilt als legitimer Output der Gruppe und drückt ihre kollektiv entschiedene Position aus. Wäre es legitim gewesen, wenn die zuerst beauftragte Frau ihren Entwurf ohne Rücksprache im Namen der Gruppe gedruckt hätte? Auch wenn das für ein externes Publikum nicht zu merken wäre, gibt es Beispiele, die zeigen, dass die Legitimität nicht hergestellt wurde, wenn die Entscheidungsprozesse nicht respektiert wurden.

12 8.-März-Demonstrationen in Österreich werden oft von regionalen Bündnissen organisiert (wie etwa dem 8.-März-Komitee in der Steiermark). Einblick in die Entwicklung der Frauentagdemonstrationen seit den 1970er Jahren gibt Maria Mesner, Viele und Verschiedene. Die „neue“ Frauenbewegung und die Frauentage, in: Frauentag! Erfindung und Karriere einer Tradition, hg. von Heidi Niederkofler-Maria Mesner-Johanna ZECHNER (Wien 2011) 171-195.

13 Luciana Duranti differenziert zwischen rechtlicher, diplomatischer und historischer Authentizität. Bewegungsunterlagen besitzen selten rechtliche Authentizität und auch nur begrenzt diplomatische Authentizität, denn die inneren und äußeren Merkmale sowie die zu beachtenden Entstehungsprozesse für Unterlagen sind meistens nicht genau festgelegt. Es ist schwer festzumachen, dass bei einem Plenumsprotokoll ein Merkmal fehlt, weswegen es diplomatisch nicht authentisch wäre und das auch aufgezeigt werden könnte. Die Entstehungsprozesse verleihen eine diplomatische Authentizität, weil nur so klar wird, wer berechtigt ist, ein Dokument überhaupt auszustellen: Luciana Duranti, Diplomatics: New Uses for an Old Science. Archivaria. The Journal of the Association of Canadian Archivists 28 (1989) 7-27; 29 (1989) 4-17; 30 (1990) 4-20; 31 (1990) 10-25; 32 (1991) 6-24; 33 (1991) 6-24, hier 28 17f. 


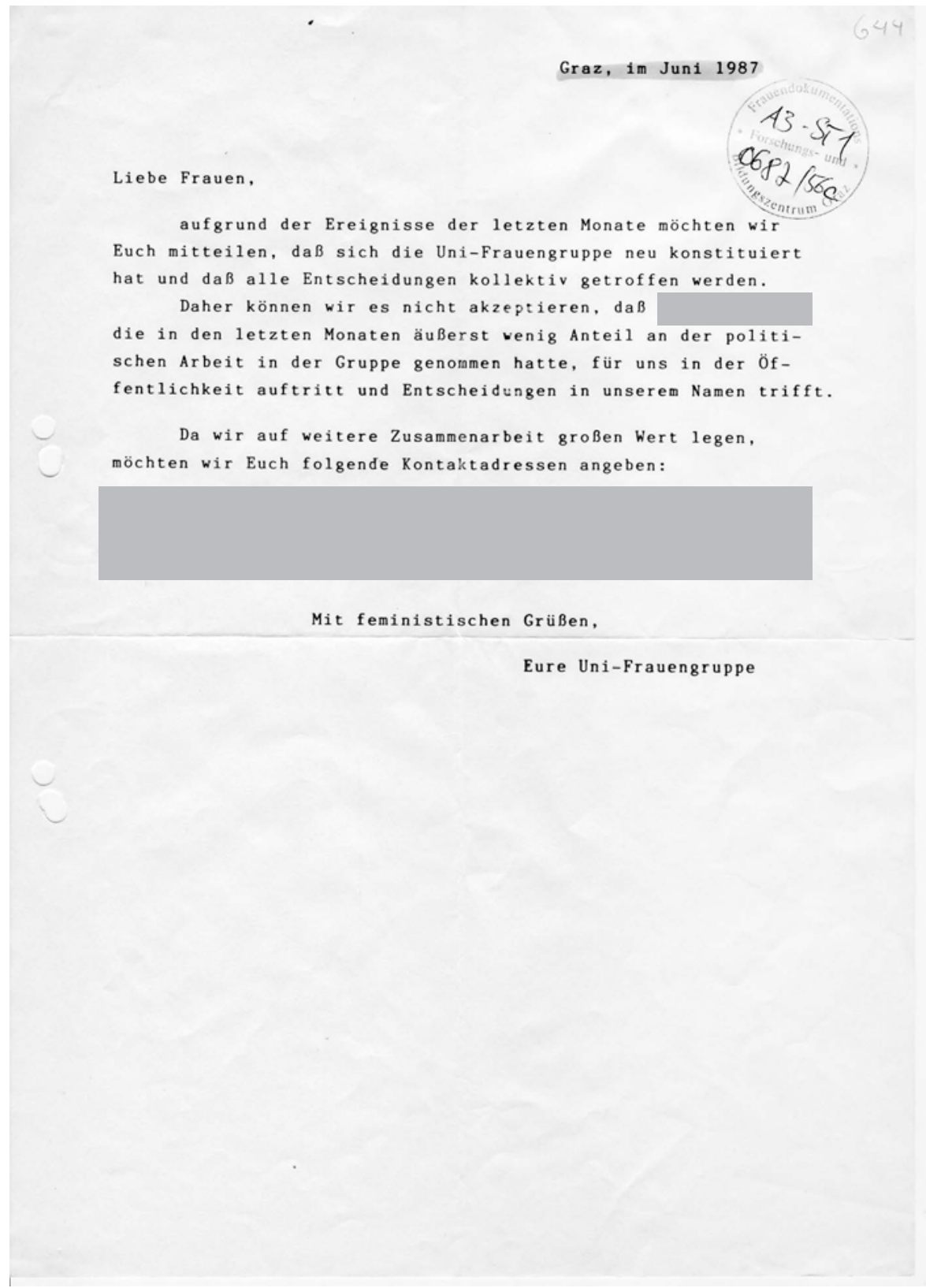

Abb. 1: Stellungname der neu konstituierten Uni-Frauengruppe (Elizabeth Kata; STICHWORT, G44 FR HA der ÖH Uni Graz, Interna, Stellungnahme der UNI-Frauengruppe, Juni 1987). 
Ich möchte ein kurzes Beispiel anführen. Die Frauenreferentin der Österreichischen Hochschuler*innenschaft $(\ddot{\mathrm{OH}})$ wurde 1986 nach Teilnahme an einer feministischen Protestaktion von der männlich dominierten ÖH der Universität Graz des Amtes enthoben $^{14}$. Daraufhin formierte sich die Uni-Frauengruppe anstelle des Frauenreferats. Diese Frauengruppe warf einem weiblichen Mitglied nach einigen Monaten vor, im Namen der Gruppe agiert zu haben, ohne der Gruppe ihr Vorhaben mitzuteilen. Die Gruppe schrieb an die Frau: Wir wollen gerne mit Dir zusammenarbeiten, aber wir wehren uns vehement gegen Deine einsamen Entscheidungen! ${ }^{15}$ Die Uni-Frauengruppe schickte daraufhin eine Stellungnahme (Abb. 1) an nahestehende Grazer Frauengruppen sowie Frauenreferate an anderen Universitäten, um sich von der Frau, ihrem öffentlichen Auftreten und ihrer Entscheidungsfindung im Namen der Gruppe zu distanzieren. Der Text erklärt: [A] ufgrund der Ereignisse der letzten Monate möchten wir Euch mitteilen, daß sich die Uni-Frauengruppe neu konstituiert hat und daß alle Entscheidungen kollektiv getroffen werden. Daher können wir es nicht akzeptieren, daß [N.], die in den letzten Monaten äußerst wenig Anteil an der politischen Arbeit in der Gruppe genommen hatte, für uns in der Öffentlichkeit auftritt und Entscheidungen in unserem Namen trifft ${ }^{16}$.

Die Stellungnahme (die mit feministischen Grüßen schließt) betont die Form der Entscheidungsfindung - Entscheidungen werden kollektiv getroffen. Was das genau bedeutet, bleibt unklar, aber offensichtlich mussten mehrere Frauen der Gruppe an dem Entscheidungsprozess teilnehmen. Der als einsam betrachteten Entscheidung einer Einzelperson fehlte es an Legitimität; sie sorgte für Unmut, so sehr, dass eine Distanzierung für notwendig gehalten wurde. In diesem Fall kam es später zu einer Aussprache, die in einem Plenumsprotokoll der Autonomen Frauenplattform Graz festgehalten wurde. Dort steht, dass der Brief auf Grund eines Mißverständnisses zustande gekommen sei. Interessanterweise hält das Protokoll auch eine Kritik an der Form der Auseinandersetzung per Brief fest: Wir glauben, daß Streitereien und Auseinandersetzungen wichtig sind [...], wollen sie aber nicht in Form von derartigen Briefen austragen und halten dies auch nicht für die geeignete Form mit Differenzen umzugehen ${ }^{17}$. Die Uni-Frauengruppe hat mit ihrer Stellungnahme nicht gegen irgendwelche formalen Regeln verstoßen, aber der Umgang wird trotzdem in Frage gestellt. In einer nicht-hierarchischen Bewegung hat niemand die Deutungshoheit, um einen bestimmten Umgang festzulegen. So kommt es immer wieder zu Konflikten zwischen der Einzelnen und der Gruppe, sowie zwischen der Gruppe und der Szene oder der Bewegung.

Welche Fassung eines Schriftstücks als „Ausfertigung“ zu gelten hat und welche Merkmale eine „authentische“ Ausfertigung ausmachen, ist oft schwierig nachzuvollziehen. Wir haben selten Konzepte mit Unterschriften oder Namenskürzeln (und sie sind nicht halbbrüchig geschrieben), aber wir können Entscheidungen mithilfe von Plenumsprotokollen und ähnlichem internem Schreibwerk ${ }^{18}$ nachvollziehen. Die Ausfertigungen besit-

${ }^{14}$ Eva TAXacher, Frauenbewegung(en) im Archiv des Doku Graz. Eine Bestandsaufnahme und kritische Reflexion (Wien 2013) 95.

15 STICHWORT. Archiv der Frauen-und Lesbenbewegung, Wien [im Folgenden: STICHWORT], G44 FR HA der ÖH Uni Graz, Interna, Brief der UNI-Frauengruppe, Juni 1987.

16 STICHWORT, G44 FR HA der ÖH Uni Graz, Interna, Stellungnahme der UNI-Frauengruppe, Juni 1987.

17 STICHWORT, G44 FR HA der ÖH Uni Graz, Interna, Protokoll der Autonomen Frauenplattform Graz, 21. 7. 1987.

${ }^{18}$ Internes Schreibwerk in Bewegungskontexten reicht von Protokollbüchern bis zu persönlichen Mit- 

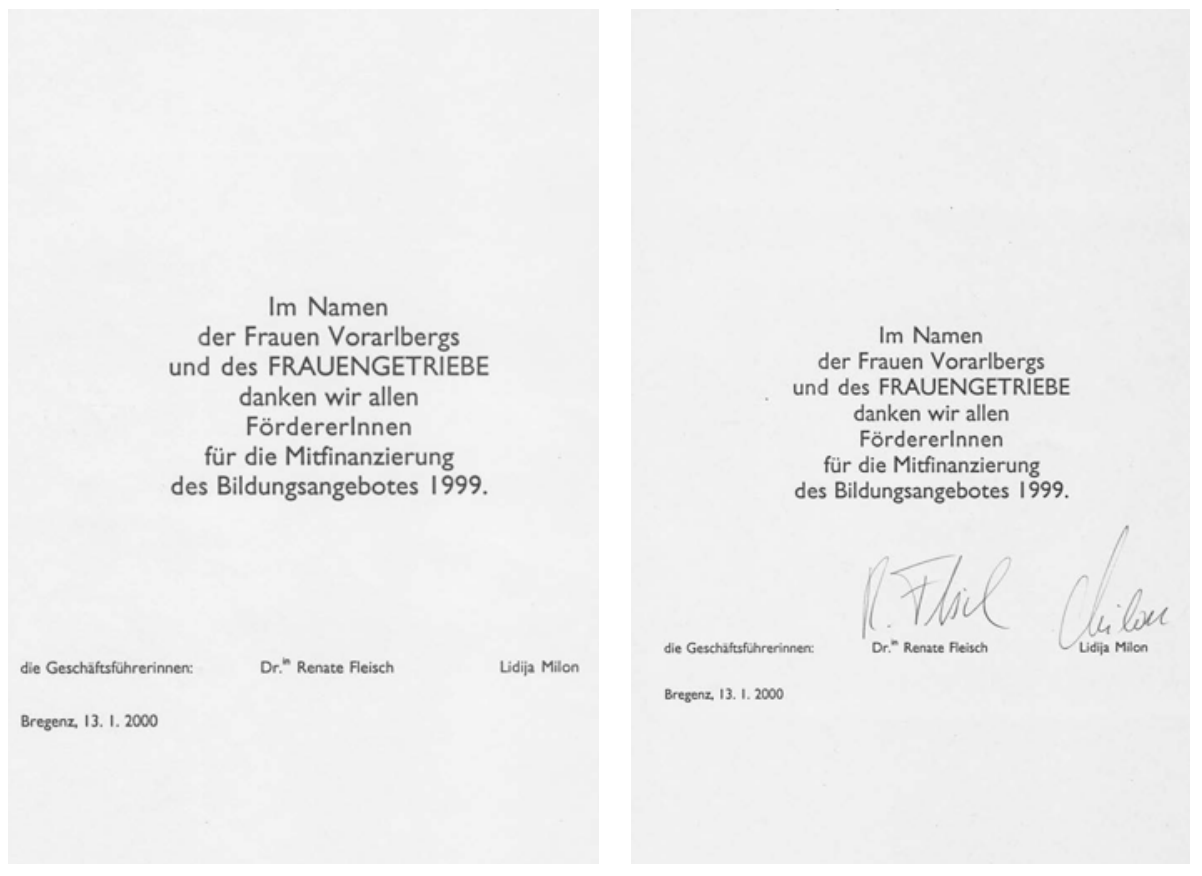

Abb. 2: Gegenüberstellung von Entwurf/nicht vollzogener Vorstufe und Ausfertigung eines Jahresberichts des Vereins Frauengetriebe (Elizabeth Kata; STICHWORT, G162 Frauengetriebe, Jahresbericht 1999).

zen oft gewisse Charakteristika, wie Logos, Impressa oder Unterschriften, die die Vollziehung der Unterlage bestätigen. So können wir „Ausfertigung“, „Entwurf“ und „Kopie“ in manchen Fällen auseinanderhalten. Wir haben hier als Beispiel zwei Fassungen eines Jahresberichts des Vereins Frauengetriebe aus Vorarlberg. Die Unterschrift unterscheidet, ganz wie bei herkömmlichen Unterlagen, das ausgefertigte Original von dem Entwurf oder der nicht vollzogenen Vorstufe (Abb. 2) ${ }^{19}$. Neben den Unterschriften wird die Ausfertigung durch den Ausdruck auf Papier mit dem - hier nicht abgebildeten - Vereinslogo erkenntlich. Der Vordruck des Logos erfolgte auf Umweltpapier, was dazu führt, dass die Entwürfe in besserem Zustand als die Ausfertigungen sind.

In anderen Fällen wird zum Beispiel bewusst mit einem falschen Impressum gearbeitet, damit etwa eine Aussage nicht negativ auf eine Gruppe zurückfällt. Falsche Impressa werden durch „lustige“ Namen (wie „Wüde Weiba“, das heißt: Wilde Weiber) oder auch symbolische Adressen (fiktive Straßen wie „3710 Frauendorf, Pflastersteinweg 47/11“20 oder Plätze, die nach feministischen Vorbildern benannt sind) gekennzeichnet. Die Subversion dieses Mechanismus zur Authentifizierung dient mehrfachen Zwecken. Einerseits weisen die Namen oder Adressen auf das politische Selbstverständnis der Gruppe hin;

schriften und befindet sich manchmal in privatem Besitz. Die Erhaltungskette beziehungsweise die Besitzverhältnisse sind, wie bei vielen Bewegungsunterlagen, nicht immer klar, und sie kommen teilweise nur durch Umwege ins Archiv.

19 STICHWORT, G162 Frauengetriebe, Jahresbericht 1999.

20 STICHWORT, G211 Frauengruppe Widerstand gegen sexuelle Männergewalt Innsbruck, Flugblatt „10. Juni = Vätertag, 10. Juni = Tätertag“. 
wenn die Adresse als „Rosa-Luxemburg-Platz“ angegeben wird, ist Rosa Luxemburg eine Identifikationsfigur. Anderseits führen die falschen Impressa dazu, dass eine Zuschreibung eines Flugblatts oder Plakats zu einer bestimmten Gruppe maßgeblich erschwert wird. Impressa sind laut Mediengesetz erforderlich, und falsche Impressa minimieren bei Nichteinhaltung die Wahrscheinlichkeit, dass man mit dem Gesetz in Konflikt gerät und eine Strafe für das Verteilen von Material ohne Impressum anfällt.

Gruppenprozesse und ihre Bedeutung für die Entstehung von Unterlagen sind oft nicht genau nachvollziehbar, entweder weil sie nie niedergeschrieben wurden oder weil Niederschriften nicht erhalten sind. Deswegen erfahren wir manchmal über die Gruppenprozesse nur durch Vermerke an den Unterlagen. In Bewegungszusammenhängen können regelmäßige Kommentare von bestimmten Gruppenmitgliedern auf die offizielle oder inoffizielle Rolle dieser Personen in der Gruppe hinweisen. Spannend für mich sind Fragen wie die folgenden: Gibt es einzelne Frauen, die - trotz des Anspruchs auf kollektive Entscheidungsfindung - Gruppenprozesse aushebeln können? Gibt es inoffizielle Vetorechte? Eine Untersuchung nach genetischer Archivalienkunde könnte Antworten auf solche Fragen liefern.

Eine Druckvorlage des Monatsprogramms des Vereins Frauengetriebe liefert ein Beispiel für die Informationen zu Geschäftsprozessen, die in Marginalien erhalten sein können. Die Druckvorlagen der Monatsprogramme sind über Jahre erhalten und bieten selten Informationen, die über den später gedruckten Text hinausgehen. Manchmal sind die Abdrucke in verschiedenen Zeitungen oder Zeitschriften ebenfalls vorhanden und belegen, dass die Vorlage auch tatsächlich gedruckt wurde. Die Druckvorlage für das Programm von Dezember 1999 enthält hingegen Anmerkungen über die Zeitschriften und Zeitungen, an die diese Vorlage geschickt werden sollte (Abb. 3). Ich erschließe die Marginalien wie folgt: Damit das Programm als viertelseitige Einschaltung am 18. November 1999 erscheinen konnte, wurde die Vorlage am 8. November ausgeschickt (ges. am 8. Nov. an: [...]). Es folgt eine Liste von regionalen (Feldkircher Anzeiger) sowie feministischen (Anschläge) Medien. Drei von den aufgelisteten Publikationen sind durchgestrichen. Bei der letzten angeführten Publikation, dem Wochenblatt Lindau, wird auch die Art der Zusendung angegeben: per Fax - an Hr. Hagel. Die Informationen werden durch ein gelbes Post-It ergänzt. Darauf steht „KULTUR - JA. [DORNBIRNER] G[E]M[EINDE]BBLATT [!] + [BREGENZER] BLÄTTLE + F[ELD]K[IRCHER] ANZEIGER: NEIN!“21. Es lässt sich nicht mit Sicherheit erschließen, ob sich das Ja oder Nein auf die Übernahme der Einschaltung bezieht oder zum Beispiel auf eine Entscheidung, überhaupt an diese Medien abzuschicken oder nicht. Trotzdem erfahren wir, welche Medien für eine Einschaltung in Betracht gezogen wurden, teilweise wie das Absenden erfolgte und welcher Zeitrahmen für das Erscheinen der Einschaltung eingeplant wurde. Da es keine Instruktionen gibt, wie die Einschaltung der Monatsprogramme zu erfolgen hatte, liefern diese Vermerke Informationen, die sonst nicht vorhanden wären und Prozesse nachvollziehbar machen.

\section{Analytische Archivalienkunde}

Analytische Archivalienkunde untersucht und beschreibt die inneren und äußeren Merkmale von Unterlagen. Manche der in der klassischen Archivalienkunde beschrie-

${ }^{21}$ STICHWORT, G162 Frauengetriebe, Druckvorlage Monatsprogramm Dezember 1999. 


\section{FRAUENGETRIEBE}

Bildungszentrum für Frauen

Veranstaltungen Dezember 1999

Lila Telefon 05574/45538

Information und Beratung für lesbische Frouen

Mo, 6. Dez. 19-21 Uhr im FRAUENGETRIEBE

Rund und schön - ein Film aber den

weiblichen Körper

Videofilm

Mo, 6. Dez. 19.30 Uhr im FRAUENGETRIEBE

Vorschau:

Sonntagsfrühstück

fir Lesben und interessierte Froven

So, 9. Jänner 2000, II Uhr im FRAUENGETRIEBE

Năhere Angaben zu den einzelnen

Veranstaltungen im Veranstaltungskalender

Herbst 1999.

Bestellen Sie den Kalender telefonisch oder

kommen Sie vorbei.

Unsere Öffnungszeiten sind:

Mittwoch und Donnerstag

jeweils $9-12 \mathrm{Uhr}$

ansonsten Anrufbeantworterin

Schillerstr. 2, 6900 Bregenz

Schillerstr. 2, 6900 Bregenz
Telefon 05574/45 538 -Fax 52035

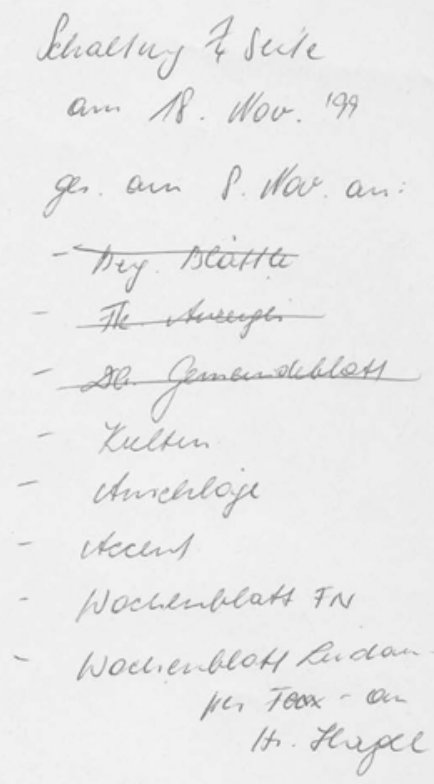

KULTR: JA

GBMLATT'

DLAOTLCE F FIC

AN 2E/Gin:

$N F / N$

Abb. 3: Druckvorlage für ein Monatsprogramm des Vereins Frauengetriebe (Elizabeth Kata; STICHWORT, G162 Frauengetriebe, Druckvorlage Monatsprogramm Dezember 1999).

benen Elemente sind allgemein in zeitgenössischen Unterlagen nicht mehr oder nur selten vorhanden, weil zum Beispiel Adelstitel und -würden mit dem Ende der Monarchie verschwanden. Andere Merkmale kommen gerade bei Bewegungsunterlagen nicht vor, weil Bewegungszusammenhänge andere Kommunikationsansprüche als Behörden haben. Bewegungen, die anti-hierarchische Ansprüche stellen, versuchen diese auch in ih- 
rer Kommunikation umzusetzen. Einerseits mündet das manchmal in einfacher Sprache, also im Ablehnen von Amtsdeutsch und bürgerlichen Normen („Spießertum“). Andererseits versuchen gerade die Frauenbewegungen, Frauen auch in der Sprache sichtbarer zu machen, indem "gegendert" wird. Frauen werden explizit angesprochen und nicht bloß „mitgemeint“ - die Sprache soll auch weibliche Lebensrealitäten widerspiegeln. Bewegungsspezifische Ausdrücke findet man anstelle herkömmlicher Grüße oder Anreden. Feministinnen* schreiben an „Liebe Frauen“ oder „Liebe FrauenLesben“ und unterschreiben „mit feministischen Grüßen“, um nur ein paar Beispiele zu geben. Die inneren Merkmale in Bewegungsunterlagen können Hinweise über das Verhältnis der Autor*innen zu den Adressat*innen sowie über die genauere politische Ausrichtung einer Gruppe geben.

Sie können auch für die Datierung herangezogen werden. Ein Beispiel für das Datieren durch innere Merkmale ergibt sich aus der Art und Weise des Genderns. Frühe feministische Texte werden nicht gegendert und sprechen von „der Frau“ in der Einzahl. Später werden Frauen in der Mehrzahl adressiert, dann „FrauenLesben“, heute vielleicht „Frauen*Lesben*Inter*Trans“ (F* $\left.{ }^{*}{ }^{*}{ }^{*} \mathrm{~T}\right)$. Die Bezeichnung $\mathrm{F}^{*} \mathrm{~L}^{*} \mathrm{I}^{*} \mathrm{~T}$ wurde meines Wissens in Wien erst im Rahmen der \#unibrennt-Proteste 2009 verbreitet, als es im Hauptgebäude der Universität Wien einen $\mathrm{F}^{*} \mathrm{~L}^{*} \mathrm{I}^{*} \mathrm{~T}-$ Raum gab. Ein Text aus Wien, der F*L*I*TPersonen anspricht, wird nicht aus den 1990er Jahren stammen. Das Gendern liefert oft einen Terminus post quem oder auch einen Terminus ante quem. Weiters weisen die unterschiedlichen Formen des Genderns heute in explizit feministischen Kreisen auf den Zugang zu Transgender-Personen hin - wer heute mit Unterstrich (_) oder Sternchen (*) gendert, will Transgender- und Intersex-Personen explizit ansprechen und inkludieren. Die inneren Merkmale in Bewegungsunterlagen sind nicht die aus der klassischen Archivalienkunde geläufigen, aber sie sind vorhanden und identifizierbar.

Bei den äußeren Merkmalen gibt es ebenfalls solche, die für Bewegungsunterlagen spezifisch sind. Auch heute kann der Schriftträger uns etwas erzählen, zum Beispiel, wenn ein Schriftstück auf Umweltpapier gedruckt wurde. Aus meinen Erfahrungen mit frauenbewegten Unterlagen würde ich sagen, dass die Blütezeit des Thermopapiers zwischen ungefähr 1998 und 2001 lag. Vor zirka 1998 hatten wenige Gruppen eigene Faxgeräte, sondern nutzten Faxgeräte von größeren NGOs mit, und Faxnachrichten wurden deswegen fotokopiert. Ab 2001 hatten die meisten Frauengruppen eigene E-Mail-Adressen. Diese Entwicklungen widerspiegeln allgemeine Änderungen in der Kommunikationstechnologie, tragen aber manchmal spezifische Züge, teilweise durch die begrenzten finanziellen Mittel feministischer Initiativen bedingt.

In Bewegungszusammenhängen wird das äußere Erscheinungsbild auch von Logos und Symbolen geprägt. Frauenzeichen $(q)$ kommen beispielsweise in vielen Formen vor; sie können auf politische Ausrichtung hinweisen sowie zur Datierung angewendet werden. Manche Frauenzeichen (etwa mit gehobener Faust) werden eher mit Radikalfeminismus assoziiert, andere mit sozialistischen Frauen und so weiter. Ein doppeltes Frauenzeichen kann Frauen in der Mehrzahl oder auch Lesben bedeuten. Ein besonderer Fall sind die Fotokopien von Zeichnungen von Franziska Becker, einer feministischen Zeichnerin. Sie tauchen oft in Grazer feministischen Protokollen der zweiten Hälfte der 1980er Jahre auf und tragen somit zur Lokalisierung und zeitlichen Einordnung dieser Dokumente bei $^{22}$. Die äußeren Merkmale von Bewegungsunterlagen sind meist sehr unterschiedlich von denen von Behördenschriftgut, aber auch sie können erklärt und aufgelöst werden.

\footnotetext{
${ }^{22}$ Beispielsweise im Protokoll der Autonomen Frauenplattform Graz (wie Anm. 17).
} 


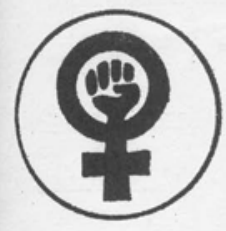

AKTION

UNABHÄNGIGER FRAUEN

\section{AUF -}

MITTEILUNGEN

Nr. 216

P.b.b.

Brscheinungsort wien

Verlangspostamt: 1010 wien

\section{frauenfors g h ung u. weiblicher lebenszusammenhang $Z 91$ \\ 1090 wien, bergg. $5 / 24$, tel. 3148544}

Das nächste Plenum findet arn 22.5.1974 un 8 whr in der Schotten= gasse statt. Eritca Fischer spricht liber das Buch von MARTA DAET.A COSTA

Das Koko trifft sich jeden Freitag un 5.30 Uhr in li.t Wien In der novea Zusamensetzung findon sich. Sylvia, Ermy, Helcn, Cirlitane, Kristin, Wirl, Susi.

Das noue Lokal wird also nun laut Plenumbesohl uß genietet, obwoh! anci andore răune, tie wir ursprüng= lich wollzen, dazv gencmen wereen milsson, Das bedentet, des bu die fonovierung der läumo vesentilicit teurer wind. In diesen Zusammenkeng esn ipe11: "Zahlt vitte die Beitrăge. an bester. per Daterauftrag, sonst gehen wir ploite,

Ingrid Strobl Ubersiede1t z' Erice Fischer

Abb. 4: Auszug aus AUF-Mitteilungen Nr. 21 (Elizabeth Kata; STICHWORT, AUF-Mitteilungen Nr. 21, Mai 1974).

Hier möchte ich einen Auszug aus einem Brief an das Koordinationskomitee der Aktion Unabhängiger Frauen (AUF) anführen, der die äußeren Merkmale (sowie auch den Inhalt) einer Aussendung der AUF (Abb. 4) kritisiert. Die Kritik an den äußeren Merk- 
malen lautet: Die Matrizen sind einzeilig (mit dem kleinsten Zeilenabstand) zu schreiben, möglichst auf einer elektrischen Maschine oder wenigstens auf einer mit kleinen Typen. Die Papiere sind beidseitig zu bedrucken (keine leeren Seiten). Beides deshalb, weil sonst unverhältnismäßig viel (teures) Papier verschwendet wird. Also kein perfektionistischer Fimmel! Beim Matrizenschreiben muß das Farbband aus der Maschine genommen werden, [w]enn die Maschine keinen eigenen Hebel hat, der das Farbband bei Bedarf aus dem Anschlagbereich entfernt. Sonst wird die Sache beim Abziehen zu schwach bzw. zu unscharf23.

Hier wird von einem AUF-Mitglied angegeben, wie die Aussendungen auszusehen haben und warum. Die äußeren Merkmale wie Zeilenabstand und beidseitiger Druck werden aus Kostengründen vorgeschrieben. Die Hinweise, wie die Matrizen zu schreiben sind, erzählen uns auch über den Druckvorgang und Einzelheiten der verwendeten Maschine. Zudem wird vorgeschrieben: Kein weißes Papier kaufen, sondern das billigste (leicht gelblich). Weiters wurde an den inneren Merkmalen die Reihenfolge kritisiert. Die Schreiberin bezieht sich auf ein Papier mit Beschlüssen zur Form der Aussendungen, das aber offenbar von niemanden gelesen wurde. Anscheinend funktionierte die Informationsweitergabe bezogen auf die Form der Aussendungen nicht oder nicht ausreichend. Die Schreiberin führt zur Einteilung der Aussendungen aus: Auf der ersten Seite sollen die Termine angekündigt werden... Plena, Feste, Treffen, Demonstrationen, Filme, Anfangstreffen neuer Gruppen, etc. Auf der zweiten Seite sollen die spezielleren Informationen folgen: Kontakttel. für geplante Aktivitäten, „Erinnerungslisten“... Falls es dritte und weitere Seiten gibt, kommen hierher die inhaltlichen Beiträge, Artikel, Flugblätter, Protokolle; und die Zahlungsaufforderungen ${ }^{24}$. Die richtige Reihenfolge soll zur Lesbarkeit beitragen und sicherstellen, dass die aktuellsten Informationen an erster Stelle sind. Die Kritik zeigt, dass eine Aussendung, die die Formen nicht beachtet, bemängelt wird - zwar wird die Legitimität nicht gänzlich abgesprochen, aber die Einhaltung gewisser formaler Kriterien gefordert.

In dem eben zitierten Beispiel werden finanzielle Gründe für gewisse Erscheinungsformen (wie die Papierauswahl) angegeben, aber auch identitätsstiftende Momente können für das Erscheinungsbild sehr wichtig sein. So finden eine bewusste Abgrenzung zu bürgerlichen Normbildern und die Ausarbeitung einer eigenen Ästhetik statt. Populär in den autonomen Frauenbewegungen waren und sind beispielsweise Flugblätter in Collage-Form (also nicht professionell layoutiert) und die Anwendung von Buchstaben in unregelmäßigen Größen in Transparenten. Es wird Wert auf die eigene Produktion beziehungsweise Produktionsfähigkeit gelegt. Flugblätter oder Aussendungen wirken dadurch vielleicht weniger „professionell“, aber sie belegen das D.I.Y. (do-it-yourself)Ethos. Sie zeigen eine eigene Kreativität, die sich mit der Zeit zu einer eigenen Tradition entwickelt. Die unregelmäßig großen Buchstaben findet man bei Transparenten aus den 1990er Jahren ebenso wie bei Transparenten aus den letzten Jahren. Feministische Zines (selbstgemachte kleine Zeitschriften, von dem Wort „Magazine“) bevorzugen immer noch Collage-Formate, und Zine-Autorinnen* verwenden oft Schreibmaschinen, obwohl Schreibmaschinen inzwischen viel weniger verbreitet sind als Computer. Die Zine-Ästhetik, die sich in den 1990er Jahren zu Zeiten von Riot-Grrrl entwickelte, blieb bis heute großteils unverändert. Insofern lohnt sich umso mehr eine tiefergehende Untersuchung der Merkmale von Bewegungsunterlagen, denn manchmal können gerade die inneren Merkmale Informationen zur Datierung und Kontextualisierung liefern, welche die

23 STICHWORT, G22 FZ Wien, Interna, Kritik an der Aussendung Nr. 21, Mai 1974.

24 Ebd. 
äußeren Merkmale auf Grund der Dauerhaftigkeit ästhetischer Traditionen nicht immer vermitteln können.

\section{Systematische Archivalienkunde}

Systematische Archivalienkunde versucht Dokumente zu typologisieren. Die systematische Archivalienkunde bereitet die meisten Schwierigkeiten bei der Untersuchung von Bewegungsunterlagen und ergibt für mich die meisten Widersprüche - gegen die klassischen Kriterien der Archivalienkunde, aber vielleicht noch mehr gegen die Ansprüche und Selbstbilder der Unterlagen-Produzierenden. Unterlagen nach hierarchischer Richtung oder hierarchischen Verhältnissen einzuteilen passt überhaupt nicht zu nicht- oder antihierarchischen Bewegungen, die keine behördliche Autorität beanspruchen können. Unterlagen nach Zweck einzuteilen wäre zwar möglich, aber die inneren und äußeren Merkmale ändern sich nach meinen Erfahrungen eher mit einer Änderung der Adressat*innen als mit dem Zweck. Dementsprechend würde ich meinen, dass Bewegungsunterlagen sich am besten nach Adressat*innen einteilen lassen. Bei der Einteilung nach Adressat*innen geht es um inhaltliche Nähe und Selbstverständnis statt expliziten Hierarchien. Ich unterscheide zwischen Schreiben an Behörden und Geldgeber*innen (sogenannte institutionelle Stellen), Unterlagen für die Öffentlichkeit (Flugblätter, Presseaussendungen und so fort), Unterlagen an andere Gruppen oder Personen innerhalb der Bewegung sowie gruppeninternen Unterlagen (worunter auch Memorialschreibwerk fallen würde, hier also etwa Plenumsprotokolle). Innerhalb dieser Einteilungen wäre eine weitere Typologisierung möglich.

Unterlagen, die an ähnliche Adressat*innen gerichtet sind, tendieren dazu, ähnliche Merkmale aufzuweisen. Egal ob ein Förderantrag (früher: Bittschrift), eine Einladung zu einer Veranstaltung oder ein Abschlussbericht, Schreiben an institutionelle Stellen werden eher förmlich gehalten. Die gesellschaftlich sonst üblichen Schreibfloskeln werden verwendet und das Erscheinungsbild wird eher „professionell“ und frei von bewegungstypischen Symbolen gehalten. Die Sprache ist wiederum anders, wenn ein größeres Publikum oder überhaupt die Allgemeinheit angesprochen wird. Da wird vielleicht appelliert, aber es wird ebenfalls angestrebt, nicht zu sehr in Bewegungsjargon zu verfallen. Noch einmal anders sieht es aus, wenn die Rezipientinnen* des Textes selbst Teil der Bewegung sind. Stellungnahmen, Aufrufe, Berichte enthalten gewisse Bewegungscodes, sowohl in den inneren als auch den äußeren Merkmalen. Die Grußformeln sind schwesterlich oder feministisch, bekannte Parolen werden wiederholt oder das Dokument wird mit Frauenzeichen geziert. Innerhalb dieses Adressat*innenkreises sind Veränderungen und Abweichungen oder auch statisch bleibende Elemente leichter zu erkennen und womöglich bedeutsam. Die Meinung bewegungsinterner Gruppen hat auch mehr Bedeutung als die der Behörden. Eine Gruppe wird mehr wertgeschätzt oder ernst genommen, wenn sie als Teil der Bewegung gilt oder die „richtige“ Position vertritt, im Gegensatz zu einer anderen Gruppe, die vielleicht eine „zu bürgerliche“ Position einnimmt, und diese Positionen kommen durch innere und äußere Merkmale oft recht deutlich zum Ausdruck. Die inneren und äußeren Merkmale der Schreiben vermitteln sowohl das Selbstbild als auch das Bild des Gegenübers und dieses wiederum vermittelt das Verhältnis oder zumindest das Verständnis des Verhältnisses zwischen Adressant*in und Adressat*in.

Diese Verhältnisse sind nicht immer klar einzuordnen. Einerseits gibt es Grenzgeher*innen, die sich nicht leicht in eine der vorgeschlagenen Kategorien einordnen 


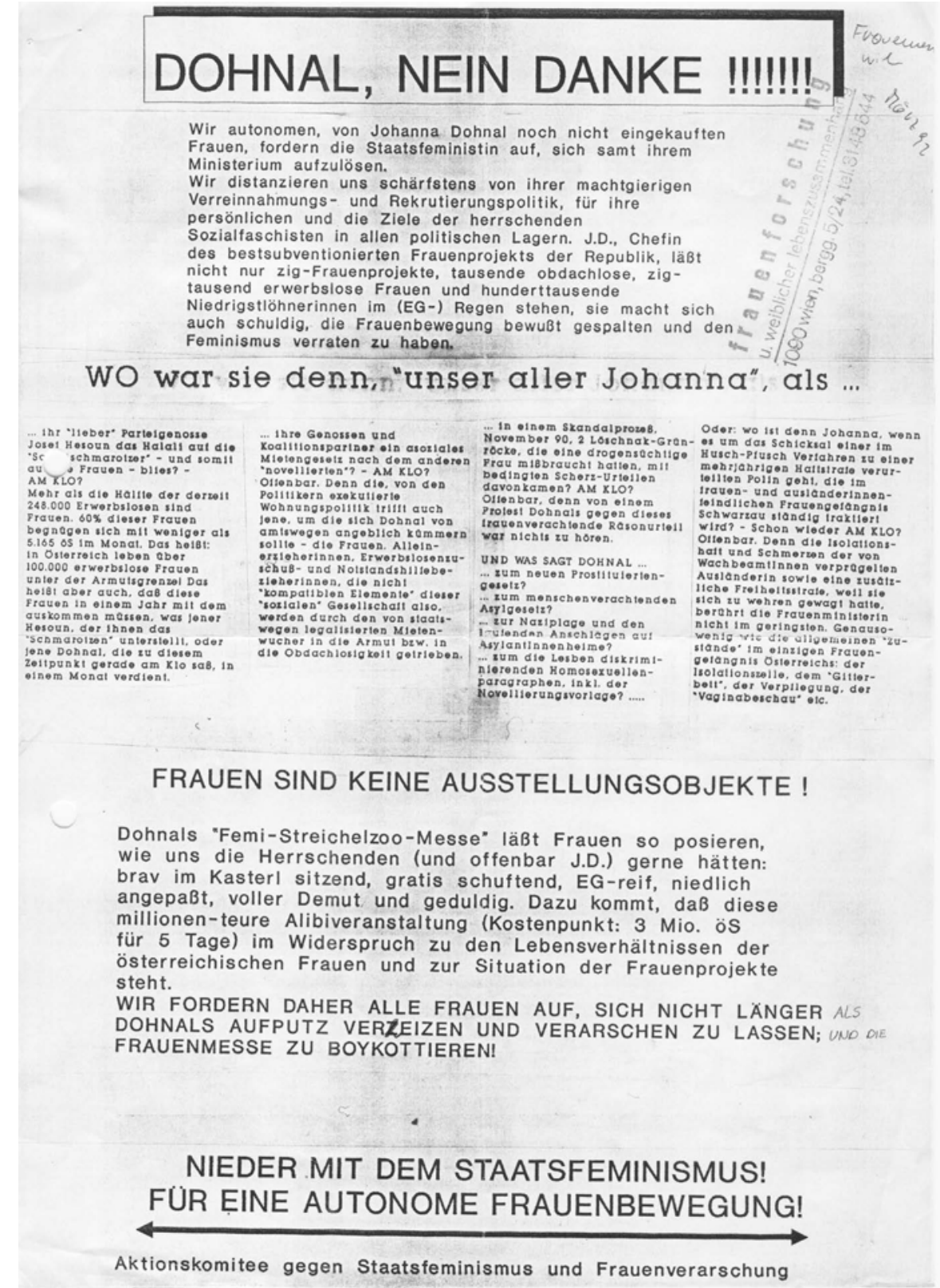

Abb. 5: Flugblatt gegen die erste Frauenmesse in der Hofburg (Elizabeth Kata; STICHWORT, 08-2.2.2. Aktionskomitee gegen den Staatsfeminismus, Dohnal, Nein Danke!, März 1992).

lassen, wie zum Beispiel den Bund demokratischer Frauen, eine kommunistische und dementsprechend parteinahe Organisation, die sehr wohl an Aktionen und Diskursen der autonomen Frauenbewegung teilnahm. Anderseits verschieben sich Verhältnisse, so 


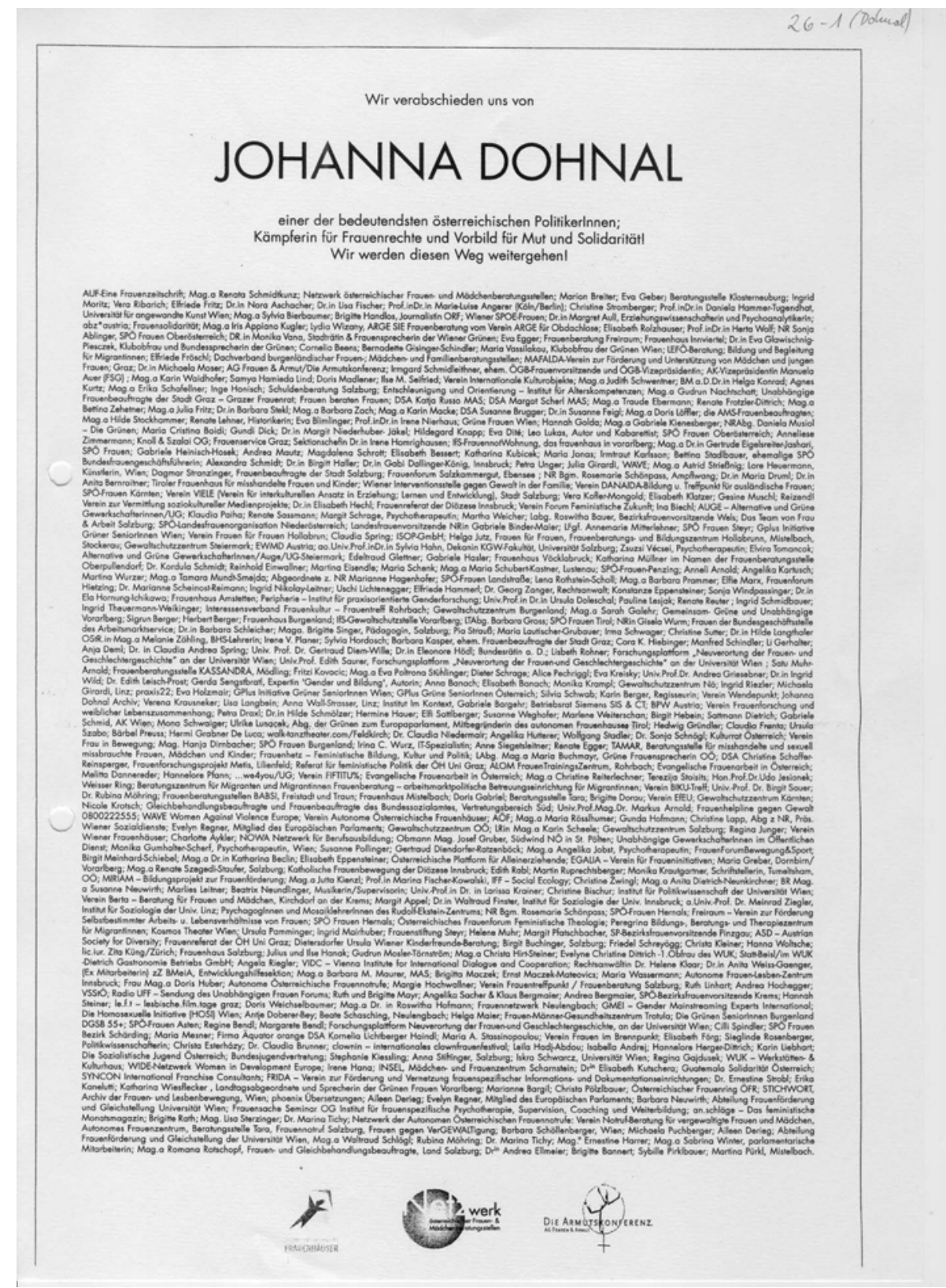

Abb. 6: Traueranzeige zum Ableben von Johanna Dohnal, unterzeichnet von vielen autonomen Frauengruppen (Elizabeth Kata; STICHWORT, 26-1 (Dohnal, Johanna), Wir verabschieden uns von Johanna Dohnal, 2010).

dass die Bewegungszugehörigkeit in Frage gestellt oder nachträglich zugeschrieben wird. Ein Beispiel für Österreich wäre Johanna Dohnal, Frauenstaatssekretärin und später Frauenministerin bis 1995. Autonome Frauengruppen betrachteten sie teilweise als eine institutionelle Figur, weil sie in der SPÖ und der Regierung verankert war. Ein Flugblatt 
mit der Überschrift Dohnal, Nein Danke! wetterte 1992 gegen die erste Frauenmesse in der Hofburg, die Dohnal mitorganisierte, und den Staatsfeminismus, den sie verkörpere $(\text { Abb. } 5)^{25}$. Doch es gab immer wieder Zusammenarbeit zwischen autonomen Frauengruppen und Dohnal, und es gibt zahlreiche Beispiele, die zeigen, dass Frauengruppen sie als Mitkämpferin betrachteten und sie entsprechend anschrieben. Als Dohnal 2010 verstarb, wurde sie mit einer Traueranzeige geehrt, die auch von etlichen autonomen Frauengruppen unterschrieben wurde (Abb. 6) ${ }^{26}$.

Eine zumindest grobe Typologisierung der Unterlagen innerhalb der Adressat*inneneinteilung wäre ein nächster Schritt in meinen Untersuchungen von Bewegungsunterlagen. So könnte man zwischen Presseaussendungen, Flugblättern, Förderanträgen, Forderungen, Unterschriftenlisten, Plenumsprotokollen, Jahresberichten und mehr unterscheiden. Es geht mir bei der Typologisierung um ein Hilfsmittel, um Unterlagen besser erkennen zu können, und nicht um einen Normalisierungs- beziehungsweise Legitimationsprozess. Bewegungsaktivist*innen wie auch Bewegungsarchivar*innen würden eine strenge Typologisierung ablehnen - eine solche würde dem negativen kulturwissenschaftlichen Bild des Archivs als Herrschaftsinstrument entsprechen, das alles, was nicht ins Bild passt, ausblendet. Archive in diesem Sinn werden von vielen in den Bewegungen abgelehnt, die sich ihnen durch eigene Archive und Gegenöffentlichkeit widerset$z^{2}{ }^{27}$. Es ist wichtig zu verstehen, wie eine Bewegung und die ihr zugehörigen Gruppen ihre Unterlagen selbst benennen und verstehen, während man gleichzeitig die einzelnen Unterlagen in einen größeren Kontext von strukturierter und formengetriebener Schriftlichkeit einbettet und versteht. Soziale Bewegungen wie auch ihr Schriftgut bekämpfen gesellschaftliche Normen oder verschieben sie, aber sie sind trotzdem von diesen geprägt beziehungsweise etablieren neue Normen. Systematische Archivalienkunde hilft die alten wie neuen Normen zu erkennen. Die Typologisierung dient nicht einer Beurteilung, ob Bewegungsunterlagen vollzogen oder „authentisch“ im administrativ-rechtlichen Sinne sind, sondern es geht darum, die Unterlagen, ihre Unterschiede und ihren Kontext verständlicher und zugänglicher zu machen. Das bedeutet vielleicht einen Bruch mit den Zielen der systematischen Aktenkunde, aber dieser Bruch ermöglicht eine weitere Anwendung der archivalienkundlichen Methoden an Unterlagen, die jenseits der administrativrechtlichen Bürokratie entstanden sind. Diese Ausbreitung der Archivalienkunde kann allen Historiker*innen, Archivar*innen und Interessierten nützlich sein.

\section{Fazit}

Dass archivalienkundliche Untersuchungen von Bewegungsunterlagen dienlich sind und von Nützer*innen auch angenommen werden, zeigt das Angebot des STICHWORT Archivs der letzten Jahre. 2016 leitete ich einen Workshop anlässlich des Frauenarchivtags am 11. Mai zur Arbeit mit Unterlagen der Neuen Frauenbewegungen. Ohne das Wort „Archivalienkunde“ zu erwähnen, zeigte ich Teilnehmerinnen* vor, wie man innere und äußere Merkmale zur Datierung heranziehen oder wie man Entscheidungsprozesse

25 STICHWORT, 08-2.2.2. Aktionskomitee gegen den Staatsfeminismus, Dohnal, Nein Danke!, März 1992.

26 STICHWORT, 26-1 (Dohnal, Johanna), Wir verabschieden uns von Johanna Dohnal, 2010.

${ }^{27}$ Ein Beispiel für diesen Zugang aus Wien liefert das Heft: Anarchivieren. Bildpunkt (Herbst/Winter 2011) $1-31$. 
anhand der Unterlagen nachvollziehen kann. Ohne meine archivalienkundlichen Untersuchungen wäre diese Art der Vermittlung nicht möglich. Teilnehmerinnen* lernten erste archivalienkundliche Skills, womit sie besser weiterrecherchieren können. Die Veranstaltung wurde von Margit Hauser und Andrea Zaremba 2017 zum Frauenarchivtag wiederholt, und inzwischen gibt es eine Veranstaltungsreihe unter dem Titel Unser Papier spricht $z u \operatorname{dir}^{28}$. Die archivfachliche Einführung in die Quellen unter Berücksichtigung von Ergebnissen der archivalienkundlichen Untersuchungen führt zu erhöhten Fähigkeiten, sich produktiv mit den Bewegungsunterlagen auseinanderzusetzen.

Ein Interesse für Archivalienkunde von Bewegungsunterlagen zeigten die Leiter*innen der Lehrveranstaltung „Aktenkunde/Vertiefung“ an der Universität Wien im Rahmen des Masterstudiums des Instituts für Österreichische Geschichtsforschung. Kathrin Kininger und Thomas Stockinger luden mich 2016 ein, über dieses Thema in ihrer Lehrveranstaltung vorzutragen, und eine erneute Einheit zu Bewegungsunterlagen fand im Sommersemester 2018 statt. Somit wird ein Stück „Archivalienkunde von unten“ in dem traditionellen Archivstudium an künftige Historiker*innen und Archivar*innen weitergegeben, was zur breiteren Akzeptanz von Bewegungsunterlagen als archivische Quellen führt, die mit den Methoden der Archivalienkunde untersucht werden können.

Dieser Beitrag sollte einige mögliche Inhalte der genetischen, analytischen und systematischen Archivalienkunde anhand von Unterlagen aus den Neuen Frauenbewegungen in Österreich aufzeigen. Diese müssen weiter ausgearbeitet werden. Auch ein Kriterienkatalog zur Bestimmung von Bewegungsunterlagen etwa nach dem Vorbild des „Kriterienkatalogs zur Bestimmung von Aktenschriftstücken“, der sich im Werk von Michael Hochedlinger findet, wäre durchaus vorstellbar ${ }^{29}$. Dieser Beitrag soll als Anstoß gelten ein archivalienkundliches Handbuch für Bewegungsquellen muss her.

Aktenkunde hat mir wichtige Werkzeuge für das Lesen, das Verstehen und die Kontextualisierung von Unterlagen, ja, auch Bewegungsunterlagen, gegeben. Ich hoffe durch eine Erweiterung der Untersuchungsgegenstände und eine Auflockerung oder Verschiebung mancher archivalienkundlicher Schwerpunkte in Bezug auf nicht-behördliche Unterlagen zu einer Archivalienkunde beizutragen, die sich als nützliche Methode für die Arbeit mit archivischen Unterlagen von sozialen Bewegungen sowie von anderen nichtstaatlichen Bereichen anbietet.

28 STICHWORT Veranstaltungen. STICHWORT Newsletter 44 (2017) 22.

29 Hochedlinger, Aktenkunde (wie Anm. 5) 236. 



\title{
Trusting Records in the Digital Age: Changing Archival Perspectives
}

\author{
Heather MacNeil
}

Technology change entails both innovation and tradition ${ }^{1}$. As the media studies scholar David Thorburn puts it, the „new grows out of the old, repeats the old, embraces, reimagines and extends the old" ${ }^{\text {" }}$. If we want to understand our emerging digital culture, he continues, we need to adopt an historical outlook on the digital environment and, along with it, a principle of continuity rather than discontinuity ${ }^{3}$. This essay is informed by Thorburn's observation and injunction: in it I trace the evolution of archival perspectives on record trustworthiness in the western world through what we might term the premodern, modern, and contemporary periods of archival history. My aim is to expose some of the roots of those perspectives and to suggest some of the ways in which they are being reimagined and extended in the digital age $\mathrm{e}^{4}$.

\section{The premodern period of archival history}

Through much of archival history, the understanding of what made and kept records trustworthy rested implicitly on generalizations about the record-making and record-keeping practices of creating organizations and the status of archival institutions as trusted repositories. Such generalizations, in turn, were rooted in, and borrowed from, a number of traditions, among them Roman law, the rationalist tradition of legal evidence scholarship, the modernist tradition of historical criticism, and the diplomatic tradition of documentary criticism. These traditions took shape between the $5^{\text {th }}$ and $19^{\text {th }}$ centuries. During this period, the trustworthiness of records was tied to their status as particular

1 This essay is a slightly revised version of the presentation delivered to the Annual Conference of the Institute of Austrian Historical Research, The Future of the Past in the Present: Archives as Lighthouses in the Information Age in Vienna, Austria, November 9-11, 2016.

2 David Thorburn, Web of Paradox, in: Rethinking Media Change. The Aesthetics of Transition, ed. David Thorburn-Henry Jenkins-Brad Seawell (Media in Transition, Cambridge [Massachusetts]-London 2003) 19-22, at 21.

3 Ibid. 21.

${ }^{4}$ In this essay I have drawn on my previous writings in this area. The overview of the premodern period of archival history is based on Heather MacNeIL, Trusting Records. Legal, Historical and Diplomatic Perspectives (The Archivist's Library 1, Dordrecht-Boston-London 2000) 1-31. The overview of the modern and contemporary periods draws, in part, on Heather MacNeIL, Trust and Professional Identity: Narratives, Counter-narratives and Lingering Ambiguities. Archival Science. International Journal on Recorded Information 11 (2011) 175-192. 
kinds of facts. In Roman antiquity, trustworthiness was associated with the truth-value of records as embodiments of legal facts and revolved, in large part, around the concept of public faith. In the Corpus Juris Civilis, public faith was conferred on a record by its being preserved in a public place, e. g. a temple, a public office, or an archives. An archives was described as ,the public place where records are deposited [...] so that they remain uncorrupted and capable of making faith [...] and so that a continuing memory of the acts to which they attest be preserved" 5 . The notion translated as „making faith" meant constituting proof of whatever they were about.

Over time, the concept of public faith came to include the preparation of records in accordance with legally prescribed forms. According to Luciana Duranti, the significance that now attached to documentary form had three consequences. First, it created a need for expertise in the compilation of certain legal documents out of which a notarial profession began to emerge. Second, probative capacity was extended only to original documents or to authenticated copies of those documents. And, third, record creators began to separate documents prepared in accordance with prescribed forms from other documents. A split between the so-called "archives treasure“, consisting of the documents embodying completed acts and endowed with public faith, and the ,archives sediment“, consisting of the documents generated in the routine conduct of affairs, became increasingly apparent. The former were consciously set aside and preserved as continuing proof of past events; the latter were left to accumulate and eventually disappear. As a consequence of this split, public faith gradually extended to documents kept in secure custody over a long period of time ${ }^{6}$.

Many of these legal concepts survived in the Middle Ages, but they were adapted to meet changing needs. For example, because archives had the capacity to confer public faith on records, only those "persons or corporations invested with sovereign power had the right to establish one in their own jurisdiction ", a right referred to as the jus archivii ${ }^{7}$. In Roman times and during the early Middle Ages, sovereignty was owned exclusively by the emperor and the pope and by persons to whom they endowed that right, such as notaries ${ }^{8}$. In the $13^{\text {th }}$ century, however, sovereignty was extended to monasteries and city-states. Thereafter, continuing custody by a monastery or city-state could endow a document with public faith. Marco Bologna has described jus archivii as „the origin of the notion of archives as instruments of power" because it ,involve[d] both the right to hold the documents created and received in the course of the creator's activity and the right to make them fully effective as original and authentic documents in any place, thereby providing them with legally binding value as evidence of what [was] written on them".

With the emergence of philology in the $14^{\text {th }}$ and $15^{\text {th }}$ centuries, the trustworthiness

5 The relevant sections of the Corpus have been compiled and translated from the Latin by Luciana DuRanti, Medieval Universities and Archives. Archivaria. The Journal of the Association of Canadian Archivists 38 (1994) 37-44, at 41. I have followed her rendering with one alteration: I have translated the Latin phrase fidem faciant as „capable of making faith“, where Duranti has translated it as „serve as authentic evidence“. See also Luciana Duranti's article in the present volume.

${ }^{6}$ Luciana Duranti, The Concept of Appraisal and Archival Theory. The American Archivist 57 (1994) $328-344$, at 332s.

7 Ibid. 332.

8 Duranti, Medieval Universities (cit. n. 5) 41.

9 Marco Bologna, Historical Sedimentation of Archival Materials: Reinterpreting a Foundational Concept in the Italian Archival Tradition. Archivaria. The Journal of the Association of Canadian Archivists 83 (2017) $35-57$, at 51 n. 34. 
of records also came to be tied to their truth-value as manifestations of historical facts. A $16^{\text {th }}$-century offshoot of philology - historical legal studies - concerned itself specifically with the credibility of records as historical testimony, translating questions concerning their relative authority, the tests of documentary authenticity, and the indications of an author's biases into a methodology of criticism ${ }^{10}$. As Barbara Shapiro notes, „[b]oth as historians and lawyers, scholars searched for reliable witnesses and sought to date documents and assess the good faith, knowledge, and credibility of those who initially had prepared them "11 . A $17^{\text {th }}$-century offshoot of philology - the diplomatic method of documentary criticism - concerned itself specifically with the authenticity of medieval charters issued by sovereign authorities in previous centuries. The original tenets of this analytical technique were outlined in Jean Mabillon's „De re diplomatica“. In it, Mabillon analyzed the material, ink, language, script, abbreviations, formulas, seals, chancery notes and so on of some 200 documents, and on the basis of that analysis he „stated what, for a particular time and place, was the correct form for a genuine document, and presented [...] the general principle of diplomatics "12. The remainder of the treatise provided proofs and illustrations of these principles and the manner in which they were to be applied, and included facsimiles of original documents, with notes and arguments demonstrating why they should be considered authentic. As Randolph Head observes, Mabillon's treatise thus served as a kind of "portable archive of authenticity“13.

Over the following two centuries criteria for determining the trustworthiness of records, not only as embodiments of legal facts, or manifestations of historical facts, but also as sources from which legal or historical facts might be inferred (the notion of evidence as inference), began to emerge and found their way into the emerging disciplines of law and history ${ }^{14}$. Legal and historical scholars also began to distinguish between two different kinds of trustworthiness. The reliability of a record referred to its truth-value as a testimonial statement of facts and it was assessed in relation to the proximity of the observer and recorder to the facts recorded. The authenticity of a record referred to its truth-value as a physical manifestation of the facts it recorded and it was assessed in relation to the document's original instantiation. By the end of the $19^{\text {th }}$ century, criteria for establishing the trustworthiness of records as evidence were firmly embedded in the law of evidence and in historical criticism. In common law jurisdictions, for example, the legal criteria were located in the business records exception to the hearsay rule, which allowed records created „in the usual and ordinary course of business" (i. e. by a person under a business duty to observe and record the facts contained in the record) to be admitted as evidence; in the „best evidence“ rule requiring the production of documentary originals as a means of verifying the integrity of records admitted as evidence; and the „ancient records“ rule which presumed the authenticity of records maintained in a natural or proper place of

\footnotetext{
${ }^{10}$ Julian H. Frankuin, Jean Bodin and the Sixteenth-Century Revolution in the Methodology of Law and History (New York-London 1963) 4.

11 Barbara J. Shapiro, Probability and Certainty in Seventeenth-Century England. A Study of the Relationships between Natural Science, Religion, History, Law, and Literature (Princeton 1983) 164.

12 James Westfall Thompson-Bernard J. Holm, A History of Historical Writing, 2 vol. (New York 1942) 219.

13 Randolph C. Head, Documents, Archives, and Proof around 1700. The Historical Journal 56 (2013) 909-930, at 913.

14 The developments described in this paragraph are discussed in more detail in MacNeIL, Trusting Records (cit. n. 4) 23-31.
} 
custody over a substantial period of time. In historical criticism they were located in the analytical techniques of external and internal criticism (the latter of which included the techniques of diplomatic criticism), and in the preference for primary sources over secondary ones.

\section{The modern period of archival history}

The modern period of archival history begins with the centralization of historical archives in national and state archival institutions across Europe over the course of the $19^{\text {th }}$ century, and the opening of those archives to the public. When European archival theory started to take shape in the late $19^{\text {th }}$ and early $20^{\text {th }}$ centuries in response to these developments, it absorbed many of the legal and historical criteria for assessing record trustworthiness and reinterpreted them for the aggregations of documents now in the custody of archival institutions. According to that emerging theory, archives possessed a circumstantial probability of trustworthiness because they were made or received by an entity in the course of carrying out its activities (a variation on "the usual and ordinary course of business") and in accordance with its own needs rather than the interests of posterity. The English archival theorist Sir Hilary Jenkinson drew a specific connection between the authenticity of archival documents and their continuous custody by a records creator and its legitimate successors (a variation on the ancient records rule). The foundational principle of provenance ${ }^{15}$ governing the arrangement of archives was based on the assumption that keeping the records of a given records creator together and in the order given to them by that creator was the best means to preserve the trustworthiness that attached to records through the circumstances of their creation, maintenance, and custody ${ }^{16}$. According to Head, the „triumph of provenance as the organizing principle for the custodial archive [...] effectively institutionalized the ius archivi in the form of archival authority " ${ }^{17}$.

For most of the $20^{\text {th }}$ century, the primary frame of reference within which the archival profession's notion of record trustworthiness operated was organizational recordkeeping. Within that framework, the circumstances of creation, maintenance and custody that are considered to provide the grounds for presuming the trustworthiness of records draw implicitly on an understanding of medieval chancery practices and the modern manifestation of these practices in Weberian-type bureaucracies. As I once put it: „Medieval chanceries exercised an exclusive competence over the creation of records issued by sovereign authorities and because the records granted privileges, elaborate and rigorous procedures were required to ensure their trustworthiness. The structure and functioning of medieval chanceries reflect, in embryonic form, five salient features of Weber's ,ideal' modern bureaucracy: (1) an unambiguous hierarchical authority structure; (2) a rationalisation of offices; (3) the specialisation of labour and specification of competencies; (4) the existence of rules, policies and procedures; and (5) the formalisation of activities by means of written documentation" 18 .

Within such environments, record reliability, i. e. trustworthiness as a statement of

15 Here the principle of provenance is understood to include both respect des fonds and respect for original order.

16 MacNeIL, Trust and Professional Identity (cit. n. 4) 177s.

17 Head, Documents (cit. n. 13) 911.

18 MacNeIL, Trusting Records (cit. n. 4) 104s. For a more detailed comparison see ibid. 104-111. 
fact, is viewed as a form of social bookkeeping ${ }^{19}$ and is established by examining the completeness of the record's documentary form and the amount of control exercised over the process of its creation. Record authenticity, i. e. trustworthiness as a record, is concerned with the record's identity and integrity, and is established by examining its status as an original or copy and the history of its transmission, maintenance, and custody over time and space. The methods for ensuring reliability and authenticity, accordingly, have tended to focus on procedural controls over record making and record keeping, the creation of central registries, the standardization of documentary forms, and an unbroken line of custody, because it has been assumed that these measures will establish the records' reliability, ensure that their identity is always fixed and explicit, and protect their integrity through time.

\section{Contemporary archival perspectives}

Since the early 1990s, technological, organizational and social changes of various kinds have thrown into question the continuing validity of many of these traditional understandings and assumptions. The strict hierarchical structure of Weberian-type bureaucracies has been largely displaced by more diffuse and fluid hierarchical structures and the shift from analogue to digital forms of information and communication has dramatically changed the nature of recordkeeping within organizations. Digital record systems are frequently multi-purpose, highly networked, and decentralized; they encompass a range of textual, visual, and auditory data that can be compiled, combined, and presented in a variety of ways, and they perform a range of functionalities, according to the needs of different users. Many systems contain a mix of raw data, information and one or more types of records. Determining whether a given electronic system even contains records (at least as they have been traditionally understood), therefore, and locating the familiar indicators of their identity - dates, classification codes, names of authors and addressees - can be a formidable challenge.

This situation is complicated further by the fact that records do not reside within these systems as fixed and unitary objects; each time a record is retrieved, a reproduction process is involved. To reflect this reality and its implications, the Preservation Task Force of the First International Research on the Preservation of Authentic Records in Electronic Systems Project (InterPARES 1) has constructed the concept of a "digital component of an electronic record“; in that construction „an electronic record is stored as one or more digital components. Digital components have no necessary relation to the elements of documentary form recognized in diplomatics analysis of records. Rather, they are determined technologically by the way the bits are stored and by the methods (software) that must be applied to reproduce the record. Reproducing an electronic record entails (1) reconstituting it, that is, reassembling its digital components if it has more than one, or extracting any digital component stored in a physical file that contains more than one such component; and (2) presenting it in proper form" 20 .

19 For a more detailed discussion of organizational records as a form of social bookkeeping see Vernon K. Dibble, Four Types of Inference from Documents to Events. History and Theory. Studies in the Philosophy of History 3 (1963/64) 203-221.

${ }^{20}$ Kenneth Thibodeau et al., Trusting to Time: Preserving Authentic Records in the Long Term, in: The InterPARES Project. The Long-term Preservation of Authentic Electronic Records: The Findings of the InterPARES Project, ed. Luciana Duranti (San Miniato 2005) 99-116, at 106. 
These acts of reassembly and re-presentation help to underline the point that digital records are best understood as ,a series of performances across time“, rather than as fixed and „stable artefacts“21. In 2002, the National Archives of Australia adopted a performance model of a digital record, according to which the „essence" of a digital record comprises the characteristics of its context, rendition and structure that need to be preserved together with the record's content to protect its authenticity. The performance model thus allows for a range of variability in the „look and feel“ and functionality of digital records over time, while preserving those record characteristics deemed „essential“ for the record to retain its meaning. This reconceptualization of a digital record has resulted in a rethinking of the link between originality and authenticity. In principle, an original record is the first complete and effective record; a traditional means of ensuring the authenticity of a record over time has been to preserve the original. However, in a digital environment, no original survives, so we have had to shift from preserving originals to preserving copies that are functionally equivalent to an original as to their consequences, and to specify the requirements such copies need to meet to achieve that equivalency.

The traditional connection between authenticity and provenance is also undergoing a reexamination. When it was first articulated in the late $19^{\text {th }}$ and early $20^{\text {th }}$ centuries, the principle was viewed as a means of fixing and stabilizing the identity and integrity of an aggregation of records at a particular historical moment - the last period in which they were actively used by the creator. In the contemporary archival literature, there is a growing recognition that we need to broaden our conceptualization of provenance to take into better account the history of record aggregations both before and after they enter archival custody. Like individual records, aggregations of records may also be seen as a series of performances that unfold over time as they are shaped and reshaped, initially by their creators and subsequently by their custodians. Such shaping and reshaping is exacerbated in the digital world where records are instantiated in variable formats, mixed and remixed, resituated and recontextualized in and through time. Since these transformations may have an impact on the records' identity and integrity, they need to be accounted for in archival description and documentation systems. In light of this acknowledgement, a number of scholars both inside and outside the archival profession are exploring the role provenance and context-related metadata might play in establishing trust in digital records specifically, and some of the means by which that metadata might be made visible to users ${ }^{22}$.

Given the ease with which digital records can be altered and the inevitability of their physical and representational transformation over time, we have also had to reassess the presumption of authenticity that has long attached to records maintained in an unbroken chain of custody from creation to preservation. An unbroken chain of custody may still help support a presumption of authenticity but, clearly, it is no longer sufficient. In the digital world, a meaningful presumption of authenticity depends on what the Inter-

${ }^{21}$ Helen Heslop-Simon Davis-Andrew Wilson, An Approach to the Preservation of Digital Records (National Archives Green Paper, Canberra 2002) 13. Archived electronic version: National Archives Green Paper: an approach to the preservation of digital records, in: PANDORA. Australia's Web Archive, http://nla. gov.au/nla.arc-49636 [1.3. 2018].

${ }^{22}$ For a sample of recent writing in this area see the essays collected in: Building Trust in Information. Perspectives on the Frontiers of Provenance, ed. Victoria L. Lemieux (Springer Proceedings in Business and Economics, Cham 2016); see also Geoffrey Yeo, Trust and Context in Cyberspace. Archives and Records. The Journal of the Archives and Records Association 34 (2013) 214-234. 
PARES Preservation Task Force terms „an unbroken chain of preservation“, which entails the implementation of processes and procedures that protect and preserve the identity and integrity of records from the moment of their creation and throughout their life ${ }^{23}$.

To address these new needs and requirements, archivists and archival institutions have reconsidered the exclusive emphasis placed on the custodial care of historical records and are directing more attention to the management of current records; shifting from post-hoc to anticipatory approaches to preservation in order to provide some assurance that the records coming into archival custody, and even those that are not, can be trusted to some degree. The consequences of that shift are discernible in the archival research that has been undertaken to identify the „significant properties“ of digital records that need to be carried forward with them through time ${ }^{24}$ and to articulate methods for protecting the trustworthiness of digital records both before and after their transfer to archival custody ${ }^{25}$. Also discernible in these efforts is evidence of a continuity principle at work as old methods are reimagined and extended to address a new reality. We can see it in the proliferation of digital recordkeeping tools and standards that are functionally analogous to those that worked in the analogue world, in the reintroduction of the familiar indicators of record identity and integrity by means of metadata, in the rediscovery of diplomatics as an analytical tool for both establishing and assessing the reliability and authenticity of digital records; in the expansion of provenance from organizing principle to contextualizing framework, and in the development of trusted digital repositories within and without traditional archival institutions.

To conclude I would like to consider continuity from one further angle. During the Middle Ages in the customary regions of France, affixing the seal of a superior jurisdictional authority was a common means of conferring legal authenticity on the documents of private persons. To provide proof of a contract the individual would present himself before a judge to formally acknowledge the execution of the contract; that acknowledgement was then recorded by the judge in the form of a letter, validated by his seal ${ }^{26}$. The practice of attesting private deeds by public or well-known seals constituted a valuable form of attestation because it gave these transactions publicity. A modern version of the publicity principle may be found in English common law in the public documents exception to the hearsay rule, where the circumstantial probability of a public document's reliability is bolstered by the requirement that it be made available to the public at all times on the grounds that „where an official record is [...] subject to public inspection, the

${ }^{23}$ Kenneth Thibodeau et al., How to Preserve Authentic Electronic Records, in: The InterPARES Project (cit. n. 20) 293-328, at 304 .

24 See, for example, David Giaretta et al., Significant Properties, Authenticity, Provenance, Representation Information and OAIS, in: iPRES 2009. The Sixth International Conference on the Preservation of Digital Objects. Proceedings. October 5-6, 2009, Mission Bay Conference Center, San Francisco, California, in: California Digital Library, https://escholarship.org/uc/item/0wf3j9cw [1/3/2018]; Significant Properties, in: National Archives. Electronic Records Archives. Advisory Committee on the Electronic Records Archives (ACERA). ACERA Presentations (8/4/2010), https://www.archives.gov/files/era/acera/pdf/significant-properties.pdf [1.3. 2018].

${ }_{25}$ For an overview of some European initiatives in this area see Stephan STRODL et al., Research on Digital Preservation within Projects Co-funded by the European Union in the ICT Programme (May 2011), in: EU Commission. CORDIS Community Research and Development Information Service. Archives, http://cordis. europa.eu/fp7/ict/telearn-digicult/report-research-digital-preservation_en.pdf [1. 3. 2018].

26 Thomas Frederick Tout, Chapters in the Administrative History of Mediaeval England. The Wardrobe, the Chamber and the Small Seals, 5 vol. (Publications of the University of Manchester - Historical Series 34 , 35, 48, 49, 57, , Manchester et al. 1920-1930) 1 121-126. 
facility and certainty with which errors would be exposed and corrected furnishes a special and additional guarantee of accuracy" 27 .

I think there is a useful connection to be made between publicity and transparency: we might think of transparency as a contemporary reimagining and extension of the publicity principle. In suggesting that connection I am drawing on an observation made by the technology commentator David Weinberger that, in the „ecology of belief" generally, and with internet resources specifically, „transparency is the new objectivity “28. According to Weinberger: „What we used to believe because we thought the author was objective we now believe because we can see through the author's writings to the sources and values that brought her to that position. Transparency gives the reader information by which she can undo some of the unintended effects of the ever-present biases". In that sense, he continues, „transparency mirrors the nature of the Web: Content is linked, public, discussed and always subject to dispute and revision" 29 .

Making visible to our users information about the various contexts in which digital records have been made, managed and preserved over time is an important means of assisting them in coming to an informed decision about whether the records they are drawing upon for their research meet their specific requirements for reliability and authenticity. Users can play a critical role in assessing the nature and degree of trustworthiness that records ought to be accorded in particular circumstances because their assessment is based on a wider range of considerations than are typically taken into account by recordkeepers, archivists and archival institutions. This is especially true when we consider that records, as „boundary objects“, are shared across diverse communities of practice and are used by each of those communities in different ways; it follows that each community will bring its own perspective to bear on questions of trustworthiness ${ }^{30}$. Thus, the archival perspective on what makes records in general and digital records in particular trustworthy in and through time is best seen as merely a starting point in a more broadly based and socially negotiated process of assessment.

Making visible the actions and decisions taken by archivists and archival institutions in the name of protecting and preserving record trustworthiness is a particularly critical means of enacting the publicity principle. By advertising our own actions and decisions we are inviting user communities to understand the archive as a construction of the past rather than a mirror of that past and paving the way for building a more broadly based apparatus of trust. As the historical anthropologist, Ann Laura Stoler, puts it, „Whether documents are trustworthy, authentic and reliable remain pressing questions, but a turn to the social and political conditions that produced these documents, [...] has altered [our] sense of what trust and reliability might signal and politically entail“ ${ }^{\text {" } 31}$.

The long-term preservation of trustworthy digital records is a historically situated pro-

27 John Henry Wigmore-James H. Chadbourn, Evidence in Trials at Common Law, 5: Sections 13601684 (Boston 1974) $620 \$ 1632(2)$.

28 David Weinberger, Transparency: The New Objectivity, KMWorld. Content, Document and Knowledge Management 18/8 (28/8/2009), http://www.kmworld.com/Articles/Column/David-Weinberger/Transparencythe-new-objectivity-55785.aspx [1.3. 2018].

29 Ibid.

30 Geoffrey Yeo, „Nothing is the Same as Something Else“: Significant Properties and Notions of Identity and Originality. Archival Science 10 (2010) 85-116, at 97-99.

31 Ann Laura Stoler, Colonial Archives and the Arts of Governance: On the Content in the Form, in: Refiguring the Archive, ed. Carolyn Hamilton et al. (Dordrecht-Boston-London 2002) 83-100, at 85. 
cess in which archivists and archival institutions make choices about what records to preserve and how to preserve them in accordance with their understanding of the nature and purpose of those records and current conventions for managing them; such understanding is shaped, in turn, by a host of assumptions and value commitments. Consequently, the choices we make, inevitably, will result in some „significant properties“ of records being protected at the expense of other, perhaps equally significant properties; and in the privileging of certain contexts of records creation, maintenance and use over others. This is why the archival scholar Geoffrey Yeo insists that „transparency - publicising the decisions we have made and not seeking to pretend that they are anything other than constructed outcomes of fallible [...] processes - is as crucial as an awareness of their consequences" 32 . In the end, the credibility of the claims archivists and archival institutions make for the trustworthiness of digital records rests on our ability to demonstrate the trustworthiness of the principles, policies and practices we have adopted to manage them. We are bound, therefore, not only to design and implement policies and practices that will support a strong circumstantial probability of record trustworthiness, but also to provide and make transparent to our user communities an honest and adequate account of the actions and decisions we have taken in the course of stewarding those records over the long term.

32 Yeo, Nothing is the Same (cit. n. 30) 111. 



\section{ÜBERLIEFERUNGSBILDUNG UND BEWERTUNG}





\title{
Nil memorabile, sed tamen asservandum
}

\section{Merkwürdigkeit und Überlieferungsbildung: Das Beispiel der Archive katholischer Ordensgemeinschaften}

\author{
Helga Penz
}

Unter Anwendung Schellenbergscher Bewertungskriterien und des Federführungsprinzips ante litteram traf der Regularkanoniker und Archivar des Augustiner-Chorherrenstifts Herzogenburg Wilhelm Bielsky um die Mitte des 19. Jahrhunderts eine Entscheidung über die Archivwürdigkeit von Schriftstücken aus der Frühen Neuzeit ${ }^{1}$. Es handelte sich um Verwaltungsschriftgut aus der Prälatur betreffend eine dem Stift inkorporierte nahegelegene Pfarre. Der kleine Faszikel enthält beispielsweise die Anordnung des Propstes Maximilian Herb vom 30. September 1701 an die Klosterkanzlei, dem Pfarrvikar eine Schuld von dreißig Gulden zu erlassen und ihm sein Mostdeputat zu kürzen. Auf dem Aktenumschlag vermerkte der Stiftsarchivar (Abb. 1): Dokumente verschiedenen Inhaltes betreffend die Pfarre St. Veit zu Inzersdorf. Nil memorabile sed tamen asservandum ${ }^{2}$. Obwohl der ohnehin nicht sehr hohe Evidenzwert des Schriftstücks also noch weit hinter seinem Informationswert zurückblieb - „nichts Erinnerungswürdiges“ -, entschied der Archivar wegen des Umstands, dass es sich um Unterlagen aus Geschäftsgängen handelte, an denen der Klostervorsteher unmittelbar und eigenhändig Anteil hatte, für die Erhaltung: „trotzdem aufzubewahren“. In einer Zeit, in der Stiftsarchivare als Historiographen agierten und Archivwürdigkeit nach Quellenwert bemessen wurde, ist dies eine erstaunliche Leistung formaler Bewertung, an deren Stabilität zu Recht zu zweifeln war. Wilhelm Bielskys Beurteilung erfolgte darum als Anweisung an seine Nachfolger im Archivamt des Klosters.

1 Theodore R. Schellenberg, Die Bewertung modernen Verwaltungsschriftguts, übers. und hg. von Angelika Menne-Haritz (Veröffentlichungen der Archivschule Marburg, Institut für Archivwissenschaft 17, Marburg 1990). Zum Federführungsprinzip vgl. Jürgen Treffeisen, Archivübergreifende Überlieferungsbildung in Deutschland. Die vertikale und horizontale Bewertung. forum-bewertung. Das interaktive Forum für alle Fragen der archivischen Überlieferungsbildung (2004), http://www.forum-bewertung.de/beitraege/1022.pdf [1. 3. 2018]. Der Vorschlag archivischer Bewertung auf Grundlage rein formaler Kriterien hat zur sogenannten „neuen deutschen Bewertungsdiskussion“ geführt: vgl. Robert Kretzschmar, Die „neue archivische Bewertungsdiskussion" und ihre Fußnoten. Zur Standortbestimmung einer fast zehnjährigen Kontroverse. Archivalische Zeitschrift 82 (1999) 7-40. Zum Stiftsarchiv Herzogenburg vgl. Helga Penz, Kloster - Archiv - Geschichte. Schriftlichkeit und Überlieferung im Augustiner-Chorherrenstift Herzogenburg in Niederösterreich 1300-1800 (ungedr. Dissertation, Universität Wien 2004), http://othes.univie.ac.at/38498/1/Diss-Text.pdf [1. 3. 2018].

2 Stiftsarchiv [StiA] Herzogenburg, H.F. 537/7. 


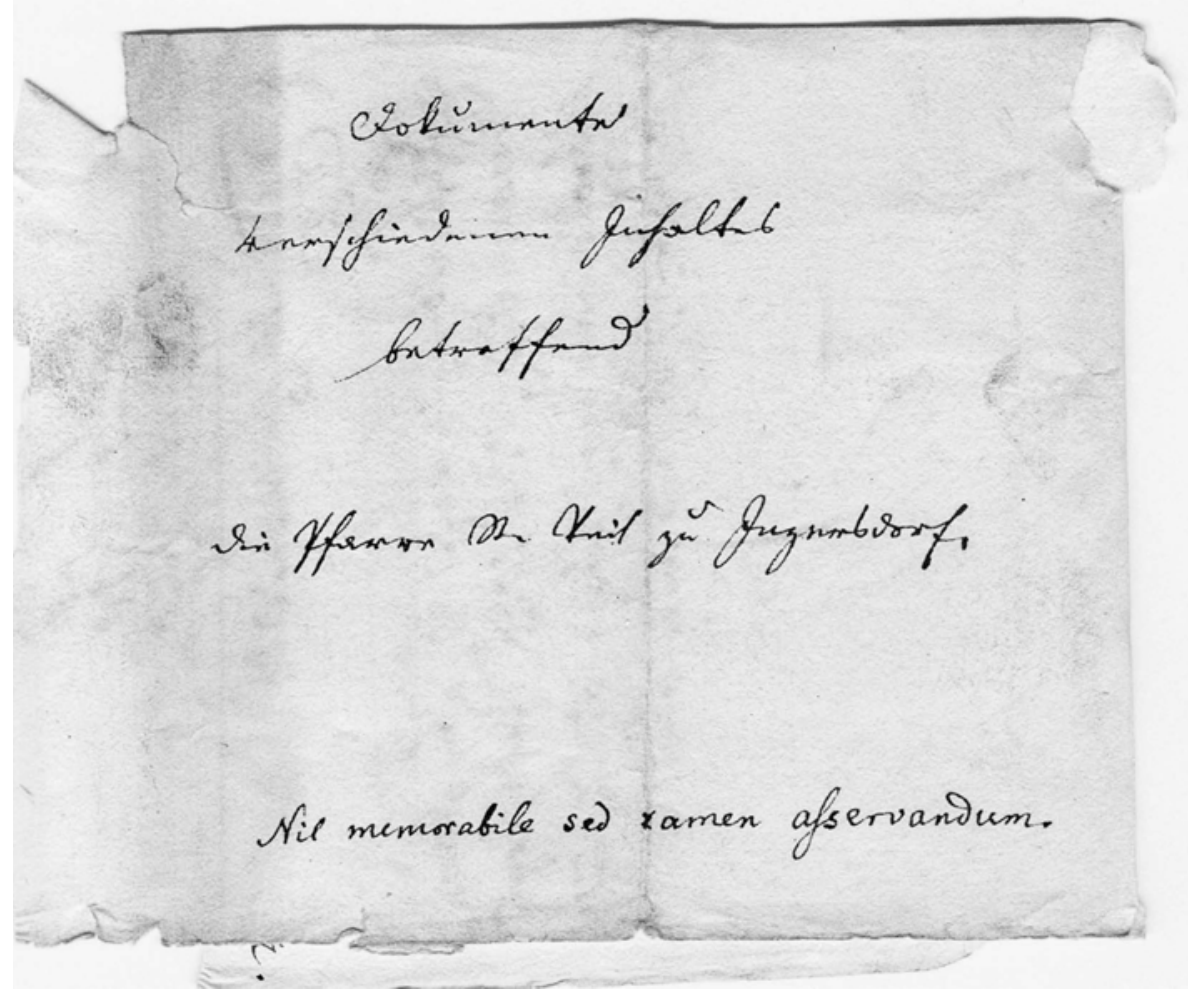

Abb. 1: Faszikelumschlag aus dem Stiftsarchiv Herzogenburg mit Vermerk des Stiftsarchivars Wilhelm Bielsky Nil memorabile sed tamen asservandum (Helga Penz; StiA Herzogenburg, H.F. 537/7).

Im Kontext der Forschungsinteressen damaliger Historiker und Historikerarchivare erschien die Aussagekraft simpler Schuldenquittungen, die in keinem Zusammenhang zu einem nennenswerten Ereignis der klösterlichen Geschichte standen, vernachlässigbar. Antiquarische Qualität, wie sie eine mittelalterliche Pergamenturkunde besaß, war zwar bereits eine Kategorie für eine positive Bewertung, aber ein frühneuzeitliches Schriftstück hatte aus der Sicht des 19. Jahrhunderts noch nicht die Patina reiner Historizität angenommen. Einem Zeitgenossen des Herzogenburger Stiftsarchivars, dem Historiker Leopold Ranke, gewährte man in Wien die Akteneinsicht für seine Forschungen zur „Verschwörung gegen Venedig 1618“ mit Hinweis auf die politische Sensibilität des Materials nur nach Intervention höchster Regierungsstellen ${ }^{3}$.

In den traditionsreichen Klöstern der Prälatenorden kommen Historizität und Aktualität auf andere, besondere Weise zur Deckung. Die Gründungserzählung bleibt im Archiv durch Überlieferung präsent: Aus Fundationsurkunden, klösterlicher Annalistik, Verbrüderungsbüchern und Nekrologen, Unterlagen zu Selig- und Heiligsprechungsprozessen der Stifter mit Viten und Mirakelsammlungen entstanden sinnstiftende Erzählungen. Der älteste namentlich bekannte Stiftsarchivar des Benediktinerklosters Lambach

3 Kasper Risbjerg Eskildsen, Leopold Ranke's Archival Turn: Location and Evidence in Modern Historiography. Modern Intellectual History 5 (2008) 425-453. 
P. Ildephons Prandtner verfasste 1731 eine Gründungserzählung mit dem Titel Nova Antiquitas, in der er eine Abschrift der mittelalterlichen Vita Adalberonis, die in der Stiftsbibliothek überliefert ist, mit Quellen aus dem Stiftsarchiv anreicherte ${ }^{4}$. Die mittelalterlichen Ursprünge seines Hauses führte er in den archivischen Zeugnissen zur Verehrung des Stifters Adalbero von Würzburg durch die Zeiten bis auf seine gegenwärtige. Klosterchroniken sind keine literarische, sondern eine archivische Textgattung und nicht selten nach der Logik der Archive organisiert. Das Narrativ klösterlicher Selbstvergewisserung wurde entlang der Überlieferung entwickelt, die auf diese Weise mit Bedeutung aufgeladen wurde.

Eine Geschichte von Überlieferungsbildung und Bewertung in Archiven katholischer Ordensgemeinschaften ist noch nicht geschrieben worden. Klosterarchive kommen in archivhistorischen Überblicken nach dem Mittelalterteil häufig nur mehr am Rande oder gar nicht mehr vor. Auch das österreichische Denkmalschutzgesetz hatte in seiner Bestimmung, dass für kirchliche Archive grundsätzlich die Vermutung eines öffentlichen Interesses ihrer Erhaltung gilt ${ }^{5}$, wohl eher die altehrwürdige Überlieferung kirchlicher und klösterlicher Einrichtungen mit langer Tradition im Blick als das Verwaltungsschriftgut moderner Sozialeinrichtungen, die von Orden geführt werden.

Im Folgenden werden Aspekte der Überlieferungsbildung vorgestellt, wie sie in Orden und Klöstern, in denen die Archivarinnen und Archivare Angehörige der Konvente und Kommunitäten sind, gestaltet wurde und wird, Bewertung also aus der Innensicht einer Institution erfolgt.

\section{Archive der Prälatenorden: Bewertung aus Betroffenheit}

In den Klöstern der sogenannten alten Orden zog man in der Frühen Neuzeit aus den Archivalien, besonders aus dem Urkundenschatz, eine Geschichte der Äbte und Klostervorsteher von den Anfängen bis in die Gegenwart. Prälatenlisten, die im 18. Jahrhundert in Handschriften angelegt wurden, erfuhren nicht selten eine Fortführung bis weit ins 20. Jahrhundert. Dem Ideal der Unverbrüchlichkeit und Stabilität in vielerlei Hinsicht bietet die Reichhaltigkeit an Altertümern Beruhigung und Bestätigung. Chroniken und Archivregister sind zum Fortschreiben angelegt, die Archive die tiefen Fundamente, auf denen noch Hohes zu bauen wäre: Behausungen der Ermächtigung.

Denkwürdigkeit ist ein Kriterium, das archivische Ordnung auch durchkreuzen kann. Der Abt des Benediktinerklosters St. Peter Amand Pachler (1657-1673) ließ für das Abteiarchiv ein Repertorium anlegen, in dessen Vorwort er sich dafür entschuldigt, dass ein ordo exactus wegen der Fülle und Vielfalt der Unterlagen nicht herstellbar gewesen $\operatorname{sei}^{6}$. In einer Lade befanden sich laut Repertorium unterschidliche denckwürdige sachen unnd acta,

${ }^{4}$ Helga Penz, Allerhand schreibereien. Lambacher Archivgeschichte im 18. Jahrhundert, in: Stift Lambach in der Frühen Neuzeit. Frömmigkeit, Wissenschaft, Kunst und Verwaltung am Fluss. Tagungsband zum Symposion im November 2009, hg. von Klaus Landa-Christoph StötTinger-Jakob WüHrer (Linz 2012) 213-223, hier 217.

5 Bundesgesetz vom 25. September 1923, betreffend Beschränkungen in der Verfügung über Gegenstände von geschichtlicher, künstlerischer oder kultureller Bedeutung (Denkmalschutzgesetz). Bundesgesetzblatt für die Republik Österreich 103 (5. 10. 1923), Nr. 533, \$2 und $\$ 4$ Abs. 2.

${ }^{6}$ Helga Penz, Erinnern als Kulturtechnik: Klosterarchive im Barock, in: Europäische Geschichtskulturen um 1700 zwischen Gelehrsamkeit, Politik und Konfession, hg. von Thomas Wallnig-Thomas StockingerInes Peper-Patrick Fiska (Berlin-Boston 2012) 91-106, hier 93. 
welche sub Amando abbate vorbeygangen unnd in andere classes archivii nit haben khönden beygelegt werden ${ }^{7}$. Was von einem Abt übrigbleibt, sperrt sich bisweilen der Einordnung in Bestehendes. Denn die Fortschreibung der Tradition bedeutet nicht Gleichförmigkeit: Was ein Abt zu seinem Anliegen macht oder wegen der Zeitumstände machen muss, hinterlässt Schriftgut, für das die classes archivii mitunter keinen vorgesehenen Platz bieten. Oberenwechsel sind in den meisten Kloster- und Ordensarchiven deutlich ablesbar und Unterlagen werden häufig nach Regierungsjahren von Prälaten und Amtsjahren von Provinziälen ausgebildet und organisiert.

Archivwürdigkeit bemisst sich in Ordensgemeinschaften auch nach dem Stellenwert im klösterlichen Lebensvollzug. Weniger die historische Aussagekraft als der Erinnerungswert geben dem Archiv Signifikanz. Die Bewertung aus Betroffenheit wurzelt in der hohen Bedeutung des Zurückblickens und des sich immer wieder neu auf den Beginn Besinnens. Das augustinische Wort vom Anfang, der immer mitgeht, wird im Archiv anschaulich und angreifbar. Briefe von Stifterinnen oder Stiftern und Ordensmitgliedern, chronikale Aufzeichnungen, Rundschreiben von Oberinnen und Oberen werden bei Tisch vorgetragen. Der verstorbenen Ordensangehörigen, bei den Äbtissinnen und Äbten oft Jahrhunderte zurückreichend, wird im täglichen Totengebet gedacht.

Vieles liegt dem Erinnerungswert und der rechtlichen Relevanz aber auch fern. Trotzdem wurden selbst Schriften aus der Kanzlei aufgehoben und erhalten, an denen nit vüll gelögen ${ }^{8}$ oder die gar von keiner wichtigkeit ${ }^{9}$ waren. Die Dignität der Archivwürde bleibt in den Stiftsarchiven jenen Unterlagen, die aus dem Parteienverkehr mit den Untertanen entstanden, zwar oft versagt, aber auch ohne diese Nobilitierung wird dem Fußvolk unter den Archivalien die Existenzberechtigung nicht entzogen, denn für einen Löschungsauftrag als formelle damnatio memoriae sind sie nicht wichtig genug. Dieser erging beispielweise für die nachgelassene Korrespondenz des Abtes der Benediktinerabtei St. Peter in Salzburg Johannes Staupitz mit seinem ehemaligen Beichtkind und Schüler Martin Luther: ein Archiv mit Reinheitsgebot und Bewertung als Purifizierung ${ }^{10}$. Das Platzproblem dürfte im 18. Jahrhundert noch keines gewesen sein, und ein nicht kleiner Teil der Überlieferung fiel - soweit sich das nachvollziehen lässt - ohnehin dem Zahn der Zeit zum Opfer. In den Kanzleien war es vom Gebrauch zum Verbrauch der Schriften nicht weit. Klosterumbauten, Übersiedelungen und kriegsbedingte Evakuierungen taten ein Übriges.

Die Klöster der Prälatenorden kannten stets zwei Archivkörper: das Kanzleiarchiv für Grundbuchamt und Hofgericht und das innere oder Prälaturarchiv. Was von Wichtigkeit und woran etwas gelegen war, verwahrte der Abt im eigenen Archiv, die Bewertung fand beim Transfer von den Händen des Hofrichters in jene des Prälaten statt. Die Trennung erfolgte nicht nach den modernen Vorstellungen von Registratur und Archiv, sondern nach Funktionalität des Schriftguts. Erst im 18. und vor allem 19. Jahrhundert wurden aus den inneren Archiven die heute bekannten Stiftsarchive, die die historische Überliefe-

\footnotetext{
7 Ebd. 95.

8 StiA Herzogenburg, H.F. 230/1, Abraitungen und auffsandungen betreffend, daran nit vüll gelögen ist. Vermerk, 18. Jahrhundert; vgl. Penz, Kloster (wie Anm. 1) 5.

9 StiA Lambach, Hs. 1, Catalog zum Stiftsarchiv (1734-1740), unfoliiert: Alte kauffbrief [...], fascicul mit alten schrifften [...] Das übrige ist gar von keiner wichtigkeit, vgl. PENZ, Allerhand schreibereien (wie Anm. 4) 215 .

${ }^{10}$ Alfred Jeremias, Johannes von Staupitz. Luthers Vater und Schüler. Sein Leben, sein Verhältnis zu Luther und eine Auswahl aus seinen Schriften (Quellen. Lebensbücherei christlicher Zeugnisse aller Jahrhunderte 3/4, Sannerz-Leipzig 1926) 69.
} 
rung des gesamten Klosters enthalten ${ }^{11}$. Die Stiftsarchivare waren die Kustoden der ihnen anvertrauten Überlieferung klösterlicher Tradition. Auf der Suche nach den Ursprüngen und Anfängen auf gesichertem geschichtswissenschaftlichen Boden widmeten sie sich besonders den Quellen des Mittelalters. Die Diplome und Urkunden waren das erste Archivgut, in dessen vorarchivische Ordnung massiv eingegriffen und das dem Diktum der Diplomatik unterworfen wurde.

Die Metamorphose vom Schrift- zum Archivgut vollzog sich bisweilen unter der Wahrnehmungsschwelle von Prälaten und Stiftsarchivaren, sodass manchmal verborgen in Faszikelbündeln echte Überreste barocken Verwaltungshandelns überliefert wurden. Im Stiftsarchiv Herzogenburg ist beispielsweise ein ehemaliger Anschlagzettel der Kanzleitür aus dem Jahr 1625 überliefert, mit dem die Einstellung des Parteienverkehrs während der Weinlese bekannt gemacht wurde ${ }^{12}$. Vielleicht hat die aufwändige kalligraphische Gestaltung des Schriftbilds, die an das Gepräge einer Urkunde erinnert, die Überlieferungschance erhöht. In vier Jahrhunderten hat dieses Archivstück jedenfalls einen enormen Zuwachs an intrinsischem Wert erfahren und ist heute im Stiftsmuseum ausgestellt.

Nicht alle Stiftsarchivare waren gewillt, so lange zu warten. Im 19. Jahrhundert betraten Historikerarchivare als Jäger und Sammler bedeutsamer Quellen das Biotop Archiv und verursachten ein Artensterben. Die Stifte mit den ambitioniertesten Historikern, die wie das Augustiner-Chorherrenstift St. Florian sogar eine eigene Historikerschule hervorgebracht haben ${ }^{13}$, erlebten die größten Verluste. Was bereits vorher - wie oben erwähnt als unwürdig und wertlos deklassifiziert worden ist, konnte nun endlich als unnützer Ballast abgeworfen werden, insbesondere serienmäßige und nicht-narrative Unterlagen der grundherrschaftlichen Verwaltung und der klösterlichen Rechnungslegung.

Trotzdem war der Gleichförmigkeit archivischer Überlieferung nicht zu entgehen. Sie gab Historikerarchivaren und Archivhistorikern in vor- und nachrevolutionärer Zeit das beruhigende Gefühl historischer Kontinuität und stabiler Entwicklung. Geschichtsschreibung entlang des Aktenlaufs spürte keinem Weltgeist nach und forderte nicht zu Positionen und Handlungen auf, sie mehrte die klösterliche stabilitas. Revolutionen fanden außerhalb der Archive natürlich trotzdem statt und schlugen auf Verwaltungshandeln und Aktenumlauf durch. Mit der Aufhebung der Grunduntertänigkeit 1848/49 wurden in den Stiftskanzleien ganze Sachmaterien obsolet und angelaufener Aktenausstoß nicht mehr gebraucht. Nach einigen Jahren wurde er zu Makulatur und recycelt, Spuren davon sind bis heute in den Archiven erhalten ${ }^{14}$. Es ist davon auszugehen, dass diese vorarchivischen Bewertungen großen Stils ohne Zuziehen des Stiftsarchivars geschahen, der vermutlich selbst am wenigsten mit dergleichen behelligt werden wollte.

${ }^{11}$ Helga Penz, Die Prälatenarchive, in: Quellenkunde der Habsburgermonarchie (16.-18. Jahrhundert). Ein exemplarisches Handbuch, hg. von Josef Pauser-Martin Scheutz-Thomas Winkelbauer (MIÖG Ergbd. 44, Wien-München 2004) 686-695.

12 Penz, Kloster (wie Anm. 1) 25.

13 Karl Rehberger, Ein Beitrag zur Vorgeschichte der „Historikerschule“ des Stiftes St. Florian im 19. Jahrhundert. Mitteilungen des Oberösterreichischen Landesarchivs 10 (1971) 210-250.

${ }_{14} \mathrm{Im}$ Stiftsarchiv Herzogenburg wurde beispielsweise ein Schreiben an das Hofgericht vom 10. Februar 1806 als Umschlag für die Rentamtsrechnungen von 1866 wiederverwendet: StiA Herzogenburg, H.4.3F. $1001 / 3$. 


\section{Archive der jüngeren Orden: Archivanordnungen}

Serienmäßigkeit, Aktenplan und Archivordnung verbesserten die Überlieferungschancen in den Ordensarchiven. Die im 16. und 17. Jahrhundert neu gegründeten Ordensgemeinschaften legten in ihren Konstitutionen großen Wert auf geordnete Schriftlichkeit für die ordensinterne Kommunikation und für die Aktenführung beim Verwaltungshandeln, in Rechtsakten und im Verkehr mit kirchlichen und weltlichen Behörden. Der

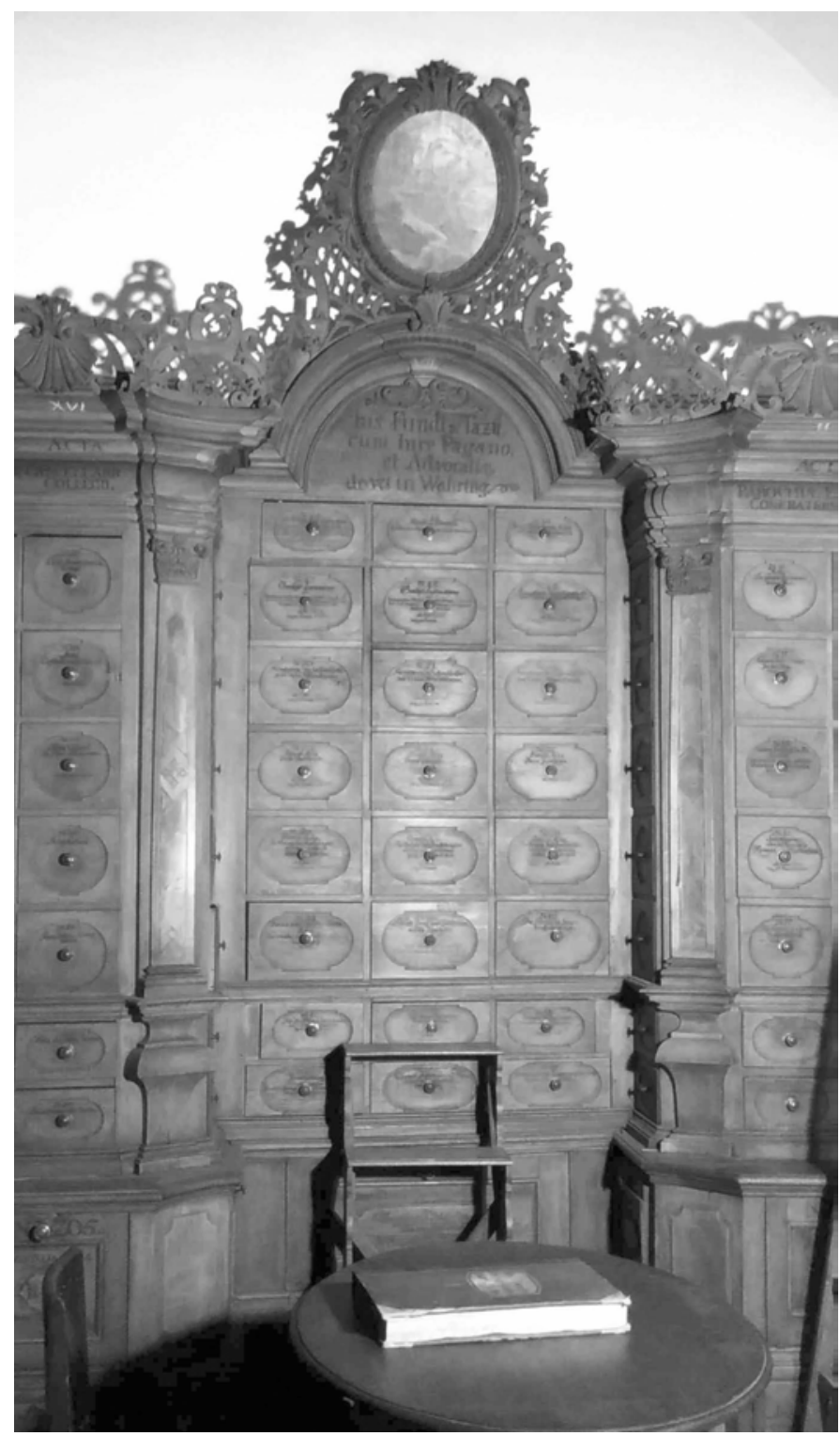

Abb. 2: Archivschrank des Barnabitenarchivs bei den Salvatorianern, Wien (Robert Passani). 
Schriftproduktion der Temporalienverwaltung wurde dabei besondere rechtliche Relevanz beigemessen, Archive wie jenes des Wiener Kollegs der Regularkleriker vom hl. Paulus, nach ihrem mailändischen Mutterkloster San Barnaba Barnabiten genannt, sind Kanzleiarchive. Die Unterlagen der Spiritualienverwaltung sind wesentlich weniger umfangreich und außerhalb dieses Archivs überliefert.

Die Archiv- und zugleich Registraturordnung des Wiener Barnabitenarchivs ist umfassend und vorausplanend. Für die Archiveinrichtung im 18. Jahrhundert wurden eigens gefertigte Einbaumöbel angepasst. Diese dienten gleichzeitig der Ablage, Wiederauffindbarkeit und Archivierung (vgl. Abb. 2). Mit den fest angebrachten Ladenbeschriftungen, die nicht nur Nummern, sondern auch Sachbezeichnungen enthalten, manifestiert sich ein Anspruch auf Dauerhaftigkeit, der eingelöst wurde: Das Barnabitenarchiv besteht auch hundert Jahre nach Weggang des Ordens aus Österreich noch immer unverändert im heutigen Kloster der Salvatorianer.

Die barnabitische Archiv-Einrichtung ist zugleich eine Archivanordnung. Für den Archivar ist sie selbsterklärend, dann jeder Vernünftiger wird nach Lesung deren Hauptrubriken von selbsten belehret werden, was diesfahls zu beobachten und zu thuen seye, heißt es in der Einleitung zu seinem Archivrepertorium ${ }^{15}$. Das „totale Archiv“ schien für alle Eventualitäten gerüstet. Was an Schriftlichkeit anfiel, wurde klassifiziert und hatte idealiter seinen Rang in Haupt- und Untergruppen. An Bewertung und Skartierung nach Erledigung war nicht gedacht, jedenfalls nicht explizit. Auch pragmatischer Schriftlichkeitskultur wohnte der Archivzweck von Erinnerung und Selbstvergewisserung inne, der nach Ablauf des Geschäftsvorgangs bestehen blieb. Wohlgeordnete Schriftlichkeitsdepots festigten die Ordnung und trotzten dem Zeitenwandel ${ }^{16}$.

\section{Archive der Frauenorden: Eigenwerte}

Die hohe Wertschätzung für geordnete Schriftgutverwaltung und Archivierung ist auch in den Frauenordensgründungen der Frühen Neuzeit nachvollziehbar. Frauenklöster wie das 1717 gegründete Wiener Kloster von der Heimsuchung Mariens administrierten allerdings keine Grundherrschaft, waren streng klausuriert und finanzierten ihren Unterhalt für die Ordensfrauen und das Mädchenpensionat neben den Kostgeldern vorwiegend aus einem kaiserlichen Stiftungskapital, dessen Zinsertrag aus der Hofkammer angewiesen wurde. Das Amt der Archivarin wurde früher häufig in Personalunion mit dem der Assistentin der Oberin, die deren Kanzlei führte, ausgeübt und ist heute zumeist die Aufgabe einer emeritierten Priorin.

Wie bei den Barnabiten oder Jesuiten machen auch die Ordenskonstitutionen der Salesianerinnen detaillierte Angaben über das Amt der Schwester, welche die Obsorge über die Schriften das Closters hat (vgl. Abb. 3): Bildung von Faszikeln für zusammengehörige Unterlagen, deren Beschriftung und systematische Ablage, Protokollierung von Entlehnungen außer Haus, enge Zusammenarbeit mit der Wirtschafterin beim Führen der Kopial- und Rechnungsbücher sowie Registrierung neuer Schriften in einem Inventarium ${ }^{17}$.

15 Wiener Barnabitenarchiv im Provinzarchiv der Salvatorianer, Archivrepertorium von 1758.

16 Markus Friedrich, Die Geburt des Archivs. Eine Wissensgeschichte (München 2013) 97f.

17 Buch deren Ordens-Gebräuchen und Directorium [...] für die geistlichen Schwestern von der Heimsuchung Mariä (Wien 1727) 55f.; vgl. Helga Penz, Vive Jésus. 300 Jahre Salesianerinnen in Wien, in: Das Kloster der Kaiserin. 300 Jahre Salesianerinnen in Wien, hg. von Helga Penz (Petersberg 2017) 13-17. 


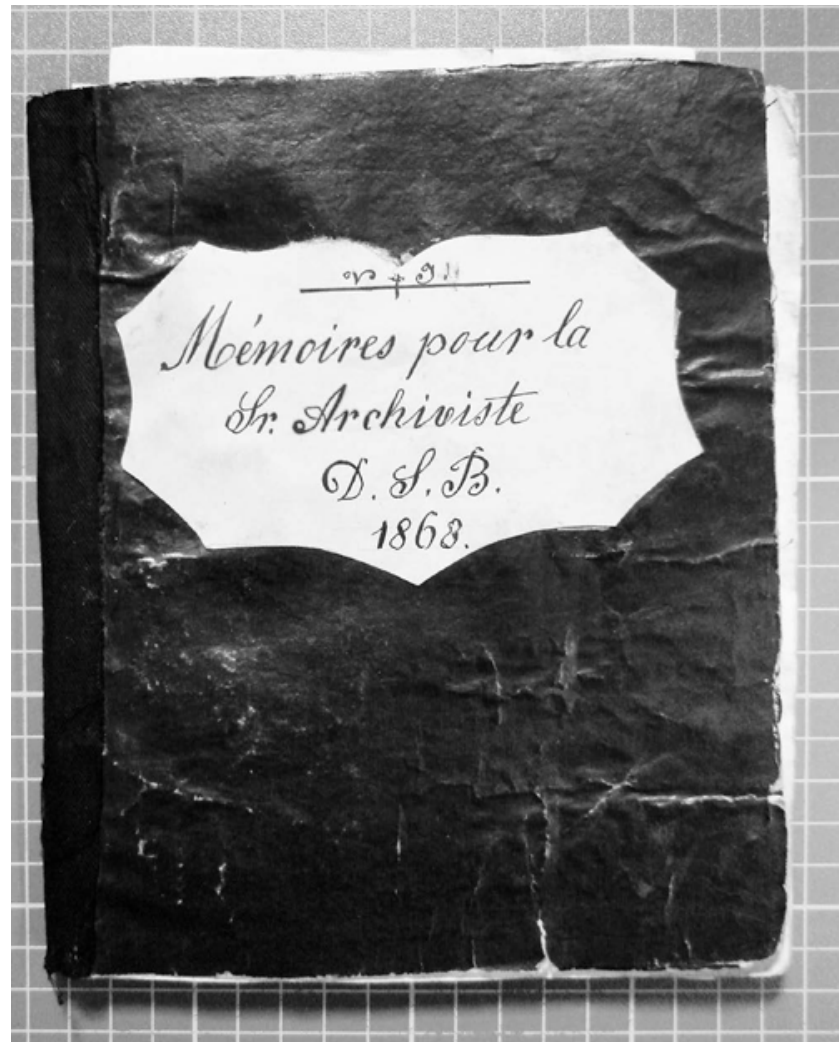

Abb. 3: „Denkwürdigkeiten“ für die Schwester Archivarin im Kloster der Salesianerinnen Wien (Helga Penz; Archiv der Salesianerinnen Wien, Lade A-XXI).

Ein Inventaire des Titres et Papiers befindet sich auch im Archiv der Salesianerinnen in Wien, es spiegelt eine Verschriftlichungsstrategie und Ablageordnung wider, die den Standards seiner Zeit entspricht. Die Bücher, die die einzelnen Klöster des Ordens zu führen hatten, sind darin ebenso aufgelistet wie, in jeweils eigenen Serien, die Urkunden, die Reliquienauthentiken, bischöfliche Genehmigungen, fürstliche Privilegien, Stiftungsbriefe, Verträge und Obligationen. Einige Bücher, die 1717 angelegt wurden, werden bis heute geführt, so das Konventbuch, das Kapitelbuch, das Noviziatsbuch und das Totenbuch.

Die hohe Kontinuität in den Aufzeichnungen machte die Archivarin zu einer Schaltstelle der Schriftlichkeit im Kloster. Die Geschäftstagebücher, die sie führte, zeigen ihre enge Vernetzung mit den Aufgaben der Mitschwestern, denen sie die Amtsbücher zum Eintrag herausgab und danach wieder empfing und bewahrte. Sie führte auch die Klosterannalen, sodass das Archiv zu einem Scharnier zwischen Verwaltungshandeln und Memorialkultur wurde. Ein Teil des Archivs blieb stets aktuell, in anderen Teilen jedoch bekamen die Unterlagen allmählich nur mehr historischen Wert. Nach dem Ersten Weltkrieg und dem Ende der Habsburgermonarchie verlor das Kloster sein Stiftungskapital und musste sowohl Apostolat wie Ökonomie neu organisieren. Die Einträge in die 1717 angelegten Archivinventare und Urkundenkopialbücher enden abrupt mit dem Jahr 1919. Was bereits im Archivraum untergebracht war, blieb zwar erhalten, doch dürfte es einiges vom Schreibtisch der Oberin nicht bis dorthin geschafft haben. 
Was archivwürdig ist, hat wesentlich damit zu tun, was wichtig für das Kloster ist. Die Aufbewahrung im Archiv soll Dinge dem Vergessen entreißen: An manche Dinge muss man sich erinnern, weil Nachweise über ihre Erledigung gefordert werden könnten, an anderes will man sich erinnern, weil es Bedeutung im Klosterleben hat. Im Archiv der Wiener Salesianerinnen mit seinen historischen Archivschränken findet man neben Laden mit der Bezeichnung „Steuern und Abgaben“, „Dokumente der Schwestern“ und „Dekrete des Ordinariats“ auch „Dokumente, die sich auf die Annalen beziehen“. Dies sind Dokumentations- und Sammlungsbestände mit Bildern, Zeitungsausschnitten und Kleindrucken, die an bedeutende kirchliche Ereignisse und Personen erinnern. Besonders viel Raum nimmt im Archiv auch die Memoria an die Stifterin, Kaiserinwitwe Wilhelmina Amalia, ein. Für das ungeübte Auge führt dieses Verständnis von Archivwürdigkeit in seinen unterschiedlichen Aspekten von Bedeutsamkeit zu Merkwürdigkeiten: zu einer verwirrend kontrastreichen Überlieferungsvielfalt, die gleichzeitig besonders in geistlichen Frauengemeinschaften schmerzhaft große Fehlstellen aufweist. Dass es im Archiv der Salesianerinnen mehr Fotos von Päpsten als von den Schwestern gibt, erhellt Bedeutsamkeit und Erinnerungswert in der Selbsteinschätzung. Hier hat man auch nicht die Vorstellung, die eigene Geschichte einer außerklösterlichen, geschichtsforschenden Nachwelt zu überliefern. Das Kloster selbst ist schon wie sein Archiv nicht auf Vorläufigkeit, sondern auf Dauer angelegt.

Kloster- und Kongregationsgründungen des 19. Jahrhunderts kennen die frühneuzeitlichen exakten und detaillierten Regelungen für Schriftgutverwaltung und Archivierung nicht mehr. Was an Unterlagen anfiel, verblieb in der Ablage der Oberin; Älteres, das man nicht mehr täglich zur Hand haben musste, wurde an einer Verwahrstelle im Klostergebäude deponiert. Archive im engeren Sinn entstanden oft erst im 20. Jahrhundert. Wechselhafte Geschicke einer Gemeinschaft in den Zeitläuften sind an den Archiven deutlich abzulesen, so etwa beim Benediktinerinnenkloster St. Gabriel. Dieses wurde 1888 in Prag gegründet, doch mussten die Ordensfrauen 1919 ins Exil nach Österreich, wo das neue Kloster 1942 von den Nationalsozialisten beschlagnahmt wurde. Die Archivverluste hatte man nach 1945 durch Aufzeichnungen von Erinnerungen und Sammlungen ergänzt: Aus Chroniken anderer Klöster wurden Abschriften angefertigt, ein kleiner Bestand „Prag zur Zeit der Gründung unseres Klosters“ wurde angelegt ${ }^{18}$. Das Archiv soll Auskunft über die Geschichte geben, Quelle und Geschichtsschreibung fallen in eins, eine scharfe Trennung zwischen prozessgeneriertem Archivgut, selbst erzeugten Dokumentationen oder Anreicherungen mit Erwerbungen aus fremden Provenienzen wurde weder angestrebt noch getroffen. Archivwürdigkeit war ein inhaltlich definiertes Kriterium.

Wer nach sinnstiftenden Narrativen in Kloster- und Ordensarchiven fragt, muss vor Augen haben, dass das Leben der Religiosen im Erwartungshorizont der Ewigkeit steht. Der Tod war darum im Kloster schon immer ein erinnerungswürdiges Ereignis. Die Schwestern von St. Gabriel haben mit „Sterbechroniken“ eine besondere Form der Totenparte hervorgebracht: Kein persönlicher und intimer Aspekt des Lebens der einzel-

18 Steiermärkisches Landesarchiv [StLA], Archiv der Abtei St. Gabriel, Karton 1. Für die Hilfestellung bei der Benützung des Bestands danke ich dem zuständigen Landesarchivar Peter Wiesflecker. Zur Archivgeschichte vgl. Peter Wiesflecker, „... ut acta et documenta archivorum [...] diligenter serventur ... “ Über Möglichkeiten der Ordnung und Verwahrung von Archiven geistlicher Gemeinschaften durch staatliche Archive. Analecta Cisterciensia 60 (2010) 275-296. 
nen Ordensfrau wurde in die Klosterchronik und den Gedächtnisort Archiv so detailliert eingeschrieben wie ihr Eintritt in die Ewigkeit ${ }^{19}$.

Aufzeichnungen aus dem Klosteralltag, besonders diesbezügliche Unterlagen von Novizen- und Küchenmeisterinnen, Ökonominnen, Sakristaninnen und auch Oberinnen sind in vielen Ordensarchiven rar. Auch im Archiv der Benediktinerinnen von St. Gabriel haben Aufzeichnungen über die Art des Ordenskleides, wie es bei Einkleidung und Profess vorzubereiten ist, oder Einteilungen für Ämter und Dienste, also Schriftstücke mit Ablaufdatum, nur aus der letzten Zeit des Klosters vor Übergabe seines Archivs an ein Landesarchiv den Status der Archivwürdigkeit erreicht. Die gut dokumentierte Begleitung des zuständigen Landesarchivars hat dabei keine unbeträchtliche Rolle gespielt.

Der 1882 gegründete Konvent der Dominikanerinnen in der Steiermark musste 2012 aufgegeben werden, die letzten im Alter schon recht fortgeschrittenen Schwestern übersiedelten in eine Pflegeeinrichtung und übergaben ihr Archiv dem Diözesanarchiv ${ }^{20}$. Nach 130 Jahren sind vier Bananenschachteln geblieben: Die Hälfte davon enthält die Kataloge der Schule, die die Ordensfrauen geführt haben, einen weiteren Teil machten die Baupläne und diesbezügliche Akten und Rechnungen aus, ein Bruderschaftsbuch, das Zeremonienbuch für die Professen und die Weiheurkunde für die Klosterkapelle bilden schon die herausragenden Einzelstücke des Rests. Schmale Überlieferung in Frauenordensarchiven ist zwar keine Überraschung, dass aus diesem Archiv aber mehr über die Schweine- und Hühnerställe als über die Schwestern zu erfahren war, machte doch stutzig. Im Diözesanarchiv selbst ist kein Übergabeprotokoll vorhanden, Rückschlüsse auf Bewertung und Skartierung daher nicht möglich. Eine Anfrage bei den noch lebenden Schwestern ergab, dass sie „Klosterchronik, Dokumente und Verträge“ mit ins Heim genommen haben.

Archivsplitter wie diese einzusammeln ist gerade bei den Frauenkongregationen eine Herausforderung, denn viele dieser Gemeinschaften, die im 19. Jahrhundert als Laiengesellschaften mit sozialem Engagement besonders in Armenfürsorge, Erziehung und Krankenpflege gegründet wurden, werden in den nächsten Jahren und Jahrzehnten erlöschen und die Anzahl der Ordensleute in Österreich wird sich wieder bei den Zahlen des 18. Jahrhunderts einpendeln.

\section{Fazit}

Archivische Überlieferungsbildung hat stets das Ziel, Vergangenes für Zukünftiges zu sichern und einer Nachwelt zur Verfügung zu stellen. In Klöstern und Ordensgemeinschaften hat man dabei wesentlich die eigenen Nachkommen im Auge gehabt. Eine Analyse historischer Bewertungsvorgänge und ihrer handelnden Personen legt verschüttete Muster von Bestandszusammensetzungen frei und bettet die archivalische Quelle in den Kontext ihrer Überlieferungsgeschichte. Bei der hier zu erörternden „Informationsgesellschaft" im Allgemeinen und in Fragen der Überlieferungsbildung im Besonderen ist mir die Transparenz dieser Kontextualität ein besonderes Anliegen. Fraglich erscheint allerdings trotz Archival Turn, wie sehr dies das Interesse der Historikerinnen und Historiker

19 StLA, Archiv der Abtei St. Gabriel, Karton 17.

${ }^{20}$ Ich danke dem Direktor des Diözesanarchivs Graz-Seckau, Matthias Perstling, für den Zugang zu diesem noch unerschlossenen Bestand. 
erregen wird, ist man doch als Archivarin sehr viel häufiger mit Anfragen nach Digitalisaten als mit solchen nach Bestandsgeschichten konfrontiert. Um der Nachwelt die Quellen für kritisches Nachfragen an die Hand zu geben, braucht es gerade bei der Sicherung der Überlieferung der vielen kleineren Archive nicht-behördlicher Institutionen, Einrichtungen, Initiativen und Unternehmen diese Transparenz. Die Geschichte und Analyse dieser Archive kann aber, wie hoffentlich gezeigt werden konnte, aktuelle Diskussionen über Bewertungsfragen sehr bereichern. 



\title{
Alles doch ganz einfach? Archivische Bewertung zwischen Wissenschaft und Bauchgefühl
}

\author{
Matthias Buchholz
}

Gern wird die archivische Bewertung als Königsdisziplin im Aufgabenkanon der Archivarinnen und Archivare bezeichnet ${ }^{1}$. In dieser Benennung schwingen Stolz und eine gewisse Ehrfurcht mit. Beides ist berechtigt. Schließlich geht es um mehr als zum Beispiel bei Aschenputtel, die Linsen aus der Asche aussortieren soll. So bedauernswert Aschenputtel ist, so hat sie doch eine beneidenswert klare Aufgabe, deren Erledigungsgrad sich vergleichsweise leicht auch ohne Expertenkommissionen und Arbeitskreise überprüfen lässt. Aber wann haben wir Archivare die Überlieferungsbildung gut oder wunschgemäß bewältigt? Ein Blick auf einige der Einflussgrößen kann zumindest etwas Licht ins Dunkel bringen.

\section{Gesetze}

Vielleicht hilft ja auch ein Blick ins Gesetz? Das österreichische Denkmalschutzgesetz definiert den Begriff der Archivalien in $\$ 25$ wie folgt: „(1) Archivalien sind Schriftgut sowie zu dokumentarischen Zwecken oder zur Information der Öffentlichkeit hergestelltes Bild-, Film-, Video- und Tonmaterial, das von geschichtlicher oder kultureller Bedeutung für die Erforschung und das Verständnis der Geschichte und Gegenwart in politischer, wirtschaftlicher, sozialer oder kultureller Hinsicht sowie bezüglich Gesetzgebung, Rechtsprechung, Verwaltung und den Schutz allgemeiner oder besonderer bürgerlicher Rechte ist. Kommt derartigen Gegenständen geschichtlich gewordenen Charakters jedoch Bedeutung dieser Art nicht zu, dann sind sie nicht Archivalien im Sinne dieses Abschnittes, und zwar auch dann nicht, wenn Sammlungen dieser Art, wie Sammlungen von musikalischen Handschriften, literarischen Schriftstücken, Ansichts- und Porträtsammlungen und dergleichen, als Archive bezeichnet werden. (2) Schriftgut sind schriftlich geführte oder auf elektronischen Informationsträgern gespeicherte Aufzeichnungen aller Art wie Schreiben und Urkunden samt den damit in Zusammenhang stehenden Karten, Plänen, Zeichnungen, Siegel, Stempel mit deren Anlagen einschließlich der Programme, Karteien, Ordnungen und Verfahren, um das Schriftgut auswerten zu können“2.

\footnotetext{
1 Es wurde der Vortragsstil beibehalten.

2 Bundesgesetz betreffend den Schutz von Denkmalen wegen ihrer geschichtlichen, künstlerischen oder sonstigen kulturellen Bedeutung (Denkmalschutzgesetz - DMSG), in: Rechtsinformationssystem RIS, https:// www.ris.bka.gv.at/GeltendeFassung.wxe?Abfrage=Bundesnormen\&Gesetzesnummer=10009184 [1. 3. 2018].
} 
Wir können uns einige Orientierungspunkte herauspicken: Geschichte, Kultur, Politik, Wirtschaft, Soziales, Gesetzgebung, Rechtsprechung, Verwaltung und den Schutz allgemeiner oder besonderer bürgerlicher Rechte. Für jeden ist etwas dabei! Das ist wenig hilfreich, liest es sich doch so, als müsse das Archiv in seiner Gedächtnisfunktion die eierlegende Wollmilchsau sein. Und in Deutschland sieht es nicht anders aus ${ }^{3}$ ! Es wird also nur ein sehr grober Rahmen vorgegeben, der von der Archivarszunft gefüllt werden muss.

\section{Berufsethos}

Vielleicht hilft uns ein Blick in den vom International Council on Archives verabschiedeten „Kodex ethischer Grundsätze für Archivarinnen und Archivare“ ${ }^{\text {“ }}$ bei der Beantwortung der Frage, was eine gute Überlieferungsbildung ausmacht? Die klare Antwort lautet: Ja und nein! Gleich in Punkt 1 heißt es: „Archivarinnen und Archivare haben die Integrität von Archivgut zu schützen und auf diese Weise zu gewährleisten, dass es ein zuverlässiger Beweis der Vergangenheit bleibt" . Der Bezug lautet also „Archivgut“ und nicht „Schriftgut“. Damit stellt sich für die Verfasser des Kodex die Frage gar nicht, ob nicht bereits jede Art von Bewertung als Eingriff in die Integrität der Überlieferung betrachtet werden muss. Je nach Naturell können wir jetzt also beruhigt oder beunruhigt sein.

Punkt 2 wird schon ein wenig deutlicher: „Archivarinnen und Archivare haben Archivmaterial in seinem historischen, rechtlichen und administrativen Kontext zu bewerten, auszuwählen und aufzubewahren, um so das Provenienzprinzip zu bewahren und die ursprünglichen Zusammenhänge der Schriftstücke zu erhalten und zu verdeutlichen “6. Das Ziel ist also neben der Bewahrung des Provenienzprinzips die Erhaltung der ursprünglichen Zusammenhänge eingedenk des historischen, rechtlichen und administrativen Kontexts. Damit werden als anerkannte Adressaten Interessenten aus den Bereichen Geschichtswissenschaft, Justiz und Verwaltung namentlich genannt. Das schließt weitere Nutzerkreise nicht aus, gewichtet sie aber geringer.

Punkt 3 lautet: „Archivarinnen und Archivare haben die Authentizität der Schriftstücke während der Bearbeitung, Aufbewahrung und Benutzung zu schützen"7. Der vierte und der fünfte Punkt sind dann ein wenig künstlich voneinander getrennt. In Punkt 4 heißt es: „Archivarinnen und Archivare haben die fortwährende Benutzbarkeit und Verständlichkeit des Archivguts sicherzustellen“8. Punkt 5 schließt wie folgt an: „Archivarinnen und Archivare haben Aufzeichnungen über ihre Bearbeitung von Archivgut zu führen und müssen in der Lage sein, diese zu begründen “9. Gerade in Bezug auf Bewertungsentscheidungen ist die Komposition von Archivgut nicht vollumfänglich zu verstehen, wenn kein Nachweis darüber besteht, was vernichtet wurde, und möglichst auch die

\footnotetext{
${ }^{3}$ Gesetz über die Nutzung und Sicherung von Archivgut des Bundes (Bundesarchivgesetz - BArchG) vom 10. März 2017, in: Das Bundesarchiv, https://www.bundesarchiv.de/DE/Navigation/Meta/Ueber-uns/ Rechtsgrundlagen/Bundesarchivgesetz/bundesarchivgesetz.html [1. 3. 2018].

${ }^{4}$ ICA Code of Ethics (German), in: ICA. International Council on Archives, https://www.ica.org/sites/ default/files/ICA_1996-09-06_code\%20of\%20ethics_DE.pdf [1.3. 2018].

5 Ebd.

6 Ebd.

7 Ebd.

8 Ebd.

9 Ebd.
} 
Begründung der Entscheidung. Insofern greift die Vorgabe zu kurz, der Archivar müsse in der Lage sein, die Entscheidung zu begründen. Das ist der feine, aber im Ernstfall entscheidende Unterschied zwischen „begründbar" und „begründet“.

Ebenfalls von Relevanz für das Thema der Überlieferungsbildung sind dann noch Punkt 8 und 9: „8. Archivarinnen und Archivare haben das spezielle Vertrauen, das ihnen entgegengebracht wird, im Interesse der Allgemeinheit zu gebrauchen und dürfen ihre Stellung nicht zum ungerechten Vorteil für sich oder andere nutzen. - 9. Archivarinnen und Archivare haben stets die Entwicklung ihres beruflichen Könnens durch systematische und ständige Fort- und Weiterbildung ihrer Berufskenntnisse zu verfolgen und die Ergebnisse ihrer Forschungen und Erfahrungen mit anderen zu teilen" ${ }^{10}$. Es ist dort zwar nicht expressis verbis zu lesen, ließe sich aber wie folgt interpretieren: Der Blick über den Tellerrand kann von Nutzen sein!

\section{Wert}

Der Archivar ist im Prozess der Unterscheidung von „archivwürdig“ und „kassabel“ mehr als bloßer Sachwalter kultureller Identität einer Gesellschaft. Durch den Akt der Überlieferungsbildung, also die Transformation von Überresten in Tradition unter Vernichtung nicht archivwürdiger Unterlagen, trägt er wesentlich zur Schaffung kultureller Identität bei. In diesem Punkt befinde ich mich in einem kleinen Deutungs- beziehungsweise Perspektivendissens zu Robert Kretzschmar, der argumentiert, dass durch den Prozess der Bewertung der Charakter der einzelnen Unterlagen als Überreste nicht entscheidend angetastet wird ${ }^{11}$. Das mag mit Blick auf das einzelne Dokument zutreffen. Aber zum einen arrangiert der Archivar schließlich eine neue Komposition der Überlieferung mit Blick unter anderem darauf, was für die Nachwelt von Interesse ist, und zum anderen scheidet er dafür gezielt Dokumente aus. Was ist dies anderes als die Schaffung von Tradition? Das ist meines Erachtens mehr als eine definitorische Petitesse, hat es doch Auswirkungen auf das professionelle Selbstverständnis der Zunft. Provokant formuliert: Der Gedanke des Erhalts des Überrestcharakters trotz des archivarischen Eingriffs nimmt dem Archivar die Verantwortung oder reduziert sie zumindest stark. Das aber ist kontrafaktisch!

Aus dem Prozess der Bewertung speist sich die Bedeutung, vielleicht sogar die Macht der Arbeit der Archivare. Das Archivgut ist Ergebnis eines zielgerichteten und begründeten (nicht nur begründbaren) Ausleseprozesses. Zunächst ist jede Bewertungsentscheidung richtig und falsch zugleich! Eine Wertsetzung ist nur möglich bei Benennung des Bezugspunktes. Nur dann kann über „richtig“ und „falsch“ diskutiert werden. So banal, wie zunächst anzunehmen wäre, scheint diese Feststellung nicht zu sein. Erst kürzlich erschien im Kontext der Archivpflege und Archivberatung wieder ein Beitrag mit Bewertungsempfehlungen, der völlig auf die Angabe der Basis für diese Empfehlungen verzichtete $^{12}$. Damit ist er im doppelten Sinne nicht diskutabel! Wenn archivische Bewertung

10 Ebd.

11 Vgl. Robert Kretzschmar, Absichtlich erhaltene Überreste. Überlegungen zur quellenkundlichen Analyse von Archivgut. Archivar. Zeitschrift für Archivwesen 67 (2014) 265-269.

12 Bewertungsempfehlungen der Arbeitsgruppe „Archivische Bewertung“ der Arbeitsgemeinschaft bayerischer Kommunalarchive, in: Kommunalarchive in Bayern, http://kommunalarchive-bayern.de/3358.html [1.3. 2018]. 
tatsächlich so simplifiziert werden kann, stellt sich die Frage, wozu es einer Ausbildung bedarf und was das ganze Gerede von der „Königsdisziplin Bewertung" soll. Natürlich „hilft“ (!) man auf diese Weise den nicht fachlich adäquat besetzten Archiven. Aber um welchen Preis? Auf diese Weise wird die mangelhafte Besetzung der kleinen Archive perpetuiert. Dies ist gleichbedeutend mit der stillschweigenden Akzeptanz der Absenkung (anzustrebender) archivischer Standards!

Es existiert bei der archivischen Bewertung zunächst kein „falsch“ und kein „richtig“. Erst durch die Benennung (und Begründung) des wertsetzenden Bezugspunktes wird die Folgerichtigkeit einer Bewertungsentscheidung diskutierbar und überprüfbar. Die eine archivische Wahrheit existiert nicht! Dies ist der Dreh- und Angelpunkt der Bewertungsdiskussion.

Werden wir von einem Touristen auf der Straße gefragt „Wohin muss ich gehen? Welchen Bus soll ich nehmen?", dann ist unsere Antwort die Frage „Wohin möchten Sie denn?“. Genau so banal verhält es sich mit der Bewertung. Um zu wissen, welche Instrumente und Hilfsmittel auszuwählen sind, muss ich das Ziel kennen. Insofern ist alles tatsächlich ganz einfach. Aber halt, der Teufel steckt im Detail! Die Benennung eines Ziels ist mitunter schwierig. Durch die Auswahl eines Überlieferungsziels werden andere vernachlässigt oder im „schlimmsten“ Fall potentiell ausgeschlossen. Das fällt nicht immer leicht, kann aber nicht umgangen werden, da die Alternative die Vollarchivierung wäre.

An diesem Punkt wird aber auch deutlich, warum die Bewertung - aus meiner Sicht völlig zu Recht - als Königsdisziplin bezeichnet wird. Sie fordert zweifellos die Kreativität der Archivare, denn es handelt sich eben nicht um das bloße Abarbeiten eines immer gleichen Bewertungskatalogs, der nach „Archivieren“, „Kassieren“ und „Überprüfen, eventuell in Auswahl“" unterscheidet. Die Archivare haben einen großen Ermessensspielraum, den es verantwortungsvoll zu nutzen gilt. Insofern ist es wichtig, sich offen und diskursiv auszutauschen. Das sind wir dem wichtigen Feld der Überlieferungsbildung schuldig!

Auf dieser Basis lässt sich auch sagen, was gute Überlieferungsbildung ausmacht. Archivische Bewertung ist keine exakte, keine objektive Wissenschaft! Ob eine Bewertungsentscheidung richtig oder falsch ist, lässt sich nur dann feststellen, wenn der wertgebende Bezugspunkt, kurz: wenn das (begründete) Ziel der Überlieferungsbildung bekannt ist. So lässt sich die Entscheidung auf dem Wege der Begründungsobjektivität evaluieren. Deshalb ist diese Erörterung des Ziels der Überlieferungsbildung die Mindestanforderung an eine Bewertungsentscheidung oder ein Bewertungsmodell. Diese Ziele werden einem zeitbedingten Wandel unterliegen, ohne dass damit vergangene Entscheidungen nachträglich korrekturbedürftig würden. Denn die neue Entscheidung ist - sollte sie begründet sein wie die vorangegangene auch - keineswegs „besser“. Und welches verquere Geschichtsverständnis stünde hinter einer permanenten Überformung der bereits bewerteten Bestände?

Eine ähnliche Frage stellt sich auf einem weiteren Gebiet. Ist als Bewertungsziel die quantitative Reduzierung der Überlieferung auf einen bestimmten Prozentsatz (wie im staatlichen Archivwesen Nordrhein-Westfalens) benannt, ohne dies zuvor inhaltlich zu unterfüttern, so ist dies aus fachlichen und berufsethischen Gründen abzulehnen. Auch die vorhandene Magazinkapazität darf nicht als Kriterium gelten. Ansonsten gäbe man den Prozess der Bewertung der Beliebigkeit preis!

Wie kann nun der Wert einer Akte, eines Schriftstückes bestimmt werden? Gibt es für Archive einen dauernden, einen ewigen Wert oder ist von der Relativität der Werte auszugehen? Ein Kontinuum in der Geschichte der Menschheit ist sicherlich das Erkennt- 
nisinteresse des Menschen, die Suche nach Wahrheit. Doch ist die Übertragbarkeit auf den archivischen Bewertungsalltag aufgrund der inhaltlichen Wandelbarkeit eingeschränkt. Eine Vielzahl unterschiedlichster Wissenschaften hat sich in den letzten Jahrhunderten entwickelt. Noch vor 150 Jahren war an Frauen- und Totalitarismusforschung, Sozialwissenschaft und Kernphysik, Informations- und Dokumentationswissenschaft et cetera noch nicht zu denken. Erkenntnisorientierte Werte haben sich im Laufe der Jahre mit verschoben. Ist der Archivar damit gleichsam ein zweifacher Sisyphos, ächzend angesichts der Aktenmassen und zugleich chancenlos bei der archivischen Bewältigung von Informationen?

In den Informationswissenschaften wird die Information charakterisiert als „die schrittweise und wiederholte Beseitigung von Ungewißheit in Problembehandlungs- und -bewältigungsprozessen, in denen die Zuführung externen Wissens (d. h. beim Problemlöser nicht verfügbaren Wissens) notwendig ist" ${ }^{13}$. Daten werden also dann zu Informationen, das heißt sie erlangen dann eine neue Qualität, wenn sie Ungewissheit beseitigen. Insofern besitzen sie einen eindeutigen Wert. Um allerdings Ungewissheit zu beseitigen, muss man wissen, worin diese besteht.

Die Archivare sind selbstverständlich ebenso äußeren Einflüssen, ja generell den Zeitläuften unterworfen wie jeder andere Zeitgenosse auch. Insofern stellt sich natürlich die Frage nach der Kontrolle dieser Macht. Hier liegt einiges im Argen! Zugespitzt formuliert: Gerade im Bereich der Überlieferungsbildung lässt sich die Zunft ungern in die Karten schauen und wird dabei vom Desinteresse ihrer Kunden sekundiert.

Ketzerisch gefragt: Wenn die Archivistik oder Archivwissenschaft nicht einmal einer Kontrolle für nötig und würdig befunden wird, ist ihr gesellschaftlicher Wert dann gering? Wie verträgt sich das mit der Konjunktur des Archivbegriffes in der Kulturtheorie? Es ist eine Frage des Berufsethos, wie wir auf zahlenmäßig geringes Interesse reagieren. Indem wir als Archivare passiv bleiben, bestätigen wir den ungünstigen Befund! Wir benötigen mehr (Selbst-)Kontrolle durch fachlichen Diskurs, der auch Kontroversen nicht scheut, zum Beispiel Diskussion über Dokumentationsprofile und Bewertungsmodelle. Neben der Bereitschaft zur Diskussion muss ein Debattenstil kultiviert werden, dessen Leitlinie lauten könnte: „Nicht Sieg sollte der Sinn der Diskussion sein, sondern Gewinn“ (Joseph Joubert).

\section{Konzept}

„Hut ab“ also vor allen Archivaren, die noch mit Freude „Ja“ zur Bewertung sagen! Immerhin sehen wir uns ja nicht nur dem einen Historiker gegenüber, dessen Forschungsinteressen wir kennen. Theoretisch könnte über alles geforscht werden. Und da ist noch gar nicht die Rede von den Genealogen, die sich bereits freuen, wenn sie herausfinden können, dass ihr Urahn am 23. März 1915 in Schnepfau (Vorarlberg) einen Sack Kartoffeln gekauft hat. Das möchte ich gar nicht verspotten, sondern sogar dazu aufrufen, die Berechtigung dieses individuellen Anliegens zumindest zu erwägen.

Das Kölner Stadtarchiv, durch den Einsturz vom 3. März 2009 tragisch zu einem Neubeginn gezwungen, hat sich diese Frage im Grundsatz beantwortet: „Als Bürgerarchiv

13 Hans-Jürgen Manecke-Thomas SeEger, Zur Entwicklung der Information und Dokumentation in Deutschland, in: Grundlagen der praktischen Information und Dokumentation. Ein Handbuch zur Einführung in die fachliche Informationsarbeit, hg. von Marianne Buder-Werner ReHFeld-Thomas SEeger-Dietmar Strauch (München u. a. ${ }^{4} 1997$ ) 16-60, hier 20. 
hat das Historische Archiv [...] die Interessen aller Bürgerinnen und Bürger zu berücksichtigen. Das bedeutet einerseits, dass Überlieferungsbildung nicht allein im Hinblick auf bestimmte, z. B. wissenschaftliche, Fragestellungen erfolgt. Diese werden vielmehr gleichberechtigt mit nicht-wissenschaftlichen Benutzungsanliegen sowohl der Familien- und Ortsgeschichte, als auch der Rechtssicherung und anderer Anliegen betrachtet. Andererseits heißt dies nicht, dass jede greifbare Quelle zu jedem Einwohner von Köln archiviert wird, um ein eventuelles Interesse befriedigen zu können. Die Überlieferungsbildung eines Bürgerarchivs ist vielmehr auch dem Wirtschaftlichkeitsgrundsatz verpflichtet, der eine schrankenlose Archivierung für beliebige Zwecke genauso verbietet wie der Datenschutzaspekt dem entgegensteht. Das Bürgerarchiv handelt daher im Interesse der Bürgerinnen und Bürger, wenn eben nicht alles dauerhaft aufbewahrt wird, sondern nur solche Träger der Überlieferung, deren Aggregationsgrad und Informationswert dies rechtfertigen. Dass sich die Identifizierung dieser Bereiche in stetiger Diskussion mit der archivischen Fachwelt, Vertretern der Wissenschaft und keineswegs zuletzt den Bürgerinnen und Bürgern zu vollziehen hat, ist dabei selbstverständlich" ${ }^{14}$.

Dabei folgt man aber auch keinen Forschungsmoden: „Der Überlieferungs-Auftrag eines Archivs kann daher nicht auf - im Zweifel schnell wechselnde - Methoden der Geschichtswissenschaft oder anderer historisch arbeitender Wissenschaften abgestellt werden. Neue Methoden der Geschichtswissenschaft finden dennoch ihre Quellen, denn ein Ziel des Dokumentationsprofils ist die Grundsicherung von Informationen zu den für die Stadtgesellschaft und mit ihr für die über Köln hinausweisende Allgemeinheit wichtigen Bereichen, soweit dazu aggregierte oder konzentrierte Quellen vorliegen. Mit diesen ist zwar nicht das letzte Detail zu erforschen, jedoch geht die Überlieferung statt dessen so in die Breite, dass Auswertungen unter den verschiedensten Gesichtspunkten möglich werden, ohne sich vorab auf einen bestimmten festzulegen" 15 .

Die Idee des Dokumentationsprofils wird aber auch kritisch gesehen. So schrieb Jürgen Treffeisen jüngst in Scrinium: „Die Bewertung findet auch bei diesem Verfahren weitestgehend bereits zum Zeitpunkt der Entstehung der Unterlagen statt. Es berücksichtigt ebenso Unterlagen außerhalb der eigenen Verwaltung und versucht eine Erfassung der lokalen Lebenswelt in systematischen Kategorien auf der Grundlage des Zeitgeschehens in der jeweiligen Kommune. Es beabsichtigt die Festlegung von Dokumentationszielen für die ermittelten Kategorien vor der Bewertung und führt eine Wertanalyse der bereits im Archiv vorhandenen Quellen bezogen auf die erarbeiteten Dokumentationsziele durch. Und es definiert einen Quellenfundus, der zum Erreichen der Dokumentationsziele archiviert werden muss. Dabei werden die gleichen Kriterien für die Bewertung von amtlicher und nichtamtlicher Überlieferung zugrunde gelegt. Die Kategorisierung der lokalen Lebenswelt führt zu einer Wertanalyse archivreifer Unterlagen unter qualitativen und quantitativen Gesichtspunkten. Eine weitergehende Darstellung und Analyse dieses Bewertungsverfahrens ist hier im vorgegebenen Rahmen nicht möglich. Ein kurzer Vergleich mit der vertikalen und horizontalen Bewertung sei aber kurz angedeutet: Bei der vertikalen und horizontalen Bewertung werden Aufgaben und die damit in Verbindung stehenden Unterlagen analysiert. Es wird analysiert, wo die aussagekräftigsten Unterlagen

\footnotetext{
14 Andrea Wendenburg-Max Plassmann, Fachkonzept für das Historische Archiv der Stadt Köln bis zum Jahr 2050. Mitteilungen aus dem Stadtarchiv von Köln 100 (2014) 63-114, hier 80f.

15 Max Plassmann, Dokumentationsprofil für das Historische Archiv der Stadt Köln. Mitteilungen aus dem Stadtarchiv von Köln 100 (2014) 115-169, hier 121.
} 
zu einer Aufgabe vorhanden sind, wo hingegen nur Doppelüberlieferung vorliegt. Dies erfolgt zunächst unabhängig von der historischen Bedeutung der einzelnen Aufgabe. Das Dokumentationsprofil geht den umgekehrten Weg. Es analysiert zuerst Inhalte. Damit wird nach meinem derzeitigen Kenntnisstand der Weg einer auswertungsoffenen Überlieferungsbildung verlassen" 16 .

Damit ist dann auch das problematische Wort von der Auswertungsoffenheit gefallen, bei dessen uneingeschränkter Verwendung man Gefahr läuft zu suggerieren, dass ein chirurgischer Eingriff berührungsfrei möglich ist.

\section{Austausch und Sensibilisierung}

Kann die Einbeziehung externen Expertenwissens von Nutzen sein? Postuliert wird das immer wieder, aber nur selten bis gar nicht umgesetzt. Was sind die möglichen Ursachen dafür? Zweifel am Nutzen? Zu wenig Zeit? Möchte man sich nicht in die Karten schauen lassen? Vermutlich ist es von allem etwas. Es besteht die Sorge, dass die wissenschaftlichen „Kunden“ für ihr jeweiliges Forschungsfeld fordern, alle Unterlagen aufzubewahren. Aber ist das ein hinreichender Grund dafür, den diskursiven Austausch von vornherein abzulehnen? Auch wenn Clemens Rehm sich 2001 skeptisch gegenüber Dokumentationsplänen zeigte, befürwortete er doch den Austausch mit Dritten. Konkret benannte er drei Gruppen: „1. Vorausgesetzt wird hier, dass bei der Erstellung von Archivierungsmodellen die betroffenen Verwaltungen einbezogen wurden. Dann bleiben noch zwei weitere große Gruppen: 2. Es wären die Verbände und Vereine zu berücksichtigen, die diejenigen vertreten, die an der Entstehung der Unterlagen beteiligt sind. In erster Linie sind hier Berufsverbände angesprochen. Es wäre sicher interessant gewesen bei den bundesweiten Bewertungsmodellen zu Unterlagen der Justiz die Anmerkungen der juristischen Verbände zu erfragen. 3. Es wären die Vereine und Verbände zu berücksichtigen, die diejenigen vertreten, die an der Auswertung von Archivgut interessiert sind: Historikerverbände und Geschichtsvereine. Es müsste eigentlich im ureigensten Interesse dieser Organisationen sein, in diesem Prozess mitwirken zu können. Wesentlich ist: Die Bewertungsentscheidung bleibt dem Archivar [...]. Das Archivwesen bleibt Herr des Verfahrens" ${ }^{17}$.

Es geht um Sensibilisierung durch die Abwägung der Meinungen und Methoden Dritter. Bei der Frage der Stichprobenziehungen und der Klärung des Begriffes „Repräsentativität" hat das beispielsweise ganz gut funktioniert ${ }^{18}$, ohne dass deshalb die Forderung nach flächendeckender Anwendung repräsentativer Verfahren erhoben wurde.

In Deutschland gab es 2006 einen konkreten Versuch, mit den Historikern in einen Austausch zu kommen. Auf dem Programm des 46. Deutschen Historikertags in

16 Jürgen Treffeisen, Zum aktuellen Stand der archivischen Bewertungsdiskussion in Deutschland - Entwicklungen, Trends und Perspektiven. Scrinium. Zeitschrift des Verbandes Österreichischer Archivarinnen und Archivare 70 (2016) 58-92, hier 74.

17 Clemens Renm, Kundenorientierung. Modewort oder Wesensmerkmal der Archive? Zu Transparenz und Partizipation bei der archivischen Überlieferungsbildung, in: Zwischen Anspruch und Wirklichkeit. Das Dienstleistungsunternehmen Archiv auf dem Prüfstand der Benutzerorientierung. Vorträge des 61. Südwestdeutschen Archivtags am 26. Mai 2001 in Schaffhausen, hg. von Hans ScHadex (Stuttgart 2002) 17-27, hier 26.

18 Vgl. Matthias Buchiolz, Archivische Überlieferungsbildung im Spiegel von Bewertungsdiskussion und Repräsentativität (Archivhefte 35, Köln 22011). 
Konstanz stand eine von Archivaren, namentlich dem Arbeitskreis Bewertung im Verband deutscher Archivarinnen und Archivare (VdA), konzipierte Sektion unter dem Titel „Geschichtsbilder der Archive / Geschichtsbilder der Wissenschaft: Dokumente und Deutungen zur Anti-Atomkraft-Bewegung der 1970er Jahre“. Die Beiträge wurden danach publiziert ${ }^{19}$. Eine Diskussion mit den Historikern kam dennoch weder während des Historikertags noch danach in Gange. Ist dies aber bereits ein Beleg für das Desinteresse unserer potentiellen Gesprächspartner? Vielleicht war das gewählte Setting untauglich und man sollte über Bewertungskonzepte anhand konkreter Überlieferungen sprechen? Oder es bedarf einer anderen Abstraktionsebene, eventuell der des Dokumentationsprofils? Vielleicht böten auch die Positionspapiere des Arbeitskreises Bewertung im VdA eine geeignete Diskussionsgrundlage? Oder muss auf der „Gegenseite“ das Interesse überhaupt erst geweckt werden?

Jedenfalls greift auf unserer Archivarsseite das Zeitargument zu kurz! Wenn die Überlieferungsbildung tatsächlich die Königsdisziplin ist, muss ihr ein entsprechendes Zeitkontingent eingeräumt werden. Salopp gesagt: Einem Hirnchirurgen wird man tendenziell mehr Zeit für eine Operation einräumen als einem Orthopäden, obwohl dessen physisches Betätigungsfeld größer ist! Eine 1996 unter rheinischen Kommunalarchiven durchgeführte Umfrage lässt vermuten, dass etwa sieben bis zehn Prozent der Arbeitszeit für Bewertungsbelange aufgewandt werden ${ }^{20}$. Ob das viel oder wenig ist, wird man trefflich diskutieren können. Aber nach „Königsdisziplin“ sieht es nun wirklich nicht aus!

\section{Transparenz}

Da die Bewertung nicht objektiv sein kann, aber immerhin Begründungsobjektivität hergestellt werden kann, ist Transparenz das A und O der professionellen Überlieferungsbildung. Nur durch sie werden Diskussion und fachliche Qualitätskontrolle überhaupt erst möglich.

Clemens Rehm formulierte 2001 noch offensiver: „Die Forderung nach Transparenz der Überlieferungsbildung und die Publikation von Modellen allein reicht meines Erachtens nicht aus. Was geschieht, wenn diese Transparenz von archivischer Seite nicht hergestellt wird, aus welchen Gründen auch immer? Wird dann die Institution zur Rechenschaft gezogen? Es ist darauf hingewiesen worden, dass nie ein Rechnungshof diesen Bereich der archivischen Arbeit durchleuchtete. Meine Frage lautet eher: Warum soll eine archivarische Entscheidung, mit weitreichenden Folgen wie die Bewertung nicht auch verwaltungsgerichtlich überprüft werden können? Selbst wenn eine Kassation nicht rückgängig gemacht werden kann, zum Verfahren der Bewertung könnten sich bei bzw. nach einem solchen Verfahren neue Grundlagen der Bewertung entwickeln“21.

Transparenz macht den Austausch mit Dritten überhaupt erst möglich. Insofern sind etwa die Positionspapiere des Arbeitskreises Bewertung im VdA (Überlieferungsbildung

191968 und die Anti-Atomkraft-Bewegung der 1970er Jahre. Überlieferungsbildung und Forschung im Dialog, hg. von Robert Kretzschmar-Clemens Rehm-Andreas Pilger (Werkhefte der Staatlichen Archivverwaltung Baden-Württemberg - Serie A 21, Stuttgart 2008).

20 Matthias Buchiolz-Angelika Raschke-Peter Weber, Vom ungeliebten und schwierigen Geschäft der archivischen Bewertung. Eine Bestandsaufnahme zur Bewertungspraxis in rheinischen Kommunalarchiven, in: Buchнolz, Überlieferungsbildung (wie Anm. 18) 320-355, hier $331 \mathrm{f}$.

${ }^{21}$ Rehm, Kundenorientierung (wie Anm. 17) $23 \mathrm{f}$. 
im Verbund; Maßstäbe für Überlieferungsqualität) ${ }^{22}$ ein Anfang, wenn es um die Benennung von Standards geht. Doch so richtig und wichtig etwa die Idee der Überlieferungsbildung im Verbund ist, so sehr bedarf sie doch der effizienten Operationalisierung. Dokumentationsprofile können hier ein wirkungsvolles Mittel der schnellen Verständigung sein. Sind diese Dokumentationsprofile mit einzelnen Bewertungsmodellen unterfüttert, ist ein schneller Austausch möglich. Ohne diese Grundlagen verbleibt die Überlieferung im Verbund auf der Ebene mehr oder weniger zufällig getroffener partikularer Absprachen.

Aber vielleicht wird uns auch von außen schon ein Teil unserer Bewertungsentscheidungen abgenommen? So wurden bislang die Auswirkungen der Informationsfreiheitsgesetze auf die Arbeit des Archivars nicht ausreichend diskutiert. Besteht eine Analogie zwischen der Nutzung eines zum Zeitpunkt der Nutzung nicht erschlossenen Bestands durch Dritte und der Nutzung von Unterlagen durch Dritte in einer Behörde im Rahmen des Informationsfreiheitsgesetzes? Wenn ja, stellt sich die Frage der Notwendigkeit der Aufbewahrung alles Eingesehenen. Oder unterscheiden wir hier zwischen wissenschaftlicher und privater Nutzung und legen damit fest, dass die wissenschaftliche Nutzung eine höhere Berechtigung aufweist?

Ganz unzweifelhaft bestimmt der Archivar bereits mit seiner Auswahl in den Bereichen Sicherung, Bewertung und Erschließung, welche Bestände überhaupt und zu welchem Zeitpunkt sie genutzt werden können. Insofern hat der Archivar konkrete Macht. Diese ist jedoch in Bezug auf den Inhalt des Archivguts naturgemäß retrospektiv. Sie ist darauf beschränkt, dem konkreten Geschehen hinterherzuhinken, also (eigentlich) zu spät zu kommen oder - freundlicher formuliert - nicht zeitgenössisch zu sein. Sie ist aber auch in anderer Hinsicht sehr beschränkt, da sie im Regelfall immer eines Mittlers, des auswertenden Nutzers, bedarf. Insoweit ist die retrospektive Macht des Archivs eine dependente, lediglich potenzielle Macht. Dennoch ist sie in der Lage, für eine gewisse nachträgliche Korrektur, vielleicht sogar späte Gerechtigkeit zu sorgen. Aber halt! Darf das - mit Blick auf den Ethikkodex - handlungsleitend sein?

\section{Fazit}

Der Titel dieses Vortrags lautet: „Alles doch ganz einfach? Archivische Bewertung zwischen Wissenschaft und Bauchgefühl“. Ist nun alles doch ganz einfach? Ja und nein! Es ist alles (eigentlich) ganz einfach, weil es keine objektive Wahrheit gibt und Bewertungsentscheidungen demnach immer richtig und falsch zugleich sind. Da es aber zum Ethos des Archivars gehört, nachvollziehbare Entscheidungen zu treffen, muss er seine Bewertungsmaßstäbe und letztlich das Ziel der Überlieferungsbildung offenlegen. An diesem Punkt wird es dann zumindest schwierig und das auch gleich auf zwei Ebenen. Einerseits müssen Begründungen erarbeitet werden, die deutlich über „Das ist wichtig!“ hinausgehen. Andererseits macht man sich mit der Offenlegung seiner begründeten Entscheidung

${ }^{22}$ Robert Kretzschmar, Positionen des Arbeitskreises Archivische Bewertung im VdA - Verband deutscher Archivarinnen und Archivare zur archivischen Überlieferungsbildung. Einführung und Textabdruck. Der Archivar. Mitteilungsblatt für deutsches Archivwesen 58 (2005) 88-94; Andreas Pilger, Ein neues Positionspapier des VdA-Arbeitskreises „Archivische Bewertung“ zur Überlieferungsbildung im Verbund. Archivar. Zeitschrift für Archivwesen 65 (2012) 6-11; DERs., Arbeitskreis Archivische Bewertung. Bewertung elektronischer Fachverfahren. Archivar. Zeitschrift für Archivwesen 68 (2015) 89-92. 
vermeintlich (!) angreifbar. Wir müssen lernen, dass sachliche Diskurse helfen, uns selbst zu vergewissern und uns zu sensibilisieren. Sie sind unsere Qualitätskontrolle! 


\title{
Digitale Bilder - Was darf, was kann bleiben?
}

\author{
Michel J. Pfeiffer
}

Digitale Produkte erreichen Tag für Tag Archive. Fürsorge und Pflichten garantieren stetige Zuflüsse. Einem Strand gleich speist sich das Archiv aus Objekten, welche am Ende ihres ursprünglichen Lebenszyklus angelangt sind. Das Angespülte wird dem Strand vom abfließenden Wasser, der Ebbe, teilweise entrissen. Der Zahn der Zeit sorgt für die entsprechende Pulverisierung, Sedimente sinken ab, Neuland entsteht. Ein natürlicher Zyklus? Was dem durchschnittlichen Abbaumechanismus nicht entspricht, verstehen wir als Überreste, als Strandgut. Solches wird regelmäßig aufgegriffen, im besten Fall als Gebrauchsgegenstand wiederverwendet, repariert, separiert oder als Kunsthandwerk erneut in einen Umlauf gebracht, ansonsten endgültig entsorgt. Die Bewertung findet hierbei zwischen ökonomischer Notwendigkeit einerseits und einem hochgradig individuellen Zugang andererseits statt. Objektivität und Nachvollziehbarkeit? Fehlanzeige. Das Spektrum zwischen diesen Polen erscheint geradezu grenzenlos, inhärent ist all diesen Zugängen jedoch eine zweckorientierte Nutzungsperspektive.

Freilich, die Gleichsetzung von Archivgut mit Strandgut ist falsch. Komparatistisch verwendet, unterstützt diese Metapher unseren gegenwärtigen Umgang mit dem Massenphänomen Bild sowie den daraus resultierenden (Bewertungs-)Ängsten. Werden Archive und weitere Kulturinstitutionen der angeschwemmten Massen digitaler Objekte - Bilder sind damit nur ein Teilaspekt - nicht Herr, so droht ihnen bestenfalls Handlungsunfähigkeit. Diese gilt es zu verhindern, andernfalls droht Messie-tum ${ }^{1}$. Eine für Archivpersonal vermutlich völlig absurde Vorstellung, in welcher sich die Wichtigkeit der unbedingten Handlungsfähigkeit manifestiert. Die Rolle des bewertenden Archivpersonals wird also nicht darin bestehen, Sandstrände von Unrat zu befreien, sie besteht darin, methodische Fragen zu ergründen, wie die digitale Kulturlandschaft zu gestalten ist und welche Rolle dabei Bilder spielen dürfen, ja müssen.

Künftig, so die These des Autors, wird es darum gehen, der Gesellschaft eine repräsentative, empirisch nutzbare, visuell codierte Datenbasis zur Verfügung zu stellen. Diese Forderung zielt auf etwas Eigenständiges ab, welches sich von der klassischen Ergänzungsleistung unterscheidet. Sie soll gar nicht erst ergänzen, sie ist Erkenntnisgrundlage. Es geht nicht

${ }^{1}$ Die kürzlich erschienene Veritas-Studie 2016 zeigt das Ausmaß der digitalen Sammelwut. $63 \%$ der Büro-Mitarbeiter und IT-Fachkräfte verstehen sich schon selbst als Datenmessies und $83 \%$ der IT-Führungskräfte sehen durch das digitale Messiesyndrom das Unternehmen selbst als gefährdet an: vgl. Daten-Messies sind überall: Veritas-Studie zeigt das Ausmaß der digitalen Sammelwut. Veritas. Pressemitteilung (27. 9. 2016), https://www.veritas.com/de/ch/news-releases/2016-09-27-veritas-research-shows-digital-hoarding-behaviouris-pervasive-with-employees-willing-to-give-up-almost-anything-but-their-data [1. 3. 2018]. 
um die „Wiederbelebung“ der Internationalen Ikonographischen Kommission, sondern um ein visuell codiertes Informationsdispositiv. Denn digitale Bilddatenbestände werden künftig empirisch breit abgestützte Längsschnittanalysen zu vielfältigen Themen ermöglichen. Erst damit werden deduktive Schlüsse, serielle Analysen und anderes möglich. Die Bilder hierzu müssen vorhanden, zugänglich und maschinenlesbar ausgezeichnet sein. Die Technologien dazu existieren. Welche Fragen die Gesellschaft an diese Datenbasis stellt, muss hier nicht beantwortet werden.

\section{Schiffbruch}

Die Installation „24 hours in photos“ des niederländischen Künstlers Erik Kessels verdeutlicht die aktuelle Beklemmung, welche mit der Masse digitaler Bilder und deren Bewertung einhergeht ${ }^{2}$. Kessels' Installation entstand aus der Menge digitaler Bildmotive, welche 2010 an einem Tag auf die Fotoplattform Flickr hochgeladen wurden. Der Künstler druckte 350.000 Motive im Format 10 x $15 \mathrm{~cm}$ aus und arrangiert sie jeweils an unterschiedlichen Ausstellungsorten (Abb. 1).

Die nicht mehr vorstellbare Masse digital codierter Bilder wird der Gesellschaft gemeinhin als apokalyptisch anmutende Flut vermittelt. Kessels transmedialisiert digitale Codes in eine erfahrbare Form, verleiht der Masse eine räumliche Dimension: „The Medium is the Message“" . Aus der Bilderflut werden handhabbare Bilderberge. In künstlerischer Freiheit erscheint diese Reflexionsmethode bestechend einfach. Chaos ist die neue Ordnung.

Die Imagination zu ertrinken mag beängstigen, doch kein Mensch wird durch die digitale „Bilderflut“ ernsthaft gefährdet. In dieser Haltung manifestiert sich vielmehr Unvermögen - darin, dass sich das Archivpersonal selbst nicht in der Lage sieht, mit den bevorstehenden Massen umzugehen, darin zu schwimmen. Paradigmatisch sei hier auf die Position des Schweizer Sammlers Peter Herzog anlässlich der Tagung „Der kostbare Augenblick. Massenfotos, Ökonomie und das Versprechen der Dauer““4 verwiesen: „Bilderflut? Da fühle ich mich wie ein Fisch im Wasser!“ Die regelmäßig ins Feld geführte Argumentation unzureichender Finanzmittel lässt das Archivpersonal weder zum Fisch mutieren, noch wird es kurzfristig zum Rettungsschwimmen befähigt. Letzteres bleibt einer spezifischen Ausbildung vorbehalten, welche zur Erhaltung der Kompetenz regelmäßiges Training voraussetzt. Die Aufstockung finanzieller Ressourcen verleitet grundsätzlich dazu, an Vertrautem festzuhalten. Das wäre eine gute Strategie, wenn sie nicht erst zur aktuellen Situation geführt hätte. Zu konstatieren ist, dass bis dato keine konsensfähige Bewertungstheorie visuell codierter Datenträger existiert, auf die man (mit größeren Budgets) erfolgreich zurückgreifen könnte.

Akzeptiert man erstmals die Rolle der Schiffbrüchigen, so ergeben sich überblickbare Handlungsalternativen: Erstens, die hoffnungsloseste, nicht lebensbejahende, ist unterzu-

2 Ein nachgeführtes Verzeichnis der Installationen von „24 hours in photos“ findet sich auf der Webseite des Künstlers: Erik Kessels, 24 Hrs in Photos, in: KESSELSKRAMER, http://kesselskramer.com/ exhibitions/24-hrs-of-photos [1.3. 2018].

3 Vgl. Marshall McLuhan, Das Medium ist die Botschaft, übers. und hg. von Martin Baltes (FundusBücher 154, Dresden 2001).

${ }^{4}$ Anlässlich dieser Tagung, die vom 12. bis 13. 5. 2016 in Luzern stattfand, entlockte Felix Türlemann dem Schweizer Sammler Herzog diese und andere Äußerungen unter dem provokanten Titel: „Man sollte alles sammeln". 


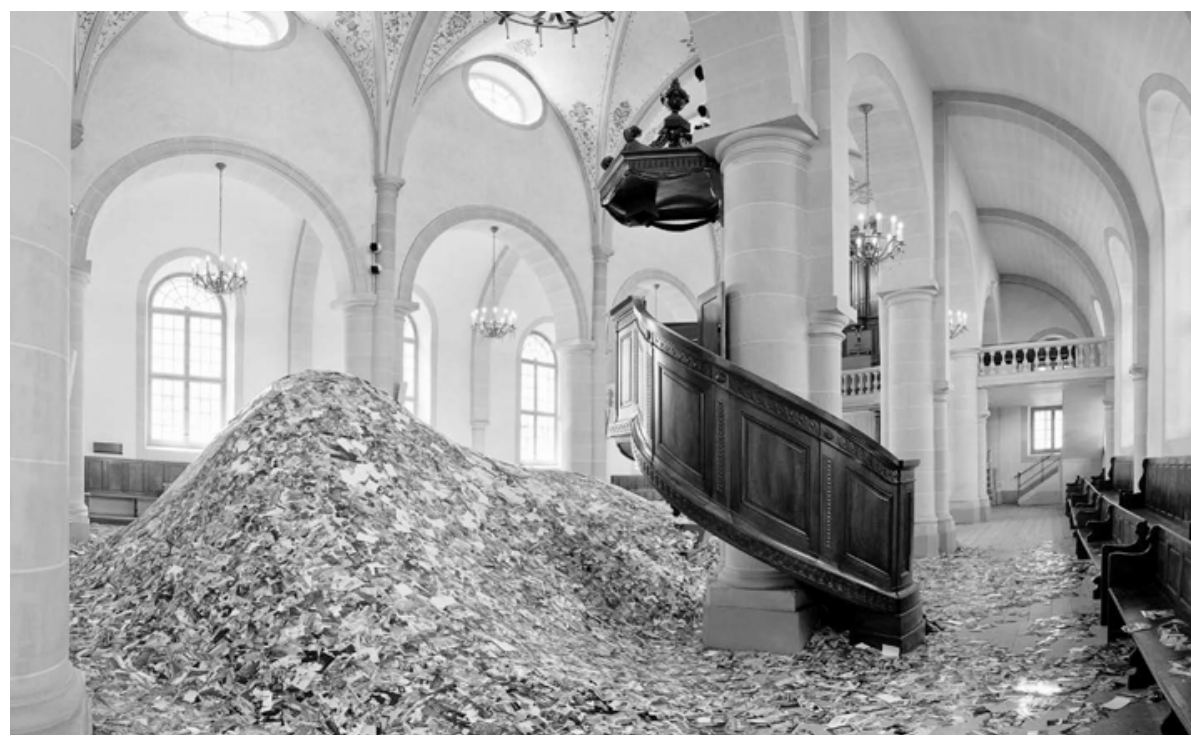

Abb. 1: Installation „24 hours in photos“ des niederländischen Künstlers Erik Kessels (Erik Kessels; KESSELSKRAMER, http://kesselskramer.com/exhibitions/24-hrs-of-photos [1. 3. 2018]).

gehen. Diese Perspektive wird hier nicht weiter verfolgt. Die zweite, minimal optimistische Handlungsperspektive besteht darin, sich an einem Auftriebskörper festzuklammern, bis die vermeintliche Rettung eintrifft. Dem entspricht die Suche nach validen Bewertungskriterien für visuelle Kulturgüter. Die Rettung entspricht einem Kann-Kriterium, dessen Eintrittswahrscheinlichkeit erfahrungsgemäß gering ist. Mittels Auftriebskörper wird der Untergang damit hinausgezögert. Das Chaos bleibt bestehen, es gilt dieses als Organisationsprinzip zu akzeptieren. Die dritte Handlungsalternative ist zugleich die gefährlichste und dennoch erfolgversprechendste. Diese Variante besteht darin zu schwimmen, mit oder ohne Schwimmhilfe, hoffentlich Richtung Ufer. Deutlich wird damit die Abhängigkeit von einer Zielkoordinate. Fest steht, zum Schwimmen braucht man Mut und Entschlossenheit. Damit sind die wichtigsten intrinsischen Faktoren genannt, welche ein idealtypischer Archivar oder eine Archivarin benötigt. Um von der nächsten „Freakwave" digitaler Daten nicht weggespült zu werden, sondern darauf erfolgreich zu gleiten, benötigt es zusätzliche Kreativität. Auf dem Wellenkamm surfend lässt sich die Flutwelle nicht nur überblicken, es besteht durchaus die Möglichkeit, daran Spaß zu entwickeln ${ }^{5}$.

$\mathrm{Zu}$ viel verlangt? Tradierte Berufsbilder verändern sich rasant; um erforderliche Kompetenzen zu entwickeln, wird lebenslanges Lernen Pflicht, oder man geht unter. Massenhafte, digitale Bilddaten sind damit keine Bedrohung für Archive, sondern für das Archivpersonal. Wir tun gut daran, diese neue Herausforderung als Chance zu entdecken. „Big Data“ ist mehr als ein Trend, Daten gelten als zukunftsweisende Ressourcen ${ }^{6}$, künftig

\footnotetext{
5 So die Ergänzung vom Direktor des Schweizerischen Bundesarchivs, Andreas Kellerhals, anlässlich des Referats zur Tagung. Siehe dazu den Beitrag von Andreas Kellerhals in diesem Band.

${ }^{6}$ Eine solche Sicht liegt etwa der Gründung des ETH-Forschungszentrums Big Data zugrunde: vgl. Erich Aschwanden, Aus Datensumpf soll Datensee werden. Die beiden ETH in Zürich und Lausanne gehen in Sachen Big Data in die Offensive. Profitieren soll davon die ganze Schweiz. Neue Zürcher Zeitung (6. 2. 2017), https://www.nzz.ch/schweiz/nationales-zentrum-fuer-datenwissenschaft-aus-datensumpf-soll-datensee-wer-
} 
auch im Archiv. Die Frage ist damit gestellt, wie diese Ressourcen künftig erfolgreich an Benutzende zu bringen sind. Aufgrund vielfältiger Technologiesprünge und der damit verbundenen kurzen Entwicklungszyklen gelten aktuelle Lösungsansätze für tendenziell träge agierende Kulturinstitutionen mit einem langfristig orientierten Aktionshorizont als risikobehaftet und sind damit „instabil“. Selbstkritisch gilt es zu reflektieren, ob wir als digital immigrants und Angehörige einer konventionellen Geisteswissenschaft überhaupt noch in der Lage sind, diese dynamischen Herausforderungen zu lösen, beziehungsweise welche Rolle wir künftig den Digital Natives einräumen werden.

\section{Auf zu neuen Ufern}

Der flächendeckende Netzausbau, die alltägliche Informationsarbeit auf dem Smartphone, die umfassende „Digitalisierung“ angestammter Berufsbilder sind Ausdruck eines beginnenden, tiefgreifenden Wandels unserer Gesellschaft. Weder die technologischen noch die gesellschaftlichen Veränderungen werden vor den Toren der Gedächtnisinstitutionen haltmachen. Sie selbst befinden sich im Umbruch, suchen Orientierung. Beispiel hierfür ist die Veränderung von Bibliotheken hin zum „Dritten Ort“" oder das museale Dilemma zwischen virtuellen Ausstellungen, um neue Besuchergruppen zu erreichen, und der Notwendigkeit, Eintritt zahlende Besuchende bei der Stange zu halten, welche das Überleben sichern sollen. Bis jetzt war es so, dass Benutzende Gedächtnisinstitutionen aufsuchen mussten, um zu den Informationsquellen selbst zu gelangen. Man kann dies als „Holschuld“ bezeichnen. Der technologische Wandel kann durchaus dazu führen, dass Benutzende aus dem Metadatenangebot künftig eine „Bringschuld“ für die Objekte selbst ableiten. Gedächtnisinstitutionen würden damit vom sogenannten pull-zum pushVersorger. Mit dem Fortschreiten dieser Entwicklungen sind mindestens zwei Dinge vorhersehbar. Langfristig besteht die Herausforderung für Gedächtnisinstitutionen darin, im Besonderen für Archive, den Kulturwandel vom „Findmittellieferanten“ zum „Contentprovider“ erfolgreich zu vollziehen. Damit sei gesagt, dass die bloße Verzeichnung von Dokumenten in einem Archivinformations- oder Bibliothekssystem, beziehungsweise das Erstellen und Zur-Verfügung-Stellen von Metadaten künftig nicht mehr ausreichen wird. Visuell codierte Kulturgüter wie Gemälde und Fotografien wurden seit jeher geschaffen, um betrachtet zu werden. Die Frage, ob sie digitalisiert werden oder nicht, ist obsolet. Auch in Museen entstehen Bestrebungen, Informationssysteme, welche ursprünglich zur Dokumentation und Verwaltung von Objekten entwickelt wurden, direkt zur Vermittlungsarbeit einsetzen. Damit ist die Hoffnung verbunden, effektivere Onlineausstellungen in virtuellen Museen zu bespielen ${ }^{8}$. Absehbar ist auch, dass Digital Natives ein verändertes Bedürfnis bezüglich der Verfügbarkeit und der Usability von Information haben. Wichtiger Erfolgsfaktor ist damit die Schnittstelle Mensch-Maschine. Was die Interoperabilität von Daten betrifft, so werden sich auch die Schnittstellen zwischen Maschinen verändern

den-ld.143922 [1. 3. 2018].

7 Robert BARTH, Die Bibliothek als Dritter Ort. Wenn Bibliotheken im 21. Jahrhundert bestehen wollen, müssen sie neue Eigenschaften in den Vordergrund stellen. biblioBE.ch. Informationen für die Schul-und Gemeindebibliotheken des Kantons Bern (19. 2. 2014), http://www.bibliobe.ch/de/fachbeitrage/die-bibliothek-alsort/die-bibliothek-als-dritter-ort.aspx [1. 3. 2018].

${ }^{8}$ Beispiele hierfür sind aktuelle Qualifikationsarbeiten an der Hochschule des Autors, unter anderen eine Arbeit für das Museum Rietberg, Zürich. 
müssen. So wird künftig sichergestellt, dass Kundinnen und Kunden ihre Daten auch in den von ihnen rezipierten Kanälen auffinden können.

Wie könnte man, vor diesem absehbaren Hintergrund, Zielkoordinaten massenhafter digitaler Bildersammlungen definieren oder gar zweckorientierte Nutzungsperspektiven erwägen? Denn im Archiv gelten inhaltliche, prospektiv gestellte Fragen als schwierig zu beantworten, da man nicht vorwegnehmen möchte, was folgende Generationen benötigen werden, so einer der archivarischen Gemeinplätze. Regelmäßig flammen dementsprechend Pflichtabgabeforderungen auf, sie gelten landläufig als „die Lösung“ inhaltlicher Probleme. Damit ist jedoch nicht auszuschließen, dass Außenstehende zum Schluss gelangen könnten, dass Archivierende um des Sammelns willen sammeln². Auch Legaldefinitionen helfen in der Fragestellung, was künftig benötigt wird oder aktuell zu sammeln ist, nicht wirklich weiter, wie das folgende Beispiel zeigt: „Archivwürdig sind Unterlagen, die rechtlich, administrativ, politisch, wirtschaftlich, historisch, sozial oder kulturell wertvoll und für eine authentische Überlieferung wichtig sind"10.

Was kann dem Archivpersonal in einer Zeit des radikalen Umbruchs Orientierung bieten, wenn es die gesetzgebenden Institutionen nicht können oder wollen? Fällt diese Aufgabe im Rahmen der entsprechenden Legaldefinitionen möglicherweise den Archiven selbst zu? Wenn ja, kann die Festlegung gesellschaftsrelevanter Zielsetzungen erste Orientierungshilfe bieten. Die Herausforderung, insbesondere für visuell codierte Kulturgüter, besteht darin, Größen festzulegen, die messbar, überprüfbar und damit nachvollziehbarer sind. Sicherlich sind vielerorts Überlieferungs- beziehungsweise Dokumentationszielsetzungen vorhanden, explizit oder implizit. Jedoch sind sie so verfasst, dass sie den Institutionen möglichst viele Interpretationsspielräume offen lassen. Die direkte Folge davon ist, dass sich diese Zielsetzungen nicht operationalisieren lassen. Das muss sich ändern, dringendst. Deutlich wird damit, dass das Bewertungsproblem „hausgemacht“ ist.

Während sich in angloamerikanischen Ländern konstruktivistisch orientierte Modelle durchsetzten, die sich an einer gesamtgesellschaftlichen Dokumentation orientierten, hielt und hält man in deutschsprachigen Ländern an positivistisch geprägten Modellen fest, die den Provenienzbegriff als Dogma der Objektivität aufrecht erhielten und noch immer erhalten. Damit können angloamerikanische Archive Orientierung bieten, welche die aktuellen Bewertungsfragen bereits überwunden haben. Der kanadische Archivar Terry Cook (1947-2014) definierte in seinem periodisch funktionierenden Macro-Appraisal-Ansatz zuerst eine Dokumentationszielsetzung, welche den Anforderungen entsprechend tiefgreifend oder breit gegliedert ist ${ }^{11}$. Wichtig sei hier vor allem die aktive Identifikation relevanter Ablieferungsstellen, Bestände und so fort. Im Unterschied zum klassischen (europäischen) Archivverständnis erfolgt die Überlieferungsbildung institutionenübergreifend. Auf Landes- oder Kantonsebene können somit alle Gedächtnisinstitutionen gemeinsam in dieselbe gesamtgesellschaftliche Dokumentationszielsetzung mit eingebunden werden. Redundanzen können somit ausgeschlossen und Zuständigkeiten,

9 Hierbei wird allzumal verkannt, dass Pflichtabgaberegelungen die Sammlungstätigkeit regeln und damit keinen Beitrag zur Bewertung liefern.

${ }_{10}$ BGS 152.4 - Archivgesetz (29. 1. 2004), $\$ 2$, Abs. 2, in: Kanton Zug - Gesetzessammlungen - Systematische Sammlung BGS, https://bgs.zg.ch/frontend/versions/1329 [1. 3. 2018].

11 Robert Kretzschmar zeigte in seinen Publikationen zur horizontalen und vertikalen Bewertung längst auf, dass dieser Ansatz im deutschsprachigen Raum umzusetzen ist: Robert Kretzschmar, Vertikale und horizontale Bewertung. Ein Projekt der staatlichen Archivverwaltung in Baden-Württemberg. Der Archivar. Mitteilungsblatt für deutsches Archivwesen 49 (1996) 257-260. 


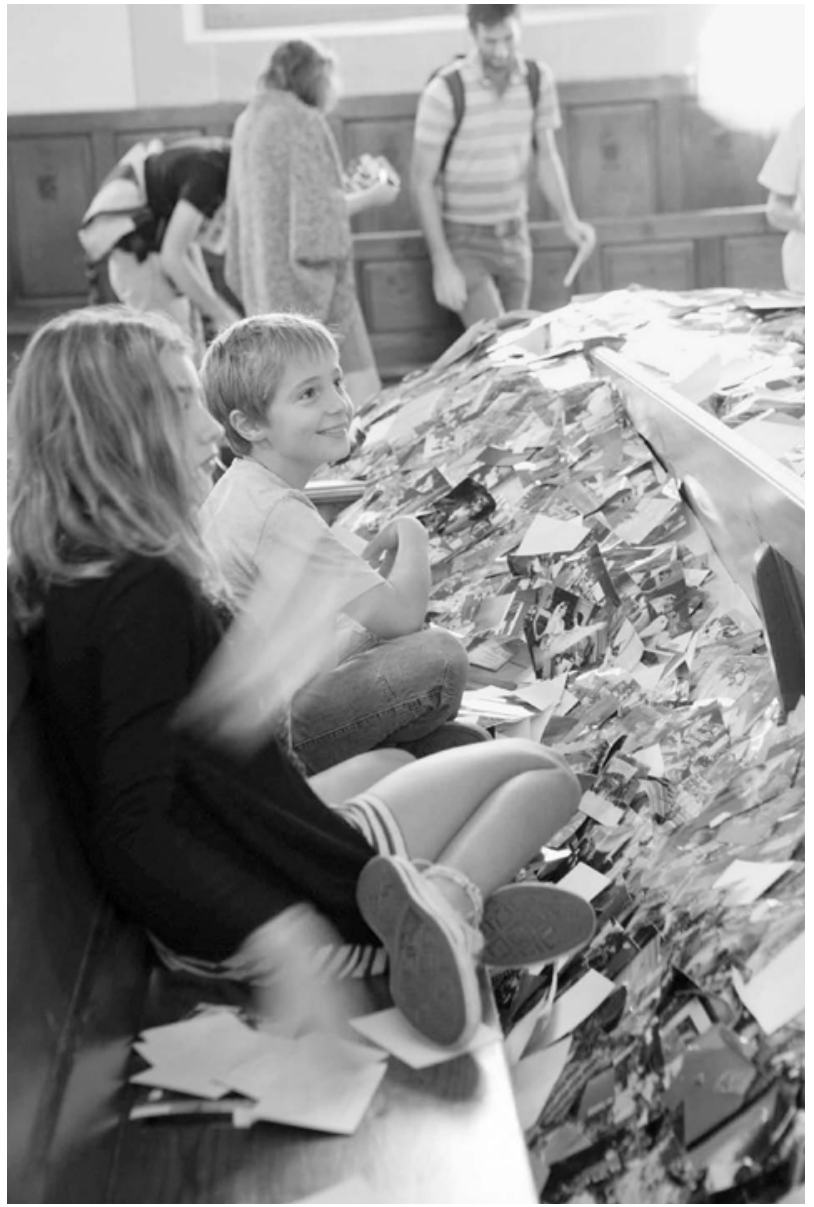

Abb. 2: Installation „24 hours in photos" des niederländischen Künstlers Erik Kessels (Erik Kessels; KESSELSKRAMER, http://kesselskramer.com/ exhibitions/24-hrs-of-photos [1.3. 2018]).

auch die Arbeitsbelastungen, Budgets und so weiter verteilt werden. Sind diese Dokumentationszielsetzungen erstmals erstellt und vereinbart, so erfolgt in den einzelnen Institutionen eine Ist-Analyse. Man schaut „im Keller“ nach, was von der zu sammelnden Zielsetzung bereits vorhanden ist. Aus der Differenz zwischen dem Dokumentationsziel und der aktuellen Bestandsanalyse wird die künftige Sammlungshypothese gebildet. In der darauffolgenden Sammlungsperiode wird das in der Sammlungshypothese festgehaltene Material erneut aktiv gesammelt, erschlossen und vermittelt ${ }^{12}$. Damit beschreiben Cook wie auch Kretzschmar eine strategisch ausgerichtete Sammlungsstrategie, deren Ergebnisse periodisch überprüfbar sind.

„Bewertung“ wird damit ein aktiver Identifikationsprozess im vorarchivischen Feld, welcher die visuelle Überlieferungsbildung - im Auftrag des gesellschaftlichen Kollektivs - direkt steuert. Es geht also um Sammlungspolitik. Auf digitale Bildbestände bezogen gilt es, die entsprechenden Provenienzen, Bestände, Teilbestände, Serien, Dossiers, ja vielleicht sogar Items festzustellen und diese mittels direkter Ansprache in das strategische

12 Terence Gordon Cook, What is Past is Prologue: A History of Archival Ideas Since 1898, and the Future Paradigm Shift. Archivaria. The Journal of the Association of Canadian Archivists 43 (1997) 17-63. 
Sammlungskonzept miteinzubeziehen. Das sind keine neuen Gedanken, in der Schriftgutverwaltung, neudeutsch Records Management, wird das seit längerem praktiziert. Gibt es Gründe, dies nicht zu tun? Werden die längst bekannten Zugänge von Cook oder Kretzschmar als nicht zielführend eingeschätzt oder sind sie nur unbequem?

Ist der Identifikations- und Planungsprozess erstmals abgeschlossen, sind es methodisch-technische Fragen, die klären, wie sich Sammlungsziele erreichen beziehungsweise wie sich die Massen identifizierter und für gesellschaftsrelevant befundener Bilddaten praxisgerecht überliefern, speichern, erschließen und vermitteln lassen (Abb. 2).

\section{Auftriebskörper und Schwimmhilfen}

Den Schiffbrüchigen hilft es durchaus, sich nach Auftriebskörpern umzuschauen, um auf ihre baldige Rettung zu warten. Trifft sie nicht ein, so können sie mit der Schwimmhilfe zumindest ihren Untergang hinauszögern. Praktisch bedeutet dieses Szenario, dass man von längst existierenden Kriterien analoger Bildbestände ausgehend bewertet. Dabei sollte man sich bewusst sein, dass man damit Bekanntes „falsch“ macht, was in der skizzierten Situation freilich zielführend erscheint, langfristig jedoch keine Lösung sein wird.

Längst konstatierte der Leiter des Historischen Archivs des Süddeutschen Rundfunks, Edgar Lersch, eine tiefe Kluft zwischen klassischen Archivstandards zur Überlieferungsbildung und der in Medienarchiven angetroffenen Realität ${ }^{13}$. Die Aufgabe von Medienarchiven sei es, visuelle Zeichenträger so zu archivieren, dass das scheinbar Vergessene adäquat erinnert und erforscht werden könne - ohne die Erkenntnishorizonte visueller Quellen zu verengen, ja gar auf deren illustrative Funktion zu begrenzen. Lersch hielt fest, dass in diesem Sektor erhebliche Versäumnisse vorlagen, was er unter anderem auf die Tradition des bibliothekarisch-dokumentarischen Sammlungsgedankens einzelner isolierter Stücke zurückführte sowie auf die Tatsache, dass ganze Provenienzen nur sehr selten übernommen werden. Dadurch werden visuelle Datenträger nur auf eine fragmentarische Weise überliefert, welche den Herstellungsprozess und den breiteren Zusammenhang nicht adäquat dokumentiert.

Tendenziell widerspiegeln sich in dieser Kritik die häufigsten Bewertungsprobleme über alle Gedächtnisinstitutionen hinweg. Nachvollziehbar wird die aktuelle Herausforderung mit einem Blick auf die nahezu 40-jährige Entwicklung des angloamerikanischen Bewertungsdiskurses, der sich in Europa, allen voran in deutschsprachigen Ländern, nur marginal niederschlug. 1979 stellte der amerikanische Kunsthistoriker und Kurator Paul Vanderbilt dem Archivpersonal grundlegende Fragen zur Überlieferungsbildung fotografischer Bestände ${ }^{14}$. Etwa, ob diese im Ganzen oder in Teilen zu überliefern sind, ob einzelne Objekte priorisiert zu behandeln wären und ob einzelne Objekte oder nur Serien zu verzeichnen sind. Vanderbilt erörterte historische und künstlerische Werte, wobei er davon ausging, dass die inhaltliche Bewertung von Fotografien denselben Kriterien wie jene von Gemälden unterliegt. Er differenzierte zwischen neun inhaltsorientierten Kriterien zur positiven Wertauslese („Frühe Datierung“, „first“ in unterschiedlichsten Bezügen, „Publizität“, anerkannter „Kontext“, „dokumentierte Eigenschaften“, „charakteristische

13 Edgar Lersch, Historische Medienarchive: Überlegungen zur archivwissenschaftlichen Theoriebildung in der Medienüberlieferung. Der Archivar. Mitteilungsblatt für deutsches Archivwesen 53 (2000) 27-34.

${ }_{14}$ Paul Vanderbilt, Evaluating Historical Photographs: A Personal Perspective (AASLH Technical Leaflet 120, Nashville 1979). 
Eigenschaften“, „Denkmäler“, „Wachstum und Veränderung“ sowie „objektive Realität“) und hielt drei Ausschlusskriterien („Überfluss“, nicht vorhandene „Prägnanz“ und „Einfallslosigkeit" ${ }^{15}$ fest. Er betonte damit inhaltliche Deutungsangebote, welche sich aus unterschiedlichen Kontexten erschließen. Bemerkenswert an seinem Vorschlag ist nicht etwa sein positivistischer Zugang, der teilweise an Ranke erinnert, sondern die indirekte Betonung der Differenz. Seine Selektion richtet sich zwar nach kontroversen inhaltsorientierten Aspekten, innerhalb derselben strebt er jedoch nach größtmöglicher Vielfalt. Die Reduktion bezieht sich damit auf redundante Information, Differenz soll erhalten bleiben. Grundsätzlich ist das ein valabler Bewertungszugang, wenn es denn gelingt, die von Lersch konstatierte Kluft zu überwinden.

Mitte der 1980er Jahre sah sich Thomas D. Norris mit der Herausforderung konfrontiert, circa eine Million Negative des Sacramento History Center zu bewerten ${ }^{16}$. Er verglich seine Lage mit derjenigen seiner Kollegen, die nach der Jahrhundertwende wissenschaftliche Kriterien zur Bewertung schriftlicher Überlieferungen erarbeitet hatten. Rückblickend hielt er fest, dass große Bestände anhand der Provenienz, wenn man das entsprechende Ordnungssystem erkennt, zu ordnen seien, denn der originäre Zugang biete - abgesehen von den bereits diskutierten epistemologischen Aspekten - sowohl eine effektive Bewertung als auch eine kostengünstigste Nutzung. Er regte an, sich der jeweiligen Stärken und Schwächen einer Sammlung bewusst zu werden. Inhaltlich verwies er darauf, Bestände von „Mug-Shots“ - damit meinte er regelmäßig wiederkehrende Motive wie den „Hund der Woche“ - zu kassieren, denn solche Fotografien seien vermutlich nicht von historischem Wert.

Zur selben Zeit erarbeitete William H. Leary weitreichende Bewertungskriterien für historische Fotografien ${ }^{17}$. In seiner UNESCO-RAMP-Studie forderte er sowohl das Archivpersonal als auch die geschichtswissenschaftlich Forschenden dazu auf, historischen Fotografien mit derselben Aufmerksamkeit zu begegnen, wie sie die Archäologie einzelnen Artefakten entgegenbrächte. Das war und ist heute noch eine starke Forderung. Angesichts der damals in den USA jährlich produzierten Bildmenge von circa zehn Milliarden Fotografien sei es für alle, die Medien archivieren, trotz Risiken und Schwierigkeiten von Akquisitionsplänen unerlässlich, strategische Maßnahmen zu treffen, um in ihrer wichtigen Vernichtungsaufgabe nicht zu versagen. Er war sich bewusst, dass die fotografische Überlieferungsbildung davon abhängig ist, ob historisch relevante Bestände auch den entsprechenden Institutionen zugeführt werden können. Gemäß Leary liegt der Wert historischer Fotografien in ihrem Informationswert. Er argumentierte, dass die Evidenz einer Organisation mit schriftlichen Unterlagen effektiver überliefert werden könne als mit Bildern, denn Bilder, welche nur die Evidenz einer Organisation abbildeten, wären „very boring and insignificant images "18. Leary bestimmte den Informationswert auf Grund von „Alter“, „Bildinhalten“ beziehungsweise „Gegenständen“, „Einzigartigkeit“, „Identifikation“ derselben für die Verzeichnung, „Qualität“ - wie Schärfe, Komposition -, aber auch „Erhaltungszustand“ für Nitrat- oder Acetatnegative sowie „Quantität“, welche Forschenden komparatistische Schlüsse ermöglichen sollte, „Zugänglichkeit“ und der Person

15 Zur Vereinfachung wurden die englischen Begrifflichkeiten durch den Autor übersetzt.

16 Thomas D. Norris, Processing Extremely Large Collections of Historical Photographs. The Midwestern Archivist X/2 (1985) 129-134.

17 William H. Leary, The Archival Appraisal of Photographs. A RAMP Study with Guidelines (Paris 1985).

18 Ebd. 19. 
des „Fotografen“. Zur Bestandsverdichtung hielt er fest, dass die Ausdünnung die effektivere Methode sei als Sampling.

Mit der RAMP-Studie, die sich mit inhaltlichen Kriterien auseinandersetzte, erschien die Bewertung visueller Quellenbestände im angloamerikanischen Raum als weitgehend gelöst, während in Europa an archivarischen Dogmen festgehalten wurde. Die Objektivität schriftlicher Überlieferungsbildung konnte nur durch die allgemeine Akzeptanz diametral organisierter Bildbestände aufrechterhalten werden, da Letzteren seit der Antike etwas Subjektives zugestanden wird. Diese positivistisch veranschlagte Objektivität von Texten wurde damit um den Preis epistemologischer Verluste, der Preisgabe des Quellenwerts visuell codierter Daten, erkauft. Insofern schien es bis zum Auftauchen der Forderung nach dem „Bild als Quelle“ legitim, Fotografien als Illustration zu verwenden ${ }^{19}$. In den auslaufenden 1980er Jahren wurde diese Bewertungsdiskussion durch technische Fragen zur aufkommenden Digitalisierung und EDV-gestützten Sammlungs- und Archivsystemen verdrängt. Im Vordergrund stand nun die technische Machbarkeit und Umsetzung von EDV-gestützten Archiv- und Digitalisierungslösungen.

Die Frage nach der Archivwürdigkeit von Fotografien wurde vom frankokanadischen Historiker und Archivar Normand Charbonneau erst nach der Jahrtausendwende erneut aufgenommen ${ }^{20}$. Der Informationswert bildete auch für ihn die Grundlage zur Bewertung von Fotografien. Er argumentierte, dass die Aussagekraft eines fotografischen Dokuments nicht direkt aus der Beziehung zur Provenienz hervorgehe, sodass das Archivpersonal deshalb nicht auf die traditionellen Methoden zurückgreifen könnte. Außerdem zeige sich, dass Archivpersonal wie auch Benutzende zur Fotografie ein fast irrationales Verhältnis aufbauten, da Bilder persönliche Erinnerungen evozierten. Für Charbonneau avancierte damit das eigene Wissen zum ersten Bewertungsinstrument, weshalb auch er das Archivpersonal aufforderte, sich im Vorfeld einer Bewertung vertiefte Kenntnisse über die Herkunft und den Entstehungszusammenhang eines überlieferungsfähigen Bestandes zu erwerben, ansonsten dieser nicht als Ganzes erfasst werden könne. Zur Beurteilung des Informationswerts hält Charbonneau die Interessen der Benutzenden für relevant, da Bilder aufgrund spezifischer Interessen recherchiert würden. Eine Position, die höchst kritisch zu beurteilen ist, denn auch diese Interessen sind transitorisch. Des Weiteren weist Charbonneau darauf hin, dass zur Beurteilung einer Fotografin oder eines Fotografen, deren Technik und deren Leistung sowie zur Identifikation der Einzigartigkeit von Bilddokumenten fotohistorische und -technische Kenntnisse unerlässlich sind. Als die notwendigsten Kriterien zur Bewertung von Fotografien nennt er: die "Lesbarkeit" von Informationen beziehungsweise des Mediums, „Benutzerbedürfnisse“ und Interessen, „Inhalte“ oder Themen, die „Produktionsabsicht“, „Kommunikationszweck“, „Ergänzungsdokumentation“, „Einzigartigkeit“ der Information, „Alter“ des Mediums sowie Alter der festgehaltenen Informationen, „ästhetische Qualität“, „Bedeutung“ für die Kunst- und Fotografiegeschichte sowie „Zugangsrestriktionen“.

19 Hierzu sei auf den zeitgeschichtlichen Diskurs der Visual History verwiesen: Gerhard JAGschitz, Visual History. Das audiovisuelle Archiv. Fachzeitschrift für Theorie und Praxis des audiovisuellen Archivwesens 29/30 (1991) 23-51; Visual History. Ein Studienbuch, hg. von Gerhard Paul (Göttingen 2006); Bilder als historische Quellen? Dimension der Debatten um historische Bildforschung, hg. von Jens JäGER-Martin KNAUer (Paderborn 2009).

20 Normand Charbonneau, The Selection of Photographs. Archivaria. The Journal of the Association of Canadian Archivists 59 (2005) 119-138. 
Neu an Charbonneaus Beitrag ist der Vorschlag, die Bewertungsentscheidung fotografischer Bestände auf der Basis von Schellenbergs Taxonomie als Top-Down-Prozess zu operationalisieren. Vom Bestand ausgehend werden Serien und Unterserien identifiziert, auf Evidenz- und Informationswerte überprüft, bis letztlich das einzelne Bild bewertet ist. Dabei berücksichtigt er, dass visuelle Überlieferungen aus unterschiedlichen Informationsträgern bestehen können, weshalb diese konsequenterweise zusammen zu bewerten seien, da die Übernahme eines Mediums die Übernahme eines anderen bedingen könne.

Die Medienarchivarin der Library of Congress, Diane Vogt-O'Connor, exemplifiziert die Ansätze von Leary und Charbonneau ${ }^{21}$. Im Fokus ihres Beitrags stehen sowohl strategische Überlegungen zum Aufbau einer fotografischen Sammlung als auch die praxisorientierte Bewertung. Sie empfiehlt die Erarbeitung detaillierter Sammlungssurveys, die unter anderem auch Interviews von Gönnern und Sammlern inkludieren. Surveys geben Auskunft über die Sammlungsgeschichten, Interessen der Sammelnden, Sammlungsthemen, gesammelte Quantität und Qualität, beschreiben innere Ordnung und Aufbewahrungsmaterialien und stecken rechtliche Restriktionen ab. Aus diesen signifikanten Sammlungsinformationen entsteht ein Bewertungsbericht, der unter Berücksichtigung des Kosten-Nutzen-Verhältnisses sowie einer Risikoabschätzung eine Akquisitionsempfehlung ausspricht oder einen Ratschlag zur Überarbeitung von Dokumentationsplänen erläutert.

Gemäß Vogt-O'Connor entstehen in öffentlichen Verwaltungen visuelle Informationsträger aus rechtlichen Gründen oder zu administrativen Zwecken. Der Primärwert ergibt sich aus den Materialien, deren Bedeutung sich aus dem Bezug des Objekts zu den Produzierenden oder Auftraggebenden entfaltet. Passfotos, Bilder von stornierten Schecks, Dokumentationen von Preisverleihungen, visuelle wissenschaftliche Rohdaten und so weiter identifizieren jemanden oder etwas. Primärwert haben jedoch auch nicht öffentliche Bilder wie Schnappschüsse aus dem Familienurlaub, so Vogt-O'Connor. Sekundärwerte beziehen sich auf visuelle Informationsträger, welche neben der primär intendierten Nutzung durch das Produktionsumfeld für die wissenschaftliche Forschung von Bedeutung sein können, wie beispielsweise dokumentarische Fotografien. Sind in Fotografien nur Primärwerte feststellbar, so stehen diese Bilder zur Disposition - während Fotografien mit Sekundärwert archivwürdig erscheinen. Typischerweise gilt es die sekundäre Aussagekraft von Fotografien anhand der vier Schlüsselfaktoren Evidenzwert, Informationswert, artefaktischer Wert (artefactual value) und Beziehungswert (associational value) zu identifizieren.

Für das Archivpersonal bedeutet dies, dass die Einschätzung des Evidenzwerts mittels historisch-kritischer Methode erfolgen muss. In Abgrenzung zu Historikerinnen und Historikern, deren Aufgabe die Überprüfung und Interpretation der visuellen Informationsträger ist, muss die Archivarin oder der Archivar in der Lage sein, das Potenzial evidenzbasierender Erkenntnishorizonte zu beobachten, zuzuordnen, um dessen Anschlussfähigkeit abschätzen zu können. Evidenz geht aus den Fragen hervor: Wer erstellte das Bild, für wen und zu welchem Zweck war das Bild bestimmt, welche Mitteilung intendierten die Produzierenden, wer wählte zuvor Bilder aus und modifizierte sie, wer stellte das Bild in welchen Kontext, wieso, wann und warum? Wurden die Fotografien und ihre Dokumentation überprüft und validiert oder auf einem anderen Weg authentifiziert ${ }^{22}$ ?

${ }^{21}$ Diane Vogt-O’Connor, Appraisal and Acquisitions, in: Mary Lynn Ritzenthaler et al., Photographs. Archival Care and Management (Chicago 2006) 78-127.

22 Vgl. ebd. 98f. 
Fotosammlungen, Bildserien oder Bildcluster enthalten oft nützliche Informationen, die weit über den ursprünglich intendierten Verwendungszweck hinausgehen. Fotografien liefern sozialhistorische Angaben über Tätigkeiten, Epochen, Ereignisse, Orte oder Prozesse, die anderweitig nicht oder nur sehr rudimentär dokumentiert sind. Fotografien halten jedoch auch unbeabsichtigte Informationen fest, die für ganz andere, unvorhergesehene Zwecke genutzt werden können. Vogt-O'Connor verweist hierzu auf langfristig angelegte Landschafts- und Naturdokumentationen, die Informationen über Umweltveränderungen liefern können. Auch Aufnahmen urbaner Zentren enthalten nicht nur Hinweise auf Architektur und deren Veränderungen im öffentlichen Raum, sie dokumentieren auch Immigrationstrends oder das Zusammenleben der Gesellschaft. Unter dem artefaktischen Wert versteht Vogt-O'Connor die Kreativität der Bildproduzierenden, ihre technischen Fähigkeiten in der Postproduktion sowie die Verwendung von Motiven, welche Kunstschaffende in Beziehung zu anderen Bildern oder in Zusammenhang mit schriftlichen Informationen setzen. Mittels des artefaktischen Werts können diese Beziehungen beobachtet werden. Dabei hielten Fotografien einen dokumentarischen Moment fest, erzeugten einen kommunikativen Akt zwischen Produzierenden und Benutzenden, und als Gegenstände materieller Kultur reflektierten sie die Möglichkeiten verfügbarer Technologien.

Des Weiteren sammeln Institutionen Fotografien von Personen, Orten oder Ereignissen, die in einer spezifischen Relation zur Organisation, ihren Beständen und ihrer Geschichte stehen. Mit dem Beziehungswert assoziiert Vogt-O’Connor Personen, wie Fotoassistierende, welche zur Produktion von Fotografien beitrugen, Sammelnde, die Kollektionen zusammengetragen haben, Personen, die Fotoalben erstellt und damit ihr eigenes Leben oder das anderer illustriert haben, sowie Forschende, die mit ihren Veröffentlichungen zu einer signifikanten Bedeutungssteigerung einer Fotografie oder eines Sammlungsbestands beigetragen haben. Zur Evaluierung dieses Werts geht VogtO'Connor von den Fragen aus, ob der zu übernehmende Bestand im Besitz einer Person war, für die sich die sammelnde Institution aufgrund ihrer Sammlungspolitik interessiert, und ob das zu bewertende Material weitere Werke einzelner Personen oder Organisationen beinhaltet, welche die Institution aktiv erwerben sollte. Vogt-O'Connor verweist auch auf monetäre Werte, die keine klassischen Archivwerte darstellen, nach denen Bilder bewertet werden können, deren Höhe aber maßgeblich für besondere Schutzmaßnahmen von Fotografien verantwortlich ist.

Bei allen erkenntnisreichen Ansätzen von Vogt-O'Connor sowie anderen angloamerikanischen Publizierenden ist die Tendenz nachfrageorientierter „Bildnutzung“ als Bewertungskriterium nicht zu verkennen. Das erscheint kritisch, denn Nachfragen sind volatil und entsprechen wiederum dem bibliothekarisch-dokumentarischen Sammlungsgedanken, den bereits Lersch kritisierte. Bildnutzung kann dann als eigenständiges Kriterium verstanden werden, wenn sie nicht die Nachfrage durch Nutzende bezeichnet, sondern als Chance verstanden wird, Bilder zu vermitteln und diese in die entsprechenden Kontexte zu stellen. Bildnutzung ist daher weit zu fassen. Letztendlich geht es um die Anschlussfähigkeit der Bildinformation. Eine historisch orientierte Medienkritik, wie dies aus Vogt-O'Connors Verständnis des Evidenzwerts hervorgeht, die sich auf eine erweiterte Lasswell-Formel bezieht, bietet hierzu das entsprechende Instrumentarium. Archivwürdig sind visuelle Informationsträger dann, wenn sich sowohl bildintrinsische als auch kontextuelle Informationen in ihrer Anschlussfähigkeit beobachten lassen. 


\section{Land in Sicht}

Vor dem Hintergrund einer positivistischen Philosophie, in der das „objektive“ Provenienzprinzip eingebettet liegt, werden grundlegende Fragen von Wertesystemen, die sich im Bewertungsdiskurs von Fotografien oder Film manifestieren, maskiert. Erschwerend kommt hinzu, dass einer systematischen Überlieferungsbildung visueller Kulturgüter eine latent ikonophobe Grundhaltung entgegensteht. In den historischen Disziplinen resultiert sie aus der philologischen Tradition der Textkritik, die letztendlich einen illustrativen Bildgebrauch zur Folge hatte. Visual History ist vielleicht ein kompensatorisches Momentum, das die jahrhundertelange Textlastigkeit der traditionellen Geschichtswissenschaft zum Ausdruck bringt. Die Debatten rund um den Visual Turn haben erst bewusst werden lassen, wie komplex visuelle Kommunikation ist und in welchen Dimensionen sie unseren Alltag beeinflusst. In staatlichen Gedächtnisorganisationen führte die ikonische Wende jedoch zu keiner grundsätzlichen Veränderung von visuellen Überlieferungsstrategien $^{23}$. Ignoriert man wahrnehmungstheoretische und kognitionsbedingte Herausforderungen der Bildbetrachtung, so ist davon auszugehen, dass visuelle Datenbestände nach wie vor in nutzungsorientierten, pertinenten Systemen verbleiben. Mag das mimetische Verständnis in der Gesellschaft tief verwurzelt sein, so lassen sich trotzdem keine schlüssigen Argumente finden, welche den damit implizierten Erkenntnisverlust rechtfertigen. Es überrascht deshalb nicht, dass nach wie vor visuell codierte Archivkörper und deren Kontexte verzerrt oder gar zerstört werden, weshalb Joanna Sassoon aufgrund der Logik der Bilder auch angloamerikanisches Archivpersonal auffordert, sich der Frage zu stellen, wie sie die Integrität und Authentizität ihrer Bildbestände gewährleisten ${ }^{24}$.

Mögen positivistisch orientierte Bewertungsschemata für schriftliche Archivalien noch lange Zeit „funktionieren“, so konturiert die Bewertung visueller Quellen vertagte Herausforderungen. Hierbei spielt es keine Rolle, ob die Bilder analog oder in digital codierter Form vorliegen. Ihnen gemein sind die Eigenschaften, dass sie von einem Individuum oder von einem Kollektiv für einen bestimmten Verwendungskontext produziert worden sind und nach ihrer primären Verwendung zur Generierung von neuem Wissen gesammelt, restauriert und archiviert oder aufgrund der ihnen nicht zugestandenen Bedeutung vernichtet werden. Die in visuellen Artefakten festgehaltenen intentionalen Zeichenprozesse zeugen damit von soziokulturellem Handeln und liefern Einblicke in Weltaneignungsstrategien aus unterschiedlichsten gesellschaftlichen Systemen und Perspektiven. Visuelle Archivwürdigkeit zeichnet sich damit durch eine entsprechende Wirkung aus. Diese gilt es zu entdecken und zu dekodieren. Sammlungsrelevant stellt sich damit die Frage danach, wo Bilder Wirkung entfalten und vor allem welche? Eine visuelle Sammlungskultur hat damit weniger mit Archivierung zu tun als damit, Material für die Überprüfung der Gegenwart bereitzustellen. Denn die Gesellschaft greift dann auf Bilder-

${ }^{23}$ Es wäre verfrüht, von einer Trendwende zu sprechen. Ausnahmen wie die Übernahme des Ringier Bildarchivs bestätigen die Regel; vgl. Ringier Bildarchiv - Ein Sicherungs- und Vermittlungsprojekt, in: Kanton Aargau - Departement Bildung, Kultur und Sport - Bibliothek \& Archiv - Staatsarchiv - Projekte, https:// www.ag.ch/de/bks/kultur/archiv_bibliothek/staatsarchiv/projekte_3/ringier_bildarchiv_1/ringier_bildarchiv. jsp [1. 3. 2018]. Umso gravierender zeigt sich an diesem Beispiel die Herausforderung zu bewerten, vgl. Über den Wert der Fotografie. Zu wissenschaftlichen Kriterien für die Bewahrung von Fotosammlungen, hg. von Andrea Voellmin-Walter Leimgruber-Nora Mathys (Baden 2013).

24 Joanna Sassoon, Beyond Chip Monks and Paper Tigers: Towards a New Culture of Archival Format Specialists. Archival Science. International Journal on Recorded Information 7 (2007) 133-145. 
sammlungen zurück, wenn sie sich nicht mehr erinnert und stattdessen Aufzeichnungen hinzuzieht, von denen sie annimmt, dass sie das beschreiben, was ihr in der Erinnerung nicht mehr präsent scheint. Fotografien, die nicht materialisiert und archiviert werden, entziehen sich damit den gesellschaftlichen Erinnerungsmöglichkeiten - unserem kulturellen Bildgedächtnis - und sind darin vergleichbar mit gesprochenen, nicht dokumentierten Worten aus vergangener Zeit ${ }^{25}$.

Sind Bilder nicht dokumentiert, sind Informationen nicht überprüfbar, bleiben sie illustrativen Zwecken vorbehalten, denn sehen heißt damit nach wie vor glauben ${ }^{26}$. Die daraus resultierende Problematik darf seit dem Diskurs rund um die Wehrmachtsausstellung vorausgesetzt werden ${ }^{27}$. Gerade intentionale Eigenschaften, die etwas über die Produzierenden, ihre Produktionsbedingungen oder über ihre Organisationsweise aussagen, sind mit dem Entstehen konstruktivistischer beziehungsweise systemtheoretischer Positionen aus der Forschung nicht mehr wegzudenken. Das gilt auch für die Bewertung selbst. Skartierungsentscheidungen fotografischer Bestände sagen vermutlich mehr über die Bewertenden aus, als der fotografische Inhalt über die entsprechende Provenienz. Diskussionen, ob nun Bewertungskriterien in Archivinformationssystemen offenzulegen sind oder nicht, erübrigen sich damit grundsätzlich. Erkenntnistheoretisch sind sie für den Nachvollzug des noch Sichtbaren relevant. Gegenläufig dazu ist auch immer die Frage, was wir als Betrachtende nicht sehen, nicht sehen können. Hierbei ist es als Rezipierende wichtig zu unterscheiden, ob etwas nicht fotografiert, nicht abgezogen, nicht gesammelt wurde oder der Skartierung zum Opfer fiel. Eine adäquate Argumentation zur Skartierung ist damit eine zwingende Dokumentationsgröße, andernfalls ist der letztmögliche Anspruch an Objektivität fallen zu lassen.

Mediensammlungen aller Art kommt damit die Aufgabe zu, visuelle Zeichenträger so zu archivieren, dass das Vergangene, scheinbar Vergessene erinnert, interpretiert und erneut vermittelt werden kann. Damit wir als Gesellschaft anhand visueller Quellen die unwiederbringliche Vergangenheit interpretieren können, benötigen wir endlich Archive, die es zulassen, Bildinformationen repräsentativ zu sammeln, zu erschließen, vorzuhalten und abzurufen ${ }^{28}$. Dies hatte schon Lersch zum Ausdruck gebracht. Hier wird ein Umdenken notwendig sein, das zu einer neuen visuellen Archivkultur führen muss und die hausgemachten Probleme löst.

Alle, die sich in Archiven, Bibliotheken, Museen und in der historischen Forschung mit visuellen Quellen befassen, müssen in die Lage versetzt werden, die Logik dieser Quellen und ihrer Systeme zu verstehen, um diese als Überreste der Vergangenheit uns, der Gesellschaft, auch künftig und digital zur Verfügung zu stellen. Das hat mit Ausbildung zu tun. Andererseits müssen sie in der Lage sein, mit dem „Mut zur Lücke“ medienspezifische Narrative zu analysieren und Geschichte zu konstruieren. Das ist ihr Geschäft.

25 In Anlehnung an die Gedanken von Timm STARL, Hinter den Bildern. Identifizierung und Datierung von Fotografien von 1839 bis 1945. Fotogeschichte. Beiträge zur Geschichte und Ästhetik der Fotografie 99 (2006) 3-88, hier 3.

26 Vgl. Dana Arnold, Sehen heißt glauben: Historiker und Bilder, in: Bilder als historische Quellen (wie Anm. 19) 27-43.

${ }^{27}$ Vgl. Miriam Y. Arani, „Und an den Fotos entzündete sich die Kritik“. Die „Wehrmachtsaustellung“, deren Kritiker und die Neukonzeption. Ein Beitrag aus fotohistorisch-quellenkritischer Sicht. Fotogeschichte. Beiträge zur Geschichte und Ästhetik der Fotografie 85/86 (2002) 97-124; Cornelia BRINK, Ikonen der Vernichtung. Öffentlicher Gebrauch von Fotografien aus nationalsozialistischen Konzentrationslagern nach 1945 (Schriftenreihe des Fritz-Bauer-Instituts 14, Berlin 1998).

28 Diese notwendige Forderung richtet sich nach innen wie nach außen. 


\section{Surfschule}

Die erste gute Nachricht besteht in der Feststellung, dass die chemische Fotografie endlich erscheint, sie ist bereits ein historisches Faktum. Mit der dargestellten Sammlungsstrategie sowie den zu konsolidierenden angloamerikanischen Bewertungsmethoden hat man durchaus intakte Überlebenschancen, die Risiken dieser Bewertungsmethoden sind bekannt. Die zweite gute Nachricht ist die, dass Kessels' Fiktion der materialisierten Flutwelle nur im Museum existiert. Niemand ertrinkt.

Die vorangegangene Darstellung sollte deutlich machen, dass sowohl die Anforderungen an zu überliefernde visuell codierte Datenbestände als auch die Qualifikation des Archivpersonals in Fragen der Bewertung und Verzeichnung zu klären sind ${ }^{29}$. Im Besonderen, wenn es befähigt werden soll, in digitalen Fluten zu schwimmen. Weder Flutwellen noch Schiffbrüche werden geplant, Vorkehrungsmaßnahmen dagegen jedoch sehr wohl. Es ist damit eine Frage des Widerstands oder danach, wofür wir als Gesellschaft unsere Energie, unsere Ressourcen einsetzen wollen: entweder gegen die Flutwelle mit retardierten Zugängen anzukämpfen oder mit entstehenden, unsicheren Technologien das Risiko einzugehen, auf der Flutwelle zu surfen ${ }^{30}$ (Abb. 3).

Vorboten des digitalen Wachstums von Bildbeständen sind soziale Medien, welche visuell codierte Daten aggregieren. Flickr, Instagram oder Imgur sind zwischenzeitlich so immens, dass angesichts des konstatierten „analogen Schiffbruchs“ es gar nicht erst denkbar erscheint, digitale Bildbewertung intellektuell leisten zu wollen ${ }^{31}$. Hinzu kommen akute und zum Teil nicht geklärte Urheberrechtsfragestellungen, welche die Komplexität um ein Vielfaches erhöhen.

Absehbar sind zwei unterschiedliche Entwicklungsmöglichkeiten: Aus der analogen Bewertungserfahrung können wichtige Kriterien herausgearbeitet werden, um künftig auf Algorithmen basierende Identifikationssysteme zu bauen, welche Archivpersonal im Sammeln von digitalen Kulturgütern oder digitalen Nachlässen unterstützen. In den letzten zehn Jahren wurden sammlungsbezogene Technologien wie das Web-Harvesting vorangetrieben, Schnittstellen und Formate entstanden ${ }^{32}$. Neueren Datums sind Fachbereiche, welche sich mit der maschinellen Informationsextraktion, der Klassifikation und Anno-

29 Forschende, welche Bilder befragen, sind hiervon nicht auszunehmen.

${ }^{30}$ An dieser Stelle könnte man sich durchaus die Frage stellen, was das Ziel von massenhaften digitalen Bildersammlungen überhaupt sein kann? Benötigt die Gesellschaft so etwas wie eine Arche Noah für Texte und Bilder, um angesichts digitaler Massenkommunikation zu überleben? Oder benötigt das Archivpersonal eine hochseetaugliche Rettungsinsel, um zu überleben?

31 Tagtäglich nutzen über eine Milliarde Menschen Facebook, täglich werden 350 Millionen Fotografien hochgeladen. Insgesamt sind es über 250 Milliarden Fotografien, welche für einen täglichen Speicherzuwachs von vier Petabyte sorgen. Im Sommer 2015 kommunizierte YouTube die Zahl von 300 Stunden Videomaterial, die jede Minute auf das Portal hochgeladen werden: Kit SMITH, 47 Facebook-Statistiken für 2016. Brandwatch Blog (25. 5. 2016), https://www.brandwatch.com/de/2016/05/47-facebook-statistiken-fuer-2016 [1. 3. 2018]; YouTube for Press, in: YouTube, https://www.youtube.com/yt/press/statistics.html [1.3. 2018]. Der Autor geht nicht davon aus, dass sich ein Wirtschaftsarchiv oder eine öffentliche Archivinstitution finden wird, die solche Bestände dereinst übernimmt oder mit aktuellen Methoden bewertet.

32 Vgl. Art. Webarchivierung, in: nestor-Wiki, https://wiki.dnb.de/display/NESTOR/Webarchivierung [1. 3. 2018]; Harvesting, in: Schweizerische Nationalbibliothek NB - NB Professionell - e-Helvetica - Infos für Fachpersonen - Technische Infos, https:/www.nb.admin.ch/snl/de/home/nb-professionell/e-helvetica/ infos-fuer-fachpersonen/technische-infos/harvesting.html [1.3. 2018]; WARC, Web ARChive file format, in: Library of Congress - Sustainability of Digital Formats: Planning for Library of Congress Collections, https:// www.loc.gov/preservation/digital/formats/fdd/fdd000236.shtml [1. 3. 2018]. 


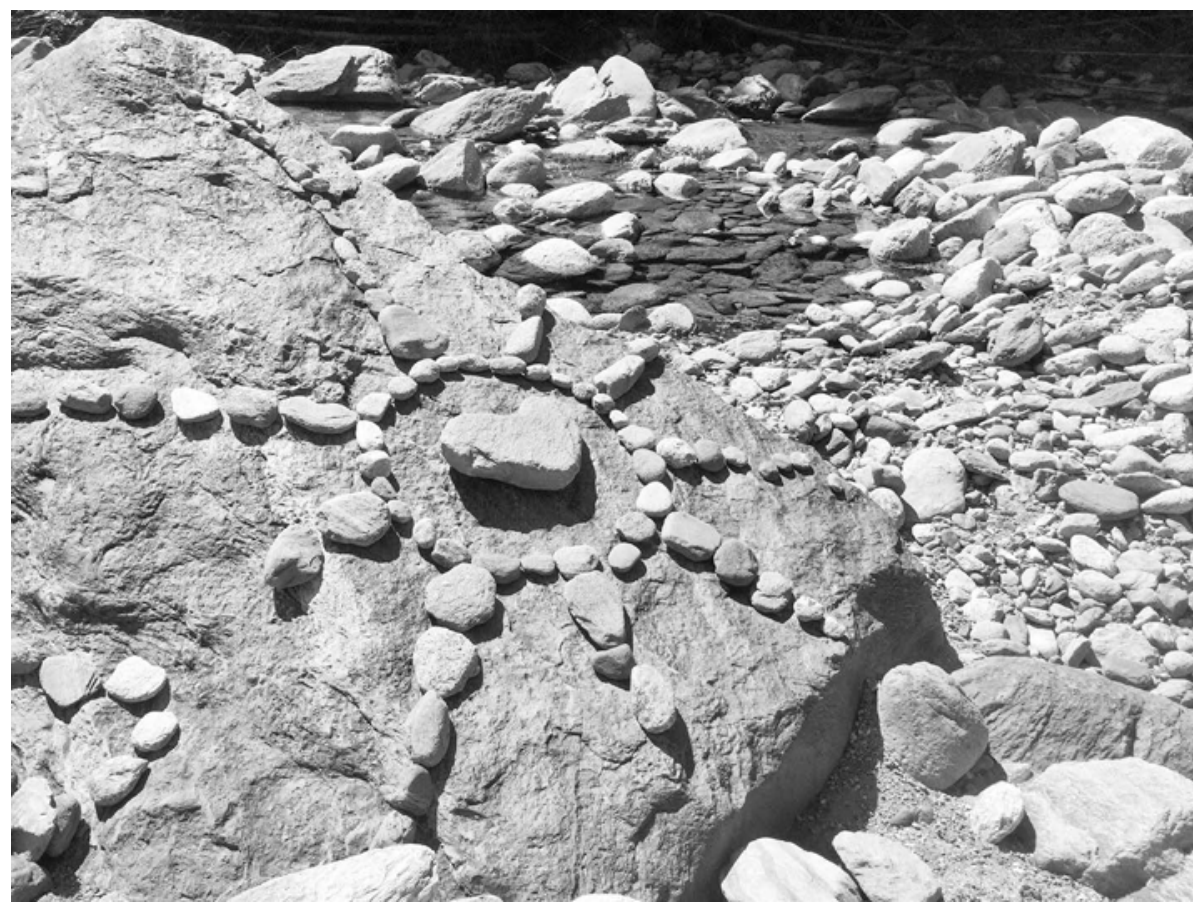

Abb. 3: „Landart“ (Michel J. Pfeiffer).

tation von Texten oder mit der Kontextualisierung von Texten zu Texten sowie Texten zu Bildern beschäftigen ${ }^{33}$. Damit lassen sich heute sogenannte Recommender-Systeme erstellen ${ }^{34}$. Aktuell wird an Maschinen geforscht, welche in der Lage sind, innert Bruchteilen von Sekunden "Spambegriffe“, welche im IPTC-Header einer Bilddatei platziert sind und nicht zum eigentlichen Bildinhalt gehören, zu detektieren und zu eliminieren. Die Summe dessen, was an der Forschungsfront entsteht, kann dazu beitragen, mathematische Bewertungsmodelle zu entwickeln, deren Resultate simulierbar werden. Damit ließen sich erstmalig Bewertungsergebnisse validieren und vergleichen. Eine weitere Entwicklungsmöglichkeit besteht darin, die skizzierten Systeme zur Sichtbarmachung von Bildern innerhalb der Flutwelle zu verwenden. Das heißt, Maschinen identifizieren auf-

33 Albert Weichselbraun-Daniel Streiff-Arno Scharl, Consolidating Heterogeneous Enterprise Data for Named Entity Linking and Web Intelligence. International Journal on Artificial Intelligence Tools. Architectures, Languages, Algorithms. AI Theory, AI Tools, AI Applications 24/2 (2015), http://dx.doi.org/10.1142/ S0218213015400084 [1. 3. 2018]; Arno Scharl et al., Semantic Systems and Visual Tools to Support Environmental Communication. IEEE Systems Journal 11 (2017) 762-771.

34 Albert Weichselbraun-Philipp Kuntschiк, Mitigating Linked Data Quality Issues in KnowledgeIntense Information Extraction Methods, in: Proceedings of the 7th International Conference on Web Intelligence, Mining and Semantics. Amantea, Italy, June 19-22, 2017, in: ACM Digital Library, https://dl.acm. org/citation.cfm?id=3102272 [1. 3. 2018]. Siehe auch die Projektwebsite: IMAGINE (Cross-modal information extraction for improved image meta data), in: HTW Chur, Hochschule für Technik und Wirtschaft, http://www.htwchur.ch/digital-science/forschung-und-dienstleistung/institut-sii/projektuebersicht/imagine. html?sword_list=uq1ebkg0vv18m2\&no_cache=1 [1.3. 2018]. 
grund der vordefinierten Kriterien, Kontexte und Fragestellungen relevante Bilder und machen diese für die jeweilig Nutzenden sichtbar. Skartierung wird damit einem „Filter“ gleichgesetzt.

Vieles, was heute in Archiven als „Aufgabe der Forschung“ verstanden wird, beispielsweise die Datierung oder die Verortung von Bildinhalten auf Objektebene, wird künftig bei digitalen Bildprodukten aufgrund verschiedenster Metadaten durch Maschinen ergänzt und vor allem plausibilisiert werden können. Schon heute bieten technische Metadatenstandards für die herstellenden Firmen die Möglichkeit, Signaturen oder Seriennummern für einzelne Geräte zu vergeben, oder sie sind ohne das Wissen der Kundschaft längst implementiert. So können Bilderproduzierende Datensätze ihrer Kameras direkt zuordnen, Agenturen identifizieren die Ablieferung der für sie fotografierenden Personen aufgrund von Logins, Seriennummern, Zeitstempeln und Georeferenzierung nahezu automatisch. Die Herkunftsbestimmung digitaler Bilddaten ließe sich auch im Archiv unter Hinzuziehung der klassischen Überlieferungsdokumentationen wesentlich vereinfachen. Freilich, eingebettete Metadatenstandards wie IPTC und EXIF lassen sich manipulieren, das ist kein Geheimnis. Nichtsdestoweniger lassen sich eingebettete Metadaten zur Steuerung der Datenströme einsetzen ${ }^{35}$. Des Weiteren ist bekannt, dass digitale fotografische Apparate beziehungsweise deren Bildsensoren charakteristische Muster aufweisen, welche mit einem individuellen Duktus vergleichbar sind. Diese lassen sich mittels statistischer Häufigkeitsverfahren analysieren und bestimmen. Mit zunehmender Rechenleistung sind solche Prüfmethoden keine Utopien. Ob die intellektuelle Plausibilisierung digitaler Bilddaten in Verbindung mit ihren Metadaten besser ist, sprich die Reliabilität der Ergebnisse eine geringere Fehlerquote aufweist als die maschinell durch Algorithmen erzeugte, wird man künftig messen können.

Von zentraler Bedeutung ist hier jedoch, auf die Notwendigkeit der Modellierung hinzuweisen. Damit man künftig Bewertungsalgorithmen entwickeln kann, benötigt es repräsentative, mustergültig verzeichnete Test- und Lerndatenkorpora, welche den archivarischen Gepflogenheiten entsprechen ${ }^{36}$. Sie sollen die wichtigsten Mediengattungen wie Fotografie, Video, Audio und Texte beinhalten. Ihre Organisationsstrukturen sollten sowohl das Provenienzprinzip berücksichtigen als auch pertinente Strukturen aufweisen. Damit sei gesagt, dass Organisationsstrukturen öffentlicher Verwaltungen bis hin zu Nachlässen abzubilden sind. Werden die Daten zudem semantisch ausgezeichnet, lassen sich kontextuelle Bezüge technisch detektieren, die Ergebnisse bemessen und damit vergleichen. Die Vision besteht also darin, mathematische Lösungen zu entwickeln, welche uns qualitative Hinweise bezüglich der Authentizität, der Integrität sowie der Originalität von exemplarisch beschriebenen Beständen vor und nach einem Bewertungsprozess liefern. Damit entstehen nachvollziehbare Handlungsalternativen, welche später auf große Massen anwendbar sind. Die modellhafte Überlieferungsbildung ist damit vollständig und nachvollziehbar dokumentierbar. Sämtliche Entscheidungen sind transparent darzustellen, wie dies Vogt-O’Connor mit dem Sammlungssurvey deutlich machte. Aus heu-

35 Ein Blick auf die Bewegungsdaten, welche durch Smartphones entstehen und Konzerne wie Google zur Berechnung der aktuellen Verkehrssituation einsetzen, beziehungsweise auf die Möglichkeit, Staus aufgrund der gespeicherten Daten vorherzusagen, lässt künftige Szenarien für die Datenmodellierung erahnen.

36 Mit dem yfcc100m besteht ein erstes, frei zugängliches Datenset von über 99 Millionen Bildern, welches durch Multimedia Commons erweitert wurde. Dieses Korpus spiegelt die archivarische Realität beziehungsweise aktuellen Anforderungen jedoch in keiner Weise wider: yfcc100m Browser, http://www.yfcc100m.org/? [1.3. 2018]; multimedia commons, https://multimediacommons.wordpress.com/ [1.3. 2018]. 
tiger Sicht ist das knapp skizzierte archivarische Testkorpus ein notwendiges technisches Desiderat, um weiterführende Entwicklungen innerhalb der Bewertungsfragestellung von digitalen Bildern voranzutreiben.

Die Historikerin oder der Historiker 2.0 wird vermutlich dieselben quellenkritischen Fragen stellen, wie wir das heute tun. Kurz: Wer hat was, wo, wann und wie, wozu, unter welchen Bedingungen getan? Was sie unterscheiden wird, ist die Kompetenz, digitale Quellen aufgrund ihrer spezifischen Eigenschaften zu befragen. Es ist offensichtlich: Wenn „wer“, „wann“ und „wo“ nicht beantwortbar sind, das heißt diese Daten weder dokumentiert noch erforschbar sind, so sind Bildinformationen nicht anschlussfähig an andere Bilder, an die jeweiligen Kontexte, in denen diese Bilder ihre Wirkung entfaltet hatten. Hierbei wurde schon skizziert, dass einige dieser Fragen technisch beantwortet werden können, aufgrund der Menge und der Komplexität künftig beantwortet werden müssen. Das bedingt sowohl neues Fachwissen auf dem Gebiet der Dateiformat-, Metadaten- oder Hardware- und Softwaregeschichte im Allgemeinen, als auch die Fähigkeit, forensische Werkzeuge zur digitalen Quellenkritik einzusetzen, im Besonderen. Mit Blick auf digitale Bildmanipulationsmöglichkeiten durch Photoshop erscheint die Forderung nach einer umfassenden Bildkritik - als komplementäre Methode zur Textkritik - durchaus vertretbar. Kurz, ein neues historisch-technisches Verständnis ist gefordert. Die Ausoder Weiterbildung zum „surfenden“ Archivpersonal in der digitalen Bilderflut erscheint unumgänglich. Andernfalls besteht die Möglichkeit, die historische als auch die archivarische Kernkompetenz künftig in der Informatik anzusiedeln. Tod der Mediävistik, lang lebe die digitale Medienarchäologie! So eine offensichtlich übertriebene Forderung, die plakativ skizziert, wohin sich die Ausbildung in der Geschichts- wie in der Archivwissenschaft entwickeln sollte. Was für ein Wagnis! 



\title{
Überlieferungsbildung und Erschließung im digitalen Bereich. Ein Arbeitsbericht aus dem Vorarlberger Landesarchiv
}

\author{
Markus Schmidgall
}

Die Wahrnehmung in der Öffentlichkeit - eine kurze Einleitung

Das Vorarlberger Landesarchiv in Bregenz darf seit seiner Gründung im Jahre 1898 auf eine nunmehr über 100-jährige Geschichte zurückblicken ${ }^{1}$. Diese Traditionslinie muss aber nun auch in Zeiten des Internets, des E-Mails und des elektronischen Verwaltungsakts fortgeschrieben werden. Welche Schwerpunkte in der Wahrnehmung des Vorarlberger Landesarchivs als sogenanntes landeskundliches Kompetenzzentrum gesetzt wurden beziehungsweise zukünftig zu setzen sind, soll im Folgenden kurz angerissen werden. Zunächst wird das Landesarchiv vorrangig durch die Beantwortung von Anfragen allgemeiner Art zur Vorarlberger Landesgeschichte wahrgenommen. Hierbei spielt es keine Rolle, zu welcher Epoche oder zu welchem Sachverhalt die Anfragenden an das Landesarchiv herantreten. In gleicher Weise spielt die Familienforschung im Zusammenhang mit bekannten Vorarlberger Biografien eine herausragende Rolle bei den an das Landesarchiv gerichteten Anfragen. In diesem Zusammenhang erfreuen sich insbesondere die online zugänglichen Pfarrmatriken ${ }^{2}$ aus den katholischen und evangelischen Pfarreien in Vorarlberg größter Beliebtheit. Einen weiteren großen Schwerpunkt in den Anfragen bilden die Häuser- und Grundstücksforschungen auf Basis der im Landesarchiv vorgehaltenen und mittlerweile verfilmten Verfachbücher, der Vorläufer der heutigen Grundbücher. In Verbindung damit stehen auch wirtschaftsgeschichtliche Aspekte rund um Gaststätten, Industriebetriebe oder den Tourismus. Weitere Themen werden durch die im Landesarchiv integrierte Musiksammlung, durch zahlreiche Nachlässe oder die übernommenen Gemeinde- und Pfarrarchive abgedeckt. Die letztgenannten Bestände bilden innerhalb des Landesarchivs insofern eine Besonderheit, als sie zu Beginn des 20. Jahrhunderts aus konservatorischen Gründen ins Haus gelangten und heute oftmals die einzige Überlieferung von Gemeinden oder Pfarrgemeinden darstellen.

\footnotetext{
${ }^{1}$ Zur Geschichte des Vorarlberger Landesarchivs vgl. Archiv und Geschichte. Festschrift 100 Jahre Vorarlberger Landesarchiv, hg. von Karl Heinz Burmeister-Alois NiederstäTter (Forschungen zur Geschichte Vorarlbergs N. F. 3, Konstanz 1998).

2 Vorarlberger Personenstandsbücher online, http://www.vla.findbuch.net/ [1. 3. 2018].
} 


\section{Die digitale Überlieferungsbildung}

Ausgehend von den vorgenannten thematischen Schwerpunkten wurden in der Vergangenheit Bewertungsentscheidungen im Rahmen einer bisher überwiegend analog erfolgten Überlieferungsbildung getroffen. Hierbei bildeten naturgemäß die Registraturen die natürlichen Quellen zur Sicherung des historisch wertvollen Schriftguts. Insgesamt ist das Vorarlberger Landesarchiv primär für die Sicherung der Unterlagen des Amtes der Vorarlberger Landesregierung inklusive diverser nachgeordneter Dienststellen und

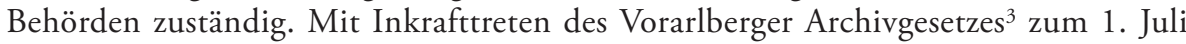
2016 wurde diese Aufgabe in einen gesetzlichen Rahmen gefasst. Im Gegensatz zur bisher praktizierten analogen Überlieferungsbildung trägt das Archivgesetz aber nun auch den Herausforderungen der Überlieferungsbildung im digitalen Bereich Rechnung. Dies ist notwendig, da bereits zur Mitte der 1990er Jahre in der Vorarlberger Landesverwaltung damit begonnen wurde, sogenannte „digitale Registraturen“ beziehungsweise später Dokumentenmanagementsysteme in die alltäglichen Arbeitsabläufe zu integrieren. Parallel zu diesen Entwicklungen wurde sowohl in Vorarlberg wie in ganz Österreich schrittweise auf den digitalen Akt nach einem einheitlich verwendeten Standard (ELAK) umgestellt. Mit dem digitalen Akt sollte zukünftig der althergebrachte „analoge“ Papierakt nahezu vollständig aus dem Arbeitsalltag verschwinden.

Der unbedingte Glaube an die Zukunft des digitalen Akts hatte um die Jahrtausendwende auch konkrete Auswirkungen auf die Baumaßnahmen des Vorarlberger Landesarchivs. Im Zuge eines angebahnten Magazinanbaus wurden die geplanten Kapazitäten nach den zu erwartenden Zuwachsraten von archivwürdigen Papierdokumenten ausgerichtet. Dementsprechend fiel der anvisierte Anbau für das Landesarchiv weitaus kleiner aus als zunächst beabsichtigt. In der Argumentation für diese Verkleinerung war von Seiten des Amts der Vorarlberger Landesregierung erkennbar, dass spätestens bis zum Jahre 2015 das sogenannte „papierlose Büro“ Wirklichkeit werden sollte. Zwischenzeitlich sind die damals neu hinzugekommenen Raumkapazitäten nahezu vollständig aufgebraucht. Allerdings ist keineswegs ein Ende der Übernahmen von Papierakten in Sicht. Es muss sogar festgehalten werden, dass der überwiegende Teil der Verwaltung dazu neigt, mehr Papier als jemals zuvor zu verwenden. Geheime Ablagen bei den einzelnen Sachbearbeiterinnen und Sachbearbeitern sowie hybride beziehungsweise parallele Überlieferungen sind die Folge. Im Angesicht einer dennoch propagierten Umstellung auf digitale Hilfsmittel wie etwa Dokumentenmanagementsysteme erschwert dies die eigentliche Überlieferungsbildung ungemein.

Mit Einführung der sogenannten digitalen Registratur in der Vorarlberger Landesverwaltung zur Mitte der 1990er Jahre wurde auch das Dokumentenmanagementsystem VOKIS eingesetzt. Zunächst nur in einigen wenigen ausgewählten Abteilungen eingeführt, wurde dieses System von der Abteilung Informatik im Amt der Vorarlberger Landesregierung letztendlich über alle Verwaltungsabteilungen inklusive des Vorarlberger Landesarchivs gespannt. Auf Basis der eGov-Suite der Firma Fabasoft aus Linz umfassten die ersten Versionen dieses Dokumentenmanagementsystems keine Module oder Applikationen zur Aussonderung beziehungsweise Archivierung von genuin digital erstellten Dokumenten oder Akten. In Kooperation mit der Abteilung Informatik im Amt der Lan-

3 Archivgesetz. Vorarlberger Landesgesetzblatt 1 (20. 1. 2016), Nr. 1, in: Rechtsinformationssystem RIS, https://www.ris.bka.gv.at/GeltendeFassung.wxe?Abfrage=LrVbg\&Gesetzesnummer=20000872 [1. 3. 2018]. 
desregierung konnte sich das Vorarlberger Landesarchiv aktiv in die Ausarbeitung und Implementierung eines Archivierungsmoduls einbringen. Dieses Modul ermöglichte es erstmals, von der jeweiligen Verwaltungsabteilung zur Aussonderung beziehungsweise Archivierung vorgesehene digitale Akten zu markieren und dem Landesarchiv digital zu „übergeben“. In weiterer Folge hatte das Landesarchiv nun die Möglichkeit, diese Akten als „archivwürdig“ oder als „nicht archivwürdig“ zu bewerten. Entsprechend der Bewertungsentscheidung „nicht archivwürdig“ wurden die digitalen Akten ohne ihre Metadaten aus dem operativen System gelöscht. Mit der Bewertungsentscheidung „archivwürdig“ versehen, verblieben die digitalen Akten zunächst in einem digitalen Zwischenarchiv, in welchem sie sowohl für die entsprechende Verwaltungsabteilung wie für das Landesarchiv einsehbar waren. An diesem Punkt fehlte letztendlich die korrekte Überführung der archivwürdigen digitalen Dokumente in ein digitales Archiv beziehungsweise Magazin, das nach dem OAIS-Modell definiert ist. Seit Mitte des Jahres 2015 ist in der Vorarlberger Landesverwaltung - wie auch in den Vorarlberger Gemeinden - das Nachfolgesystem V-DOK im Einsatz. Dieses gänzlich neu konzipierte Dokumentenmanagementsystem verfügt zum derzeitigen Stand noch nicht über ein entsprechendes Archivierungsmodul zur Aussonderung und Archivierung von digitalen Akten. Neben der anvisierten Implementierung eines solchen Moduls ist es das Ziel des Vorarlberger Landesarchivs, zukünftig die aufgezeigten Archivierungsprozesse in standardisierter Form in diesem System zu hinterlegen. Dies soll mithilfe von diversen Projekten realisiert werden.

\section{Die Probleme der digitalen Überlieferungsbildung}

Wie bereits angedeutet, ergeben sich aus der Einführung von digitalen Hilfsmitteln wie Registratur- oder Dokumentenmanagementsystemen für die digitale Überlieferungsbildung diverse Probleme. Mit der vollständigen Aufgabe von (zentralen) Kanzleistrukturen innerhalb der Landesverwaltung und der nachgeordneten Übertragung der Aufgaben von Registraturen beziehungsweise deren Personal an die Abteilungssekretariate zerfielen vielfach die bis dahin vorhandenen Strukturen innerhalb der Schriftgutverwaltung ${ }^{4}$. Erschwerend kommt hinzu, dass nunmehr die Aktenplanerstellung beziehungsweise -veränderung nicht mehr zentral exekutiert oder korrigiert wird, sondern von den jeweiligen Abteilungen vollzogen werden kann. Zusätzlich erschweren die hohe Fluktuation und die nicht selten eklatant schlechte Ausbildung des Personals der heutzutage zentral organisierten Abteilungssekretariate eine kontinuierlich unbefriedigende Schriftgutverwaltung. Dies hat beispielsweise zur Folge, dass nur mehr rudimentär oder vielfach auch gar nicht mehr Metadaten vergeben werden. Des Weiteren sind die gesetzlichen Rahmenbedingungen, denen die Abteilungen unterliegen, vielfach unbekannt oder werden geflissentlich ignoriert. Die vier herausragenden Kriterien Authenticity, Reliability, Integrity und Useability einer funktionierenden Dokumenten- beziehungsweise Schriftgutverwaltung, welche nach DIN ISO 15489-15 klar definiert sind, werden sehenden

\footnotetext{
${ }^{4}$ Vgl. Heinz Hoffmann, Behördliche Schriftgutverwaltung. Ein Handbuch für das Ordnen, Registrieren, Aussondern und Archivieren von Akten der Behörden (Schriften des Bundesarchivs 43, München 22000).

5 Allgemein vgl. Schriftgutverwaltung nach DIN ISO 15489-1. Ein Leitfaden zur qualitätssicheren Aktenführung, hg. von Alexandra Lutz (Kommentar: Information, Dokumentation, Berlin-Wien-Zürich 2012). Zugeschnitten auf Archive vgl. Zwischen analog und digital - Schriftgutverwaltung als Herausforderung für Archive. Beiträge zum 13. Archivwissenschaftlichen Kolloquium der Archivschule Marburg, hg. von Alexandra
} 
Auges umgangen. Auch ist festgeschrieben, dass Metadaten beizugeben sind und die vorhandenen Geschäftsprozesse für eine eindeutige Identifikation genau dokumentiert sein müssen.

Was bedeutet dies nun sowohl für Archive ${ }^{6}$ wie für Verwaltungen? Die Dokumentenverwaltung nach DIN ISO 15489-1 ist vor allem als Führungsaufgabe zu verstehen. Sie beinhaltet somit eine permanente Kontrolle der Einhaltung der einschlägigen Regelwerke (zum Beispiel Kanzleiordnungen) sowie die Verwendung von übergreifenden Systemen für die Schriftgutverwaltung. Gerade für den letzten Punkt ist ausdrücklich anzumerken, dass sich diese Systeme für eine funktionierende Schriftgutverwaltung nicht zwangsläufig auf den digitalen Bereich beschränken müssen. Im Zuge einer um sich greifenden Zusammenführung von verschiedenen Systemen für eine Schriftgutverwaltung ist eine funktionierende Schriftgutverwaltung auf analoger Basis unabdingbar. Gerade vor dem Hintergrund dieser beschriebenen Zusammenführungen ist auch die regelmäßige Ausbildung aller mit der Aktenführung beziehungsweise Dokumenten- oder Schriftgutverwaltung betrauten Mitarbeiterinnen und Mitarbeiter notwendig. Diese regelmäßige Ausbildung kann etwaige Fluktuationen beispielsweise im Bereich des Personals in den Sekretariaten abfangen oder gar ausgleichen.

\section{Die Agenda „Vorarlberger Landesarchiv 2015“ und ihre Folgen}

Parallel zu diesen Entwicklungen der Schriftgutverwaltung, welche auch in der Vorarlberger Landesverwaltung deutlich zu Tage traten, reagierte das Vorarlberger Landesarchiv seit dem Jahre 2010 auf diese Tendenzen mit einem konzentrierten Arbeitsprogramm. Mithilfe der sogenannten Agenda „Vorarlberger Landesarchiv 2015“ sollten innerhalb von fünf Jahren in verschiedenen Bereichen erste Rahmenbedingungen geschaffen werden, die es dem Landesarchiv und der Landesverwaltung erlauben würden, die Entwicklungen der digitalen Schriftgutverwaltung neu und positiv zu gestalten. Hierfür konnten fünf größere Bereiche identifiziert werden: Organisatorisches und Rechtsgrundlagen ${ }^{7}$; Personal; EDV und Digitales Langzeitarchiv; Erschließung (analog wie digital); sowie Raumkapazitäten. Im Folgenden soll insbesondere der Bereich „EDV und Digitales Langzeitarchiv" näher betrachtet werden. Innerhalb dieses Bereiches wurde im Jahre 2013 das Programm „Digitales Langzeitarchiv Vorarlberg“ initiiert. Neben dem Vorarlberger Landesarchiv ist hier insbesondere die Abteilung Informatik im Amt der Vorarlberger Landesregierung involviert. Das Programm wurde in mehrere Projekte unterteilt. Den Anfang bildete das Projekt A mit der Erarbeitung eines Organisationskonzeptes für ein digitales Langzeitarchiv. Hierin sollte zunächst der Ist-Zustand der Schriftgutverwaltung sowohl im analogen wie im digitalen Bereich analysiert werden. In einem folgenden Schritt waren allgemein gültige Normen und Standards der Schriftgutverwaltung für die Abläufe innerhalb der Landesverwaltung anzupassen und zu integrieren. Die Landesverwaltung

Lutz (Veröffentlichungen der Archivschule Marburg, Institut für Archivwissenschaft 49, Marburg 2009).

${ }^{6}$ Zur Bedeutung der Schriftgutverwaltung für Archive vgl. Robert Kretzschmar, Auf dem Weg in das 21. Jahrhundert: Archivische Bewertung, Records Management, Aktenkunde und Archivwissenschaft. Archivar. Zeitschrift für Archivwesen 63 (2010) 144-150.

7 In diesem Punkt kam es unter anderem zur Ausarbeitung des Vorarlberger Archivgesetzes und der neuen Archivordnung. 


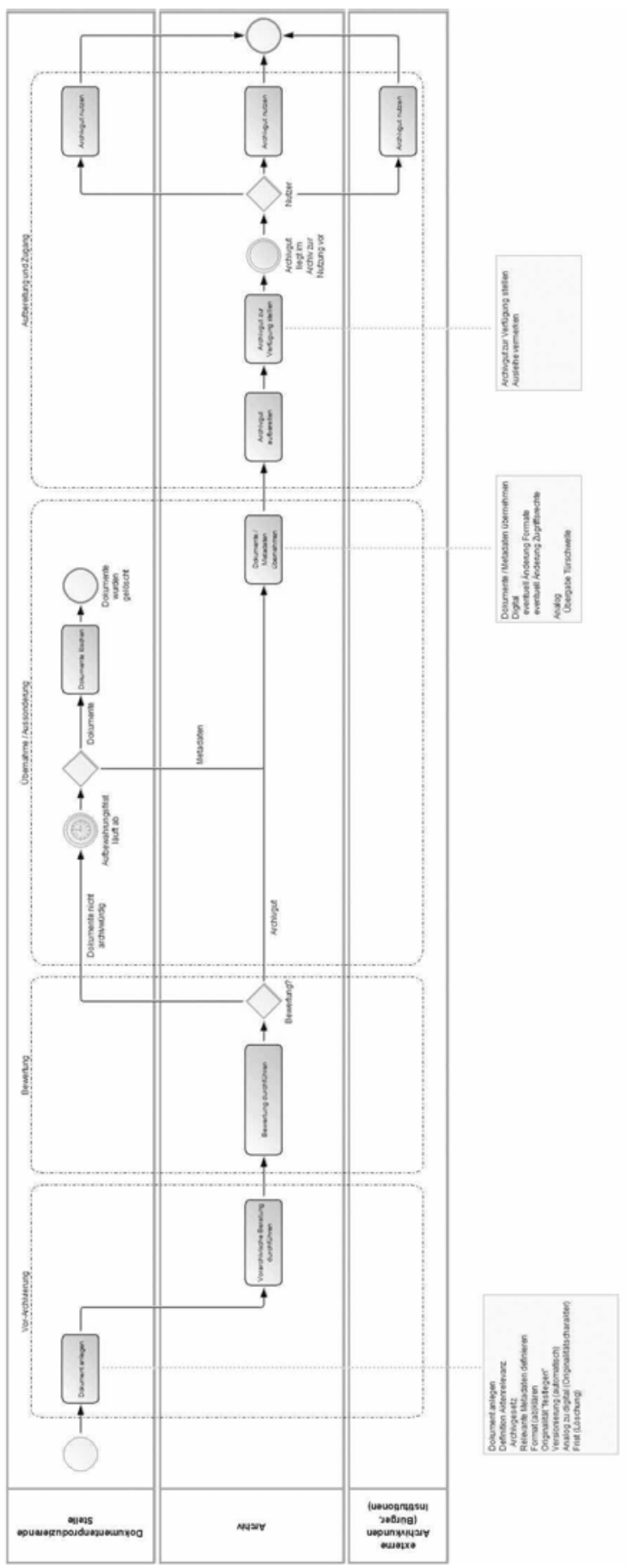

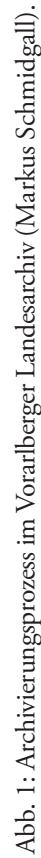


selbst hat als Aufgabe ein langfristig angelegtes Records Management ${ }^{8}$ gefordert. Des Weiteren wurden Übernahmen von als Archivgut bewerteten Unterlagen und Dokumenten aus der Landesverwaltung an das Landesarchiv standardisiert (Abb. 1). Wie bereits angedeutet, sind Arbeitsumstellungen und Prozessoptimierungen im Bereich der Schriftgutverwaltung permanente Aufgaben der Führungsebene. Somit ist ersichtlich, dass dieses Teilprojekt weit über das Jahr 2015 hinaus Bestand haben wird. Für das Vorarlberger Landesarchiv ist die Tätigkeit innerhalb dieses Teilprojektes die Chance, langfristig auf die Entwicklungen im Bereich der Schriftgutverwaltung einzuwirken. Die positiven Effekte, die sich daraus ergeben können, werden zukünftig die Übernahmen von landesgeschichtlich bedeutsamen Quellen erleichtern.

Neben einer sukzessiven Anpassung der sogenannten Behördenberatung - der prospektiven Bewertung und Übernahme von Schriftgut aus der Landesverwaltung - muss sich auch die Infrastruktur des Landesarchivs an die Gegebenheiten der Zeit anpassen. Auch wenn die Mitarbeiterinnen und Mitarbeiter des Landesarchivs in der Vergangenheit sehr gute Arbeit bei der Bewältigung ihrer Aufgaben geleistet haben, so muss dennoch konstatiert werden, dass sich gerade im Bereich der Behördenberatung sowohl auf Seiten der Landesverwaltung wie auf Seiten des Landesarchivs einige Baustellen angestaut haben. Ein Beispiel: Obwohl mit der Einführung von elektronischen Vorgangsbearbeitungssystemen in der Vorarlberger Landesverwaltung bereits vor über einer Dekade begonnen wurde, konnten diese Systeme bis heute nicht bis in alle Winkel der Verwaltung ausgerollt werden, so dass ein allumfassender Zufluss elektronischen Archivmaterials zukünftig wohl nicht gegeben sein wird. Die Gründe hierfür sind mannigfaltig: Technikphobie, fehlende Akzeptanz oder auch mangelnde Durchsetzung und Kontrolle durch Vorgesetzte haben einen Wildwuchs an heterogenen Arbeitsweisen entstehen lassen, der auch die Außenwirkung der Verwaltung nachhaltig schädigen kann. Ebenso ist der Öffentlichkeit die kostenintensive Anschaffung, Pflege und Wartung von solchen Vorgangsbearbeitungssystemen nur mehr schwer vermittelbar - insbesondere dann, wenn diese nicht umfassend eingesetzt werden. Demgegenüber erscheint die Einführung des neuen Vorgangsbearbeitungssystems V-DOK in enger Kooperation mit den Vorarlberger Gemeinden als neuer Hoffnungsschimmer. Es bleibt in diesem Zusammenhang zu hoffen, dass die Einführung Hand in Hand mit einer umfangreichen Schulung möglichst aller Mitarbeiterinnen und Mitarbeiter der Landesverwaltung einhergeht!

\section{Das Vorarlberger Archivinformationssystem}

Im Gegensatz zu diesem langfristig angelegten Projekt konnte das parallel initiierte Projekt B „Vorarlberger Archivinformationssystem“ recht zügig umgesetzt und abgeschlossen werden. Mit dem Projektbeginn wurde von verschiedenen Seiten der Wunsch geäußert, dass es zum Aufbau eines Archivinformationssystems (AIS) für das gesamte Land Vorarlberg kommen sollte. Dieses sollte demnach dem Land wie den Gemeinden zur Verfügung stehen. Im Angesicht der überschaubaren Größe des Landes Vorarlberg erachtete man eine solche Überlegung für sinnvoll. Weiter versprachen sich die

${ }^{8}$ Dazu ist von der Arbeitsgruppe Records Management des Verbandes Österreichischer Archivarinnen und Archivare ein Leitfaden erstellt worden: Christine Gigler-Barbara Kerb-Juliane Mikoletzky-Markus Schmidgall-Karin Sperl, Leitfaden Records Management. Einsatz und Gebrauch für Archive in Österreich (2017). Scrinium. Zeitschrift des Verbandes österreichischer Archivarinnen und Archivare 72 (2016) 155-190. 
Projektpartner" auch Synergieeffekte im vorarchivischen Bereich, da es bereits zu diesem Zeitpunkt zum Aufbau eines einheitlichen Dokumentenmanagementsystems im Land gekommen war.

Um gerade in den Punkten „Erschließung“ und „EDV und Digitales Langzeitarchiv“ den Anschluss an die fachlichen Entwicklungen nicht zu verpassen, entschloss sich das Vorarlberger Landesarchiv zur Evaluierung und Ausschreibung eines AIS. Dieses Vorgehen deckte sich mit einer Teilanforderung aus dem Programm „Digitales Langzeitarchiv Vorarlberg". Hierin wurde nach geeigneten Partnern für die Implementierung eines AIS gesucht. Nach einer fachlichen wie auch technischen Überprüfung konnten verschiedene Anbieter aus der Schweiz und aus Deutschland zu einer Produktpräsentation nach Bregenz eingeladen werden. Die Anbieter hinterließen allesamt einen qualitativ hochwertigen Eindruck. Nach einer mehrwöchigen Verhandlungsphase mit allen Anbietern und einem internen Abgleichungsverfahren bezüglich der technischen Machbarkeit und Umsetzung fiel die Entscheidung auf die Firma AUGIAS-Data aus Senden (Nordrhein-Westfalen). Die Firma AUGIAS-Data administriert derzeit rund 1.200 Archive vorwiegend im kommunalen Bereich des gesamten deutschsprachigen Raums. In Österreich zählt etwa das Niederösterreichische Landesarchiv ${ }^{10}$ zu den Kunden von AUGIAS. Das nunmehr ausgewählte Produkt „AUGIAS-Archiv“ bietet hierfür eine wichtige Komponente an. Neben dem Vorarlberger Landesarchiv, wo das AIS als Gesamtdatenbank fachlich administriert wird, wurden zusätzliche „Archivstandorte“ für die jeweils beteiligten Kommunalarchive eingerichtet. Diese sind aber keineswegs als „Filialarchive“ des Landesarchivs zu verstehen, sondern arbeiten vollkommen autonom und werden auch in den jeweiligen Kommunalarchiven betreut. Das Landesarchiv agiert lediglich als technischer Standortverwalter im Hintergrund. Mit dieser Standortstruktur ist es den beteiligten Archiven möglich, von verschiedenen Standorten aus allesamt ihre jeweiligen Datensätze in ein und dieselbe Datenbank einzuarbeiten und selbstständig zu verwalten. Hierbei können sowohl die Supervisorinnen und Supervisoren der einzelnen Standorte als auch alle anderen Archivmitarbeiterinnen und Mitarbeiter lediglich nur in ihrem jeweiligen Segment Datensätze anlegen, bearbeiten und für eine Recherche freigeben. Die Daten der anderen Beteiligten sind nicht einsehbar. Lediglich im Falle einer archivübergreifenden Recherche werden zur Fragestellung passende Rechercheergebnisse aus anderen Standorten angezeigt. Insbesondere diese übergreifende Suchfunktion für alle beteiligten Archive in Vorarlberg war das Hauptkriterium für den Erwerb eines AIS.

Bei allen Aufgabenstellungen und auch technischen Möglichkeiten ist aber ebenso seit der Einführung des AIS im Frühsommer 2014 darauf zu achten, dass einzelne Schritte sorgsam geplant und in Etappen vollzogen werden. Das Landesarchiv strebte keine überstürzte Einführung an, bei der im Anschluss an verschiedenen Baustellen gleichzeitig hätte gearbeitet werden müssen. Vielmehr wurden alle Mitarbeiterinnen und Mitarbeiter des Landesarchivs im Rahmen einer zweitägigen Schulung an die neue "Arbeitsumgebung" herangeführt. Ebenso konnten bereits die ersten zehn Anwendenden aus den beteiligten Kommunalarchiven mit der Unterstützung von AUGIAS-Data geschult werden. Wei-

9 An diesem Projekt waren das Vorarlberger Landesarchiv, der Arbeitskreis Vorarlberger Kommunalarchive, die Abteilung Informatik im Gemeindeverband Vorarlberg und die Abteilung Informatik im Amt der Vorarlberger Landesregierung beteiligt.

${ }^{10}$ Landesarchiv, in: Land Niederösterreich, http://www.noe.gv.at/Bildung/Landesarchiv-.html [1. 3. 2018]. Weitere Kunden in Österreich sind beispielsweise das Oberösterreichische Landesarchiv in Linz und das Stadtarchiv Innsbruck. 


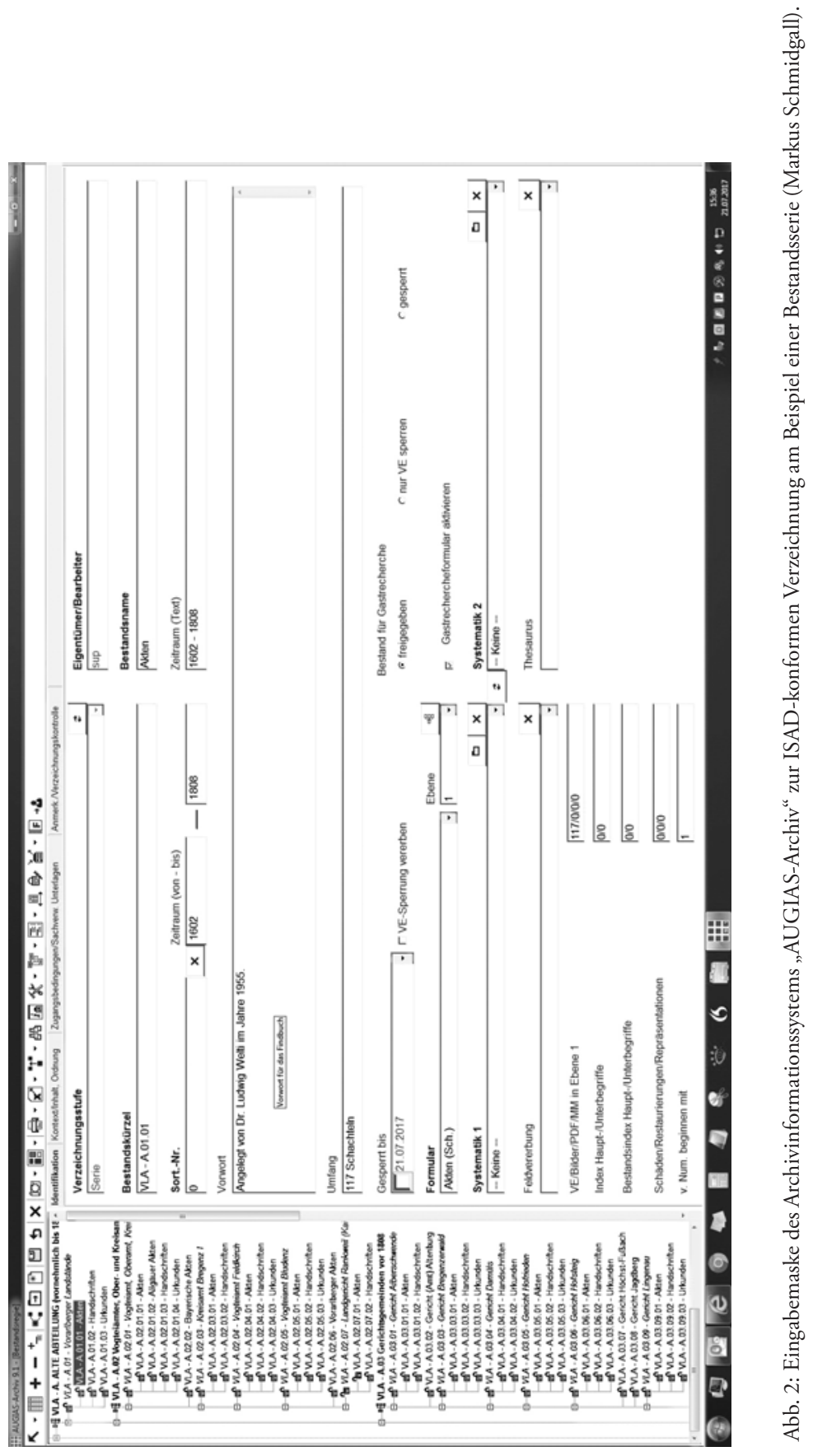


tere Funktionalitäten und Updates werden im laufenden Betrieb eingeführt und umgesetzt. Kontinuierliche Unterstützungsmaßnahmen auch von Herstellerseite rundeten die Einführungsphase ab. Insgesamt war es das Ziel des Landesarchivs, dass die eigentlichen Begünstigten dieser Neuerung - die Benützenden der Archive - keinen Qualitätsverlust bemerken. Gerade bei der Beantwortung von Anfragen sowohl per E-Mail als auch vor Ort im Lesesaal hofft das Landesarchiv auf eine spürbare Beschleunigung der Bearbeitung. Möglich machen soll dies die Bestände übergreifende Suche innerhalb des AIS, welche zunächst nur den Mitarbeiterinnen und Mitarbeitern des Landesarchivs zur Verfügung stehen wird. In einer zukünftigen Ausbaustufe sollen die Benützenden nicht nur vor Ort im Lesesaal, sondern auch am heimischen Computer die Möglichkeit zur selbstständigen Suche in den Beständen des Landesarchivs und der beteiligten Kommunalarchive haben. Aufgrund der gemeinsamen Plattform eines landesweit ausgerollten Archivinformationssystems kommen die Archive in Vorarlberg diesem Ziel nun einen ersten Schritt näher. Zukünftig wird die gemeinsame Abstimmung und Zielsetzung etwa in den Bereichen der Erschließung und der Präsentation zwischen Land und Kommunen noch enger sein.

\section{Die Aufgabe: (Neu-)Erschließung}

Inwieweit betrifft dies nun das Vorarlberger Landesarchiv? Das Landesarchiv steht per Definition am Ende jedes Lebenszyklus eines Akts ${ }^{11}$. Das Landesarchiv verwahrt darüber hinaus bis heute umfangreiche Bestände der so genannten Landesregistratur, also Schriftgut, welches noch nicht als Archivgut übernommen wurde. Ebenso finden sich im allgemeinen Hausarchiv des Landhauses umfangreiche Altbestände an Akten aus nahezu allen Abteilungen der Landesverwaltung, welche weder Teil der Landesregistratur sind noch einer eingehenden Bewertung durch das Landesarchiv unterzogen wurden. Nicht selten reichen einzelne Akten bis in die 1950er Jahre zurück. Auch unterhalten einzelne Abteilungen eigene „Hausarchive“, welche über das gesamte Landhaus verstreut in Heizungskellern oder anderen völlig ungeeigneten Räumen untergebracht sind. Diese Altbestände in Zusammenarbeit mit den jeweiligen Abteilungen spürbar zu verringern, bleibt eine der Hauptaufgaben der Abteilung Dokumentenverwaltung im Landesarchiv. Demgegenüber erscheint die schiere Masse an Unterlagen auch bei einer konsequent durchgehaltenen Bewertungsquote von unter zwei Prozent überwältigend.

An diesem Punkt stand das Vorarlberger Landesarchiv vor der Entscheidung zwischen einem „Weiter so ...“ oder der Suche nach Möglichkeiten zur besseren Bewältigung zumindest der zeitnah anstehenden Übernahmeprozesse. Ein AIS bietet hierzu vielfältige Möglichkeiten bei der Übernahme von Altbeständen aus der Landesverwaltung. Mithilfe einer schrittweisen Erfassung und Verzeichnung der bisher übernommenen und erschlossenen Bestände der Landesverwaltung ist es beispielsweise möglich, die bevorstehenden Übernahmen zukünftig standardisiert zu planen und abzuwickeln. Hierbei werden ebenso räumliche wie personelle Rahmenbedingungen mit in die Planungen einbezogen. Parallel zu den Erschließungsmaßnahmen im Bereich der Landesverwaltung müssen auch die Verzeichnungsmaßnahmen von Beständen aus der so genannten „Alten Abteilung“ des Landesarchivs weiter vorangetrieben werden. Hier handelt es sich um Schriftgut, welches von den ersten Überlieferungen in Form von Urkunden etwa aus dem Bestand des

\footnotetext{
11 Archivgesetz (wie Anm. 3), \$3.
} 
Klosters Mehrerau aus dem 12. Jahrhundert bis in das Jahr 1850 reicht. Im Angesicht der politischen und der damit in Verbindung stehenden administrativen Umwälzungen zur Mitte des 19. Jahrhunderts bildet dieses umfangreiche Schriftgut das „alte Vorarlberg“ ab. Neben der Alten Abteilung ergänzen die Bestände des nichtstaatlichen Archivguts (unter anderem Gemeinde- und Pfarrarchive, Nachlässe oder auch Parteienarchive) und Sonderbestände beziehungsweise Sammlungen (Fotos, Karten, Siegel, Wappen et cetera) die Überlieferung der Vorarlberger Geschichte und damit das kulturelle Erbe des Landes. Sukzessive werden all diese Bestände mit ihren reichhaltigen Überlieferungen in das Archivinformationssystem eingearbeitet und dauerhaft gepflegt (Abb. 2). Neben der bloßen Verzeichnung dieser Bestände können parallel auch Restaurierungsmaßnahmen oder Reproduktionsaufträge detailliert geplant werden. Ebenso kann mithilfe des Archivinformationssystems das Magazin des Landesarchivs, welches sich seit dem Anbau aus dem Jahr 2002 über acht Stockwerke erstreckt, Bestände übergreifend verwaltet werden.

\section{Die Standardisierung mit ISAD $(\mathrm{G})$}

"Standardisierung heißt, dass man Theorie und Best Practices nicht immer neu,erfinden' muss. Standards sind das Ergebnis von reflektiertem Berufswissen. Sie erlauben eine Vereinheitlichung, Verbesserung und Erleichterung der archivischen Arbeit, mithin die Professionalisierung der archivischen Tätigkeit"12. In diesem Zusammenhang ist nochmals auf den Begriff der Standardisierung und Professionalisierung im Archivwesen einzugehen. Vor rund einem Jahrzehnt haben die Mitarbeiterinnen und Mitarbeiter des Vorarlberger Landesarchivs damit begonnen, dessen Bestände im Internet abrufbar zu machen. Parallel zogen auch einzelne Kommunalarchive in Vorarlberg nach. Österreichweit gehörte Vorarlberg damit zu den Vorreitern. In der Zwischenzeit haben das Österreichische Staatsarchiv in Wien, alle österreichischen Landesarchive und auch einige größere Kommunalarchive nachgezogen und ihre Bestände im Internet präsentiert. Um es gleich vorwegzunehmen: Diese Entwicklungen stellen ausdrücklich keinen Bieterwettbewerb nach dem Motto „Wer hat den schönsten Internetauftritt?" dar, sondern ein standardisiertes und damit professionelles Angebot an unsere „Kundinnen und Kunden“, die Benützenden. Die Zeit der Geheimarchive gehört längst der Vergangenheit an und sollte tunlichst auch nicht wiederbelebt werden. Zugänglichkeit, Transparenz und Professionalität in allem, was wir tun, ist der Dreiklang eines modernen Archivbetriebs. Das neue Archivinformationssystem wird das Vorarlberger Landesarchiv zukünftig in diesem Anspruch weiter unterstützen und voranbringen. Dieser Leitgedanke, welcher aus den Umsetzungsempfehlungen zu ISAD $(G)$ des Verbandes Österreichischer Archivarinnen und Archivare (VÖA) entlehnt ist, vermittelt einen Eindruck von den Herausforderungen und Aufgaben, denen sich das Vorarlberger Landesarchiv mittelfristig verstärkt widmen wird.

Was bedeutet aber in diesem Zusammenhang „Standardisierung“ und mit welchen Hilfsmitteln kann das Landesarchiv diese erreichen? Und welche Aufgaben erwachsen dem Archiv hieraus? Nicht erst seit den einschlägigen Publikationen aus der jüngeren

12 Karin SperL et al., Umsetzungsempfehlungen zu ISAD $(G)$ und ISDIAH. Scrinium. Zeitschrift des Verbandes Österreichischer Archivarinnen und Archivare 68 (2014) 113-179, hier 117; vgl. ISAD(G) - Internationale Grundsätze für die archivische Verzeichnung, übers. und hg. von Rainer BrüNING-Werner HeEgEWALDTNils BRÜвасн (Veröffentlichungen der Archivschule Marburg, Institut für Archivwissenschaft 23, Marburg 32006). 
Vergangenheit werden die Vor- und Nachteile einer einheitlichen beziehungsweise standardisierten Erschließung nach international vereinbarten Grundsätzen im Archivwesen breit diskutiert. Es stellt sich hierbei die Frage, welchen Nutzen ein einheitlich erschlossenes Archivgut für unsere „Kundinnen und Kunden“ hat: die Menschen, die zu wissenschaftlichen und auch zu nichtwissenschaftlichen Zwecken die Archive besuchen. Daran anschließend wird unweigerlich die Frage an die Archive herangetragen, ob der hierfür notwendige personelle wie finanzielle Aufwand in einem vernünftigen Verhältnis zu den daraus resultierenden Ergebnissen steht. Nach einer Untersuchung im Brandenburgischen Landeshauptarchiv in Potsdam kann es durchschnittlich bis zu 53 Jahre (!) dauern, ehe erschlossenes oder digitalisiertes Archivgut erstmals wieder von den Nutzern im Lesesaal angefordert wird ${ }^{13}$. Bei dieser Zahl wird deutlich, dass die Entscheidungen für oder auch gegen konkrete Erschließungsmaßnahmen oftmals mehrere Generationen überdauern können. Gleichwohl bieten die vereinbarten Grundsätze zur standardisierten Erschließung von Archivgut auch Möglichkeiten zum zielgerichteten Umgang mit Massenschriftgut. Diese „Massenerschließung“ kann natürlich auch dem Kreis der wissenschaftlichen, aber eben auch der nichtwissenschaftlichen Benützenden im Archiv zugutekommen. Gerade im Hinblick auf die immer breitere Ausrichtung der Geschichtswissenschaften bieten schnelle und einfache Zugänge zu ganzen Beständen viele neue Möglichkeiten zu Forschungsansätzen. Aber ist dies im Sinne des Erfinders? Überfordert eine breitere, ja oftmals aufgeblähte Überlieferung die Benützenden am Ende nicht doch? Ist eine Konzentration auf bestimmte Fragestellungen überhaupt möglich, wenn wir als Archive immer mehr und komplexere Überlieferungszusammenhänge anbieten? Und wird die Komplexität nicht noch erhöht, wenn wir standardisierte Daten in vielerlei Portalen anbieten? Auch wenn die spezielle Situation der Überlieferung in Vorarlberg nicht unbedingt mit anderen österreichischen Landesarchiven zu vergleichen ist, so zeigt sie doch die besondere archivische Verzahnung auf Landes- wie Kommunalebene in Vorarlberg.

Wohl auch aus diesem Grund kam es aufgrund einer Anregung von Seiten des Arbeitskreises Vorarlberger Kommunalarchive im Sommer 2012 zur Bildung einer Arbeitsgruppe mit Landes- und Kommunalarchivarinnen und -archivaren. Ziel dieser Arbeitsgruppe ist es, die Vereinheitlichung der Erschließung nach ISAD $(G)$ weiter voranzutreiben. Neben der gegenseitigen Hilfestellung sollen auch Vorbereitungen für eine angedachte zukünftige gemeinsame Präsentation aller Vorarlberger Archivbestände getroffen werden. Denkbar wäre darüber hinaus auch die Kooperation mit dem Diözesanarchiv Feldkirch ${ }^{14}$ und dem Vorarlberger Wirtschaftsarchiv ${ }^{15}$. Einmal mehr wird bei bisher allen gemeinsamen Arbeiten ersichtlich, dass gerade in einem so kleinen und übersichtlichen Bundesland wie Vorarlberg „Insellösungen“ oder Ähnliches als nicht mehr zeitgemäß empfunden werden. Gemäß dem zitierten Credo waren sich alle Beteiligten einig, dass aufgrund der jahrelangen Erfahrungen nach den bestmöglichen Lösungen für gemeinsame Problem- und Fragestellungen gesucht werden müsse. Ausdrücklich sollte es aber nicht zu einer generellen Nivellierung der Vorarlberger Archive kommen. Archivische Alleinstellungsmerkmale gilt es auch weiterhin zu betonen, zu behaupten und auszubauen.

13 Mario Glauert, Dimensionen der Digitalisierung. Kosten, Kapazitäten, Konsequenzen, in: Digital und analog. Die beiden Archivwelten. 46. Rheinischer Archivtag, 21.-22. Juni 2012 in Ratingen. Beiträge, hg. von Claudia Kauertz (Archivhefte 43, Bonn 2013) 48-59, hier 54.

14 Archiv der Diözese Feldkirch, http://www.kath-kirche-vorarlberg.at/organisation/archiv [1. 3. 2018].

15 Wirtschaftsarchiv Vorarlberg, http://wirtschaftsarchiv-v.at/ [1.3. 2018]. 
Die erwähnte Umsetzungsempfehlung der VÖA-Arbeitsgruppe zur standardisierten Erschließung nach ISAD $(\mathrm{G})$ darf als höchst lohnenswerte Hilfestellung im österreichischen Archivwesen bezeichnet werden. Die Empfehlung zielt hierbei nicht nur auf einheitliche Erschließungsmaßnahmen ab, sondern bezieht sich bereits auch auf die mögliche einheitliche Präsentation der Ergebnisse. Gleichzeitig berücksichtigt ISAD $(G)$ bisher verwendete Verzeichnisstrukturen und ergänzt sie in sinnvoller Weise. In Verbindung mit dem EAD-Standard erreicht ISAD $(\mathrm{G})$ sein volles Wirkungsspektrum, wenn verschiedenste Bestände oder ganze Archive miteinander verknüpft werden. Technisch bieten diese Standards sehr viele Möglichkeiten, die bereits in der einen oder anderen Form auch in Österreich erfolgreich umgesetzt werden konnten. Die Reaktionen der einschlägigen Benützendengemeinden waren überwiegend positiv. Der rege Austausch von positiven wie negativen Kritiken zeigt, dass auch diese „moderne“ Form des wissenschaftlichen Austausches ihren berechtigten Platz zwischen Konferenzen, Kolloquien, altehrwürdigen Editionsreihen und dergleichen gefunden hat.

Andererseits böten sich gerade eine standardisierte Erschließung und die damit einhergehenden Maßnahmen für eine Priorisierung innerhalb eines Archivs an. Hier ist ein reger Austausch zwischen Archiven und der Forschung gefordert. Oftmals werden groß angelegte Erschließungsmaßnahmen (vorzugsweise im Rahmen von Gedenkjahren oder Ähnlichem) ohne vorherige Konsultation der Forschung angestrengt. Ebenso initiiert die Forschung Projekte unabhängig vom jeweiligen Erschließungsstand der in Frage kommenden Bestände. Relevante Archivbestände bleiben unberücksichtigt, was am Ende eines Forschungsvorhabens die Beteiligten nicht selten noch weiter voneinander entfernt von den daraus resultierenden Mehrarbeiten ganz zu schweigen. Ein Austausch über Forschungsvorhaben findet de facto nicht statt. Die Klärung der archivischen Arbeiten im Rahmen eines universitären Forschungsprojekts sollte in stärkerem Maße als zwingende Voraussetzung behandelt werden.

Bleibt die Frage, ob die Verwendung der Standards in einem ausgewogenen Verhältnis zum hierfür nötigen Arbeitsaufwand steht. Bei aller möglichen Entlastung durch diese neuen technischen Hilfswerkzeuge sind unsere archivischen Grundaufgaben wie Erschließung und Nutzbarmachung als vorrangig zu betrachten. Eine Vielzahl von Portallösungen im Stile Potemkin'scher Dörfer bleibt in Zeiten knapper Kassen der breiten Öffentlichkeit schwer vermittelbar und trägt nur bedingt zur Verbesserung des Bildes unseres ehrbaren Berufsstandes bei. Demgegenüber mehren Zugänglichkeit, Offenheit und Fachwissen unser Ansehen bei unserer überschaubaren, aber treuen Klientel. In diesem Sinne kann eine Priorisierung von standardisierten Erschließungsmaßnahmen auch den Fokus auf besonders hervorzuhebende Bestände oder Archivalien lenken. Allem Anschein nach sind erste Zugangs- und Anpassungsschwierigkeiten der Benützenden mit einer tiefgreifenden Standardisierung sehr wohl überwindbar. Viele Archive gehen bei der Bewältigung ihrer Aufgaben andere Wege und reaktivieren zum Beispiel wieder allgemein zugängliche Paläografiekurse oder erläutern die Zusammenhänge der Verwaltungsorganisation des späten 19. Jahrhunderts anhand ereignisreicher Bau- oder Polizeiakten. Da sich viele solcher Maßnahmen in der Praxis bewährt haben, wäre auch die Ausarbeitung einer Hilfestellung im landesweiten Kontext denkbar. Der Ehrlichkeit halber muss es den Benützenden aber auch vielfach zugemutet werden, sich selbstständig in komplexere Fragestellungen einzuarbeiten. Das Vorarlberger Archivinformationssystem bietet den zukünftigen Benützenden hierfür eine Hilfestellung. 


\section{INFORMATIONSAUFBEREITUNG UND VERMITTLUNG}





\title{
Leuchtturm oder GPS - welche Orientierung in der Infosphäre?
}

\author{
Andreas Kellerhals
}

Die Anfrage zu diesem Beitrag zur Zukunft der Vergangenheit im Informationszeitalter dank Archiven als Leuchtfeuern erreichte mich im Sommer während der Ferienzeit. Entsprechend waren die Zeitungen voll von redaktionell kaschierter Tourismuswerbung ${ }^{1}$. Zufälligerweise las ich etwas über das portugiesische Dorf Cascais und „Santa Marta, de[n] unverdrossene[n] Leuchtturm“; früher betrieben „drei Wärter den Leuchtturm in achtstündigen Schichten [...]. Längst orientieren sich die Frachter an GPS und Satelliten, trotzdem brennen die Leuchttürme weiter; die Seeleute wollen das so. Wenn einer ausgehe, $[. .$.$] riefen sie von den Schiffen sofort an" - soweit die Journalistenpoesie { }^{2}$. Die Türme sind funktional auf eine Orientierungshilfe beim Ausfall des GPS reduziert, aber sie sind auch zu „Sehnsuchtsorte[n]“ geworden ${ }^{3}$.

Die Analogie zur Erschließungsarbeit der Archive schien mir offensichtlich: In beiden Fällen geht es um letztlich obsolete Orientierungstechniken, an denen aber aus Gründen der Nostalgie festgehalten wird. Ich werde im Folgenden dieses Spannungsverhältnis zwischen dem traditionellen Findbuch und den neuen Archivinformationssystemen (AIS) als unterschiedlichen Produkten der Erschließung ausloten ${ }^{4}$.

Die herkömmlichen Findbücher als rein symbolische Orientierungshilfen unterscheiden sich zwar von den neuen AIS als praktischen Orientierungsinstrumenten, aber beide sind im übertragenen Sinne Sehnsuchtsorte für Archivare und Archivarinnen, das Findbuch ein nostalgischer, das AIS als dessen Gegenpol ein utopischer.

Wie zukunftsweisende Orientierungssysteme, Archivinformationssysteme als ArchivGPS, aussehen sollen, zeichnet sich allmählich ab; unsere Auseinandersetzung mit de-

${ }^{1}$ An Stelle geschlechterneutraler Formulierungen wechseln hier männliche und weibliche Formen ab. Für alle Stellen, die einen bibliographischen Nachweis verdienten, aber keinen haben, verweise ich auf Georg Christoph Lichtenberg: „ICh vergesse das meiste, was ich gelesen habe, [...]; ich weiß aber soviel, [es] trägt nichtsdestoweniger zur Erhaltung meines Geistes [...] bei. 'Sudelbücher Heft J (133)“ (Art. Georg Christoph Lichtenberg, in: Wikiquote, https://de.wikiquote.org/wiki/Georg_Christoph_Lichtenberg [1. 3. 2018]).

2 Jean-Martin Büttner, Das absolute Meer. Der Bund (20. 6. 2016) 31.

3 Walter JäGGI, Lichterlöschen im Leuchtturm. Der Bund (10. 8. 2016) 29: „Auf Ferienfotos, Postkarten und Reiseprospekten machen Leuchttürme nach wie vor eine gute Figur. In der Schifffahrt aber verschwinden sie buchstäblich vom Radar".

4 Vergleichbare Spannungsverhältnisse prägen auch andere archivische Tätigkeitsfelder, von der Entstehung und Sicherung von (digitalen) Informationen bis zu deren Vermittlung im virtuellen Lesesaal mit der Arbeit mit Quellen in ausschließlich digitaler respektive digitalisierter Form ohne weiteren Zugang zum Papier, vielleicht - horribile dictu - sogar bis zur Digitalisierung mit anschließender Vernichtung des Papiers. 
ren Gestaltung hat also ein elementar kreatives Element. Erschließung heute ist als das Ausprobieren neuer Ansätze nicht nur das Arbeiten an der Sache, sondern immer auch eine Arbeit am Instrument und dessen (Weiter-)Entwicklung. Die Thematik situiert sich in allgemeiner Perspektive an der heutigen Bruchlinie zwischen traditionellem „on-site“und virtuellem „on-line“-Archiv.

Interessant ist, dass das Interesse der archivtheoretischen Diskussion an Erschließung ebenfalls wiedererwacht ist, nachdem Erschließungsfragen seit den 1950er Jahren, angesichts des exponentiellen Wachstums der Archivbestände, durch Bewertungsfragen in den Hintergrund gedrängt worden waren ${ }^{5}$. Die Diskrepanz zwischen exponentiellem Bestandeswachstum und entsprechend rasant zunehmendem Erschließungsrückstand kulminiert in der Feststellung: „Cataloguing is a function which is not working "6. Hier gilt es mittels neuer Erschließungsansätze einen Ausweg zu finden, um wenigstens den Erschließungsrückstand nicht weiter anwachsen zu lassen, wenn möglich diesen sogar abzubauen.

Wenn angesichts sinkender Speicher- und zu verantwortender Erhaltungskosten die Möglichkeit einer Totalüberlieferung wieder geprüft werden muss, könnte die Funktion der letztlich reversiblen und laufend erweiterbaren Überlieferungsformung durch Erschließung wieder in den Vordergrund treten. Sie ersetzte dann die irreversible, aber möglicherweise nicht zwingend zielführende Überlieferungsbildung durch Selektion und Vernichtung. In einem neuen informationstechnischen Kontext bedarf es eben nicht zwingend der Bewertung und Kassation, um die „Menge der Aufzeichnungen auf ein handhabbares Maß“ zu reduzieren, um das, was „für die Auswertung wertvoll sein könn[te]“ auch sichtbar zu machen, kurz: Es gibt möglicherweise sanftere Wege, um „von geschlossenem Schriftgut zu auswertungsbereitem Archivgut“ zu kommen ${ }^{7}$. Multidimensionale Erschließung könnte, als laufender Prozess mit offener Beteiligungsmöglichkeit für viele konzipiert, laufend neue Zugänge eröffnen, weil Archivgut nicht an sich, sondern für etwas verzeichnet wird und damit, schrittweise, immer bessere Übersichtlichkeit entsteht, welche es den Benutzenden erlaubt, die jeweils relevante Menge an Informationen zu identifizieren.

\section{Erschließung in Theorie und Praxis - die 1990er Jahre}

Meine theoretische Initiation ins Archivwesen anfangs der 1990er Jahre war maßgeblich von Eckhart G. Franz’ „Archivkunde“8 und Menne-Haritz’ „Schlüsselbegriffe[n]

\footnotetext{
5 John Ridener, From Polders to Postmodernism. A Concise History of Archival Theory (Duluth 2009).

${ }^{6}$ Mark A. Greene-Dennis Meissner, More Product, Less Process: Revamping Traditional Archival Processing. The American Archivist 68 (2005) 208-263, hier 211: „Certainly, the vast breadth of contemporary manuscript sources and the increasing size of these collections since the 1950 s contribute to creating huge backlogs".

7 Angelika Menne-Haritz, Archivische Bewertung. Der Prozess der Umwidmung von geschlossenem Schriftgut zu auswertungsbereitem Archivgut. Schweizerische Zeitschrift für Geschichte 51 (2001) 448-460, hier 450 .

${ }^{8}$ Eckhart G. Franz, Einführung in die Archivkunde (Einführung Geschichte, Darmstadt ${ }^{8} 2010$ ). Die Teile zur Informatik im Archiv scheinen mir schon beim Erscheinen der letzten aktualisierten Auflage 2007 weit hinter der Realität zurückzuliegen und allenfalls nur historisch interessant. Alle anderen Teile aber sind nicht zuletzt wegen ihrer prägenden Wirkung auf Generationen von Archivaren relevant, wenn auch sachlich durchaus kritisch zu befragen.
} 
der Archivterminologie" "9 geprägt; beide Titel blieben auch noch bis weit in die 2000er Jahre prägend. Das Findbuch erscheint dort als Krönung archivischer Arbeit, apodiktisch formuliert: „Ergebnis der Erschließung ist der auswertbare Bestand, repräsentiert im Findbuch" ${ }^{10}$. Das zwingt zur Umkehrvermutung: Ein Bestand ohne Findbuch ist nicht auswertbar. Wenn das stimmte, wäre der größere Teil des Archivguts nicht nutzbar - eine Feststellung, welche wiederum so nicht stimmt.

Die reale Praxis des Schweizerischen Bundesarchivs (BAR) kontrastierte stark mit dieser Theorie. Es wurden schon damals angesichts des schnellen Wachstums der Bestände keine Findbücher mehr erstellt. Das BAR gehörte zu den vielen Archiven, in welchen „Findbücher als kommentierte, klassifizierte Normverzeichnung“ gerade nicht mehr das "Standardarbeitsziel“" waren ${ }^{11}$.

Somit öffneten in Tat und Wahrheit einige ältere Findbücher und hauptsächlich laufend neu hinzukommende Ablieferungsverzeichnisse den Zugang zum Archivgut. Letztere waren Ausschnitte (Teilmengen) aus den Aktenplänen der anbietungspflichtigen Stellen, welche die in einer Ablieferung real ins Archiv transferierten Akten auflisteten: Aktenzeichen, Titel, Zeitraum. Angesichts der 1.000 bis 2.000 Laufmeter Zuwachs pro Jahr war das für das Bundesarchiv die ohne Zeitverzögerung bestmöglich realisierbare Erschließung. Diese Form der Erschließung war eine gemeinsame Anstrengung der anbietungspflichtigen Stellen und des Archivs ${ }^{12}$. Immerhin bemühte sich das BAR durch seine Einflussnahme im sogenannten vorarchivischen Bereich, bei der Aktenführung in den Bundesämtern, positiv auf langfristig klare Ordnung und verständliche inhaltliche Beschreibung, etwa durch Setzung allgemein verständlicher Dossiertitel (Aktentitel), hinzuwirken ${ }^{13}$.

Diese Informationen über Struktur (Tektonik) und Inhalt des Archivguts, letztlich organisatorisch und prozessual kontingent portioniert, wurden ergänzt durch eine sogenannte Kompetenzenkartei. Diese lieferte zusätzlich ein Verzeichnis der Zuständigkeiten der Bundesstellen, also Hinweise auf die funktional-legale Provenienz des Archivguts ${ }^{14}$. Die Inhaltserschließung durch die Detailfindmittel gewinne in Kombination mit der

9 Angelika Menne-Haritz, Schlüsselbegriffe der Archivterminologie. Lehrmaterialien für das Fach Archivwissenschaft (Veröffentlichungen der Archivschule Marburg, Institut für Archivwissenschaft 20, Marburg $\left.{ }^{3} 2000\right)$.

10 Ebd. 64, s. v. „Erschließung“.

11 Angelika Menne-Haritz, Geleitwort zur 1. Auflage von 1994, in: ISAD(G) - Internationale Grundsätze für die archivische Verzeichnung, übers. und hg. von Rainer BRÜNIng-Werner HeEgEwALDT-Nils BRÜВасн (Veröffentlichungen der Archivschule Marburg, Institut für Archivwissenschaft 23, Marburg ${ }^{2} 2002$ ) 4-11, hier 5, 7. Es fehlten in der Schweiz damals auch „durch [...] zentralisierte, gemeinsame Ausbildung [...] kanonisierte Regelwerke“ zu deren Erarbeitung.

${ }_{12}$ Die Erschließung von 1.000 Laufmetern neuem Archivgut pro Jahr hätte gemäß unseren praktischen Erfahrungen Erschließungsarbeiten für gut zehn Archivarinnen, ein Drittel des damaligen Gesamtpersonalbestandes, bedeutet (bei einer empirisch gemessenen Repertorisierungsleistung von zehn Laufzentimetern pro Tag).

13 Streng genommen ist mit Aktentiteln keine wirkliche Inhaltsbeschreibung geleistet. Die Präzision dieser Titel in Bezug auf die Inhalte darf zu Recht in Zweifel gezogen werden.

${ }^{14}$ Ein weiteres Problem, das sich aus mangelndem IT-Verständnis ergeben hat: Wenn auch in der Sache Organisationseinheit (Provenienz) und Kompetenzträger nur grundsätzlich identisch sind (Problem zum Beispiel bei Rechtsnachfolge), so variierte die Bezeichnung doch selbst dort, wo es eigentlich kein Problem gab (Organisationseinheit gemäß alltäglichem Sprachgebrauch, beispielsweise „Oberzolldirektion“, im Vergleich zur Bezeichnung des Kompetenzträgers gemäß Rechtserlassen: „Eidgenössische Zollverwaltung“); bei noch nicht ausreichend intelligenten IT-Systemen stellten solche Dissonanzen eine maschinell unüberwindbare Hürde dar. 
Kompetenzenkartei an Präzision, so die ursprüngliche Idee. Die Kompetenzenkartei sei nichts anderes als „eine Gliederung des nach Provenienzprinzip organisierten Archivs nach Sachgruppen"15.

Erschließung im BAR erfolgte also in einem Spannungsfeld zwischen theoretischer Wertschätzung von und gleichzeitiger Abstinenz bei der Inventarisierung oder Repertorisierung; was an Erschließung noch geleistet wurde, wurde kooperativ und pragmatisch wahrgenommen. Das war primär der Überlastung geschuldet und dem Willen, keine neuen Pendenzen zu Lasten künftiger Archivarinnen und Archivare anzuhäufen.

Es kam aber dabei auch Widerstand gegen den ebenso unausrottbaren wie unerfüllbaren Perfektionswillen der damaligen Archivare zum Ausdruck, welche immer wieder rasch und gerne monierten, die vorhandenen Findbücher, obwohl mit viel Aufwand erstellt, befriedigten weder die Archivbesucher und Archivbesucherinnen noch sie selbst wirklich und sie müssten umgehend und dringend überarbeitet und verbessert werden. Es wäre aber klar eine vergebliche Anstrengung gewesen, eine Verschleuderung von Ressourcen, diese Perfektionierungsspirale der damaligen Erschließung, welche die Nachfrage in der Regel ausblendete, weiter voranzutreiben ${ }^{16}$.

\section{Frühe Digitalisierung als Extrapolation des Vergangenen}

Alternativ wurde seit den frühen 1990er Jahren über die Digitalisierung der vorhandenen Findmittel diskutiert. Man mag sich streiten, auf wann die Anfänge der Informationsgesellschaft zu datieren sind ${ }^{17}$. Eine kontinuierliche Diskussion setzte in den Archiven sicher erst relativ spät, in den 1980er Jahren, ein, als im Rahmen des Internationalen Archivrates die (möglichen) Auswirkungen der Informations- und Kommunikationstechnologie auf die Archivierung diskutiert wurden ${ }^{18}$. Heute, mehr als dreißig Jahre später, wirken einige der damaligen Diskussionen ziemlich fremdartig, wenn man an die schnelle und damals, auch in archivinformatischen Kreisen, kaum richtig prognostizierte Entwicklung der Informatik denkt.

15 Erich Schärer, Die systematische Kompetenzenkartei des Bundesarchivs (Studien und Quellen 2, Bern 1976) 113-154, hier $125 \mathrm{f}$.

16 Umberto Eco, Die Karte des Reiches im Maßstab 1:1, in: Ders., Platon im Striptease-Lokal. Parodien und Travestien (München 1993) 88-101. Ausgangspunkt für diese Überlegungen ist Jorge Luis Borges, Von der Strenge der Wissenschaft, in: Ders., Werke in 20 Bänden, 9: Borges und ich. Kurzprosa und Gedichte 1960, übers. und hg. von Karl August Horst-Gisbert Haefs (München-Wien 1993) 131. Illustrativstes Beispiel dieses unersättlichen Perfektionswillens ohne Rücksicht auf wirtschaftliche Rahmenbedingungen war beispielsweise die Forderung nach noch detaillierterer Erschließung des Pertinenzbestandes E 27, Landesverteidigung 1848-1950, nachdem dieses Archivgut im Umfang von 870 Laufmetern gerade erst mit einem zwölfbändigen Repertorium (1978) erschlossen worden war. Der Umfang dieses Repertoriums von 0,63 Laufmetern und über 23.000 Einträgen ins Verhältnis zu den 870 Laufmetern Archivalien gesetzt ergibt einen Maßstab von rund 1:1400 beziehungsweise, ausgehend vom kondensierten Ausdruck des Inhaltes in der Archivdatenbank Swiss Archives von nur noch bescheidenen 10 Laufzentimetern, von 1:8700 - kartographisch gesehen eine sehr gute Wanderkarte. Hier weiter in die Tiefe zu gehen, mag für den Facharchivar verlockend sein, der Publikumsnutzen bleibt mehr als fraglich.

17 Als erstes Projekt im Stile der Digital Humanities kann wohl die Erarbeitung des Index verborum der Werke von Thomas von Aquin ab 1949 verstanden werden: vgl. Susan Hockey, The History of Humanities Computing, in: A Companion to Digital Humanities, hg. von Susan Schreibman-Ray Siemens-John Unsworth (Blackwell Companions to Literature and Culture 26, Malden 2004) 3-19, hier 4.

${ }^{18}$ Ein Ergebnis dieser Diskussionen ist das Buch von Charles M. Dollar, Die Auswirkungen der Informationstechnologien auf archivische Prinzipien und Methoden, übers. und hg. von Angelika Menne-Haritz (Veröffentlichungen der Archivschule Marburg, Institut für Archivwissenschaft 19, Marburg 1992). 
Ziel einer möglichen Digitalisierung war - aus heutiger Perspektive - die Transponierung, nicht die Transformation der Erschließung und ihrer Produkte ${ }^{19}$. Damals pflegte man gerne die Vorstellung, dass sich die Informatik den archivischen Bedürfnissen anpassen, den archivischen Prinzipien unterwerfen müsse, nicht umgekehrt. Selbst die Vorstellung, dass sich beides gegenseitig positiv beeinflussen und sich so ein kreativer Austausch entwickeln könnte, war noch nicht selbstverständlich ${ }^{20}$.

So führte etwa die Digitalisierung der Kompetenzenkartei zu einer reinen digitalen Abbildung der Karteikarte und entsprechender Dateneingabe im Stil einer Schreibmaschine mit fixem Wagenrücklauf am Zeilenende. Das Resultat ist eine völlig wirre Datenstrukturierung und Aufsplittung von Begriffen in zufällige Wortteile, kurz: aus heutiger Sicht - leider - kaum brauchbar. Das Vorhaben wurde eingestellt, obwohl methodisch der Ansatz bestandsunabhängiger Erschließung der Behörden und ihrer Zuständigkeiten vielversprechend gewesen war und noch ist.

Bei den Detailfindmitteln setzte sich zum Glück ein Datenbankansatz, also nicht eine Online-Findbuch-Lösung durch. Diese Detailfindmittel lagen 2004 - nach sechs Projektjahren - fertig digitalisiert vor; neue kamen schon damals praktisch nur noch in digitaler Form dazu. Parallel zu diesem Digitalisierungsprojekt wurde ein Archivinformationssystem gesucht und implementiert, welches möglichst viele Funktionen abdecken und von vielen Archiven in der Schweiz eingesetzt werden können sollte. Datenaustausch über Institutionengrenzen war schon damals ein Thema, sollte aber sozusagen durch eine Softwarebrücke zwischen Informationssilos gelöst werden ${ }^{21}$.

\section{Kontinuierliche Digitalisierung: Kontinuität bis zur Perspektivenumkehr}

Diese frühe Digitalisierung versuchte die bekannte analoge Welt möglichst genau digital nachzubilden. Viele Archive bemühten sich, die Informationstechnologie (IT) archivkonform einzusetzen, ohne deren neue Möglichkeiten zu erkennen, geschweige denn echt zu nutzen. Spätestens heute muss man aber zur Kenntnis nehmen, dass die IT innovativer ist als anfänglich gedacht. Archivierung ist also im Kontext des sozialen

19 Selbstverständlich drehte sich die Diskussion auch um Fragen nach der künftigen Form von Unterlagen, nach der digitalen Gestaltung von Aktenplänen und Ordnungssystemen etc., und wie solche Unterlagen ins BAR übernommen und langfristig sicher aufbewahrt werden könnten. Die sichtbar werdende Dissoziation von Inhalt und Form wurde beispielsweise lebhaft debattiert. Man hat heute fast den Eindruck, dass sich damit die Diskussion aus dem frühen 20. Jahrhundert wiederholt hat, als die Arbeit mit Karteikarten und Schreibmaschine wegen ihrer zu beklagenden „Dissoziation von Körper und Schrift“ kritisch betrachtet und diese Dissoziation beklagt worden war, dieses Verhältnis sich aber bald verselbstverständlicht hatte: vgl. Peter BECKER, Kulturtechniken der Verwaltung. Forschungsbericht (2010), in: Schweizerisches Bundesarchiv - Service und Publikationen - Verwaltungsgeschichte - Forschungsberichte, https:/www.bar.admin.ch/dam/bar/de/dokumente/verwaltungsgeschichte/forschungsberichtbeckerkulturtechniken.pdf.download.pdf/forschungsberichtbeckerkulturtechniken. pdf [1. 3. 2018], mit Verweis auf Stefan NeLlen, Mechanisierte Sekretäre. Verwaltung im Zeichen der Schreibmaschine, in: Sprachvollzug im Amt. Kommunikation und Verwaltung im Europa des 19. und 20. Jahrhunderts, hg. von Peter Becker (1800 | 2000. Kulturgeschichten der Moderne, Bielefeld 2011) 247-274.

${ }^{20}$ Menne-Haritz, Geleitwort (wie Anm. 11) 7: „Die Karteikarte von Papritz ist zum Rohmodell vieler Datensatzstrukturen geworden“.

${ }^{21}$ Ein System, das unterschiedlichste Funktionen erfüllt, erfüllt fast zwangsläufig alle nur beschränkt gut. Es scheint heute angezeigt, verschiedene Systeme zu kombinieren (Erschließung, Logistik etc.), so dass für jede Funktion die beste Lösung gewählt werden kann. Der Datenaustausch ist längst nicht mehr von den eingesetzten Softwarelösungen abhängig. 
und semantischen Netzes wie alle E-Government-Vorhaben der disruptiven Macht der IT ausgesetzt ${ }^{22}$. Dieser Weg war lang, die Erkenntnis stellte sich nur schrittweise ein.

Das BAR als vollständig digitales Archiv imaginiert, verlangte zuerst den Aufbau eines digitalen Speichers (digital repository) mit entsprechenden Ablieferungs- und Informationsintegrationsprozessen. Sicherung und Erhaltung von Archivbeständen sind nun einmal Voraussetzung für jede Nutzung ${ }^{23}$. Zugangsseitig folgt die Digitalisierung der Daten, damit Online-Nutzung möglich wird - für originär digitale Informationen ist das zwangsläufig ohnehin die einzig sinnvolle Zugangsform. Das ist der einzige Weg, die enorm aufwändige Parallelität medial unterschiedlich ausgerichteter Arbeitsprozesse auf ein längerfristig verkraftbares Maß an Aufwand zu reduzieren. 2010 ging die Anwendung Swiss Archives in Betrieb, welche als ersten Schritt die Suche in allen gesammelten Findmitteln ermöglicht ${ }^{24}$. Herkömmliche, aber sachlich nicht begründbare Limitierungen der Recherche entfallen seither auch für die Benutzer weitgehend, wenn auch die Menge angezeigter Resultate aus technischen Gründen auf 1.500 Treffer beschränkt ist, was grundsätzlich nach wie vor unbefriedigend bleibt ${ }^{25}$.

Der vollständig digitale Zugang ist jetzt geplant und im weiteren Aufbau: Benutzerinnen und Benutzer werden nicht nur online recherchieren, sondern die Unterlagen auch in digitalisierter oder digitaler Form auf einer im Aufbau befindlichen Plattform konsultieren können oder müssen. Wenn sie Hilfe brauchen, werden sie - beziehungsweise können sie schon heute - mit dem Archivpersonal chatten oder via geteilte Bildschirme Unterstützung erhalten. So rasch wie möglich soll die digitale Konsultation oder Verarbeitung von Archivgut die ausschließlich mögliche werden, das heißt, der virtuelle „Lesesaal“ wird den herkömmlichen Lesesaal ablösen.

Diese schrittweise Entwicklung führte zu einer Perspektivenumkehr und verändert unsere Sicht auf Erschließung grundsätzlich.

\section{Freies Navigieren in der Infosphäre: Einladung zu fünf Grenzüberschreitungen als Aufbruch zu neuen Ufern?}

Ich habe die Entwicklung des BAR während der bisherigen digitalen Transition geschildert, die praktische Seite und etwas die theoretische Rezeption in der Praxis. Noch bleibt es offen, wie ein informationelles GPS - speziell in der Archivwelt - aussehen wird. Erste Tendenzen zeichnen sich jedoch ab. Darauf aufbauend stelle ich fünf Thesen auf:

${ }^{22}$ Aus der zahlreichen Literatur vgl. Ian McLoughlin-Rob Wilson-Mike Martin, Digital Government @ Work. A Social Informatics Perspective (Oxford 2013), mit Verweisen auf teilweise erstaunlich „alte “ Literatur.

23 Das digitale Magazin wurde 2009 in Betrieb genommen. Schon vorher waren die Anwendung und das Format SIARD zur Archivierung relationaler Datenbanken betriebsbereit.

${ }^{24}$ Einzig die Suche nach Personennamen bei vermutetem Vorhandensein von besonders schützenswerten Personendaten im Sinne des Datenschutzgesetzes ist nicht möglich beziehungsweise muss vor Ort im BAR erfolgen.

${ }^{25}$ Damit dieses Ziel erreicht werden konnte, wurde um 2010 viel Aufmerksamkeit auf die Erschließung gelegt, leider aber mit wenig Erfolg. Ganz im alten Geiste wurde Perfektionismus weiter groß geschrieben und beispielsweise zur Umsetzung der archivischen $\operatorname{Norm} \operatorname{ISAD}(\mathrm{G})$ mit ihren 26 Deskriptionselementen hausinterne Erschließungsregeln erarbeitet, welche über 700 Anweisungen umfassten; entsprechend komplex war die Datenbankstruktur unseres Archivinformationssystems mit zahlreichen Datenfeldern, welche realiter praktisch nie genutzt wurden. Die Mitarbeitenden hätten die Anwendung dieses hypertrophen Regelwerks wohl als demotivierende Zwangsjacke verstanden und es ist alles andere als klar, ob die Benutzerinnen und Benutzer den qualitativen Unterschied wirklich gemerkt hätten. 
Erstens: Out of the box

Es geht nicht primär um Erschließung, sondern darum, Zugänge zum Archivgut zu eröffnen oder das Archiv Teil des globalen Informationsozeans werden zu lassen - etwa in freier Interpretation der Eröffnungs-Keynote von John Hocking am ICA-Kongress in Seoul: „Out of the box, into the world!“ ${ }^{26}$ - raus aus den Silos, rein in die Welt. Interessierten Wege zum Archivgut auszuschildern, muss aber ergänzt werden durch die Ausschilderung von Wegen in die Welt für unser Archivgut. Archivgut will nicht nur gesucht werden, es soll gefunden werden, es soll dort auftauchen, wo es gebraucht werden kann.

Das bedeutet letztlich, dass wir uns von der Vorstellung einer - geschlossenen - Archivwelt und der Dominanz einer institutionellen, inhalts- und materialbezogenen Binnenperspektive verabschieden. „Out of the box“ ist eine erste Grenzüberschreitung und zwingt zu weiteren.

\section{Zweitens: Suche ist nicht nur Vorarbeit}

Die herkömmliche Sichtweise über Archivarbeit tönt bei Eckhart Franz etwa so: Der Archivbesuch sollte „tunlichst“ mit einer Anfrage ans Archiv und einer „gründliche[n] Vorbereitung" beginnen. Zu dieser gehört mitnichten nur das Studium der Findbücher; dazu gehören vielmehr die Konsultation von „[a]llgemeine[n] Nachschlagewerke[n] und Veröffentlichungen der einzelnen Archive, Beständeübersichten, Inventare[n] und Findbücher[n]“ wie auch von „Abhandlungen und Quellenpublikationen zum jeweiligen Thema [und der] Sekundärliteratur"27.

Der Historiker Winfried Schulze sieht das ähnlich: Erst „[w]enn man in Archiven die Formalien der Anmeldung und Beratung überstanden“ habe, „Archivinventar und Findbücher konsultiert“ worden seien „und endlich das gewünschte Aktenfaszikel [...] auf dem Tisch“ liege, könne „ein neues Abenteuer der Auseinandersetzung mit den Überresten der Vergangenheit" beginnen ${ }^{28}$.

Eckhart Franz relativiert immerhin in praktischer Betrachtung die Bedeutung der Findbücher klar zugunsten einer multiperspektivischen Eröffnung des Zugangs zum Archiv. Beide Sichtweisen trennen aber klar das Suchen nach von der Arbeit mit dem Archivgut. Suche ist ihnen nur Vorarbeit. Auch diese Grenze ist zu überwinden, diese Grenzziehung ist aufzulösen.

\section{Drittens: Das Archiv unter Konkurrenzbedingungen}

In die Welt hinauszutreten, erhöht die Sichtbarkeit. Das verstärkt aber auch den Konkurrenzdruck und bedeutet einen Verzicht auf die Position einer für nicht umgehbar gehaltenen Institution, wie sie lange durch ein - vermeintliches? - Informationsmonopol begründet worden war. Dabei führt bereits die Frage, wie man Archivgut findet, in die

${ }^{26}$ John Hocking, Keynote Speech: Out of the Box, Into the World, in: ICA. International Congress on Archives - Congress Highlights, https://www.ica.org/en/john-hocking-s-keynote-speech-out-of-the-box-intothe-world [1.3. 2018]: „[The] Work [of archivists] connects past and future, problems to solutions, and people with people“.

27 Franz, Einführung (wie Anm. 8) 111-115, hier $111 \mathrm{f}$.

28 Winfried Schulze, Einführung in die Neuere Geschichte (Stuttgart ${ }^{5} 2011$ ) 31, 45. 
Irre. Fragen zielen aus unterschiedlichsten Gründen - Neugier, Wissensbedürfnis und vieles mehr - und mit verschiedensten Absichten auf Unbekanntes - nicht zwingend auf Archivgut. Nur Eingeweihte und Fachleute fragen sofort nach Archivgut und dem richtigen Archiv für das Archivgut, das sie interessiert. Aber auch Antworten auf offene Fragen können in vielen Fällen im Archiv gefunden werden, selbst wenn man daran nicht zuerst gedacht hat. Die Antwort aus dem Archiv sieht sich aber mit vielen konkurrierenden Angeboten - unterschiedlicher Qualität - konfrontiert ${ }^{29}$. Auf Fragen muss aber zuerst einmal überhaupt der Hinweis geliefert werden, dass zu ihrer Beantwortung etwas in einem Archiv zu finden ist.

Meistgenutztes Suchinstrument ist heute Google. Dieses Portal prägt mit seinem Angebot ebenso maßgeblich unser Suchverhalten wie unsere Erwartungen an Suchinstrumente. Suchen heißt hier, nach komplexer, aber intransparenter Methodik Fragen mit vorhandenen Informationen abgeglichen zu bekommen. Das Suchen kann dann laufend verfeinert werden. Suche ist ein iterativer Prozess laufender Informationsgewinnung dank ständiger Konkretisierung und Verfeinerung der Suchziele.

Man kann jetzt lange klagen, dass über „Internetsuchmaschinen [...] ein gezielter Zugriff auf digitalisiertes Kulturgut aus Gedächtnisinstitutionen wie Bibliotheken, Archiven [...] wegen der meist sehr großen und undifferenzierten Treffermenge kaum möglich" sei. In der Tat: Mit Google finden wir Archivgut selten präzise und nur zufällig - aber manchmal finden wir es eben auch ${ }^{30}$.

Die Vision, vermeintliche Schwächen von Google \& Co. durch spezialisierte Kulturportale zu kompensieren, scheint mir trotz aller Mängel der großen Suchmaschinen illusorisch ${ }^{31}$. Die algorithmische Stärke von Google dürften archivische Anwendungen kaum egalisieren, selbst wenn eine andere Aufbereitung der Inhalte voranginge. Findbuchstrukturen in tektonische Strukturbäume umzuformen, verbessert zumindest die Suchmöglichkeiten heute nicht maßgeblich.

Mit Blick auf notwendige Grenzüberschreitungen: Wir müssen nicht nur nach archivischer Theorie erschließen, sondern Archivgut auch optimal für Google-Algorithmen aufbereiten. Das wäre dann Zugangseröffnung vom Suchbedürfnis her gedacht ${ }^{32}$.

29 Christa Wormser-Hacker-Thomas Mand, Information Seeking Behaviour (ISB), in: Grundlagen der praktischen Information und Dokumentation. Handbuch zur Einführung in die Informationswissenschaft und -praxis, hg. von Rainer Kuhlen-Wolfgang Semar-Dietmar Strauch (Berlin-Boston ${ }^{6} 2013$ ) 97-108.

30 Ellen Euler-Monika Hagedorn-Saupe-Gerald Maier-Werner Schweibenz-Jörn Sieglerschmidt, Kulturportale im Web. Eine Einführung, in: Handbuch Kulturportale. Online-Angebote aus Kultur und Wissenschaft, hg. von Ellen Euler-Monika Hagedorn-SAupe-Gerald Maier-Werner Schweibenz-Jörn SiegLERSCHMIDT (Berlin-Boston 2015) 3-11, hier 3.

31 Vgl. Jeff Jarvis, Was würde Google tun? Wie man von den Erfolgsstrategien des Internet-Giganten profitiert (München ${ }^{2} 2012$ ).

32 Bei Angelika Menne-Haritz, Archive, in: Grundlagen der praktischen Information und Dokumentation (wie Anm. 29) 596-608, hier 605, wird dieser Aspekt der „selbstgesteuerte[n] Relevanzentscheidung “ auch hervorgehoben. Als schon ältere Mahnung vgl. Rainer KuHlen, Die Konsequenzen von Informationsassistenten. Was bedeutet informationelle Autonomie oder wie kann Vertrauen in elektronische Dienste in offenen Informationsmärkten gesichert werden? (Suhrkamp-Taschenbuch Wissenschaft 1443, Frankfurt am Main 1999), wo gleich zu Beginn die „zentrale Frage“ gestellt wird, „was ,Abnehmen von Informationsarbeit bedeutet: Entlastung oder Entmündigung?": ebd. 5. Neuer: Dirk Helbing, Es braucht eine neue Aufklärung. ResearchGate (September 2016), https://www.researchgate.net/publication/307606887_Es_braucht_eine_neue_Aufklarung [1.3. 2018] 1, der meint: „Es braucht eine neue Aufklärung, weil die Digitalisierung zur Zeit zu einer Entmündigung führt" - selbstverschuldete Unmündigkeit ist nun sicher nicht Ziel und Sinn der heutigen technischen Möglichkeiten. 


\section{Viertens: Metadaten $=$ Daten $=$ Metadaten}

Nächste zu überwindende Grenze: Was ist Suchinstrument, was Archivgut? Digitales und digitalisiertes Archivgut kann mit heutigen Instrumenten in einem Suchprozess direkt befragt und genutzt werden. Die Grenzen zwischen Daten und Metadaten, zwischen Dokumenten und Daten verwischen sich, wie sich die Grenze zwischen Suchen als Vorarbeit und der sogenannten eigentlichen Archivarbeit auflöst ${ }^{33}$. Auch die Konzentration auf die Rekonstruktion eines Aktes aus den Unterlagen wird relativiert, weil diese immer auch Informationen enthalten, die Aufschluss über etwas potentiell Wichtiges oder Interessantes jenseits von bürokratischen Zuständigkeiten und Entscheidungen liefern. Solche Daten machen einen großen Teil des Archivguts aus, sie entstehen aber auch durch die Datafizierung von Informationen.

Und umgekehrt: Metadaten als Daten ausgewertet, geben der Suche auch eine zusätzliche Dimension. Das „Abenteuer der Auseinandersetzung mit den Überresten der Vergangenheit" beginnt eben schon während des Suchens, nicht erst nach dem - oft vermeintlichen - Finden. Es braucht also das Angebot einer Werkbank, auf der Daten klassisch gesucht, aber auch sortiert, annotiert, verknüpft, ausgewertet und die Ergebnisse der Suche auch festgehalten und gespeichert werden können und so weiter.

So lässt sich der Katalog des Schweizerischen Bundesarchivs auf opendata.swiss als offene Behördendaten herunterladen und als Datenbank auswerten ${ }^{34}$. Ein solches Vorgehen zeigt dann beispielsweise, dass im Katalog des Schweizerischen Bundesarchivs die Begriffe Österreich - Autriche - Austria beziehungsweise Wien - Vienne - Vienna für den Zeitraum 1848 bis 1998 rund $10.000 \mathrm{Mal}$ auftauchen. Dieses Ergebnis bedarf dann noch der Bereinigung, denn auch „JugoslaWIEN“ oder „MoldaWIEN“ wird gefunden, ebenso „Vienne“ in Frankreich. Netto bleiben immer noch 6.459 sogenannte Verzeichnungseinheiten, und diese wiederum lassen sich dank Provenienzinformationen verschiedenen Themenbereichen zuordnen. Damit kann eine Quellenlandschaft bezogen auf ein Forschungsthema strukturiert erfasst und mögliche vielverheißende Forschungsinteressen identifiziert werden ${ }^{35}$.

Mit diesem als Datenbank veröffentlichen Katalog ist ein wichtiger Schritt vom Findbuch nicht nur zum Online-Findbuch getan, sondern weiter zu einem Angebot von Informationen in offenen, weiternutzbaren Formaten. Ganz im Geiste der Open-(Government)Data-Ideen steht jetzt noch ein nächster Schritt an: die Publikation des Katalogs und möglichst vieler Metadaten als Linked Open Data. Das erst wäre dann die Integration der

33 Als Beispiel einer frühen Grenzverwischung kann das folgende Inventar gelten: Friedrich KücH, Politisches Archiv des Landgrafen Philipp des Großmütigen von Hessen. Inventar der Bestände (Publikationen aus den K. Preußischen Staatsarchiven 78, Leipzig 1904). Robert Kretzschmar spricht es als Quellenedition an: Robert Kretzschmar, Akten- und Archivkunde im Tübinger Netzwerk Landesgeschichte. Ein Plädoyer für eine zeitgemäße Archivalienkunde, in: Netzwerk Landesgeschichte. Gedenkschrift für Sönke Lorenz, hg. von Dieter R. Bauer-Dieter Mertens-Wilfried Setzler-Susanne Borgards (Tübinger Bausteine zur Landesgeschichte 21, Ostfildern 2013) 91-109, hier 93 (diesen Hinweis habe ich im Rahmen der Tagung von Dr. Holger Berwinkel, Berlin, erhalten). Insofern diese Unentschiedenheit zwischen Inventar und Edition eine nachträglich interpretatorische, nicht eine original intentionale ist, bezeichne ich sie lieber als Grenzverwischung denn als Grenzüberschreitung, welche als Schritt eine klare(re) Absicht braucht.

34 Swiss Archives - Archivdatenbank des Schweizerischen Bundesarchiv, in: opendata.swiss - Organisationen - Schweizerisches Bundesarchiv BAR, https://opendata.swiss/de/dataset/swiss-archives-archive-databaseof-the-swiss-federal-archives [1. 3. 2018].

35 Andreas Kellerhals, Neues Licht auf die schweizerisch-österreichischen Beziehungen 1848-1998? Modernisierte archivische Findmittel als Instrumente historischer Heuristik und Mäeutik. MÖStA 55 (2011) 245-271. 
Archivinhalte ins semantische $W_{e b}^{36}$. Die Ebene der Linked Open Data (LOD) haben wir heute zwar anvisiert, aber noch nicht wirklich erreicht. Sie bleibt auch künftig ein wichtiges Ziel.

\section{Fünftens: Öffnung als mentaler Turn}

Nicht nur das Archivgut muss sich in die offene Informationswelt integrieren, auch die Archivare und Archivarinnen müssen sich nach außen wenden. Kooperative Erschließung zusammen mit den Aktenproduzenten ist für uns schon lange Tatsache. Heute in der Zeit des sozialen Webs kann und muss diese Kooperation ausgeweitet werden: crowd-working, folksonomy sind Stichworte dazu. Die Erschließung der Fotosammlung der Swissair darf hier als gelungenes Beispiel angeführt werden: Die anfänglich oft (zu) knappen, teilweise auch irreführenden Bildbeschreibungen haben dank der Mitwirkung einer großen interessierten Gruppe von Mitwirkenden eine enorme Ausweitung und Präzisierung erhalten ${ }^{37}$.

Mit solchen Projekten kann ein Reservoir an Fachkenntnissen und Sachverstand angezapft werden, welches kein Archiv sich intern leisten könnte, das heißt damit kann eine einschränkende Grenze knapper Ressourcen überschritten werden.

\section{Neue Zugänge}

Schlussfolgernd ist klar: Das Findbuch als Ergebnis der Erschließung hat ausgedient, trotz der fast mantraartig beschwörenden Wiederholung, es sei nach wie vor das zentrale Instrument. Es wird sogar betont, welchen „wichtige[n] Stellenwert [...] die Buchform“ mit ihren klassischen Elementen des Inhaltsverzeichnisses, der Einleitung, der Liste der verzeichneten Einheiten, der Indices und der eventuellen Anhänge habe. Wenn also die „elektronische Form des Online-Findbuchs [...] die Buchform“ beibehält, dann weil damit auch die Abgrenzung der einzelnen erschlossenen Bestände gegeneinander sozusagen physischen Ausdruck finde. Obwohl auch in dieser Perspektive die „horizontal übergreifende Suche [und] die vertikale Integration der Findmittel“ gefordert wird, überwiegt doch die Stärkung der informationellen Festungen, deren gegenseitige Bezüge nicht aktiv erfassbar gemacht werden sollen ${ }^{38}$. Ein solches Verständnis des Online-Findbuchs ist für mich die Antithese jeder Öffnung, jeden Aufbruchs zum Verlassen selbst gebauter Silos.

Wenn das Findbuch als Resultat der Erschließung ausgedient hat, so sind doch noch lange nicht alle Informationen, die bei der Erarbeitung von Findbüchern, bei der Erschließung von Archivgut bis heute benutzt werden, wertlos geworden - im Gegenteil!

36 Damit könnte in der Sterne-Bewertung publizierter Daten das Maximum von fünf Sternen erreicht werden, vgl. $5 *$ offene Daten, http://5stardata.info/de/ [1. 3. 2018]. Als Testseite kann besucht werden: Archival Linked Open Data, http://data.alod.ch/ [1. 3. 2018]. Dort wird demnächst ein Teil des Katalogs des Schweizerischen Bundesarchivs als Linked Open Data in RDF (Resource Description Format) zugänglich gemacht werden.

37 Zur Projektbeschreibung und zu den Ergebnissen vgl. Nicole Graf, Die Freiwilligen der Swissair im Bildarchiv der ETH-Bibliothek. ETH-Bibliothek | Crowdsourcing. Aktuelles und Erfahrungen aus der Community (9. 5. 2016), https://blogs.ethz.ch/crowdsourcing/2016/05/09/die-freiwilligen-der-swissair-im-bildarchiv-ethbibliothek/ [1. 3. 2018].

38 Menne-Haritz, Archive (wie Anm. 32) $604 f$. 
Auch neue Zugangsöffner können nicht von beliebigen Daten ausgehen. Ich gehe davon aus, dass funktionale (Zuständigkeiten) und organisatorische Provenienz (Informationsproduzenten), die Praxis der Verwaltungsarbeit (Geschäftsverwaltung) und die Entstehungsgeschichte des Archivguts (Bewertung, Transfer et cetera) zentrale Informationen zu einem klaren Verständnis von Archivgut bleiben. Diese müssen aber nicht mehr bestandeszentriert erarbeitet werden, sondern können als besondere Arbeiten und eigenständige Informationssammlungen separat und schlimmstenfalls - ressourcenbedingt auch asynchron geleistet werden.

Selbständig erarbeitete Kompetenzgeschichten, Organisationsgeschichten, Geschichten bürokratischer Praxis oder Mediengeschichten - kurz: wesentliche Elemente einer breit verstandenen Verwaltungsgeschichte - können also schrittweise neue wesentliche Elemente zu einer besseren und präziseren Orientierung beisteuern ${ }^{39}$.

\section{Schluss}

Letztlich gilt: „Weder eine Inventarliste noch ein Findbuch, nicht einmal die Digitalisierung eines ganzen Archivs oder seine Faksimilierung verrät, was ein findiger, ideenreicher Forscher, vielleicht erst in hundert Jahren, einmal aus den Quellen erarbeiten, in ihnen entdecken und aus ihnen herauslesen wird“ 40 . Es ist deshalb mehr als verständlich, wenn immer wieder „die teils geringe Aussagekraft der Erschließungsinformationen [...] bemängelt" wird ${ }^{41}$.

Records in Contexts ${ }^{42}$, der Entwurf eines neuen ICA-Standards für Erschließung, verschiebt den Akzent nun logischerweise von der Multi-Level- auf die multidimensionale Erschließung. Das passt, weil Archivgut immer ein Multi-Use-Informationsgut ist. Für

39 Vgl. Forschungsberichte, in: Schweizerisches Bundesarchiv - Service und Publikationen - Verwaltungsgeschichte, https:/www.bar.admin.ch/bar/de/home/service-publikationen/verwaltungsgeschichte/forschungsberichte.html [1. 3. 2018]. Vom BAR (Stefan Nellen) wird gemeinschaftlich mit dem Institut für Österreichische Geschichtsforschung (Peter Becker, Andreas Enderlin und Thomas Stockinger) sowie dem Max-Planck-Institut für europäische Rechtsgeschichte in Frankfurt am Main (Peter Collin) die Online-Zeitschrift Administory herausgegeben.

40 Dietmar Schenk, Kleine Theorie des Archivs (Stuttgart $\left.{ }^{2} 2014\right) 56$.

${ }^{41}$ Christian Reinhardt-Sebastian Mundt, Nutzerstudie 2016 des Hessischen Landesarchivs. Erste Ergebnisse, in: Hessisches Landesarchiv, https://andesarchiv.hessen.de/sites/landesarchiv.hessen.de/files/ content-downloads/Nutzerstudie\%202016\%20Ergebnisse_final-20160818_0.pdf [1. 3. 2018] 2. Ähnlich Pierre BRODARD, Guide de recherche historique pour les relations internationales aux Archives fédérales suisses (1946-1978). Konzept zu einer Masterarbeit MAS ALIS (Bern 2016): „Toutefois, lorsqu’ils débutent leurs recherches, ces derniers peuvent rencontrer de nombreux obstacles. Les chercheurs doivent se familiariser avec les instruments traditionnels et les bases de données archivistiques dont le fonctionnement est très différent de celui d'un moteur de recherche classique ou d'un catalogue de bibliothèque. Dans un premier temps, ils peuvent rencontrer des difficultés à trouver les dossiers s'ils ne connaissent pas la terminologie de l'époque. A l'inverse, ils peuvent être submergés par un grand nombre de résultats de recherche. Le risque est alors important qu'ils commandent un grand nombre de dossiers qui ne correspondent pas forcément à leurs attentes. Ces difficultés viennent notamment de la structure des fonds qui reflètent l'organisation interne du service producteur de l'époque. Ce n'est donc qu'après un temps d'apprentissage que le chercheur va réussir à mieux comprendre le fonctionnement des fonds et les dossiers les plus pertinents. Cette période d'apprentissage représente également un investissement important en ressources pour les archivistes chargés de la médiation entre les utilisateurs et les fonds archivistiques. Il est donc dans l'intérêt de chacune des deux parties que les chercheurs deviennent rapidement indépendants".

42 Records in Contexts - Conceptual Model, in: ICA. International Congress on Archives - International Standards, http://www.ica.org/en/egad-ric-conceptual-model [1.3. 2018]. 
die Archive geht es dabei darum, das Ziel „description facilitates discovering“ im Auge zu behalten. Erschließung muss nicht immer nur Starthilfe für langwierige Archivarbeiten sein; es gilt Informationen dort zur Verfügung zu stellen, wo sie gebraucht werden, seien das auch kurzfristige und - mit nostalgischem Blick betrachtet - oberflächliche Nutzungsinteressen.

In diesem Zusammenhang muss noch einmal das Stichwort Linked Open Data bemüht werden. Solche Daten bieten verlässliche Verknüpfbarkeit von Informationen und bauen auf langfristig zuverlässiger digitaler Verfügbarkeit von Archivgut auf. LOD machen Archive auf ganz neue Art und Weise und potentiell auch in unerwarteten Kontexten anschlussfähig, und sie tragen zur präziseren Orientierung und durch ihre langfristig gesicherte Verfügbarkeit zur Orientierungssicherheit bei ${ }^{43}$.

Auch solch modernisierten Zugänge zu schaffen, bedeutet Arbeit. Der Aufwand für diese neue Entwicklung, für die Datafizierung der Erschließungsinformationen - prototypisch in Karteiform gesammelt - ist nicht zu unterschätzen. Es ist nicht zwingend mehr Aufwand als bei traditioneller, aber nicht zukunftsfähiger Erschließung; man muss aber die Ressourcen im Archiv neu organisieren, die Arbeitsprozesse anpassen.

Wo möglich sollte man auch auf schon bestehende Metadatensammlungen - oder Datensammlungen mit Metadatenfunktion für die Archive - zurückgreifen. Mit in Programmiersprachen wie Python geschulten Archivaren und Archivarinnen dürfte auch der „technische Turn“ leicht zu schaffen sein. Dazu kommt, dass immer neue IT-Anwendungen entwickelt werden, welche zum Glück ebenfalls helfen, Metadaten zu extrahieren und zusammen zu verbinden, wie beispielsweise das Projekt „Social Networks and Archival Context" zeigt ${ }^{44}$.

Und wenn sich die Bedeutung der herkömmlichen Metadaten für das Suchen relativiert, so behalten sie doch ihr Gewicht für das Verständnis und die Interpretation des Gefundenen. In einer Welt mit „information at your fingertips“ ${ }^{45}$, mit einer unmittelbaren Verfügbarkeit von Informationen, ist der Bedarf an zuverlässiger Einordnungskompetenz groß. Fähigkeit zur Quellenkritik wird zu einer Grundanforderung an alle in der Informationsgesellschaft, wenn wir so viel finden und nicht mehr wissen, was wir genau finden. Quellenkritik erinnert uns auch daran, dass Daten, vermeintlich Gegebenes, eigentlich immer Fakten, Gemachtes, sind, nie absichtslos geschaffen, nie absichtslos festgehalten und tradiert oder genutzt. Ob es so gesehen wirklich Überreste oder nur Traditionsquellen gibt, mag genauer geprüft werden. Unmittelbar gilt es, Imprägnierungsmittel gegen naive Faktengläubigkeit, heute oft potenziert als Glaube an die höhere Aussagekraft visualisierter Fakten, anzubieten.

Informationen, die ich nicht mehr zum Suchen brauche, bleiben trotzdem unverzichtbar für das Verstehen. Suchen und Finden sollen ja nicht nur Verfügbarkeit nachweisen, sondern (intellektuelle) Zugänglichkeit und Verstehen ermöglichen. Es ist also auf

43 Als gute Einführung in die LOD-Praxis vgl. Seth van Hooland-Ruben Verborgh, Linked Data for Libraries, Archives and Museums. How to Clean, Link and Publish Your Metadata (London 2014).

${ }_{44}$ Thomas J. Lynch, Social Networks and Archival Context Project: A Case Study of Emerging Cyberinfrastructure. Digital Humanities Quarterly 8/3 (2014), http:/www.digitalhumanities.org/dhq/ $\mathrm{vol} / 8 / 3 / 000184 / 000184 . \mathrm{html}$ [1.3. 2018].

45 So der Titel der Keynote von Bill Gates auf der Computermesse Comdex, Las Vegas 1994: vgl. Christoph Dernbach, Bill Gates 1995: „Information at Your Fingertips (2005)“. Mr. Gadget (20. 5. 2009), http:// www.mr-gadget.de/future-tech/2009-05-20/zurueck-in-die-zukunft-die-vision-von-bill-gates-aus-demjahr-1994 [1.3. 2018]. 
ausreichende Signaldichte bei jedem archivischen GPS zu achten, und zwar unabhängig davon, ob wir auf hoher Informationssee oder in seichten Gewässern surfen ${ }^{46}$.

Und: So kann man das Archiv sogar in den Alltag von Jedermann bringen, beispielsweise in einer Art lexikalischem Angebot wie dem Wien Geschichte Wiki, beispielsweise zum Thema Wiener Kongress oder in anderen Apps, beispielsweise im touristisch-kulturwirtschaftlichen Bereich mit der App fieldtrip. Dokumente hier zeit- und ortspräzise verfügbar zu machen, bereichert die Umweltwahrnehmung und schafft Mehrwert: Wien und der Wiener Kongress - beim Vorbeigehen am Bundeskanzleramt als dem Tagungsort des Wiener Kongresses könnte dann entsprechende historische Information und auch ein Bild der Schlussakte angeboten werden, wiederum zurückverlinkt zum Wien Geschichte Wiki. Vorteil der LOD ist, dass man nicht jeden Bezug selber herstellen muss, man muss nur die Daten nutzbar anbieten.

Kritik am Leuchtturmsystem der Findbücher und Begeisterung für neue Orientierungssysteme im Stile von GPS - Archivinformationssysteme - bedeuten nicht, dass alles Bisherige wertlos geworden ist. Ich plädiere keineswegs dafür, das Erarbeiten von Findbüchern dem UNESCO-Übereinkommen zur Erhaltung des immateriellen Kulturerbes zu unterstellen und etwa - in der Schweiz - in die Liste der gefährdeten lebendigen Traditionen aufzunehmen, zusammen mit dem Appenzeller Witz, der Basler Fasnacht und der Brienzer Holzschnitzerei. Ich halte mich lieber an die oft zitierte Einsicht von Tomasi di Lampedusa: „Wenn wir wollen, dass alles bleibt wie es ist, muss sich alles ändern“ ${ }^{47}$. Nutzen wir unser reichhaltiges Wissen, unsere enormen Erfahrungen und unsere Daten, aber modernisieren wir unsere Arbeitsweisen, erneuern wir unsere Produkte, gestalten wir das Suchen und Finden neu. So stärken wir die Stellung der Archive in der Informationsgesellschaft als einer Gesellschaft informationell mündiger Bürgerinnen und Bürger!

46 Aleida Assmann, Druckerpresse und Internet - von einer Gedächtniskultur zu einer Aufmerksamkeitskultur. Archiv und Wirtschaft. Zeitschrift für das Archivwesen der Wirtschaft 36 (2003) 5-12, hier 10.

47 Giuseppe Tomasi di Lampedusa, Der Leopard, übers. von Charlotte Birnbaum (München 2004) 35. 



\title{
Methoden der künstlichen Intelligenz und ihre Anwendung in der Erschließung von Textinhalten
}

\author{
Brigitte Krenn
}

Archivierung im 21. Jahrhundert geht Hand in Hand mit Digitalisierung. Entsprechend liegen immer mehr Daten in maschinenlesbarer Form vor und sind, theoretisch, bereit für vielfältigste computergestützte Untersuchungen. Ziel des folgenden Beitrags ist es, einen grundlegenden Einblick in Bereiche der Computerlinguistik beziehungsweise Sprachtechnologie zu vermitteln, die sich mit der Verarbeitung geschriebener Sprache befassen. Dabei werden zunächst computerlinguistische Verarbeitungsschritte vorgestellt, wie man von der unstrukturierten Zeichenkette zum morphosyntaktisch analysierten Satz gelangt und welche sprachlichen Ressourcen und Verarbeitungswerkzeuge dabei verwendet werden. Außerdem wird ein kurzer Exkurs ins maschinelle Lernen unternommen. Ein weiterer Abschnitt des Beitrags beschäftigt sich einerseits mit dem Auffinden von Texten (information retrieval) und andererseits mit der Untersuchung ihrer Inhalte (Informationsextraktion). Der letzte Abschnitt des Texts widmet sich Fragestellungen, die für die Erschließung digital archivierter Dokumente von besonderem Interesse sind, wobei die Darstellungen anhand einer Reihe von praxisbezogenen Fragen und aus einer computerlinguistischen Perspektive heraus erfolgen. Diese Vorgehensweise soll Archivarinnen und Archivaren als Gedankenanregung dienen, wo in ihrer Arbeit sprachtechnologische Methoden von Interesse sein können, und des Weiteren erste methodische Hinweise für die Verwendung von computerlinguistischen Ressourcen und Werkzeugen liefern ${ }^{1}$.

Sprachtechnologie (language technology - LT) beziehungsweise Sprachverarbeitung (natural language processing - NLP) ist ein breites Feld von Grundlagen- und angewandter Forschung, das sich mit verschiedensten Aspekten der Verarbeitung (Analyse und Generierung) gesprochener (speech) und geschriebener Sprache (text) beschäftigt, wobei sich der vorliegende Beitrag auf die Analyse von Texten konzentriert. Dabei geht es vor allem darum, Methoden, Ansätze und Vorgehensweisen zu beschreiben, die dazu geeignet sind, verschiedenste Arten von Information aus unstrukturierten Texten zu extrahieren.

Unstrukturierter Text ist digitalisierter Text (Daten) ohne formale Struktur, siehe zum Beispiel nachfolgenden Textausschnitt aus Bertha von Suttners „Langeweile“ nach der Wiedergabe im „Projekt Gutenberg“:

${ }^{1}$ Fachbegriffe werden, soweit sie im Deutschen gebräuchlich sind, übersetzt. Die originale, das heißt englische Terminologie wird in jedem Fall verwendet, um einen eindeutigen Bezug zur typischerweise englischsprachigen Terminologie der Forschung in Computerlinguistik und Künstlicher Intelligenz herzustellen. 
In dieser Stimmung habe ich mich zum Schreibtisch geschleppt, um meine Langeweile zu schildern. Das ist so eine Art, den Feind bei den Hörnern zu nehmen. "Hab' ich dich nun, graues, bleiernes Gespenst, mußt du mir jetzt Rechenschaft darüber geben, warum du mich plagst - warum du mich um die gute Meinung bringen willst, die ich mir selbst zu zollen pflegte? «2

Semistrukturierte Daten sind Daten mit strukturierender Information, aber auch Metainformation, wie zum Beispiel Dokumente in den Formaten $\mathrm{HTML}^{3}$ oder $\mathrm{XML}^{4}$, siehe zum Beispiel zum obigen Textausschnitt den HTML-Sourcecode von der Webseite:

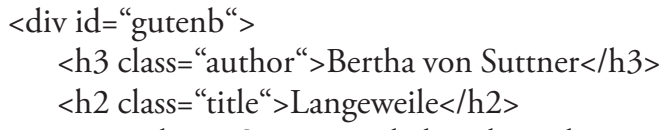

$<$ p $>$ In dieser Stimmung habe ich mich zum Schreibtisch geschleppt, um meine Langeweile zu schildern. Das ist so eine Art, den Feind bei den Hörnern zu nehmen. »Hab' ich dich nun, graues, bleiernes Gespenst, mußt du mir jetzt Rechenschaft darüber geben, warum du mich plagst - warum du mich um die gute Meinung bringen willst, die ich mir selbst zu zollen pflegte? «</p> $</$ div $>$

In diesem Beispiel semistrukturierten Textes finden sich einerseits Bereiche mit Metainformation wie der Information, dass es sich um einen Textausschnitt aus dem Projekt Gutenberg handelt (<div id="gutenb" $>$ ), die Autorin (class="author") des vorliegenden Textes Bertha von Suttner und der Titel (class= "title") des Textes „Langeweile“ ist. Die Labels $h 3$ und h2 sagen dem Browser, dass der Text in der mit h3 gekennzeichneten Klammer in kleinerem Font erscheinen soll als der Text in der $h 2$-Klammer. Die Zeichenketten $\langle p\rangle$ und $\langle/ p\rangle$ sagen, dass der damit eingerahmte Text einen Absatz darstellt. Innerhalb des Absatzes ist der Text unstrukturiert und kann unter Zuhilfenahme von sprachtechnologischen Methoden und Werkzeugen (tools) weiter automatisch analysiert und interpretiert werden.

\section{Von der Zeichenkette zum strukturierten Satz}

Mittels verschiedener Verarbeitungsschritte wird unstrukturierter Text mit Struktur beziehungsweise Zusatzinformation versehen. Dieser grundlegende Prozess soll anhand der Darstellung in Abbildung 1 näher erläutert werden.

Im Folgenden gehen wir davon aus, dass der Text digitalisiert und in ASCII-lesbarer Form vorliegt. Das heißt, für den Computer ist der Text mit all seinen Metainformationen eine unstrukturierte Abfolge von Zeichen. Unser Textbeispiel könnte wie folgt aussehen:

$<$ div id="gutenb" $><$ h3 class="author" $>$ Bertha von Suttner $</$ h3 $><$ h2 class= "title" $>$ Langeweile $</$ h2 $><$ p $>$ In dieser Stimmung habe ich mich zum Schreibtisch

2 Bertha von Suttner, Langeweile. Spiegel online. Projekt Gutenberg-DE, http://gutenberg.spiegel.de/ buch/langeweile-2595/1 [9. 2. 2018].

3 HTML5 Tutorial, in: w3schools.com, https://www.w3schools.com/html/ [9. 2. 2018].

4 XML Tutorial, in: w3schools.com, https://www.w3schools.com/xml/ [9. 2. 2018]. 


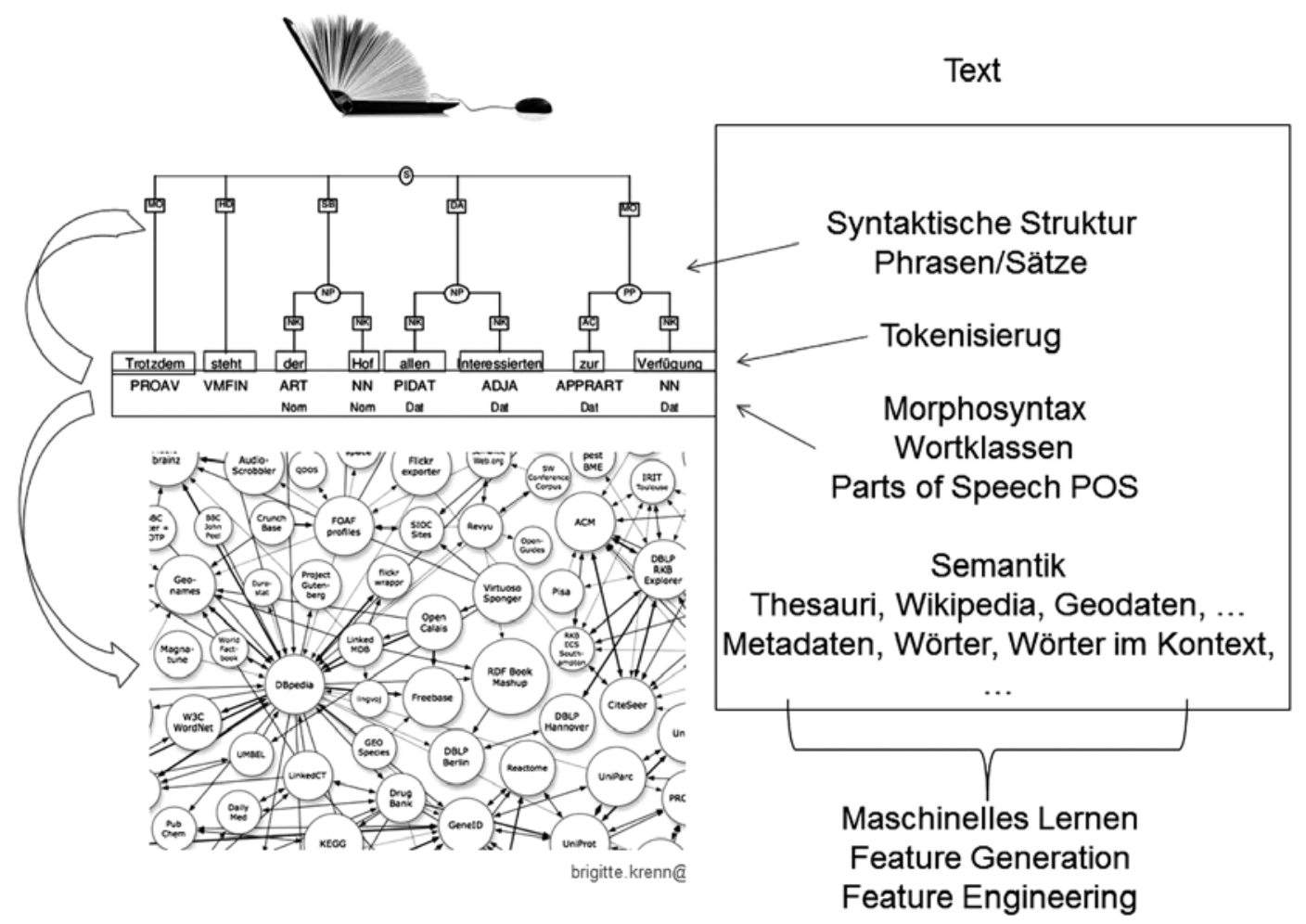

Abb.1: Verarbeitungsstufen in der Textanalyse (Brigitte Krenn).

geschleppt, um meine Langeweile zu schildern. Das ist so eine Art, den Feind bei den Hörnern zu nehmen. "Hab’ ich dich nun, graues, bleiernes Gespenst, mußt du mir jetzt Rechenschaft darüber geben, warum du mich plagst - warum du mich um die gute Meinung bringen willst, die ich mir selbst zu zollen pflegte? «</ $\mathrm{p}></$ div $>$

In einem ersten Schritt wird dieser Text nun um jene Elemente, die nicht Teil des Textes sind - wie beispielsweise den HTML-Code - bereinigt und tokenisiert. In unserem Fall bedeutet das, dass alle Metainformation (hier zum Beispiel die Zeichenketten in Spitzklammern) gelöscht beziehungsweise durch ein Leerzeichen ersetzt wird. Dieser Schritt resultiert in folgender Zeichenkette:

Bertha von Suttner Langeweile In dieser Stimmung habe ich mich zum Schreibtisch geschleppt, um meine Langeweile zu schildern. Das ist so eine Art, den Feind bei den Hörnern zu nehmen. "Hab' ich dich nun, graues, bleiernes Gespenst, mußt du mir jetzt Rechenschaft darüber geben, warum du mich plagst - warum du mich um die gute Meinung bringen willst, die ich mir selbst zu zollen pflegte?» 
Tokenisierung ist der Verarbeitungsschritt, um aus einer Zeichenkette einzelne Wörter zu extrahieren. Dabei ist es damit nicht getan, alles das, was zwischen zwei Leerzeichen oder zwischen einem Leerzeichen und einem potentiellen Satzzeichen steht, als Wort zu betrachten, sondern es müssen eine Reihe von Teilaspekten berücksichtigt werden, wie zum Beispiel: Trennstriche, die zwei Teile eines Wortes verbinden, sind von Gedankenstrichen zu unterscheiden; Punkte am Satzende von Punkten als Teil von Abkürzungen oder als Teil von speziellen Ausdrücken wie beispielsweise URLs (gutenberg.spiegel.at), Zahlen (2.005), Datumsangaben (21.12.2013). Bei Leerzeichen zwischen Ziffern ist zu entscheiden, ob es sich um eine oder mehrere Zahlen handelt. Ein Hochkomma kann als Anführungszeichen, aber auch als Apostroph fungieren. Eigennamen möchte man als eine Einheit identifizieren und nicht als Aneinanderreihung voneinander unabhängiger Wörter. Alles in allem ist Tokenisierung eine Voraussetzung für die weitere Verarbeitung eines Textes 5 .

Texte werden nicht nur in ihre Wörter zerlegt, sondern man möchte in der weiteren Verarbeitung auch auf Satzeinheiten zugreifen können. Sentence Splitting ist der Vorgang, der einen Text in seine Einzelsätze zerlegt. Dazu werden Satzzeichen und das Wissen über gängige Abkürzungen in der Sprache beziehungsweise der zu verarbeitenden Domäne und Textsorte eingesetzt. Oft werden Tokenisierung und Sentence Splitting in einem Verarbeitungsschritt durchgeführt, wobei sowohl regelbasierte als auch auf maschinellem Lernen basierende Methoden angewandt werden ${ }^{6}$.

Part-of-Speech (PoS) Tagging ist der Vorgang, der eine Sequenz von Wörtern mit einer zugehörigen Sequenz von eindeutigen Part-of-Speech Tags versieht, das heißt morphosyntaktischen Kategorien wie Nomen, Verb, Artikel, Adjektiv und dergleichen. Jedem Wort im laufenden Text wird eine eindeutige morphosyntaktische Kategorie (PoS-Tag) zugeordnet. Zur Illustration siehe das Beispiel in Abbildung 1, wo jedem Wort ein PoSTag zugeordnet ist:

\begin{tabular}{|l|l|l|l|l|l|l|l|}
\hline Trotzdem & steht & der & Hof & allen & Interessierten & zur & Verfügung \\
\hline PROAV & VMFIN & ART & NN & PIDAT & ADJA & APPRART & NN \\
\hline
\end{tabular}

Die PoS-Tags stammen aus dem Stuttgart-Tübingen Tagset (STTS) ${ }^{7}$ : PROAV steht für Pronominaladverb, VMFIN steht für ein Funktionsverb (hier im Funktionsverbgefüge „zur Verfügung stehen“), ART für bestimmten oder unbestimmten Artikel, NN für Nomen, PIDAT für attribuierendes Indefinitpronomen ohne Determiner, ADJA für attributives Adjektiv (hier nominalisiert verwendet), APPRART für Präposition mit Artikel.

PoS-Tagging ist Ausgangspunkt für viele Anwendungen in der Textverarbeitung. Typischerweise basiert PoS-Tagging auf maschinellen Lernverfahren, wobei die entsprechenden Modelle auf manuell annotierten Texten mit Millionen von Wörtern trainiert werden. Diese Texte stammen aus Treebanks, das sind Textkorpora, die mit PoS und syn-

\footnotetext{
5 Jonathan J. Webster-Chunyu Kit, Tokenization as the Initial Phase in NLP, in: Proceedings of the Fifteenth International Conference on Computational Linguistics. Nantes, August 23-28, 1992, 4: Notes de projet sans démonstrations, hg. von Christian Boitet (Grenoble 1992) 1106-1110.

${ }^{6}$ Für die Diskussion eines auf maschinellem Lernen basierenden Ansatzes vgl. Katrin ToManEK-Joachim Wermter-Udo Hahn, Sentence and Token Splitting Based on Conditional Random Fields, in: Proceedings of the 10th Conference of the Pacific Association for Computational Linguistics (Melbourne 2007) 49-57.

7 STTS Tag Table (1995/1999), in: Institut für Maschinelle Sprachverarbeitung. Lexika, http://www.ims. uni-stuttgart.de/forschung/ressourcen/lexika/TagSets/stts-table.html [9. 2. 2018].
} 


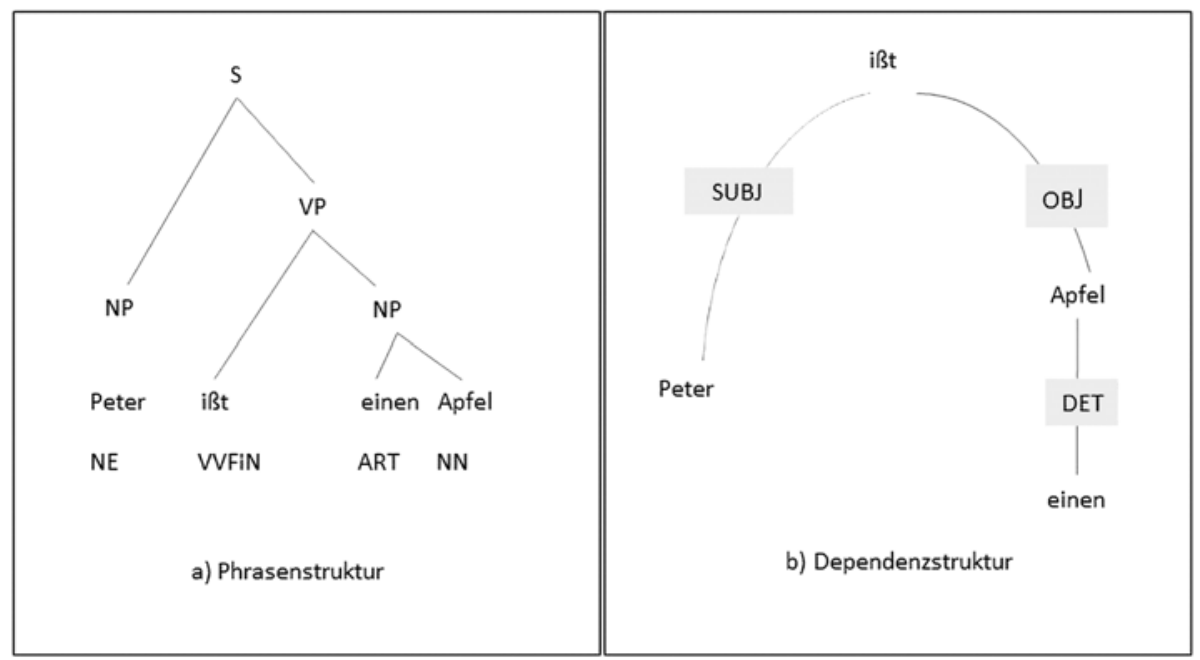

Abb. 2: Syntaktische Strukturen (Brigitte Krenn).

taktischer Struktur annotiert und manuell korrigiert sind. Beispiele für eine deutsche und eine englische Treebank sind das Tiger Corpus $^{8}$ und die Penn Treebank ${ }^{9}$. Ein Beispiel für einen auf maschinellem Lernen basierten Part-of-Speech Tagger wurde von Helmut Schmid 2013 beschrieben ${ }^{10}$. Georg Heigold, Günter Neumann und Josef van Genabith untersuchen neuronale Lernmodelle zum morphologischen Tagging, das heißt, es werden nicht nur PoS annotiert, sondern auch morphologische Informationen wie Numerus, Genus und Kasus, Person, Tempus und Modalität ${ }^{11}$.

Weitere Schritte in der sprachtechnologischen Analyse sind Phrase Chunking und Parsing. Beim Phrase Chunking oder Partial Parsing werden typischerweise Nominalund Präpositionalphrasen identifiziert. Dies kann entweder mittels manuell erstellter Regeln zur NP- und PP-Grammatik der jeweiligen Sprache basierend auf PoS-Sequenzen oder mittels maschineller Lernverfahren und Training auf Treebanks bewerkstelligt wer$\operatorname{den}^{12}$.

Unter Parsing oder Full Parsing versteht man die vollständige syntaktische Analyse eines Satzes. Dabei werden generell zwei Ansätze unterschieden: Phrasenstruktur-Parsing

8 TIGER Corpus, in: Institut für Maschinelle Sprachverarbeitung. Corpora, http://www.ims.uni-stuttgart. de/forschung/ressourcen/korpora/tiger.en.html [9. 2. 2018].

9 Treebank-3, in: Linguistic Data Consortium, https://catalog.ldc.upenn.edu/ldc99t42 [9. 2. 2018].

${ }^{10}$ Helmut Schmid, Probabilistic Part-of-Speech Tagging Using Decision Trees, in: New Methods in Language Processing, hg. von Daniel B. Jones-Harold Leslie Somers (Studies in Computational Linguistics, London 1997) 154-164.

11 Georg Heigold-Günter Neumann-Josef van Genabith, An Extensive Empirical Evaluation of Character-Based Morphological Tagging for 14 Languages, in: EACL 2017. 15th Conference of the European Chapter of the Association for Computational Linguistics. April 3-7, 2017, Valencia, Spain. Proceedings of Conference, 1: Long Papers, hg. von Phil Blunsom-Alexander Koller (Stroudsburg 2017) 505-513.

12 Erik F. TJong KIm SAng-Sabine Buchrolz, Introduction to the CoNLL-2000 Shared Task: Chunking, in: CoNLL '00. Proceedings of the 2nd Workshop on Learning Language in Logic and the 4th Conference on Computational Natural Language Learning. Lisbon, Portugal - September 13-14, 2000 (New Brunswick 2000) 127-132. 
(phrase structure parsing) und Dependenz-Parsing (dependency parsing). Während bei Ersterem die Wörter eines Satzes in syntaktischen Einheiten (Satz S, Nominalphrase NP, Verbalphrase VP) gruppiert werden (Abbildung 2a), werden bei einer Dependenzstrukturanalyse funktionale Beziehungen (Subjekt SUBJ, Objekt OBJ, Determiner/Artikel DET) zwischen 2 Wörtern (ißt-Peter, ißt-Apfel, Apfel-einen) hergestellt, womit Bedeutungszusammenhänge direkt sichtbar werden (Abbildung 2b).

Gegenwärtige Parser basieren typischerweise auf maschinellem Lernen und sind auf Baumbanken (Treebanks) trainiert. Da die Fehlerrate beim vollständigen Parsing mit der Komplexität der zu analysierenden Sätze steigt, wird in der Praxis Partial Parsing anstelle von Full Parsing eingesetzt. Hier sind die Ergebnisse zuverlässiger und für viele Anwendungen in der Informationsextraktion ist es ausreichend, auf Teilstrukturen anstelle von vollständigen Satzstrukturen zu arbeiten ${ }^{13}$.

\section{Sprachressourcen und sprachtechnologische Werkzeuge}

Sprachressourcen (language resources) ist ein Überbegriff für Wortlisten, Lexika, Terminologiedatenbanken, Thesauri, verschiedene Sammlungen an Textkorpora wie beispielsweise Treebanks, aber auch Textsammlungen ohne spezielle Annotierungen wie zum Beispiel parallele Korpora, die Texte und deren Übersetzung in eine andere oder mehrere andere Sprachen enthalten und für das Trainieren von Modellen für die maschinelle Übersetzung dienen, um nur ein paar Beispiele zu nennen. Es gibt eine Reihe von Initiativen, die der Bereitstellung solcher Ressourcen dienen, siehe etwa das Linguistic Data Consortium LDC ${ }^{14}$, das entsprechende Europäische Pendant ELRA/ELDA ${ }^{15}$ oder die ebenfalls europäische Initiative CLARIN ${ }^{16}$ mit einem Schwerpunkt auf Ressourcen für Geistes- und Sozialwissenschaften.

Für die Be- und Verarbeitung der Sprachressourcen gibt es eine Reihe von Werkzeugen (software tools), wie Tokenizer, Part-of-Speech Tagger, Partial Parser zur syntaktischen Analyse von Nominal- oder Präpositionalphrasen, Full Parser zur syntaktischen Analyse ganzer Sätze. Verschiedene Softwarebibliotheken stehen dafür zur Verfügung, etwa Core NLP oder NLTK ${ }^{17}$. Diese Tools wurden typischerweise für die computerlinguistische

13 Sabine Buchholz-Erwin Marsi, CONLL-X Shared Task on Multilingual Dependency Parsing, in: Proceedings of the Tenth Conference on Computational Natural Language Learning. New York City, New York - June 08-09, 2006, hg. von Lluis Marquez-Dan Klein (Stroudsburg 2006) 149-164; Djamé SEdDaH et al., Overview of the SPMRL 2013 Shared Task: Cross-Framework Evaluation of Parsing Morphologically Rich Languages, in: SPMRL-2013. Fourth Workshop on Statistical Parsing of Morphologically Rich Languages. Proceedings of the Workshop. 18 October 2013, Grand Hyatt Seattle, Seattle, Washington, USA, hg. von Yoav Goldberg-Yuval Marton-Ines Rehbein-Yannick Versley (Stroudsburg 2013) 146-182; Lingpeng Kong-Nathan Schneider-Swabha Swayamdipta-Archna Bhatia-Chris Dyer-Noah A. Smith, A Dependency Parser for Tweets, in: EMNLP 2014. The 2014 Conference on Empirical Methods in Natural Language Processing. Proceedings of the Conference, October 25-29, 2014, Doha, Qatar, hg. von Bo PANG-Walter Daelemans (Stroudsburg 2014) 1001-1012.

${ }_{14}$ Language Resources, in: Linguistic Data Consortium, https:/www.ldc.upenn.edu/language-resources [9. 2. 2018].

15 European Language Resources Association, http://www.elra.info/en/ [9. 2. 2018].

16 CLARIN - European Research Infrastructure for Language Resources and Technology, https://www. clarin.eu/ [9. 2. 2018].

17 Stanford CoreNLP - Natural language software, http://nlp.stanford.edu/software/corenlp.shtml [9. 2. 2018]; Natural Language Toolkit, http://www.nltk.org/ [9. 2. 2018]. Für weitere Beispieltools vgl. 5 Open 
Lehre entwickelt und werden im akademischen Bereich oft als Basiskomponenten in der Entwicklung von Prototypen verwendet.

Mit dem Aufkommen der Forschungsrichtung Digital Humanities, das heißt der Anwendung und Entwicklung von computergestützten Werkzeugen speziell für die Geisteswissenschaften, sind auch eine Reihe von Toolsammlungen speziell für Digital Humanities entstanden ${ }^{18}$.

\section{Exkurs: Der Einsatz von maschinellem Lernen}

Maschinelles Lernen (machine learning - ML) ist heute aus der Sprachtechnologie und der Künstlichen Intelligenz insgesamt nicht mehr wegzudenken. Die ML zugrundeliegende Idee ist, dass der Computer ausgehend von einem Beispieldatensatz, dem Trainingskorpus, bestimmte Aufgaben lernt, wie das Gruppieren von Dokumenten anhand ihrer inhaltlichen Ähnlichkeiten (Dokumenten-Clustering oder Dokumentenklassifikation). Das gelernte Modell wird dann auf neue Daten angewendet. Im ML werden zwei Strategien verfolgt: überwachtes (supervised) oder unüberwachtes (unsupervised) Lernen. Bei Ersterem enthalten die Trainingsdaten Annotierungen, wie das korrekte Ergebnis aussehen soll, etwa bei der Dokumentenklassifikation sind die Trainingsdaten mit Information hinsichtlich ihrer Klassenzugehörigkeit versehen. Dies ist beim unüberwachten Lernen nicht der Fall. Hier soll das Lernverfahren selbständig Muster in den Trainingsdaten erkennen. Am Beispiel von Dokumenten-Clustering bedeutet das, der Lernalgorithmus findet Klassen aufgrund von Ähnlichkeiten zwischen den einzelnen Datensätzen (Dokumenten).

Es gibt eine breite Auswahl an ML-Werkzeugen und Bibliotheken. Dazu gehören unter anderem Apache Mahout ${ }^{19}$, die Java-Bibliotheken Weka und MOA (letztere speziell für Data Mining von Input Streams) ${ }^{20}$, scikit-learn (Pyton) ${ }^{21}$, TensorFlow, Theano, Caffe, Torch, wobei die letzteren vier vor allem für Deep Learning interessant sind ${ }^{22}$.

Deep Learning ist ein Oberbegriff für die verschiedensten Ansätze zum maschinellen Lernen mit Artifiziellen Neuronalen Netzen (ANNs), die mehr als einen Neuronenlayer haben. Deep-Learning-Algorithmen haben in letzter Zeit eine weite Verbreitung gefunden, da sie in verschiedenen Bereichen der Künstlichen Intelligenz zu mit anderen Verfahren konkurrenzfähigen oder diese übertreffenden Ergebnissen geführt haben, zum Beispiel in der Bild- und Sprachverarbeitung. Insbesondere in der Sprachverarbeitung

Source Tools for Taming Text, in: opensource.com, https://opensource.com/business/15/7/five-open-sourcenlp-tools [9. 2. 2018].

18 TAPoR 3. Discover research tools for studying texts, http://tapor-test.artsrn.ualberta.ca/home [9. 2. 2018]; Digital Humanities Tools, http://dhresourcesforprojectbuilding.pbworks.com/w/page/69244319/Digital [9. 2. 2018].

19 MAHOUT. For Creating Scalable Performant Machine Learning Applications, http://mahout.apache. org/ [9. 2. 2018].

20 Weka 3: Data Mining Software in Java, in: WEKA. The University of Waikato, http://www.cs.waikato. ac.nz/ml/weka/ [9. 2. 2018]; MOA. Machine Learning for Streams, https://moa.cms.waikato.ac.nz/ [9. 2. 2018].

21 scikit-learn, https://github.com/scikit-learn [9. 2. 2018].

22 TensorFlow. An Open-Source Machine Learning Framework for Everyone, https://www.tensorflow.org/ [9. 2. 2018]; theano, http://deeplearning.net/software/theano/ [9. 2. 2018]; Caffe. Deep Learning Framework by BAIR, http://caffe.berkeleyvision.org/ [9. 2. 2018]; Torch. A Scientific Computing Framework for LuaJIT, http://torch.ch/ [9. 2. 2018]. 
gehören dazu die Spracherkennung (speech recognition), das Erkennen semantischer Ähnlichkeiten, aber auch die maschinelle Übersetzung ${ }^{23}$. Eine wichtige Voraussetzung für Deep Learning ist das Vorhandensein sehr großer Datenmengen (Millionen von Texten, Milliarden von Wörtern), von denen gelernt werden kann.

\section{Zum Auffinden von Texten und zur Untersuchung ihrer Inhalte}

Zwei wichtige Bereiche in der Untersuchung von Texten sind Informationsauffindung (information retrival - IR) und Informationsextraktion (information extraction - IE). IR dient dazu, aus einer Menge an Dokumenten jene zu identifizieren, die auf eine spezielle Suchanfrage passen. Teilbereiche von IR sind Querying, Dokumentenklassifikation und Dokumentenclustering. Mittels IE werden verschiedenste Arten von Informationen aus unstrukturierten Texten identifiziert. Dazu gehört das Auffinden von sogenannten Named Entities wie beispielsweise Personennamen, Firmennamen, Ortsnamen und dergleichen. Voraussetzung für IR sowie für IE ist, dass die Daten in maschinenlesbarer Form vorliegen. Beide Bereiche werden im Folgenden näher besprochen.

Im weiteren Sinne bedeutet Information Retrieval das Auffinden von einzelnen Dokumenten in einer Sammlung (bis zu Millionen oder Milliarden) von Dokumenten basierend auf bestimmten Informationsanforderungen, die in sogenannten Queries formuliert werden. Querying befasst sich mit dem Abbilden einer Suchanfrage auf einzelne Dokumente, das heißt dem Berechnen, wie gut eine Suchanfrage auf ein einzelnes Dokument passt. Dies ist auch ein Teilaspekt dessen, was Suchmaschinen wie Google im Internet machen.

Der Information-Retrieval-Prozess beginnt damit, dass eine Benutzerin eine Anfrage (query) in das IR-System eingibt. Eine Query kann aus einem beziehungsweise idealerweise mehreren Suchwörtern oder -phrasen bestehen oder auch aus ganzen Sätzen, welche vom System letztendlich auf Suchwörter, -phrasen abgebildet werden.

Query Expansion ist dabei ein wichtiges Mittel, um die von der Benutzerin abgesetzte Suchanfrage zu erweitern. So werden dabei etwa Tippfehler korrigiert und flektierte Wörter auf ihre Stammform reduziert (stemming): Beispielsweise werden „Haus, Hauses, Häusern, Häuser" jeweils auf die Stammform „Haus“ abgebildet. Synonyme und ähnliche Wörter werden hinzugenommen. Dazu werden semantische Ressourcen verwendet, wie WordNet oder ConceptNet ${ }^{24}$, und in jüngerer Zeit Word Embeddings. Das sind Vector-Space-Modelle, wobei Wörter aufgrund ihrer Ähnlichkeiten beziehungsweise Verschiedenheiten basierend auf ihrem Auftreten in sehr großen Textkorpora (Milliarden von Wörtern) miteinander gruppiert oder voneinander unterschieden werden ${ }^{25}$.

23 Yann LeCun-Yoshua Bengio-Geoffrey Hinton, Deep Learning. Nature. International Journal of Science 521 (2015) 436-444.

24 WordNet. A Lexical Database for English, https://wordnet.princeton.edu/ [9. 2. 2018]; ConceptNet. An Open, Multilingual Knowledge Graph, http://conceptnet.io/ [9. 2. 2018]; vgl. Ming-Hung Hsu-MingFeng TsaI-Hsin-Hsi Chen, Query Expansion with ConceptNet and WordNet: An Intrinsic Comparison, in: Information Retrieval Technology. Third Asia Information Retrieval Symposium, AIRS 2006. Singapore, October 16-18, 2006. Proceedings, hg. von Hwee Tou NG-Mun-Kew Leong-Min-Yen Kan-Donghong Ji (Lecture Notes in Computer Science 4182, Berlin-Heidelberg 2006) 1-13.

25 Piotr Bojanowski-Edouard Grave-Armand Joulin-Tomas Mikolov, Enriching Word Vectors with Subword Information. Transactions of the Association for Computational Linguistics 5 (2017) 135-146; Mohannad Almasri-Catherine Berrut-Jean-Pierre Chevallet, A Comparison of Deep Learning Based Query Expansion with Pseudo-Relevance Feedback and Mutual Information, in: Advances in Information Retrie- 


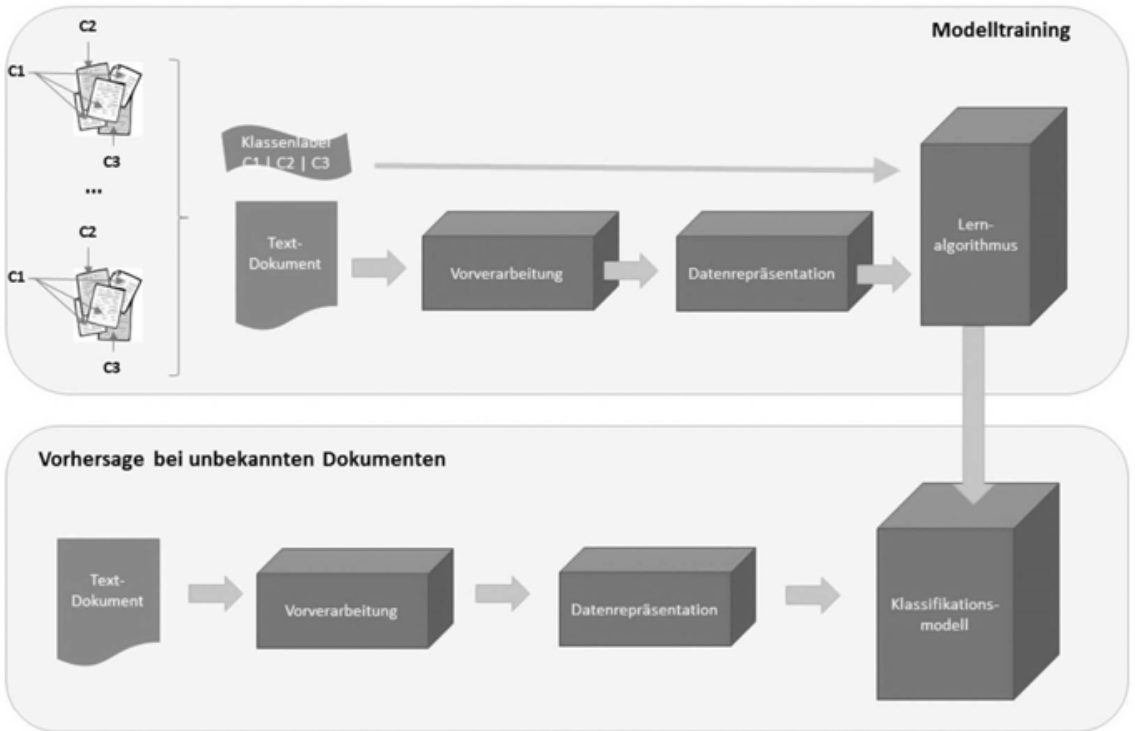

Abb. 3: Document Classification (Brigitte Krenn).

Dokumentenklassifikation (document classification) ist ein Teilbereich von maschinellem Lernen, bei dem basierend auf einer Sammlung von bereits (typischerweise manuell) klassifizierten Dokumenten ein Modell trainiert wird, mit Hilfe dessen für neue Dokumente entschieden wird, ob beziehungsweise wie ähnlich sie den bereits klassifizierten Dokumenten sind. Bei dieser Art von Lernverfahren handelt es sich um überwachtes Lernen (supervised learning). Abbildung 3 gibt einen schematischen Überblick über die Verarbeitungsschritte in der Dokumentenklassifikation. Für das Modelltraining werden bereits klassifizierte Daten benötigt. In unserem Beispiel sind die Dokumente, von denen gelernt wird, in drei Klassen (C1, C2, C3) unterteilt. Die Textdokumente werden (i) vorverarbeitet, unter anderem werden sogenannte Stopwords (Artikel, Präpositionen und dergleichen) entfernt; für den Text charakteristische Terme werden identifiziert, semantische Information wird extrahiert; (ii) die aus den Texten extrahierte Information wird modelliert (Datenrepräsentation), beispielsweise als Merkmalsvektoren; (iii) Lernalgorithmen werden angewandt, um Modelle zu bilden, die die in den Dokumenten enthaltene Information so modellieren, dass Dokumente aus derselben Klasse nahe beieinander liegen, einander ähnlich sind und Dokumente aus unterschiedlichen Klassen sich möglichst voneinander unterscheiden. Das dabei gelernte Klassifikationsmodell wiederum wird eingesetzt, um neue, vom Modell bisher nicht gesehene Texte zu klassifizieren („Vorhersage bei unbekannten Dokumenten“).

Dokumenten-Clustering (document clustering) ist das Gegenstück von Klassifikation und gehört in den Bereich unüberwachtes Lernen (unsupervised learning). Docu-

val. 38th European Conference on IR Research, ECIR 2016. Padua, Italy, March 20-23, 2016. Proceedings, hg. von Nicola Ferro et al. (Lecture Notes in Computer Science 9626, Cham 2016) 709-715; Tomas Mikolov-Ilya Sutskever-Kai Chen-Greg S. Corrado-Jeffrey Dean, Distributed Representations of Words and Phrases and their Compositionality, in: Advances in Neural Information Processing Systems 26. 27th Annual Conference on Neural Information Processing Systems 2013. December 5-10, Lake Tahoe, Nevada, USA. Proceedings, hg. von Chris Burges et al., 4 Bde. (Red Hook 2014) 4 3111-3119. 


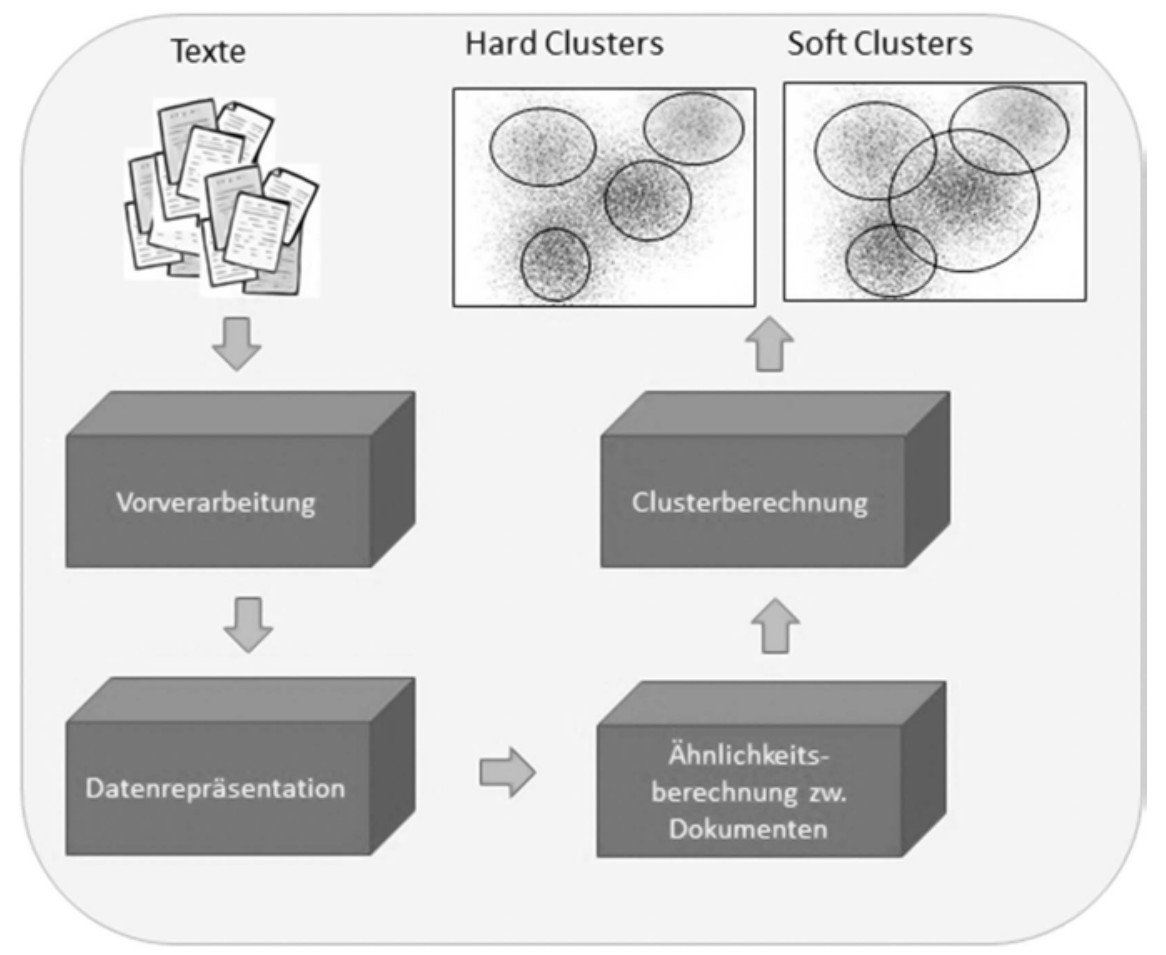

Abb. 4: Document Clustering (Brigitte Krenn).

ment Clustering bedeutet das Gruppieren von Dokumenten anhand ihrer inhaltlichen Ähnlichkeit. Ähnliche Dokumente werden in eine Gruppe, ein Cluster zusammengelegt. Abbildung 4 illustriert die Verarbeitungsschritte im Document Clustering. Input sind unklassifizierte, unannotierte Texte, die vorverarbeitet und deren Information typischerweise mittels Merkmalsvektoren repräsentiert wird. Auf Basis der Merkmalsvektoren werden Ähnlichkeits- und Clusterberechnungen durchgeführt. Je nach Clusteringansatz werden die Texte eindeutig einem Cluster (hard clustering) oder mehreren Clustern (soft clustering) zugeordnet. Es existiert eine Vielzahl von Klassifikations- und Clusteringmethoden ${ }^{26}$. Für Beispiele von ML-Bibliotheken sei auf den Exkurs am Ende des vorhergehenden Abschnitts verwiesen.

26 Jiaming Xu et al., Self-Taught Convolutional Neural Networks for Short Text Clustering. Neural Networks 88 (2017) 22-31; Manisha Kannvdiya-Kailash Patidar-Rishi Singh Kushwaha, A Survey on: Different Techniques and Features of Data Classification. International Journal of Research in Computer Applications and Robotics 4/6 (2016) 1-6; Adil FAHAD et al., A Survey of Clustering Algorithms for Big Data: Taxonomy and Empirical Analysis. IEEE Transactions on Emerging Topics in Computing 2 (2014) 267-279; Data Clustering. Algorithms and Applications, hg. von Charu C. AgGarwal-Chandan K. Reddy (Chapman \& Hall/CRC Data Mining and Knowledge Discovery Series, Boca Raton 2014); Charu C. Aggarwal-Chengxiang Zhai, A Survey of Text Classification Algorithms, in: Mining Text Data, hg. von Charu C. Aggarwal-Chengxiang Zhai (New York-Dordrecht-Heidelberg-London 2012) 163-222; Christopher D. Manning-Prabhakar RaGHavan-Hinrich SchüTZe, Introduction to Information Retrieval (Cambridge u. a. 2008). 
Informationsextraktion (IE) ist ebenso wie IR ein breit untersuchtes Gebiet in der Sprachtechnologie mit einer Reihe von Methoden, Werkzeugen und Bibliotheken. Für beide Gebiete gilt, wie für alles in der sprachtechnologischen Verarbeitung von Texten: Je besser die einzelnen Verarbeitungskomponenten und Schritte an die zu verarbeitende Art von Texten angepasst sind, desto besser werden die Ergebnisse sein.

IE ist ein Überbegriff für verschiedene Methoden und Werkzeuge, die dazu dienen, strukturierte Information aus unstrukturierten Daten, zum Beispiel Texten, aber auch Bildern, Audio- und Videomaterial zu extrahieren. Dazu gehören unter anderem die Extraktion von Eigen-, Firmen- und Ortsnamen, Informationen zu Ereignissen, Datums- und Zeitangaben, Themen, Meinungen und dergleichen. Typische Aufgabenbereiche der IE sind: das Erkennen von Eigen-, Orts- und Firmennamen (named entity recognition), das Auflösen von referenziellen Ausdrücken (coreference resolution), die Extraktion semantischer Beziehungen (relationship extraction) sowie Term- und Terminologieextraktion.

Named Entity Recognition (NER) umfasst die Teilbereiche der Identifizierung von Eigennamen, Firmennamen, Ortsnamen, Datums- und Zeitangaben einerseits - das heißt, es geht darum zu erkennen, ob eine bestimmte Zeichenkette ein Name (named entity) ist oder nicht - und deren eindeutiger Zuordnung zu Entitäten (Personen, Firmen, Orten und so fort) in der Welt andererseits. Coreference Resolution bezieht sich auf die Zuordnung von aktuellen Nennungen im Text zu bereits vorher im Text identifizierten Entitäten. Siehe dazu das nachfolgende Beispiel aus einem Text der Website des Steiermärkischen Landesarchivs:

Das Steiermärkische Landesarchiv sammelt und bewahrt die schriftlichen und bildlichen Quellen zur Geschichte des Landes Steiermark und hält sie zugänglich ${ }^{27}$.

Dieses Beispiel enthält zum einen zwei Named Entities, die durch NER erkannt und markiert werden könnten: die Zeichenketten Steiermärkische Landesarchiv sowie Steiermark. Zum anderen liegt eine referentielle Beziehung vor, nämlich eine anaphorische Referenzbeziehung zwischen dem Pronomen sie und der Phrase schriftlichen und bildlichen Quellen zur Geschichte des Landes Steiermark. Diese zu erkennen, wäre Aufgabe der Coreference Resolution.

Relationship Extraction bezieht sich auf die Identifizierung von Beziehungen zwischen Entitäten, zum Beispiel: Eine Person hat eine bestimmte Funktion, arbeitet für eine Organisation, eine Firma hat eine bestimmte Adresse und dergleichen. Dies kann am nachfolgenden Beispiel illustriert werden:

An Stelle des verstorbenen Landtagsabgeordneten Prof. Dr. Alois Dienstleder wird Landtagsabgeordneter Hugo Mrazek als Mitglied in den Gemeinde-und Verfassungsausschuß gewählt28.

Darin finden sich folgende Beziehungen zwischen Named Entities und Funktionen: Prof. Dr. Alois Dienstleder ist Landtagsabgeordneter und verstorben, Hugo Mrazek ist Landtagsabgeordneter, der Gemeinde- und Verfassungsausschuß hat Mitglieder, Hugo Mra-

27 Das Land Steiermark - Steiermärkisches Landesarchiv, http://www.landesarchiv.steiermark.at/ [9. 2. 2018].

28 Steiermärkischer Landtag, I. Periode, 1946, 5. Sitzung am 1. März 1946, Beschluss Nr. 16, in: Das Land Steiermark - Steiermärkisches Landesarchiv - Landtagsprotokolle seit 1861, http://www.landesarchiv. steiermark.at/cms/dokumente/12083702_111932290/08b184b3/LTProt-Be-1945-1949-Nr-1-178.pdf [9. 2. 2018]. 
$z e k$ wird Mitglied des Gemeinde- und Verfassungsausschusses. Für die Identifizierung dieser Art von Beziehungen ist eine syntaktische Analyse des Satzes von Bedeutung. Da Full Parsing von komplexeren Satzstrukturen fehleranfällig und entsprechend nicht verlässlich ist, kann diese Art von Bedeutungsbeziehungen in der Praxis nur unter Zuhilfenahme verschiedenster Arten von Information (syntaktisch, lexikalisch, ontologisch) und von Heuristiken behandelt werden.

In der Term- beziehungsweise Terminologieextraktion geht es einerseits darum, wichtige Begriffe für einen Textkorpus zu identifizieren, um beispielsweise Wissensdomänen zu konzeptualisieren, entsprechende Domänenontologien und Terminologielexika aufzubauen. Andererseits geht es darum, die Begriffe zu identifizieren, die charakteristisch für einen bestimmten einzelnen Text sind und die diesen von den restlichen Dokumenten in der Datenbasis unterscheiden. Damit lässt sich herausfinden, um welche speziellen Themen es in einem bestimmten Text geht. Ein Standardmaß dafür ist TFIDF (term frequency-inverse document frequency $)^{29}$.

Eine spezielle Art von Termen sind Mehrwortausdrücke (multi-word expressions MWE), das heißt zwei oder mehrere Wörter, die überzufällig häufig miteinander vorkommen. Typische MWE sind englische Komposita (sun tan, bus stop), Named Entities („New York“, „Dresdner Bank“), Kollokationen („blinder Passagier“, „zur Verfügung stellen“) und bildliche Ausdrücke („zu Fall bringen“, „am Herzen liegen“). Für ihre Identifikation wird eine Reihe von Maßen angewandt ${ }^{30}$.

Um Terme aus Textdaten zu extrahieren, werden diese erst mit PoS-Tags versehen, die PoS-getaggten Texte werden typischerweise mittels Phrase Chunking in Nominal(NP), Verbal- (VB) und Präpositionalphrasen (PP) zerlegt. Auf diese Weise können syntaktisch plausible Termkandidaten (zum Beispiel Adjektiv-Nomen, Nomen-Nomen, Verb-Nomen, Verb-PP) identifiziert und das Rauschen in den Daten reduziert werden. Ein Minimalansatz ohne Einbeziehung morphosyntaktischer Information hingegen ist, aus einem Fließtext alle Wörter, die einer geschlossenen Klasse angehören, zu löschen. Das sind jene Wörter einer Sprache, die vollständig aufgezählt werden können; dazu gehören insbesondere Artikel, Pronomen, Präpositionen und Partikel. Danach werden N-Gramme (typischerweise Uni-, Bi- und Tri-Gramme) von adjazenten Wörtern gebildet und deren Auftretenshäufigkeiten im Textkorpus als Ausgangsmaterial für die weiteren Berechnungen verwendet. Bezogen auf den Beispielsatz „Peter ißt einen Apfel“ sieht das wie folgt aus:

\begin{tabular}{|l|l|l|}
\hline Uni-Gramme & Bi-Gramme & Tri-Gramme \\
\hline Peter ißt Apfel & Peter ißt ißt Apfel & Peter ißt Apfel \\
\hline
\end{tabular}

\section{Überlegungen zur Erschließung digital archivierter Dokumente}

Basierend auf den in den vorhergehenden Abschnitten besprochenen Methoden soll näher darauf eingegangen werden, was zu tun ist, um aus ASCII-lesbaren Texten Informationen zu extrahieren, beziehungsweise wie man Textbestände inhaltlich gruppieren kann, zum Beispiel wenn es keine Metadaten gibt oder die vorhandenen Metadaten nicht

29 Manning-Raghavan-Schütze, Introduction (wie Anm. 26).

30 Siehe zum Beispiel: www.collocations.de. Computational Approaches to Collocations, http://www.collocations.de/ [9. 2. 2018]. 
mehr ausreichend interpretierbar sind. Dieser Abschnitt ist nun als Leitfaden gedacht. Im Folgenden werden zwei Aufgabenstellungen besprochen. Erstens sollen Textdokumente anhand von Ähnlichkeiten gruppiert werden; zweitens soll ermittelt werden, welche Information in einzelnen Dokumenten enthalten ist.

Als erstes werden folgende Vorüberlegungen angestellt; diese beziehen sich auf die Bestandsaufnahme hinsichtlich des Vorhandenseins notwendiger computerlinguistischer Ressourcen und Werkzeuge.

- Welche Ressourcen sind vorhanden, welche müssen beschafft oder aufgebaut werden?

- Um welche Sprachen handelt es sich?

- Habe ich diachrone oder synchrone Texte?

- Aus welcher Zeit und Sprachvariante stammen meine Texte?

- Habe ich entsprechende Sprachressourcen für die jeweilige (historische) Sprachvariante?

- Welche meiner Verarbeitungsschritte und Werkzeuge sind sprachabhängig, weil sie zum Beispiel grammatikalisches Wissen modellieren?

- Welche meiner Verarbeitungsschritte und Werkzeuge sind sprachunabhängig, weil sie beispielsweise auf maschinellem Lernen beruhen?

- Habe ich genügend Daten für maschinelles Lernen? Wie viele Texte habe ich? Welche Lernmethoden kann ich anwenden? Zum Beispiel brauchen Methoden des Deep Learning viel größere Mengen an Trainingsdaten (Hunderttausende, Millionen und Milliarden von Trainingsinstanzen), während andere Lernmethoden, wie Support Vector Machines (SVM) oder Random Forrests, um nur zwei Beispiele zu nennen, für Lernen anhand von kleineren Datensätzen (Hunderte oder Tausende Trainingsinstanzen) geeignet sind.

\begin{tabular}{|l|l|}
\hline \multicolumn{2}{|l|}{ Checklisten } \\
\hline Ressourcen & Werkzeuge \\
\hline Lexika & Tokenizer \\
Wortlisten & Lemmatizer \\
Stop Words & Part of Speech Tagger \\
Named Entities: & Phrase Chunker \\
Eigennamen & Parser (Phrasenstruktur, Dependenzstruktur) \\
Orts-, Ländernamen & Namenerkennung \\
Firmennamen & Datumserkennung \\
usw. & Adresserkennung \\
& usw. \\
\hline
\end{tabular}

Ausgehend von unterschiedlichen Fragen und deren Beantwortung kann man mit vorhandenen Daten unterschiedliche Dinge tun. Nachfolgend werden einige Beispiele für solche Verwendungszwecke gegeben und die dazugehörigen Fragen und ihre Beantwortung durchexerziert.

Beispiel 1: Ausnutzen von Metadaten zur Gruppierung von Texten, als Grundlage (i) zur Erstellung von Terminologielexika, (ii) zum Lernen von Textklassifikationsmodellen. Um herauszufinden, ob die vorhandenen Metadaten überhaupt geeignet sind, die Texte 
thematisch zu gruppieren, sind erst einmal folgende Fragen positiv zu beantworten: Haben mehrere Texte dieselbe themenspezifische Beschlagwortung? Bin ich mir sicher, dass die Beschlagwortung konsistent verwendet wurde? Ist das der Fall, können die gleich beschlagworteten Texte derselben Gruppe oder Klasse zugeordnet und entsprechende Klassenlabel vergeben werden. Somit entsteht ein Datensatz mit klassifizierten Daten, das heißt, jedes Dokument aus dem Datensatz ist aufgrund seiner Beschlagwortung einer, eventuell auch mehreren thematischen Klassen zugeordnet. Ein solcher Datensatz kann für verschiedene Zwecke verwendet werden, unter anderem zur Extraktion themenspezifischer lexikalischer Ressourcen. Damit entsteht ein Terminologielexikon, das in der Informationsextraktion verwendet werden kann. Der Datensatz kann aber auch für maschinelles Lernen verwendet werden, als Trainingsmaterial zum Lernen von Klassifiern. Abhängig von der Anzahl der Texte pro Klasse und davon, ob Texte nur einer einzigen oder mehreren Klassen zugeordnet sind, sind unterschiedliche mathematische Modelle zum Lernen von Klassifiern geeignet ${ }^{31}$.

Beispiel 2: Verwendung textsortenspezifischer Dokumente zur Erstellung von Speziallexika. Gegeben ist eine Reihe von Texten, die derselben Textsorte angehören, beispielsweise einer bestimmten Art amtlicher Dokumente. Dieser Datensatz eignet sich zum Beispiel als Ressource für die Erstellung von textsortenspezifischen Lexika (Terminologie, Named Entities, Datumsangaben und dergleichen). Des Weiteren können Methoden des Dokumentenclusterings angewendet werden, um zu versuchen, den Datensatz noch einmal weiter in inhaltliche Subkorpora zu unterteilen. Dies einerseits, um zu untersuchen, wie homogen oder heterogen der Datensatz ist, und andererseits, um die Texte in den resultierenden Clustern wiederum für die Erstellung spezifischer Lexika zu verwenden - vorausgesetzt, die resultierenden Cluster werden aus archivarischer Sicht für sinnvoll erachtet. Gibt es eine Vorstellung, wie viele Themengruppen vorkommen, können Clustering-Methoden mit einer im Voraus bestimmten Anzahl von Clustern verwendet werden, andernfalls müssen Methoden eingesetzt werden, welche die Clusteranzahl anhand der Trainingsdaten dynamisch selbst bestimmen. Je nachdem, ob man möchte, dass jedes Dokument genau einem (hard clustering) oder mehreren (soft clustering) Clustern zugewiesen wird, sind andere Clusteringmethoden zu verwenden ${ }^{32}$.

Beispiel 3: Erstellung zeit- beziehungsweise regionalspezifischer Lexika. Stammen die Texte aus derselben Zeit oder aus einer bestimmten Region, kann dieser Datensatz für die Erstellung entsprechender lexikalischer Ressourcen verwendet werden. Dokumentenclustering kann ebenfalls eingesetzt werden, um weitere Gruppierungsmöglichkeiten der Dokumente zu untersuchen.

Beispiel 4: Nutzen von Textclustering für die Sichtung von (großen) Mengen an Dokumenten. Sind Hunderte, Tausende, Hunderttausende oder gar Millionen von Dokumenten vorhanden, von denen man nicht genau weiß, welche einander ähnlich sind, können diese unter Einsatz von Textclusteringverfahren gruppiert werden. Die so entstandenen Gruppen können dann wiederum mittels Methoden der Informationsextraktion weiter untersucht werden.

Beispiel 5: Nutzen von Textklassifikation zur Einordnung „neuer“ Texte. Hat oder bekommt man Texte, von denen man nicht weiß, ob und wie sie zu bestehenden Texten passen, eignen sich Methoden der Textklassifikation für deren Einordnung in bestehende

\footnotetext{
31 Siehe dazu weiter oben die Ausführungen zum Information Retrieval.

32 Siehe dazu weiter oben die Ausführungen zum Information Retrieval.
} 
Dokumentengruppen. Vorausgesetzt, ein Teil der bestehenden Texte ist bereits inhaltlichen Gruppen zugeordnet (klassifiziert), kann mittels Dokumentenklassifikation festgestellt werden, ob ein weiteres („neues“) Dokument zu einer bereits vorhandenen Klasse passt. Gibt es eine Reihe von Dokumenten, die nicht beziehungsweise nur einer Klasse „other" zugeordnet werden können, kommt wiederum Dokumentenclustering zum Einsatz, um diese Texte zu gruppieren. Inhaltlich weiter analysiert werden die Texte dann mittels Methoden der Informationsextraktion. Somit kann herausgefunden werden, welche Inhalte in den Clustern vorkommen.

Beispiel 6: Identifizieren wichtiger Wörter und Phrasen. Eine Möglichkeit, um herauszufinden, worum es in einzelnen Texten geht, ist das Identifizieren wichtiger Wörter und Phrasen. Um dies zu bewerkstelligen, sind verschiedene Arbeitsschritte nötig, dazu gehören etwa Tokenisierung, Lemmatisierung (Stemming), die Eliminierung von Stopwords, Satzzeichenbehandlung und das Erkennen phrasaler Muster. Schließlich können mittels Wort- und Phrasenhäufigkeiten Terme identifiziert werden. Dabei geht es nicht nur um die relative Häufigkeit eines Wortes oder Lemmas im vorliegenden Text, sondern darüber hinaus um Maße wie TFIDF.

Beispiel 7: Identifizieren wichtiger Sätze. Eine weitere Möglichkeit, um einen ersten Eindruck vom Inhalt eines Textes zu bekommen, ist das Identifizieren jener Sätze, die die im Text enthaltene Information möglichst gut widerspiegeln. Ein sehr einfacher Ansatz wurde von Hans Peter Luhn im Jahr 1958 beschrieben ${ }^{33}$. Zuerst werden jene Wörter identifiziert, die charakteristisch (signifikant) für das betrachtete Dokument sind. Luhn verwendet dabei die Auftretenshäufigkeit eines Wortes, ein modernerer und ausgefeilterer Ansatz ist zum Beispiel das TFIDF-Maß, bei dem das Gewicht eines Wortes für ein Dokument relativ zu einer gegebenen Menge von Dokumenten (Dokumentenbasis) berechnet wird. Der TFIDF-Wert ist umso höher, je charakteristischer das Wort für den gegebenen Text ist; Wörter, die in vielen Texten der Dokumentenbasis vorkommen, sind nicht charakteristisch für das einzelne Dokument. In einem zweiten Schritt wird gezählt, wie viele signifikante Wörter in einem Satz innerhalb einer Sequenz auftreten, das heißt, es dürfen maximal vier nicht signifikante Wörter zwischen zwei signifikanten stehen, um eine Sequenz zu bilden. Signifikanzwerte werden wie folgt berechnet: Das Quadrat der signifikanten Wörter in einer Sequenz wird dividiert durch die Gesamtanzahl der Wörter in der Sequenz (Abbildung 5). Sätze mit den höchsten Signifikanzwerten werden als repräsentativ für das Dokument betrachtet. Dieses Beispiel dient in erster Linie dazu, ein erstes Verständnis der lexikalischen Grundlagen zu vermitteln, wie wichtige Sätze identifiziert werden können. Die in der Praxis eingesetzten Programme sind viel

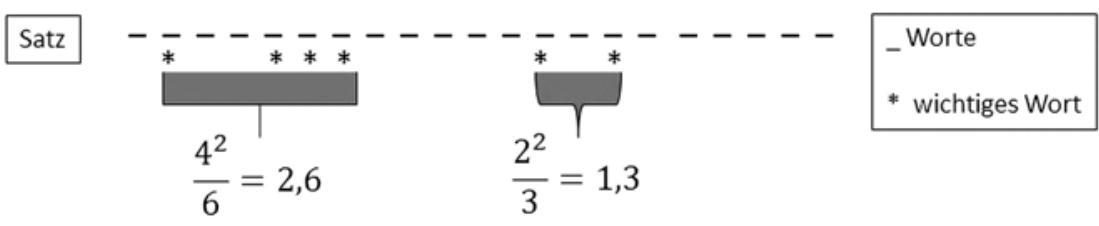

Abb. 5: Identifikation wichtiger Sätze nach Hans Peter Luhn (Brigitte Krenn)

33 Hans Peter Lunn, The Automatic Creation of Literature Abstracts. IBM Journal of Research and Development 2 (1958) 159-165. 
komplexer und berücksichtigen weitere Faktoren, wie die Position des Satzes im Text, bestimmte Terme als Suchwörter, Named Entities, semantische Informationen und vieles mehr. Der spezifische Forschungsbereich, der sich mit diesen Themen beschäftigt, heißt Extractive Summarization. Dabei geht es um die Auswahl von Sätzen aus einem Text, die den Hauptinhalt des Textes möglichst gut zusammenfassen. Aktuell ist dieser Bereich besonders bedeutend für die automatische Zusammenfassung von Nachrichtentexten. Die Literatur zu diesem Thema ist entsprechend vielfältig ${ }^{34}$.

\section{Resümee}

Im vorliegenden Beitrag wurden grundlegende Methoden der Sprachtechnologie zur Verarbeitung von Texten vorgestellt und deren Nutzbarmachung für die archivarische Tätigkeit diskutiert. Hierbei wurde das Augenmerk insbesondere auf die automatische Identifizierung von Ähnlichkeiten zwischen Texten (Dokumentenclustering und Dokumentenklassifikation) gelegt, weiters auf das Erstellen von lexikalischen Ressourcen (Erstellung von Wortlisten und Termkandidatenlisten basierend auf ausgewählten Datensätzen) sowie auf die Erschließung von Textinhalten (Informationsextraktion und automatische Textzusammenfassung). Ausgangspunkt war, dass die zu untersuchenden beziehungsweise zu verarbeitenden Texte und etwaige Metainformation bereits in maschinenlesbarem Format vorliegen. Der gesamte für die archivarische Praxis äußerst wichtige Bereich der Datendigitalisierung inklusive der Optical Character Recognition (OCR), das heißt der Umwandlung von Bildern handschriftlicher oder maschinengeschriebener Sprache in ein maschinenlesbares Format, wird im vorhandenen Beitrag nicht diskutiert, sondern als notwendige Voraussetzung für die weitere Verarbeitung betrachtet. Die vorgestellten Methoden und Vorgehensweisen sind dazu gedacht, erste Ideen zu liefern, wie Sprachtechnologie in der archivarischen Praxis von Nutzen sein kann.

Es ist jedoch auch deutlich zu sagen, dass tragfähige Lösungen des Zusammenspiels von Expertenwissen in der Sprachtechnologie, im maschinellen Lernen und im Archivwesen bedürfen. In Abhängigkeit vom vorliegenden Archivgut und den zu verfolgenden Zielsetzungen liegen die Lösungen entweder mehr auf Seiten kommerzieller Softwaresysteme zur Archivierung, dem Dokumentenmanagement und der Dokumentenanalyse, die gegebenenfalls um einschlägige, alltagstaugliche Funktionalitäten erweitert werden müssen, oder bedürfen, in bestimmten Fällen, hoch spezialisierter, forschungsgetriebener Ansätze. Für die Zukunft ist davon auszugehen, dass neue Berufsbilder entstehen werden, die archivarische und sprachtechnologische Expertise miteinander verknüpfen, was letztendlich auch in der Ausbildung von Archivaren seinen Niederschlag finden wird.

${ }^{34}$ Überblicke bieten Vishal Gupta-Gurpreet Singh Lehal, A Survey of Text Summarization Extractive Techniques. Journal of Emerging Technologies in Web Intelligence 2 (2010) 258-268; Ani Nenkova-Kathleen McKeown, A Survey of Text Summarization Techniques, in: Mining Text Data (wie Anm. 26) 43-76. 


\title{
Parallelangebot oder Wegweiser in die Zukunft? Das weltweit umfangreichste Stadtgeschichte-Wiki und seine Interaktion mit dem Wiener Archivinformationssystem WAIS
}

\author{
Christoph Sonnlechner
}

„Die Archive werden sich unter den veränderten Bedingungen nur behaupten können, wenn sie ihrerseits ihre Binnenstrukturen, vor allem in Gestalt von Kooperationen und Verbünden, weiter ausbauen und dabei anschlussfähig für das übergreifende Gefüge bleiben. Dies gilt in gleicher Weise für die Archivierung genuin digitaler Unterlagen wie

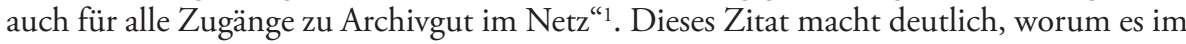
Folgenden gehen soll, in welchem größeren Kontext das konkret zu Zeigende zu verorten ist. Wenn die Frage gestellt wird, wie das Wien Geschichte Wiki ${ }^{2}$ mit dem Wiener Archivinformationssystem WAIS ${ }^{3}$ interagiert, dann ist angesprochen, wie Archive in der digitalen Welt, im Informationszeitalter agieren können und sollen. Es geht um die Frage von Agieren versus Reagieren beziehungsweise Interagieren. Was soll digital angeboten werden und wem? Was ist primär? Sollen Archive besser alleine oder im Verbund auftreten?

\section{Die Herausforderung der Archive durch das Digitale}

Es ist wohl unbestritten, dass Archive derzeit um ihre Rolle in der Informationsgesellschaft ringen. Die US-amerikanische Archivarin Kate Theimer hat nicht zuletzt in ihrer Keynote-Lecture anlässlich der Tagung „Archive 2.1“ in Stuttgart 2014 klar angesprochen, dass die Nutzer im Mittelpunkt unseres Handelns als Archivare stehen müssen ${ }^{4}$.

\footnotetext{
1 Robert Kretzschmar, Archive als digitale Informationsinfrastrukturen. Stand und Perspektiven. Archivar. Zeitschrift für Archivwesen 66 (2013) 146-153, hier 153.

2 Wien Geschichte Wiki, http://www.geschichtewiki.wien.at [1.3. 2018].

3 Wiener Archivinformationssystem - WAIS, http://www.wais.wien.at [1. 3. 2018].

${ }^{4}$ Kate Theimer, The Future of Archives is Participatory: Archives as Platform, or A New Mission for Archives. ArchivesNext (3. 4. 2014), http://archivesnext.com/?p=3700 [1. 3. 2018]. Nachzulesen sind diesbezügliche grundlegende Gedanken in Kate Theimer, Web 2.0 Tools and Strategies for Archives and Local History Collections (London 2010); DIEs., What is the Meaning of Archives 2.0? The American Archivist 74 (2011) 58-68; DIEs., A Different Kind of Web. New Connections between Archives and Users (Chicago 2011); DIES., Interactivity, Flexibility and Transparency: Social Media and Archives 2.0, in: The Future of Archives and Recordkeeping. A Reader, hg. von Jennie Hill (London 2011) 123-143. Ein umfassender Literaturüberblick zur Thematik kann hier unterbleiben, da ein solcher online und regelmäßig aktualisiert zur Verfügung steht: Bastian Gillner, Archiv 2.0 - ein Überblick zu Links und Literatur. Archive 2.0 (28. 4. 2013), http://archive20. hypotheses.org/622 [1.3. 2018].
} 
Bastian Gillner genauso wie Joachim Kemper, beide aus der seit ein paar Jahren aktiven Gruppe webaffiner Archivare, haben in Vorträgen und Aufsätzen die proaktive Rolle von Archivaren im digitalen Zeitalter eingefordert. Max Plassmann formuliert die künftige Rolle von Archivaren so: „Archivarinnen und Archivare werden (wie Bibliothekare) zu unkenntlichen Maklern von Information und können ihre inhaltlichen Kompetenzen immer weniger direkt den Nutzern anbieten. Nutzer recherchieren vielmehr in einer Datenbank oder einem Portal selbst, finden die gesuchten Quellen und schauen sie sich zu ihrer Freude oder zur wissenschaftlichen Auswertung an, ohne dass Archivare dies außerhalb von Logdateien mitkriegen und ohne dass eine archivische Beratung gesucht wird"5. Nutzungsgewohnheiten und Lebenswelten ändern sich. Menschen, die zunehmend Dienstleistungen im Internet nutzen - ob Online-Banking, Online-Steuererklärungen, Online-Einkäufe oder News-Konsum online -, erwarten auch von Archiven entsprechende digitale Angebote. „Der heimische Schreibtisch, aber auch Smartphones und Tablets werden zu ,Außenstellen ' des Lesesaals, mit,Öffnungszeiten“ rund um die Uhr. Diese Entwicklung erscheint unumkehrbar. Ein Negieren oder auch nur eine bewusste Verzögerung der Entwicklung ist wenig hilfreich“, skizziert Joachim Kemper die Zukunft der Archivnutzung ${ }^{6}$. Archive sind aufgerufen, sich mit der Frage zu beschäftigen, wie sie den Zugang zu Archivgut beziehungsweise die Benutzung ganz allgemein in Zukunft gestalten wollen und werden.

Diese Entwicklung sehend, haben Archive seit Jahren Webpages erstellt und digitale Angebote entwickelt. Das wichtigste davon ist zumeist ein Archivinformationssystem. Daneben gibt es aber auch noch entkoppelte Karten- und Fotosammlungen sowie Zimelienschauen, Dokumentationen und vieles mehr. Jedes Archiv schnürt ein individuelles Paket und hofft, potenzielle Nutzer damit zufrieden zu stellen. Was wollen Nutzer aber eigentlich? Diese Frage muss Archivare beschäftigen. Wie kann die nutzergerechte, digitale Archivnutzung der Zukunft aussehen?

Das Hessische Landesarchiv führte diesbezüglich im Herbst 2016 eine breit angelegte, extern begleitete Nutzerzufriedenheitsumfrage durch. Erste Ergebnisse wurden am Deutschen Archivtag in Koblenz 2016 präsentiert $^{7}$. An oberster Stelle steht der Wunsch nach Verbesserung der Usability des Archivinformationssystems. Auch stark gewünscht ist der Ausbau des digitalen Angebots.

Die Usability vieler Archivinformationssysteme lässt zu wünschen übrig - aus vielerlei Gründen. Für Nutzer sind Ordnungsprinzipien oft fremd, das Provenienzprinzip ist ebenso unbekannt wie die zum Verständnis notwendige Verwaltungsgeschichte. Es wird häufig veraltete beziehungsweise Fachsprache verwendet. Kapituliert der Informationssuchende vor der Suche in der Tektonik und verwendet das meist angebotene allgemeine

5 Max Plassmann, Archiv 3.0? Langfristige Perspektiven digitaler Benutzung. Archivar. Zeitschrift für Archivwesen 69 (2016) 219-223, hier 221.

${ }^{6}$ Joachim Kemper, „Anfragen“ über Soziale Medien, Blogposts, Chats, Twitter \& Co.? Aspekte einer virtuellen Nutzerberatung im Web 2.0. Archivar. Zeitschrift für Archivwesen 69 (2016) 224-227, hier 224.

7 Christian Reinhardt-Sebastian Mundt, Nutzerstudie 2016 des Hessischen Landesarchivs. Erste Ergebnisse, in: Hessisches Landesarchiv, https://landesarchiv.hessen.de/sites/landesarchiv.hessen.de/files/contentdownloads/Nutzerstudie\%202016\%20Ergebnisse_final-20160818_0.pdf [1. 3. 2018]. Christian Reinhardt und Sebastian Mundt präsentierten die Resultate auch in einem Vortrag „Die Nutzerumfrage 2016 des Hessischen Landesarchivs - Ziele, Methoden, Ergebnisse“ im Rahmen der Zweiten Gemeinsamen Arbeitssitzung „Archive als Kompetenzzentren - gut aufgestellt für die Zukunft?“ des Deutschen Archivtags in Koblenz am 30. 9. 2016. 


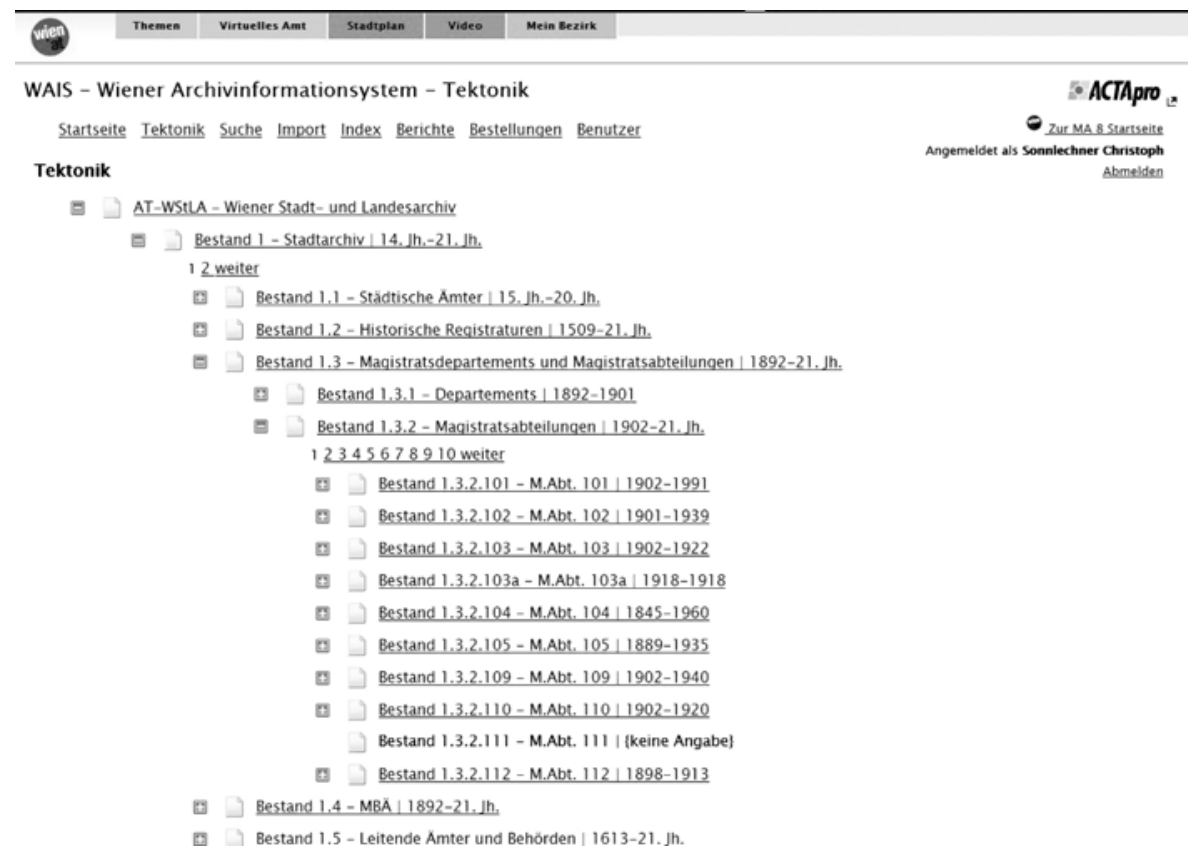

Abb. 1: Das Wiener Archivinformationssystem - WAIS. Ansicht des geöffneten Tektonikbaums, der die Bestandsstruktur nüchtern und technisch wiedergibt (Christoph Sonnlechner; Wiener Archivinformationssystem - WAIS, http://www.wais.wien.at [1.3. 2018]).

Suchfeld, erhält er oftmals unstrukturierte und ungefilterte Ergebnisse, die nur schwer zu interpretieren sind. Jörg Fischer hat es auf den Punkt gebracht, indem er die fehlende Verknüpfung mit dem Wissensziel des Nutzers als Manko benannte ${ }^{8}$. Die Kommunikation von Archiven und potenziellen Nutzern funktioniert im Digitalen noch nicht wunschgemäß. Wie aber könnte eine derartige Kommunikation beschaffen sein, um den Nutzer zu den erwünschten Informationen gelangen zu lassen? Sicher nicht funktionieren wird die Übertragung beziehungsweise Abbildung des analogen Lesesaals ins Digitale. Dort ist es nämlich dann tatsächlich wie in einem analogen Lesesaal: Der Nutzer kann bestenfalls Digitalisate einsehen. Die gravierenden technischen und gesellschaftlichen Veränderungen der vergangenen Jahre, die ihren Ausdruck in den sogenannten Sozialen Medien finden, werden dabei schlichtweg ignoriert. Bastian Gillner konstatiert eine erhebliche Verbesserung der Nutzungsbedingungen, „[...] wenn in einem virtuellen Lesesaal nicht nur Digitalisate einsehbar wären, sondern es dem Nutzer auch möglich wäre, (inter)aktiv und kommunikativ, personalisiert und/oder kollaborativ mit dem Archivgut zu agieren“".

8 In seinem Vortrag „Optimierung durch Konzentration - oder: Wie und warum sich Archivarinnen/ Archivare im digitalen Zeitalter neu erfinden (müssen)“ im Rahmen der Zweiten Gemeinsamen Arbeitssitzung des Deutschen Archivtags 2016 (wie Anm. 7).

9 Bastian Gillner, Archive im digitalen Nutzerkontakt. Virtuelle Lesesäle, soziale Medien und mentale Veränderungszwänge. Archivar. Zeitschrift für Archivwesen 66 (2013) 406-415, hier 412. In diesem Aufsatz findet sich auch sehr nützliche weiterführende Literatur zum Thema. Siehe dazu auch online und regelmäßig aktualisiert Gillner, Archiv 2.0 (wie Anm. 4). 
Ein virtueller Lesesaal der Zukunft sollte im besten Fall also solche Funktionen erfüllen.

Das Wiener Stadt- und Landesarchiv verfügt seit nunmehr zehn Jahren über ein Archivinformationssystem, das WAIS (Wiener Archivinformationssystem), über das auch elektronisch archiviert werden kann. Wie so viele derartige Systeme wirkt es - einmal geöffnet - nüchtern und technisch (Abb. 1). Es bietet neben der Suche im Tektonikbaum auch eine allgemeine Suchfunktion - mit all den bereits oben genannten Nachteilen, insbesondere der unstrukturierten Auflistung von Treffern. Das derart erzielte Ergebnis lässt den Suchenden oft ratlos zurück. Ein „Durchklicken“ würde vielleicht das erhoffte Ergebnis erbringen, führt vielleicht aber auch auf einen falschen Pfad, jedenfalls aber beim unkundigen Nutzer oftmals zu Frustration.

\section{Möglichkeiten der Unterstützung bei der Online-Recherche}

Das Landesarchiv Baden Württemberg hilft dem derartig Such-Unkundigen seit 2015 über sogenannte Online-,Rechercheführer“ zur Personen- und Ortssuche weiter. Auch oft nachgefragte Themen wie „Heimerziehung“ oder „Eisenbahn“ können über ein Themenportal recherchiert werden, ebenso wie „Archivalientypen“" ${ }^{10}$. Die Online-Nutzerorientierung des Landesarchivs Baden Württemberg ist auch in Hinblick auf die Verknüpfung diverser digitaler Angebote als Vorbild herauszustellen. Unter anderem werden Digitalisate aus dem Archivinformationssystem mit LEO, dem landeskundlichen Portal Baden-Württemberg, verlinkt ${ }^{11}$. LEO-BW ist das einzige deutsche landeskundliche Portal, das federführend von einem Archiv betreut wird. Alle anderen Portale liegen zumeist in den Händen der jeweiligen Landesbibliotheken oder vereinzelt bei anderen Institutionen unter Mit- beziehungsweise Zuarbeit der Landesarchive ${ }^{12}$. LEO-BW kontextualisiert Quellen, führt Bestände aus verschiedenen Institutionen des Landes zusammen und schafft damit einen Mehrwert. Die Suche kann orts-, personen- oder objektbezogen erfolgen. Alle Suchzugänge ermöglichen eine weitere Strukturierung der Ergebnisse über untergeordnete Suchfilter. Das Portal wendet sich an alle Bürger des Landes, will aber auch Materialien für Schulen bereitstellen. Wissenschaftlich Forschende erhalten einen vernetzten Zugang zu landeskundlichen Angeboten.

Viele deutsche Bundesländer haben für übergeordnete historische Recherchen den Weg über ein die digitalisierten Bestände verbindendes Portal gewählt. Solche Portale können verschieden organisiert sein. Neben LEO-BW seien das Landesgeschichtliche Informationssystem Hessen (LAGIS) ${ }^{13}$ und sachsen.digital ${ }^{14}$ beispielhaft genannt, insbe-

${ }^{10}$ Rechercheführer, in: Landesarchiv Baden Württemberg, https://www.landesarchiv-bw.de/web/57399 [1.3. 2018].

${ }^{11}$ LEO-BW - Landeskunde entdecken online. Das vernetzte landeskundliche Informationssystem für Baden-Württemberg, in: Landesarchiv Baden Württemberg, https://www.landesarchiv-bw.de/web/50999 [1. 3. 2018]; LEO-BW. Landeskundliches Informationssystem Baden-Württemberg, http://www.leo-bw.de/ [1. 3. 2018]. Über LEO-BW können zu einem recherchierten Ort Basisinformationen aus dem baden-württembergischen Ortslexikon, aus Literatur, Archivbeständen, statistischen Daten und Landkarten sowie Bildmaterial abgerufen werden ebenso wie biografische Informationen zu Persönlichkeiten. Zudem finden sich Digitalisate von Fotos, Karten, Videos, Handschriften, Büchern und Urkunden sowie Hinweise auf Sehenswürdigkeiten, Museen und Gedenkstätten. Siehe dazu auch den Beitrag von Robert Kretzschmar.

12 Eine Zusammenstellung der landesgeschichtlichen und landeskundlichen Internet-Portale (kurz AG Regionalportale) findet sich unter: Internet-Portal „Westfälische Geschichte“, http://www.lwl.org/westfaelischegeschichte/portal/Internet/ [1.3. 2018].

13 Landesgeschichtliches Informationssystem Hessen (LAGIS), http://www.lagis-hessen.de/ [1. 3. 2018].

${ }_{14}$ Sachsen.digital. Das Portal zur Geschichte, Kultur und Landeskunde Sachsens, http://www.sachsendi- 

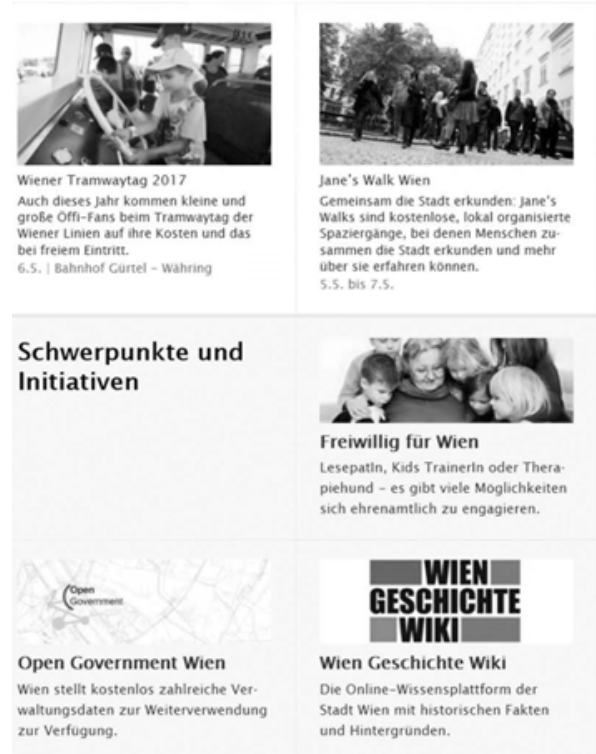
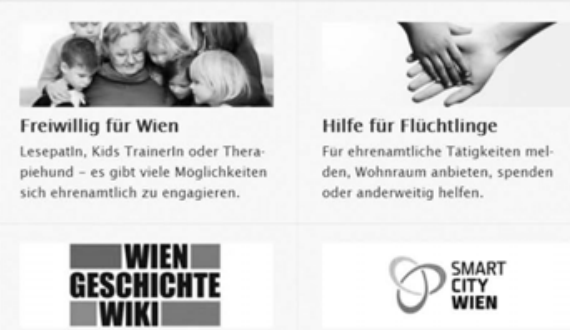

Wien Geschichte Wiki

Die Online-Wissensplattform der Stadt Wien mit historischen Fakten und Hintergrunden.

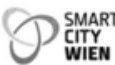

\section{Smart City Wien}

Intelligente, soziale und ressourcen. ithre Burgerinnen.
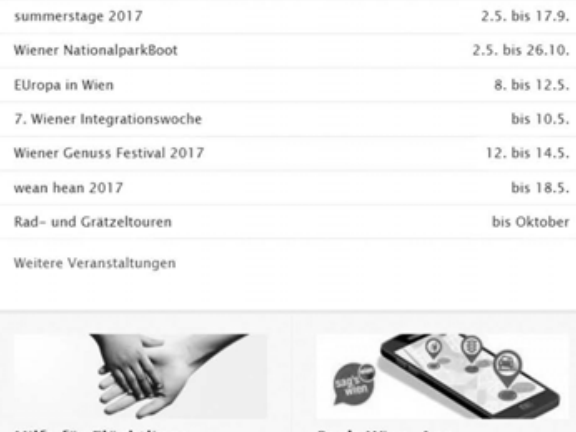

Sag's Wien-App

Jederzeit von unterwegs ein Anliegen, eine Gefahrenstelle oder eine Storung an die Stadtverwaltung melden. schonene Losungen fur die Stadt und

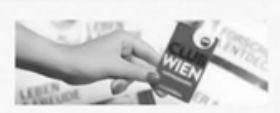

CLUB WIEN

Jetzt kostenlos Mitglied werden, zahtreiche Vergunstigungen genieben und Wien kenneniernen.

Abb. 2: Startseite des offiziellen Webauftritts der Stadt Wien. Wien Geschichte Wiki findet sich prominent platziert unter den wichtigsten Services der Stadt (Christoph Sonnlechner; Stadt Wien, https://www.wien. gv.at/ [1.3. 2018]).

sondere aber das bayerische Bavarikon ${ }^{15}$, das durch ein auf Wiki-Technologie basierendes „Historisches Lexikon Bayern“ ergänzt wird. Das Wiener Stadt- und Landesarchiv versucht Ähnliches über den Weg eines Wikis, über das Wien Geschichte Wiki. Wien Geschichte Wiki ist eine historische Wissensplattform der Stadt Wien. Die Grundlage bildet das „Historische Lexikon Wien“ von Felix Czeike" ${ }^{16}$. Das Wiki führt Wissen von Expertinnen und Experten aus Stadtverwaltung und Öffentlichkeit zusammen. Mit Stand November 2017 gibt es mehr als 39.000 Beiträge und über 6.000 Abbildungen. Das Wiki wird vom Wiener Stadt- und Landesarchiv (Magistratsabteilung 8) und der Wienbibliothek im Rathaus (Magistratsabteilung 9) gemeinsam finanziert und betrieben. Das Wien Museum fungiert als Kooperationspartner. Technisch umgesetzt wurde das Wiki vom KDZ - Zentrum für Verwaltungsforschung mittels der Open-Source-Software Semantic MediaWiki. Gehostet wird das Wiki von der Abteilung für Automationsunterstützte Datenverarbeitung, Informations- und Kommunikationstechnologie der Stadt Wien (Magistratsabteilung 14). Das Wiki ist eine Mitmachplattform. Externe Experten können sich registrieren und Inhalte textlicher und bildlicher Natur einbringen. Diese werden von einem Redaktionsteam, bestehend aus Experten für Stadtgeschichte, überprüft. Es werden nur Texte akzeptiert, die einem wissenschaftlichen Standard gerecht werden. Getroffene Aussagen müssen über Quellen und Literatur belegbar sein (Abb. 2).

gital.de/startseite/ [1.3. 2018].

15 Bavarikon, https://www.bavarikon.de/ [1. 3. 2018].

16 Felix Czeıke, Historisches Lexikon Wien, 6 Bde. (Wien 1992-2004). 
Wien Geschichte Wiki ist ein semantisches Wiki ${ }^{17}$, das Inhalte in derzeit sieben Kategorien - Personen, Topografische Objekte, Bauwerke, Organisationen, Ereignisse, Karten und Sonstiges - in Form von Einzelartikeln erfasst. Zu jedem Artikel gibt es einen (Haupt-) Text und semantische Datenfelder. Die Daten dieser Felder sind aus der Datenbank über programmierte Abfragen auswertbar. Mit Stand April 2017 haben sich knapp 1.000 Personen im Wiki als Mitarbeiterinnen und Mitarbeiter registriert. Das Wiki ist seit seinem Online-Stellen im September 2014 um knapp 10.000 Artikel gewachsen (2014: ca. 27.500). Es hat, ebenfalls mit Stand April 2017, pro Tag zwischen 4.500 und 5.000 Besucher (Unique Clients), im Monat über 100.000. Der Oktober 2017 verzeichnete über 145.000 Unique Clients. Die Tendenz ist deutlich steigend. Seit September 2014 gab es knapp 90 Millionen Zugriffe auf das Wiki (Ende November 2017). Aufgrund der für die Messung relevanten Anzahl von Einzelartikeln ist das Wien Geschichte Wiki das größte Regionalwiki der Welt ${ }^{18}$. Alle Abbildungen sowie Inhalte, die nach dem 15. Dezember 2013 ins Wiki hochgeladen wurden, können unter der Lizenz CC BY-NCND weiterverwendet werden ${ }^{19}$.

\section{Motivation}

Das Wiener Stadt- und Landesarchiv hat sich zur Einrichtung und zum Betrieb des Wikis aus verschiedenen Gründen entschieden. In $\$ 5$ Abs. 6 des Wiener Archivgesetzes wird in Bezug auf die Aufgaben des Wiener Stadt- und Landesarchivs Folgendes angeführt: „Durchführung von wissenschaftlichen Forschungen zur Wiener und vergleichenden Stadtgeschichte und Förderung des Verständnisses für die Geschichte der Stadt Wien durch einschlägige Veröffentlichungen, Ausstellungen, Führungen und andere Veranstaltungen "20. Diese Bestimmung ist der gesetzliche Auftrag beziehungsweise bildet die Grundlage für den Betrieb des Wien Geschichte Wikis. Dies gilt einerseits für die Aufarbeitung von historischen, stadtgeschichtlichen Inhalten, andererseits für die Vermittlung in Form eines zeitgemäßen, elektronischen Mediums. Die Entscheidung, ein eigenständiges semantisches Wiki aufzubauen und nicht in die Wikipedia einzuarbeiten, wurde

17 Semantic MediaWiki, https://www.semantic-mediawiki.org/wiki/Semantic_MediaWiki [1. 3. 2018]. Semantic MediaWiki eignet sich im Übrigen auch als Archivsoftware: „Mediawiki eignet sich für Archive und Museen, da es als Open-Source-Software vollständig dokumentiert ist und aufgrund seiner Verbreitung eine sehr günstige Zukunftsprognose und Langzeitstabilität verspricht. Die Semantic-Mediawiki-Extension erweitert das Mediawiki zur Datenbank, in welcher auch auf Archive und Inventare zugeschnittene Datenmodelle (z. B. ISAD $(G)$ ) abgebildet werden können. Es existieren Schnittstellen für den Daten-Export und -Import. Bilder und Dateien können integriert werden. Mediawiki-Installationen sind ausgereift und zuverlässig, sowie performant und skalierbar" (Art. Archivsoftware, in: Wikipedia. Die freie Enzyklopädie, https://de.wikipedia. org/wiki/Archivsoftware [1. 3. 2018]). Es handelt sich also um eine kostengünstige und zukunftsträchtige Lösung für kleinere Archive ohne komplexe Erfassungs- und Darstellungsbedürfnisse. Für größere Archive von Landesverwaltungen etc., die auch elektronisch archivieren, reichen die Funktionalitäten nicht aus.

18 Art. Regiowiki, in: Wikipedia. Die freie Enzyklopädie, https://de.wikipedia.org/wiki/Regiowiki [1. 3. 2018]; Art. Node:Ranking, in: Stadtwiki, http://allmende.stadtwiki.info/wiki/Node:Ranking [1. 3. 2018 ].

${ }_{19}$ Impressum, in: Wien Geschichte Wiki, https://www.geschichtewiki.wien.gv.at/Impressum [1. 3. 2018].

${ }^{20}$ Gesetz betreffend die Sicherung, Aufbewahrung und Nutzung von in Eigentum oder Verwahrung der Stadt Wien befindlichem Archivgut (Wiener Archivgesetz - Wr.ArchG). Landesgesetzblatt für Wien 55 (17. 10 2000), https://www.wien.gv.at/recht/landesrecht-wien/rechtsvorschriften/html/i1300000.htm [1. 3. 2018]; vgl. Ferdinand Opll, Das Wiener Archivgesetz und seine Auswirkungen auf die Umsetzung archivischer Aufgaben. Scrinium. Zeitschrift des Verbandes Österreichischer Archivarinnen und Archivare 56 (2002) 26-33. 
bewusst getroffen, um die redaktionelle Eigenständigkeit und technische Optimierung auf die Bedürfnisse einer Stadtverwaltung zu gewährleisten. Mit der Eigenständigkeit ist die Möglichkeit zu technischen An- und Einbindungen in bereits bestehende OnlineInfrastrukturen der Stadt sowie zu Schwerpunktsetzungen im Inhaltlichen gegeben, wie noch zu zeigen sein wird.

Das Archiv leistet mit seiner Arbeit einen Beitrag zur Identität der Stadt. Durch die gezielte Erarbeitung von Artikeln zu gewissen Themenkomplexen, die zum Beispiel in Hinblick auf Gedenkjahre und Jubiläen erfolgt, kann die Stadt Schwerpunkte setzen und an der Interpretation der eigenen Geschichte maßgeblich mitwirken. Nicht zuletzt wird das Straßennamenlexikon im Zusammenwirken mit dem Kulturamt der Stadt seit 2016 ausschließlich im Wien Geschichte Wiki geführt. Alle Wiener Verkehrsflächen sind mit ihrer Geschichte abrufbar. Die Gedenkkultur der Stadt bildet sich auch über die Führung des Gedenktageindex (Gedenken an Personen) durch die Wienbibliothek ab. 2017 wurde zudem eine neue Kategorie angelegt, die Gedenkzeichen aller Art zum Inhalt hat von der Gedenktafel über den „Stolperstein“ bis hin zum Grenzstein. Das Wiki soll auf Wunsch des Presse- und Informationsdienstes der Stadt Wien künftig möglichst alle historischen Inhalte, die sich derzeit noch auf dem Internetauftritt der Stadt finden, aufnehmen.

Im konkreten Zusammenwirken mit dem Archivinformationssystem ist aber vor allem auf einen Leitsatz aus dem Leitbild des Archivs hinzuweisen, der unter der Überschrift „Wir machen Erinnern möglich“ besagt: „Wir leiten Sie durch den von uns gebauten Informations- und Wissensraum “21. Das Wiki ist neben der persönlichen Beratung im Lesesaal das Instrument, um Interessierte dort hinzuführen, wo sie über Unterlagen des Archivs entweder online ein Ergebnis erhalten oder zu den Beständen hingeleitet werden, in denen sie recherchieren müssen, um zum Ergebnis zu gelangen. Der gebaute Wissensraum bildet sich im Wesentlichen durch das WAIS ab. Das Archivinformationssystem ist klar strukturiert und bildet über seine Tektonik die Provenienz der Bestände $\mathrm{ab}$. Die Ordnung erfolgt immer hierarchisch von der Bestandsgruppe über den Bestand zur Serie, dem Akt und schließlich zum Einzelstück. Um im Zuge einer Recherche mithilfe des allgemeinen Suchfelds nicht frustriert zu werden, muss in der Tektonik gesucht werden. Eine derartige Suche erfordert aber zwangsläufig Kenntnisse der Verwaltungsgeschichte, ja sogar der archivischen Ordnungsgeschichte, um erfolgreich zu sein. Die archivische Ordnung weist einen hohen Komplexitätsgrad auf, der auch im System selbst nicht reduzierbar ist. Lediglich ein begleitender thematischer Zugang wie zum Beispiel über einen Rechercheführer oder das Wien Geschichte Wiki kann Orientierung geben.

\section{Beispielhaftes aus dem Wien Geschichte Wiki}

Im Folgenden soll anhand einiger Beispiele verdeutlicht werden, wie die Interaktion zwischen dem Wiki und dem Archivinformationssystem im Konkreten funktioniert. Die tägliche Auskunftspraxis des Wiener Stadt- und Landesarchivs lieferte die ersten Schwer-

${ }^{21}$ Leitbild des Wiener Stadt- und Landesarchivs, in: Stadt Wien - Kultur \& Freizeit, https://www.wien. gv.at/kultur/archiv/leitbild.html [1. 3. 2018]; vgl. Ferdinand OpLL, „Einsicht in Vergangenheit - Aussicht auf Erinnerung“. Zur Erarbeitung eines Leitbildes für ein Archiv. Scrinium. Zeitschrift des Verbandes Österreichischer Archivarinnen und Archivare 61/62 (2007/2008) 130-149. 
punkte der Aufbereitung für die praktische Nutzung. Sehr häufig wird nach der Tätigkeit einer Abteilung zu einer gewissen Zeit gefragt, zum Beispiel des Kulturamts in der NS-Zeit. Im WAIS ist eine derartige Suche nicht ganz einfach. Gibt man „Kulturamt“ in das allgemeine Suchfeld ein, erhält man eine unstrukturierte Masse an Treffern, durch die man sich Durchklicken könnte, um hoffentlich nach nicht allzu vielen Umwegen auf die richtige Spur zu gelangen. Die gezielte Suche im Tektonikbaum führt ohne Kenntnisse der Verwaltungs- und Archivgeschichte nicht weiter. Das Problem liegt in der Komplexität. Das Kulturamt trägt heute die offizielle Bezeichnung „Magistratsabteilung 7 - Kultur“. Über die Jahrzehnte haben die Bezeichnungen und Zahlen aber gewechselt. Deshalb trägt jene Abteilung, welche die Kulturagenden in der Stadt wahrnimmt, im WAIS die stabile Archivbezeichnung „M.Abt. 350“. Das herauszufinden, bedarf des Nachfragens oder kombinierten Suchens und Rückschließens im WAIS. Genau um solch komplexe Sachverhalte einfach zugänglich zu machen, bietet sich das Wiki an. Es soll den Zugang zu Archivgut ermöglichen, die Auffindbarkeit erleichtern. Der Vorteil des Wikis gegenüber dem WAIS im Falle einer Suche liegt auf Hand: Die Wiki-Inhalte sind im Gegensatz zum WAIS Google- beziehungsweise Suchmaschinen-indexiert und hoch gerankt, weil es sich um eine wien.at-URL handelt, das Wiki intern stark verlinkt ist, und weil es täglich sehr viele Änderungen gibt, was ein besseres Listing bewirkt. Die Inhalte sind also leicht auffindbar. Zudem entspricht die Suchlogik im Wiki dem bekannten und vertrauten Suchverhalten. Im Fall des Kulturamts kann einfach der Begriff „Kulturamt“ ins Suchfeld eingegeben werden. Das Wiki verlinkt automatisch auf den Artikel „Magistratsabteilung 7 - Kultur“ weiter. Dort findet man dann einen Abriss der Verwaltungsgeschichte. Unter anderem erfährt man, dass die Kultur 1939 in der Hauptabteilung III angesiedelt war. Auch wird ein kurzer Überblick über das Tätigkeitsfeld geboten. Schließlich findet sich auch ein Link zu den Beständen dieser Magistratsabteilung - der besagten „M.Abt. 350“ - und ihrer Vorgängerabteilungen im WAIS $^{22}$. Damit ist der Suchende am Ziel, kann online die erschlossenen Bestände studieren und Akten zur Einsichtnahme direkt über das WAIS in den Lesesaal bestellen.

Sehr häufig wird nach Verlassenschaftsabhandlungen in Wien gefragt. Abhandlungsverfahren konnten über die Jahrhunderte von sehr vielen verschiedenen Einrichtungen durchgeführt werden, je nach dem Zeitpunkt des Todes beziehungsweise der Zugehörigkeit zu einer Personengruppe. Der Wiki-Artikel „Verlassenschaftsabhandlung“ gibt einen Überblick darüber und bietet zudem eine Tabelle, in der sich die gesuchte Person verwaltungstechnisch verorten lässt. Der Service des Wikis besteht nun vor allem auch darin, dass nicht nur in die Bestände des Wiener Stadt- und Landesarchivs verlinkt wird, sondern auch punktgenau in die Archivinformationssysteme anderer Archive, wie zum Beispiel des Österreichischen Staatsarchivs oder des Niederösterreichischen Landesarchivs, geleitet wird, wo ebenfalls Verlassenschaftsabhandlungen von in Wien Verstorbenen verwahrt werden (Abb. 3) ${ }^{23}$. Zur Unterstützung der Suche nach Verlassenschaftsabhandlungen im Wiener Stadt- und Landesarchiv werden zudem Abteilungseinteilungen und Verzeichnisse online zur Verfügung gestellt und mit dem Wiki verlinkt. Diese Art des Überblicks und der Hinführung zum gesuchten Bestand könnte über ein Archivinformationssystem nicht geboten werden.

22 Art. Magistratsabteilung 7 - Kultur, in: Wien Geschichte Wiki, https://www.geschichtewiki.wien.gv.at/ Magistratsabteilung_7___Kultur [1.3. 2018].

23 Art. Verlassenschaftsabhandlung, in: Wien Geschichte Wiki, https:/www.geschichtewiki.wien.gv.at/ Verlassenschaftsabhandlung [1.3. 2018]. 


\begin{tabular}{|c|c|c|}
\hline Personenkreis & zustāndige Behörde & Verwahrung des Schriftgutes heute \\
\hline $\begin{array}{l}\text { Mitglieder des Kaiserhauses und } \\
\text { regierender/furstlicher Häuser }\end{array}$ & $\begin{array}{l}\text { Hofkanzlei bzw. Staatskanzlei, } \\
\text { Obersthofmaschallamt (1783-1918) }\end{array}$ & $\begin{array}{l}\text { - Testamente / Verlassenschaftsabhandlungen: } \\
\text { OeSTA/HHStA HA OMaA 216 }\end{array}$ \\
\hline Personenkreis & Zuständigkeiten in Wien bis 1783 & Verwahrung des Schriftgutes heute \\
\hline $\begin{array}{l}\text { Behördenchefs und Beamte der } \\
\text { landesfurstlichen Zentralbehörden, } \\
\text { Angehorige des Hofstaates und } \\
\text { Hofbedienstete }\end{array}$ & $\begin{array}{l}\text { Obersthofmarschallamt (bis } 1783 \text {; } \\
\text { 1749-1763 Niederosterreichische } \\
\text { Regierung) }\end{array}$ & $\begin{array}{l}\text { - Testamente adelig: OeSTA/HHSTA HA OMAA } \\
643 \text { - }^{[3]} \\
\text { - Testamente unadelig: WStLA, Alte Ziviljustiz } 0 \\
\text { - Abhandlungen adelig: } \\
\text { 1. Splitter OeSTA/HHSTA HA OMaA } 643 \text { E } \\
\text { 2. ONB } \\
\text { - Abhandlungen unadelig: OeSTA/HHSTA HA } \\
\text { OMaA } 622\end{array}$ \\
\hline Angehorige der Hofjägerei & $\begin{array}{l}\text { Oberstjagermeisteramt (bis 1749, danach } \\
\text { Niederösterreichische Regierung) }\end{array}$ & $\begin{array}{l}\text { - Testamente/Abhandlungen: OeStA/HHStA HA } \\
\text { OJaA } 69 \text { ए }\end{array}$ \\
\hline $\begin{array}{l}\text { Niederösterreichische Landstände, Beamte } \\
\text { der landständischen Verwaltungen in } \\
\text { Osterreich unter der Enns }\end{array}$ & $\begin{array}{l}\text { Landmarschallamt (ab } 1764 \text { "altes" } \\
\text { Niederösterriechisches Landrecht) } \\
\text { 1500-1783 }\end{array}$ & $\begin{array}{l}\text { - Abhandlungen und Testamente: Splitter } \\
\text { OeStA/HHStA LA OLMA } \\
\text { - Abhandlungen Splitter: ONB }\end{array}$ \\
\hline $\begin{array}{l}\text { Landesfurstliche Landesbeamte, nicht- } \\
\text { landsässige Adelige, Neuadelige, höhere } \\
\text { Geistlichkeit, kaiserliche Pfarrer und } \\
\text { Benefiziaten, Großkaufleute }\end{array}$ & Niederosterreichische Regierung (bis 1783) & $\begin{array}{l}\text { - Testament adelig: Reste (ab ca. 1750) AVA } \\
\text { - Testamente unadelig: WStLA, Alte Ziviljustiz a } \\
\text { - Abhandlungen adelig: Splitter ONB } \\
\text { - Abhandlungen unadelig: WStLA, Alte } \\
\text { Ziviljustiz a }\end{array}$ \\
\hline $\begin{array}{l}\text { Bedienstete der Reichskanzlei und des } \\
\text { Reichshofrats, Reichshofratsagenten, } \\
\text { Residenten von Reichsständen }\end{array}$ & $\begin{array}{l}\text { Reichskanzlei und Reichshofrat (bzw. } \\
\text { Justizkommission) }\end{array}$ & $\begin{array}{l}\text { - Testamente und Abhandlungen: HHStA: } \\
\text { - OeStA/HHStA RHR RK Testamente o } \\
\text { - OeStA/HHStA RHR RK Justizkommission } 2\end{array}$ \\
\hline $\begin{array}{l}\text { Rechtssachen von Exterritorialen (1754 bis } \\
1767 \text { ) }\end{array}$ & Obersthofmarschallamt & - OESTA/HHSTA HA OMaA 19 ש \\
\hline $\begin{array}{l}\text { Niedere Geistliche der Diozesen Wien und } \\
\text { Passau (Osterreichischer Anteil) }\end{array}$ & $\begin{array}{l}\text { Passauisches Offizialat und Wiener } \\
\text { erzbischofliches Konsistorium }\end{array}$ & $\begin{array}{l}\text { - Testamente/Abhandlungen: WStLA, Alte } \\
\text { Ziviljustiz } \mathrm{a} \\
\text { - Diozesanarchiv Wien } \mathrm{a}\end{array}$ \\
\hline $\begin{array}{l}\text { Angehorige der Universitat Wien (und } \\
\text { bestimmte Professionisten) }\end{array}$ & Universitătskonsistorium & $\begin{array}{l}\text { - Testamente/Abhandlungen: } \\
\text { 1. Universitatsarchive } \\
\text { 2. WStLA, Alte Ziviljustiz a (Doktoren der } \\
\text { Rechte 1754-1783) }\end{array}$ \\
\hline
\end{tabular}

Abb. 3: Tabelle aus dem Artikel „Verlassenschaftsabhandlung“ im Wien Geschichte Wiki. Über die Tabelle wird man zum jeweiligen Bestand im betreffenden Archivinformationssystem geleitet, wo sich weitere Informationen und Bestellmöglichkeiten finden (Christoph Sonnlechner; Verlassenschaftsabhandlung, in: Wien Geschichte Wiki, https:/www.geschichtewiki.wien.gv.at/Verlassenschaftsabhandlung [1. 3. 2018]).

Sehr stark nachgefragt werden im Archiv Behelfe zur Umlegung historischer Adressen, insbesondere Konskriptionsnummern seit dem 18. Jahrhundert, aber auch Hausschilder. Als Archivalien kommen dafür diverse Karten der Stadt, Vorstädte und Vororte in Frage. Zudem müssen Häuserkataster konsultiert werden. Im Zusammenspiel der Unterlagen kommt man im Allgemeinen recht schnell zu einem Ergebnis. Das Wiki bietet über die Seite „Häusernummerierung" 24 einen historischen Abriss über die Entwicklung der geografischen Verortung von Menschen in der Stadt. Es werden die wichtigsten Phasen der Nummerierungen in der Stadt und den einzelnen Vorstädten dargestellt. Der Mehrwert des Wikis besteht allerdings in einer umfassenden Tabelle, welche den einzelnen Verwaltungseinheiten relevantes Kartenmaterial und Häuserkataster zeitlich zuordnet, sodass der Suchende sofort eine Konkordanz der gesuchten Adresse zur Verfügung gestellt bekommt (Abb. 4). Die Karten werden zum Download über das WAIS sowie auch georeferenziert über Wien Kulturgut ${ }^{25}$ angeboten, je nach Verwendungszweck. Der aktuelle Stadtplan

${ }_{24}$ Art. Häusernummerierung, in: Wien Geschichte Wiki, https://www.geschichtewiki.wien.gv.at/ H\%C3\%A4usernummerierung [1.3. 2018].

25 Wien Kulturgut, in: Stadt Wien - Kultur \& Freizeit, https://www.wien.gv.at/kultur/kulturgut/ [1. 3. 2018]. Wien Kulturgut ist der digitale Kulturstadtplan der Stadt Wien. Er ermöglicht den Zugang zu we- 


\begin{tabular}{|c|c|c|c|c|c|c|c|}
\hline Konskriptionsbezirk & Bezink & 1. Nummerierung & 2. Nummerierung & & 3. Nummerierung & 4. Nummerierung & Orientierungsnammert \\
\hline \multirow[t]{3}{*}{ Alservorstadt } & 8,9 & 1770 & 1795 & - & 1821 & - & 1862 \\
\hline & Huuserkataster & Ponty (1779) & Cutjahr (1816) & - & Behsel (1829) & Hauserschema $(1847)$ & Schlessinger (1875) \\
\hline & Karte & Naget (1773) A & Roscher $(1812)$ e & - & Behsel (1825) a & Stadtplan (1858) a & Stadtplan (1887) A \\
\hline \multirow[t]{3}{*}{ Atchan } & 9 & 1770 & $=$ & - & $=$ & $=$ & 1862 \\
\hline & Huuserkataster & Ponty (1779): & Cutjahr (1816) \& & - & Behsel (1829) & Hauserschema $(1847)$ & schlessinger (1875) \\
\hline & Karte & Naget (1773) a & Roscher $(1812) e$ & - & Behsel (1825) a & Stadtplan (1858) a & Stadtplan (1857) a \\
\hline \multirow[t]{3}{*}{ Alterchenfeld } & 7,8 & 1770 & 1795 & - & - & - & 1862 \\
\hline & Mauserkacaster & Ponty (1779) & Cutjahr (1816)\& & - & Behsel (1829) & Hauserschema $(1847)$ & Schlessinger $(1875) \&$ \\
\hline & Karte & Nagel (1773) A & Roscher (1812) \& & - & Behsel (1825) a & 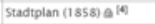 & Stadtplan $(1837)$ A \\
\hline \multirow[t]{3}{*}{ Breitenteld } & 8 & -19 & 1802 & - & 1821 & - & 1862 \\
\hline & nauserkataster & - & Cutjahr (1816)e & - & Behsel (1829) & Hauserschema $(1847)$ & schlessinger (1875) \\
\hline & Karte & - & Roscher (1812) & - & Behsel (1825) a & Stadtplan $(1858) a^{16}$ & Stadteplan (1887) a \\
\hline \multirow[t]{3}{*}{ Erdberg } & 3 & 1770 & 1795 & - & 1820 & - & 1862 \\
\hline & Mauserkacaster & Ponty (1779): & Cutjahr $(1816)$ es & - & Behsel (1829) \&f & Hauserschema $(1847)$ e & Schlessinger (1875) \\
\hline & Karte & Nagel (1773) A & Roscher (1812) है & - & Behsel (1825) a & Stadtplan (1858) A & Stadtplan $(1887)$ a \\
\hline \multirow{3}{*}{ Gumpendorf } & 6 & 1770 & 1795 & 1807 & 1821 & 1830 & 1862 \\
\hline & Hauserkataster & Ponty (1779) \& & Grestaver 0180510 & Cutjahr (1816) & Behsel (1829) of & Hauserschema $(1847)$ e & schlessinger $(1875)$ if \\
\hline & Karte & Naged (1773) a & Crimm (1805) & Roscher (1812) & Behsel (1825) a & 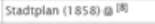 & Stadtplan (1887) \\
\hline \multirow{3}{*}{ Himmelpfortgrund } & 9 & 1770 & 1795 & - & - & - & 1862 \\
\hline & Heuserkataster & Ponty (1779) \& & Cutjahr (1816)e & - & Behsel (1829) to & Hauserschema $(1847)$ ? & Schlessinger (1875) De \\
\hline & Karte & Nagel (1773) a & Roscher (1812) & - & Behsel (1825) a & Stadtplan (1858) a & Stadtplan (1887) \\
\hline \multirow[t]{3}{*}{ Hundsturm } & 5,10 & 1770 & 1795 & 1816 & 182119 & 1829 & 1862 \\
\hline & nauserkatasser & Ponty (1779) & Fraisi (1812) & Cunjahr (1816) & Cuejaht (1821) & $\begin{array}{l}\text { Behsel (1829) of } \\
\text { Hauserschema (1847) }\end{array}$ & Schlessinger $(1875)$ \\
\hline & Karte & Nagel (1773) a & Roscher $(1812)$ & - & Behsel (1825) a & Stadtplan (1858) an & Stadtplan (18s7) a \\
\hline \multirow[t]{4}{*}{ Hungelbrunn } & 4.5 & 1770 & - & - & - & - & 1862 \\
\hline & Mauserkataster & Ponty (1779) & Cutjahr $(1816) \&$ & - & Behsel (1829) & Hauserschema $(1847)$ & schlessinger $(1875)$ \\
\hline & Karte & Nagel $(1773) a^{(111)}$ & Roscher $(1812){ }^{112]}$ & - & Behsel (1825) a [1]] & Stadtplan $(1858) a^{|14|}$ & Stadtplan (18s7) \\
\hline & & Nagel $(1773) Q^{(119)}$ & - - 161 & - & Behsel (1825) a ${ }^{117}$ & Stadtplan (1858) a C18 & Stadtplan $(1857) a$ \\
\hline
\end{tabular}

Abb. 4: Tabelle des Wiki-Artikels „Häusernummerierung“. Die Tabelle leitet in den gewünschten, online stehenden Häuserkataster sowie die benötigte Karte weiter (Christoph Sonnlechner; Häusernummerierung, in: Wien Geschichte Wiki, https://www.geschichtewiki.wien.gv.at/wiki/H\%C3\%A4usernummerierung [1.3. 2018]).

kann mit der jeweiligen historischen Karte überlagert werden. Mithilfe der Möglichkeit, eine historische Karte mit einem halb-transparenten Layer der aktuellen Stadtkarte zu überblenden, sieht man sofort, welcher aktuellen Orientierungsnummer beispielsweise eine (historische) Konskriptionsnummer entspricht. Mithilfe der Verknüpfung mit den online von der Wienbibliothek im Rathaus zur Verfügung gestellten Häuserkatastern können auf Knopfdruck auch zusätzliche Informationen wie Pfarr- und Grundherrschaftszugehörigkeit recherchiert werden, was für die Personen- und Hausgeschichtsforschung von großer Bedeutung ist.

\section{Neue Funktionalitäten}

Neben den herausgegriffenen Anwendungen, welche mittels der Verknüpfung mit dem WAIS sowie anderen webbasierten Informationsportalen einen Mehrwert herstellen, gibt es im Wiki noch weitere Funktionalitäten, die der Wissensproduktion dienen können und Archivmaterial erschließen. Zuerst sei auf das Taggen eingegangen. Das Wiki bietet die Möglichkeit, einerseits Scans von Archivalien über das WAIS einzubinden, aber auch diese direkt ins Wiki hochzuladen und zu taggen. Beispielhaft ist dies erstmals im

sentlichen Identitätsmerkmalen der Stadt: Umfangreiches Kartenmaterial zeigt die kulturgeschichtliche und stadtplanerische Entwicklung Wiens von der Frühzeit bis in die Gegenwart. Detaillierte Informationen über verschiedene Kulturgüter wie Gebäude, Denkmäler, archäologische Objekte, historische Stadtpläne, stadtgeschichtliche Objekte, Kunstwerke und vieles mehr können auch über Suchmasken abgerufen werden. Das Portal wird in Zusammenarbeit von Kulturabteilung (MA 7), Wiener Stadt- und Landesarchiv (MA 8), Architektur und Stadtgestaltung (MA 19) sowie Museen der Stadt Wien - Stadtarchäologie betrieben. Zielsetzung ist die Aufbereitung und Präsentation von raumbezogenen Kulturgütern in zeitgemäßer Form. 


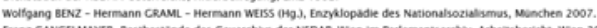

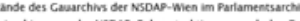

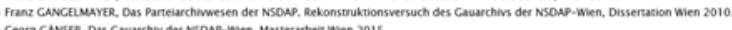

Ceorg CANSER, Das Gauarchiv der NSDAp-Wen. Masterarbea Wien 2015.

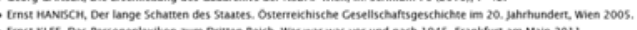

- Inst KLLE, Das Personenlexikon zum Dertsen Reich. Wer wat was vor und nach 1945, Frankfurt am Main 2011.

- Robert LY OEg, Organisationsbuch der NSOM, Manchen 1936.

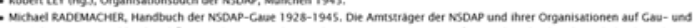
Kreisebene in Deuschland und Oreerreich somie in den Reschsgaven Danzig-wescpreufea, Sudetenland und Wartheland, vecha 2000. - Christane ROThe LNOER, Die Antinge der Wiener 5s, wien 2012.

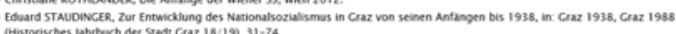
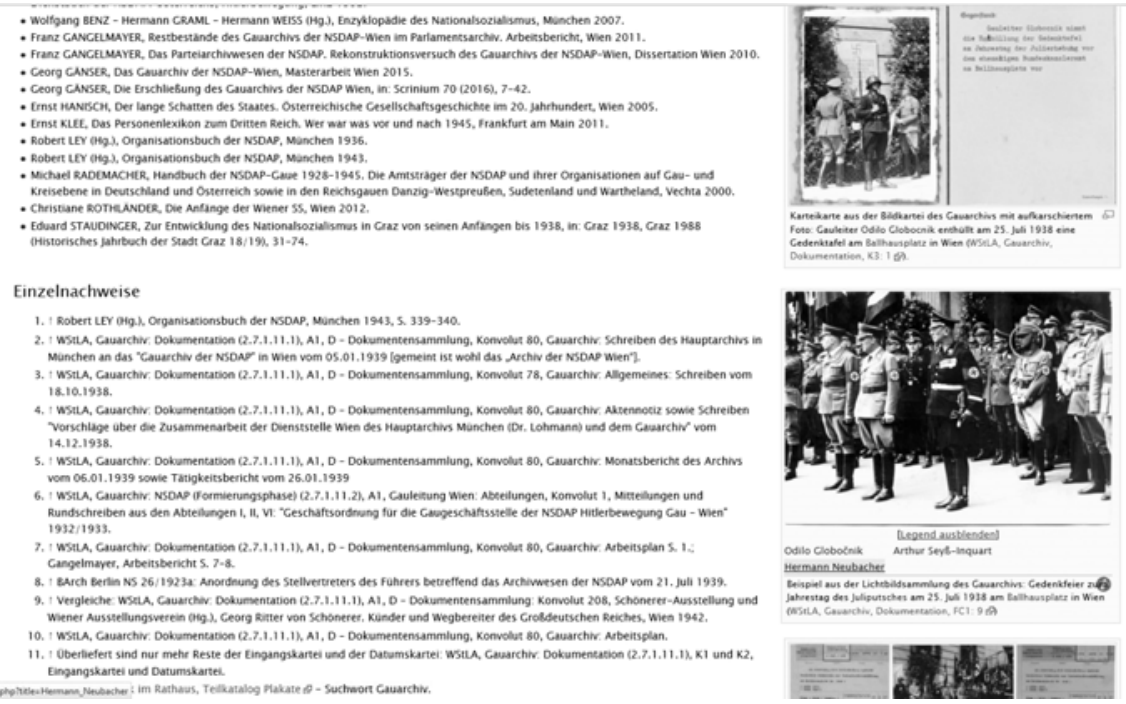

Deter

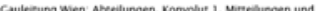

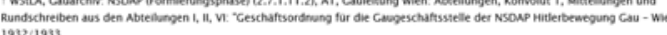

Cangelimaver, Arteitsbericht 5.7.

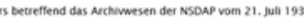

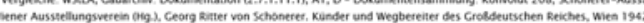

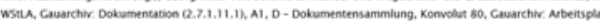

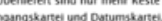

Abb. 5: Ansicht der ersten getaggten Abbildung im Wien Geschichte Wiki, die sich im Artikel „Gauarchiv der NSDAP Wien“ findet (Christoph Sonnlechner; Gauarchiv der NSDAP Wien, in: Wien Geschichte Wiki, https://www.geschichtewiki.wien.gv.at/Gauarchiv_der_NSDAP_Wien [1.3.2018]).

Wien Geschichte Wiki im Rahmen des Artikels „Gauarchiv der NSDAP Wien“ geschehen ${ }^{26}$. In diesem Artikel wird ein Bestand des Wiener Stadt- und Landesarchivs beschrieben, seine Teilbestände dargestellt sowie in den Kontext von Institutionen (Provenienzstellen) und verantwortlichen Personen gestellt, zu denen es ebenfalls Artikel gibt, die verlinkt sind. Illustriert wird der Artikel durch Flugblätter und Fotos aus dem Bestand. Ein Foto aus der Lichtbildsammlung des Gauarchivs, welches die Gedenkfeier zum Jahrestag des Juliputsches am 25. Juli 1938 am Ballhausplatz in Wien zeigt ${ }^{27}$, wurde getaggt (Abb. 5). Identifizierte Personen sind auf diese Weise elektronisch markiert. Klickt man auf eine solche Markierung, wird man zu demjenigen Artikel geleitet, der Entsprechendes zur Person ausführt, im konkreten Fall beispielsweise zu Odilo Globočnik, Arthur SeyßInquart und Hermann Neubacher. Klickt man zum Beispiel auf den Wiener NS-Bürgermeister Neubacher, so erhält man nicht nur eine Biografie des Funktionärs, sondern unter „Quellen“ auch gleich den Hinweis, dass es einen Volksgerichtsakt zu dieser Person gibt. Ein Link führt wiederum direkt ins WAIS, wo sich Basisinformationen zum Akt und die Bestellmöglichkeit in den Lesesaal finden.

Die Möglichkeit des Taggens gibt es im Wien Geschichte Wiki seit 2016. Dadurch kann kollaborative Erschließung von Unterlagen erfolgen. Eine weitere Variante von Partizipation von Außenstehenden ist damit eröffnet. Obschon derzeit (2017) noch wenig

${ }^{26}$ Art. Gauarchiv der NSDAP Wien, in: Wien Geschichte Wiki, https://www. geschichtewiki.wien.gv.at/ Gauarchiv_der_NSDAP_Wien [1.3.2018].

27 WStLA, NSDAP Wien, Gauarchiv Wien, Dokumentation, FC1, Li - Lichtbildsammlung, 1, NSDAP: Ereignisse und Personen: 9 - Gedenkfeier: Juliputsch | 25.07.1938, in: Wiener Archivinformationssystem WAIS, https://www.wien.gv.at/actaproweb2/benutzung/archive.xhtml?id=Akt+++++9E0540D0-924D-473 7-B867-EEC320661E4Alanm08ga1\#Akt 9E0540D0-924D-4737-B867-EEC320661E4Alanm08ga1 [1.3. 2018]. 


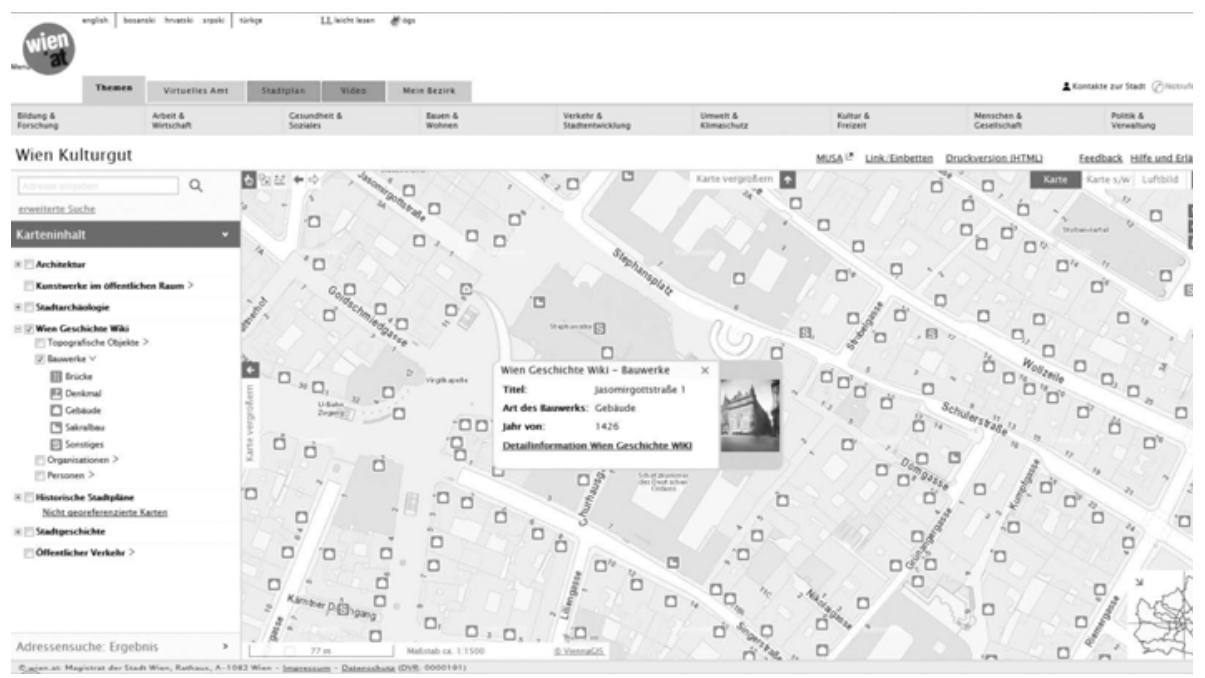

Abb. 6: Ansicht des seit Mitte 2017 zur Verfügung stehenden Wiki-Layers auf der georeferenzierten Plattform Wien Kulturgut. Über diesen Layer werden die verortbaren Informationen des Wikis erstmals über die Suche im Raum verfügbar (Christoph Sonnlechner; Wien Kulturgut, https://www.wien.gv.at/kultur/kulturgut/ [1. 3. 2018]).

genutzt, eröffnet das Tool neue Möglichkeiten. Angedacht werden könnte die Platzierung von Digitalisaten (Fotos, Karten, Listen ...) im Wiki zum Zwecke des gemeinschaftlichen Erschließens. Die Dokumente wären dann im Wiki hochgeladen. Aus dem WAIS - über das die unkommentierten Digitalisate zur Verfügung stünden - könnte aufs Wiki verlinkt werden, wo sich die getaggten, erschlossenen Dokumente befänden. Mittlerweile gibt es eine Vielzahl von erfolgreichen Beispielen solchen Crowdsourcings. Das bekannteste ist wohl die Erschließung des Swissair-Fotoarchivs durch ehemalige Swissair-Mitarbeiter über ein Projekt der verwahrenden Bibliothek der ETH Zürich ${ }^{28}$. Es handelt sich hier um einen klassischen Fall des Einbringens von Expertenwissen aus der Community in die Erschließung. Erfolgreich sind solche Projekte meistens dann, wenn es sich um ein konkretes, überschaubares Projekt mit klar definiertem Expertenkreis handelt. Nur durch die Vorgabe von gewissen strukturierenden Leitlinien und begleitende Moderation des Prozesses ist eine qualitativ hochwertige Erschließung erwartbar, die archivische Standards erfüllen kann. Der große Vorteil im Fall des Wikis ist zudem, dass die Digitalisate darin getaggt online stehen, wohingegen die Digitalisate im Archivinformationssystem unangetastet bleiben. Damit kann ein Bedenken vieler Archivare, nämlich Nicht-Archivare in den eigenen Daten(banken) kaum kontrollierbar und womöglich nicht standardisiert kommentieren zu lassen, hintangehalten werden.

${ }^{28}$ Nicole Graf, Die Freiwilligen der Swissair im Bildarchiv der ETH-Bibliothek. ETH-Bibliothek | Crowdsourcing. Aktuelles und Erfahrungen aus der Community (9. 5. 2016), https://blogs.ethz.ch/crowdsourcing/2016/05/09/die-freiwilligen-der-swissair-im-bildarchiv-eth-bibliothek/ [1. 3. 2018]. Gute Erfahrungen mit Crowdsourcing haben auch das Stadtarchiv Speyer bei der Identifikation von Fotos und das Landesarchiv Baden-Württemberg bei der Erschließung von Kriegsgräberlisten gemacht. Siehe dazu und auch zu nordeuropäischen Beispielen die Tagungsdokumentation zu Archive 2.1 in Stuttgart: Konferenz 2014. Archive 2.0 (2014), http://archive20.hypotheses.org/tagung-2014 [1. 3. 2018]. Dort ist auch der maßgebliche Vortrag online zur Verfügung gestellt: Nicole Graf, Crowdsourcing. Neue Möglichkeiten und Grenzen für Bildarchive (Offene Archive 2.1). Archive 2.0 (23. 6. 2014), http://archive20.hypotheses.org/1775 [1. 3. 2018]. 
Im Herbst 2017 wurde ein nächster neuer Zugang zu historischer Information eröffnet: In Wien Kulturgut wurde ein Wiki-Layer online gestellt. Damit kann die Suche auch über den Raum erfolgen. Alle geografisch verortbaren, mit Adressen versehenen Artikel werden nach Kategorien (Bauwerke, Topografische Objekte ...) auf dem Wiener Stadtplan mittels Icon angezeigt. Fährt man mit der Maus auf das Icon, erscheint eine Bubble mit den wesentlichen Informationen samt Abbildung, soweit vorhanden. Wünscht man mehr Information, klickt man darauf und wird zum jeweiligen Artikel geleitet (Abb. 6).

\section{Fazit}

Das WAIS ist und bleibt jenes Instrument, mit dem der Archivbestand verwaltet und geordnet abgebildet wird, Archivalien für die Einsichtnahme durch den Nutzer bestellt beziehungsweise direkt aufgerufen werden können und - vor allem wenn es sich um authentische elektronische Unterlagen handelt - mit dem der Archivierungsprozess maßgeblich begleitet wird. Das Wien Geschichte Wiki ist hingegen ein kollaborativer Grenzgang zwischen Citizen Science und amtlicher, korrekter, überprüfter Information. Über dieses System wird versucht, strukturiertes stadt- und verwaltungsgeschichtliches Wissen leicht zugänglich anzubieten. Im Rahmen des Online-Lexikons werden stadthistorische Betreffe, Archivbestände und auch Einzelquellen erläutert, strukturiert und zusammengeführt. Information wird aufbereitet und vermittelt. Schließlich wird punktgenau unter anderem ins WAIS und damit beispielsweise zur Verzeichnung eines Archivales inklusive Bestellmöglichkeit zur Einsichtnahme und gegebenenfalls inklusive Möglichkeit zur Ansicht eines Digitalisats verlinkt; aber nicht nur dorthin, sondern auch in andere Archivund Bibliothekssysteme.

Das Informationszeitalter mit all seinen digitalen Möglichkeiten bietet Chancen - gerade Archiven, die oftmals über geringe Beachtung und Ignoranz ihnen gegenüber klagen. Sie können nunmehr sichtbar werden und Leistungen sowie Angebote transparent machen. Das Wiener Stadt- und Landesarchiv verfügt über ein Bündel von Online-Angeboten: WAIS, Wiki, INFODAT - Informationsdatenbank des Wiener Gemeinderats und Landtags ${ }^{29}$, Wien Kulturgut, ein OGD (Open Government Data)-Paket ${ }^{30}$ und einen Social Media-Auftritt. Über diese Kanäle und im Verbund mit der rechtlichen Festlegung auf die Zurverfügungstellung von Archivgut unter einer CC-BY-Lizenz eröffnen sich Möglichkeiten. Archivgut wird über das Wiki jedenfalls bereits kontextualisiert und kann künftig kommentiert und getaggt, über Facebook ${ }^{31}$ auch geteilt werden. Gerade das Wiki eröffnet - wie gezeigt - neben der Möglichkeit des Embeddings von Archivgut auch

29 Informationsdatenbank des Wiener Landtages und Gemeinderates (Infodat Wien), https://www.wien. gv.at/infodat/ [1.3. 2018].

${ }^{30}$ Zur Open-Government-Strategie der Stadt Wien vgl. Für eine offene Stadt. Open Government Wien, https://open.wien.gv.at/site/ [1.3. 2018].

31 Das Wiener Stadt- und Landesarchiv hat sich nach einer längeren Phase der Diskussion und Beobachtung im Herbst 2016 dazu entschlossen, einen Facebook-Auftritt zu etablieren. Gründe für den Schritt waren einerseits, dass gewisse Gruppen von Interessierten wie etwa die Digital Natives vorwiegend mit einem solchen Auftritt erreicht werden können, andererseits auch Foren wie die geschlossene Gruppe „Archivfragen“ entstanden sind, auf denen sich Archive nutzbringend austauschen. Dazu vgl. Angela STilwell, Die FacebookGruppe Archivfragen (Offene Archive 2.1). Archive 2.0 (13. 6. 2014), http://archive20.hypotheses.org/1743 [1.3. 2018]. Der Facebook-Auftritt des Wiener Stadt- und Landesarchivs wird von der Öffentlichkeitsarbeit des Archivs betreut. Mehr als die Hälfte aller Posts nimmt ihren Ausgang bei Artikeln des Wien Geschichte Wikis. 
die Möglichkeit des Crowdsourcings. Es lässt sich über die digitalen Funktionalitäten ein Mehrwert erzielen, der weit über die statische Präsentation digitalisierter Quellen hinausgeht. 


\title{
Zum Verhältnis von klassischen Formen der Archiverschließung und den Zugängen der Digital Humanities zum Information Retrieval
}

\author{
Georg Vogeler
}

Was sind die digitalen Geisteswissenschaften? Das Forschungsgebiet entwickelt sich zunehmend zu einer akademischen Disziplin, die etabliertes Wissen in Studiengängen vermittelt und einen angeregten Forschungsdiskurs in Tagungen und Publikationen führt. Dabei ist die inhaltliche Ausrichtung immer noch sehr vielfältig, denn die Schwerpunkte können in der Verarbeitung von Texten und natürlicher Sprache ebenso liegen wie in der digitalen Repräsentation und formalen Erschließung von (nicht nur) schriftlichen Objekten des Kulturerbes. Eine mögliche Beschreibung des Tätigkeitsbereichs der Digitalen Geisteswissenschaften ist die Untersuchung des digitalen und digitalisierten Kulturerbes in seiner menschlichen Aneignung, seiner geisteswissenschaftlichen Reflexion, seiner hermeneutischen Kontextualisierung, seiner informationslogischen Formalisierung, seiner technischen Realisierung und seiner statistischen Berechnung. Pragmatischer können die digitalen Geisteswissenschaften als Auseinandersetzung von Geisteswissenschaftlerinnen mit Computern verstanden werden - auch wenn in der Fachdiskussion die Bezeichnung „Humanities Computing“ seit etwa zehn Jahren durch die „Digital Humanities“ ersetzt worden ist ${ }^{1}$. Im Folgenden soll ein Blick der digitalen Geisteswissenschaften auf das Problem der archivischen Erschließung geworfen werden. Es geht für die digitalen Geisteswissenschaften darum, sich zu fragen, was spezifisch an den Informationen in den Archiven und dem Informationsbedürfnis des Benutzers von Archiven ist, was die Kommunikation zwischen Archiv und Benutzer von Bibliotheken, Banken, Meteorologen oder Gendatenbanken unterscheidet, und wie diese Kommunikation vom Computer geformt wird und werden könnte.

Es kann also zum Beispiel aus dem allgemeinen Wissen über Informationsvisualisierungen auf den Spezialfall von Archiverschließung geschlossen und die Frage gestellt werden, ob Techniken wie Word Clouds, Statistiken, geographische Karten, hierarchische Bäume oder Netzwerke in der Kommunikation zwischen Archiv und Archivbenutzer

1 Zur langfristigen Entwicklung vom „Humanities Computing“ zu den „Digital Humanities“ vgl. Patrik Svensson, Humanities Computing as Digital Humanities. Digital Humanities Quarterly 3/3 (2009), http://www. digitalhumanities.org/dhq/vol/3/3/000065/000065.html [1.3. 2018]. Der Beitrag ist Teil einer längeren Serie zur Geschichte der Digital Humanities: DERs., The Landscape of Digital Humanities. Digital Humanities Quarterly 4/1 (2010), http://www.digitalhumanities.org/dhq/vol/4/1/000080/000080.html [1. 3. 2018]; DERs., From Optical Fiber To Conceptual Cyberinfrastructure. Digital Humanities Quarterly 5/1 (2011), http://www.digitalhumanities. org/dhq/vol/5/1/000090/000090.html [1. 3. 2018]; DERs., Envisioning the Digital Humanities. Digital Humanities Quarterly 6/1 (2012), http://www.digitalhumanities.org/dhq/vol/6/1/000112/000112.html [1. 3. 2018]. 
sinnvoll eingesetzt werden könnten. Ein Forschungsergebnis der digitalen Geisteswissenschaften ist dabei, dass ein Blick auf die Objekte des Kulturerbes - im Fall der Archive also Archivalien, im reicher erforschten Fall literaturwissenschaftlicher Analysen Texte nicht mehr ein detailliertes Studium des Einzelobjekts ist, sondern ein in statistischen und graphischen Darstellungen aggregiertes „distant reading“2.

Für die Erschließung von Kulturerbeobjekten beziehen sich die Digitalen Geisteswissenschaften besonders auf das Forschungsfeld der „Informationsrückgewinnung“, in dem Informatiker, Informationswissenschaftler und Computerlinguisten daran arbeiten, wie man den Computer benutzen kann, um textuelle Inhalte, die sich nicht in einer einfachen Zeichenkette eindeutig abbilden lassen können, in großen Datenmengen finden zu können. Das Forschungsfeld existiert schon seit der Mitte des 20. Jahrhunderts und findet insbesondere im Feld der Websuchmaschinen, aber auch für digitale Bibliotheken Anwendung 3 . Obwohl die von den Archivaren im Zuge der Erschließung erstellten „Metadaten “ für Archivalien für diese Methoden der Informationsrückgewinnung einschlägiges Material bilden, sind diese Methoden im Archivkontext bislang noch nicht systematisch diskutiert worden ${ }^{4}$.

Im Folgenden möchte ich ein paar Ideen und Methoden aus den digitalen Geisteswissenschaften für die Kommunikation zwischen Archiv und Benutzer in der Archiverschließung vorstellen. Es wird dabei ebenso um eher kreative Experimente gehen wie um etablierte Verfahren der Informationsrückgewinnung, die in der Archiverschließung noch wenig Verbreitung gefunden haben. Ich stelle dabei den fachwissenschaftlichen Benutzer in den Vordergrund, der das Archiv als Teil einer historischen Forschungsarbeit besucht. Welche der etablierten Verfahren für die Kommunikation zwischen diesem und den Archiven sinnvollerweise berücksichtigt werden können, ergibt sich aus dem Verhältnis zwischen vorhandenen Technologien, allgemeinen Nutzererwartungen und grundsätzlichen Erkenntnissen über die Theorie der Geschichtswissenschaft und des Archivwesens. In der Zusammenschau stellt sich heraus, dass sich die etablierten Verfahren gut mit archivischen Konzepten in der Gestaltung der Systeme zur Informationsrückgewinnung verbinden lassen, obwohl sie in den existierenden Archivinformationssystemen erstaunlich wenig verbunden werden.

Beginnen möchte ich mit zwei Beispielen, die der klassischen Form der Archiverschließung zuwiderzulaufen scheinen. In etablierten Formen der Archiverschließung ord-

2 Der Begriff ist von Franco Moretti geprägt, der es als computergestützte literaturwissenschaftliche Methode dem klassischen „close reading“ gegenüberstellte: Franco Moretri, Graphs, Maps, Trees. Abstract Models for a Literary History (London-New York 2005); DERs., Distant Reading (London-New York 2013).

3 Zum Forschungsfeld, das auch unter der englischen Bezeichnung „Information Retrieval“ geläufig ist, vgl. Christopher D. Manning-Prabhakar Raghavan-Hinrich Schütze, Introduction to Information Retrieval (Cambridge u. a. 2008); Winfried GöDERT-Klaus LepsKY-Matthias NAGELSCHMidT, Informationserschließung und Automatisches Indexieren. Ein Lehr- und Arbeitsbuch (Berlin-Heidelberg 2012); Qiaozhu MeI-Dragomir RAdEv, Information Retrieval, in: The Oxford Handbook of Computational Linguistics, hg. von Ruslan Mitкоv [Online-Vorabpublikation der 2. Auflage], http://doi.org/10.1093/oxfordhb/9780199573691.013.022 [1. 3. 2018]; Marijn Koolen-JaAP Kamps-Vincent De Keijzer, Information Retrieval in Cultural Heritage. Interdisciplinary Science Reviews 34 (2009) 268-284.

${ }^{4}$ Eine Fallstudie bieten Silvia Schenkolewski-Kroll-Assaf Tractinsky, Archival Description, Information Retrieval, and the Construction of Thesauri in Israeli Archives. Archival Science. International Journal on Recorded Information 6 (2006) 69-107. In letzter Zeit sind dagegen die Forschungen zur automatischen Extraktion von Text aus Bildern von handschriftlichen Dokumenten in den Vordergrund gerückt; siehe insbesondere das READProjekt: READ. Recognition and Enrichment of Archival Documents, https://read.transkribus.eu/ [1. 3. 2018]. 
nen Archivarinnen das Archivmaterial mit ihrem historischen Fachwissen sinnhaft und organisieren es in einer Hierarchie. Eine hierarchische Organisation bietet sich insbesondere deshalb an, weil sich die Archivalien im Laufe ihrer Entstehung als Gruppen organisiert haben: Aus Einzelschriftstücken werden Akten, Akten werden in Schrankfächern gelagert, die in Räumen stehen und so die physische Registratur bilden ${ }^{5}$. Die Archiverschließung kann nun diese Vorordnungen benutzen, um Archivalien auch auf höheren Aggregationsstufen beschreiben und verwalten zu können. Aus dieser physisch vorgefundenen Ordnung kann man nun eine sachliche ableiten - falls die Archivalien nicht ohnehin schon nach sachlichen Kriterien gelagert waren. Das Bestreben der Archivare, den historischen Zustand der Akten zumindest beim Übergang im Archiv abzubilden (respect des fonds), bevorzugt solche Organisation auch in der Beschreibung. Zusätzlich entwickeln Archivare aber auch eigene hierarchische Ordnungen: In der Archivtektonik werden die Archivbestände zum Beispiel nach Zeitschichten, Behördenorganisation oder Geographie hierarchisch angeordnet. In der inneren Ordnung können Akten auf eine vereinheitlichte Registraturordnung oder auf eine von den Archivaren als nützlich erachtete Ordnung verteilt werden, insbesondere wenn die ursprüngliche innere Ordnung kaum mehr nachvollziehbar ist ${ }^{6}$. Im Folgenden sollen Zugangswege zu Archivalien vorgestellt werden, die als radikale Gegenentwürfe zu solchen Erschließungshierarchien gelten könnten: erstens Experimente mit Zugangswegen, die auf dem Muster der Textanordnung, Vertonung der Scans oder dem puren Zufall beruhen, und zweitens der sogenannte „Google-Suchschlitz“, also ein Suchinterface, das nur eine Volltextsuche auf Grund vom Benutzer frei gewählter Schlagwörter kennt, wobei die Treffer dieser Suche nach im Detail unbekannten Algorithmen so sortiert werden, dass die „relevantesten“ Treffer an den Anfang gereiht werden.

Ein Beispiel für einen besonders kreativen Zugang bietet die Webseite des John Latham Archive. John Latham (1921-2006) war ein rhodesisch-britischer Künstler, der als Maler und Bildhauer und insbesondere als Konzept- und Performancekünstler gewirkt hat $^{7}$. Die Webseite verwendet die drei Brüder Karamasow Mitya (für Dimitri), Ivan and Alyosha (für Alexej) als Metaphern für verschiedene Zugriffsarten: Mitya lebt in den Tag hinein und kann deshalb mit einer mehr oder wenig zufälligen Zusammenstellung von Bildern der Archivalien zum Stöbern angeregt werden. Ivan ist ein vernünftiger Zeitgenosse, der eine systematische facettierte Suche mit vordefinierten Beschreibungskategorien bevorzugt. Alyosha entwickelt sein Verständnis der Inhalte des Archivs intuitiv an ungewohnten Darstellungsformen wie einer Übersetzung der Daten in Töne ${ }^{8}$. Einen ebenso kreativen Zugang bietet das online digitalisierte Archiv des Verlags Bloodaxe Books ${ }^{9}$. Der Verlag widmet sich zeitgenössischer Poesie. Die Archivwebseite bietet deshalb als zentralen Zugriffsweg die Werke der verlegten Autoren an. Die im Archiv verwahrten Manu-

\footnotetext{
5 Zur Komposition als zentralem Prinzip der behördlichen Schriftgutorganisation vgl. Johannes PAPRITZ, Archivwissenschaft, 4 Bde. (Marburg ${ }^{2} 1983$ ) 1 248-254.

${ }^{6}$ Zur Komplexität der Erschließungspraxis und zur Funktion von Provenienz- und Registraturprinzip darin vgl. Bodo UhL, Die Bedeutung des Provenienzprinzips für Archivwissenschaft und Geschichtsforschung. Zeitschrift für bayerische Landesgeschichte 61 (1998) 97-121.

7 Zu Latham jüngst: A World View: John Latham, hg. von Amira GaD-Joseph Constable (London 2017).

${ }^{8}$ Athanasios Velios, Creative Archiving. A Case Study from the John Latham Archive. Journal of the Society of Archivists 32 (2011) 255-271.

9 The Bloodaxe Archive, http://bloodaxe.ncl.ac.uk/ [1. 3. 2018].
} 


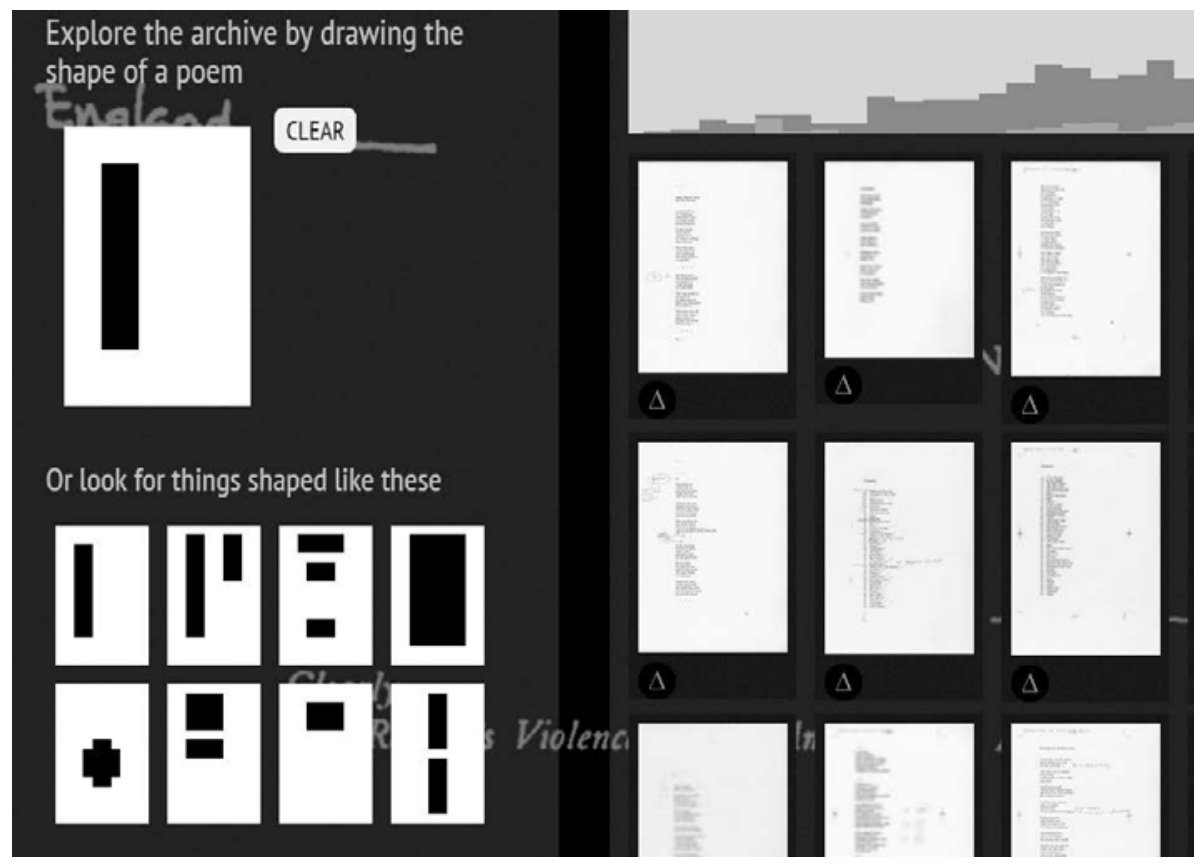

Abb. 1: bloodaxe Zugang über Layoutmuster, http://bloodaxe.ncl.ac.uk/explore/\#/shapes

skripte sind als Bilder und als automatisch eingelesene Volltexte zugänglich. Das Archiv kann deshalb die Häufigkeit von Stichwörtern als Suchkriterium verwenden, aber sie auch als Netzwerkgraphik von Kookkurrenzen darstellen. Die Bilder ermöglichen zusätzlich auch den besonders ungewohnten Zugang über die Verteilung des Textes auf der Seite: Texte mit schmalen Kolumnen grenzen sich von die ganze Seite füllenden Texten oder zentrierten Anordnungen ab (Abb. 1) ${ }^{10}$.

Derartige Projekte sind inspiriert vom archivierten Material, verwenden aber nicht die Standardverfahren archivischer Erschließung, um den Benutzer zu seinem Ziel zu führen. Dabei werden archivische Regelwerke und Erschließungsnormen sehr wohl angewendet: Das John Latham Archive verweist in seinem „vernünftigen“ Zugang unter dem Namen „Ivan“ explizit darauf, ISAD $(\mathrm{G})$-konforme Erschließungsdaten zu verwenden ${ }^{11}$. Derartige Projekte sind nicht nur von der kreativen Leistung der Künstler inspiriert, deren Archivalien verfügbar gemacht werden, sondern auch von einer Forschungsposition der Digitalen Geisteswissenschaften: Geisteswissenschaftliche Forschung ist nicht eine regelgeleitete, reproduzierbare Tätigkeit, sondern eine diskursive Auseinandersetzung mit den zu untersuchenden Objekten. Dabei ist die Benutzerschnittstelle kulturell geprägt wie die Dokumente selbst ${ }^{12}$. Die Gestaltung dieser Schnittstelle ist also auch eine Aussage für sich. Die

${ }^{10}$ Tom Schofield et al., Archival Liveness. Designing with Collections Before and During Cataloguing and Digitization. Digital Humanities Quarterly 9/3 (2015), http://www.digitalhumanities.org/dhq/ vol/9/3/000227/000227.html [1.3. 2018].

${ }_{11}$ IA, in: Archive as Event, http://www.ligatus.org.uk/aae/ivan [1. 3. 2018].

12 Johanna Drucker, Performative Materiality and Theoretical Approaches to Interface. Digital Humanities Quarterly 7/1 (2013), http://www.digitalhumanities.org/dhq/vol/7/1/000143/000143.html [1. 3. 2018]. 
beiden Webportale weisen darauf hin, dass ihr Material sich nicht nur nach den gleichen Kriterien, wie sie schon der Archivbildner angewendet hatte, ordnen lässt.

Diese experimentellen Zugriffsformen sind deshalb auch den Benutzern nicht gerade geläufig. Sehr viel geläufiger ist ein anderer Gegenentwurf zur Gestaltung des Information Retrieval in den Archiven: Der „Google-Suchschlitz“ ist die in der Benutzerkultur wohl am besten etablierte Form der Interaktion mit Informationsressourcen. Er steht primär für eine einfache Volltextsuche, die meist auch in modernen Archivsystemen angeboten wird, neben einer hierarchisch organisierten Archivtektonik oder dem Angebot von kompletten Findbüchern, die zur Lektüre zumindest der Einleitung, aber auch der Kontexte einzelner Einträge einladen. Geoffrey Yeo argumentiert nun, dass die modernen Informationstechnologien sowohl auf der Seite der Benutzer wie der Bestandsbildner die klassischen archivischen Erschließungsmethoden überflüssig machen werden: Forscher organisieren, so beobachtet er, ihre Informationen als flache Datensammlungen ohne hierarchische Ordnung und greifen ebenso primär auf Volltextsuchen zu wie die Bürokratien, die mit der Einführung von allgemeiner Bürosoftware ihre Akten auch zunehmend als flache Datenbank von Dokumenten verstehen, aus der man mit einer Volltextsuche am leichtesten relevante Informationen extrahieren kann ${ }^{13}$. Man könnte seine Annahmen über das dominante Verhalten zur Informationsorganisation zum Beispiel mit dem Hinweis auf hierarchisch organisierte Dateisysteme oder Systeme zur Abbildung von Geschäftsprozessen in Frage stellen. Ich möchte im Folgenden aber eine andere Perspektive einnehmen und danach fragen, ob der Google-Suchschlitz wirklich ein Konzept der Informationsrückgewinnung ist, das radikal anders als das archivischer Erschließung ist.

Der Google-Suchschlitz ist nämlich erst durch verschiedene technische Maßnahmen im Hintergrund so erfolgreich geworden. Es ist nicht so sehr die Reduktion auf eine nicht-hierarchische Volltextsuche, sondern es sind die Algorithmen, die relevante Treffer zu den Suchwörtern ermitteln. Sie beruhen darauf, dass aus den Suchwörtern auf Suchintentionen geschlossen wird, die sich in statistisch messbaren Eigenschaften des sogenannten „Suchraums“, das heißt der Gesamtheit der durchsuchten Objekte, wiederfinden lassen. Normalerweise werden Suchraum, Suche und zu durchsuchende Dokumente dabei einfach als eine unstrukturierte Ansammlung von Wörtern (bag of words) verstanden. Darin kann dann von der Häufigkeit der Wörter in einem Dokument in Relation zur Häufigkeit der Wörter im gesamten Suchraum auf die Relevanz der Wörter für die Beschreibung des Dokuments geschlossen werden. Erkenntnisse der Computerlinguistik haben diesen Ansatz nennenswert weiterentwickelt, indem sie morphologische und lexikalische Normalisierungen sowohl im Suchraum wie in der Suchanfrage vornehmen. Die Relevanz von Dokumenten für eine Suchanfrage ist aber auch Resultat von menschlichen Entscheidungen. In den modernen Suchmaschinen wird die Verweisstruktur des Internet benutzt, um auf diese Entscheidungen automatisch zurückzuschließen: Die Anzahl von Dokumenten, die auf ein Dokument verweisen, ihr Status im Gesamtnetzwerk (viele oder wenige Verweise) oder ihr Host (etwa Universitätsserver) sind Indizien, nach denen Google die Relevanz von Webseiten für eine Suchanfrage zu beurteilen versucht. Mit der Einführung des Google Knowledge Graph ist die Suche intern noch semantisch erweitert worden. Es wird überprüft, ob die Suchtermini in einer Faktendatenbank zu finden sind, aus der sich alternative Bezeichnungen oder Disambiguierungen ableiten lassen. Diese

13 Geoffrey Yeo, Contexts, Original Orders, and Item-Level Orientation. Responding Creatively to Users' Needs and Technological Change. Journal of Archival Organization 12 (2015) 170-185. 
semantische Übersetzung von Suchen ist ein Forschungsbereich im Feld der künstlichen Intelligenz, wird aber experimentell auch schon in Bibliotheksinformationssystemen angewendet ${ }^{14}$. Ähnlich sind auch komplexere statistische Methoden noch nicht im Alltag der Suchmaschinen angekommen. Verfahren des sogenannten „Topic Modeling“ zum Beispiel mit der sogenannten Latent Dirichlet Allocation ${ }^{15}$ versuchen, mit statistischen Methoden Wörter in einem Corpus an Hand ihrer Nachbarschaften so zu gruppieren, dass sich sinnvolle Zusammenhänge ergeben. Die hinter einer Volltextsuche stehenden Algorithmen sind also komplexer als die einfache Eingabeform es vermuten lässt. Auf welche Kriterien diese Algorithmen achten, ist aber offen. Es könnten also auch für die Archive relevante Kriterien verwendet werden.

Die vordergründige Annahme einer auf nicht-archivische Suchformen fokussierten Benutzergruppe ist auch an einer anderen Stelle zu hinterfragen: Passen sich die modernen Historikerinnen entweder ganz der archivischen Erschließungsweise an oder hegen sie nur naive Erwartungen aus der nicht-archivischen Welt der Suchmaschinen? Wohl kaum. Peter Haber geht zum Beispiel in seiner umfassenden Analyse des Verhältnisses von Geschichtswissenschaften zu den digitalen Informationstechnologien genau davon aus, dass „Jede Suche [...] geprägt [wird] vom Vorwissen, das der Suchende mitbringt, von der Kenntnis der entsprechenden Zusammenhänge und Fachbegriffe, von der Erfahrung und der Routine im Umgang mit den Hilfsmitteln" ${ }^{16}$. Amerikanische Archivwissenschaftlerinnen haben schon zu Beginn des Jahrtausends diese Position empirisch untermauern können ${ }^{17}$. Yakel und Torres bescheinigen den Historikerinnen, dass sie in der Auseinandersetzung mit der Überlieferung eine „archival intelligence“ entwickeln ${ }^{18}$. Hea Lim Rhee entwirft 2012 ein Modell archivalischer Suche, in dem nach der Suche (searching stage) die accessing stage (Zugriff) und die processing stage (Verarbeitung) neue Suchanfragen anstoßen können, also in eine erneute searching stage münden ${ }^{19}$. Es entsteht dabei etwas, das nennenswerte Ähnlichkeiten zum hermeneutischen Zirkel hat. Ich möchte diese iterative oder explorative Methode als „lernende Suche“ bezeichnen und anregen, dass sie in der Diskussion über die Gestaltung von Informationssystemen einen festen Platz bekommt, denn sie ist ein Kern geschichtswissenschaftlicher Forschungspraxis, in der Wissen über das Forschungsobjekt schon während der Suche des Quellenmaterials erzeugt wird.

Eine zweite Perspektive auf die Historiker im Archiv ergibt sich aus geschichtstheoretischen Überlegungen der jüngeren Vergangenheit. In der Debatte um die Rolle der Geschichtsdarstellung für die Geschichtswissenschaft hat sich herausgestellt, dass die Erzäh-

${ }_{14}$ Etwa in der Zusammenarbeit zwischen der Sächsischen Landes- und Universitätsbibliothek Dresden mit der Firma AvantgardeLabs: vgl. Jens Miтtelbach-Robert Glass, A Library Data Management Platform Based on Linked Open Data. ResearchGate (Dezember 2014), http://doi.org/10.13140/RG.2.1.1178.0965 [1.3. 2018].

15 David M. BleI-Andrew Y. NG-Michael I. Jordan, Latent Dirichlet Allocation. Journal of Machine Learning Research 3 (2002/03) 993-1022.

16 Peter Haber, Digital Past. Geschichtswissenschaft im digitalen Zeitalter (München 2011) 163.

17 Wendy M. Duff-Catherine A. Johnson, Accidentally Found on Purpose: Information-Seeking Behavior of Historians in Archives. The Library Quarterly. Information, Community, Policy 72 (2002) 472-496.

18 Elizabeth Yakel-Deborah A. Torres, AI: Archival Intelligence and User Expertise. The American Archivist 66 (2003) 51-78.

19 Hea Lim RheE, Modelling Historians' Information-seeking Behaviour with an Interdisciplinary and Comparative Approach. Information Research 17/4 (2012), http://InformationR.net/ir/17-4/paper544.html [1.3. 2018]. 
lung eine elementare Form der Aneignung von Vergangenheit ist, und zwar unabhängig von der Gesamtstruktur der historischen Darstellungen durch Wissenschaftler ${ }^{20}$. Für die Archive bedeutet das, dass auch bei der Konsultation von Archivmaterial narrative Muster angewendet werden: Der Verlauf einer Verhandlung in der Korrespondenz, der Bericht über eine behördliche Maßnahme in internem Schriftgut, die vor Gericht verhandelten Einzelschicksale, die Reaktion auf einen politischen Vorschlag - die alle auch als Teile von analytischen Studien verwendet werden können. Neben einer analytischen, kategorisierenden Erschließung könnte eine moderne Archiverschließung also auch Narrationen als Suchwege unterstützen.

Die Kernfrage archivischer Erschließung aus der Perspektive der digitalen Geisteswissenschaften ist also nicht die Frage, ob in anderen Lebensbereichen entwickelte Technologien und Formen der Benutzung von Informationsressourcen auch auf die Archivwelt übertragen werden sollten, sondern ob und wie diese spezifische Art der Auseinandersetzung mit dem in den Archiven bewahrten Kulturerbe mit digitalen Mitteln unterstützt werden kann. Archivisches Information Retrieval muss sich also nicht an angebliche Standards der Suchmaschinentechnologie anpassen, sondern Archivinformationssysteme müssen die Technologien auswählen oder neu entwickeln, die am besten zu den Methoden der Geschichtswissenschaft und des Archivwesens passen.

Wie oben skizziert, leitet die etablierte archivische Ordnungslehre und Erschließungspraxis aus dem respect des fonds, dem „Provenienzprinzip“, dem „Registraturprinzip“ und der „Archivtektonik“ hierarchische Modelle der Erschließung ab. Damit sind fünf theoretische Ansprüche verbunden, die begründen, warum diese Organisation der Informationen die bestmögliche ist:

- Der Archivbildner hat die bestmögliche Informationsstruktur gefunden.

- Die Organisation nach Provenienz (und nach Registratur) bildet die Entstehungszusammenhänge ab.

- Einzelstücke sind ohne ihren Entstehungskontext nicht verständlich.

- Die Hierarchie erleichtert die Suche.

- Eine hierarchische Ordnung erleichtert die Erschließungsarbeit.

Unter diesen fünf Gründen sind zwei, die einer näheren Betrachtung mit den Erfahrungen der Informationserschließung großer digitaler Ressourcen nicht gut standhalten. Clay Shirky hat 2005 den Sieg der Suchmaschinen gegenüber hierarchischen Webverzeichnissen nachvollzogen und verallgemeinert: Er argumentiert sowohl mit der konkreten Entwicklung in der Erschließung von Webressourcen wie grundsätzlich. Er erklärt den Siegeszug der Suchmaschinen, die über Stichwörter und Verweise zwischen den Ressourcen die Informationsbedürfnisse der Benutzer befriedigen, gegenüber hierarchischen Webverzeichnissen wie dem Yahoo/DMOZ-Katalog mit der Mehrdeutigkeit der Objekte: Texte sind nicht physische Objekte, die genau einen Platz im Regal haben müssen, sondern können auf verschiedene Themen verweisen. Unter den Bedingungen von Hypertext ist dann die Hierarchie nur noch ein Verweis unter vielen. Klassifikationen funktionieren nur, wenn es um ein kleines Corpus mit klaren abgrenzbaren Kategorien geht. Über diese

${ }^{20}$ Eine Zusammenfassung bietet Wolfgang HardTwig, Formen der Geschichtsschreibung. Varianten historischen Erzählens, in: Geschichte. Ein Grundkurs, hg. von Hans-Jürgen Goertz (Reinbek 1998) 169-188, hier 174f.; vgl. Achim Saupe-Felix Wiedemann, Narration und Narratologie. Erzähltheorien in der Geschichtswissenschaft, Version: 1.0, in: Docupedia-Zeitgeschichte (28. 1. 2015), http://docupedia.de/zg/ saupe_wiedemann_narration_v1_de_2015 [1.3.2018]. 
Kategorien müssen diejenigen, die erschließen, ebenso Bescheid wissen wie die Benutzer der Erschließung ${ }^{21}$.

Aus informationspraktischer Sicht ist die hierarchische Organisation also ein Hilfsmittel, das zur Organisation von Papierakten in Behörden mit professionellen Registraturen nützlich war und so auch helfen kann, die Akten im Archiv wiederzufinden. Die hierarchische Ordnung der Akten in Aktenplan und Archivverzeichnung war nützlich - vielleicht sogar essentiell -, um Einzelstücke in der Menge der Papierdokumente $\mathrm{zu}$ finden. Für die Benutzer war die Ordnung schon immer fremd und musste erst erlernt werden. Unter den Bedingungen elektronischer Datenverwaltung ist diese Ordnung nicht mehr die gegebene. Geoffrey Yeo hat 2015 darauf hingewiesen, dass der mediale Übergang auch andere - und eventuell bessere - Erschließungswege zur Verfügung stellt. Diese kommen zunehmend zum Einsatz, sowohl auf Seite der Aktenproduzenten in der digitalen Aktenführung als auch auf Seite der wissenschaftlichen Benutzer in ihren Forschungsdatenbanken. Beide verwenden kategoriale Beschreibungen von Einzeldokumenten, als inhaltliche Beschreibung meist in Form von Schlagwörtern. Hinzu kommt, dass die Erschließungskategorien der Archivbildner sich nur selten mit den Erschließungskategorien der Forscher decken, die Einzeldokumente also von den Benutzern in neue Kontexte eingeordnet werden. Er schließt daraus, dass zwei der oben angeführten Gründe für eine hierarchische Erschließung wegfallen: Der Archivbildner hat weder notwendig die bestmögliche Informationsstruktur gefunden, noch erleichtert eine hierarchische Klassifikation notwendig die Suche ${ }^{22}$. Damit reduzieren sich die Gründe für den Erhalt einer hierarchischen und an der Organisation der Dokumente wie beim Archivbildner orientierten Erschließung auf die Abbildung von Entstehungszusammenhängen, auf die Kontextualisierung der Akten und auf die Arbeitspragmatik. Diese Gründe sind gut genug, die spezifischen Eigenschaften einer solchen Erschließung in der digitalen Umgebung fruchtbar zu machen.

Die Verfahren der Suchmaschinen zur Gewichtung der Treffer und zur Suchexpansion sind zunächst einmal nicht auf Archive übertragbar. Das liegt einerseits daran, dass Archive gewöhnlich keine umfangreichen Texte, sondern nur kurze beschreibende Metadaten den Maschinen zur statistischen Bewertung bereitstellen können. Auch eine Gewichtung auf Grund von Verweisen erscheint durch die geringe Anzahl von expliziten Verweisen in den Beschreibungen vernachlässigbar. Bei einer näheren Betrachtung wird jedoch deutlich, dass zumindest eine morphologische Anreicherung und eine Gewichtung auch für Suchmaschinen in Archivbeschreibungen sinnvoll sein können. Thomas Grotum hat so 2015 in seiner Stellungnahme zur Veröffentlichung des Archivportal-D die Zusammenarbeit zwischen Archivwissenschaft und Digital Humanities eingefordert: „Bei der Entwicklung entsprechender Werkzeuge im Rahmen der Digital Humanities könnten Archive und Forschung kooperieren "23. Aus Sicht der Digital Humanities scheint der Einsatz von Lemmatisierungswerkzeugen in den Beschreibungsdaten eine

${ }^{21}$ Clay Shirky, Ontology is Overrated: Categories, Links, and Tags, in: Clay Shirky's Writings About the Internet (2005), http://shirky.com/writings/ontology_overrated.html [1.3. 2018].

22 Yeo, Contexts (wie Anm. 13).

23 Thomas Grotum, Schritt für Schritt ins Paradies? Anforderungen an Archivportale aus der Sicht eines Historikers, in: Netz werken. Das Archivportal-D und andere Portale als Chance für Archive und Nutzung. Beiträge zum 19. Archivwissenschaftlichen Kolloquium der Archivschule Marburg, hg. von Irmgard Christa Becker-Gerald Maier-Karsten Uhde-Christina Wolf (Veröffentlichungen der Archivschule Marburg, Hochschule für Archivwissenschaft 61, Marburg 2015) 251-268, hier 268. 
Erweiterung der archivischen Erschließungssysteme, die dem gewöhnlich in lexikalischen Grundformen suchenden Benutzer die Suche nennenswert erleichtert. Dabei kann auf etablierte Forschungsergebnisse im Bereich der Computerlinguistik zurückgegriffen werden. Weniger etabliert sind Methoden, den Suchraum zusätzlich noch um Referenzen auf Lexika zu erweitern, die etwa Über- und Unterbegriffe verzeichnen können, wie zum Beispiel in den WordNets ${ }^{24}$, oder Wissen aus der historischen Semantik einfließen zu lassen ${ }^{25}$.

Um das Prinzip der Treffergewichtung sinnvoll anzuwenden, müssen andere Kriterien als die von Google verwendeten Links angewendet werden. Mit der Archivtektonik bietet die klassische Archiverschließung dafür einen guten Kandidaten. Die verschiedenen Hierarchieebenen, insbesondere aber der Provenienzzusammenhang, sind nämlich Formen vereinfachter Informationserfassung: Die Erschließungsangaben eines Bestandes sind für die meisten darin enthaltenen archivalischen Einheiten gültig. Ein Treffer in einer Bestandsbeschreibung repräsentiert also eine Vielzahl an Treffern in Aktenverzeichnungen insbesondere dann, wenn diese Akten selbst noch gar nicht verzeichnet sind. Auch für die Sucherweiterung kann eine hierarchische Ordnung von Archivgut nutzbar gemacht werden, wenn nämlich auch die Beschreibungen der Teile eines Bestandes als Deskriptoren des Gesamtbestandes verstanden werden. Wenn diese Einsichten über die Funktion der Archivtektonik in die Gewichtung von Suchtreffern integriert werden, dann sollte eine Suche nach „Enteignung Jüdischer Besitz“ im Archivportal-D den Bestand B 323 im Bundesarchiv („Treuhandverwaltung von Kulturgut bei der Oberfinanzdirektion München") vor dem Schriftverkehr mit dem Reichsleiter Martin Bormann (B 323/102) aus dem Bestand sortieren. Eine solche archivisch geprägte Methode des Information Retrieval wendet also archivfachliches Wissen auf die vorhandenen technischen Konzepte an: aus dem „Google-Suchschlitz“ wird ein „Archivsuchschlitz“.

Das Archivportal-D hat zum Beispiel in seinem Suchinterface einen weiteren Schluss aus den spezifisch archivischen Bedingungen der Erschließung gezogen. Die Benutzer suchen zwar per Stichwortsuche in der Gesamtheit der Erschließungstexte und bekommen einzelne Verzeichnungseinheiten, egal welcher Erschließungsebene, als Treffer geliefert. Sie können die Trefferliste aber nach weiteren archivischen Erschließungskategorien einschränken. In dieser facettierten Suche sind die Provenienz, die Verzeichnungsstufe und der Archivalientyp im zweiten Suchschritt ebenso zusätzlich anwendbare Kategorien wie Schlagwörter, Personen, Orte oder Zeiträume. Diese Lösung nutzt die Funktion des Provenienzprinzips und den Erhalt der originalen hierarchischen Gliederung als Abbild der Entstehungsumstände und als Erläuterung des Kontextes von Einzelstücken.

Die Erschließungstiefe dem Benutzer als Suchkriterium anzubieten und sie als Kriterium für die Gewichtung der Treffer zu verwenden, kann in der Erschließungspraxis dazu motivieren, ausführliche Bestandsbeschreibungen langwierigen Einzelaktverzeichnungen vorzuziehen. Die Erleichterung der Erschließungsarbeit durch eine hierarchische Organisation wird also durch die auf Mathematik aufbauende Möglichkeit verstärkt, summarische Beschreibungen dem Benutzer mit dem gleichen Gewicht anzubieten wie

${ }^{24}$ Das älteste dieser hierarchischen Lexika ist das englische Wordnet, erstellt und gepflegt an der Princeton University: Wordnet. A Lexical Database for English, https://wordnet.princeton.edu/ [1. 3. 2018]. Für die deutsche Sprache wird eine entsprechende Ressource an der Universität Tübingen erstellt: GermaNet. A German WordNet, http://www.sfs.uni-tuebingen.de/GermaNet/ [1. 3. 2018].

${ }^{25}$ Zum Problem der Formalisierung von Wissen alleine auf Grundlage von modernem Sprachgebrauch vgl. Kim H. Veltman, Towards a Semantic Web for Culture. Journal of Digital Information 4/4 (2004), http:// journals.tdl.org/jodi/article/view/113 [1. 3. 2018]. 
eine Vielzahl von Einzelverzeichnungen in einem Bestand. Es ergibt sich daraus jedoch die Forschungsaufgabe, realistisch einzuschätzen, welches Gewicht eine Bestandsbeschreibung erhalten soll. Dazu wären die Deskriptorenräume ausführlicher Bestandsbeschreibungen mit der Gesamtheit der Deskriptoren für die enthaltenen kleineren Verzeichnungseinheiten zu vergleichen und gegebenenfalls Schlüsse für die Praxis der Bestandsbeschreibung zu ziehen.

Provenienz- und Registraturprinzip sind auch in der Archivfachwelt nicht unumstritten, weil sie nur den Zustand der Aktenorganisation bei der Übernahme im Archiv abbilden. Die Entwicklung der Aktenorganisation als Teil der Behördengeschichte bleibt dabei unsichtbar ${ }^{26}$. Es ist aber akzeptiert, dass Provenienz- und Registraturprinzip ebenso wie andere Erschließungsmethoden einen zum Verständnis der Akten notwendigen Kontext liefern ${ }^{27}$. Aus der oben angeführten erkenntnistheoretischen Position heraus geht es also darum, diesen Kontext - wie alle von den Archivaren ermittelten Kontexte - zum Teil der Narrationen werden zu lassen, in denen die geschichtswissenschaftlichen Benutzer die Archivalien rezipieren. Damit meine ich nun nicht die eher theoretische Position, dass auch die Verfahren der Archivare prinzipiell auch Meistererzählungen realisieren ${ }^{28}$. Es geht vielmehr um die konkrete Rezeption der Archivalien als Zeugnisse von menschlichen Handlungen, die in eine sinnvolle Abfolge gebracht werden, um sie zu verstehen. Die Benutzer sollten sich aus der archivischen Erschließung Narrative konstruieren können, die ihnen helfen, zu den Einzelakten zu gelangen, und die ihnen helfen, sich eigene Narrationen aufzubauen, um zu historischen Erkenntnissen zu kommen. Können die - offensichtlich narrativen - Behörden- und Bestandsgeschichten gemeinsam mit kategorialen Beschreibungen wie Aktentitel, Laufzeit oder Indizes mit Hilfe von Informationstechnologien so umorganisiert werden, dass sie diese Prozesse unterstützten?

Die Digital Humanities haben dazu kein Patentrezept anzubieten. Sie engagieren sich zwar im Bereich der Narratologie, sind aber derzeit noch vorwiegend damit beschäftigt, den Befund aufzunehmen: Digitale Techniken werden vorwiegend dazu eingesetzt, Er-

26 Insbesondere Peter Scott und Mario Fenyo argumentierten dafür, auf der den Fonds untergeordneten Ebene der Serien zu erschließen: Peter Sсотт, The Record Group Concept: A Case for Abandonment. The American Archivist 29 (1966) 493-504; Mario D. Fenyo, The Record Group Concept: A Critique. The American Archivist 29 (1966) 229-239. Aus den systematischen Überlegungen hinter dieser Kritik leiten sich etwa Positionen zur funktionalen Beschreibung von Archivgut ab: Peter Horsman, Wrapping Records in Narratives. Representing Context through Archival Description, in: ITS. International Tracking Service - News - 12.10.2011 Internationale Archivtagung beim ITS, https://www.its-arolsen.org/fileadmin/user_upload/Metanavigation/ Aktuelles/2011_PDF/04_Horsman_text.pdf [1. 3. 2018]. Zur Geschichte der Archivtheorie: Terence Gordon Соок, What is Past is Prologue: A History of Archival Ideas since 1898, and the Future Paradigm Shift. Archivaria. The Journal of the Association of Canadian Archivists 43 (1997) 17-63. Weiters vgl. Jefferson BaILeY, Disrespect des Fonds: Rethinking Arrangement and Description in Born-Digital Archives. Archive Journal 3 (2013), http://www.archivejournal.net/essays/disrespect-des-fonds-rethinking-arrangement-and-descriptionin-born-digital-archives/ [1.3. 2018].

${ }^{27}$ Heather MacNeIL, The Context is All: Describing a Fonds and its Parts in Accordance with the Rules for Archival Description, in: The Archival Fonds: From Theory to Practice, hg. von Terence M. EASTwOOD (Ottawa 1992) 195-225.

28 Wendy M. Duff-Verne Harris, Stories and Names: Archival Description as Narrating Records and Constructing Meanings. Archival Science. International Journal on Recorded Information 2 (2002) 263-285; oder weniger allgemein: Heather McNeIL, Picking Our Text. Archival Description, Authenticity, and the Archivist as Editor. The American Archivist 68 (2005) 264-278. Archivare haben aber auch schon praktische Konsequenzen aus der Erkenntnis zu ziehen versucht: Melanie Feinberg, Designing Collections for Storytelling: Purpose, Pathos, and Poetry. Information Research 15/3 (2010), http://www.informationr.net/ir/15-3/colis7/colis701. html [1. 3. 2018]. 
zählstrukturen in literarischen Texten aufzufinden, zu dokumentieren und zu annotieren. Forschungen zur aktiven Erzeugung von Narrativen (story telling) finden mit Studien zur künstlichen Intelligenz statt. Sie sind aber noch nicht sehr weit fortgeschritten ${ }^{29}$.

Dennoch können etablierte archivische Erschließungsmethoden einen narrativen $\mathrm{Zu}$ gang zu den Archivalien unterstützen. Erstens müssen sie ernst nehmen, dass die Organisation der Archivalien im Bestand Kontext für ihre Bestandteile liefert. Der Informationsgewinn aus der Organisation von Einzelakten in Bestände liegt für die Archivbenutzer darin, dass sie sich damit über Tätigkeiten der historischen Akteure informieren können: Die Behörden agieren in ihrem Schriftgut, sie kommunizieren, entscheiden, ordnen an, verbieten. Wo sie nicht direkt durch das Schriftgut agieren, dokumentieren sie doch ihr Handeln, das Handeln der in ihnen beschäftigten Personen oder das Handeln derjenigen, die mit ihnen kommunizieren. Die klassische hierarchische Erschließung auf Bestandsebene nach $\operatorname{ISAD}(\mathrm{G})$ berücksichtigt das mit den Angaben zur Geschichte des Bestandsbildners und zum Inhalt des Bestandes - historische Erzählungen, die bei Lektüre beim Archivbenutzer ein Verständnis von den im Bestand zu erwartenden Informationen erzeugen. Das ICA schlägt für diese Bedürfnisse zwei weitere Standards vor: ISAAR(CPF) für die Beschreibung der Bestandsbildner und ISDF für die Aufgaben und Tätigkeiten der Akten erzeugenden Instanzen ${ }^{30}$. ISAAR(CPF) hat im XML-Schema EAC-CPF konkretisiert einigermaßen Verbreitung in der Archivwelt gefunden. ISDF ist bislang nur von akademischem Interesse, vermutlich auch weil es an konkreten technischen Implementationen fehlt, mit denen diese Informationen in die digitalen Abläufe alltäglicher Archivarbeit integriert werden können.

Die Datenmodellierung, das heißt die Entwicklung von modellhaften Konzepten und ihre Abbildung in konkrete technische Lösungen, ist nun ein zentrales Forschungsfeld der Digital Humanities ${ }^{31}$. Die archivische Erschließung hat mit den Kategorien von ISAD $(G)$ ein relativ flaches Modell entwickelt. Die verbale Beschreibung der Bestände - beziehungsweise der Einzelakten - enthält viele Informationen, die anders organisiert werden könnten, um sie für weitere Suchanfragen zugänglich zu machen. Sie werden für einen „erzählenden“ Zugang zum Archivmaterial besonders attraktiv, wenn sie Verbindungen zu Beschreibungen in anderen Erschließungseinheiten identifizieren. Dafür steht mit Schlagwortindizes ein etabliertes Mittel zur Verfügung: Die klassischen Angaben zu Personen und Orten helfen, ihr Vorkommen auch bestandsübergreifend leicht zusammenzuordnen. Technische Potentiale ergeben sich daraus, mit modernen Mitteln der Wissensorganisation zu verschlagworten.

Die Erschließungsarbeit, die zurzeit bei den Staatlichen Archiven Bayerns an den

29 Pablo Gervás, Story Generator Algorithms, in: Handbook of Narratology, hg. von Peter HüHN-Jan Christoph Meister-John Pier-Wolf Schmid, 2 Bde. (Berlin-Boston 2014) 2 825-835; David Herman, Cognitive Narratology, in: ebd. 1 46-64.

30 ISDF: International Standard for Describing Functions, in: ICA. International Council on Archives International Standards, http://www.ica.org/en/isdf-international-standard-describing-functions [1. 3. 2018]; ISAAR(CPF): International Standard Archival Authority Record for Corporate Bodies, Persons and Families, 2nd Edition, in: ICA. International Council on Archives - International Standards, https://www.ica.org/en/ isaar-cpf-international-standard-archival-authority-record-corporate-bodies-persons-and-families-2nd [1. 3. 2018].

31 Willard McCarty, Humanities Computing (Basingstoke-New York 2005) 29-72; DERs., Modeling: A Study in Words and Meanings, in: A Companion to Digital Humanities, hg. von Susan Schreibman-Ray Siemens-John Unsworth (Blackwell Companions to Literature and Culture 26, Malden-Oxford-Carlton 2004) 254-270. 
Wiedergutmachungsakten der Nachkriegszeit geleistet wird, ist ein gutes Beispiel dafür, worauf es hierbei zu achten gälte ${ }^{32}$. Die für die Registratur der Akten angelegten Karteikarten sind eine gute Basis für die Erschließung. Sie enthalten Informationen wie die Namen der Verfolgten und der Anspruchsberechtigten, die jetzigen Eigentümer und ihre Vorgänger. Diese Informationen sind zentral für die Klarstellung von Restitutionsansprüchen an enteignete Kunst. In der summarischen Beschreibung eines Aktentitels und einfacher Personenschlagwörter gehen diese strukturellen Informationen aber nur in einen nicht weiter untergliederten Aktenbetreff ein. Die bayerischen Archivarinnen haben in den Erfassungsrichtlinien für die Rollen der an den Verwaltungsverfahren beteiligten Person ein kontrolliertes Vokabular („Antragsteller“, „Antragsgegner“, „Verfolgter“, „Berechtigter") entwickelt, das der externe Dienstleister bei der Digitalisierung der Karteikarten zu verwenden hat. Diese pragmatische Lösung tilgt also nicht etwa textuelle Information, sondern ordnet sie nur anders an, reduziert die Struktur der Vorlage. Das scheint die Folge eines technischen Systems zu sein, nämlich der verwendeten Eingabemaske für die Erschließungsdaten. Die benutzte Software nutzt aber nicht die an sich vorhandenen technischen Methoden, denn die digitalen Hilfsmittel zur Modellierung erlauben es, über die einfache Kategorisierung als „Person“, „Ort“ oder „Sachschlagwort“ hinauszugehen $^{33}$. Mit den Methoden der Klassenbeziehungen können nämlich Verallgemeinerungen in Wissensrepräsentationen so ausgedrückt werden, dass die Erschließungsinformationen sowohl in der allgemeinen Kategorie „Person“ wie in einer speziellen Kategorie als „Verfolgter" auffindbar sind. In der Museumswelt ist zum Beispiel mit dem Standard des CIDOC-CRM ein Modell entwickelt worden, das solche Verallgemeinerungen bis auf die abstrakte Unterscheidung von temporalen und nicht-temporalen Konzepten mitdenkt ${ }^{34}$.

Das CIDOC-CRM setzt auf einem Basismodell auf, das sich in den digitalen Geisteswissenschaften zunehmender Beliebtheit erfreut: der Repräsentation von strukturierten Daten als Graphen, das heißt als Beziehungen zwischen zwei Knoten. Dieses Modell lässt sich auf eine sehr einfache intellektuelle Struktur abbilden, nämlich einfache Aussagesätze aus „Subjekt“ (Knoten), „Prädikat“ (Beziehung) und „Objekt“ (Knoten). Dieses Modell ist zum Beispiel Grundlage der Aktivitäten des W3C im Bereich des sogenannten „Semantic Web“, in dem strukturierte Daten über Web verknüpfbar und austauschbar gemacht werden ${ }^{35}$. In der Archivwelt ist dieser Ansatz mit der Arbeit an „Records in Contexts" ( $\mathrm{RiC}$ ) in Angriff genommen worden. $\mathrm{RiC}$ ist ein konzeptionelles Modell, das die Dokumentation und ihre Organisation sowie die Akteure, ihre Rollen, Aufgaben und Aktivitäten zueinander in Beziehung setzt. Es kann damit sowohl die Aktivitäten bei der Entstehung der Akten, ihre Organisation in der Registratur und im Archiv abbilden als

32 Ich danke Herrn Dr. Julian Holzapfl für den Einblick in die Erschließungspraxis und die fruchtbare Diskussion über die dahinter stehenden Konzepte.

33 EAD 2002 sieht im Bereich für die Beschreibung mit kontrollierten Vokabularien neben den Kategorien persname, geogname und subject auch noch Organisations- und Familiennamen (corpname, famname), Berufe und Funktion (occupation, function) sowie archivalische Genres (genreform) vor: Encoded Archival Description Tag Library, Version 2002, in: The Library of Congress - Standards - EAD, http://loc.gov/ead/tglib/elements/ controlaccess.html [1.3. 2018].

${ }^{34}$ CIDOC-CRM Conceptual Reference Model, http://cidoc-crm.org/ [1. 3. 2018]. Das offizielle Dokument zur Definition des Standards ist: Definition of the CIDOC Conceptual Reference Model, Version 6.2.2, September 2017, hg. von Patrick Le Boeuf et al., in: CIDOC-CRM Conceptual Reference Model - Resources, http://cidoc-crm.org/sites/default/files/2017-09-30\%23CIDOC\%20CRM_v6.2.2_esIP.pdf [1. 3. 2018].

35 Semantic Web, in: W3C. World Wide Web Consortium - Standards, https://www.w3.org/standards/ semanticweb [1.3. 2018]. 
auch die Dokumentation zu bestimmten Personen, Behörden und Aktivitäten durchsuchbar machen. Noch sind die vorgeschlagenen fast 800 Beziehungen zwischen den Objekten wenig entwickelt. Sie zeigen aber deutlich, welches Potential sie für eine gänzlich anders konzipierte Suche bieten: RiC-R204 definiert zum Beispiel „Agent authored Record“ oder RiC-R511 „Activity resulted in Record“36.

Aus einer solchen Konzeption der archivischen Beschreibung ergeben sich Zugangsformen, welche das Wissen über die Archivalien, über ihren Inhalt und über ihren Entstehungskontext den assoziativen und narrativen Modellen der Forscher näher bringen. In verschiedenen Archiven sind Experimente mit einschlägigen Darstellungsmethoden ihrer Erschließung gemacht worden. Das Staatsarchiv Thurgau hat zum Beispiel seine Beständeübersicht als Netzplan visualisiert ${ }^{37}$. Weiter gehen die Archivabteilung der New York Public Library ${ }^{38}$ und ein Portal zu Bologneser Archivalien des 19. und 20. Jahrhunderts unter dem Namen „Città degli archivi“"39. Beide übersetzen die Deskriptoren (Personen, Organisationen, Orte, Inhalte und Ereignisse) der Archivalien in eine graphische Visualisierung aus Knoten und Beziehungen. Die Benutzer können sich damit zu jedem beliebigen Knoten die einschlägigen Archivalien, aber auch die Beziehungen zu anderen Deskriptoren anzeigen lassen, und sich so durch die Assoziationsketten der Erschließung hangeln. Während das Bologneser Projekt die Beziehungen auch, wie in RiC vorgeschlagen, konkret benennt, sind die Beziehungen zwischen den New Yorker Archivalien nicht klassifiziert. Dafür dienen dort auch die Verzeichnungseinheiten als Knoten, die zu weiteren Deskriptoren führen. Dieser Unterschied ist relevant für die Frage danach, welche Konsequenzen digitale Instrumente für die archivische Erschließung haben: Die amerikanische Darstellung benutzt nämlich auch die Organisation der in den Dokumenten enthaltenen Information durch die Archivbildner und die Archive. Sie zeigt, dass sich die Informationen, die in der Erschließungshierarchie und in den Standards archivischer Erschließung enthalten sind, den Vorgehensweisen des lernenden Suchens annähern können, wenn sie in andere Präsentationsformen gebracht werden.

Am Ende dieses Beitrags kann ich deshalb zu den eher experimentellen Formen der Präsentation von archivischer Erschließung zurückkehren und ein Beispiel anführen, bei dem sich ein Designer Gedanken über die digitale Präsentation von Archiverschließung gemacht hat. Mitchell Whitelaw hat Visualisierungen der National Archives of Australia entworfen, die als Zugangsweg zu den einzelnen Archivalien benutzt werden können. In einer ersten Form visualisiert er die Größe der Bestände je Bestandsbildner. Whitelaw verwendet dafür sowohl die Zahl der Verzeichnungseinheiten als auch die Zahl der Regalmeter und bildet damit eine innere Hierarchie der Erschließung ab, die auf abstrakterer Ebene bleiben kann und damit wenige Verzeichniseinheiten für umfangreiches Archivmaterial erzeugt, oder mehr Details aufnimmt, die sich auf eine geringe Menge von Papier verteilen. Dass eine solche Visualisierung natürlich den unterschiedlichen Lagerungsbedingungen von zum Beispiel Urkunden und Papier Rechnung trägt, ist ein Hinweis auf die Notwendigkeit einer archivfachlich informierten Form der Darstellung. Eine zweite

36 Records in Contexts - Conceptual Model, in: ICA. International Congress on Archives - International Standards, http://www.ica.org/en/egad-ric-conceptual-model [1.3. 2018].

37 Urban StÄHeli, Archivnetzplan. Ein neues Findmittel für die Online-Suche in Archivbeständen. Informationswissenschaft. Theorie, Methode und Praxis 4/1 (2016) 266-278.

38 Terms Explorer, in: The New York Public Library. Archives and Manuscripts, http://archives.nypl.org/ terms [1.3. 2018].

39 archIVI. Città degli archivi, http://www.cittadegliarchivi.it [1.3. 2018]. 
Visualisierung listet die in den Titeln vorkommenden Wörter so auf, dass sie zum einen auf ihre Häufigkeit über die Zeit und zum anderen auf mit ihnen gemeinsam vorkommende Wörter verweisen ${ }^{40}$. Gerade diese zweite Visualisierung nähert sich wieder den narrativen Elementen historischer Forschung an, indem die Texte der Archivverzeichnung aufgebrochen und in neue Zusammenhänge gestellt werden. Die Benutzer bekommen also gewissermaßen alle möglichen Geschichten erzählt, die sich aus den Aktentiteln bilden lassen.

Aus Sicht der Digital Humanities gestaltet sich das Verhältnis von klassischen Formen der Archiverschließung und den Zugängen der Digital Humanities zum Information Retrieval also als Anspruch, nicht die etablierten hierarchischen Formen durch flache Erschließung zu ersetzen, sondern die Gründe und das Potential der etablierten Formen von Erschließung im Information Retrieval der Archivinformationssysteme nutzbar zu machen. Die Anforderung an ein solches muss also nicht nur heißen, einen hierarchischen Zugang etwa über die Archivtektonik zu ermöglichen, sondern diese auch in der Suchumgebung zum Beispiel über Indizes oder die Stichwortsuche zu berücksichtigen. Wenn eine Stichwortsuche eine Gewichtung der Treffer nötig macht, dann ist dabei die Bedeutung der übergeordneten Erschließungsebenen entsprechend zu berücksichtigen. Umgekehrt kann mit einer solchen Berücksichtigung auch die Archivpraxis guten Gewissens die Erschließung von Beständen, Bestandsgruppen, Aktengruppen und anderer Aggregationen von Einzelarchivalien bevorzugen, da Treffer in diesen Beschreibungen hervorgehoben werden. Es ist sogar zu überlegen, ob nicht die Aktentitel innerhalb einer Aggregation im Suchraum durch die Beschreibung übergeordneter Einheiten angereichert werden sollten. Experimente mit der Visualisierung von hierarchischer Erschließung geben weitere Beispiele, wie sie informativ in die Präsentation der Archivinformationen integriert werden kann. Darüber hinaus unterstützt Archiverschließung auch narrative Zugänge, wenn die Bezüge, die sich aus Schlagwörtern, Erschließungshierarchien und verbaler Kontextualisierung zum Beispiel in Form von Behörden- und Bestandsgeschichte ergeben, den Benutzern als Lesepfade durch das Archivgut angeboten werden können. Hier ist noch Forschungsbedarf, der einer Zusammenarbeit zwischen Digital Humanities und Archivwissenschaft eine rosige Zukunft verspricht.

40 Series Browser, in: NAA. National Archives of Australia - About us -Research Grants, http://www.naa. gov.au/about-us/grants/ian-maclean/series-browser.aspx [1.3. 2018]. 


\title{
Archival Information System: A Bridge Between Past, Present and Future
}

\author{
Miroslav Novak
}

Introduction

In the archival community it is well known that archivists should constantly consider different theoretical and practical issues related to their everyday work in view of the many changes in modern society. New archival tasks and new ways of using information technology in archives ${ }^{1}$, the development of professional experiences and standards, the amount and quality of captured descriptions of archival entities, and the archiving of e-re$\operatorname{cords}^{2}$ are only a few of the issues of today's archival theory and practice. In this context particular attention should be paid to solutions in archival information systems.

Archivists generally assume that the term ,archival information system “ is well known and understood among professionals and the public alike. But in practice, users still have some basic questions about this topic, especially those who are coming into contact with archives for the first time.

Today's archival institutions run different information systems with large quantities of captured and organized data ${ }^{3}$. The majority of data related to archival material is accessible, but certain subsets are not available for online use due to different reasons: national or international legislation on data protection, preservation-related issues and so on. From the user's point of view it is important to understand the dynamics of changes in these data statuses. They change in the course of time analogously to the roles and policy of the archival system and the national laws for data protection ${ }^{4}$.

\footnotetext{
${ }^{1}$ Susanne Fröhlich-Elisabeth Schöggl-Ernst, Digitale Archivierung in Österreich. Atlanti. Review for Modern Archival Theory and Practice 25/1 (2015) 265-274.

2 Lajos Körmendy, Information Society, E-Records and the New Archival Science. Atlanti. Review for Modern Archival Theory and Practice 25/1 (2015) 141-152.

3 At the end of 2016, there were on the Archives Portal Europe more than 253.900.000 descriptive units of archival material captured from 6.861 archival institutions in Europe: Archives Portal Europe, http://www. archivesportaleurope.net/ [1.3. 2018].

4 Elena Romanova, Regulation of Access to Personal Data in the Archives: Issues, Trends and Challenges. Atlanti. Review for Modern Archival Theory and Practice 26/1 (2016) 109-115.
} 
Following the international archival professional guidelines ${ }^{5}$, users normally expect that the archival information systems will function as a bridge which will help them to fulfil all their information needs when searching for the archival material for their histori$\mathrm{cal}$, genealogical or other researches. On the other hand, archivists have, of course, a more complex view of what is defined as an archival information system. Following this logic, it is basically a complex entity for managing different information needs in an archival institution.

\section{The Archivist's Professional Point of View}

In order to do their job properly, archivists need many different types of information from different sources. These relate not only to acquisitions or to managing the content and the context of the archival material, but also to other archival professional activities, including access and usage of archival material according to the applicable legislation. Because of this, the complexity and connectivity of such a system can be highly demanding. On the theoretical level this can be seen in the model of an Open Archival Information System (OAIS) ${ }^{6}$. However, various archival professional discussions based on that model show that even archivists do not always have a very precise and clear view of all the possibilities, and the related benefits and needs, of such a system.

In all archival institutions archival information systems have experienced a remarkable development. This success has multiple reasons: changes in social conditions, and in some cases also in political regimes; standardization in the archival professional field; the rapid development of information technology, especially computer-supported communications and related use of the Internet in different public and private services ${ }^{7}$. One of the basic challenges of today's archival science is related to the question of whether the archival community is able to find appropriate and long-term stable solutions for new professional issues or not. Furthermore, are archivists able to give proper answers to various and sometimes sensitive professional questions related to archival material in a modern information society?

In archival practice it is obvious that archival institutions have implemented different, but compatible, technological infrastructure such as computers, printers, and scanners. In the process archivists have also acquired new technological skills and knowledge that they can share on an international level. But a simple question arises: have they also developed the needed methods ${ }^{8}$ of archival professional work and obtained new knowledge related to the new technological possibilities and users' needs? These questions are closely

\footnotetext{
5 Universal Declaration on Archives, in: ICA. International Council on Archives, http://www.ica.org/en/ universal-declaration-archives [1.3. 2018].

${ }^{6}$ Reference Model For An Open Archival Information System (OAIS). Recommended Practice CCSDS 650.0-M-2. Magenta Book, June 2012, in: The Consultative Committee for Space Data Systems. The Official Web Site. Publications - Magenta Books: Recommended Practices, https://public.ccsds.org/pubs/650x0m2. pdf [1. 3. 2018].

7 Bogdan Florin Popovici, Electronic Records Management in Romania: More Electronic-, Less RecordsManagement. Atlanti. Review for Modern Archival Theory and Practice 25/1 (2015) 183-192.

8 Zdenka Semlič RajH-Izet Š ABotić-Alenka ŠAuperL, Znanstvenoraziskovalno delo v arhivistiki: značilnosti uporabe dveh raziskovalnih metod [Research in Archival Science: Characteristics of Two Research Methods]. Tehnični in vsebinski problemi klasičnega in elektronskega arhiviranja 12 (2013) 125-144, http://www. pokarh-mb.si/uploaded/datoteke/Radenci/Radenci2013/11_Semlic_Sabotic_Sauperl_2013.pdf [1.3.2018].
} 
connected with the challenges related to the quality of the product of archival professional work, especially the description of the archival material and other entities.

Each archival institution holds unique archival material. In many cases this is the main reason for developing specific solutions in information management and other fields. This is understandable, as one of the basic results of that development should be an archival information system which is a suitable tool for archivists in supporting their particular information needs. Another important aspect of this development is captured data or information which the archivists create during their archival professional activities and process or store in the archival information system. These data should be considered as the "product" of archival professional activities.

Many cases from the last decades show us the complexity of the development and usage of archival information systems and data that are captured in them. Let us take a closer look at some of the cases in Slovenia. Until the mid-1980s, information systems used in Slovenian archives were based on paper technology. Descriptions were handwritten or typewritten. Owing to a short-term economical approach, archival material was described only down to the level of technical units such as archival boxes. Exceptions were made for important archival material like medieval deeds, which might be described from fonds or collection down to item level. Moreover, it was common practice in Slovenian archives to use original finding aids, that is, journals or indexes once kept by the creators of the fonds, as archival finding aids for searching archival material in the reading room. All these various types of metadata are more or less preserved on paper until today, and only a small amount of them have been transferred into the electronic archival information systems.

By the beginning of the 1990s, all archives in Slovenia had acquired their first computers. At that time these were basically used as sophisticated typewriters (WordStar, Word for DOS, Display Writer) and as platforms for small databases in Turbo Pascal, Dbase3+ or Clipper with a few thousand records?.

The crucial development in this area was brought about by the systematic implementation of the international standard $\operatorname{ISAD}(G)^{10}$. It was introduced as a professional framework for describing archival material on different platforms, especially those designed for local area networks. From a technical point of view, diverse software solutions were chosen. Custom software or personal information managers such as askSam ${ }^{11}$ were used. With these tools archivists could create and use different databases in the form of thousands of logically interconnected files. Several hundred thousand records of archival material were created using these programs. Some of these have since been transferred into the current information system based on scopeArchiv ${ }^{12}$; others are still waiting to be migrated.

9 Miroslav NovaK, Tehnološko-tehnična podpora arhivskemu strokovnemu delu v Pokrajinskem arhivu Maribor [Technological and Technical Support of Archival Professional Work in the Regional Archives Maribor], in: Hraniti in ohraniti. 100 let načrtnega zbiranja in ohranjanja arhivskega gradiva ter 70 let delovanja profesionalne arhivske ustanove v Mariboru. Jubilejni zbornik (Maribor 2003) 375-427.

10 The Slovenian translation of the Ottawa $1994 \operatorname{ISAD}(\mathrm{G})$ became available in 1998: Splošni mednarodni standardi za arhivsko popisovanje. Mednarodni standardi za arhivski zapis o ustvarjalcih arhivskega gradiva: pravnih osebah, fizičnih osebah in družinah, trans. Olga Pıvк (Ljubljana 1998), in: Arhiv Republike Slovenije Zakonodaja in dokumenti, http://www.arhiv.gov.si/fileadmin/arhiv.gov.si/pageuploads/zakonodaja/isadg1.pdf [1. 3. 2018].

$11 \operatorname{askSam}^{\mathrm{TM}}$. Um mehr zu wissen, http://www.asksam.de [1. 3. 2018].

12 Archivinformationssystem scopeArchiv, http://www.scope.ch/de/produkte/moduluebersicht.html $[1 / 3 / 2018]$ 
The development and short-term usage of the InfoArh system from 2003 to 2007 in some Slovenian archives helped archivists to understand how to create and manage records of archival material in a complex archival information system ${ }^{13}$. Furthermore, similar descriptions were also made with MS Office programs like MS Word, MS Excel or various open-source equivalents.

In 2008, the scopeArchiv platform was implemented in the Archives of the Republic of Slovenia, and in 2009 in all six regional archives ${ }^{14}$. This platform has allowed Slovenian archivists to create or import more than one hundred thousand records of description of archival material per year since then (fig. 1) ${ }^{15}$. This amount of created records has called for important new professional decisions not only regarding technology and communications, but also on the level of establishing professional processes, standardizing descriptions, controlled dissemination of information about the usage of archival material, research on the results of archival professional work with special emphasis on re-use of captured data, education for new needs and exchange of professional experiences, and so forth.

\section{Number of captured Units of Description in SIRAnet from $2010-2016$}

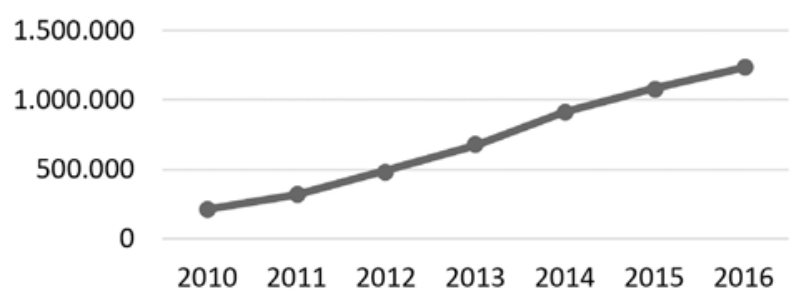

Fig. 1

The relatively fast development of the available technology has had a direct impact on archival theory and its practical implementations in the archival institutions. It may seem that in some institutions only one archival information system is used. Yet in practice several parallel archival information systems may coexist. In relatively small archival institutions, like the Regional Archives Maribor (fig. 2), several different technological solutions with many personal modifications dating from the last three decades are to be found:

- personal or shared information systems which are obsolete and no longer in use,

- personal or shared information systems which are obsolete but still in use, and

- the current mutual archival information system.

13 Miroslav NovaK-Vladimir Drobnjak, Zasnova in strateške usmeritve slovenskega vzajemnega arhivskega informacijskega sistema [The Design and Strategic Orientation of the Slovenian Mutual Archive Information System]. Tehnični in vsebinski problemi klasičnega in elektronskega arhiviranja 1 (2002) 322-333.

${ }_{14}$ The Slovenian regional archives have organized a mutual archival information system called SIRAnet, powered by scopeArchiv: SIRAnet, http://www.siranet.si [1.3. 2018].

15 The system developed over the last seven years currently encompasses, within a single instance of the database, more than 1.2 million units of description, more than 1.1 million containers per level, about 900.000 descriptors per level, 158.000 containers, 45.000 locations, 41.000 files per level, 33.000 descriptors, etc. 


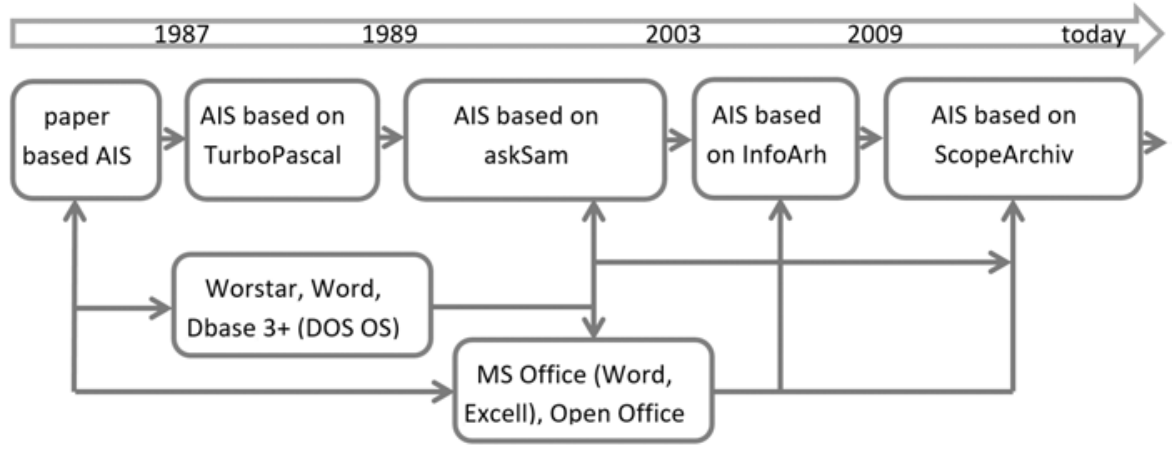

Fig. 2: Model of implemented AIS and directions of data migrations in the case of the Regional Archives Maribor, 1980s to present (Miroslav Novak).

Obsolete information systems exist as independent solutions that are logically not interconnected with the current system. Some of them run on outdated technical infrastructure, which in turn causes huge problems when faced with the need to harmonize data with the $\operatorname{ISAD}(\mathrm{G})$ standards and migrate it into the current information system. Obsolete infrastructure (such as programs working only in a DOS environment), non-standardized descriptions of archival material at the box level, lack of data or incomplete data in the description for understanding the context and content of the described archival material, problems with the code tables used in obsolete infrastructure, and typing errors are only a few of the problems with descriptions generated under formerly used information systems. From today's perspective they have multiple impacts. One of these is limited access to basic information sources, not only for the archivists, but also for the users in the archive.

Some of the problems with data capture into the current information system can be observed in the results of special surveys focused on the creation of the titles of units of description. The first survey was performed in the middle of $2013^{16}$ and the second at the end of 2016. The results show that titles for units on the higher levels of description are on average half as long as those of units at lower levels (fig. 3). This means that the information value of unit titles varies with the level of description, resulting in imbalances in the database in terms of information value, structure and data potentials ${ }^{17}$.

The surveys also took a closer look at differences in the length of titles of units of description between the six regional archives where the descriptions were created. All unit titles were in Slovenian. The results clearly show that archivists in different archives create titles of very dissimilar average length for comparable units of description. It is known

16 Miroslav NovaK-Zdenka Semlič Rajh, Merjenje rezultatov uspešnosti izgradnje arhivskih podatkovnih zbirk s kvantitativno-kvalitativno metodo na primeru podatkovne zbirke SIRAnet [Measurement of Performance Results of the Construction of Archival Information Systems by Quantitative and Qualitative Methods, Using the Example of the Information System SIRAnet], in: Standardizacija (p)opisov arhivskega gradiva in uskladitev strokovnih praks v slovenskih javnih in cerkvenih arhivih. Zbornik referatov, ed. Jure VolČJAK (Ljubljana 2013) 19-39.

17 Similar results are presented by Zdenka SEmLIČ RAJH-Alenka ŠAUPERL, Analiza oblikovanja vsebine zajetih podatkov v podatkovni bazi SIRAnet [Content Analysis of Data Captured in the SIRAnet Database]. Tehnični in vsebinski problemi klasičnega in elektronskega arhiviranja 12 (2013) 145-157, http://www.pokarhmb. si/uploaded/datoteke/Radenci/Radenci2013/12_Semlic_Sauperl_2013.pdf [1.3.2018]. 


\section{Average number of used words in title of the UD in the year 2013 and 2016}

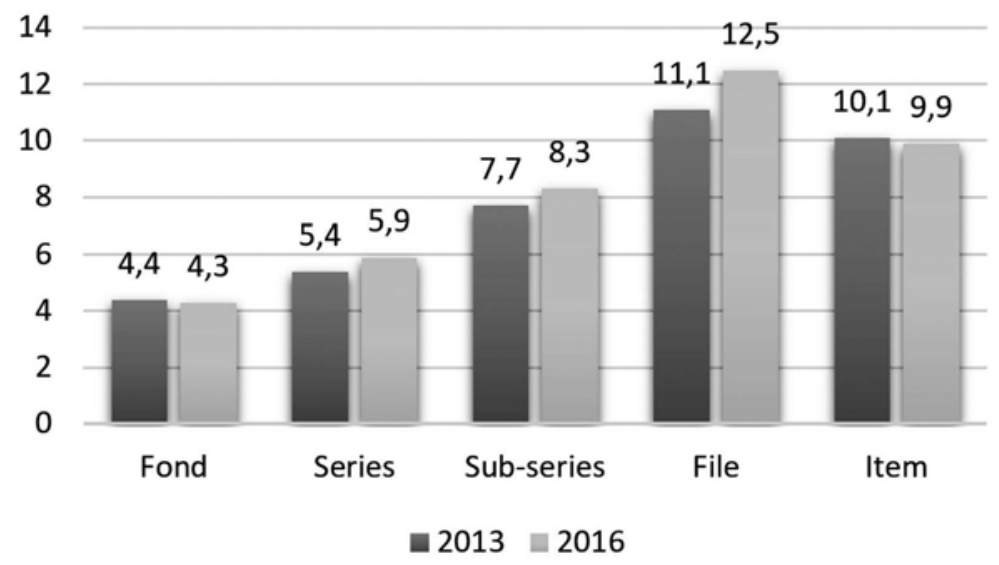

Fig. 3: Average number of words used in titles of units of description, 2013 and 2016 (Miroslav Novak).

\section{Average number of characters used in title of the UD by archive in the year 2013 and 2016}

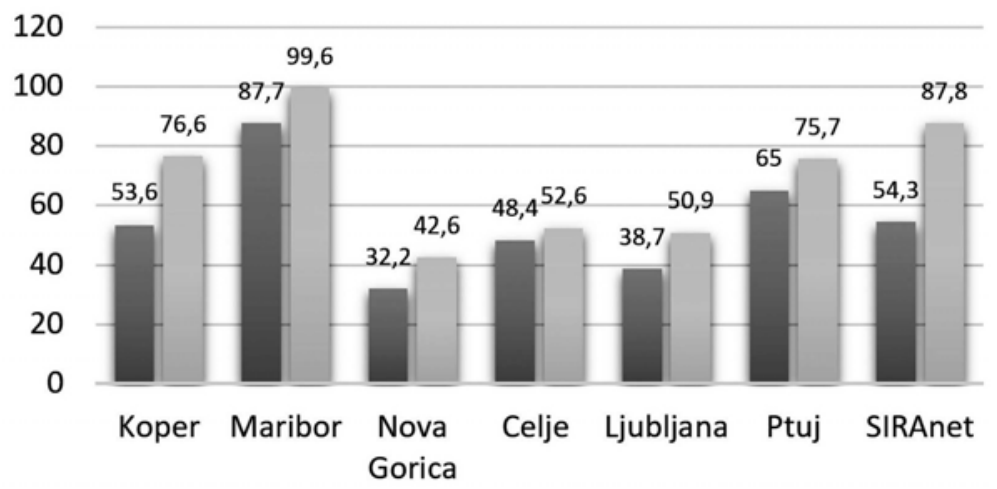

- $2013 \square 2016$

Fig. 4: Average number of characters used in titles of units of description, by archive, 2013 and 2016 (Miroslav Novak).

that the methods of archival description depend on several factors, including conceptions of the types of archival material, traditions of description in particular archives, the level of developed professional practice in a given institution, and more. Of course the personal touch of each archivist should be taken into account, but nevertheless, the results of the survey show significant variation between archives that cannot be explained by individual habits alone (fig. 4). This result is important for the further development of the current 
New and Returning Visitors

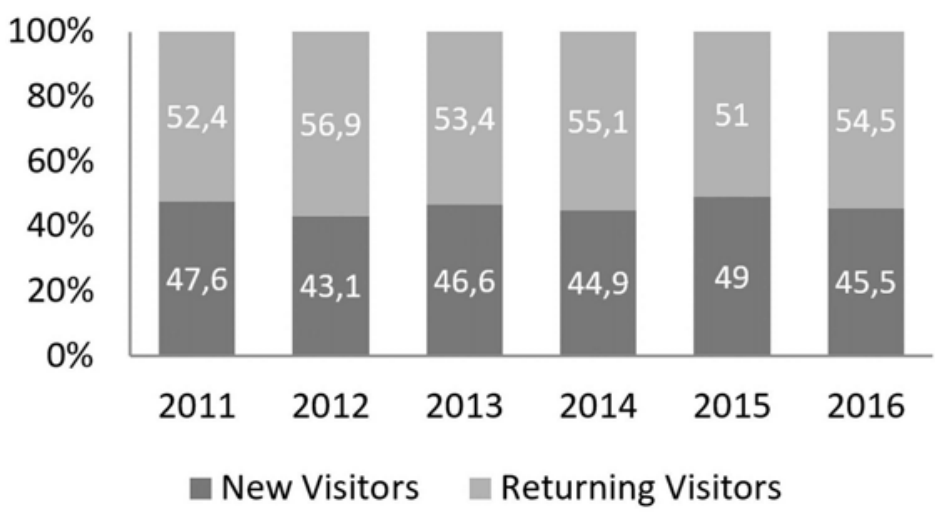

Fig. 5: New and returning users of SIRAnet, 2011-2016 (Miroslav Novak).

mutual information system because the information derived from unit titles from all six regional archives appears in that system in more than ten different contexts. The necessary balance in this regard needs to be achieved.

Understanding what happens with captured data used in different contexts, especially those published on the Internet, is as important as understanding the behaviour of users. Let us take a brief look at new and returning visitors. The statistics from 2011 until 2016 show comparable results (fig. 5). More than half of all users of the SIRAnet Query are considered new visitors. The question remains whether the new visitors understand our descriptions or not. Let us observe these questions from an international perspective.

The Regional Archives Maribor recently transferred data from its database to the Archives Portal Europe. Several tens of thousands of records in the Slovenian language became a small drop in an ocean of data containing almost two hundred and fifty million records in the archival portal. After the data were imported into the portal, several questions arose. How can users translate records from Slovenian, and from other languages that are not widely used, into understandable English or German? We assume that technical assistance could be provided using machine-supported translation. This question points to the more general one of whether users understand the content of units of description. Consequently two short surveys were conducted, one at the end of $2014^{18}$ and another at the end of 2016. A search was carried out for information relating to the Austrian capital city. The results show that there are many hits - numbering in the tens of thousands - in the German, French and Hungarian languages. For other languages the results are limited to less than 2.000 hits.

${ }^{18}$ Miroslav NovaK, Arhivski portal Evrope - prilika za arhivski profesionalni razvoj na lokalnom, nacionalnom i globalnom nivou [Archives Portal Europe - Opportunity for Archival Professional Development at Local, National and Global Level]. Arhivski Zapisi. Časopis za arhivsku teoriju i praksu 21/1 (2014) 113-124. 


\begin{tabular}{|l|l|l|}
\hline $\begin{array}{l}\text { Search terms (names for the city of } \\
\text { Vienna in different languages) }\end{array}$ & Number of hits 2014 & Number of hits 2016 \\
\hline Wiedeń (Polish) & 144 & 199 \\
\hline Vídeň (Czech) & 595 & 595 \\
\hline Viedeň (Slovak) & 45 & 45 \\
\hline Bécs (Hungarian) & 21.502 & 21.503 \\
\hline Beč (Croatian or Bosnian) & 1 & 7 \\
\hline Dunaj (Slovenian) & 116 & 209 \\
\hline Wien (German) & 34.355 & 49.731 \\
\hline Vienna (English) & 948 & 1.466 \\
\hline Vienne (French) & 8.007 & 13.943 \\
\hline Viena (Romanian, Spanish, Portuguese) & 344 & 476 \\
\hline Виена (Bulgarian) & 19 & 817 \\
\hline Беч (Serbian) & 0 & 1 \\
\hline SUM & 66.076 & 88.992 \\
\hline
\end{tabular}

Such a search is complicated by the fact that many variants of the name of the city are used in different languages. Some are quite similar to each other, such as the names in Polish (Wiedeń), Czech (Vídeň) and Slovak (Viedeň); those in Hungarian (Bécs), Serbian, Croatian or Bosnian (Вес̌ or Беч); or those used in French (Vienne), English and Italian (Vienna), Romanian, Spanish, Portuguese (Viena) and Bulgarian (Виена); but between such groups the divergences are considerable and the identity is not necessarily recognizable to users. The results of the survey show that in 2014 about $52 \%$ and in 2016 about $56 \%$ of the total hits for all of these search terms were generated using the search term "Wien“. Other forms of the name produced fewer hits, in some cases obviously showing only a very small fraction of relevant records. The correspondence between hits and records is not exact, given that some records may contain more than one of the search terms.

Another problem appears due to the lack of authority records. In Slovenia, the name Beč also indicates a village in the municipality of Cerknica, and it could also be a Slovenian family name. In Croatia this name indicates a village in the municipality of Bosiljevo. Similarly the Slovenian name for Vienna, Dunaj, is identical to the Slovakian name of the river Danube, as well as that of a Polish village near Warsaw. In Slovenia, it too could be used as a family name. The German form Wien is the name of a river in Austria, a town and a community in different parts of the United States of America, as well as a family name ${ }^{19}$. From this point of view it is not possible to say how many hits are truly related to the Austrian capital city of Vienna and how many to other entities with the same name in different European languages.

We can conclude that the Archives Portal Europe is a great achievement in the integration of archival professional work by European archivists. At this level it can serve as

\footnotetext{
19 Ibid.
} 
an excellent tool for searches that indicate the location of original archival material. At the same time, it could also be used as the object of professional research in archival science for identifying and evaluating different methods that are in use and approaches to archival professional work in Europe - especially in the description of various kinds of archival material. From several surveys on descriptions in local or national archival information systems in Europe, it is well known that compliance with the $\operatorname{ISAD}(\mathrm{G})$ standard is in many cases relatively weak. These weaknesses are manifested in different ways, for example in the design of reference codes, in the definitions of levels of description, or in the use of data fields which are not compatible with the $\operatorname{ISAD}(\mathrm{G})$. These results can be surprising, given that the $\operatorname{ISAD}(\mathrm{G})$ has existed for more than 25 years and that it is translated and available in all European languages.

\section{Conclusions}

The European archival community strives to offer trustworthy and user-friendly archival information systems. But there are several questions to be considered. One of them is: What needs to be changed in each national archival strategy or in everyday archival professional work, so that the local or national archival information systems will serve users as a bridge between past and future? Or is it necessary to create more sophisticated and better descriptions of archival material? Part of the answers to these questions can be found in European legislation. The re-use of public information requires minimal synchronization of national rules and practice from archives. What exactly is meant by those requirements is a matter of discussion. The fact remains that according to the regulation, descriptions of archival material are defined as products which can be broadly exchanged, not only on national levels and not only within the public sector.

Archivists should be the decisive voices in governing all processes of archival professional work. These must be carried out according to precise requirements and standards. Whether we consider examples of small mutual archival systems like the Croatian system Arhinet ${ }^{20}$, the Swiss network Archives Online ${ }^{21}$ and the Slovenian SIRAnet, or large unified systems like that of the Romanian National Archives ${ }^{22}$ and National Archives UK's Discovery portal covering over 2.500 archives across the $\mathrm{UK}^{23}$, or the Archives Portal Europe on the international level, all these cases show us the long-term importance of standardization on all levels of description and in all parts of archival information systems. The quality of the product of archival professional work, the long-term stability of the systems, and public faith in the archival community are also all closely connected with standardization. These factors play important roles in achieving good results from the point of view of the users of archival information systems. On the basis of this realization, we can summarise and highlight a few important points:

- Archival information systems have a direct impact on archivists and other personnel in archives. Because of this it is important to understand different relations between information systems and personnel supporting them.

- Necessary changes to archival systems should always be manageable and rational. Spe-

\footnotetext{
${ }^{20}$ Nacionalni arhivski informacijski sustav, http://arhinet.arhiv.hr/default.aspx [1. 3. 2018].

21 Archives Online, http://www.archivesonline.org/Search [1.3. 2018].

22 Arhivele Naționale, http://cautare.arhivelenationale.ro/cautare/ro/search [1. 3. 2018].

23 The National Archives - Discovery, http://discovery.nationalarchives.gov.uk/ [1. 3. 2018].
} 
cial attention should be given to data as the product of interaction between the system and archivists when changing the system.

- Collected and organized data must fulfil many requirements and standards on various levels: technical, linguistic, informational, and others. Archivists should be aware of all the consequences when archival descriptions are not compatible with professional rules.

- If archivists use the same methodological approaches to description as they did using paper-based archival information systems, this can lead to lack of data for creating useful information in current systems. The consequences can include poor computer support for data search and data sorting, limited results of machine-supported translations of content, difficulties in data usage especially for disabled persons, problems in data interoperability and connectivity on the logical and physical level, and others.

- In many cases the following problems are crucial: spelling mistakes, inconsistencies in establishing links between records, use of abbreviations, usage of Roman numerals, logical errors in capturing contents, inappropriate allocation of normative values, and the like. All these problems cause defects in captured data in the archival information system.

For further improvement of the existing archival information systems it is important to establish a secure and stable two-way communication between systems and users and between archivists and users. Here the so called „cross-domain “information retrieval seems to be important, and with it the related answers to users' questions.

The global archival community is trying to address the new needs and the challenges of the modern age in the area of archives management. A special group of archivists (EGAD), supported by the ICA, has prepared a new concept of generating information about archival material. At this point it is important that archivists discuss the new concept. For its development it will be crucial to perform different tests and to observe and evaluate its implementation in everyday work.

The new paradigm of archival descriptions is also addressed to records managers and others involved in the management of documents in the agencies and in other creators of archival material. This could also be perceived as shifting part of the task of description from the archives to the creators. But in order to achieve this goal, archivists need special professional developments, and research conducted by creators. Furthermore, records managers need to be educated and professional solutions ought to be customized. 
DAS ARCHIV IN SEINER UMWELT 



\title{
Das „neue“ Archivdenken und die geisteswissenschaftlichen Grundlagen der Archivwissenschaft
}

\author{
Dietmar Schenk
}

Über das Informationszeitalter und seinen Einfluss auf das Archivwesen wird unentwegt geschrieben und debattiert; dagegen bleibt weithin unbeachtet, dass sich gleichzeitig die Stellung der Archiv wis s e n s ch a ft im Spektrum der Disziplinen wandelt - mit unabsehbaren Folgen für die öffentliche Wahrnehmung der Archive. In den Kultur- und Geisteswissenschaften ist eine Hinwendung zum Thema „Archiv“ zu verzeichnen, deren Auftakt mittlerweile zwei Jahrzehnte zurückliegt. Durch diesen Archival Turn sieht sich die archivarische Berufswissenschaft einem „neuen“ Archivdenken gegenüber; sie ist nicht mehr die alleinige Instanz, die für den Gegenstandsbereich des Archivs und des Archivierens die fachliche Kompetenz reklamiert.

Das schlägt sich auch auf dem Buchmarkt nieder: Jüngst erschien ein „Handbuch Archiv"; es gehört zur Handbuch-Reihe des Metzler-Verlags, die an den Universitäten viel genutzt wird. Die Herausgeber sind nicht Archivare oder Archivwissenschaftler; nur vier von 28 Beiträgen stammen von Facharchivaren ${ }^{1}$. Und der Band entstand nicht etwa an der Archivschule in Marburg an der Lahn, sondern am Deutschen Literaturarchiv in Marbach am Neckar, einer ganz und gar bibliothekarisch geprägten Einrichtung - was man außerhalb der Szene nicht wissen kann. Pointiert ausgedrückt: Der Archival Turn hat dazu geführt, dass Archivarinnen und Archivare bei ihrem ureigensten Thema kaum mehr zu Wort kommen².

Noch vor nicht allzu langer Zeit war in Fachkreisen oft zu hören, dass die Ausarbeitung eines Handbuchs der Archivwissenschaft wünschenswert sei; es war geradezu eine Redensart, hierauf hinzuweisen. Doch kam es nicht zur Behebung dieses Desiderats. Zwar gibt es eine verdienstvolle „Praktische Archivkunde“; eine stärker theoretisch angelegte Darstellung, die archivwissenschaftliche Ansätze mit institutionell untermauerter Autorität ins Feld der Wissenschaft tragen könnte, fehlt jedoch. Die neuerdings am „Archiv“

\footnotetext{
${ }^{1}$ Handbuch Archiv. Geschichte, Aufgaben, Perspektiven, hg. von Marcel Lepper-Ulrich RaulfF-Tanja KunZ-Simone Waidmann-Julia Katharina Waltke (Stuttgart 2016). Von den verantwortlichen Herausgebern ist Ulrich Raulff, Direktor des Marbacher Archivs, Kulturwissenschaftler und Journalist; Marcel Lepper ist Germanist. Von facharchivarischer Seite sind Michael Hollmann, Angelika Menne-Haritz, Andreas Pilger und Hartmut Weber beteiligt.

2 Zum Vergleich: Die Herausgeber des entsprechenden „Handbuchs Bibliothek“ sind Professoren der Bibliotheks- und Informationswissenschaft; vgl. Handbuch Bibliothek. Geschichte, Aufgaben, Perspektiven, hg. von Konrad Umlauf-Stefan Gradmann (Stuttgart-Weimar 2012).

3 Praktische Archivkunde. Ein Leitfaden für Fachangestellte für Medien- und Informationsdienste. Fachrichtung Archiv, hg. von Norbert Reimann-Wolfgang BocKhorst-Hans-Jürgen Höötmann (Münster ${ }^{3} 2014$ ).
} 
interessierten Wissenschaftler dagegen zögern nicht; sie füllen, zumindest äußerlich gesehen, die Lücke aus.

Und wie stehen die Repräsentanten der Archivwissenschaft zu dieser Entwicklung? Die Direktorin der Archivschule Marburg, Irmgard Christa Becker, schrieb in der Zeitschrift Archivar eine Besprechung, in der sie das neue Handbuch fast überschwänglich lobt; in ihrem abschließenden Satz bezeichnet sie es „als Bestandsaufnahme einer Diskussion, an der die Archive im Sinne der Archivgesetzgebung, die in Deutschland die meisten Archive stellt [!], endlich teilnehmen sollten! “4

Diese Empfehlung kommt im Jahr 2016 allerdings ein wenig spät; auf dem Höhepunkt der Debatten ums „Archiv“ ungefähr ein Jahrzehnt früher wäre sie aktuell gewesen. Außerdem stellt sich die Frage, ob nicht gerade die Archivschule Marburg aufgefordert ist, zur Auseinandersetzung mit dem Archival Turn selbst einen Beitrag zu leisten ${ }^{5}$. Müsste sie sich nicht in ihrer Eigenschaft als Hochschule für Archivwissenschaft um die Grundlagen des Faches, die hier berührt sind, und den eher theoretischen Zweig des archivarischen Fachgesprächs in besonderem Maße kümmern? Im Übrigen scheint Becker vorauszusetzen, dass die Fachkollegen bislang angesichts der interdisziplinären Herausforderung ganz und gar geschwiegen hätten, was aber nicht der Fall ist.

Gewiss sind es nicht viele, die sich bislang mit dem „neuen“ Archivdenken auseinandergesetzt haben, doch gibt es immerhin einige. Zu ihnen gehört der Verfasser dieses Aufsatzes. Er absolvierte die Marburger Archivschule und leitet seit 25 Jahren ein „Archiv im Sinne der Archivgesetzgebung“. Den Dialog, zu dem Becker aufruft, führt er seit langem; Ergebnisse liegen vor ${ }^{6}$.

Die nachfolgenden Ausführungen widmen sich der angedeuteten Konstellation deshalb auf der Basis langjähriger Erfahrungen, nicht zuletzt in der archivarischen Praxis. Zunächst wird skizziert, wie der kulturwissenschaftliche Archiv-Diskurs in Deutschland bisher verlaufen ist; dabei wird auch nachvollzogen, wie die Archivwissenschaft auf ihn reagierte - oder eben nicht. Da die Berufswissenschaft der Archivare in ihrem derzeitigen Zustand im Verhältnis zum „neuen“ Archivdenken nicht gut aufgestellt ist, bleibt

${ }^{4}$ Irmgard Christa Becker, [Rezension zu:] Handbuch Archiv. Geschichte, Aufgaben, Perspektiven. Hrsg. Von Marcel Lepper und Ulrich Raulff. J. B. Metzler Verlag, Stuttgart 2016. X, 294 S., Abb., geb. 69,95€. ISBN 978-3-476-02099-4. Archivar. Zeitschrift für Archivwesen 69 (2016) 408f., hier 409.

5 Die wenig ambitionerte Haltung, die Becker zu erkennen gibt, kam auch in ihrer mündlichen Stellungnahme in der Diskussion meines Vortrags am 11. November 2016 in Wien zum Ausdruck. In den Wortmeldungen aus dem Kreis der Zuhörer wurde die Frage aufgeworfen, ob es wünschenswert sei, dass die Archivwissenschaft ein universitäres Standbein besitze. Becker erklärte daraufhin freimütig, dass der Auftrag der Archivschule Marburg nur die Lehre, nicht aber eigene Forschung umgreife; diese sei nur im Rahmen von Drittmittel-Projekten punktuell möglich. Wie jedoch kann Unterricht auf wissenschaftlichem Niveau ohne den Geist der Forschung lebendig und innovativ sein?.

${ }^{6}$ Dietmar Schenk, Kleine Theorie des Archivs (Stuttgart 2008, 22014); DERs., „Aufheben, was nicht vergessen werden darf". Archive vom alten Europa bis zur digitalen Welt (Stuttgart 2013). Darüber hinaus ist gemeinsam mit Rainer Hering, dem Direktor des Landesarchivs Schleswig-Holstein, ein Sammelband entstanden, der auf eine Tagung „Macht und Ohnmacht der Archive. Archivarische Praxis, Archivtheorie und Kulturwissenschaft heute“ 2011 in Berlin zurückgeht: Wie mächtig sind Archive? Perspektiven der Archivwissenschaft, hg. von Rainer Hering-Dietmar Schenk (Veröffentlichungen des Landesarchivs Schleswig-Holstein 104, Hamburg 2013). Vor kurzem ist zudem eine Publikation mit den Resultaten eines von der Deutschen Forschungsgemeinschaft geförderten Projekts erschienen, in dem exemplarisch erprobt wurde, wie eine fruchtbare Zusammenarbeit der Archivwissenschaft mit der Musikwissenschaft unter den Bedingungen des Archival Turn aussehen kann: Archive zur Musikkultur nach 1945. Verzeichnis und Texte, hg. von Antje KalCHER-Dietmar Schenk (Kontinuitäten und Brüche im Musikleben der Nachkriegszeit, München 2016). 
nichts anderes übrig, als einen Blick in die Geschichte des Faches zu werfen; dort lassen sich verschüttete Potentiale ausmachen. Im Zuge einer Rekonstruktion „klassischer“ archivwissenschaftlicher Positionen wird der Gedankengang auf ein Schlüsselproblem hin zugespitzt: das Desiderat einer zeitgemäßen Bestimmung des Archivbegriffs. Die Ausführungen münden in ein Plädoyer für eine Archivwissenschaft, die sich ihres geisteswissenschaftlichen Anteils wieder bewusst wird.

\section{Das „neue“ Archivdenken und das Schweigen der Archivwissenschaft}

Die Formel vom „Archival Turn“ muss man sich nicht unbedingt zu eigen machen; zweifellos handelt es sich um eine oft gedankenlos gebrauchte Floskel des akademischen Jargons. Immerhin markiert sie derzeit stattfindende Veränderungen: Es ist ein deutlich gewachsenes Interesse für Phänomene des Archivs und des Archivierens im gesamten Spektrum der Kultur- und Geisteswissenschaften zu beobachten, das mit der Archivwissenschaft und den ihr traditionell nahestehenden Gebieten der Geschichtswissenschaft ursprünglich nichts zu tun hat, jedoch die herkömmlichen Archive als Gegenstand der Betrachtung nicht außen vor lässt.

Am Beginn stand Jacques Derridas berühmte Londoner Rede von 1994; sie ist beinahe schon historisch geworden ${ }^{7}$. Ausgearbeitet zu einem kleinen Buch mit dem Titel „Mal d'Archive“, auf Englisch: „Archive Fever"8, ging der damals ausgelöste Impuls um die Welt. Es gab eine Phase, in der - anknüpfend an Derrida und auch im Rückgriff auf Michel Foucaults „Archäologie des Wissens“" denken in betonter Abgrenzung vom herkömmlichen Archivbegriff verbreitet war. Diese Welle ist heute wohl allmählich vorbei. Postmoderne und poststrukturalistische Positionen, die mit dem „Archiv“-Diskurs eng verbunden waren, werden an den Universitäten zwar immer noch fortgeschrieben; in der Ära des „Neuen Realismus“ (Markus Gabriel) ${ }^{10}$ haben sie allerdings den Reiz verloren, intellektuell avantgardistisch zu sein. Für die Archivwissenschaft einschlägiger sind jedoch Forschungen, die empirisch besser abgesichert $\operatorname{sind}^{11}$.

Inhaltlich gesehen ist der Gesichtspunkt der „Archivmacht“ betont worden. Sie wirke sich, so die These, bei der „Konstruktion von Wissen“ aus ${ }^{12}$. Die Möglichkeiten, so etwas wie Wahrheiten zu erkennen und unverrückbare Tatsachen zu ermitteln, seien

7 Gehalten am 5. Juni 1994 während einer Konferenz „Memory: The Question of Archives“ im Freud Museum, London. Das Museum führte 2014 eine Jubiläumstagung „20 Years of Archive Fever“ durch.

8 Jacques Derrida, Mal d'archive. Une impression freudienne (Paris 1995); deutsche Übersetzung: DERs., Dem Archiv verschrieben. Eine Freudsche Impression (Berlin 1997).

9 Michel Foucault, L'archéologie du savoir (Paris 1969); deutsche Übersetzung: Ders., Die Archäologie des Wissens (Frankfurt am Main ${ }^{7} 1995$ ).

${ }^{10}$ Gabriels plakative Formel soll an dieser Stelle nicht als These dienen, sie sei lediglich als Symptom erwähnt: vgl. Markus Gabriel, Fünf Jahre Neuer Realismus. Wider die postmoderne Flucht vor den Tatsachen. Neue Zürcher Zeitung (19. 6. 2016). Siehe ganz aktuell auch die Zeitdiagnose von Hans Ulrich Gumbrecht, Wirklichkeitsdämmerung. Rückkehr zum philosophischen Realismus. Neue Zürcher Zeitung (25. 8. 2016).

11 Einen Überblick bietet die Sammelrezension von Annika Wellmann, Theorie der Archive - Archive der Macht. Aktuelle Tendenzen der Archivgeschichte. Neue Politische Literatur. Berichte über das internationale Schriftum 57 (2012) 385-401.

12 Archiv - Macht - Wissen. Organisation und Konstruktion von Wissen und Wirklichkeiten in Archiven, hg. von Anja Horstmann-Vanina Kopp (Frankfurt am Main-New York 2010). Es handelt sich um die Ergebnisse eines von der Deutschen Forschungsgemeinschaft geförderten Projekts an der Universität Bielefeld. 
beschränkt; ja, der Begriff der Wahrheit im herkömmlichen, als naiv hingestellten Verständnis schien obsolet zu sein ${ }^{13}$. Diese Auffassung hat zur kritischen Auseinandersetzung mit der jahrhundertealten Einbindung des Archivwesens in die Sphäre von Staat und Herrschaft angeregt, greift aber letztlich zu kurz: Die Vielfalt der Archive sowohl in der Gegenwart als auch im Verlauf der Geschichte wird nicht genügend erfasst. Bereits im Mittelalter standen Archive nicht nur den Mächtigsten zur Verfügung; sie kontrollierten und sicherten, zum Beispiel in den mittelalterlichen Stadtrepubliken Italiens, den stabilen Zugang zu Information auch für die Bürgerschaft, lange bevor die Geheimarchive der Fürstenstaaten in den Vordergrund rückten ${ }^{14}$. Es ist nicht zufällig, dass sehr alte Archive gerade aus Klöstern und Städten stammen, die des Schutzes ihrer Rechte gegenüber Stärkeren bedurften.

Inzwischen sind die Fragestellungen jedoch vielfältiger geworden. Im Nachgang zu der von Derrida angestoßenen Diskussion hat sich heute insgesamt eine reflektierende Einstellung zu Phänomenen des Archivs und des Archivierens verbreitet. Im Grunde genommen wird mit ihr aber nur eine Entwicklung nachvollzogen, welche die historisch orientierten Disziplinen insgesamt durchlaufen haben: Sie sind in den letzten Jahrzehnten in gewisser Weise theoretischer geworden. Zum einen stützen sie sich auf Hypothesen und Modelle, die oft aus systematisch vorgehenden Wissenschaften wie etwa der Soziologie entlehnt sind; solche heuristischen Vorgaben werden bekanntlich gern als Theorien bezeichnet. Und mit ihrem Gebrauch geht zudem oftmals ein kritisches Nachdenken über die Historie selbst einher. Reinhart Koselleck sprach Anfang der 1970er Jahre in der damaligen Krise der Geschichtswissenschaft von "Theoriebedürftigkeit “"15 und brachte damit ein Desiderat auf den Punkt, dem später tatsächlich Rechnung getragen wurde.

Im weiteren Sinne verstanden, ist das „neue“ Archivdenken mittlerweile sehr heterogen. Was an Gemeinsamkeit nach dem Abklingen der Derrida- und Foucault-Rezeption bleibt, ist ein tendenziell gewachsenes Interesse am „Archiv“, verbunden mit einer erweiterten Bedeutung des Wortes, das oft prononciert als Metapher eingesetzt wird. Kennzeichnend ist, dass die Forschung im Archiv von der Forschung über das Archiv begleitet wird und beides Hand in Hand geht. Im Wort „Archivforschung“ ist diese doppelte Perspektive gefasst ${ }^{16}$. In der Sprache der geschichtswissenschaftlichen Tradition ausgedrückt, rücken damit Aspekte der Historik in den Blick; das heißt, die Grundlagen des menschlichen Umgangs mit der Vergangenheit werden, soweit sie in Bezug auf die Archive von Belang sind, nicht länger übersehen oder als selbstverständlich vorausgesetzt ${ }^{17}$.

${ }^{13}$ Hierzu kritisch Dietmar Schenk, „Archivmacht“ und geschichtliche Wahrheit, in: Wie mächtig sind Archive? (wie Anm. 6) 21-43. Jetzt auch in französischer Übersetzung: DERS., Pouvoir de l'archive et vérité historique. Écrire l'histoire 13/14 (2014) 35-53.

${ }_{14}$ Zusammenfassend und mit Hinweisen auf weitere Literatur: Schenk, Aufheben (wie Anm. 6) 57-64. Mit ähnlichem Tenor befasst sich mit der frühen Neuzeit: Markus Friedrich, Die Geburt des Archivs. Eine Wissensgeschichte (München 2013).

15 Reinhart Koselleck, Über die Theoriebedürftigkeit der Geschichtswissenschaft, in: Theorie der Geschichtswissenschaft und Praxis des Geschichtsunterrichts. Acht Beiträge, hg. von Werner Conze (Stuttgart 1972) 10-28; DERs., Wozu noch Historie? Historische Zeitschrift 212 (1971) 1-18.

16 Arne Klawitter, Archivforschung. Das Archiv als Ausgangspunkt einer philosophischen Kulturarchäologie. Waseda-Blätter 23 (2016) 104-114.

${ }_{17}$ Um ein Eingehen auf die gegenwärtige geschichtstheoretische Diskussion, das notwendigerweise unvollständig und unzulänglich bliebe, zu vermeiden, sei stellvertretend ein älteres, nach wie vor lesenswertes Werk genannt, und zwar das meines früh verstorbenen Lehrers Karl Georg FABER, Theorie der Geschichtswisenschaft (Beck'sche schwarze Reihe 78, München $\left.{ }^{3} 1974\right)$. Siehe den seinem Gedenken gewidmeten Aufsatz: Dietmar 
Als sich der Archival Turn vor langem international abzeichnete, beteiligten sich in Nordamerika auch Archivare an der lebhaft geführten Debatte ${ }^{18}$. Ein hervorstechendes Beispiel sei genannt: Der Archivwissenschaftler Terry Cook in Kanada entwarf ein postmodern inspiriertes Konzept der Archivwissenschaft ${ }^{19}$. Anders in Deutschland: Hier dominierte in der Kulturwissenschaft zunächst eine Lesart, deren Abstand zur Archivpraxis und zur herkömmlichen Archivwissenschaft riesig war ${ }^{20}$. Für Archivare stellte sich dieser Diskussionsstand zweifellos nicht gerade als einladend dar: Die einen verstehen sich von vornherein als bloße Praktiker, so dass ihnen jede Art von Theorie ein wenig suspekt ist. Diejenigen, die sich als Historiker-Archivare begreifen, sind meist durch das Studium der historischen Hilfswissenschaften, der Mediävistik oder der Landesgeschichte geprägt; im Rahmen ihrer Fachgebiete finden sich offenkundig kaum Anknüpfungspunkte, um auf den „neuen“ Archiv-Diskurs produktiv einzugehen.

In der deutschen Archivwissenschaft herrschte deshalb wohl die stillschweigende Annahme vor, dass der Archival Turn und seine Folgen ein angewandtes Fachgebiet wie die archivarische Berufswissenschaft nichts angingen. Diese Einschätzung beruht aber, wie im Übrigen auch noch anhand eines Beispiels aus der archivarischen Praxis gezeigt wird ${ }^{21}$, auf einem Irrtum. Was aber ist angesichts dessen zu tun? Der Archivwissenschaft fehlt es an Aufmerksamkeit für Inhalte, mit denen Brücken zum modernen Archiv-Diskurs geschlagen werden könnten. Sowohl die verwaltungsnahe als auch die informationswissenschaftliche Orientierung des Faches ${ }^{22}$ steht dem „neuen“ Archivdenken fern; beide Richtungen vernachlässigen insgesamt die eher theoretisch angelegten Themen der Archivwissenschaft. Dieser fehlt es deshalb an konzeptioneller Kraft, um mit dem Archival Turn konstruktiv oder auch kontrovers umzugehen.

Seit Jahrzehnten hat man im Rahmen der Archivwissenschaft die jeweils gegebenen konkreten Probleme administrativer, technischer und organisatorischer Art behandelt, auf die Weiterentwicklung des Faches als einer Disziplin, die systematische Fragen aufwirft und sich mit den Geisteswissenschaften berührt, aber verzichtet. So fehlt es an neuen Ideen, ja an einem Blick aufs Ganze mit der Fähigkeit zur begrifflichen Synthese; es entsteht der Eindruck, dass das archivarische Wissen in ein buntes Puzzle archivpraktisch relevanter Einzelheiten zerfällt.

Mangelndes Profil führt jedoch zur Unkenntlichkeit. Wer Wissenschaft und Öffentlichkeit heute beobachtet, gewinnt unweigerlich den Eindruck, in einer verkehrten Welt

Schenk, Über Archivlandschaften, in: Archivlandschaft Rheinland. 49. Rheinischer Archivtag, 18.-19. Juni 2015 in Pulheim-Brauweiler. Beiträge, hg. von Claudia Kauertz (Archivhefte 46, Bonn 2016) 12-25.

18 So in dem umfangreichen Sammelband: Archives, Documentation, and Institutions of Social Memory. Essays from the Sawyer Seminar, hg. von Francis X. Blouin jr.-William G. Rosenberg (Ann Arbor 2006). Die beiden Herausgeber, ein Archivar und ein Historiker, haben später - als Frucht ihrer Begegnung - gemeinsam ein Buch verfasst: Francis X. Blouin jr.-William G. Rosenberg, Processing the Past. Contesting Authority in History and the Archives (Oxford u. a. 2011).

19 Terence Gordon Cook, Fashionable Nonsense or Professional Rebirth: Postmodernism and the Practice of Archives. Archivaria. The Journal of the Association of Canadian Archivists 51 (2001) 14-35.

20 So etwa Cornelia Vismann, Akten. Medientechnik und Recht (Fischer Taschenbuch 14927, Frankfurt am Main 2000); Wolfgang ERnst, Das Rumoren der Archive. Ordnung aus Unordnung (Merve 243, Berlin 2002).

21 Siehe unten im Abschnitt „Echt und einmalig“.

22 Erstere an der Archivschule Marburg, die eine verwaltungsinterne Ausbildung durchführt, Letztere an den archivbezogenen Studiengängen des Fachbereichs Informationswissenschaften der Fachhochschule Potsdam. 
zu leben: Die Archivare schweigen. Dagegen nehmen Medientheoretiker, Kulturwissenschaftler und als Trittbrettfahrer auch Bibliothekare das Wort „Archiv“ in Beschlag - und mit ihm oft genug die Sache selbst ${ }^{23}$. Es ist überdies ungünstig, dass die Wissenschaft, die dem Metier der Archivare zugrunde liegt, zum Teil nicht einmal mehr beim Namen genannt wird, sondern hinter dem Inkognito der Informationswissenschaft verschwindet ${ }^{24}$.

Unter den skizzierten Umständen hat sich in Deutschland eine Arbeitsteilung eingespielt, die das Archivwesen, im Einklang mit derzeitigen Positionen der Archivwissenschaft, auf die bloße Technik des Archivierens zurückdrängt. Daraus droht eine - typisch deutsch anmutende - Spaltung zwischen „Geist“ und „Technik“ zu entstehen. Archivtheoretischer Spekulation steht eine Praxis gegenüber, die zur Gedankenlosigkeit neigt.

Theorie und berufspraktische Orientierung lassen sich aber nicht einfach auseinanderdividieren und in zwei Disziplinen aufteilen. Im akademischen Elfenbeinturm fehlt es an konkreter Kenntnis der Vorgänge des Archivierens und selbst der archivalischen Bestände; eine ahistorische Lehre vom Archivmanagement dagegen lässt wissenschaftlichen Weitblick vermissen und klammert wesentliche Aspekte aus. Schon deshalb ist eine Archivwissenschaft, die den ihr gegebenen Rahmen ausfüllt, unverzichtbar.

\section{Potentiale der „klassischen“ Archivkunde}

Auf das „neue“ Archivdenken wird im Folgenden - über das Wenige bisher Gesagte hinaus - inhaltlich nicht näher eingegangen ${ }^{25}$. Stattdessen soll ausgelotet werden, wie sich die Archivwissenschaft für geisteswissenschaftliche Ansätze wieder öffnen kann. Dem heutigen Stand der archivarischen Berufswissenschaft, die dem Archival Turn ratlos gegenübersteht, werden in einer Rückblende die Potentiale der „klassischen“ Archivwissenschaft zur Seite gestellt.

Der letzte Anlauf, die Archivwissenschaft systematisch zu entwickeln und sogar in der Art eines Handbuchs darzustellen, liegt bereits vierzig Jahre zurück; er ging aus der Archivschule Marburg hervor, die sich damals auf eine große Tradition berief. In der 1976 erschienenen vierbändigen „Archivwissenschaft“ von Johannes Papritz ist gleich auf den ersten Seiten eine Ursprungserzählung zu lesen: Die Marburger Schule sei durch ihren Vorgänger von 1894 ein Ableger des Instituts für Österreichische Geschichtsforschung, und Papritz erinnerte daran, dass dieses unter dem Einfluss der École des Chartes gestanden hatte ${ }^{26}$. Durch die Abfolge Paris, Wien, Marburg war die kleine hessische Universitätsstadt mit der großen Welt der Geschichtswissenschaft verbunden.

Es ist bemerkenswert, dass Papritz auf diese Genealogie Wert legte. Zwar spielten damals die historischen Hilfswissenschaften einschließlich der Diplomatik in der archivari-

${ }^{23}$ Hierfür ein aktuelles Beispiel: Der Berliner Arbeitskreis Information (BAK) kündigt für den 26. 1. 2017 einen Vortrag „Hybrid-Erschließung im Deutschen Schrift- und Buchmuseum. Wie erschließt man Archivgut, Museumsgut und Buchbestände mit RDA in einer Datenbank?“ an. Resource Description and Access (RDA) ist ein bibliothekarisches Regelwerk; das genannte Museum gehört zur Deutschen Nationalbibliothek. Vom Bibliothekswesen gehen immer wieder Bestrebungen aus, archivarische Aufgaben zu integrieren; so sinnvoll das im Einzelfall sein mag, so problematisch ist es, wenn daraus ein Trend wird. Denn es zeigt sich immer wieder, dass es trotz gegenteiliger Beteuerungen an Verständnis für archivische Belange fehlt.

24 So am Fachbereich Informationswissenschaften der Fachhochschule Potsdam.

25 Das ist an anderer Stelle zumindest ansatzweise geschehen: Schenk, Archivmacht (wie Anm. 13).

${ }^{26}$ Hier wurde die zweite Auflage benutzt: Johannes PAPritz, Archivwissenschaft, 4 Bde. (Marburg ${ }^{2} 1983$ ) $14-7$. 
schen Ausbildung noch eine große Rolle, und sie sind ein Bindeglied zu jener großen Tradition. Innerhalb des von ihm gelehrten Faches, der „Archivwissenschaft“, vollzog Papritz jedoch den Bruch mit der Geschichtswissenschaft. Die historischen Hilfswissenschaften verstand er als universitäre Disziplinen; er rechnete sie nicht zur Archivwissenschaft „im engeren Sinne “27, die in seinem voluminösen Werk ausgebreitet wird. Er leugnet, dass dieses Fach, abgesehen von Randgebieten, zur „historischen Wissenschaft“ gehört $^{28}$. So wandte er sich entschieden gegen ein fachliches Dispositiv, in dem die Archivwissenschaft eine geisteswissenschaftliche Dimension besessen hätte. Freilich hält er mit seinem Euvre - übrigens anders als seine Nachfolger - am Anliegen der systematischen Durchdringung des Stoffgebiets fest.

Dennoch zeichnet sich bei ihm die heute dominante Auffassung ab, die in die gegenwärtige Krise geführt hat. Das Verständnis von archivarischer Kompetenz, das zugrunde liegt, sieht im Archivar lediglich einen Experten für das Management von Archivalien, nicht für das Verständnis archivalischer Quellen; im zugehörigen Fachgebiet wird darauf verzichtet, die Grundlagen der Disziplin auf dem jeweiligen Stand der geisteswissenschaftlichen Diskussion zu behandeln. Fritz Wolff - ein Lehrer der Archivwissenschaft an der Archivschule Marburg, auf dessen soliden Unterricht noch der Schein von Papritz' System fiel - attestiert diesem in einem lesenswerten biographischen Porträt, dass er eine Generation tüchtiger Verwaltungsfachleute in die Archive entlassen habe ${ }^{29}$. Doch darf die Kehrseite nicht verschwiegen werden: Die Archivwissenschaft ist um wesentliche Aspekte beschnitten worden. Die Rationalität des archivarischen Handelns erschöpft sich eben nicht im „rationellen Kräfteeinsatz“, so sehr Papritz diesen Gesichtspunkt betonen $\operatorname{mag}^{30}$.

Seine Antipathie gegenüber der Historie, um die Wortwahl Reinhart Kosellecks noch einmal aufzugreifen ${ }^{31}$, ist aufgrund ihrer leicht durchschaubaren Motive fragwürdig: Aspekte der Geschichtlichkeit des menschlichen Tuns und Lassens wurden in der Nachkriegszeit gern ausgeblendet, wobei die Motive außerhalb der Fachwissenschaft lagen. Allzu viele Zeitgenossen besaßen das Bedürfnis, über die NS-Zeit zu schweigen, sei es, dass sie traumatisiert waren und die Rückschau angesichts des Geschehenen unerträglich gewesen wäre, sei es, dass sie - wie Johannes Papritz selbst - belastet waren, so dass man über die Vergangenheit lieber hinwegging ${ }^{32}$. Geschichte und historische Bildung verloren

27 Ebd. 118.

28 Ebd. 132.

29 Fritz Wolff, Archivwissenschaft und Archivpraxis bei Johannes Papritz, in: Archivische Erschließung Methodische Aspekte einer Fachkompetenz. Beiträge des 3. Archivwissenschaftlichen Kolloquiums der Archivschule Marburg, hg. von Angelika Menne-Haritz (Veröffentlichungen der Archivschule Marburg, Institut für Archivwissenschaft 30, Marburg 1999) 11-24. Als Teilnehmer am 24. wissenschaftlichen Lehrgang der Archivschule Marburg habe ich Wolffs Unterricht 1989/90 erhalten und von ihm sehr profitiert.

30 PApritz, Archivwissenschaft (wie Anm. 26) 393.

31 Vgl. Koselleck, Wozu noch Historie (wie Anm. 15).

32 Papritz war einer der Organisatoren der propagandistischen, insbesondere gegen Polen gerichteten „Ostforschung “: vgl. Michael Burleigh, Germany Turns Eastwards. A Study of Ostforschung in the Third Reich (Cambridge-New York 1988); Nils BRÜBACH, Johannes Papritz - eine Archivarsbiographie, in: Archivische Erschließung (wie Anm. 29) 25-38. Das Archivwesen hat die Aufarbeitung der Zeit des „Dritten Reiches“ vor einem Jahrzehnt auf einem Archivtag zum Hauptthema gemacht; vgl. Das deutsche Archivwesen und der Nationalsozialismus. 75. Deutscher Archivtag 2005 in Stuttgart, hg. von Robert Kretzschmar et al. (Tagungsdokumentationen zum Deutschen Archivtag 10, Essen 2007). Die einschlägigen Forschungen gehen weiter; hingewiesen sei auf die Beiträge zum Schwerpunktthema „Archive und der Nationalsozialismus“ in Heft 70/4 (2017) der Zeitschrift Archivar unter der Gastherausgeberschaft von Rainer Hering. Es bleibt das Desiderat, 
insgesamt an Attraktivität, und der deutsche Historismus mit seinem weit verbreiteten Geschichtsoptimismus, einem Erbe des 19. Jahrhunderts, brach zusammen. Mancher Angehörige der älteren Generationen beklagte das als „Verlust der Geschichte“33, ohne die Zeitstimmung verändern zu können. Infolge von Nationalsozialismus, Weltkrieg und Holocaust war das Ende der älteren deutschen Geschichtsauffassung unvermeidlich.

Als sich das Geschichtsinteresse seit den späten 1970er Jahren in breiten Kreisen der Bevölkerung zurückmeldete und der Geschichtswissenschaft deshalb ein Aufschwung beschert war, hatte sich die Archivwissenschaft jedoch von den historisch orientierten Wissenschaften bereits so weit gelöst, dass sie von deren Entwicklung abgekoppelt blieb. In diesen bildeten sich neue Ansätze aus, die den Historismus älterer Prägung ebenso wie den Positivismus hinter sich ließen, ohne dass sich dies auf die Archivwissenschaft auswirkte. Heute fehlen ihr deshalb wichtige Voraussetzungen, um auf den kulturwissenschaftlichen „Archiv“-Diskurs eingehen zu können.

Im Zuge einer historischen Bestandsaufnahme archivwissenschaftlicher Positionen stößt man nun in der Zeit v or 1945 auf einen Ansatz, der auf der Höhe der Geisteswissenschaft der damaligen Zeit stand und in dieser Hinsicht Maßstäbe setzt. Er darf heute nicht mehr übersehen werden. Der Weg zurück führt zu einem Wechsel des Schauplatzes: von Marburg nach Berlin-Dahlem. Dort entstand 1930 in Verbindung mit dem Geheimen Preußischen Staatsarchiv das Institut für Archivwissenschaft und geschichtswissenschaftliche Fortbildung (IfA), das für die Entwicklung der Archivwissenschaft wichtige Anstöße gab ${ }^{34}$. Vorbild war auch hier das Wiener Institut für Österreichische Geschichtsforschung ${ }^{35}$. Mit Berlin-Dahlem ist die - fast vergessene, in den Einzelheiten bis dato nicht einmal bekannte - Konzeption einer historisch orientierten, mit Geschichtstheorie und Philosophie im Dialog stehenden Archivwissenschaft verbunden, gegen die sich Papritz in der Nachkriegszeit so vehement abgrenzte.

Das Dahlemer Institut wurde noch in den Jahren der Weimarer Republik mit Unterstützung des sozialdemokratischen Ministerpräsidenten Preußens, Otto Braun, gegründet. Doch versank es nach der nationalsozialistischen „Machtergreifung“ schnell in den Niederungen des „Dritten Reiches“. Diese Zeitumstände dürfen aber nicht dazu führen, dass die dort verfolgten Ideen übersehen werden. Denn im Umkreis dieses Instituts sind damals aus der archivarischen Praxis heraus zwei Grundsteine für das Gebäude der Archivwissenschaft gelegt worden, die nach wie vor tragfähig sind: Heinrich Otto Meis-

archivwissenschaftliche Positionen der deutschen Nachkriegszeit mit Blick auf ihre vergangenheitspolitische Bedingtheit näher zu beleuchten, wie es in anderen Disziplinen bereits geschehen ist; hierzu vgl. Dietmar ScHENK, Die deutsche Archivwissenschaft im Nationalsozialismus und in der Nachkriegszeit. Anmerkungen zu einer wenig beachteten Problematik. Archivar. Zeitschrift für Archivwesen 70 (2017) 402-411.

33 So der konservative Althistoriker Alfred Heuss, Verlust der Geschichte (Kleine Vandenhoeck-Reihe 82, Göttingen 1959). Geschichte wird hier als ein Gegenstand der Identifikation, nicht der kritischen Auseinandersetzung angesehen.

${ }^{34}$ Neben dem Aufsatz von Pauline Puppel, Die „Heranziehung und Ausbildung des archivalischen Nachwuchses“. Die Ausbildung am Institut für Archivwissenschaft und geschichtswissenschaftliche Fortbildung in Berlin-Dahlem (1930-1945), in: Archivarbeit im und für den Nationalsozialismus. Die preußischen Staatsarchive vor und nach dem Machtwechsel von 1933, hg. von Sven Kriese (Veröffentlichungen aus den Archiven Preußischer Kulturbesitz - Forschungen 12, Berlin 2015) 335-370, immer noch Wolfgang Leesch, Das Institut für Archivwissenschaft und geschichtswissenschaftliche Fortbildung (IfA) in Berlin-Dahlem, in: Brandenburgische Jahrhunderte. Festgabe für Johannes Schultze zum 90. Geburtstag, hg. von Gerd Heinrich-Werner Vogel (Veröffentlichungen des Vereins für Geschichte der Mark Brandenburg 35, Berlin 1971) 219-254.

35 Leesch, Institut (wie Anm. 34) 226-229. 
ners „Aktenkunde“36 und Adolf Brennekes „Archivkunde“. Gerade weil das Gebiet einer Archivalienkunde, die an die Aktenkunde ebenso wie an die Diplomatik anknüpft, in diesem Band mit mehreren Beiträgen vertreten ist, darf ein Wort zur Archivkunde nicht unterbleiben. Sie ist genauso wichtig, und nur zusammengenommen konstituieren beide Fachgebiete jene „Formenkunde“, die im Mittelpunkt der deutschen archivwissenschaftlichen Tradition steht.

Brenneke, der Begründer der Archivkunde, war ein niedersächsischer Archivar, der 1930 ans Geheime Preußische Staatsarchiv in Berlin berufen wurde. Als Lehrer am Dahlemer Institut entwarf er von 1931 an eine anspruchsvolle Theorie und Geschichte der Archive, die er bis zu seinem Tod anderthalb Jahrzehnte lang immer weiter ausbaute. Seine Konzeption ist durch Wolfgang Leeschs posthume, aus einer Vorlesungsnachschrift hervorgegangene Bearbeitung in den Grundzügen bekannt; diese erschien 1953 - übrigens dank Meisners Unterstützung - in der DDR ${ }^{37}$. Leesch gelang es nicht, die Veröffentlichung des Buches im Westen Deutschlands zu erreichen, was auch an Papritz reservierter Haltung lag. Nicht ganz frei von einem anti-intellektuellen Ressentiment, hielt dieser die Konzeption Brennekes für allzu theoretisch, ja „verstiegen“"38. Sein Fehlurteil bedarf der Revision. Schauen wir also näher hin.

Dass der „Brenneke-Leesch“ eine Darstellung aus zweiter Hand ist, die aus quellenkritischer Sicht als unzureichend angesehen werden muss, fiel bislang offenkundig nicht auf. Als das Buch vor mehr als einem halben Jahrhundert erschien, konnte man Leeschs Ausarbeitung als Versuch der Aktualisierung eines nur wenige Jahre zurückliegenden archivwissenschaftlichen Entwurfs lesen und als solchen akzeptieren. Wer sich jedoch heute in Brennekes Gedankengebäude vertiefen möchte, gelangt rasch an den Punkt, an dem er gern einmal originale Texte kennenlernen würde. Es ist im Übrigen ein selbstverständliches Erfordernis wissenschaftlicher Sorgfalt, dass grundlegende Schriften eines Pioniers der Archivwissenschaft in authentischer Form zugänglich gemacht werden, und solche liegen vor.

Als mir vor ungefähr zehn Jahren die unbefriedigende editorische Situation von Brennekes Euvre bewusst wurde, begann ich mit Nachforschungen in archivalischen Quel$\operatorname{len}^{39}$. Erst in ihnen zeigte sich, wie weit seine archivwissenschaftliche Konzeption reicht. In unveröffentlichten Texten wird eine über die Vorlesungen hinausführende Stufe der Ausarbeitung sichtbar; Brenneke entwickelte seine historisch fundierte Theorie der Archive Schritt für Schritt weiter. So entstand eine Archivistik, die der Historik zur Seite

${ }^{36}$ Heinrich Otto Meisner, Aktenkunde. Ein Handbuch für Archivbenutzer mit besonderer Berücksichtigung Brandenburg-Preußens (Berlin 1935).

37 Adolf Brenneke, Archivkunde. Ein Beitrag zur Theorie und Geschichte des europäischen Archivwesens, bearbeitet nach Vorlesungsnachschriften und Nachlaßpapieren und ergänzt, hg. von Wolfgang LEESCH (Leipzig 1953).

38 Johannes Papritz, [Rezension zu:] Adolf Brenneke, Archivkunde. Ein Beitrag zur Theorie und Geschichte des europäischen Archivwesens, bearbeitet nach Vorlesungsnachschriften und Nachlaßpapieren und ergänzt von Wolfgang Leesch. Mit einer Photographie und einem Lebensbild Adolf Brennekes. Leipzig, Koehler \& Amelang 1953. XIX, 542 S. Archivalische Zeitschrift 52 (1956) 237-244, hier 238. Papritz bezeichnet Brennekes Gedanken als „eigenwillige, knorrige [...], sich zeitweilig bis zur Verstiegenheit steigernd“.

39 Der Nachlass befindet sich im Landesarchiv Niedersachsen, Standort Hannover, Hann. 91 Nachlass Adolf Brenneke. Einschlägig sind darüber hinaus folgende Archivalien: Geheimes Staatsarchiv Preußischer Kulturbesitz, Berlin, I. HA Rep. 178 (Generaldirektion der Staatsarchive), insb. Personalakten Adolf Brenneke; LWL-Archivamt für Westfalen (Münster), Nachlass Wolfgang Leesch; Berlin-Brandenburgische Akademie der Wissenschaften, Nachlass Heinrich Otto Meisner. 
steht. Die wichtigsten nachgelassenen Schriften - es sind Artikel zu einem Sachwörterbuch für die deutsche Geschichte, das zeitbedingt nicht zustande kam - sind inzwischen als Buch erschienen ${ }^{40}$; sie ergänzen nun den nach wie vor lesenswerten „BrennekeLeesch".

In aller Kürze zusammengefasst, ergibt sich auf dieser Quellengrundlage folgendes Bild: In der Stimmung der „Krisis des Historismus“ (Ernst Troeltsch) ${ }^{41}$ griff Brenneke seit den 1920er Jahren gedankliche Motive der historischen Geisteswissenschaft seiner Zeit auf, um sie schließlich als Lehrer für „Archivkunde“ zu einer veritablen Synthese zusammenzuführen. Sein archivwissenschaftliches Denken steht ganz im Zeichen des Historismus; vor allem knüpfte er an Johann Gustav Droysens Historik, an Friedrich Meineckes Verständnis des Historismus als Weltanschauung und an die geisteswissenschaftliche Psychologie Eduard Sprangers, eines Schülers von Wilhelm Dilthey, an.

Das waren führende Wissenschaftler, die sich im geistigen Leben ihrer Zeit als Bezugsgröße für eine anspruchsvolle, historisch orientierte Archivwissenschaft damals anboten. Im Jahr 1936 erschien Meineckes große ideengeschichtliche Studie „Die Entstehung des Historismus“, und im Jahr darauf wurde Droysens berühmte, zwischen 1857 und 1882 gehaltene Vorlesung zur „Enzyklopädie und Methodologie der Geschichte“ erstmals in ausführlicher Form zugänglich. Sprangers Schrift „Lebensformen“, auf die sich Brenneke ebenfalls stützte, war seit langem in mehreren Auflagen greifbar ${ }^{42}$. Brenneke eignete sich die bei diesen Autoren ausgebreitete idealistische Geschichtsauffassung an und verknüpfte sie mit archivfachlichen Themen, insbesondere mit seinem auf Aspekte der archivischen Ordnung konzentrierten Bild der Archivgeschichte. Methodisch wirkte sich zudem die komparatistische Vorgehensweise des Verfassungshistorikers Otto Hintze aus, die ihm schon durch die Vermittlung von Heinrich Otto Meisner, einem Hintze-Schüler, bekannt gewesen sein muss; Brenneke nutzte sie für sein Konzept einer allgemeinen, vergleichenden Archivgeschichte.

Die Krise des Historismus äußerte sich nicht zuletzt darin, dass selbst unter Geisteswissenschaftlern der beklemmende Eindruck verbreitet war, historisches Denken führe in letzter Konsequenz zur Relativierung aller Werte und damit zur Orientierungslosigkeit im Lebensalltag $^{43}$ : Da sich alles wandelte, schien es nichts mehr zu geben, woran man sich halten konnte. Von diesem Krisenbewusstsein war Brenneke persönlich wohl gar nicht erfasst. Doch übernahm er den reflexiven Zug im Umgang mit der Historie, der im Zeichen der Krise aufgekommen war, und trug ihn in die Archivwissenschaft hinein. Seine

40 Adolf BrenneKe, Gestalten des Archivs. Nachgelassene Schriften zur Archivwissenschaft, hg. und mit einem Nachwort versehen von Dietmar Schenk (Veröffentlichungen des Landesarchivs Schleswig-Holstein 113, Hamburg 2018), online: dx.doi.org/10.15460/HUP.LASH.113.183 [12. 12. 2018].

${ }^{41}$ Ernst Troeltsch, Die Krisis des Historismus. Die neue Rundschau 33 (1922) 572-590.

42 Friedrich Meinecke, Die Entstehung des Historismus, 2 Bde. (München-Berlin 1936); Johann Gustav Droysen, Historik. Vorlesungen über Enzyklopädie und Methodologie der Geschichte, hg. von Rudolf HüBner (München-Berlin 1937); Eduard SPranger, Lebensformen. Geisteswissenschaftliche Psychologie und Ethik der Persönlichkeit (Halle $\left.{ }^{6} 1927\right)$. Was Droysens Historik angeht, so wird heute meist auf die frühe Fassung der Vorlesung zurückgegriffen, die in einer jüngeren Edition vorliegt: Johann Gustav Droysen, Historik. Historisch-kritische Ausgabe 1: Rekonstruktion der ersten vollständigen Fassung der Vorlesungen (1857). Grundriß der Historik in der ersten handschriftlichen (1857/1858) und in der letzten gedruckten Fassung (1882), hg. von Peter Leyh (Stuttgart-Bad Cannstadt 1977).

43 So bereits Friedrich Nietzsche, Vom Nutzen und Nachteil der Historie für das Leben. Zweite Unzeitgemäße Betrachtung [1874], in: DERs., Sämtliche Werke. Kritische Studienausgabe, hg. von Giorgio ColLIMazzino Montinari (München 1988) 1 243-334. 
Überlegungen zeichnen sich nicht nur durch Gelehrsamkeit und Gedankenreichtum aus; sie sind darüber hinaus beispielhaft für eine geisteswissenschaftlich ausgerichtete Archivwissenschaft.

Brennekes Ansatz besitzt heute eine gewisse Relevanz. Die Thematisierung des „Werkzeugs des Historikers", wie der Lübecker Archivar Ahasver von Brandt das methodische Instrumentarium der Geschichtsforschung bezeichnet hat ${ }^{44}$, stand im 19. Jahrhundert, nicht zuletzt bei Droysen, im Kontext der Historik; noch Ernst Bernheims berühmtes „Lehrbuch", das in weiten Partien einer Erläuterung der historischen Methode gewidmet ist, umfasst umgekehrt auch einen geschichtsphilosophischen Teil ${ }^{45}$. Dass dieser Problemkreis im Wesentlichen auf eine bloße Handwerkslehre reduziert wurde, entspricht dem Stand der Geschichtswissenschaft in der Epoche des Positivismus, in der Geschichte mit methodischer Strenge erforscht, das Tun und Lassen des Historikers als solches aber nicht thematisiert wurde: Den Geist der Forschung sah man als selbstverständlich an.

Diese Zeit ist aber seit langem vorbei, und „Positivismus“ wurde - übrigens nicht ganz zu Recht - ein negativ besetztes Schlagwort. Eine Archivwissenschaft, die dem Stand der Geisteswissenschaft im 21. Jahrhundert entsprechen wollte, kann jedoch der erwähnten „Theoriebedürftigkeit“ der historisch orientierten Disziplinen ${ }^{46}$ nicht mehr ausweichen. Wenn ihr Rechnung getragen würde, käme es zu einer Annäherung an die Geschichtstheorie, die im Dahlemer Institut und insbesondere bei Brenneke bereits in den Blick gerückt war. Über das Verhältnis zwischen Archivistik und Historik müsste gerade heute, natürlich in engster Verbindung mit Aspekten der Quellenkunde und der Methodologie, gründlich nachgedacht werden ${ }^{47}$.

Brennekes Entwurf lebt von Anregungen, die er zum Teil bereits in der Weimarer Zeit in sich aufnahm. Doch fällt seine Ausarbeitung mitten in die NS-Zeit, ja in den Zweiten Weltkrieg. Im Sommer 1943 im Alter von 68 Jahren pensioniert, arbeitete er bis zu seinem Tod wenige Monate nach Kriegsende im Januar 1946 an seiner weitgreifenden Konzeption der Archivwissenschaft. Misstrauisch fragt man sich: Ist diese vom Ungeist der NS-Zeit erfasst worden? Eine genaue Interpretation zeigt aber, dass Brennekes Denken durch und durch historistisch ist; es lässt sich deshalb nicht als nationalsozialistisch qualifizieren ${ }^{48}$. Ein noch in der Kaiserzeit geprägter Archivar, der zwar die Staatsgläubigkeit und das Geschichtsvertrauen der deutschen Historiker-Elite teilte, aber ideologisch betrachtet kein „Nazi“ war, zog damals eine facettenreiche Bilanz der Archivwissenschaft seiner Epoche. In gewisser Weise war Brenneke ein konservativer Anwalt „historischer

\footnotetext{
${ }_{44}$ Ahasver von Brandt, Werkzeug des Historikers. Eine Einführung in die Historischen Hilfswissenschaften (Stuttgart-Berlin-Köln-Mainz ${ }^{18} 2012$ ).

45 Ernst Bernheim, Lehrbuch der Historischen Methode und der Geschichtsphilosophie. Mit Nachweis der wichtigsten Quellen und Hilfsmittel zum Studium der Geschichte (Leipzig ${ }^{5} 1908$ ).

46 Vgl. Koselleck, Theoriebedürftigkeit (wie Anm. 15).

47 Hierzu vgl. Marc Bloch, Apologie pour l'histoire ou Métier d'historien (Cahiers des Annales 3, Paris 1949); deutsche Übersetzung: DeRs., Apologie der Geschichte oder Der Beruf des Historikers, hg. von Lucien Febvre (München 1985). Dieser unvollendete Entwurf einer Historik räumt dem Aspekt der „Kritik“ breiten Raum ein. Heute liegt eine kritische Ausgabe vor: DERs., Apologie pour l'histoire ou metier d'historien. Édition critique, hg. von Étienne Bloch (Paris 1993); deutsche Übersetzung: Apologie der Geschichtswissenschaft oder Der Beruf des Historikers, hg. von Peter Schöttler (Stuttgart ${ }^{2} 2008$ ).

48 Dazu jetzt Dietmar Schenk, Archivwissenschaft im Zeichen des Historismus - ein Nachwort, in: BrenNEKE, Gestalten des Archivs (wie Anm. 40). Zu den Kriterien der Unterscheidung, ja zur „Paradigmenkonkurrenz" zwischen Historismus und Nationalsozialismus vgl. Friedrich JAEGER-Jörn Rüsen, Geschichte des Historismus. Eine Einführung (München 1992) 95-112, hier 97.
} 
Vernunft“ (Jörn Rüsen) ${ }^{49}$; sein Verständnis von Rationalität ist ein ganz anderes, viel weiter gefasstes als dasjenige, das Papritz vertrat.

In den USA ist mit Blick auf die konstitutive Phase der Archivwissenschaft im frühen 20. Jahrhundert vom „Golden Age of Archival Theory“ gesprochen worden ${ }^{50}$. Angesichts der NS-Zeit wird eine solche Formulierung in Deutschland kaum jemandem über die Lippen kommen; der älteren, geisteswissenschaftlichen Tradition des Faches fehlt es, geschichtlich bedingt, an Glanz. Anknüpfungspunkte für eine anspruchsvolle Archivwissenschaft gibt es in der Tradition der deutschen „Archivkunde“ aber dennoch. Sie ist verschüttet, weil sie der Geistlosigkeit des „Dritten Reiches“ und der Geschichtsvergessenheit der Nachkriegszeit zum Opfer fiel.

Die heutige Archivwissenschaft steht vor der Aufgabe, hier wieder anzuknüpfen. Sie muss sich die Einsichten aus der konstitutiven Phase des Faches von neuem aneignen, um sich von den Verengungen, die in den Jahrzehnten nach 1945 eingetreten sind, zu befreien. Wie die - im Vorigen nur skizzierte - historische Rekonstruktion heute fruchtbar gemacht werden kann, soll im Folgenden anhand einer kurzen Erörterung des Archivbegriffs, eines Kernproblems des Faches, angedeutet werden.

\section{Facetten des Archivbegriffs}

Die Konturen dessen, was früher bündig „Archiv“ hieß, verschwimmen heute - nicht zuletzt im Zuge der digitalen Revolution. Das in den letzten Jahren aufgekommene „neue“ Archivdenken knüpft an die zu Tage tretende Offenheit der „archivischen Sphäre“ an, und es trägt seinerseits dazu bei, sprachliche Konventionen aufzulösen.

Die Schlüsselfrage, die sich für die Archivwissenschaft im Informationszeitalter stellt, lautet deshalb - anders als für die Geschichtswissenschaft im Jahr 1970 - nicht etwa „Wozu noch Archive?"51, sondern „Was heißt heute eigentlich ,Archiv'?". Was die Definition des Grundbegriffs der Archivwissenschaft angeht, so müssen schlicht und einfach Grenzlinien gezogen werden. Wenn offen bliebe, was als „Archiv“ anzusprechen ist, verlöre das Fach die Bestimmung seines Gegenstands.

Der metaphorische Gebrauch des Wortes „Archiv“, auf den wir heute stoßen, ist kein Phänomen des 21. Jahrhunderts; er begleitete die Entfaltung der historischen Archive von den Anfängen vor zweihundert Jahren $a^{52}$. Heute ist jedoch eine qualitativ neue Stufe erreicht: Die „Archive“ der digitalen Welt können für junge Leute der primäre Berührungspunkt mit der „archivischen Sphäre" sein; es sind nicht mehr unbedingt die herkömmlichen Papier-Archive mit ihrem Staub und ihren verborgenen Schätzen. Längst prägen die Gegebenheiten des Informationszeitalters die Vorstellungen, die mit dem Wort „Archiv“ assoziiert werden und die es anschaulich machen. Und die erweiterte Bedeutung, die sich im Alltag der digitalen Welt verbreitet hat, ist längst ins Feld der Wissenschaft gewandert; nicht zuletzt aufgrund dieser semantischen Verschiebung hat

49 Jörn RüsEN, Grundzüge einer Historik 1: Historische Vernunft. Die Grundlagen der Geschichtswissenschaft (Göttingen 1983).

${ }^{50}$ Richard J. Cox, Lester J. Cappon and the Relationship of History, Archives, and Scholarship in the Golden Age of Archival Theory. The American Archivist 68 (2005) 74-112.

${ }^{51} \mathrm{Vgl}$. Koselleck, Wozu noch Historie (wie Anm. 15). Es handelt sich um einen Vortrag auf dem Deutschen Historikertag 1970, der auf die damalige Krise der Geschichtswissenschaft eingeht.

52 Vgl. Schenk, Aufheben (wie Anm. 6) 38-52. 
die Archivwissenschaft ihr Monopol bei der Thematisierung der „archivischen Sphäre“ eingebüßt.

Hintergrund und Anlass des nachfolgenden Versuchs, hervorstechende Merkmale von Archiven aus archivwissenschaftlicher Sicht herauszuarbeiten, ist diese Konstellation. Wenn der Gedankengang im Folgenden also auf die Frage nach den charakteristischen Merkmalen des „Archivs der Archivwissenschaft“ zugespitzt wird, so geht es nicht abstrakt um terminologische Klärungen. Vielmehr soll der seiner Bedeutung entleerte Archivbegriff, der heute in Umlauf gekommen ist, überwunden und konkretisiert werden.

\section{Aus Geschäften wird Geschichte. Archivalien als geschäftliche Unterlagen}

Anknüpfend an das Vorige, sei mit Adolf Brennekes Begriffsbestimmung, also einer „klassischen“ Auffassung, begonnen. Auch in der Schlüsselfrage, was genau unter einem „Archiv“ zu verstehen ist, bezog sich der Begründer der „Archivkunde“ auf Johann Gustav Droysen. In Anspielung auf dessen berühmtes Diktum von den „Geschäften“, aus denen „Geschichte“ wird ${ }^{53}$, bestimmte er Archivalien als „Überreste geschäftlicher Willensakte" 54 . Damit legte er eine klar umrissene Definition vor, die am ursprünglichen Zweck der Dokumente orientiert ist. Sie setzt die bis heute gegebene Verbindung zwischen Archiv und vorarchivischem Bereich voraus: Ein Archiv besitzt die Zuständigkeit für bestimmte Einrichtungen, bei denen geschäftliche Unterlagen erwachsen, zusammengestellt und aufbewahrt werden, und es übernimmt diese, sobald sie dort nicht mehr laufend gebraucht werden. Der Inhalt dieser Bestände ist durch das Aufgabengebiet des Bestandsbildners, das heißt durch die Art seiner Geschäfte, vorgezeichnet.

Jede vorarchivische Ablage ist zumindest rudimentär organisiert. Damit Archivgut entstehen kann, muss es so etwas wie eine Geschäftsstelle geben, die ihre Tätigkeit in schriftlicher Form ausübt und Unterlagen in gewissen Grenzen aufbewahrt; eine angegliederte Registratur und, in einem zweiten Schritt, das Archiv nehmen die betreffenden Dokumente zu gegebener Zeit auf. Obwohl die Archive, Brenneke zufolge, auch noch nach Jahrhunderten ihre Bindung an die Sphäre der Geschäfte behalten, in der die Archivalien ursprünglich gebraucht wurden, existiert doch - eben dank Droysen - eine Klammer zwischen Geschäften und Geschichte. So bietet Brenneke eine elegante Lösung an, um die „archivische Sphäre“, die er im schriftlichen Niederschlag der Geschäfte aufgehen lässt, trotzdem mit der geschichtlichen Welt zu verknüpfen.

Ihm kann nicht vorgeworfen werden, dass er die Archive der Staaten und der Kommunen, also der öffentlichen Hand, vorzieht; der „archivischen Sphäre“, wie er sie versteht, ordnet er etwa auch Familien- und Wirtschaftsarchive zu. Soweit es um den Zweck der Archive geht, stand die Betonung des geschäftlichen Charakters des Archivguts aber schon zu Brennekes Lebzeiten im Begriff, anachronistisch zu werden; denn die Rückbindung der Archivalien an ihren Ursprung lockerte sich doch auch schon in der ersten Hälfte des 20. Jahrhunderts mit der Zunahme von Nutzungen durch unterschiedliche Historiker, vom Familienforscher bis zum Universitätsprofessor. Es bildete sich allmählich der Typus des historischen Archivs aus ${ }^{55}$. Dass sich die Belange der Geschichtswis-

53 Droysen, Historik. Vorlesungen (wie Anm. 42) 322: „was heut ein Geschäft ist, gilt, wenn es wichtig genug war, nach einem Menschenalter für ein Stück Geschichte“.

54 Adolf Brenneke, Archiv, in: Ders., Gestalten des Archivs (wie Anm. 40).

55 Vgl. Schenk, Theorie (wie Anm. 6) 11-13. 
senschaft und der Erinnerungskultur auch auf Bewertungsentscheidungen auszuwirken begannen, berücksichtigte Brenneke noch kaum.

Sein Archivbegriff ist dennoch nicht völlig veraltet. Jene administrativen, rechtlichen, amtlichen oder kommerziellen Dokumente, die er als geschäftlich kennzeichnet, sind für die Archive nach wie vor zentral. Angelika Menne-Haritz, Direktorin der Archivschule Marburg in den 1990er Jahren, definierte die Archivwissenschaft demzufolge als „Wissenschaft für die Nutzbarmachung von Unterlagen aus Verwaltungsprozessen [...] durch die Aufbereitung zu Archivgut" ${ }^{46}$; Robert Kretzschmar hielt daran fest, dass hier die „Kernfunktion" der Archive benannt wird"

Mit dieser Aussage wird gar nicht geleugnet, dass ein wesentlicher Zweck der Archive heute darin besteht, Geschichtsquellen bereitzuhalten, denn die fraglichen Unterlagen werden, so Menne-Haritz, „als Evidenz und Information“ nutzbar gemacht. Dem amerikanischen Archivar Theodore Schellenberg zufolge, auf den dieses Begriffspaar zurückgeht ${ }^{58}$, schaffen Archive Evidenz, indem sie Verwaltungshandeln nachweisbar halten und damit Transparenz herstellen, während der informative Wert von Archivalien weit darüber hinausführt. Einstmalige administrative Unterlagen sind unabhängig von ihrem Ursprungszweck in Bezug auf unterschiedlichste Fragestellungen, die an sie herangetragen werden, aussagekräftig; dank dieser Eigenschaft erweisen sie sich als Überreste - ganz im Sinne der historischen Quellenkunde.

Doch werden Menne-Haritz zufolge alle Unterlagen, die nicht aus „Verwaltungsprozessen" stammen, dem Bereich eines bloß ergänzenden Sammelns zugeordnet, der innerhalb des Aufgabengebiets der Archive deutlich an zweiter Stelle steht. Diese Abstufung ist, jedenfalls nach Auffassung mancher heutiger Archivare, nicht ganz befriedigend. Und ein weiterer Punkt kommt verstärkend hinzu: Es gibt Eigenschaften von Archiven, die für diese zwar kennzeichnend, aber nicht spezifisch sind; das lädt dazu ein, das Wort „Archiv“ auf Gegenstände zu übertragen, die charakteristische Attribute ebenfalls besitzen, aber nicht aus der Sphäre der Geschäfte stammen. Hierauf wird im Folgenden eingegangen.

\section{Herkunftszusammenhänge. Archivische Strukturen und die Entwicklungsdynamik des historischen Archivs}

Brenneke befasste sich in seinem archivwissenschaftlichen Entwurf ausgiebig mit einem Gesichtspunkt, der sich bei der Bearbeitung älteren Archivguts aufgedrängt hatte: Er betrachtete die Herkunft der Archivalien und - in Verbindung damit - die "Gestaltung“ der Archive. Das heißt, die Untersuchung galt den Formen der im Laufe der Zeit entstandenen Zusammenstellungen aufgezeichneter und abgelegter Information in Registratur und Archiv. Diese analysierte Brenneke in weiten historischen Linien von der Spätantike bis zu seiner Gegenwart.

56 Angelika Menne-Haritz, Schlüsselbegriffe der Archivterminologie. Lehrmaterialien für das Fach Archivwissenschaft (Veröffentlichungen der Archivschule Marburg, Institut für Archivwissenschaft 20, Marburg 1992) 40 .

57 Robert Kretzschmar, Auf dem Weg in das 21. Jahrhundert: Archivische Bewertung, Records Management, Aktenkunde und Archivwissenschaft. Archivar. Zeitschrift für Archivwesen 63 (2010) 144-150, hier 149.

58 Vgl. Theodore R. Schellenberg, Modern Archives. Principles and Techniques (Melbourne 1956); deutsche Übersetzung: DERs., Akten- und Archivwesen der Gegenwart. Theorie und Praxis, übers. und hg. von Gudrun Banzhaf-Ernst Posner-Georg Winter (Archiv und Wissenschaft 2, München 1961). 
Der Grund für diese Fragerichtung ergab sich aus der archivarischen Praxis: Das Provenienzprinzip verlangt, dass archivalische Bestände entsprechend der Herkunft der Unterlagen gebildet und gegeneinander abgegrenzt werden; auch die innere Ordnung eines Bestandes soll - nach dem Registraturprinzip - so erhalten bleiben, wie sie sich vorarchivisch ausgebildet hat. Das ist zumindest insoweit sinnvoll, als auf diese Weise eine halbwegs gute Auffindbarkeit der einzelnen Dokumente gewährleistet werden kann. Die vorgefundene Struktur der Archivalien ist im Übrigen ein Wert an sich, weil sich an ihr die ursprüngliche Funktion des jeweiligen Schriftguts ablesen lässt: Sie ist ihrerseits eine Spur der Vergangenheit. Angesichts dessen zieht die „Formenkunde“, die das Archivgut in seinem gewachsenen Zusammenhang analysiert, die Aufmerksamkeit der Archivwissenschaft auf sich.

Die „Archivkunde“ Brennekes befasst sich demnach auf subtile Weise mit der Vernetzung der Archivalien untereinander. Papritz „Archivwissenschaft“ folgte ihm in dieser Hinsicht mit eigenen Ideen. Während Brenneke in der Geschichte der Archive unterschiedliche „Archivgestaltungstypen“ identifizierte ${ }^{59}$, spricht Papritz von „Strukturen“ und konzentriert sich, stärker gegenwartsbezogen und an Fragen des Records Management interessiert, auf die „Organisationsformen des Schriftgutes in Kanzlei und Registratur "60. Angesichts des Gewichts der „archivwissenschaftlichen Strukturlehre“ (Robert Kretzschmar) ${ }^{61}$, die beide in jeweils eigenständiger Weise entfalteten, lag es nahe, den Archivbegriff von der Existenz solcher vorarchivisch gegebenen Formen her zu verstehen.

Brenneke war in dieser Hinsicht zurückhaltend; zurecht merkte er an, dass der Erhalt der bereits bestehenden Ordnung, also die Bewahrung des Herkunftszusammenhangs, vor Einführung des Provenienzprinzips in den Archiven gar nicht angestrebt wurde. Demzufolge sind viele ältere Bestände, die in Archiven verwahrt werden, strukturell betrachtet gar keine archivischen Fonds. Dennoch wird der Umstand, dass vorgängige „Formen" im eben beschriebenen Sinne vorhanden sind, später als abgrenzendes Merkmal der Archive herangezogen. So schreibt zum Beispiel Eckhart Franz in seiner weit verbreiteten „Einführung in die Archivkunde“: „Was die Archive von Bibliotheken, Museen und anderen Dokumentationsinstituten abhebt, ist [...] der besondere funktionale Zusammenhang des organisch erwachsenen Archivguts"62.

Die deutsche Archivwissenschaft des 20. Jahrhunderts betont also eine charakteristische Eigenschaft von Archiven: ihre durch die jeweilige Provenienz vorgegebene Form, die auch als „Gestalt“ oder „Struktur“ gefasst wird. In ihr hat sich die ursprüngliche Funktion der Dokumente niedergeschlagen. Dieses Merkmal liegt quer zur Bestimmung des Archivbegriffs, die im vorigen Abschnitt behandelt wurde; es kann auf geschäftliche Unterlagen zutreffen, aber auch auf andere Komplexe von Dokumenten wie etwa Nachlässe, die häufig auch Material nicht-geschäftlicher Art wie etwa Manuskripte und persönliche Korrespondenz

59 Brenneke, Archivgestaltungstypen, in: Ders., Gestalten des Archivs (wie Anm. 40).

60 Papritz, Archivwissenschaft (wie Anm. 26) 1123.

61 Kretzschmar, Auf dem Weg (wie Anm. 57) 147.

${ }^{62}$ Eckhart G. Franz, Einführung in die Archivkunde (Die Geschichtswissenschaft 12, Darmstadt ${ }^{3}$ 1989) 2. Übrigens ist die „Strukturlehre“ auch archivpraktisch von hoher Relevanz. Als Anleitung für das Ordnen und Verzeichnen von Archivalien ist Brennekes Ansatz bis heute gewinnbringend; die Vorgehensweise, die seinem Konzept gemäß ist, unterscheidet sich markant von der Anwendung bibliothekarischer oder dokumentarischer Schemata, die von außen an das Material herangetragen werden. Der genuin archivwissenschaftliche Ansatz ist ihnen, soweit es um die Bearbeitung von Archiven im beschriebenen Sinne geht, überlegen. Hierzu vgl. Dietmar Schenk, Darf das Archivale beim Verzeichnen gelesen werden?, in: Archive zur Musikkultur (wie Anm. 6) $64-70$. 
umfassen. Der strukturale Begriff des Archivs, der sich hier andeutet - er steht in Opposition zu dem der Sammlung -, führt aber bereits in die Unübersichtlichkeit einer durchaus weitläufigen „archivischen Sphäre“. Er kann ohne Weiteres auf bestimmte Komplexe nichtgeschäftlicher Unterlagen angewandt werden und trägt dadurch zu einer Ausweitung des archivarischen Aufgabengebiets bei, die seit dem 19. Jahrhundert tatsächlich eingetreten ist.

Die Entwicklung nahm, sehr knapp resümiert, folgenden Verlauf: Indem die Archive in den Dienst der Geschichtswissenschaft und der Erinnerungskultur traten, verloren die administrativen und rechtlichen Belange des Archivträgers ihr bisheriges Monopol; sie waren nicht mehr der alleinige Zweck des Archivierens. Dieser Wandel, der von der Öffnung der Archive für externe Nutzer begleitet war, wirkte sich mit der Zeit auch auf die Ziele und Wege der Überlieferungsbildung aus; es entstand die paradoxe Situation, dass Archive zu sammeln begannen. Heute geschieht dies zum Teil sogar unter der Vorgabe von Zielen, die in einem Dokumentationsprofil niedergelegt sind. Der Zweck, künftige Geschichtsquellen zu bewahren, beeinflusste fortan den Bestandsaufbau ${ }^{63}$.

Die Vorgänge des Archivierens im engeren Sinne, des Sammelns und des Dokumentierens lassen sich seitdem nicht mehr fein säuberlich voneinander trennen; sie überlagern sich in der beruflichen Tätigkeit des Archivars. Die von Menne-Haritz und auch von Kretzschmar favorisierte Definition des Archivs erodiert. Schon deshalb ist das Wort „Archiv", selbst wie es in archivarischen Fachkreisen benutzt wird, vieldeutig. Ein historisches Archiv, das gemäß eines Dokumentationsprofils arbeitet ${ }^{64}$, ist in gewisser Weise eine Dokumentationsstelle für Geschichte und zugleich eine Sammlung archivalischer Quellen. Die bereits sehr weit gehende Entgrenzung des Archivbegriffs, die hier erkennbar wird, hat nichts mit dem Archival Turn und ebenso wenig mit der digitalen Revolution zu tun.

Eine halbwegs organisierte Ablage und Aufbewahrung begünstigt die ÜberlieferungsChance von Dokumenten ${ }^{65}$; gerade deshalb wird man aber die Sphäre des Alltags und der informellen Kommunikation nicht grundsätzlich aus der Domäne von Archiven entlassen wollen, die sich um die Sicherung der Geschichtsquellen der Zukunft bemühen. Andernfalls würde der Bereich der „formellen“ Schriftlichkeit bevorzugt. Dies vermeiden zu wollen, ist ein weiterer Grund, der dafür spricht, den Begriff des Archivs über die Sphäre der Geschäfte hinaus auszuweiten; Spuren des Alltags wären sonst ausgeklammert. Auch diese sind jedoch - einschließlich der heute massenhaft verbreiteten, digital aufgezeichneten Bilder und Töne - als geschichtliche Zeugnisse wertvoll; sie sollten in geeigneter Auswahl gesammelt und aufbewahrt werden.

Den Archivbegriff auf die gesamte pragmatische Schriftlichkeit zu beziehen - auf jenes Gebiet also, das der Mediävist Hagen Keller für seine Forschungen ins Auge gefasst

$63 \mathrm{Zu}$ dieser langfristig vonstatten gehenden „Entgrenzung“ vgl. ScHENK, Aufheben (wie Anm. 6). In meiner archivgeschichtlichen Studie habe ich die angesprochene Entwicklung vorrangig unter dem Gesichtspunkt der archivarischen Intervention, die sie nach sich zieht, behandelt. Es stellt sich nämlich die Frage nach ihrer Legitimität: Wie können die Eingriffe, die von Archivaren durch Bewertungen und Aktivitäten des Sammelns vorgenommen werden, mit ihrer Neutralitätspflicht vereinbart werden? Dass es aus archivwissenschaftlicher Sicht notwendig ist, das Wort „Archiv“ definitorisch einzuhegen, stand nicht im Vordergrund. Insofern der vorliegende Aufsatz sich mit diesem Problem befasst, führt er die früheren Überlegungen einen Schritt weiter.

${ }^{64}$ Die Befürworter dokumentarischer Ansätze innerhalb der Archivwissenschaft benutzen gern das Argument, dass die Unterlagen der öffentlichen Hand mit Blick auf eine umfassende historische Überlieferungsbildung nicht privilegiert sein dürften. So jüngst Enno STAнL, Über Dokumentationsprofile kultureller Bestände. Das Beispiel Musik, in: Archive zur Musikkultur (wie Anm. 6) 51-56.

65 Arnold Esch, Überlieferungs-Chance und Überlieferungs-Zufall als methodisches Problem des Historikers. HZ 240 (1985) 529-570. 
hat ${ }^{66}$-, bietet sich deshalb an. Dann erstreckt sich die „archivische Sphäre“ allerdings weit bis ins Feld der Mitteilungen und Nachrichten hinein; sie überschneidet sich dann mit jenem großen Bereich, den Brenneke „literarisch“ genannt und ins Aufgabengebiet der Bibliotheken verwiesen hatte ${ }^{67}$. Bereits vor dem Archival Turn ist der Archivbegriff im offenen Terrain der historischen Archive vielschichtig und zum Teil paradox geworden.

\section{Echt und einmalig. Über die Authentizität von Archivalien und Archiven}

Eine Episode aus dem Umkreis meiner eigenen archivarischen Tätigkeit sei angeführt, um die heutigen Veränderungen des Archivbegriffs zu illustrieren, die alles noch komplizierter machen: Am 5. März 2009, zwei Tage nach dem Einsturz des Kölner Stadtarchivs, stand im Berliner Tagesspiegel ein Hintergrundbericht, der eine lange Aufzählung betroffener Bestände enthielt ${ }^{68}$. Eine der Erwähnungen ließ mich stutzen: die des Nachlasses des Medientheoretikers Vilém Flusser. Denn nicht lange zuvor, 2007, war an der Universität der Künste Berlin ein Vilém-Flusser-Archiv eröffnet worden. In derselben Zeitung wurde es übrigens wenig später unter dem Titel „Das Archiv des Medien-Propheten“ ausführlich vorgestellt; dort stand klipp und klar, dass es von Köln nach Berlin umgezogen sei ${ }^{69}$.

Was stimmt denn nun? Die Aussagen widersprechen sich. In der Folgezeit erfuhr ich, dass sich der Nachlass tatsächlich im Kölner Stadtarchiv befand; in Berlin waren, abgesehen von Flussers Reisebibliothek, im Wesentlichen nur Kopien vorhanden. Eine aktuelle Recherche im digitalen Historischen Archiv Köln und im Archivportal-D ergibt, dass der Flusser-Nachlass dort weiterhin verbucht ist ${ }^{70}$. Die in Berlin befindliche Arbeits- und Forschungsstelle wird dennoch als Archiv bezeichnet; dabei bleibt ausgeklammert, dass es eine andere Einrichtung gibt, welche die Funktion des Depots erfüllt. Wenn man nun erfährt, dass vor kurzem ein „Spiegelarchiv“ des Flusser-Archivs in São Paulo, Brasilien, eingerichtet wurde, so wird vollends erkennbar, dass sich der Archivbegriff hier von der Idee des echten und einmaligen Dokuments entfernt hat ${ }^{71}$.

Es entspricht der Alltagserfahrung mit digitalen Medien, dass es angesichts der relativen Unabhängigkeit elektronischer Information von ihrem materiellen Träger und der

${ }^{66}$ Hagen Keller, Die Entwicklung der europäischen Schriftkultur im Spiegel der mittelalterlichen Überlieferung. Beobachtungen und Überlegungen, in: Geschichte und Geschichtsbewußtsein. Festschrift Karl-Ernst Jeismann zum 65. Geburtstag gewidmet von Kollegen und Freunden der Universität Münster, hg. von Paul Leidinger-Dieter Metzler (Warendorf 1990) 171-204; Ders., Vom „heiligen Buch“ zur „Buchführung“. Lebensfunktionen der Schrift im Mittelalter. FMSt 26 (1992) 1-31.

${ }^{67}$ Erst mit dieser Erweiterung des Archivbegriffs rücken übrigens die Literaturarchive in den Horizont des Archivwesens.

${ }^{68}$ Christiane Peitz, Köln: Schatz im Schutt. Nach dem Einsturz des Kölner Stadtarchivs. Warum mittelalterliche Handschriften im Wikipedia-Zeitalter so kostbar sind. Tagesspiegel (5. 3. 2009).

69 Ute Bongartz, UdK Berlin: Das Archiv des Medien-Propheten. Tagesspiegel (31. 7. 2009). Das FlusserArchiv gelangte durch Siegfried Zielinski, jetzt Rektor der Hochschule für Gestaltung Karlsruhe, an die Universität der Künste Berlin.

${ }^{70}$ Bestand 1608. Flusser, Vilém - ca. 1950-1990, in: Das digitale Historische Archiv Köln, http://historischesarchivkoeln.de:8080/actaproweb/archive.xhtml?id=Best++++00041843HupElko\#Best__ 0004184 3HupElko [1. 3. 2018]. Anita Jóri, Mitarbeiterin des Flusser-Archivs, hat mich freundlicherweise mit einer Mail vom 28. 3. 2017 auf meine Anfrage hin darüber informiert, dass sich die Sachlage nicht geändert hat. Sie betont den Wert des in Berlin vorhandenen Materials: die originale Bibliothek, weitere Einzelstücke sowie Videomaterial und Tonaufzeichnungen.

${ }^{71}$ Vgl. Vilém Flusser Archiv, http://www.flusser-archive.org [1. 3. 2018]. 
Leichtigkeit des Abspeicherns und Kopierens nicht selbstverständlich ist, dass in einem konkreten informationellen Kontext ein „Original“ überhaupt zu identifizieren ist; auf den ersten Blick könnte man meinen, dass die Auszeichnung eines authenticum hinfällig geworden ist. Bislang waren das Archivwesen und die Materialität einer Überlieferung mit intrinsischem Wert (intrinsic value ${ }^{72}$ zwei Seiten einer Medaille; eine archivische Institution umfasste demzufolge stets einen Speicher authentischer Dokumente oder Gegenstände als ihren Mittelpunkt. Beim Flusser-Archiv hat sich das Wort „Archiv“ vom normativen Konzept der Authentizität gelöst.

Diese begriffliche Dehnung provoziert Missverständnisse. Problematisch wird es für Archive, die zur Bewahrung des kulturellen Erbes beitragen, wenn eine Kopien-Sammlung mit einem echten Archiv in Konkurrenz tritt und der Unterschied zwischen beidem nicht mehr deutlich ist. Das Kölner Unglück zeigt aber gerade, dass für die Archivierung im elementaren Sinne des bloßen Aufbewahrens - die Lagerung an sicherer, geschützter und bewachter Stelle - gesorgt werden muss, damit die Spuren der Vergangenheit erhalten bleiben. Das gilt im Übrigen auch für digitale Daten, wie jeder weiß, dessen Stick oder Laptop schon einmal einen Defekt hatte und der die darauf befindlichen Dateien versehentlich oder aus Leichtsinn nicht genügend gesichert hatte. Der Sprachgebrauch, mit dem wir heute konfrontiert sind, lenkt von diesem elementaren Tatbestand ab. Dass heutzutage alles Mögliche mit der Autorität und dem Gestus der Wissenschaft in die „archivische Sphäre" rückt, vom Abfall bis hin zur Bibliothek ${ }^{73}$, mag intellektuell bestechend sein, birgt für das Archivwesen aber auch Gefahren. Der für die Archivwissenschaft konstitutive Begriff des Archivs wird dadurch verwässert.

Infolge der digitalen Revolution und des Archival Turn rückt also ein weiteres Merkmal von Archivgut in den Blick. Es ist keineswegs neu, muss jedoch als ein Kennzeichen gerade heute betont werden: Archivalien sind authentische Überreste der Vergangenheit ${ }^{74}$. Diese Aussage lädt freilich ihrerseits zum metaphorischen Gebrauch ein. Denn mit ihr wird eine notwendige, aber keine hinreichende Bedingung dafür formuliert, dass ein bestimmtes Dokument als ein Archivale gelten kann; auch Denkmäler, archäologische Funde und Kunstwerke besitzen die genannte Eigenschaft. Den Begriff der Authentizität zu bedenken, ist eine Aufgabe, die gerade mit Blick auf den medialen und informationstechnischen Wandel unserer Zeit geboten ist $^{75}$.

Die in digitalen Medien übliche, sehr schnelle Veränderbarkeit von Aufzeichnungen ist nämlich nicht immer erwünscht. Menschliche Gemeinschaften sind auf einen verbind-

72 Aus archivarischer Sicht: Angelika Menne-Haritz-Nils BrüBach, Der intrinsische Wert von Archivund Bibliotheksgut. Kriterienkatalog zur bildlichen und textlichen Konversion bei der Bestandserhaltung (Veröffentlichungen der Archivschule Marburg, Institut für Archivwissenschaft 26, Marburg 1997).

${ }^{73} \mathrm{Vgl}$. Aleida Assmann, Erinnerungsräume. Formen und Wandlungen des kulturellen Gedächtnisses (C. H. Beck Kulturwissenschaften, München 1999) 213-217, 343-347; Bibliothek als Archiv, hg. von Hans Erich BöDEKER-Anne SAADA (Veröffentlichungen des Max-Planck-Instituts für Geschichte 221, Göttingen 2007).

74 Vgl. Robert Kretzschmar, Absichtlich erhaltene Überreste. Überlegungen zur quellenkundlichen Analyse von Archivgut. Archivar. Zeitschrift für Archivwesen 67 (2014) 265-269. Das Wort „Original“ ist sehr vieldeutig. In der Marburger Tradition ist es verpönt; ob und inwieweit es aus heutiger Sicht archivwissenschaftlich brauchbar ist, müsste einmal ausgelotet werden.

75 Erste Überlegungen hierzu finden sich etwa bei Andreas Hedwig, Wozu brauchen wir authentische Archivalien? Archive im Dienste der Demokratiesicherung, in: Lebendige Erinnerungskultur für die Zukunft. 77. Deutscher Archivtag in Mannheim, hg. von Heiner Sснміт et al. (Tagungsdokumentationen zum Deutschen Archivtag 12, Fulda 2008) 269-279. 
lichen, zuverlässigen und stabilen „pool of information“ (Bernard Williams) angewiesen, aus dem alle Mitglieder schöpfen können ${ }^{76}$. Die Archive sind ein solcher Informationsspeicher; ihre Aufgabe besteht darin, die enthaltenen Dokumente in ihrer Authentizität und Integrität langfristig zu schützen.

Eine archivwissenschaftliche Theorie der Authentizität ist deshalb ein Desiderat. Sie würde sich mit Aspekten der ,archivischen Sphäre“ befassen, die bislang weitgehend als eine unbefragte Selbstverständlichkeit angesehen und deshalb kaum einer kritischen Betrachtung unterzogen wurden. Heute ist es jedoch notwendig, Basis-Eigenschaften wie die Echtheit von Archivalien auch im Rahmen der Archivwissenschaft näher zu untersuchen. Am Konzept des authentischen Dokuments muss jeder festhalten, dem das materielle Kulturerbe, vor allem aber ein von der regulativen Idee der Wahrheit bestimmter Umgang mit der Vergangenheit am Herzen liegt.

Die Authentizität von Archivalien und Archiven ist eine wesentliche Voraussetzung dafür, dass Tatsachen-Wahrheiten (vérités de fait) ${ }^{77}$ gefunden werden können; man muss sich auf Zeugnisse stützen, um die Möglichkeit zu gewinnen, mit der Vergangenheit in einem Verfahren der Spurensicherung und der Beweiserhebung mit Blick auf Erkenntnis präzise umzugehen. Die Ermittlung von Aussagen darüber, dass bestimmte Sachverhalte mit einem hohen Grad an Wahrscheinlichkeit zutreffend sind, muss sich auf echte Dokumente als Beleg und Nachweis stützen. Die Diplomatik hat sich hartnäckig mit dem notorischen Verdacht der Fälschung auseinandergesetzt, der sich bei einer sehr großen Zahl von mittelalterlichen Urkunden als berechtigt erwiesen hat; seit der Begründung der Disziplin im 17. Jahrhundert hat sie erstaunliche Resultate erzielt. Die Probleme mit fake news, die sich heutigentags aufgetan haben, demonstrieren die Relevanz des Themas.

Der Wert von Archivalien fußt auf der im Alltag verwurzelten, aber auch rational begründbaren Überzeugung, dass es wichtig ist, Tatsachen-Wahrheiten über die Vergangenheit anhand echter Dokumente feststellen zu können; entsprechende Aussagen und Behauptungen müssen dauerhaft überprüfbar gehalten werden. In einem jahrhundertelangen Prozess des Erfahrungsgewinns hat sich aufgrund des Bedürfnisses, derartige Fakten zu kennen, eine Kulturtechnik herausgebildet, die schon vor dem 19. Jahrhundert - dem Zeitalter der Geschichte - mit dem Unterhalt von Archiven und mit der Archivarbeit aufs Engste verknüpft war: die historische Methode oder Quellenkritik.

Die Praxis der Kritik ist auf „originale“ Dokumente angewiesen; sie muss auf Spuren des Vergangenen zurückgreifen können, die aussagekräftig sind - und diese findet man nicht zuletzt in Archiven. Das Wissen um die Leistungen der Archive, die das „historische Material“ (Droysen) ${ }^{78}$ bereit- und in gewisser Weise rein halten, muss angesichts der sogenannten post-faktischen Tendenzen unserer Gegenwart wachgehalten werden.

76 Bernard Williams, Truth and Truthfulness. An Essay in Genealogy (Princeton 2002) 43.

$77 \mathrm{Zu}$ diesem bereits bei Leibniz vorkommenden Begriff vgl. Hannah ArendT, Wahrheit und Politik [1961], in: DIES., Zwischen Vergangenheit und Zukunft. Übungen im politischen Denken I (München-Zürich 1994) 327-370.

78 Droysen, Historik. Vorlesungen (wie Anm. 42) 426. 


\section{Schlussfolgerungen}

Die ganz und gar vorläufigen Ausführungen zum Archivbegriff müssen an dieser Stelle abgebrochen werden. Sie hatten im Rahmen des vorliegenden Aufsatzes lediglich den Zweck, die Richtung einer möglichen Argumentation anzugeben, die den grundlegenden Begriff des Archivs angesichts der heutigen Situation der Zeit aufschlüsselt.

Fassen wir also zusammen: Die digitale Revolution wird von einem fächerübergreifenden wissenschaftlichen Diskurs um das Thema „Archiv“ begleitet und gleichsam orchestriert. Der heutigen Offenheit und Unübersichtlichkeit der „archivischen Sphäre“ entspricht die thematische Weite der Hinwendung zu Phänomenen des Archivs und des Archivierens in den Wissenschaften. Das „neue“ Archivdenken kann den Archivaren aber nicht gleichgültig sein, denn es ist einflussreich und wirkt in die Öffentlichkeit hinein. Zugleich ist das Wort „Archiv“ in Gefahr, durch seine Beliebtheit beliebig zu werden. Angesichts unterschiedlichster Verwendungen fragt man sich: Was heißt heute eigentlich „Archiv“? Die Merkmale und Eigenschaften von Archiven in Verbindung mit den Zwecken des Archivierens aus archivwissenschaftlicher Sicht herauszuarbeiten, ist eine dringliche Aufgabe geworden - selbst dann, wenn man an der einzigen kompakten Definition festhalten wollte, die es gibt, nämlich der Anbindung des Archivs an die Sphäre der Geschäfte.

In archivarischen Fachkreisen werden die eher theoretisch angelegten Aspekte der Archivwissenschaft, die hier berührt sind, kaum diskutiert ${ }^{79}$. Dadurch entfremdet sich das Fach immer mehr von geisteswissenschaftlichen Fragestellungen und Arbeitsweisen, was sich in der archivarischen Praxis ungünstig auswirkt. Der Gedanke, dass Archiv- und Geschichtswissenschaft zusammengehören, ist zwar nicht ganz verloren. Robert Kretzschmar zum Beispiel wies darauf hin, dass sich quellenkundliche, hilfswissenschaftliche und archivwissenschaftliche Inhalte gegenseitig ergänzen und befruchten ${ }^{80}$. Angesichts des Archival Turn ergeben sich allerdings für die Archivwissenschaft neue Herausforderungen, wenn sie der traditionellen Verbundenheit der „archivischen Sphäre“ mit der Historie gerecht werden will. Die Archivwissenschaft muss ein viel breiteres disziplinäres Feld als Adressat in Betracht ziehen als die historischen Grund- oder Hilfswissenschaften.

In diesem weiteren Horizont ist es erforderlich geworden, sehr elementar, aber reflektiert zu erläutern, was unter Archivarbeit zu verstehen ist. In der Konsequenz der vorigen Ausführungen soll abschließend thesenartig zusammengefasst werden, was nottut.

Erstens: Die Archivwissenschaft kann sich in ihrem Verhältnis zu den Kultur- und Geisteswissenschaften nicht mehr darauf beschränken, Historikern darin behilflich zu sein, dass ein quellenkritisch nutzbares methodisches Werkzeug bereitsteht. Das Fach steht vor der Aufgabe, die archivarische Praxis als wichtigen Aspekt des Erinnerns und des Umgangs mit der Vergangenheit im Kontext kultur- und geisteswissenschaftlicher Debatten aus archivarischer Perspektive zu beschreiben und zu begründen.

Zweitens: Um dieser Aufgabenstellung gerecht zu werden, ist es erforderlich, fallen gelassene Stränge in der Tradition der Archivwissenschaft wieder aufzugreifen. Im Zuge einer historischen Rekonstruktion stößt man auf die archivische „Formenkunde“, die nicht nur die Archivalienkunde, sondern auch die „Archivkunde“ umfasst: die Lehre von den

79 Für eine nachdenkliche Bilanz vgl. Robert Kretzschmar, Quo vadis - Archivwissenschaft? Anmerkungen zu einer stagnierenden Diskussion. Archivalische Zeitschrift 93 (2013) 9-32.

80 Vgl. Kretzschmar, Auf dem Weg (wie Anm. 57). 
„Gestaltungen“ und „Strukturen“ der Archive. Dieses Gebiet muss in vollem Umfang, das heißt als Nukleus einer Theorie und Geschichte der Archive, zurückgewonnen werden.

Drittens: Daraus ergibt sich, dass das lange vernachlässigte Gebiet der Archivgeschichte nicht mehr nur das Aschenbrödel der Archivwissenschaft sein kann. Es ist seiner Funktion nach mehr als eine „Subdisziplin“, deren Pflege zwar wünschenswert, aber letztlich entbehrlich ist ${ }^{81}$. In ihrer Verbindung mit Archivtheorie verdient es die Geschichte der Archive, als Problemgeschichte rehabilitiert zu werden. Archivtheorie ohne historische Tiefe bleibt hohl; archivgeschichtlichen Fragestellungen fehlt ohne archivtheoretische Basis der Kompass.

Es steht außer Frage, dass sich die archivarische Berufswissenschaft an der Pragmatik des Archivierens ausrichten muss; doch kommt es zu einer Verengung des Blickfeldes, wenn die vielfältigen Aspekte historisch informierter Reflexion ausgespart bleiben. Nur um den Preis des Bedeutungsverlusts kann die Archivwissenschaft auf eine Berücksichtigung ihres geisteswissenschaftlichen Anteils verzichten; der Erfolg des „neuen“ Archivdenkens weist darauf unmissverständlich hin.

81 Wilfried Reininghaus, Archivgeschichte. Umrisse einer untergründigen Subdisziplin. Archivar. Zeitschrift für Archivwesen 61 (2008) 352-360. Dazu kommentierend: Schenk, Aufheben (wie Anm. 6) 15-18. Reininghaus setzt sich für eine stärkere Berücksichtigung archivgeschichtlicher Fragen innerhalb der Archivwissenschaft ein, was zu begrüßen ist; wie eine Verknüpfung mit dem Gebiet der Archivtheorie heute aussehen könnte, thematisiert er allerdings nicht. 



\title{
Externe Kommunikation und digitale Vermittlung - heraus aus der archivischen „Staubecke“?
}

\author{
Joachim Kemper
}

\begin{abstract}
„Da war noch der schwarze, hagere Archivar Ibrahim Effendi, von dem es hieß, er sei unbestechlich. Ein schweigsamer, zurückhaltender Mann, der sich nur um seine zahlreichen Kinder und um die Korrespondenz und das Archiv des Wesirs kümmerte. Sein Leben war ein Kampf mit ungeschickten, pflichtvergessenen Schreibern [...] und mit den Papieren, die, wie verhext, nie in Ordnung kamen. Den Tag verbrachte er in einem halbdunklen Zimmer voller Schubladen und Regale. Hier herrschte eine nur ihm bekannte Ordnung. Verlangte man von ihm die Abschrift eines Dokuments oder irgendeinen alten Brief, geriet er jedesmal in Aufregung, als geschähe etwas ganz Unerwartetes, Unerhörtes [...]“1.
\end{abstract}

\section{Heraus aus der archivischen „Staubecke“}

Im folgenden Beitrag ${ }^{2}$ wird vielfach vom „Staub“ der Archive die Rede sein, und dies auch noch in vielen Varianten. Selbst in den Titel des Beitrags hat sich, wenn auch in Anführungszeichen sowie mit einem deutlichen Fragezeichen versehen, die „Staubecke“ eingeschlichen. Und wenn es auf den ersten Blick auch wenig originell erscheint: Wenn man als Archivarin oder Archivar die Gelegenheit hat, vor einem sogenannten „Laien“Publikum zu sprechen, dann wird klar, dass die „Archive“ entsprechend konnotiert sind und selbst Literaturnobelpreisträger wie der sicherlich in Archiv- und Registraturfragen nicht unkundige frühere Diplomat Ivo Andrić waren vor solchen Anwandlungen nicht gefeit. Verkürzt gesagt, handelt es sich um ein eigentlich diffuses Bild, gerne auch in Funk, Fernsehen und mittlerweile auch im Netz transportiert - staubige Keller, muffige Aktenablagen der Kriminalpolizei mit schrulligen „Archivaren“, die ohne erkennbaren Sinn in Stehordnern oder Zeitungssammlungen „recherchieren“. Im nachgerade besten Fall wird das Archiv mit geheimnisvollen Gewölben oder alten Papieren und Urkunden in Verbindung gebracht: Das wäre dann die (Fantasy-)Harry-Potter-Variante, um im Bild zu bleiben ${ }^{3}$.

${ }^{1}$ Ivo Andrić, Wesire und Konsuln, übers. von Hans Thurn (Frankfurt am Main-Wien-Zürich 1963) 268.

2 Es handelt sich um die leicht überarbeitete und mit den nötigen Nachweisen versehene Fassung des Vortrags am 11. 11. 2016 (mit Ergänzungen im Frühjahr 2018). Die seinerzeitige Präsentation ist weiterhin abrufbar: Joachim Kemper, Externe Kommunikation und digitale Vermittlung (9. 11. 2016), in: SlideShare, https://de.slideshare.net/JoachimKemper/externe-kommunikation-und-digitale-vermittlung [1. 3. 2018].

3 Zur ersten Variante ein passendes, recht aktuelles Zitat aus der Beschreibung von „Lotte Jäger und das 
„Dem Staub der Jahrhunderte entreißen - Offene Archive!“, so lautete der Titel einer Session auf der 10. re:publica in Berlin im Mai 20164. Die re:publica ist die europaweit größte Konferenz rund um die Themen Digitalität, Soziale Medien und nicht zuletzt Netzpolitik. Gut und gerne 8.000 Besucher kamen dazu nach Berlin. Darunter dürften sich, und dies ist eine eher optimistische Schätzung, vielleicht ein Dutzend Archivarinnen und Archivare befunden haben. Warum war dies so? Eine Erklärung fällt schwer, zumal die kulturellen Themen in Berlin keineswegs zu kurz kamen und digitale Fragen eigentlich zum modernen Handwerkszeug der Archivarinnen und Archivare zählen sollten. Die re:publica ist vom Programm her prinzipiell auch offen für Archive, Bibliotheken und Museen, und dies bei einer ganz erheblichen medialen Verbreitung. Was lag da näher, als einen Archiv-Versuchsballon loszulassen, also eine Session einzureichen? Hierzu fanden sich neben dem Verfasser dieses Beitrags zwei Kolleginnen bereit ${ }^{5}$. Bei näherem Hinsehen zeigte sich sehr schnell, dass es das noch nie gegeben hatte - es gab bisher keinen (!) archivischen Vortrag, keine archivaffine Diskussionsrunde oder andere Präsentation von Archivarinnen und Archivaren bei der re:publica. Und dabei haben gerade die Archive im Kontext der re:publica viel zu sagen, etwa mit Bezug zu zeithistorischen, gesellschaftsoder informationspolitischen Themen; Ähnliches gilt in Bezug zum Beispiel zur digitalen Langzeitarchivierung, zu Fragen von Aktenvernichtungen in Behörden und so weiter die Beispiele ließen sich fortsetzen. Dieser Sachstand wird nicht besser, wenn man auf die Bibliotheken und Museen schaut (ganz zu schweigen, wenn historische oder kulturvermittelnde Themen berücksichtigt werden): Hier wechselten sich die Rednerinnen und Redner auf der re:publica in den vergangenen Jahren regelmäßig ab, und dies teils mit sehr großem Publikumszuspruch.

Die Archiv-Session musste für den re:publica-Kontext kurz und „knackig“ betitelt und beschrieben werden. Eine archivwissenschaftliche Ausarbeitung zu Bewertungsmodellen für die Unterlagen der Sozialgerichtsbarkeit oder der Polizei hätte zwar sicher Eingang in archivfachliche Zeitschriften gefunden, aber bei der re:publica wären die Chancen auf die Annahme doch erheblich gesunken. Mithin war also „Übersetzungsarbeit“ zu leisten, die nicht wenigen der gut und wissenschaftlich ausgebildeten Archivarinnen und Archivare leider oft abzugehen scheint. Die eingereichten Thesen der Sektion sind (genauso wie die Präsentationsfolien) weiterhin im Netz verfügbar ${ }^{6}$. Die Präsentation und die Live-Diskussion vor Ort handelten von der geringen öffentlichen Wahrnehmung der Archive, auch eben im Vergleich zu vielen anderen Kulturguteinrichtungen (damit gemeint sind keineswegs nur die „Flaggschiffe“ unter den Museen, die auch im musealen Kontext kaum erreichbar sind!); es ging um die Ausrichtung nach den Bedürfnissen und

tote Mädchen“ (ZDF 2016): „Lotte Jäger (Silke Bodenbender) war zwölf Jahre bei der Mordkommission. Ihrer Psyche tat das nicht gut. Jetzt versucht sie, es ruhiger angehen zu lassen: als Sonderermittlerin für ungeklärte Fälle. Ihr Kollege Schaake (Sebastian Hülk) ist Akten-Fetischist, hockt im staubigen Archiv-Keller und versorgt Lotte mit den nötigen schmutzigen Informationen aus grauer Vorzeit“. Zitiert nach: Rainer Tiтtelbach, Reihe „Lotte Jäger und das tote Mädchen“. tittelbach.tv. Der Fernsehfilm-Beobachter (8. 1. 2017), http://www.tittelbach.tv/programm/reihe/artikel-4224.html [1.3. 2018].

${ }^{4}$ Elisabeth Steiger-Joachim Kemper-Antje Diener-Staeckling, Dem Staub der Jahrhunderte entreißen: Offene Archive!, in: re:publica. The Most Inspiring Festival for the Digital Society, https://re-publica.com/ de/16/session/dem-staub-jahrhunderte-entreissen-offene-archive [1. 3. 2018].

5 Dr. Antje Diener-Staeckling (LWL-Archivamt Münster) und Elisabeth Steiger M. A. (Universität Köln, EU-Projekt co:op).

${ }^{6}$ re:publica presentation (30. 4. 2016), in: SlideShare, https://de.slideshare.net/OffeneArchive/republicapresentation [1.3.2018]. 
Interessen unserer Nutzerinnen und Nutzer. Schließlich sollten auch die eigentlich beachtlichen digitalen Gehversuche in vielen Bereichen des Archivwesens Erwähnung finden - darunter die digitale Erweiterung vieler Archive um Angebote der Sozialen Medien und Blogs. Hier stand und steht die Konferenzreihe „Offene Archive“ im Hintergrund, die nach Stationen in Speyer (2012), Stuttgart (2014) und Siegen (2015) im Jahr 2017 erneut stattfand - diesmal im Landesarchiv Nordrhein-Westfalen (19. bis 20. Juni) ${ }^{7}$. Der Begriff „digitale Erweiterung“ ist ein eigentlich auch wunderbar auf die Archive anwendbarer Terminus aus dem Feld der Museen: Nicht selten kümmern sich eigens dafür abgestellte Kuratorinnen und Kuratoren um den Ausbau der digitalen Aktivitäten sowie der externen Kommunikation ihres Museums und arbeiten an der Implementierung einer digitalen Strategie; teils werden die Strategiekonzepte auch vorab der Öffentlichkeit zur Diskussion und Weiterentwicklung zur Verfügung gestellt ${ }^{8}$.

Nochmals zurück zur re:publica des Jahres 2016: Die erste archivische „Session“ der Konferenzreihe fand, was durchaus bezeichnend für den Stellenwert der Archive in der Öffentlichkeit zu sein scheint, im relativ kleinen Rahmen statt. Während bei Stargästen wie dem bekannten Blogger Sascha Lobo mehrere Tausend Zuhörer anwesend waren und selbst die Bibliotheksveranstaltung vor mehreren Hundert Gästen stattfand, freuten wir uns über knapp 60 Besucherinnen und Besucher. War die aktive Teilnahme an der re:publica nun mehr ein Erfolg oder eher ein Misserfolg? Die Antwort ist ganz klar: ein Erfolg - auch wenn offensichtlich ist, dass das „Heraus aus der archivischen Staubecke“, so wenig diese Ecke eigentlich staubig ist, beschwerlich ist. Auf dieser grundlegenden und viel beachteten digitalen Konferenz ist für Archive noch viel Luft nach oben, in vielerlei Hinsicht. Im Übrigen hatte die re:publica auch im Jahr 2017 eine Archiv-Sektion im Programm? .

\section{„Für Öffentlichkeitsarbeit habe ich keine Zeit“}

Der Wege aus der „Staubecke“ sind viele. Sehr viele davon können (und: müssen!) digital unterstützt oder vor allem digital gegangen werden. Ein bezeichnendes Beispiel, an das der Verfasser immer wieder denken muss: Am Rande eines regionalen Archivtags im deutschen Südwesten kreiste in einer Pause einmal das Gespräch rund um das Thema Öffentlichkeitsarbeit. Die damaligen Gesprächspartnerinnen und -partner betreuten ihre Einrichtungen ohne weitere personelle Unterstützung - Ein-Personen-Archive sind

\footnotetext{
7 Die Dokumentation aller bisherigen Konferenzen, teils inklusive Videoaufnahmen und Volltexten von Vorträgen: Offene Archive 2.0 bis 2.2. Archive 2.0, https://archive20.hypotheses.org/offene-archive-2-0-bis-2-2 [1. 3. 2018].

${ }^{8}$ Christian Gries, Digitale Strategien für Museen. Iliou Melathron (14. 1. 2016), http://blog.iliou-melathron.de/digsmus/ [1.3. 2018]. Dass im Vergleich dazu im Archivwesen eine digitale Strategie nochmals etwas deutlich anderes darstellt, muss an dieser Stelle nicht weiter ausgeführt werden. Stichworte wären unter anderen die elektronische Archivierung von digitalen Unterlagen, mithin die Integration eines digitalen Magazins in die Gesamtheit der Digitalisierungsaktivitäten, oder die Implementierung eines digitalen Lesesaals beziehungsweise von Tools zur Nutzerführung und Interaktion - also Fragen, die vor kurzem auch im Schwerpunktthema „Virtueller Lesesaal“ in Heft 69/3 (2016) der Zeitschrift Archivar zur Debatte standen.

9 Joachim Kemper, RE:PUBLICA 2017: Archiv-Meetup, 8. Mai. Archive 2.0 (4. 1. 2017), https://archive20.hypotheses.org/3991 [1. 3. 2018]; Elisabeth Steiger-Antje Diener-Staeckling-Joachim Kemper, Im Hinterzimmer der digitalen Revolution. Oder: Warum Archive wie Fallschirme sind (Archiv-Meetup), in: re:publica. The Most Inspiring Festival for the Digital Society, https://re-publica.com/de/session/im-hinterzimmer-digitalen-revolution-oder-warum-archive-fallschirme-sind-archiv-meetup [1.3. 2018].
} 
ja gerade im kommunalen Bereich häufig anzutreffen. Thema der kleinen Runde waren PR-Maßnahmen in allen Schattierungen: also vom analogen Flyer über öffentliche Führungen, Vorträge, eine Homepage, bis hin zur möglichen Nutzung der Sozialen Medien. Eine Aussage, die damals fiel, lautete sehr prägnant: „Für Öffentlichkeitsarbeit habe ich keine Zeit!“ Es gehe darum, das Archiv beim Eintritt in den Ruhestand so fachgerecht erschlossen, verpackt und geordnet zu hinterlassen, dass dieses zur Not auch unbesetzt noch Jahrzehnte überdauern könne. Hier wurde das (an sich fachlich ehrenwerte und korrekte!) Prinzip des säurefreien Kartons in der Compactus-Anlage gegen jegliche Formen der Kommunikation gewendet; und es wurde vergessen, dass auch interne Kommunikation gegenüber dem Archivträger zur Öffentlichkeitsarbeit zählt und unabdingbar für das Überleben eines Archivs ist. Eine weitere Gesprächspartnerin der genannten kleinen Runde sei an dieser Stelle namentlich erwähnt: Andrea Rönz, Leiterin des aufgrund ihrer Initiative digital äußerst aktiven kleinen Stadtarchivs in Linz am Rhein. Das Archiv ist auf gleich mehreren Web-2.0-Kanälen aktiv, was auch für die Planung und Organisation von Ausstellungen und Publikationen gilt. Trotzdem sind, wie der Verfasser aus eigener Anschauung gesehen hat, im Magazin des Archivs auch säurefreie Kartons vorhanden (und eigentlich nur solche) - aber selbst diese sind schon im Blog und Facebook-Auftritt des Stadtarchivs präsentiert worden ${ }^{10}$.

Für den deutschsprachigen Raum könnten an dieser Stelle mittlerweile zahlreiche Archive, darunter auch große staatliche Archive und noch mehr Stadtarchive, aber auch Kirchen- oder Universitätsarchive genannt werden, die in den letzten Jahren im partizipativen Web 2.0 aktiv geworden sind. Auf staatlicher Ebene zählen hierzu auch das Schweizerische Bundesarchiv (ein durchaus herausragendes Beispiel ${ }^{11}$ ) und ebenso das Österreichische Staatsarchiv - dieses ist das vermutlich erste deutschsprachige Archiv mit einer eigenen Facebook-Präsenz gewesen; mittlerweile wird auch intensiv und gut getwittert $^{12}$. Auch das deutsche Bundesarchiv ist seit einigen Monaten in die „Blogosphäre“ eingetaucht beziehungsweise wendet sich neuen Nutzergruppen $\mathrm{zu}^{13}$. Vielfach finden sich umfassendere digitale Ansätze, die neben Web-2.0-Tools wie Facebook und Twitter auch beispielsweise Aspekte von nutzergenerierter Erschließung (Crowdsourcing), virtuelle oder interaktive Präsentationen, Smartphone-Applikationen für die Bildungsarbeit oder ganz generell eine intensive Orientierung an neuen Nutzungs- und Recherchegewohnheiten beinhalten können. Stichworte wären: Online-Beratung, Chats, Imagefilme und Erklärvideos, ebenso wie das leider gar nicht immer so banale WLAN im Lesesaal; auch die Nutzung des Smartphones für das Fotografieren im Lesesaal kann hier dazu gezählt werden ${ }^{14}$. Zweifellos wird vielfach noch experimentiert; nicht selten bleibt ein Auftritt bei Facebook für längere Zeit der einzige Schritt in das „Neuland“ der Sozialen Medien.

${ }_{10}$ Den besten Überblick bietet: Stadtarchiv Linz am Rhein. Archiv \& Stadtgeschichte im Web 2.0, https:// archivlinz.hypotheses.org/ [1.3. 2018].

11 Schweizerisches Bundesarchiv, https://www.bar.admin.ch/bar/de/home.html [1. 3. 2018].

12 Österreichisches Staatsarchiv, in: Facebook, https://www.facebook.com/oesterreichischesstaatsarchiv/ [1. 3. 2018]; Österreichisches Staatsarchiv @Staatsarchiv, in: Twitter, https://twitter.com/Staatsarchiv [1. 3. 2018].

13 Weimar - Wege zur Demokratie. Ein neues Quellenportal des Bundesarchivs entsteht-Machen Sie mit!', https://blogweimar.hypotheses.org/ [1. 3. 2018]; „Eine gelungene Aktion“ - Erster Geocaching-Event im Bundesarchiv. Archive 2.0 (1. 9. 2016), https://archive20.hypotheses.org/3684 [1. 3. 2018].

${ }_{14}$ Ganz abgesehen davon ist mancherorts das bloße Mitführen von Handy, Smartphone \& Co immer noch im Archiv-Lesesaal gänzlich untersagt. 
$\mathrm{Zu}$ konstatieren ist aber grundsätzlich, dass bei den meisten Kolleginnen und Kollegen im Archivbereich mittlerweile das Unverständnis oder gar „Entsetzen“ ausbleibt - von der interessierten Öffentlichkeit ganz zu schweigen. Als an der Sache interessierter Archivar beziehungsweise interessierte Archivarin wird man auch nicht mehr regelmäßig darauf hinweisen müssen, dass Facebook allein mehr Beitragsaufrufe hat als das gesamte Netz zusammen (sieht man von der Google-Suchmaschine ab), was vielleicht allein schon Rechtfertigung genug sein dürfte.

\section{Social Media - Chance oder Gefahr?}

Sehr schön lässt sich die Entwicklung anhand der Deutschen Archivtage in den letzten Jahren ablesen: Erstmals richtig zur Sprache kam das Thema Web 2.0 im Jahr 2009 in Regensburg. Mario Glauert erntete dort, als er Beispiele und Konzepte aus dem angelsächsischen Raum und Westeuropa präsentierte, noch weitgehend Kopfschütteln (zumindest war dies die "gefühlte“ Reaktion, die der Verfasser dieses Beitrags auch im Saal erlebte ${ }^{15}$. Es hat dann einige Zeit und erste Gehversuche in der Praxis gebraucht ${ }^{16}$, ehe immerhin im Rahmen der großen Informationsveranstaltung auf dem Archivtag des Jahres 2012 eines dieser Praxisbeispiele kurz präsentiert werden konnte. Bezeichnenderweise stand die damalige Veranstaltung noch unter der Frage „Social Media: Chance oder Gefahr?" ${ }^{17}$. Wer fünf Jahre später den Deutschen Archivtag in Koblenz besuchte, der wird positiv vermerkt haben, dass die Frage von 2012 klar in Richtung „Chance“ beantwortet wird beziehungsweise dass die Sozialen Medien als nützliche und wichtige Notwendigkeit angesehen werden - auch seitens des archivischen Berufsverbandes VdA. Mehrfach wurde im Rahmen der Sektionen und Vorträge dieses Archivtags auch auf die Beiträge der Konferenzreihe „Offene Archive“ verwiesen, die - wie weiter oben erwähnt - seit dem Jahr 2012 bereits viermal stattfinden konnte ${ }^{18}$. In diesem Zusammenhang fand auch erstmals im deutschsprachigen Raum ein „BarCamp“ für Archive statt ${ }^{19}$. Ohne jegliche Wertung muss man feststellen, dass im Bereich des deutschen Bibliothekswesens im Jahr 2017 bereits das zehnte „BibCamp“ stattfindet ${ }^{20}$; es wird jeweils von unterschiedlichen Hochschulen und deren Studierenden organisiert und ist ziemlich fest institutionell etabliert, was man selbst vom „BarCamp“ der deutschsprachigen Historiker, dem „histocamp“, noch kaum sagen kann ${ }^{21}$. Immer stand im Rahmen von „Offene Archive“ auch der Blick über den Tellerrand, also der europäische Kontext, im Mittelpunkt: Sei es der Nachweis, dass auf

15 Mario Glauert, Archiv 2.0. Vom Aufbruch der Archive zu ihren Nutzern (30. 9. 2009), in: SlideShare, https://de.slideshare.net/MarioGlauert/archiv-20-vom-aufbruch-der-archive-zu-ihren-nutzern [1. 3. 2018].

16 Joachim Kemper-Jörg Fischer-Katharina Hasenfratz-Thomas Just-Jana Moczarski-Andrea Rönz, Archivische Spätzünder? Sechs Web 2.0-Praxisberichte. Archivar. Zeitschrift für Archivwesen 65 (2012) 136-143.

17 Joachim Kemper, Stadtarchiv Speyer@Web.2.0. Aus der Social-Media-Praxis eines Kommunalarchivs. Archive 2.0 (24. 9. 2012), https://archive20.hypotheses.org/225 [1. 3. 2018].

18 Siehe oben Anm. 7.

19 Konferenz 2017 + Archivcamp. Archive 2.0 (22. 6. 2017), https://archive20.hypotheses.org/konferenzarchivcamp-2017 [1. 3. 2018], mit Verweisen zur Videoberichterstattung und zu Berichten über Konferenz und „ArchivCamp“.

20 10. Bibcamp 2017 in Hannover. \#Bib10(11.11.2016), https://bibcamp.wordpress.com/2016/11/11/10bibcamp-2017-in-hannover/ [1.3. 2018].

${ }^{21}$ Histocamp. Das Barcamp für alle, die an und mit Geschichte arbeiten, https://www.histocamp.de/ [1. 3. 2018]. 
Instagram neben Cristiano Ronaldo oder Taylor Swift auch Archive in Dänemark sich ansprechend präsentieren können; seien es die von den Niederlanden und Dänemark ausgehenden, internationalen Twitter-Aktionstage oder Crowdsourcing-Projekte und Chat-Pilotprojekte aus der Schweiz. Konferenz und „ArchivCamp“ 2017 waren übrigens erstmals offizielle Veranstaltungen des neu gegründeten Arbeitskreises „Offene Archive“ im VdA ${ }^{22}$.

Nicht nur im Rahmen der genannten Konferenzreihe sowie bei den vergangenen Deutschen Archivtagen zeigt sich seit einiger Zeit, dass sich das Archivwesen zumindest etwas aus der im Beitrag mehrfach zitierten „Staubecke“ zu bewegen scheint. Dies liegt nicht zuletzt auch daran, dass in den Regionen, bei regionalen Veranstaltungen und Archivtagen die Archive-2.0-Thematik aufgegriffen wurde und wird. Schöne Beispiele hierfür sind die großen Archivtage im Rheinland und in Westfalen, ebenso auch der Südwestdeutsche Archivtag und einige andere regionale Formate in Deutschland; hier finden sich dann auch teils Blogs zu den Archivtagen, die der Vor- und Nachberichterstattung und dem LiveBloggen während der Veranstaltung dienen. Ganz aktiv ist man beispielsweise in Westfalen, besonders beim LWL-Archivamt, wo man zusätzlich auch ein fachbezogenes Blog des Archivamts und ergänzende Web-2.0-Kanäle aufgebaut hat und mit Erfolg betreibt ${ }^{23}$.

Wichtig waren und sind auch Fortbildungen: In Deutschland stehen dafür neben der Archivschule Marburg und der Fachhochschule Potsdam gerade auch die Programme der Archivämter im Rheinland und in Westfalen zur Verfügung. Durchaus lehrreich sind auch die Abschlussarbeiten in Marburg und Potsdam, die sich einzelnen Aspekten und Projekten widmen. Dass Abschlussarbeiten oft in Kooperation mit dem ausbildenden Archiv entstehen, ist für diese Archive dann sicher kein Schaden ${ }^{24}$. Wenn man im Bereich der Fortbildungen allerdings sehenden Auges durch das Netz „spaziert“, dann wird man um die zahlreichen Angebote und Ideen aus dem Umfeld der anderen Gedächtniseinrichtungen und überhaupt des Kulturmarketings kaum herum kommen. Dort erfährt man letztlich auch (zugespitzt formuliert), was die Archive und die Archivarinnen und Archivare in vielleicht ein paar Jahren auch für sich entdecken könnten. Auch Richtlinien und Handreichungen zum archivischen Umgang mit Social Media sind mittlerweile erarbeitet worden ${ }^{25}$. Die Aktualität solcher Vorgaben muss jedenfalls regelmäßig überprüft werden. Unabhängig davon sind allgemeine Social-Media-Guidelines, wie sie ja oft in Verwaltungen geschrieben werden, ein gutes Mittel, um Fehler im Umgang mit dem Medium zu vermeiden ${ }^{26}$.

22 Joachim Kemper, Neuer VdA-Arbeitskreis: Offene Archive. VdA-blog.de (15. 2. 2017), http://www.vdablog.de/blog/2017/02/15/neuer-vda-arbeitskreis-offene-archive/ [1. 3. 2018]. Der Arbeitskreis bereitet derzeit neben anderen Veranstaltungen auch erstmals ein „BarCamp“ im Rahmen des Deutschen Archivtags vor (25.9. 2018, Rostock).

${ }^{23}$ archivamt blog. Neues aus dem Archivwesen in Westfalen-Lippe, https://archivamt.hypotheses.org/ [1. 3. 2018].

${ }^{24}$ Mit Kathrin Baas und Sebastian Tripp seien hier zwei Absolventen der Archivschule Marburg genannt, die am 20. Juni 2017 bei „Offene Archive“ ihre Transferarbeiten vorstellten.

${ }^{25}$ Aktuelle Handreichung der deutschen BKK für den kommunalarchivischen Bereich: Joachim KEMPERUlrich Niess-Andrea RöNZ-Annekatrin SchALler-Susanne SchlössER-Gerold BöNNEN-Horst GeHrIngER, Handreichung zum Umgang mit Sozialen Netzwerken (Social Media) im Bereich der Historischen Bildungsund Öffentlichkeitsarbeit im Archiv, in: BKK. Bundeskonferenz der Kommunalarchive beim Deutschen Städtetag, http://www.bundeskonferenz-kommunalarchive.de/empfehlungen/Handreichnung_social_media_Endfassung.pdf [1. 3. 2018].

${ }^{26}$ Leitlinien des Deutschen Städtetages zur Kommunikation der Städte im Bereich Social Media (München 2014), in: Deutscher Städtetag, http://www.staedtetag.de/imperia/md/content/dst/siteuebergreifend/2014/leitlinien_social_media_2014.pdf [1.3.2018]. 
Einen positiven Weg zurückgelegt hat der Verband deutscher Archivarinnen und Archivare $(\mathrm{VdA})^{27}$ : Dort erarbeitete eine AG nicht nur ein neues Konzept für die Public Relations des Verbandes unter Einbeziehung seiner damals noch in den Anfängen steckenden Social-Media-Kanäle auf Facebook und Twitter. Seitens des VdA wurde außerdem ein eigenes Blog aufgesetzt. Der Dachverband der deutschen Archivarinnen und Archivare hat sich seither deutlich positioniert - dies beginnt, wie schon ausgeführt, bei den Tagungsprogrammen zum Deutschen Archivtag, in denen die Sozialen Medien des VdA und der Twitter-Hashtag des Archivtags alles andere als „,versteckt" werden; und dies endet hoffentlich auch nicht mit der Etablierung eines neuen VdA-Arbeitskreises zum Thema „Offene Archive“ ${ }^{28}$. Auch die größte deutsche Fachzeitschrift Archivar hat sich nun schon mehrmals in den letzten Jahren des Themas angenommen und entsprechenden Beiträgen größeren Raum geboten ${ }^{29}$.

Auch ein Blick zu den anderen Gedächtniseinrichtungen, vor allem zu Museen und Bibliotheken, lohnt sich. Gleichwohl muss konstatiert werden, dass sich Archive damit in einen „Wettstreit" begeben, den sie kaum „gewinnen“ können - schließlich ist ja von teils sehr differierenden (auch: finanziellen) Ausgangssituationen, Zielgruppen, Kernaufgaben und zu verwahrenden beziehungsweise an die Nutzerinnen und Nutzer zu bringenden Objekten auszugehen. Mit den großen „Tankern “im Bereich der Museen und Bibliotheken können und müssen Archive auch nicht konkurrieren; aber: Bibliotheken und $\mathrm{Mu}$ seen haben in aller Regel die Zeichen der Zeit deutlich früher erkannt. Ein Grund hierfür mag übrigens darin liegen, dass dort die öffentliche Wahrnehmung, Besucherzahlen in digitaler und analoger Form, Ausleihen (und was auch immer noch) wesentlich überlebenswichtiger sind als im archivischen Umfeld? Andererseits sind nun einmal die Zeiten vorbei, in denen den Archivarinnen und Archivaren ihre jeweilige Institution mit deren Kernaufgaben genug war - und in denen Fragen beispielsweise nach Benutzerzahlen beziehungsweise Aufgaben wie Ausstellungen, pädagogischen Aktivitäten sowie Bildungsund Öffentlichkeitsarbeit vielfach als Randnotizen galten. Ein Großteil der Öffentlichkeit „verwechselt" zweifellos Archive, Bibliotheken und Museen - was bar jeder Fachkenntnis auch gar nicht so sehr unmöglich erscheint. Diese Schwierigkeiten der Unterscheidung zeigen eigentlich vor allem eines: Eine Absolution von der Notwendigkeit einer prägnanten externen Öffentlichkeitsarbeit können sich Archive eigentlich kaum leisten. Wir Archivarinnen und Archivare sollten dann aber herausstellen, was unsere Einrichtungen so besonders macht. Gleichzeitig kann uns der Blick auf Museen und Bibliotheken weiterbringen; dies gilt übrigens auch für die Aktivitäten mancher Archive in anderen Staaten.

Es folgen abschließend einige Beispiele, die auch für die archivische Arbeit von Interesse sind. Dass bereits in dem einen oder anderen Fall Archivarinnen und Archivare beziehungsweise deren Einrichtungen mitarbeiten - dies macht Hoffnung für die Zukunft.

Zuerst soll ein Hinweis auf „Hackdays“ oder „Hackathons“ erfolgen: Es handelt sich um spartenübergreifende oder auch sehr spezifische Veranstaltungen, die von Kollaboration leben und ihren Schwerpunkt eigentlich im Feld der thematischen Soft- oder Hard-

${ }^{27} \mathrm{VdA}$ - Verband deutscher Archivarinnen und Archivare e. V., https://www.vda.archiv.net/aktuelles.html [1. 3. 2018]. Auch beim schweizerischen Dachverband sind übrigens ähnliche Ansätze, wie hier geschildert, deutlich zu erkennen: Vernetzung, in: VSA-AAS. Verein Schweizerischer Archivarinnen und Archivare, http:// vsa-aas.ch/social-vsa/vernetzung/ [1. 3. 2018].

28 Kemper, Neuer VdA-Arbeitskreis (wie Anm. 22).

29 Zuletzt im Heft 69/3 (wie Anm. 8). Im Heft 71/1 (2018) steht mit dem Thema „Archive im Web 2.0“ das archivische Bloggen im Mittelpunkt. 
wareentwicklung haben. Auch der kulturelle Bereich wird mittlerweile einbezogen, indem von verschiedenen Einrichtungen offene, also frei verwendbare Datensets etwa aus Bibliotheken, Archiven oder Museen zur Verfügung gestellt werden, die dann von Teams aus Informatikern, kulturell Interessierten sowie zum Beispiel Grafikern bearbeitet und weiter entwickelt werden. Der erste Kultur-Hackathon in Deutschland („Coding da Vinci“) fand im Jahr 2014 statt; im Jahr 2015 nahmen an der zweiten Ausgabe von „Coding da Vinci“ immerhin 33 Kulturinstitutionen teil ${ }^{30}$. Vertreten waren große Institutionen wie die Deutsche Nationalbibliothek und das Deutsche Museum, aber auch einige kleinere Museen; dazu kamen lediglich zwei Archive. Die circa 150 „hackenden“ Teilnehmerinnen und Teilnehmer entwickelten etwa 20 Projekte, die schließlich von einer Jury bewertet wurden. Zu den institutionellen Organisatoren von „Coding da Vinci“ zählte neben der Wikimedia Deutschland e. V. unter anderem auch die Deutsche Digitale Bibliothek. Zum Anspruch der Veranstaltung gehört, dass die Kultureinrichtungen ihre Daten nach Möglichkeit freigeben sollen (gemäß dem Anspruch der OpenGLAM-Initiativen ${ }^{31}$ ). Mit „Coding da Vinci“"vergleichbare Veranstaltungen fanden zuletzt zum Beispiel auch in der Schweiz statt ${ }^{32}$; auch im virtuellen Urkundenportal „Monasterium“ wurden in sogenannten „MOMathons“ Urkundendatensätze bearbeitet und verbessert ${ }^{33}$.

Ein Ziel der bereits in diesem Beitrag genannten „BarCamps“ ist, die vorherrschenden Tagungsgewohnheiten und die üblichen „frontalen“ Vorträge „aufzulockern“ beziehungsweise ein partizipativeres Angebot zu schaffen. Insofern wären auch archivische „BarCamps“ zumindest als Ergänzung zu herkömmlichen Konferenzen eine gute Möglichkeit, aus dem vielfach üblichen fachlichen „Elfenbeinturm“ herauszukommen. Rein „informierende" Konferenzen werden in Zeiten des Digitalen an Bedeutung verlieren. Der Zugang $\mathrm{zu}$ Informationen vor, während und nach den Veranstaltungen ist niederschwellig und zugleich vielfältig ${ }^{34}$.

Schließlich folgt noch ein Blick auf weitere, durchaus „klassische“ Veranstaltungsformate, die durch die Nutzung der Sozialen Medien einen erheblichen Mehrwert erhalten können: Das Bildernetzwerk Instagram zählt zu den am rasantesten wachsenden Web2.0-Tools. Bereits im Juni 2016 wurde die Marke von 500 Millionen Nutzern erreicht; täglich werden knapp 60 Millionen Beiträge (Bilder) hochgeladen ${ }^{35}$. Instagram ist eine weitgehend mobile Anwendung. Längst haben auch Kultureinrichtungen (darunter allerdings nur vergleichsweise wenige Archive) die Bedeutung des Netzwerkes erkannt. „Spaziergänge“ oder Führungen, mithilfe der App bebildert, sind mittlerweile nicht un-

30 \{COD1NG DA V1NC1\}. Der Kultur-Hackathon, https://codingdavinci.de/ [1. 3. 2018].

31 OpenGLAM, https://openglam.org/ [1.3. 2018].

32 HackCappelli - Cappelli Hackathon. Philosophische Fakultät-Blog Digitale Lehre und Forschung (12. 8. 2016), http://www.phil.uzh.ch/elearning/blog/blog/2015/09/28/hackappelli-cappelli-hackathon/ [1. 3. 2018]; Kathi Lambrecht, Kultur-Hackathon: Schweizer Kulturerbe, neu programmiert. SRF (2. 3. 2015), https:// www.srf.ch/kultur/netzwelt/kultur-hackathon-schweizer-kulturerbe-neu-programmiert [1. 3. 2018]; Beate Stermann, Swiss Open Cultural Data Hackathon 2017 - Registration is Open. OpenGLAM.ch (22. 5. 2017), https://glam.opendata.ch/swiss-open-cultural-data-hackathon-2017-registration-is-open/ [1. 3. 2018 ].

33 Elisabeth Steiger, Der dritte offizielle MOMathon. Archive 2.0 (5. 12. 2016), https://archive20.hypotheses.org/3903 [1.3.2018].

${ }^{34}$ Hier nur der Hinweis, dass aktuell auch beim Deutschen Museumsbund im Rahmen der Bundesvolontärstagung 2017 erstmals mit diesem Format experimentiert wurde: Bundesvolontärstagung 2017, in: Facebook, https://www.facebook.com/events/667128700128018/ [1. 3. 2018].

35 Art. Instagram, in: Wikipedia. Die freie Enzyklopädie, https://de.wikipedia.org/wiki/Instagram\# Bedeutung [1.3. 2018]. 

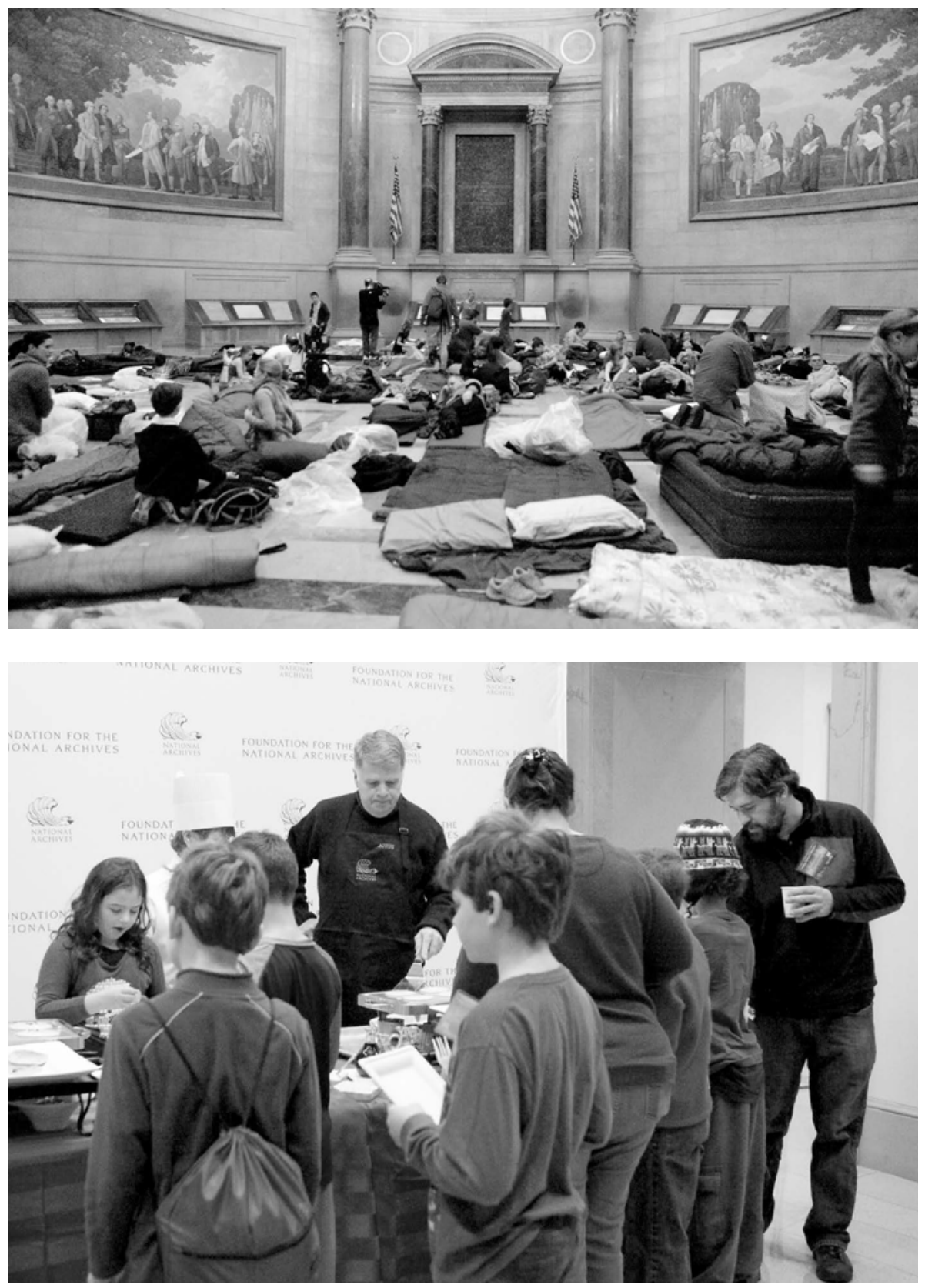

Abb. 1 und 2 (Joachim Kemper)

gewöhnlich. Ganz ähnlich funktionieren „Tweetups“ (wobei dort der Fokus mehr auf den Texten liegt) oder auch umfänglichere Social-Media-Veranstaltungen. Die Einrichtungen laden Blogger, Twitterer und nicht zuletzt Instagrammer ins Haus und gewähren diesen einen vertieften Einblick, auch hinter die Kulissen; im besten Fall befinden sich unter den 
Besuchern auch einige „Influencer“ mit großer Reichweite. Resonanz und Außenwirkung können selbst bei kleineren Veranstaltungen beeindruckend sein ${ }^{36}$. Es handelt sich in allen Fällen um digitale und zugleich analoge Formen der Vermittlung; neben der Führung oder dem Rundgang vor Ort steht man auch im Austausch mit partizipierenden oder auch fragenden „Mit-Lesern“ im Netz. Als Archivar oder Archivarin muss man nun gar nicht so sehr ins Grübeln kommen, welche Formate man denn überhaupt anbieten kann. Vieles liegt auf der Hand oder steht in der Jahresplanung schon fest: vom Tag der Offenen Tür über den deutschlandweiten „Tag der Archive“ beziehungsweise den Internationalen Archivtag bis hin zu Großveranstaltungen wie den Museumsnächten - um einige Beispiele zu nennen.

Aber dies ist nicht das Ende der Fahnenstange. Der Verfasser freut sich, und damit schließt dieser Beitrag, auf das erste deutschsprachige Archiv, das nach dem mittlerweile mehrfachen Vorbild der amerikanischen National Archives Foundation einen archivpädagogischen „Sleepover“ (Abb. 1 und 2) durchführen wird ${ }^{37}$ !

\footnotetext{
36 An dieser Stelle nur der Verweis auf den kleinen aber erfolgreichen Tweetup „Archivperlen“ des Instituts für Stadtgeschichte Frankfurt am Main am 24. 6. 2016: Joachim KEMPER, Rückblick: Tweetup \#Archivperlen (24. Juni 2016). Archive 2.0 (27. 6. 2016), https://archive20.hypotheses.org/3410 [1.3. 2018]. Im Rahmen der Konferenz „Offene Archive“ referierte am 20. 6. 2017 Dr. Christian Gries (Landesstelle für die nichtstaatlichen Museen in Bayern) zu diesem Thema; vgl. Elisabeth STEIGER, „Ich bin ganz gerührt beim Anblick der Goldenen Bulle“ - über aktive digitale Vermittlung bei Social Events in Archiven (Dr. Christian Gries) - Vorschau: Offene Archive 2.3 (1). Archive 2.0 (3. 5. 2017), https://archive20.hypotheses.org/4327 [1. 3. 2018].

37 Sleepover at the National Archives, in: National Archives Foundation, https://www.archivesfoundation. org/sleepover/ [1. 3. 2018]; vgl. Joachim KeMPER, Übernachten im Archiv: History, Heroes \& Treasures. $A r-$ chive 2.0 (4. 2. 2016), https://archive20.hypotheses.org/3012 [1.3. 2018].
} 


\title{
Was ist ein erfolgreiches Archiv?
}

\author{
Mario Glauert
}

Die in der Überschrift formulierte Frage mag zunächst einfach klingen, ist aber offenbar schwierig zu beantworten, denn auch unsere Fachkunde hat bislang keine hinreichende Antwort gefunden. Dies ist umso erstaunlicher, als die Frage oder vielmehr die Antwort darauf naturgemäß unsere berufliche Einstellung und Überzeugung prägt. Was wir für erfolgversprechend halten, leitet uns bei unserer täglichen Arbeit im Archiv, direkt oder indirekt, bewusst oder unbewusst, definiert unsere Wertmaßstäbe und Ziele, und ist die Basis, auf der wir Visionen, Leitbilder, Strategien oder Konzeptionen entwickeln. Es ist eine fachliche, ethische und vielleicht auch politische Grundlage unseres archivarischen Handelns.

Was ist ein erfolgreiches Archiv? Zugegeben, die Frage wäre schon dann schwierig zu beantworten, wenn man das Adjektiv weglässt: Was ist ein Archiv? Auch wenn man die Vielfalt der Begriffsbedeutungen im weiteren Sinne außer Acht lässt, fällt es nicht leicht, einheitliche Maßstäbe für die Ergebnisse und Leistungen der Archive aller Sparten, Typen und Formen zu formulieren. Selbst die Archive einer Sparte setzen sehr unterschiedliche Schwerpunkte. Kein Stadtarchiv ist wie das andere, und interkommunale Vergleichszirkel, wie sie in den 1990er Jahren in Deutschland eingerichtet wurden, erwiesen eher die Unterschiede und Vielfalt individueller Ausprägungen als allgemeingültige Benchmarks ${ }^{1}$.

Es gibt nicht $\mathrm{d}$ a s Archiv. Und dennoch würden wohl die meisten Archivarinnen und Archivare behaupten, es gäbe einen Grundkonsens gemeinsamer Wertvorstellungen und Ziele. Der vor zwanzig Jahren 1996 vom ICA verabschiedete „Code of Ethics“2 vereint zahlreiche solcher „Grundsätze“: Archivare sollen etwa die Integrität, den Kontext, die Provenienz und die Authentizität des Archivgutes bewahren, sollen ihre Arbeit transparent dokumentieren, sich für eine weitest mögliche Benutzung der Archivalien einsetzen, Zugänglichkeit und Datenschutz gleichermaßen gewährleisten, sich regelmäßig fortbilden und spartenübergreifend zusammenarbeiten.

In Deutschland regeln die Archivgesetze des Bundes und der Länder die Aufgaben der öffentlichen Archive. Aber wie die ethischen Grundsätze des ICA geben auch sie keine Maßstäbe vor, keine Kennzeichen, Kriterien oder Kennzahlen, um den Erfolg eines Archivs bei der Umsetzung dieser Aufgaben zu messen oder zu beurteilen.

1 Wirkungsvolle Strukturen im Kulturbereich. Zwischenbericht zum Städtevergleich der Stadtarchive der Städte Bielefeld, Dortmund, Mannheim, Münster und Wuppertal, hg. von Marga PröHL (Gütersloh 1995) 9. Außerdem vgl. Katharina Tiemann, Der Städtevergleich der Stadtarchive - Über die Zukunft der Stadtarchive in Zeiten der Erprobung neuer Steuerungsmodelle im Kulturbereich. Archivpflege in Westfalen und Lippe 42 (1995) 17-19.

2 Text in verschiedenen Sprachen unter: ICA Code of Ethics, in: ICA. International Council on Archives, http://www.ica.org/en/ica-code-ethics [1.3. 2018]. 
Warum ist das wichtig? Dreht man die Frage einmal um, wird die Bedeutung der Antwort offensichtlich: Woran wollen wir selbst, woran soll unsere tägliche Arbeit im Archiv gemessen und beurteilt werden? Oder konkreter gefragt: Welche Erfolge sollte unser Träger, unsere vorgesetzte Stelle belohnen?

Klare Vorstellungen und Maßstäbe sind also nicht nur für unsere eigene allmorgendliche Entscheidung wichtig, welchen der vielen Aufgabenberge wir vorrangig priorisieren und managen, kurz: anpacken wollen. Sie bestimmen unser Selbstverständnis und damit auch unser Selbstbewusstsein im Auftreten und in der Kommunikation gegenüber dem Träger und natürlich auch gegenüber unseren Kunden, Nutzern, Anspruchsgruppen oder Stakeholdern allgemein.

Die meisten Archivträger wüssten auf die Frage, was für sie ein erfolgreiches Archiv ist, wohl auch keine hinlängliche Antwort. Selbst wenn Zielvereinbarungen zwischen Archiven und ihren Trägern beziehungsweise vorgesetzten Stellen weithin üblicher werden, sind hinreichend definierte und verabredete Aufgaben, Aufträge und Ziele, die ein Controlling von Leistungen, die Darstellung von Erfolgen und auch die Begründung von Forderungen ermöglichen würden, noch eher selten. Mit schwierigen Folgen: Wenn die Erwartungen unklar sind, sind es auch die Aufgaben und Ziele, und damit auch die Kosten oder der Personalbedarf. Besonders fatal wird dies, wenn die bestehenden Anforderungen, neue Aufgaben oder aufgelaufene Rückstände zusätzlichen Bedarf für Haushaltsmittel, Personal oder Magazinflächen begründen sollen ${ }^{3}$.

Und Bedarf, Rückstände und neue Aufgaben gibt es in allen Archiven genug. Die Fähigkeit zur Priorisierung, eine archivfachliche Kernkompetenz, ist täglich gefordert. Sie wird im 21. Jahrhundert besonders in den Aufgabenbereichen Erschließung, Digitalisierung und Erhaltung von Archivgut geradezu zu einer Schlüsselfrage erfolgreicher archivischer Arbeit werden und zugleich zur Nagelprobe überzeugender Kommunikation mit Unterhaltsträgern und Geldgebern.

\section{Eine Frage der Perspektive}

Der vorliegende Beitrag soll sich mithin der schwierigen Antwort auf die einfache, aber wichtige Frage annähern, was die Kennzeichen, Kriterien oder Kennzahlen erfolgreicher archivischer Arbeit sein könnten. Trotz eines gewissen fachlichen, ethischen oder juristischen Konsenses über die grundlegenden Aufgaben von Archiven, welcher Art sie auch immer sind, ist die Antwort natürlich abhängig von der Perspektive des Betrachters. Für die Unterhaltsträger eines Archivs stehen zumeist wirtschaftliche und KostenAspekte im Vordergrund, und vielleicht erwarten sie auch bilderreiche Ausstellungen, Events, Schülerarbeit und Jubiläumsbände (ohne Fußnoten), vielleicht auch spektakuläre Funde - die allgemeine archivische Kärrnerarbeit ist dagegen sicher weniger ansprechend und gewollt.

Welche Vorstellungen die unterschiedlichen Nutzer-, Kunden- oder Anspruchsgruppen der Archive vom Unterhaltsträger und den anbietungspflichtigen Stellen über die verschiedenen Benutzerkreise bis hin zu den unterschiedlichen Mitarbeitern des Archivs

\footnotetext{
3 Meinhard Мотzко, Standardisierung und Zertifizierung von Aufgaben und Leistungen in Archiven, in: Archivmanagement in der Praxis, hg. von Mario GLauert-Hartwig Walberg (Veröffentlichungen der Landesfachstelle für Archive und öffentliche Bibliotheken im Brandenburgischen Landeshauptarchiv 9, Potsdam 2011) 57-67, hier 58.
} 
oder seinen Kooperationspartnern haben, ist bislang noch überraschend unerforscht. Hier fehlt eine klassische Konsumforschung, die über eine Benutzerumfrage oder archivische Stakeholder-Analyse ${ }^{4}$ hinausgeht.

$$
\text { Input - Output - Kennzahlen }
$$

Traditionell haben Archive ihre Bedeutung und Leistungsfähigkeit vor allem am Input gemessen, am Umfang ihrer Bestände in laufenden Metern, der Zahl ihrer Mitarbeiter, an Standorten, Nutzungsflächen, Magazinreserven oder Haushaltsmitteln ${ }^{5}$. Weitere übliche Zahlen für Leistungsvergleiche sind Benutzerzahlen, Benutzertage oder Anfragezahlen; hinzukommen die Menge des übernommenen Schriftguts im Jahr, neue Erschließungseinheiten oder auch fertiggestellte Findbücher. Der Umfang neu verpackter Unterlagen wurde ebenso in Archivstatistiken herausgestellt wie die Menge von restaurierten, verfilmten oder digitalisierten Archivalien.

Die Problematik solcher statistischen „Kennzahlen“ für die Beurteilung erfolgreicher archivischer Arbeit liegt auf der Hand: Schon die Definition unserer grundlegenden Maßeinheiten, etwa des „laufenden Meters“, ist im deutschsprachigen Archivwesen nicht abgestimmt ${ }^{6}$.

Andere Kennzahlen sind zwar in der Praxis weitgehend einheitlich, taugen aber sicher nicht als Maßstab für ein erfolgreiches Archiv: Hohe Zahlen bei den „Benutzungstagen“ (also der Summe der Benutzer pro Tag) können wohl auf die breite öffentliche Wirkung von Archiven, deren hohe Besucherattraktivität und ein großes öffentliches Interesse an ihren Angeboten und Beständen hindeuten. Sie können aber auch das Ergebnis ungenügender Findhilfsmittel, kurzer Öffnungszeiten, fehlender schriftlicher Auskunftsangebote oder langer Bestellfristen sein, welche die Benutzer zwingen, ein Archiv häufiger aufzusuchen, als dies etwa bei guten Recherchemöglichkeiten im Vorfeld des Besuches, langen Öffnungszeiten des Lesesaals oder kurzen Bereitstellungszeiten von Akten erforderlich wäre. Ein Archiv, das seine Bestände vollständig digitalisiert und im Internet zur Verfügung gestellt hat, wäre aus Sicht der meisten Nutzer sicher ein „sehr erfolgreiches“ Archiv, im Ranking der Benutzertage würde es hingegen an letzter Stelle stehen ${ }^{7}$.

Die traditionellen Input-Angaben sind zweifellos ein wichtiger Bestandteil der archivischen Statistik. Sie erlauben aber keine sachgerechte Beurteilung der Leistungsfähigkeit eines Archivs. Zudem sagen sie nichts aus über die Effizienz der Prozesse und Produkte. Für eine solche eher auf den Output statt auf den Input fokussierte Beurteilung sind immer Personal-Leistungs-Vergleiche oder Kosten-Leistungs-Vergleiche erforderlich ${ }^{8}$. Erst

${ }^{4}$ Martina Wiech, Strategisches Management für Archive, in: Archivmanagement (wie Anm. 3) 13-35, hier 25-28.

5 Vgl. Hartmut Weber-Renate Köhne-Lindenlaub, Archivmanagement, in: Handbuch für Wirtschaftsarchive. Theorie und Praxis, hg. von Evelyn Kroker-Renate KöHne-Lindenlaub-Wilfried Reininghaus-Ulrich S. SoÉnius (München 22005) 259-274, hier 265.

6 Martin Luchterhandt, Der „laufende Meter“. Zum Wesen archivischer Mengenangaben. Archivalische Zeitschrift 92 (2011) 61-71.

7 Mario Glauert, Kennzahlen und Benchmarking für Archive. Brandenburgische Archive. Berichte und Mitteilungen aus den Archiven des Landes Brandenburg 26 (2009) 25-34, hier 25 f.

${ }^{8}$ Der Ausschuss Betriebswirtschaftliche Steuerung der Konferenz der Leiterinnen und Leiter der Archivverwaltungen des Bundes und der Länder (KLA) hat dazu jüngst eine Empfehlung erarbeitet: Andreas HedwIG et al., Empfehlung zur Systematisierung von Querschnitts- und Fachleistungen sowie relevanter Kennzahlen in 
sie ermöglichen die Einschätzung, ob wir, um es mit Hartmut Weber zu formulieren, nicht nur die richtigen Dinge tun, sondern ob wir die richtigen Dinge auch richtig tun?.

Die Kosten-Leistungs-Rechnung, die viele Archive in Deutschland bereits seit längerem gemeinsam mit ihren Trägern praktizieren, erlaubt die Ermittlung solcher PersonalLeistungs-Relationen und die Angabe von Kosten für alle aufgestellten Produkte eines Archivs. Damit kann die Effizienz von Archivprozessen ermittelt und - einheitliche Messgrößen vorausgesetzt - verglichen werden ${ }^{10}$.

Allerdings ermöglicht auch die Kosten-Leistungs-Rechnung keine Aussage über die fachliche Qualität der erbrachten Leistungen und Produkte. Sie dient vor allem der internen Kontrolle und Steuerung, sie analysiert nur zurückliegende Prozesse und beschränkt sich allein auf Kostenaspekte (Input - Output). So wenig wie die oben genannten Angaben einer Archivstatistik erlauben Produktkosten eine Einschätzung, ob die gesetzten Ziele erreicht wurden, die archivische Arbeit also im Sinne der eingangs formulierten Frage archivfachlich, nicht betriebswirtschaftlich, „erfolgreich“ war.

Für einen Leistungsvergleich oder Benchmarking besser geeignet wären abgestimmte Kennzahlenkataloge, die - etwa auf der Basis einer „Balanced Score Card“ - ausgewählte archivische Aufgabenbereiche und Handlungsfelder zusammenstellen ${ }^{11}$. Im Gegensatz zu den Archiven haben die Bibliotheken auf der Grundlage der Deutschen (DBS) beziehungsweise Österreichischen Bibliotheksstatistik (ÖBS), die auf der DIN EN ISO 2789 „Internationale Bibliotheksstatistik“ beruhen, mit dem „Bibliotheksindex“ (BIX) bereits vor vielen Jahren ein gemeinsames Benchmarking-Instrument etabliert, das in komprimierter Form die spezifischen Stärken und Schwächen jeder Bibliothek in einem Ranking ablesbar machte ${ }^{12}$. Auch für Museen gibt es seit März 2016 solche international vereinbarten, einheitlichen Vorgaben für statistische Angaben ${ }^{13}$. Für die Archive hingegen fehlt im deutschsprachigen Raum bis heute ein abgestimmter Kennzahlen-Katalog ${ }^{14}$.

Solche abgestimmten Kennzahlen sind unabdingbar, wenn man nicht nur intern den Erfolg archivischer Arbeit, also das Erreichen von Soll- oder Ziel-Zahlen, messen und

Archiven, in: Das Bundesarchiv - Partner - KLA - Betriebswirtschaftliche Steuerung, https://www.bundesarchiv.de/imperia/md/content/bundesarchiv_de/fachinformation/ark/2017-02-01_kla-bs_empfehlungen_systematisierung_querschnitts_und_fachleistungen_komplett.pdf [1.3.2018].

9 Weber-Köhne-Lindenlaub, Archivmanagement (wie Anm. 5).

10 Andreas Hedwig, Betriebswirtschaftliches Finanzmanagement, in: Archivmanagement (wie Anm. 3) 91-114; DERs., Moderne Steuerungsinstrumente in den Archiven - Fluch oder Chance? Versuch einer Standortbestimmung, in: Ziele, Zahlen, Zeitersparnis. Wie viel Management brauchen Archive? Beiträge zum 20. Archivwissenschaftlichen Kolloquium der Archivschule Marburg, hg. von Irmgard Christa Becker-Dominik Haffer-Valeska KoAL (Veröffentlichungen der Archivschule Marburg, Hochschule für Archivwissenschaft 63, Marburg 2016) 13-58.

11 Mario Glauert, Was ist ein gutes Archiv? Ein Kennzahlen-Index für das Rating von Archiven, in: 5. Norddeutscher Archivtag, 12. und 13. Juni 2012 in Lübeck, hg. von Rainer Hering (Bibliothemata 27, Nordhausen 2013) 147-162.

12 Der BIX wurde zwar 2015 mangels ausreichender Finanzierungslage eingestellt; vgl. Einstellung des BIX. BIX. Der Bibliotheksindex - Aktuelles (1. 10. 2015), http://www.bix-bibliotheksindex.de/ [1. 3. 2018]. Allerdings hat zwischen Februar 2016 und März 2017 eine Expertengruppe Vorschläge erarbeitet, die Deutsche Bibliotheksstatistik um die Möglichkeit von Leistungsmessungen und -vergleichen weiterzuentwickeln; vgl. Arbeitsbereiche und Dienstleistungen des knb im Überblick, in: Bibliotheksportal - Das Kompetenznetzwerk für Bibliotheken (knb), https://bibliotheksportal.de/wir-ueber-uns/arbeitsbereiche/ [1.3. 2018].

13 ISO 18461:2016-03, Information und Dokumentation - Internationale Museumstatistik.

${ }_{14}$ Der Entwurf einer internationalen Archivstatistik (ISO/CD 19580, Information and Documentation International Archives Statistics) ist allerdings in Vorbereitung. Es bleibt indes abzuwarten, ob sich die dort gemachten Vorschläge am Ende durchsetzen werden. 
überprüfen möchte, sondern auch nach außen den Erfolg des Archivs - auch im Vergleich $\mathrm{zu}$ anderen Archiven - verdeutlichen und sichtbar machen möchte oder muss. Das Problem solcher Leistungsvergleiche ist indes, dass sie nicht nur abgestimmte Kennzahlen erfordern (also: Was ist ein „laufender Meter“"?), sondern auch ein gemeinsam vereinbartes Ziel- und Wertesystem voraussetzen, also wieder die (schwierige) Antwort auf die (einfache) Frage: Was ist ein erfolgreiches Archiv?

Es genügt mithin nicht zu definieren, dass die im Archiv eingesetzten Verpackungen den qualitativen Anforderungen der DIN ISO 16245 „Schachteln, Archivmappen und andere Umhüllungen aus zellulosehaltigem Material für die Lagerung von Schrift- und Druckgut aus Papier und Pergament" genügen müssen oder die technische Bearbeitung von Archivgut einem definierten Prozess entsprechen und am Ende nur Produktkosten von höchstens $240 €$ pro laufenden Meter ergeben darf. Denn ob ein Archiv insgesamt eine Verpackungsquote von $50 \%, 80 \%$ oder $100 \%$ erreichen muss, um als „erfolgreich“ zu gelten, ist damit noch nicht bestimmt ${ }^{15}$.

\section{Die Besonderheiten von Archiven}

Bei der Beurteilung von Erfolgen sind zudem einige spezifische Besonderheiten zu beachten, die Archive von anderen Institutionen, Kultureinrichtungen, Non-Profit-Organisationen, Behörden oder Wirtschaftsunternehmen unterscheiden ${ }^{16}$ :

- Archive sind Langzeitinstitutionen - sie müssen also nicht kurzfristig erfolgreich sein, wie ein Unternehmen oder ein Projekt. Ihr Erfolg zeigt sich erst in der Zukunft. Erfolgreiches Archivieren schafft erst in 50 oder 100 Jahren ein erfolgreiches Archiv. Archivare arbeiten somit immer für den Erfolg ihrer Nachfolger.

- Die öffentlichen Archive, zumindest in Deutschland, sind eine Pflichtaufgabe und können sich auf einen sicheren gesetzlichen Rahmen berufen, im Gegensatz etwa zu Bibliotheken und Museen. Selbst einem völlig „erfolglosen“ Stadtarchiv (wie auch immer dies aussehen mag) droht allenfalls die Verlagerung ins nächstgrößere Archiv aber das ereilt mitunter auch Archive, die fachlich erfolgreich geleitet werden.

- Die gesetzlichen Vorgaben engen die Spielräume der Archive aber auch ein, denn nahezu alle Fachaufgaben sind damit auch Pflichtaufgaben und lassen wenig Freiraum für individuelle strategische Entscheidungen. Archive sind somit nur bedingt Herr ihrer Aufgaben.

- Hinzu kommt, dass die archivischen Aufgaben einander bedingen: Keine Aufgabe darf länger zugunsten einer anderen vernachlässigt werden. Archive können sich also nicht von einzelnen, weniger erfolgversprechenden „Geschäftsfeldern“ oder „Produktsegmenten“ trennen, können nicht ungeliebte, unansehnliche oder wenig erfolgreiche Produktionsschritte auslassen.

${ }^{15}$ Vgl. Kennzahlen und Benchmarking zur Bestandserhaltung in Archiven. Tabellenwerk der Konferenz der Leiterinnen und Leiter der Archivverwaltungen des Bundes und der Länder (KLA). Ausgearbeitet vom Bestandserhaltungsausschuss der KLA (März 2016). Einführung und Erläuterung zum Tabellenwerk, in: Das Bundesarchiv - Partner - KLA - Bestandserhaltung, https://www.bundesarchiv.de/imperia/md/content/bundesarchiv_de/ fachinformation/ark/2016-08-01_kla_tabellenwerk_kennzahlen_benchmarking_einfuehrung.pdf [1. 3. 2018 ].

16 Mario Glauert, Archivmanagement. Schwierige Antworten auf einfache Fragen, in: Archivmanagement. Ressourcen nutzen, Potentiale erkennen. Frühjahrstagung der Fachgruppe 8 im Verband deutscher Archivarinnen und Archivare e. V., 19. bis 21. März 2014 in Berlin, hg. von Birgit RehSE-Irina Schwab (Wissenschaftsarchive 4, Leipzig 2015) 29-43, hier 34-37. 
- Zwischen der Ausführung von Fachaufgaben wie Übernahme, Bewertung, Erschließung oder Bestandserhaltung auf der einen und der Nutzung durch die Kunden auf der anderen Seite besteht gewöhnlich eine große zeitliche Lücke. Archive können daher nur bedingt auf Kundenwünsche reagieren. Ein patron-driven acquisition, eine kundengesteuerte Medienerwerbung wie in Bibliotheken, ist für sie nicht möglich. Sie müssen ihre finalen Entscheidungen zum künftigen Überlieferungsangebot als Propheten einer ungewissen Marktentwicklung treffen.

- Archive sind dabei konkurrenzlos. Sie stehen aufgrund ihrer unikalen Überlieferung untereinander nicht im Wettbewerb (allenfalls beim Erwerb von Nachlässen oder Sammlungen), was sie etwa von wissenschaftlichen oder öffentlichen Bibliotheken unterscheidet.

- Archive sind ihrer Natur nach ständig wachsende Institutionen, unabhängig von ihrem Erfolg oder ihrer Güte. Ihre Bestände wachsen auch, oder vielleicht sogar insbesondere dann, wenn sie fachlich schlecht (also nicht erfolgreich) geführt werden. Und mit den Beständen vermehren sich jedes Jahr auch die Kosten, Flächen- und Personalbedarf - und allzu oft eben auch die Rückstände.

- Archive sind Non-Profit-Organisationen: Sie zahlen sich vielleicht aus, aber sie rechnen sich nicht ${ }^{17}$. Das setzt allen betriebswirtschaftlichen Ansätzen zur Erfolgskontrolle ihrer Wirtschaftlichkeit und Effizienz klare Grenzen.

- Archive sind eben nicht wirtschaftlich, niemals kostendeckend, ihr return on investment ist - gerechnet in herkömmlichen Bilanzperioden - immer negativ.

- Archive können sich ihre Bestände nur bedingt aussuchen: Sie verwalten sperrige, schwierige Materialien mit geringem Anschauungswert, die sich den meisten Menschen nicht auf Anhieb erschließen. Unsere Produkte sind also nur sehr beschränkt markt- und marketingfähig.

- Archive haben auch kaum Einfluss auf ihre „Rohstofflieferanten“ und die Qualität und Quantität ihrer „Rohwaren“. Sie können ihre Lieferanten auch nicht wechseln diese ihr Archiv übrigens auch nicht, aber das nur am Rande.

\section{Erfolgsmessung in der archivischen Praxis}

Wie kann unter diesen besonderen Voraussetzungen der Erfolg archivischer Arbeit in der Praxis bestimmt oder gemessen werden? Kennzahlen allein können sicher nicht der Maßstab sein ${ }^{18}$. Neben der Quantität des Outputs, die für Statistiken, Berichte und Bilanzen, das Controlling oder Benchmarking von hoher Bedeutung ist, ist immer auch die Qualität im Blick zu behalten, die Gegenstand der Evaluierung von Ergebnissen oder der Auditierung von Prozessen ist.

Zudem ist der Erfolg eines Archivs sicher nicht mit betriebswirtschaftlichen Kriterien allein zu messen. Die betriebswirtschaftliche Betrachtung muss um eine archivfachliche Gegenrechnung ergänzt werden ${ }^{19}$. Der Maßstab für ein erfolgreiches Archiv kann nur ein

${ }^{17}$ Formulierung nach Oliver ScheYtт, Die Archive in der Kulturpolitik der Städte. Rede auf dem Rheinischen Archivtag in Euskirchen am 13. Juni 2002. Kulturpolitische Mitteilungen 99 (2002) 62-64, hier 64.

18 Burkhard Nolte, Kennzahlen, Kennzahlensysteme und Benchmarking - Nutzen und Grenzen im Archiv, in: Archivmanagement (wie Anm. 3) 69-89.

19 Dazu die Niederschrift einer Podiumsdiskussion auf dem 82. Deutschen Archivtag 2012: Irmgard Christa Becker-Mario Glauert-Anja Kruke-Ulrich Niess-Ulrich S. Soénius, Archive als Profit-Center?, 
archivfachlicher sein. Allerdings konnte unsere Fachkunde einen solchen bislang noch nicht anbieten.

Kennzahlen und Qualitätskontrollen setzen etablierte und abgestimmte Standards voraus. Im Archivbereich gibt es jedoch nur wenige Normen, die als Maßstab für erfolgreiche archivische Arbeit genutzt werden könnten, etwa im Bereich der Digitalen Archivierung oder der Bestandserhaltung. Für die Erfassung, Übernahme und Bewertung, für die Erschließung, Ordnung und Verzeichnung gibt es keine abgestimmten Normen, Standards oder Kriterien, die eine qualitative Bewertung der Ergebnisse und Abläufe dieser zentralen Archivprozesse oder der Bestandsqualität ermöglichen würden.

Ähnlich sieht es auch für die Bereitstellung und Benutzung oder unsere Dienstleistungsqualität insgesamt aus. Die Public Services Quality Group (PSQG) des britischen National Council on Archives hat 2008 zwar einen über fünfzig Seiten umfassenden „Standard for Access to Archives" veröffentlicht ${ }^{20}$, der aber im deutschsprachigen Raum kaum rezipiert wurde. Ein vergleichbarer Standard, der sogar explizite Vorgaben für regelmäßige Audits der Bereitstellungs- und Nutzungsbedingungen von Archiven anbietet, besteht in Deutschland nicht. Und so gibt es in Deutschland zwar zahlreiche Stadtbibliotheken, welche das Qualitätssiegel „ServiceQualität Deutschland“ führen²1, aber kein Stadtarchiv. Deutsche Archive verlangen somit zwar von ihren Dienstleistern zunehmend den Nachweis einer Zertifizierung nach DIN ISO 900122, arbeiten selbst aber nicht danach.

\section{Nutzung als Maßstab erfolgreicher Archivarbeit?}

Woran sollte man dann also die Ergebnisse und die Qualität archivischer Arbeit fachlich verlässlich „messen“? Wenn man im kollegialen Kreis die Frage erörtert, was aus Sicht der Archivarinnen und Archivare ein erfolgreiches Archiv ausmacht, wird ein Kriterium in nahezu allen Antworten an vorderer Stelle genannt: die Benutzung. Der Erfolg archivarischer Arbeit, so heißt es, erweist sich am Ende vor allem in der Nutzung des Archivgutes, sie ist Ziel und Zweck allen archivarischen Handelns, gleichsam der Sinn des Archivs.

Stimmt das? Ich denke, es lohnt sich, ein wenig über die Frage nachzudenken, ob der Maßstab für ein erfolgreiches Archiv tatsächlich seine Nutzung ist oder sein sollte, und welche Folgen das für unsere Arbeit hätte.

Dabei ist schon die Frage, was zur „Benutzung“ gezählt werden muss, keine leichte. Die Deutsche wie Österreichische Bibliotheksstatistik kämpft seit Jahren mit dem Problem, wie man die Nutzung von Bibliotheken messen kann und soll. Was ist der Unterschied zwischen einer Beratung und einer Auskunft? Ist ein Besucher, der in der Bibliothek seine eigenen Bücher durcharbeitet, ein aktiver Nutzer? Wie messe ich die Nutzung

in: Kulturelles Kapital und ökonomisches Potential - Zukunftskonzepte für Archive. 82. Deutscher Archivtag in Köln, hg. von Heiner Sснміт et al. (Tagungsdokumentationen zum Deutschen Archivtag 17, Fulda 2013) 197-222, hier 218.

${ }^{20}$ David Mander et al., A Standard for Access for Archives (2008), in: ARA. Archives \& Records Association UK \& Ireland - Publications - Other Useful Publications, http://www.archives.org.uk/images/documents/ access_standard_2008.pdf [1.3.2018].

${ }^{21}$ Vgl. ServiceQualität Deutschland. Das Q macht den Unterschied, https://www.q-deutschland.de/ [1. 3. 2018].

22 DIN EN ISO 9001:2015-11, Qualitätsmanagementsysteme - Anforderungen. 
von Online-Angeboten oder Apps ${ }^{23}$ ? Zur Nutzung in Archiven zählen jedenfalls keineswegs nur die Besucher im Lesesaal, sondern natürlich auch die Auskunftsanfragen, die Ausstellungs- und Vortragsbesucher, die Führungsteilnehmer, die Klicks in der Datenbank oder die Visits auf der Webseite ${ }^{24}$.

Macht man die Benutzung zum Maßstab erfolgreicher archivarischer Arbeit, muss man sich vergegenwärtigen, dass der Erfolg eines Archivs damit von vielen Faktoren und Entscheidungen abhängig ist, die das Archiv selbst kaum oder gar nicht beeinflussen kann. Jedes Archiv kann am Ende nur so gut sein wie die Verwaltungen und Stellen, die seine künftigen Unterlagen produzieren. Das gilt sowohl für den Inhalt und die Vollständigkeit der Aufzeichnungen wie für die Dauerhaftigkeit ihrer Trägermedien. Akten und Daten können im Archiv nicht mehr besser werden.

Zudem resultiert die tatsächliche Nutzung erfahrungsgemäß weniger aus dem Angebot der Archive als aus einem Nutzerinteresse, das primär nicht die Archive steuern, sondern Forschungstrends, gesellschaftliche Umbrüche, politische Themen oder Skandale, rechtliche oder administrative Änderungen oder ganz persönliche Anliegen. Die Änderung der Personenstandsgesetze beispielsweise oder die Reform der Grundbuchordnungen hat für die aktuelle Nutzungsstatistik der deutschen Archive viel größere Auswirkungen als jede archivarische Fachentscheidung. Und über lange Zeiträume betrachtet, tragen Feuer, Wasser, Kriege und andere Katastrophen weit mehr zur Überlieferungsbildung bei als Archivarinnen und Archivare. Der Überlieferungs-Chance und dem ÜberlieferungsZufall, die Arnold Esch 1985 als methodisches Problem des Historikers beschrieben hat ${ }^{25}$, stehen demnach Nutzungs-Chance und Nutzungs-Zufall als methodisches Problem des Archivars gegenüber.

Nutzung als Erfolgsindikator ist mithin nur sehr bedingt planbar oder im Rahmen eines archivischen Controllings steuerbar: Der Erfolg stellt sich erst Jahrzehnte, mitunter erst Jahrhunderte später ein, und eine hohe Nutzung heute sagt vor allem etwas aus über die erfolgreiche Arbeit der Archivare gestern.

Öffentliche Archive sind in Deutschland eine Pflichtaufgabe und aufgrund ihrer unikalen Bestände konkurrenzlos. Auch wenn Archive im Kundendienst und in der Servicequalität, in der Bereitstellung ihrer Unterlagen für die Benutzung und der Verbesserung ihrer Usability ihre zentrale Aufgabe und Berechtigung sehen, besteht dazu letztlich kein existenzieller Zwang zum Erfolg. Im strengen archivrechtlichen (auch wirtschaftlichen) Sinne brauchen Archive keine Benutzer, keine Benutzung, um zu bestehen - und manche verhalten sich auch noch so.

${ }^{23}$ Nachzulesen in den veröffentlichten Protokollen der DBS-Steuerungsgruppe: Protokolle, in: DBS. Deutsche Bibliotheksstatistik - Wissenswertes und Links - Steuerungsgruppen, Arbeitsgruppen und Protokolle, https://wiki1.hbz-nrw.de/display/DBS/Protokolle [1.3. 2018].

${ }^{24}$ Berücksichtigt sind diese Kriterien in einem nun von den archivarischen Berufsverbänden der USA und Kanadas angenommenen Standard: Standardized Statistical Measures and Metrics for Public Services in Archival Repositories and Special Collections Libraries, in: SAA. Society of American Archivists - Standards, https:// www2.archivists.org/standards/standardized-statistical-measures-and-metrics-for-public-services-in-archivalrepositories [1.3. 2018].

25 Arnold Esch, Überlieferungs-Chance und Überlieferungs-Zufall als methodisches Problem des Historikers. HZ 240 (1985) 529-570. Ergänzend dazu zuletzt Thomas HaYe, Verlorenes Mittelalter. Ursachen und Muster der Nichtüberlieferung mittellateinischer Literatur (Mittellateinische Studien und Texte 49, LeidenBoston 2016). 
Exkurs: Was wäre ein „wirtschaftlich“ erfolgreiches Archiv?

Ein kleines Gedankenexperiment als Exkurs: Was wäre ein wirtschaftlich erfolgreiches Archiv? Oder konkreter formuliert: Was würden Archive ändern, wenn sie mit der Benutzung ihren gesamten Archivunterhalt verdienen müssten, und nicht nur eine geringe Kostendeckungsquote durch Lesesaal- und Kopiergebühren oder Verkaufserlöse von Siegelabgüssen und Archivpublikationen zu erreichen hätten ${ }^{26}$ ?

Wenn ein Archiv mit Nutzung sein Geld verdienen müsste, würde es:

- vor allem die Erschließung und damit die Trefferquote von Begriffssuchen verbessern müssen, das heißt mehr Index-Einträge statt nur Titel und „Enthält-Vermerke“. Nur Treffer bringen Traffic und damit Einnahmen.

- Archive würden sicher noch mehr Archivgut digitalisieren und online präsentieren. Natürlich müssten sie auch für digitalisiertes Archivgut eine Indexierung oder OCR bieten: Reine Images digitalisierter Aktenseiten lassen sich kaum vermarkten, da sie für die meisten Menschen mindestens transkribiert, wenn nicht übersetzt werden müssen.

- Vermutlich würden solche Archive Beratungen, Recherchen und Auskünfte rund um die Uhr über alle Kanäle der Sozialen Medien anbieten und, wenn sie geschäftstüchtig sind, zugleich auch für die Bestände anderer Archive.

- Der Lesesaal wäre wie in vielen wissenschaftlichen Bibliotheken selbstverständlich an sieben Tagen der Woche 24 Stunden geöffnet.

- Dem Nutzer könnte man außerdem mit seiner gewünschten Akte zugleich weitere Archivalien mit dem Hinweis anbieten: „Kunden, die diese Akte bestellt haben, haben auch folgende Archivalien eingesehen".

- Das archivische Angebot würde seinen Kunden den Zugang zu vielfältigen historischen Informationsressourcen ermöglichen und ähnlich den neuen „Fachinformationsdiensten“ (FID) ein Portal und eine Plattform zu allen Fragen rund um die Archivbestände eröffnen.

- Potentielle Themen und Ausstellungen, die sich an einem breiten Publikumsgeschmack ausrichten, nicht am letzten Findbuch, könnten als History-Serie nicht nur im Fernsehen, sondern auch über einen Youtube-Kanal angepriesen werden. Alle einschlägigen Wikipedia-Artikel sollten konsequent um Links zu passenden Archivalien in der Online-Datenbank ergänzt werden.

- Vielleicht würde das Archiv auch Neukunden mit Geschenken werben, einen historischen Verein gründen oder versuchen, die Rahmenpläne für das Schulfach Geschichte um obligatorische Archivbesuche zu erweitern.

- Vermutlich würde ein solches Archiv auch anders bewerten. Die Übernahme von deutlich mehr nicht-amtlichem Schriftgut, wahrscheinlich überhaupt von weniger Schriftgut und alten Verwaltungsakten, dafür viel mehr Fotos, Filmen, AV-Medien, Webseiten und anderen optisch und akustisch ansprechenden Medienformaten, wäre anzunehmen. Vielleicht würden solche Archive elektronische Unterlagen gar nicht mehr bewerten und auf die großen Einnahmen aus Big-Data-Anfragen künftiger Forschergenerationen hoffen.

Das kleine Gedankenspiel zeigt, dass eine hohe und intensive Nutzung so eindeutig

26 Vgl. Mario Glauert, Archivbenutzung im Digitalen Zeitalter. Brandenburgische Archive. Berichte und Mitteilungen aus den Archiven des Landes Brandenburg 33 (2016) 3-9. 
und kompromisslos offensichtlich doch nicht unserer Vorstellung von einem erfolgreichen Archiv entspricht, auch wenn es durchaus einzelne Archive gibt, die in diese Richtung voranschreiten.

Zudem ist die Idee, dass die vielfältige Nutzung von Archiven ein Erfolgsindikator archivarischer Arbeit ist, noch relativ jung und keineswegs allgemein gültig: Archive haben sich erst in den letzten Jahrzehnten von bestandsorientierten zu nutzungsorientierten Einrichtungen entwickelt ${ }^{27}$. Die freie und offene Nutzung von Archiven ist ein ethisches, ein politisches Ziel, aber natürlich auch heute keineswegs selbstverständlich.

Ein erfolgreiches „Archiv 2.0“ wird nicht mehr bei der klassischen Nutzung als Einbahnstraße stehen bleiben können, sondern muss die Mitwirkung und Interaktion der Nutzer mit dem Archiv ermöglichen. Und spätestens hier zeigen sich dann die auch heute im demokratischen Mitteleuropa noch weithin bestehenden Grenzen einer radikalen Nutzerorientierung der Archive ${ }^{28}$.

\section{Nutzen statt Nutzung}

Wenn vor allem die hohe und breite Benutzung ein erfolgreiches Archiv ausmachen würde, wäre schließlich die Frage berechtigt, ob auch eine erfolglose Recherche in einem Archiv eine Nutzung und damit einen Erfolg darstellt? Ein Archiv ist nicht automatisch dann erfolgreich, wenn es viele Suchanfragen an seine Online-Datenbank zählt, sondern doch wohl nur dann, wenn daraus Bestellungen, Lesesaalbesuche und die Auswertung von Archivgut erwachsen, schließlich neue Erkenntnisse, Forschungsergebnisse und Publikationen und am Ende sogar eine gesellschaftliche Wirkung für Bildung, Wissenschaft, Kultur, Rechtssicherheit oder Demokratie. Ein erfolgreiches Archiv ist ein Archiv, das nützt - und nicht nur benützt wird.

Der Erfolg eines Archivs sollte sich daher weniger am Output, seinen Leistungen und Produkten, bemessen, als vielmehr an seiner Wirkung, am Outcome oder Impact, also am Nutzen, nicht nur an der Nutzung. Ein erfolgloses Archiv wäre dann ein wirkungsloses Archiv, selbst wenn es solide fachliche Arbeit leistet.

Doch was ist der Nutzen oder die Wirkung von Archiven? Was sollen, was wollen Archive bewirken? Und noch schwieriger: Wie misst man die Wirkung von Archiven? Auch bei diesen Fragen sind uns die Bibliotheken einmal mehr deutlich voraus, denn über die schwierigen Probleme der Wirkungsmessung bis zur Berechnung des ökonomischen Wertes von Bibliotheken diskutieren unsere Kolleginnen und Kollegen dort schon seit über fünfzehn Jahren. Seit 2014 liegt mit der ISO 16439 sogar eine internationale Norm zu Metho-

27 Die Tradition ist auch in (West-)Deutschland noch nicht so alt. In Deutschland wenig rezipiert wurde der Beitrag von Eckhart G. Franz, What Makes an Archives Successful. The „House of History“ Concept. Journal of the Society of Archivists 16 (1995) 71-76. Als das Staatsarchiv Marburg in den 1950er Jahren erstmals Findbücher veröffentlichte, war das noch höchst umstritten. Und noch in den 1960er Jahren wurde in einzelnen deutschen Landesarchiven die Bereitstellung von Archivalien davon abhängig gemacht, ob der Benutzer die notwendige Qualifikation zum Lesen der Akten mitbrachte. Noch vor 30 Jahren hätte man in dem Archiv in Potsdam, in dem der Autor dieser Zeilen heute arbeiten darf, sicher nicht die möglichst breite und umfassende Nutzung als Ziel erfolgreicher Archivarbeit formuliert. Die Staatsarchive der DDR hatten, wie die Archive in anderen Diktaturen einst und heute, ganz andere Aufgaben und Vorgaben.

${ }^{28}$ Mario Glauert, Archiv 2.0. Vom Aufbruch der Archive zu ihren Nutzern, in: Archive im digitalen Zeitalter. Überlieferung - Erschließung - Präsentation. 79. Deutscher Archivtag in Regensburg, hg. von Heiner Sснмiтt et al. (Tagungsdokumentationen zum Deutschen Archivtag 14, Fulda 2010) 43-54. 
den vor, um die Wirkung (Impact) von Bibliotheken zu ermitteln. Dies erfolgt, kurz gesagt, indem die von der Bibliothek vermuteten Effekte verglichen werden mit der durch Nutzerbefragungen erhobenen Wirkung und den tatsächlich in der Zukunft beobachteten Nachwirkungen ${ }^{29}$. Allerdings sind die Schwierigkeiten dieser Wirkungsmessung offensichtlich:

- Die Wirkungen treten oft erst langfristig ein.

- Sie sind vielfach immateriell und schwer zu quantifizieren.

- Die Ergebnisse von Befragungen zeigen die subjektive Sicht der Befragten, nicht immer den wirklichen Effekt.

- Zudem erfordern die Methoden zum Teil einen nicht unerheblichen Arbeitsaufwand.

\section{Wirkungsmessung von Archiven}

In den USA und Kanada fanden 2009/10 zwei parallele, groß angelegte Erhebungen zum wirtschaftlichen Nutzen von Archiven statt, deren Ergebnisse 2012 vorgestellt wurden $^{30}$. Auch diese Studien, die im deutschsprachigen Raum kaum beachtet wurden, zeigten indes, dass die Wirkungsmessung für Archive noch viele schwierige Fragen zu klären hat, selbst wenn man an etablierte Methoden anknüpfen kann ${ }^{31}$ :

- Was sind die beabsichtigten Wirkungen der Archive selbst beziehungsweise ihrer Unterhaltsträger?

- Wer sind - im weiteren Sinne - die Nutzer von Archiven? Wer profitiert, hat einen Nutzen von ihnen?

- Wie berücksichtigt man Wirkungen und Effekte, die erst in Jahrzehnten oder Jahrhunderten auftreten?

- Wie weit reicht der Einfluss von Archiven?

- Und wie bemisst man den wirtschaftlichen Wert einer Information oder Akte, die, würde sie nicht archiviert werden, gar nicht mehr verfügbar wäre?

29 Roswitha Poll, Was dabei herauskommt: Wirkungsforschung für Bibliotheken. Zeitschrift für Bibliothekswesen und Bibliographie 53 (2006) 59-70; Gudrun Witzler, Wert und Wirkung von Bibliotheken. Bibliothek, Forschung und Praxis 32 (2008) 279-292; Roswitha Poll, Kann man den Einfluss von Bibliotheken messen? Die Norm ISO 16439. Bibliothek, Forschung und Praxis 38 (2014) 232-238.

30 Elizabeth Yakel-Wendy M. Duff-Helen Tibbo-Adam Kriesberg-Amber Cushing, The Economic Impact of Archives: Surveys of Users of Government Archives in Canada and the United States. The American Archivist 75 (2012) 297-325.

$31 \mathrm{Zu}$ ersten Ansätzen für eine Wirkungsmessung von Archiven vgl. Caroline WAVELL-Graeme BaxterIan Johnson-Dorothy Williams, Impact Evaluation of Museums, Archives and Libraries: Available Evidence Project (Aberdeen 2002); Eilean Hooper-Greenhill, Measuring Learning Outcomes in Museums, Archives and Libraries: The Learning Impact Research Project (LIRP). International Journal of Heritage Studies 10 (2004) 151-174; Sarah J. Horton-Jaqueline Spence, Scoping the Economic and Social Impact of Archives (2006), in: Cadair. Aberystwyth University Open Access Repository, http://hdl.handle.net/2160/1808 [1. 3. 2018]; Impact of Community Archives. A 2007 Report Commissioned by the Community Archives Development Group (now CAHG), in: Community Archives and Heritage Group - Resources, http://www.communityarchives.org. $\mathrm{uk} /$ content/resources/resources/impact-of-community-archives-getting-started [1. 3. 2018]; Wendy M. DuffJoan M. Cherry, Archival Orientation for Undergraduate Students. An Exploratory Study of Impact. The American Achivist 71 (2008) 499-529; Wendy M. Duff-Andrew Flinn-Karen Emily Suurtamm-David A. Wallace, Social Justice Impact of Archives: A Preliminary Investigation. Archival Science. International Journal on Recorded Information 13 (2013) 317-348; Lertchai WasananikornkulchaI, Valuing Archives: From Nonmarket Valuation to Input-output Analysis, in: Research Libraries UK - UDC Hub, http://www.rluk.ac.uk/ about-us/blog/valuing-archives-non-market-valuation-input-output-analysis/ [1. 3. 2018]; Elizabeth Joan Kelly, Altmetrics and Archives. Journal of Contemporary Archival Studies 4 (2017) 1-21. 
Das britische Nationalarchiv stellte Anfang 2016 einen „Wirkungsraster“ vor, der insbesondere Kommunalarchiven helfen soll, anhand von Beispielen ihre positive Wirkung vor Ort etwa für „Health and Well-being“, „Stronger and Safer Communities“ oder „Learning and Education“ herauszustellen ${ }^{32}$. Ziel ist es, die vielfältigen Wirkungen der Archive messbar und damit für die lokalen Entscheidungsträger sichtbar zu machen sowie die eigenen Prioritäten stärker auf die beabsichtigten Wirkungen auszurichten. Dabei wird zwischen übergreifenden und langfristigen strategischen Wirkungen, mittelfristigen Effekten und den unmittelbaren Ergebnissen der archivischen Serviceangebote unterschieden.

Auch in Deutschland oder Österreich dürften die gesellschaftlichen und politischen Forderungen an die Archive, Wert, Nutzen und Wirkung ihrer erfolgreichen Arbeit nachzuweisen, in den kommenden Jahren eher zunehmen. Die (schwierige) Antwort auf die (einfache) Frage nach den Kennzeichen, Kriterien und Kennzahlen erfolgreicher archivarischer Arbeit wird dafür eine entscheidende Voraussetzung sein.

\section{Ausblick}

Öffentliche Archive stehen in Deutschland als gesetzliche Pflichtaufgabe nicht unter dem Leistungs- und Erwartungsdruck wie Bibliotheken oder Museen, die ihre gesellschaftliche Wahrnehmung und Wirkung mit Zahlen nachweisen und beharrlich bewerben müssen. Sie sind konkurrenzlos. Sicher ein Grund dafür, dass Archive sich bei Fragen des Leistungsvergleichs, der Wirkungsmessung oder Konsumforschung bisher wenig profilieren mussten.

Archive haben es aber auch schwerer als Bibliotheken oder Museen, ihre unmittelbare Wirkung nachzuweisen: Sie prägen nicht das Renommee einer Stadt oder Region, sie sind keine Touristenmagnete, sie sind kein Instrument der Wirtschaftsförderung (nicht einmal in einem Wiener Einkaufszentrum ${ }^{33}$ ) oder eine Maßnahme zur Verbesserung der Infrastruktur. Ihre Arbeit bringt angesichts der geringen Nutzerzahlen nur wenig Wählerstimmen oder Follower.

Dass Archive Teil und Bewahrer der kulturellen Identität sind, Bildung und Wissenschaft durch die dauerhafte Zugänglichmachung von Informationen fördern, die Rechte der Archivträger und Bürger wahren, die Nachvollziehbarkeit von Verwaltungsentscheidungen sicherstellen oder die Authentizität und Integrität des kulturellen Erbes gewährleisten, wird zwar weithin postuliert. Die archivfachliche Diskussion wird sich aber vermutlich auch der Frage zuwenden müssen, wie man diesen Anspruch nicht nur einlösen, sondern die beabsichtigten Wirkungen auch nachweisen kann.

32 A Guide to Developing an Outcomes Framework for Archives, in: The National Archives - Archives Sector - Finding Funding - Generating Income, http:/www.nationalarchives.gov.uk/documents/archives/22.01.16_Measuring_outcomes_for_archives.pdf [1.3.2018].

33 Die Tagungsstätte, das Wiener Stadt- und Landesarchiv, ist seit 2001 baulich direkt mit einer ShoppingMall, dem Wiener Gasometer, verbunden. 


\section{Autorinnen und Autoren}

Dr. Holger Berwinkel

Universitätsarchiv Göttingen

Niedersächsische Staats- und Universitätsbibliothek

Papendiek 14

37070 Göttingen

Deutschland

berwinkel@sub.uni-goettingen.de

Dr. Matthias BuchHOLZ

Bundesstiftung zur Aufarbeitung der SED-Diktatur

Kronenstraße 5

10117 Berlin

Deutschland

m.buchholz@bundesstiftung-aufarbeitung.de

Dr. Prof. Luciana Duranti

School of Library, Archival and Information Studies

The University of British Columbia

470-1961 East Mall

Vancouver, BC V6T 1Z1

Canada

luciana.duranti@ubc.ca

Prof. Dr. Mario Glauert

Brandenburgisches Landeshauptarchiv

Am Mühlenberg 3

14476 Potsdam

Deutschland

mario.glauert@blha.brandenburg.de

Elizabeth KaTA, MA

IAEA Archives

Vienna International Centre

PO Box 200

1400 Wien

Österreich

e.kata@iaea.org 
Andreas Kellerhals

ehem. Direktor des Schweizerischen Bundesarchivs

Ringoltingenstraße 3

3006 Bern

Schweiz

andreas.kellerhals@outlook.com

Dr. Joachim Kemper

Stadt- und Stiftsarchiv Aschaffenburg

Wermbachstraße 15

63739 Aschaffenburg

Deutschland

joachim.kemper@aschaffenburg.de

Mag. Dr. Brigitte KRENN

Austrian Research Institute for Artificial Intelligence (OFAI)

Freyung 6/3

1010 Wien

Österreich

brigitte.krenn@ofai.at

Prof. Dr. Robert KRETZSCHMAR

Landesarchiv Baden-Württemberg

Eugenstraße 7

70182 Stuttgart

Deutschland

robert.kretzschmar-kretzschmar@web.de

Prof. Heather MacNeIL, MA, MAS, PhD

University of Toronto, Faculty of Information

140 St. George Street

Toronto, Ontario M5S 3G6

Canada

h.macneil@utoronto.ca

Dr. Miroslav NovaK

Pokrajinski arhiv Maribor

Glavni trg 7

2000 Maribor

Slowenien

miro.novak@pokarh-mb.si 
Helga Penz

Stiftsarchiv Herzogenburg

Prandtauerring 2

3130 Herzogenburg

Österreich

archiv@stift-herzogenburg.at

Prof. Dr. Michel J. Pfeiffer M.A.

Hochschule für Technik und Wirtschaft Chur

Pulvermühlestrasse 57

7004 Chur

Schweiz

michel.pfeiffer@htwchur.ch

Dr. Dietmar SCHENK

Universität der Künste Berlin, Universitätsarchiv

Postfach 120544

10595 Berlin

Deutschland

dietmar.schenk@udk-berlin.de

Dr. Markus Schmidgall

Vorarlberger Landesarchiv

Kirchstraße 28

6900 Bregenz

Österreich

markus.schmidgall@vorarlberg.at

Mag. Dr. Elisabeth SchögGL-ERnst MAS

Steiermärkisches Landesarchiv

Karmeliterplatz 3

8010 Graz

Österreich

elisabeth.schoeggl-ernst@stmk.gv.at

Mag. Dr. Christoph Sonnlechner MAS

Wiener Stadt- und Landesarchiv

Rathaus

1082 Wien

Österreich

christoph.sonnlechner@wien.gv.at 
Mag. Dr. Thomas Stockinger MAS

Universität Wien, Institut für Österreichische Geschichtsforschung

Universitätsring 1

1010 Wien

Österreich

thomas.stockinger@univie.ac.at

Prof. Dr. Georg Vogeler

Universität Graz, Zentrum für Informationsmodellierung

Elisabethstraße 59/III

8010 Graz

Österreich

georg.vogeler@uni-graz.at

Mag. Mag. Dr. Jakob WüHRER

Oberösterreichisches Landesarchiv

Anzengruberstraße 19

4020 Linz

Österreich

jakob.wuehrer@ooe.gv.at 


\title{
Personen-, Sach- und Ortsregister
}

\author{
Stephanie Rosenkranz
}

Folgende Begriffe sind aufgrund der Häufigkeit/der hohen Frequenz ihres Auftretens nicht mit eigenen Seitenverweisen aufgenommen: Akten, Archiv, Archivgut, Archivische Tätigkeiten, Bestand, Digitales Zeitalter, Historische Wissenschaften, Informationszeitalter.

Die Registereinträge orientieren sich an dem am häufigsten verwendeten Begriff. Abkürzungen und Äquivalente in englischer und schließlich in deutscher Sprache werden in Klammer angeführt. Sofern englische Termini kein Äquivalent in der deutschen Fachsprache haben, werden sie ohne Übersetzung im Register vermerkt. Institutionen sind - soweit möglich - unter ihrem jeweiligen geografischen Standort ausgewiesen. Gegebenenfalls führt ein Querverweis zum Hauptlemma. Dasselbe gilt für alle Unterlemmata mit hoher Frequenz im Text.

Abgabepflicht 72, 127

Accountability (transparency; Rechenschaft, Transparenz) $16,21,23,29,34,37,96 f ., 110 f ., 114-116$, $120-122,138,150,152,238,257,268$

Adalbero von Würzburg 103

Administory, Zeitschrift 165 Anm. 39

Akteneditor 58

Aktenkunde 18, 20, 45, 57-69, 71-73, 87, 152, 233

- Äußere Merkmale 64, 69, 78, 80-83, 86, 92

- Aktenstilistik 65

- Analytische Aktenkunde (analytical techniques) 92f., 95

- Entstehungsstufe 58f., 61, 64, 68, 71

- Genetische Aktenkunde 58, 64, 66

- Innere Merkmale 58f., 64, 69, 78, 80, 82f., 86, 92

- Systematische Aktenkunde $65 f$.

Aktenpläne 106, 143, 157, 159 Anm. 19, 206

Aktentitel 157, 208, 210, 212

Aktenzeitalter 60

Algorithmen 23, 25, 136, 138, 162, 175, 177, 201, 203f.

- Bewertungsalgorithmen 136, 138

- Lernalgorithmen 175, 177

Amerika (anglo-amerikanischer Raum), siehe auch Nordamerika, United States of America 32, 36, 127, 129-131, 133f., 136, 204, 251

Amerikanisches Nationalarchiv siehe Washington

Anbietungspllicht 127, 157, 258

Andrić, Ivo 247

Antike, Archiv in der 16, 31, 90, 238

Arbeitsgruppe „Archivische Bewertung“ der Arbeitsgemeinschaft bayerischer Kommunalarchive 115
Arbeitskreis „Aktenkunde des 20. und 21. Jahrhunderts“ 45, 57

Arbeitskreis „Archivierung von Unterlagen aus digitalen Systemen “ 46

Arbeitskreis „Bewertung“ im Verband deutscher Archivarinnen und Archivare 120

Arbeitskreis „Information“, Berliner 230 Anm. 23

Arbeitskreis „Offene Archive“ 252f.

Arbeitskreis „Vorarlberger Kommunalarchive“ 147 Anm. 9, 151

Archivalienkunde 20, 48f., 51-55, 63, 71-73, 78, 80, 83, 86f., 233, 244

- Analytische Archivalienkunde 73, 78-83, 87

- Genetische Archivalienkunde 73-79, 87

- Systematische Archivalienkunde 73, 83-87

Archivalientyp 207

Archival Turn 16, 110, 225-227, 229f., 240-242, 244

Archivbauten 32, 37, 90, 142, 144, $149 f$

- Archiveinrichtung (Archivmöbel, Archivschränke) 107, 109

- Archivraum 108

Archivbegriff 9-16, 19, 27-29, 31-33, 37f., 86, 89f., 92, 108-110, 114, 117f., 227-230, 236-245, 247, 257-259, 261, 263f., 266, 268

Archiv-Diskurs, kulturwissenschaftlicher 226-232, 244

Archive, Arten von

- Bewegungsarchiv 71f.

- Bürgerarchiv 27, $117 f$.

- Digitales Archiv 46, 143, 160

- Diözesanarchiv 110 
- Ein-Personen-Archiv $249 f$.

- Familienarchiv 237

- Forschungsarchiv 27

- Gauarchiv 195

- Geheimarchiv 150, 228

- Gemeindearchiv 141, 146, 150

- Hausarchiv 149

- Herrschaftsarchiv 90

- Historisches Archiv 237, $240 \mathrm{f}$.

- Kommunalarchiv 27, 115 Anm. 12, 120, 147, 149f., 267

- Kreisarchiv 53

- Landesarchiv 27, 42, 110, 150f., 188

- Literaturarchiv 241 Anm. 67

- Medienarchive 129

- Nationalarchiv 92

- Online-Archiv (virtuelles Archiv) 13, 156

- Parteienarchiv 150

- Pfarrarchiv 141, 150

- Privatarchiv 22

- Religionsgemeinschaften, Archive (Ordensarchive) $23,27,32,53,90,101-111,228,250$

- Spiegelarchiv 241

- Staatliches Archiv 53, 72, 92, 237, 250

- Stadtarchiv 32, 53, 228, 237, 250, 257

- Stadtstaaten, Archive 90

- Universalarchiv 27

- Universitätsarchiv 53, 250

- Wirtschaftsarchiv 237

Archivgesetz 22, 72, 127, 144, 214, 221, 226, 261

- Bundesarchivgesetze 21, 257

- Deutsches Bundesarchivgesetz (BArchG) 114

- Landesarchivgesetze 21, 257

- Vorarlberger Archivgesetz 142, 144 Anm. 7, 149 Anm. 11

- Wiener Archivgesetz 190

Archivgut (archival holdings, archival material, records; Archivalien)

- Amtsbücher 52, 63 Anm. 41, 108

- Annalen 102, $108 \mathrm{f}$.

- Archivregister 103

- Bauakten 110

- Bewegungsunterlagen 71-87

- Bildbestände, analoge 129, $131 \mathrm{f}$.

- Bilddatenbestände allgemein (visuell kodierte Datenbestände) 131f., 134-136

- Bilddatenbestände digital 124, 128f., $131 \mathrm{f}$.

- Bilder (Fotografien, visuell codierte Kulturgüter) 18, 23, 51, 109, 123-139, 164, 190 Anm. 17, 195, 196 Anm. 28, 265

- Blogs 46, 250, $252 \mathrm{f}$.

- Briefe (Korrespondenz) 104, 205, 239, 247

- Bruderschaftsbücher 110

- Chroniken (Klosterchroniken) 103f., 110

- Digitale Archivalien (digital records; elektronisches -, digitales Archivgut) 19, 22, 24, 33, 43, 45-47, 50, 52, 60, 93-97, 123, 136, 139, 142f., 146, 160,
163, 169, 180f., 185, 197, 213, 240-242, 265

- Digitalisate 26, 59, 111, 163, 187f., 194, 196198, 202, 259, 265

- Druckvorlagen 78

- Entnazifizierungsakten 52

- Fachverfahren (elektronisches Fachverfahren) 46 48

- Feministische Unterlagen 71

- Flugblätter (Informationsmaterial) 72, 74, 78, 82, 85f., 195

- Gerichtsakten 205

- Grundbücher 141

- Handschriften 18, 103, 240

- Heiligenviten 102

- Historische Karten (Landkarten) 52, $193 \mathrm{f}$.

- Inventare (indexes) 107f., 161, 163 Anm. 33, 165, 190 Anm. 17, 215

- Jahresberichte 72

- Kapitelbücher 108

- Karteikarten 159, 210

- Kataster 193f.

- Konventbücher 108

- Kopialbücher $107 f$.

- Kopien (copy, reproduction) 61, 65, 69, 77, 80, 93f., $241 f$.

- Kriegsgräberlisten 196 Anm. 28

- Memorialschreibwerk (Memorienschreibwerk, interne Schriftstücke) 61, 65, 76, 83, 106, 205

- Mirakelsammlungen 102

- Mitschriften 76, 77 Anm. 18

- Musiksammlung 141

- Nachlässe 141, 150, 239, 241

- Nekrologe 102, 104

- Noviziatsbücher 108

- Personalakten 52

- Pfarrmatriken 141

- Plakate 72, 78, 82

- Positionspapiere 72, 76

- Prälatenlisten 103

- Protokolle (journals) 61, 64, 67-69, 74, 76, 80, 83, 215

- Rechnungsbücher 107

- Reliquienauthentiken 108

- Repertorien 103, 107

- Rundschreiben 104

- Selbstzeugnisse 51, 60

- Stenogramme 62, 68

- Sterbechroniken $109 f$.

- Steuerakten 51, 52 Anm. 66

- Urbare 52

- Urkunden 18, 48, 52, 60, 64, 67, 91f., 95, 102f., $105,108,110,150,243$

- Verbrüderungsbücher 102

- Verfachbücher 141

- Verkehrsschriftstücke 61, 65, 106

- Web-Unterlagen 52

- Weisungen 64 
- Zeitungsausschnitte 109

- Zeremonienbücher 110

- Zines 82

Archivierungsmodul 143

Archivinformationssystem (AIS; archival information system) 15, 24-26, 126, 135, 146-150, 155, 159, 160 Anm. 25, 167, 186, 188, 191f., 196f., 200, 203-205, 212-219, 221f.

- Institutionen übergreifende Recherche 147, 192, 197

- Institutionen übergreifender Datenaustausch (Softwarebrücke) 159

- Open Archival Information System (OAIS) 143, 214

- Webmodul eines Archivinformationssystems 15

- Wiener Archivinformationssystem (WAIS) 185, 188, 191-197

Archivische Standards (archival standards; Standards) 14, 26, 34, 129, 144, 146, 150-152, 196, 202, 211, 213-216, 221f., 257, $262 \mathrm{f}$.

- CIDOC-CRM 210

- DIN ISO 15489-1 143f.

- DIN ISO 16245261

- DIN ISO 9001263

- EAD-Standard 152

- ISAAR(CPF) 209

- ISAD(G) 150-152, 160 Anm. 25, 190 Anm. 17, 202, 209, 215, 217, 221

- ISDF 209

- Kodex ethischer Grundsätze für Archivarinnen und Archivare (ICA Code of Ethics) 114, 257

- Metadatenstandards 138

- Records in Contexts (ICA-Standard-Entwurf) 26, $165,210 f$.

- Standard for Access to Archives 263

- Standardized Statistical Measures and Metrics for Public Services in Archival Repositories and Special Collections Libraries (Society of American Archivists) 264 Anm. 24

Archivische Tätigkeiten (archiving; Archivierung)

- Aufbewahrung (storage) 32-37, 190 Anm. 17, 242

- Behördenberatung 146

- Beratung (Online-Beratung) 186, 191, 250, 252, 263, 265

- Bereitstellung (Aufbereitung, Zugänglichmachen) 13-15, 17, 19, 21, 24, 25-27, 29, 33f., 36f., 114, 135, 150-152, 160-166, 186-188, 191-197, 216, 219, 238, 250, 254, 257, 263-265, 268

- Bestandsbeschreibungen 207-209

- Bewertung 19, 21-23, 29, 36, 51, 66, 87, 101111, 113-121, 123-136, 138f., 142, 143, 146, $149,156,238,263,265$

- Digitale Archivierung (archiving of e-records; elektronische Archivierung) 46, 53, 93-95, 169, 188, 190 Anm. 17, 213, 249 Anm. 8, 263

- Erhaltung (maintenance, preservation; Restaurie- rung, Schutz) 18, 19, 21, 33f., 37, 92, 94f., 101, 121, 133, 150, 160, 190 Anm. 17, 242, 258, 263

- Erschließung (Ordnen, Verzeichnen) siehe Erschließung

- Öffentlichkeitsarbeit (Publizität, Vermittlung) 14f., 19, 25, 27f., 86f., 126, 128, 146, 151f., 161, 190-197, 216, 219, 244, 248-256, 258, 265

- Reproduktionsaufträge 150

- Skartierung (Kassation) 66, 105, 107, 110, 115, 120,130, 135, 138, 143, 156

- Taggen (elektronisches Markieren) 194-197

- Übernahme (Übergabeprotokolle, Ablieferungsverzeichnisse) $21,110,142 \mathrm{f} ., 146,149,157,208$, 259, 263

- Verlinken 194-197

- Vernetzung von Informationen 25

- Vernichtungsauftrag 23, 130

- Verwahrung (custody) 21, 32-34, 36, 90, 92 , 94f., 121

- Wissensmanagement 49

Archivmacht 227f.

Archivmanagement 28 Anm. 89, 230

Archivordnung $106 f$.

Archivpersonal (archivists, record keepers; ArchivarInnen) 9, 11-14, 16-19, 21-24, 27-29, 31-34, 44-46, 48, 51, 53-55, 63, 70-72, 86f., 92, 9497, 101, 103, 105, 107f., 110f., 113-117, 120f., 123-125, 127, 129-132, 134-136, 139, 146f., 149-151, 155, 158, 160, 164, 165 Anm. 41, 166, 169, 184-186, 196, 200f., 205f., 208, 210, 213f., 216-218, 220, 222, 225f., 229-231, 238, 240, 244, 247-253, 256-261, $263 f$.

Archivportal Europa (Archives Portal Europe) 213 Anm. 3, 219-221

Archivrecht 16

Archivsoftware 190 Anm. 17

Archivstatistik, Internationale (ISO/CD 19580) 260 Anm. 14

Archivtag siehe Koblenz, Regensburg, Trier

Archivtektonik 25f., 49, 110, 132, 157, 165 Anm. 41, 186f., 191f., 201, 203, 205-208, 212, $238 f$.

Archivterminologie 157

Archivtradition 23

Archivträger 27, 240, 250, 258, 260, $267 f$.

Archivwissenschaft (archival science, archival theory; Archivkunde, Archivistik) 10, 15 Anm. 36, 16 Anm. 38, 27f., 51, 55, 58, 63, 66, 87, 92, 117, 139, 156, 206, 212, 214, 216, 221, 225-239, 243-245

- Archivalische Strukturlehre 51, 63, 66, $239 \mathrm{f}$.

- Archivarische Berufswissenschaft 225f., 229f., 245

Archivwürdigkeit 22f., 101, 104, 109f., 115, 118, 127, 131-134, 143

Arhinet 221

Ash, Timothy Garton 59

askSam 215

Ausschuss „Digitale Archive“ 46 Anm. 36 
Ausstellungswesen 71, 250, 265

- Virtuelle Ausstellungen (Onlineausstellungen) 126

Australien 33

- National Archives of Australia 19 Anm. 54, 33, 94, 211

Auswertungsoffenheit 119

Authentizität (authenticity, integrity; Integrität) 31-34, 61, 69-71, 73f., 76-78, 86, 90-96, 114, $127,132,134,138,143,241-243,257,268$

Automationsunterstützte Auswertung 24

Baas, Kathrin 252

Baden-Württemberg 48

BarCamps (ArchivCamps, BibCamps) 251f., 254

Barnabiten siehe Wien

Barrera, Giulia 59

Bavarikon 189

Bayern 210

Bearbeitungsspuren (Vermerke) 58, 61f., 64f., 68f., 78, 101

- Marginalien 78

Bearman, David 33

Beck, Friedrich 46

Becker, Franziska 80

Becker, Irmgard Christa 226

Becker, Peter 165 Anm. 39

Benchmarking 259-261

Benediktinerinnen siehe Graz, St. Gabriel

Benutzende (user; Benutzung) 19, 23-27, 29, 33f., 37, 49f., 54, 57, 71f., 86, 94, 96, 111, 114, 117 119, 121, 126f., 131-133, 138, 141, 149-152, 156, 158, 160-163, 165f., 176, 185-188, 190 Anm. 17, 192f., 197, 199-208, 212-214, 217, 219-222, 240, 248-250, 253, 258f., 262-268

- archival intelligence 204

- Benutzerschnittstelle 202, 222

- Lernende Suche 204, 211

- Nutzerzufriedenheitsumfrage des Hessischen Landesarchivs 186

Berg, Heinrich 10

Berlin 241, 248

- Berlin-Brandenburgische Akademie der Wissenschaften 233 Anm. 39

- Geheimes Preußisches Staatsarchiv 232f.

- Institut für Archivwissenschaft und geschichtswissenschaftliche Fortbildung (IfA) 232f., 235

- Universität der Künste Berlin 241

- Vilém Flusser Archiv $241 f$.

Bern

- Schweizerisches Bundesarchiv (BAR) 125 Anm. 5, 157-160, 165 Anm. 39, 250

Bernheim, Ernst 235

Berwinkel, Holger 19f., 45, 71f.

Beschlagwortung 182

- Schlagwörter 201, 206f., 210, 212

- Schlagwortindizes 209

- Schlagwortlisten 26
Beständestruktur siehe Archivtektonik

Bestandsanalyse 128

Bestandsbildende Stelle (creators of the fonds; $\mathrm{Ar}$ chivbildner) 26, 33-34, 164, 205, 209-211, 215, 222, 237, 248, 258, 264

Bestandsbildung 58

Bestandsverdichtung 131

- Sampling 131

- Ausdünnung 131

Beweiskraft (probative capacity) 20, 90, 114, 243

Bewertungsalgorithmen siehe Algorithmen

Bewertungspläne (Bewertungsmodelle) 22, 117, 121, $137 \mathrm{f}$.

Bewertungstheorie 22f.

Bibliothekarisch-dokumentarischer Sammlungsgedanke 129, 133

Bibliotheksindex (BIX) 260

Bibliotheksstatistik, Internationale (DIN EN ISO 2789) 260

Bibliothekssystem 126, 197, 204

Bibliotheks- und Informationswissenschaft $225 \mathrm{Anm}$. 2,230

Bielsky, Wilhelm 101

Big Data $125 f$.

Bilddaten 23

Bildverarbeitung 175

Bloodaxe Archive 201-203

Bösch, Frank 43f., 54

Bologna, Marco 90

Bormann, Martin 207

Brandenburg-Preußen 58

- Brandenburgisches Landeshauptarchiv 151

Brandt, Ahasver von 60, 64, 235

Braun, Otto 232

Bregenz 141, 147

- Vorarlberger Landesarchiv 141-152

- Zisterzienserabtei Wettingen-Mehrerau, Archiv 150

Brenneke, Adolf 233-239, 241

Buchholz, Matthias 21

Büroreform 60-62, 66

Bütikofer, Nikolaus 55

Bukarest

- Romanian National Archives 221

Casanova, Eugenio 32

Charbonneau, Normand $131 \mathrm{f}$.

Città degli archivi 211

Clipper 215

Cloud 35-37

Collin, Peter 165 Anm. 39

Computerlinguistik 25, 169-184, 200, 203, 207

- Information extraction siehe IE

- Information retrieval siehe IR

Computer-Zeitalter 69

Cook, Terence Gordon (Terry) 127-129, 229

Corpus iuris civilis 31, 90 
Crowdsourcing 189, 195f., 198, 250, 252

Custodialism 33, 36

Czeike, Felix 189

\section{Dänemark 252}

Dässler, Rolf 46

Damnatio memoriae 104

Datafizierung 163, 166

Datenbanken (database; Online-Datenbanken) 46, 59, 147, 159, 163, 186, 190, 196f., 203, 215-217, 219, $265 f$.

- INFODAT (Informationsdatenbank des Wiener Gemeinderats und Landtags) 197

- Swiss Archives, Archivdatenbank 158 Anm. 16, 160, 221 Anm. 34

Datenmodellierung 138, $209 \mathrm{f}$.

Datenschutz 37, 118

Datenzentren (Data centers; Server) 34-37

Datierung 80, 82, 86, 129, 138

Dbase 3+ 215

Derrida, Jacques 227f.

Deutsche Bibliotheksstatistik (DBS) 260

Deutsche Demokratische Republik 233, 266 Anm. 27

Deutsche Digitale Bibliothek 254

Deutsche Forschungsgemeinschaft (DFG) 45

Deutschland 32, 54, 119, 147, 188, 226, 229f., 233 , 236, 252, 254, 257, 260f., 266 Anm. 27, 268

- Deutsche Nationalbibliothek 230 Anm. 23, 254

Diener-Staeckling, Antje 248 Anm. 5

Dienstleder, Alois 179

Digitale Bibliotheken 200

Digitale Hilfswissenschaften 19

Digitale Konsultation 160

Digitale Langzeitarchivierung 12 Anm. 20, 34, 93-97, 144, 147, 248

- Migrierung archivalischer Daten (migration of digital material) 21, 34, 215, 217

Digitale Registratur, siehe auch Dokumentenmanagementsysteme, 142

Digitales Archiv siehe Archiv

- AUGIAS-Archiv 147

- Digitaler Speicher (digital repository) 160

- OAIS-Modell 143

- V-DOK 143

Digitales Kulturgut 162, 199, 205

Digitales Zwischenarchiv 143

Digitale Übergabe (Digitale Übernahme) 143

Digital Humanities (Humanities Computing), Digitale Geisteswissenschaften 17, 26, 43, 158 Anm. $17,175,199-212$

Digital Immigrants 126

Digitalisierung 12-14, 21, 25, 66, 126, 131, 158160, 165, 169, 184, 210, 249, 258, 265

- OCR (Optical Character Recognition) 184, 265

Digital Natives 126, 197 Anm. 31

Dilthey, Wilhelm 234
DIN siehe Archivische Standards

Discovery portal, The National Archives (UK) 221

Distant Reading 200

Dohnal, Johanna 85f.

Dokumentationsbestand 109

Dokumentationsplan (Dokumentationsprofil, Dokumentationsziel) 118-121, 127f., 132, 240

Dokumentenmanagementsystem (DMS; Vorgangsbearbeitungssystem, Registraturmanagementsystem) 142-144

- Dokumentenmanagementsystem VOKIS 142

- Elektronische Vorgangsbearbeitungssysteme der Vorarlberger Landesverwaltung 146

- Vorgangsbearbeitungssystem V-DOK 143, 146

Dresden

- Sächsische Landes-, Staats- und Universitätsbibliothek Dresden (SLUB Dresden) 204 Anm. 14

Droysen, Johann Gustav 234f., 237, 243

Duranti, Luciana 13, 47 Anm. 46, 90

EAD 2002 (Encoded Archival Description Tag Library, Version 2002) 210 Anm. 33

Edition 57, 163 Anm. 33

EDV-gestützte Archiv- und Digitalisierungslösungen 131

EDV-gestützte Sammlungs- und Archivsysteme 131

EGAD 222

Ehrmann-Hämmerle, Christa 21

Eichmann, Adolf 68

Elektromagnetische Nachrichtentechnik $62 \mathrm{f}$.

Elektronische Datenverwaltung 206

Elektronischer Akt (ELAK; electronic records) 22, 33f., 52, 142

Elektronischer Lesesaal 59

E-Mail 63, 80, 141

Enderlin, Andreas 165 Anm. 39

England 95

Erschließung (Ordnen, Verzeichnen) 15, 17, 19, 24-26, 29, 58, 71, 94f., 103-108, 121, 126, 128, 130, 135f., 138, 144, 147, 149-152, 155-162, 164-166, 169, 180-182, 184, 194-196, 199212, 215-218, 221f., 258f., 263, 265

- Ordnen 14, 19, 24, 203, 239, 263

- Verzeichnen 24, 26, 126, 129f., 136, 149f., 157, 163, 197, 206-208, 211f., 239, 263

- Hierarchische Ordnung 205-207

Esch, Arnold 264

Ethik 14, 114, 121

Europa 36, 127, 129, 131, $220 f$.

Evidenzwert 23

Fachinformationsdienste (FID) 265

Faxnachrichten 80

Federführungsprinzip 101

Feldkirch

- Diözese Feldkirch, Archiv 151

- Vorarlberger Wirtschaftsarchiv 151 
Fernschreiber 62, 69

- Chiffrierung 62

- Lochstreifen 62

- Reinschrift 62

Findbehelfe (finding aids; Findbücher) 25, 49, 50 Anm. 59, 155, 157f., 160-165, 167, 203, 215, 259, 266 Anm. 27

- Archiv-Kataloge 163

- Beständeübersichten 49, 211

- Detailfindmittel 157-159

- Kompetenzenkartei 157-159

- Netzplan 211

- Online-Findmittel 54, 160, $163 f$.

- Rechercheführer 49, 188, 191

- Repertorien 158

Fischer, Jörg 187

Flickr, Fotoplattform 124

Flusser, Vilém 241

Foucault, Michel 227f.

Frankfurt am Main

- Institut für Stadtgeschichte 256 Anm. 36

- Max-Planck-Institut für europäische Rechtsgeschichte 165 Anm. 39

Frankreich (France) 95

Franz, Eckhart G. 156, 161, 239

Frauenfeld

- Staatsarchiv Thurgau 211

Frauenorden, Archive siehe Religionsgemeinschaften, Archive

Frauen- und Lesbenbewegung 20, 71-87

Fritsch, Ahasver 32

Gabriel, Markus 227

Gates, Bill 166 Anm. 45

Gedächtnisinstitution (Dokumentationsinstitution) 11, 49, 126-129, 134f., 162, 239, 248, 252-254

- Bibliothek 49, 126, 135, 162, 188, 199, 239, 241f., 248f., 251, 253f., 260-263, 265f., 268

- Museum 49, 126, 135, 188 Anm. 11, 190 Anm. 17, 210, 239, 248f., 253f., 260f., 268

- Virtuelles Museum 126

Gegen-Gedächtnis 16

Geisteswissenschaften 225, 227, 229-231, 234-236, $244 \mathrm{f}$.

Genabith, Josef van 173

Geschäftsgang (Entscheidungsprozess) 58, 60-67, 69, 72-74, 76, 78f., 83, 86f., 101, 107, 144, 165, 203, 206, 237f., 244

Geschichtsforschung siehe Historische Wissenschaften

Geschlechterspezifische Sprache 71

Gesellschaftlicher Diskurs 15

Gillner, Bastian 186f.

Glaubwürdigkeit (reliability, trustworthiness; Informationsgüte, Reliabilität) 14, 20, 31f., 36f., 89-97, 143, 221

Glauert, Mario 28, 251
Globočnik, Odilo 195

Google siehe Suchmaschine

Graz

- Benediktinerinnenkloster St. Gabriel, Archiv $109 f$.

- Diözese Graz-Seckau, Archiv 110 Anm. 20

- Konvent der Dominikanerinnen in der Steiermark, Archiv 110

- Steiermärkisches Landesarchiv 10, 179

Grotum, Thomas 206

Gruber, Stefanie 10

Haber, Peter 204

Hackathons (Hackdays) $253 \mathrm{f}$.

Hamburg

- Historikertag 44

Hannover

- Landesarchiv Niedersachsen 233 Anm. 39

$\mathrm{Ha}$, Martin 58f.

Hauser, Margit 87

Head, Randolph 91f.

Heigold, Georg 173

Henning, Eckart 46

Herb, Maximilian 101

Hering, Rainer 226 Anm. 6

Herzog, Peter 124

Herzogenburg 101, 105

- Stiftsarchiv 101f., 105

- Stiftsmuseum 105

Hessisches Landesarchiv 186

Heydrich, Reinhard 68

Hill, Jennie 12

Hintze, Otto 234

Historikerarchivar 102, 105, 229

Historikertag siehe Hamburg, Konstanz

Historikerverband 42 Anm. 9, 119

Historiographie 101-103, 105, 109

Historische Hilfswissenschaften (Historische Grundwissenschaften) 17-20, 41-46, 48, 51, 53f., 57f., $60,66,69,71,229-231,244$

- Aktenkunde siehe Aktenkunde

- Archivalienkunde siehe Archivalienkunde

- Diplomatik (diplomatic criticism) 67, 91f., 95, 105, 230, 233, 243

- Materialwissenschaft 43, 48?

- Paläographie 152

- Quellenkunde 46f., 52, 54, 59, 235, 238

Historische Kommission für Westfalen 47

Historische Wissenschaften (Geschichtsforschung, HistorikerInnen)

- Archival divide 17, 19, 119f., 152, 232

- Archivforschung 228

- Archivgeschichte (archival history) 51, 89-93, 103-111, 192, 233f., 237-240, 245

- Archivische Ordnungsgeschichte 191

- Behördengeschichte 73, 208, 212

- Bestandsgeschichte 101-111, 208, 212

- Digitale Geschichtswissenschaften 43 Anm. 13 
- Familienforschung 49, 141, 194, 237

- Fotografiegeschichte 131

- Genealogie 117, 214, 230

- Geschichte Sozialer Bewegungen 73

- Hausgeschichtsforschung 194

- Historische Rechtswissenschaften (historical legal studies) 91

- Kanzleigeschichte 51, 59, 69

- Konsumforschung 258f., 268

- Kulturgeschichte (historische Kulturwissenschaft) 47 Anm. 40, 67, 194 Anm. 25, 225, 227, 229f., 244

- Kunstgeschichte 131, 133

- Landeskunde (Landesgeschichte) 42, 141, 146, 188, 229

- Landschaftsdokumentationen (Natur-, Umweltdokumentationen) 133

- Neuzeitforschung (Frühe Neuzeit, Neuzeit) 18, 43, 58, 67, 101-110, 232

- Mediävistik (Mittelalter) 18, 43, 58, 67, 92, 95, 102f., 105, 139, 229, 241

- Medienarchäologie 47 Anm. 40, 139

- Mentalitätsgeschichte 67

- Numismatik 49

- Sozialgeschichte 133

- Soziologie 228

- Stadtgeschichte 133, 189-191, 193f., 197

- Technologiegeschichte 133, 139

- Verwaltungsgeschichte 60, 165, 186, 191f., 197

- Visual History 134

- Zeitgeschichte (Zeitgeschichtsforschung) 18, 20, 58-60, 62, 66, 69, 120, 130f., 231f., 235f., 248

Historizität 102

Hochedlinger, Michael 20, 67, 72 Anm. 5, 87

Hocking, John 161

Holzapfl, Julian 210 Anm. 32

H-Soz-Kult 44

ICA Code of Ethics siehe Rechtsnormen

IE (Information extraction; Informationsextraktion) 169, 176f., 179, 181-184

- Coreference resolution 179

- Extractive Summarization 184

- Named entity recognition (NER) 179-182

- Relationship extraction 179

- Terminologieextraktion 179f., 182

- TFIDF (term frequency-inverse document frequency) 180,183

Indices 164, 208f., 212, 265

InfoArh system 216

INFODAT siehe Datenbanken

Informationsanbieter 11, 13

Informationsportale (Informationsplattformen) 25, 53f., 76, 80 Anm. 22, 149, 151f., 160, 162, 186, 188f., 193f., 201-203, 206, 215f., 219, 265

- Archivportal-D 206f., 241

Informationsspeicher 11
Informationsverwalter 11

Informationsvisualisierungen siehe SuchinterfaceDesign

Informationswissenschaft 12, 230

Institut für Archivwissenschaft und geschichtswissenschaftliche Fortbildung (IfA) siehe Berlin-Dahlem

Interdisziplinarität 49, 51

International Council on Archives (ICA; Internationaler Archivrat) 114, 158, 209, 222

Internet 141, 214, 219

- semantic web 164, 210

Internet Ressourcen (internet resources; Webressourcen) 96, 205

InterPARES 1 (Preservation Task Force of the First International Research on the Preservation of Authentic Records in Electronic Systems Project) 93, 95

IR (Information retrieval; Informationsrückgewinnung) 26, 169, 176, 179, 182 Anm. 31-32, 200, 203, 205, 207, 212, 222

- Dokumenten-Clustering (document clustering) 176-178, 181, 183

- Dokumentenklassifikation (document classification) 176f., 183

- Querying 176

- Semantische Ressourcen 176

- Stemming (Lemmatisierung) 176, 181-183

ISAD $(\mathrm{G})$ siehe Archivische Standards

Italien, Stadtrepubliken 228

Jenkinson, Hilary 32, 92

Joergens, Bettina 44

John Latham Archive 201-203

Jóri, Anita 241 Anm. 70

Joyce, Patrick 27

Jus archivii (archival right) 32, 90, 92

Justinian (Kaiser) 31

Kanada 229, 264 Anm. 24, 267

Kanzlei (chancery) 63, 69, 92, 239

- Kanzleiarchiv 107

- Kanzleiordnungen 144

- Kanzleireform 20

- Kanzleistrukturen 143

- Kanzleitechnik 66

- Klosterkanzlei 101, 104f., 107

- Mittelalterliche Kanzleipraxis (medieval chancery practices) 92

Kata, Elizabeth 20

Kategorisierung (Klassifizierung, Typologisierung) 17-19, 50-52, 57, 63, 66, 83, 86, 102, 107, 205, $209 \mathrm{f}$.

KDZ - Zentrum für Verwaltungsforschung siehe Wien

Keitel, Christian 46, 50

Keller, Hagen 240

Kellerhals, Andreas 25, 125 Anm. 5 
Kemper, Joachim 28, 186

Kessels, Erik 124, 136

Keyler, Regina 50

Kininger, Kathrin 87

Kissinger, Henry 59

Kloosterhuis, Jürgen 20

Koblenz

- Archivtag 43 Anm. 9, 186, 251

- Deutsches Bundesarchiv 250

Köln 241

- Historisches Archiv der Stadt Köln 117f., 241f., 241

- LVR - Archivberatungs- und Fortbildungszentrum im Rheinland 251

- Universität 248 Anm. 5

Kolonialismus, Archive $15 f$.

Konferenz der Leiterinnen und Leiter der Archivverwaltungen des Bundes und der Länder (KLA) 46 Anm. 36, 259 Anm. 8, 261 Anm. 15

Konferenzreihe „Offene Archive“ (Nordrhein-Westfälisches Landesarchiv, Siegen, Speyer, Stuttgart) 249

Konstanz

- Historikertag $119 \mathrm{f}$.

Koselleck, Reinhart 228, 231

Kretzschmar, Robert 19, 115, 127 Anm. 11, 128f., 188 Anm. 11, 238-240, 244

Küch, Friedrich $58 f$.

Künstliche Intelligenz 204

Kulturelle Identität 21, 268

Lambach

- Stiftsarchiv $102 \mathrm{f}$.

- Stiftsbibliothek 103

Landesarchiv siehe Archiv

Landesarchiv Baden-Württemberg 19, 41f., 48, 188, 196 Anm. 28

Landesarchiv Nordrhein-Westfalen, siehe auch Konferenzreihe „Offene Archive“, 47, 50f., 66, 249

Landesgeschichtliches Informationssystem Hessen (LAGIS) 188

Lasswell-Formel 133

Latham, John 201

Leary, William H. 130-132

- UNESCO-RAMP-Studie $130 \mathrm{f}$.

Leesch, Wolfgang 233f.

Legitimität, Legitimation 74, 76, 82, 86

Leistungsfeststellung, archivische 257-268

LEO-BW (Landeskunde entdecken online - BadenWürttemberg) 41, 48f., 55, 188

Lernalgorithmen siehe Algorithmen

Lersch, Edgar 129f., 133, 135

Linz

- Oberösterreichisches Landesarchiv 10, 29

Linz am Rhein

- Stadtarchiv 250

Lokale Netzwerke (local area networks) 215
London 227

- The National Archives, UK 268

Lorenz, Sönke 54

Luhn, Hans Peter 183

Luther, Martin 104

Luzern 124 Anm. 4

Mabillon, Jean 91

Machtausübung (Politik) 15f., 32, 90, 96, 102, 115, $117,121,160,214,228$

- Informationspolitik 248

- Sammlungspolitik siehe Überlieferungsbildung

MacNeil, Heather 20f., 47 Anm. 46

Makulatur 105

Marbach am Neckar

- Deutsches Literaturarchiv 225

Marburg 230

- Archivschule 54, 225f., 229 Anm. 22, 230f., 238, 252

- Staatsarchiv 58, 266 Anm. 27

Marckhgott, Gerhart 29

Maribor 216, 219

- Regional Archives Maribor 216, 219

Markup-Sprachen

- HTML 170f.

- XML 170

- XML-Schema EAC-CPF 209

Massenschriftgut (Massenakten) 22, 47, 50-52, 60, 66, 117, 142, 149, 151, 156

- Datenmassen, digitale 123, 125f., 136, 138

- Masse digitaler Bilder 123-125, 127, 129, 136, 139

Materielle Diversifizierung 12

Mechanisierung 69

Medienbruch (Hybridakten) 12, 47f., 66, 142

Mediengesetz 78

Medienrevolution 20

Meiburg, Anette 72

Meinecke, Friedrich 234

Meisner, Heinrich Otto 20, 48, 57f., 60, 66f., 69, 72, $232 \mathrm{f}$.

Menne-Haritz, Angelika 156, 238, 240

Metadaten (metadata; Metainformationen) 22, 24 26, 49, 62, 94f., 126, 138, 143f., 163, 166, 169, 171, 180-182, 184, 200, 206, 213, 215-219, 221

Mittelalter, Archive 32, 90, 228

MOMathon 254

Monasterium, Urkundenportal 254

Moretti, Franco 200 Anm. 2

Mrazek, Hugo 179f.

München

- Deutsches Museum 254

- Generaldirektion der Staatlichen Archive Bayerns 46 Anm. 37

- Staatliche Archive Bayerns 54, $209 f$.

Mundt, Sebastian 186 Anm. 7 
Münster

- LWL-Archivamt für Westfalen 233 Anm. 39, 248 Anm. 5, 252

National Archives Foundation 256

National Archives of Australia siehe Australien

National Council on Archives, UK 263

Naumann, Kai 46

Nellen, Stefan 165 Anm. 39

Neubacher, Hermann 195

Neumann, Günter 173

Neuß, Erich 65

Neuzeit, Archiv in der 15, 32, 91, 101-110

New York

- Public Library, Archiv 211

Niederlande 252

Nordamerika, siehe auch Amerika, United States of America 229

Nordrhein-Westfalen 47, 68, 116, 147

Normdatensatz 26

Norris, Thomas D. 130

Novak, Miroslav 26

NSA-Untersuchungsausschuss des Deutschen Bundestages 59 Anm. 18

OAIS siehe Archivinformationssystem

OCR siehe Digitalisierung

Öffentliche Wahrnehmung der Archive 225, 247 256, 259, 266-268

Öffentlichkeitsarbeit siehe Archivische Tätigkeiten

Österreich 52, 55, 85, 142, 152, 268

Österreichische Bibliotheksstatistik (ÖBS) 260

opendata.swiss 163

OpenGLAM-Initiativen 254

Open Government Data (OGD) 197

Ordnen siehe Erschließung

Pachler, Amand 103

Papierakt (analoger Akt) 142

Papierzeitalter (paper technology) 57, 62, 215

Papritz, Johannes 230-233, 236, 239

Paris 230

- École nationale des chartes 230

- Französisches Staatsarchiv (Archives nationales) 32

Penz, Helga 23

Perstling, Matthias 110 Anm. 20

Pertinenz (Pertinenzprinzip) 22, 83, 138, $158 \mathrm{f}$.

Pfeiffer, Michel 23

Philipp, Landgraf von Hessen 58

Plassmann, Max 186

Pold, Erwin 10

Politik siehe Machtausübung

Polley, Rainer 67

Portalverbund 24, 26

Postcustodialism 33, 36

Potsdam 151

- Fachhochschule 229f., 252
Pragmatische Schriftlichkeit 48, 52, 67, 107, 240 f.

Prandtner, Ildephons 103

Primärquellen (primary sources) 92

Primärwert 23, 50f., 132

Provenienz (provenance; Entstehungskontext, Provenienzprinzip, respect des fonds) 17, 21-23, 25f., 33f., 49-51, 58f., 63, 82, 86, 92, 94f., 110, 114, 127-131, 134f., 138, 157f., 163, 165, 186, 191, 195, 201, 205-208, 237-239, 257

Public Library of US Diplomacy 59 Anm. 14

Public Services Quality Group (PSQG, NCA) 263

Publizität (publicity; Öffentlichkeit) 95-97, 129

Quellenbegriff 18

Quellenfunktion 20

Quellenkritik (historical -, documentary criticism) 18, 44f., 59, 68f., 71, 91f., 96, 135, 139, 166, 233, $243 \mathrm{f}$.

- Digitale Quellenkritik 139

- Medienkritik 133

Quellenkunde 17-19, 41f., 48-53, 244

- Archivalische Quellenkunde 42, 46, 48f., 53-55

Quellenwert 101f.

Ranke, Franz Leopold von 102, 130

Rechenschaft siehe accountability

Rechtsnormen

- Datenschutzgesetze (legislation on data protection) 160 Anm. 24, 213, 221, 257

- Österreichisches Denkmalschutzgesetz 21f., 103, 113

Records Management siehe Schriftgutverwaltung

Regensburg

- Archivtag 251

Registratur 58, 63, 104, 142f., 205f., 210, 237-239

Registraturgut (archives sediment, current records) 32, 51, 66, 72 Anm. 4, 89f., 95, 149, 201, 247

- Registraturordnung 107, 201

Registraturprinzip 66, 201 Anm. 6, 205, 208, 239

Rehm, Clemens $119 f$.

Reinhardt, Christian 186 Anm. 7

Reininghaus, Wilfried 49

Reliabilität siehe Glaubwürdigkeit

Religionsgemeinschaften, Archive siehe Archive

Renz, Johannes 46

Restaurierung siehe Archivische Tätigkeiten, Erhaltung

Rey, PJ 35

Rhee, Hea Lim 204

Rheinland, LVR - Archivberatungs- und Fortbildungszentrum siehe Köln

$\mathrm{RiC}$ (Records in Contexts) siehe Archivische Standards

Ringier Bildarchiv 134 Anm. 23

Rönz, Andrea 250

Roither, Monika 10

Romanian National Archives siehe Bukarest 
Rüsen, Jörn 236

sachsen.digital, Das Portal zur Geschichte, Kultur und Landeskunde Sachsens 188

Salesianerinnen siehe Wien

Salzburg

- Stiftsarchiv der Erzabtei St. Peter 104

Sammlung (Sammlungsgut) 72 Anm. 4, 113, 133-136, 240

- Mediensammlung 135

- Sammlungsbestände 109, 150

Sammlungsbezogene Technologien 136

- Web-Harvesting 136

Sammlungsstrategien 128f., 132, 136

- Sammlungssurveys 132, 138

St. Florian 105

- Augustiner-Chorherrenstift, Stiftsarchiv 105

St. Gallen 46 Anm. 31

- Staatsarchiv 46 Anm. 31

St. Pölten

- Niederösterreichisches Landesarchiv 147, 192

São Paulo 241

Sassoon, Joanna 134

Schäfer, Udo 67

Schellenberg, Theodore 23, 238

- Schellenbergsche Bewertungskriterien 101, 132

Schenk, Dietmar 28

Schiele, Johanna 10

Schlemmer, Martin 68

Schleswig

- Landesarchiv Schleswig-Holstein 226 Anm. 6

Schlotheuber, Eva 44, 54 f.

Schmidgall, Markus 22

Schmid, Gerhard 68

Schmid, Helmut 173

Schreibmaschine (typewriter) 82, 159 Anm. 19, 215

Schriftgutverwaltung (Records Management) 22, 24, 28, 46f., 51, 53, 95, 129, 143f., 146, 149, 157, 201 Anm. 5, 216, 222, 239

- Digitale Schriftgutverwaltung 144, 205

Schulze, Winfried 161

Schwarz, Karin 46

Schweiz 52, 55, 147, 159, 167, 252, 254

scopeArchiv Plattform (platform) $215 \mathrm{f}$.

Sekundäre Überlieferungsform 59

Sekundärquellen (secondary sources) 92

Sekundärwert 23, 50f., 132

Seoul 161

Seyß-Inquart, Arthur 195

Shapiro, Barbara J. 91

Shirky, Clay 205

SIARD (Software Independent Archiving of Relational Databases) 160 Anm. 23

Siegen siehe Konferenzreihe „Offene Archive“

SIRAnet 216f., 219, 221

Slowenien (Slovenia) 26, 215-217

- Archiv der Republik Slowenien (Archives of the Republic of Slovenia) 216
Smartphone-Applikationen 250, 254, 264

Social bookkeeping 93

Society of American Archivists (SAA) 264 Anm. 24

Soziale Medien (social media; Soziale Plattformen) 25, 28 Anm. 88, 136, 166, 186, 197, 248-256, 265

Sonnlechner, Christoph 25

Speyer siehe Konferenzreihe „Offene Archive“

- Stadtarchiv Speyer 196 Anm. 28

Sprachtechnologie (LT; language technology) 169-184, 199

- Dokumenten-Clustering 175, 182, 184

- Dokumentenklassifikation 175, 181f., 184

- Language Resources (Sprachressourcen) 174

- Latent Dirichlet Allocation 204

- Lemmatisierungswerkzeuge $206 f$

- Maschinelles Lernen (ML; machine learning) 169, 172-175, 177, 181f., 184, 204

- Morphologisches Tagging 173

- ML-Werkzeuge (tools) und -Bibliotheken 175, 178

- Parsing 173f., $180 f$.

- Part-of-Speech (PoS) Tagging 172f., $180 f$.

- Phrase Chunking 173, 180 f.

- Sentence Splitting 172

- Softwarebibliotheken 174

- Stuttgart-Tübingen Tagset (STTS) 172

- Tokenisierung 171f., 181, 183

- Toolsammlungen für die Digital Humanities 175

- Topic Modeling 204

- Treebank (Baumbank) 172-174

Sprachverarbeitung (NLP; natural language processing) 169-176

- Maschinelle Übersetzung 174, 176

- Spracherkennung (speech recognition) 176

Spranger, Eduard 234

Staatsarchiv siehe Archiv

Statistik, Archivische 259f., 262

Staupitz, Johannes 104

Steiger, Elisabeth 248 Anm. 5

Stichwortsuche 207, 212

Stockinger, Thomas 55, 87, 165 Anm. 39

Stoler, Ann Laura 96

Story telling 204-209, 212

Stuckart, Wilhelm 69

Sturm, Patrick 63

Stuttgart, siehe auch Konferenzreihe „Offene Archive", 55, 185, 196 Anm. 28

- Hauptstaatsarchiv Stuttgart 49

- Ministerium für Wissenschaft, Forschung und Kunst Baden-Württemberg 41

- Wirtschaftsarchiv Baden-Württemberg 53

Suchinterface-Design (Informationsvisualisierungen) 165 Anm. 41, 199, 201-203

Suchmaschine (Internetsuchmaschine) 162, 176, 192, 200f., 203-206

- Google 162, 176, 192, 201, 203, 207, 251

- Hierarchische Webverzeichnisse 205 
Süddeutscher Rundfunk, Historisches Archiv 129

Südwestdeutsche Archivalienkunde 19, 41f., 48-55

Suttner, Bertha von 169f.

Technischer Turn 166

Telegrafie 63

Textverarbeitung 170-172, 199

Theimer, Kate 185

Thermopapier 80

Thibodeau, Kenneth 34

Thorburn, David 89

Tomasi di Lampedusa, Giuseppe 167

Torres, Deborah A. 204

Transparenz siehe accountability

Trier

- Archivtag 49

Tripp, Sebastian 252

Troeltsch, Ernst 234

Trusted digital repositories (TDR) 34f., 95

Tübingen 54

- Institut für Geschichtliche Landeskunde und Historische Hilfswissenschaften, Universität Tübingen 48, $53 \mathrm{f}$.

- Universität 42 Anm. 7, 48

Turbo Pascal 215

Türlemann, Felix 124 Anm. 4

Überlieferungsbildung 21-23, 45, 52f., 103-111, 113-121, 127, 129, 131, 134, 138, 142, 151, 156, 240, 262, 264

- Digitale Überlieferungsbildung $142 \mathrm{f}$.

- Sammlungspolitik 128f., 133, 240

- Visuelle Überlieferungsbildung 128, 130, 134

- Visuelle Überlieferungsstrategien 134

Überlieferungsform (Medium, Überlieferungsträger) 61-63, 69f., 77f., 80, 82, 93, 124, 132, 159 Anm. $19,242,264$

- Zwischenmaterial $62 \mathrm{f}$.

Umweltpapier 80

United States of America (USA), siehe auch Amerika, Nordamerika, 130, 236, 264 Anm. 24, 267

Universal Declaration on Archives 214

Urheberrecht 136

U. S. Department of State 59

Vanderbilt, Paul 129

Verband der Historiker und Historikerinnen Deutschlands (VHD) 44f., 55, 57

Verband deutscher Archivarinnen und Archivare (VdA) 120, 251, 253

Verband Österreichischer Archivarinnen und Archivare (VÖA) 10, 150, 152

Verein Schweizerischer Archivarinnen und Archivare (VSA-AAS) 253 Anm. 27

Verwaltungsschriftgut (business records) 20, 22, 28, $32,47,51,53,60-69,73,80,91,142-144,149$, 237-239, 244, 265
- Elektronische Bürokommunikation 62

- Elektronischer Verwaltungsakt 141

- Papierloses Büro 142

- Verwaltungsschriftgut von Klöstern 101, 103, 105-108

Verzeichnen siehe Erschließung

Virtueller Lesesaal 160, 187, 249 Anm. 8

Visual Turn (Ikonische Wende) 134

Visuelle Archivkultur 135

Vogeler, Georg 26

Vogt-O’Connor, Diane 132f., 138

Vollarchivierung (Totalüberlieferung) 116, 156

Volltextsuche 201, 203f., 207, 212

Vorarlberg 141f., 146, $150 \mathrm{f}$.

Vorarlberger Landesarchiv siehe auch Bregenz

- Agenda „Vorarlberger Landesarchiv 2015“ 144

- Projekt „Digitales Langzeitarchiv Vorarlberg“ 144-147

- Projekt „Vorarlberger Archivinformationssystem (AIS)“ 146f., 152

WAIS siehe Archivinformationssystem

Wannseekonferenz 68

Washington 59

- Amerikanisches Nationalarchiv (US National Archives and Records Administration, NARA) 59

- Library of Congress (USA) 132

Weber, Hartmut 260

Weber, Max $92 \mathrm{f}$.

Weinberger, David 96

Wert von Archivgut 23, 116-118, 129-134, 267

Whitelaw, Mitchell 211

Wien 53, 80, 86 Anm. 27, 102, 150, 167, 190-195, 226 Anm. 5, 230, 268

- Abteilung für Automationsunterstützte Datenverarbeitung, Informations- und Kommunikationstechnologie der Stadt Wien (MA 14) 189

- Gasometer 268

- Institut für Österreichische Geschichtsforschung 10, 16, 53 Anm. 70, 87, 165 Anm. 39, 230, 232

- KDZ - Zentrum für Verwaltungsforschung 189

- Kloster von der Heimsuchung Mariens, Archiv (Salesianerinnen in Wien) 107-109

- Kolleg der Regularkleriker vom hl. Paulus, Archiv (Wiener Barnabitenarchiv) 107

- Kulturabteilung der Stadt Wien (MA 7) 10, 194 Anm. 25

- Museen der Stadt Wien 194 Anm. 25

- Österreichisches Staatsarchiv 150, 192, 250

- Provinzarchiv der Salvatorianer 107

- STICHWORT, Archiv der Frauen- und Lesbenbewegung 71, 73, 86

- Universität Wien 80, 87

- Wien Bibliothek im Rathaus 189, 191, 194

- Wiener Stadt- und Landesarchiv 10, 25, 188-195, 268

- Wien Museum 189 
Wien Geschichte Wiki 25f., 167, 185, 189-198

Wien Kulturgut, Digitaler Kulturstadtplan 193f., 197

Wiesflecker, Peter 109 Anm. 18

WikiLeaks $58 f$.

Wikimedia 254

Wikipedia 265

Wiki-Technologie 189

- Wiki-Layer 197

- Semantic Media Wiki, Open-Source-Software $189 f$.

Wilhelm II., deutscher Kaiser 60

Wilhelmine Amalia (von Braunschweig-Lüneburg), Kaiserin 109

Williams, Bernard 243

Wirtschaftlichkeit (Finanzierung) 258-262, 265-267

Wolff, Fritz 231

Wurthmann, Nicola 44

Yakel, Elizabeth 204

Yeo, Geoffrey 97, 203, 206

Zaremba, Andrea 87

Zielinski, Siegfried 241 Anm. 69

Zugänglichkeit (availability, usability; Verfügbarkeit, Nutzbarkeit) 15, 29, 31f., 36f., 44, 49, 52, 54 , 58f., 92, 95f., 102, 121, 126f., 131, 135, 143, $147,150,152,160-166,185-188,192-197$. 213, 240, 254, 257, 263-266

- Digitale Verfügbarkeit 166, 186-188, 254

- Freedom of Information Act 59

- Informationsfreiheitsgesetze 121

- Linked Open Data (LOD) 163f., 166

- Online-Nutzbarkeit (accessible for online use) 162f., 185f., 191-197, 213, 250, 263-265

- Online-Präsenz (Webpages) 186, 250-253, 265

Zürich

- ETH (Eidgenössische Technische Hochschule) Zürich, Bibliothek 196

- ETH-Forschungszentrum „Big Data“125 Anm. 6

- Fotoarchiv der Swissair 196

- Museum Rietberg 126 Anm. 8 